\title{
Desymmetrization of 1,3-Diones by Catalytic Enantioselective Condensation with Hydrazine
}

Binmiao Yang, Jun Dai, Yixin Luo, Kai Kiat Lau, Yu Lan,* Zhihui Shao,* and Yu Zhao*

\section{Supporting Information}

\section{Contents}

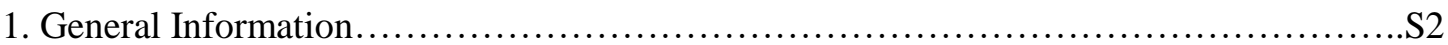

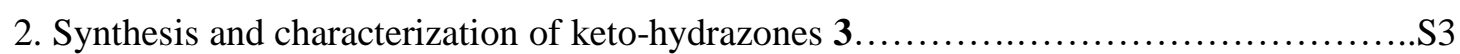

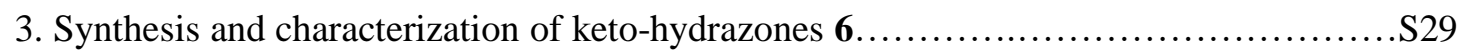

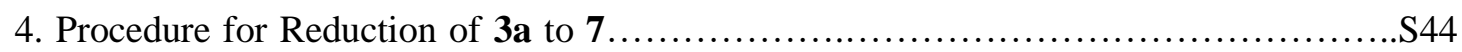

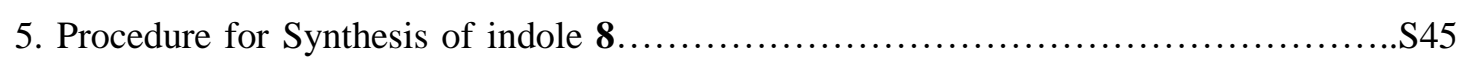

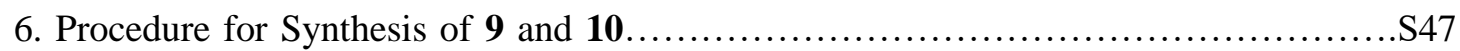

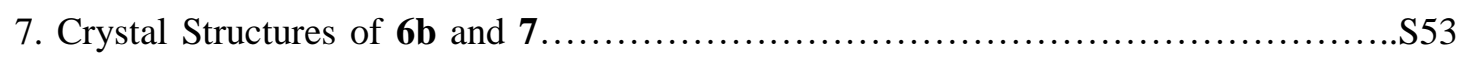

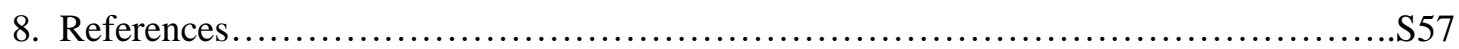

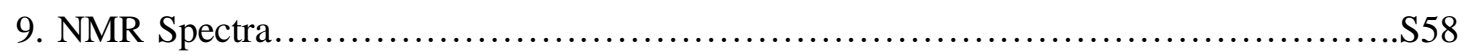

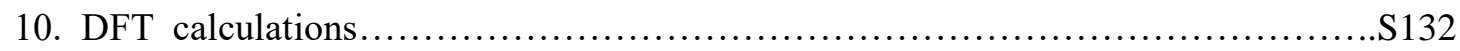


1. General information. ${ }^{1} \mathbf{H}$ and ${ }^{13} \mathbf{C}$ NMR spectra were recorded on a Bruker AMX500 $(500 \mathrm{MHz})$ spectrometer or BrukerAM $400(400 \mathrm{MHz})$ spectrometer. Chemical shifts were reported in parts per million (ppm), and the residual solvent peak was used as an internal reference: ${ }^{1} \mathrm{H}$ (chloroform $\delta$ 7.26; DMSO $\delta 2.50$; acetone- $d_{6} \delta 2.05$ ), ${ }^{13} \mathrm{C}$ (chloroform $\delta 77.0$; DMSO $\delta 39.5$; acetone- $\left.d_{6} \delta 205.87,30.60\right)$. Data are reported as follows: chemical shift, multiplicity ( $\mathrm{s}=$ singlet, $\mathrm{d}=$ doublet, $\mathrm{t}=$ triplet, $\mathrm{q}=$ quartet, $\mathrm{m}=$ multiplet, $\mathrm{br}=$ broad), coupling constants (Hz) and integration. Melting point (MP) was obtained on Buchi B-540. For thin layer chromatography (TLC), Merck pre-coated TLC plates (Merck 60 F254) were used, and compounds were visualized with a UV light at $254 \mathrm{~nm}$. High resolution mass spectra (HRMS) were obtained on a Finnigan/MAT 95XL-T spectrometer. Optical rotations were recorded on an mrc AP81 automatic polarimeter. Enantiomeric excesses (ee) were determined by HPLC analysis on Agilent HPLC units, including the following instruments: pump, LC-20AD; detector, SPD-20A; column, Chiralcel IB, IC, IE. AD-H, OD-H or AS-H.

All reactions were carried out under nitrogen atmosphere. All commercially available reagents listed below were used as received for the reactions without any purification. Phenylhydrazine, (bromomethyl)benzene, 1-(bromomethyl)-4-methylbenzene, 2-(bromomethyl)naphthalene, 1-(bromomethyl)-2-methylbenzene, 1-(bromomethyl)-3-methylbenzene, 1-(bromomethyl)-3-chlorobenzene, 1-(bromomethyl)-2-methoxybenzene, 1-(bromomethyl)-4-nitrobenzene, 1-(bromomethyl)-4-chlorobenzene, 2-methylcyclopentane-1,3-dione, 2-methylcyclohexane-1,3-dione, 3-methylpentane-2,4-dione, 4-methylheptane-3,5-dione, $\mathrm{NaBH}_{4}$. Liquid reagents were handled with a micropipette. THF, toluene, $\mathrm{CHCl}_{3}$ and $\mathrm{MeCN}$ were dried on alumina columns using a solvent dispensing system. 1,3-Diones $\mathbf{1}$ and $\mathbf{5}$ were synthesized following the reported procedure. ${ }^{1}$ Hydrazine $\mathbf{2}$ was synthesized by the reported procedure. $^{2}$ 


\section{Synthesis and characterization of keto-hydrazones 3.}

\subsection{Optimization studies}

The initial screening of various hydrazines were conducted with 1.3-dione 1a in the presence of CPA1 and $5 \AA$ MS in MeCN at $23{ }^{\circ} \mathrm{C}$ for $24 \mathrm{~h}$. Hydrazine 2a (R = 1-naphthyl) worked out as the optimal with $>98 \%$ conversion and $85 \%$ ee.

\section{Scheme S1. Screening of hydrazines $2^{\mathrm{a}}$}<smiles>CC1(Cc2ccccc2)C(=O)CCC1=O</smiles>

$1 \mathrm{a}$

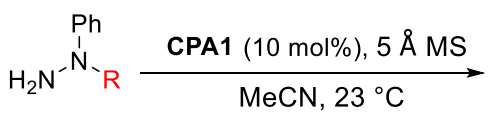

2

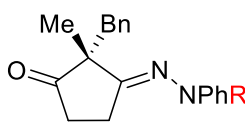

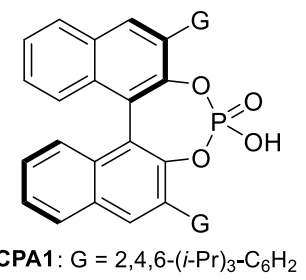

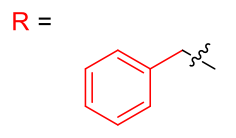

$90 \%$ conv., $50 \%$ ee<smiles>CC(C)(C)Cc1ccccc1F</smiles>

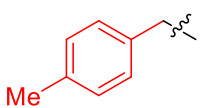

$40 \%$ conv., $45 \%$ ee

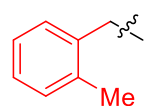

$>98 \%$ conv., $47 \%$ ee

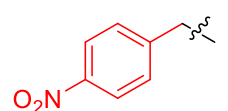

$35 \%$ conv., $49 \%$ ee

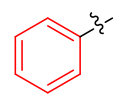

$>98 \%$ conv., $74 \%$ ee

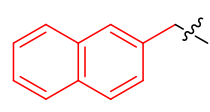

$40 \%$ conv., $50 \%$ ee

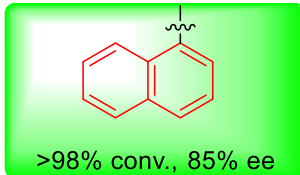

aReaction conditions: 1a $(0.12 \mathrm{mmol}), \mathbf{2}(0.1 \mathrm{mmol}), \mathbf{C P A 1}(10 \mathrm{~mol} \%)$ and $5 \AA \mathrm{MS}(40 \mathrm{mg})$ in MeCN $(1.0 \mathrm{~mL})$ at $23^{\circ} \mathrm{C}$ for $24 \mathrm{~h}$. Conversion determined by crude ${ }^{1} \mathrm{H}$ NMR. Enantiomeric excess (ee) was determined by HPLC analysis using a chiral stationary phase. 


\subsection{Reaction condition optimization}

Table S1. Optimization of Desymmetrization of $1 a^{a}$

\begin{tabular}{|c|c|c|c|c|c|c|}
\hline entry & CPA & solvent & MS & yield $(\%)^{\mathrm{b}}$ & ee $(\%)^{\mathrm{c}}$ & $\begin{array}{c}\text { Yield of } \\
4(\%)^{d}\end{array}$ \\
\hline 1 & CPA1 & toluene & $4 \AA$ & 83 & 56 & 6 \\
\hline 2 & CPA1 & $\mathrm{CHCl}_{3}$ & $4 \AA$ & 81 & 47 & 8 \\
\hline 3 & CPA1 & c-hexane & $4 \AA$ & 87 & 41 & trace \\
\hline 4 & CPA1 & THF & $4 \AA$ & 80 & 44 & 6 \\
\hline 5 & CPA1 & toluene & $3 \AA$ & $86^{f}$ & 64 & trace \\
\hline 6 & CPA1 & toluene & $5 \AA$ & 84 & 83 & 5 \\
\hline 7 & CPA2 & toluene & $5 \AA$ & 67 & 14 & trace \\
\hline 8 & $\mathrm{CPA}_{3}$ & toluene & $5 \AA$ & 95 & 85 & trace \\
\hline 9 & $\mathrm{CPA}_{4}$ & toluene & $5 \AA$ & 53 & 30 & trace \\
\hline 10 & $\mathrm{CPA}_{5}$ & toluene & $5 \AA$ & trace & - & trace \\
\hline 11 & CPA6 & toluene & $5 \AA$ & 57 & 14 & 5 \\
\hline 12 & $\mathrm{CPA}_{7}$ & toluene & $5 \AA$ & 72 & 39 & trace \\
\hline 13 & CPA8 & toluene & $5 \AA$ & 67 & 17 & trace \\
\hline $14^{e}$ & $\mathrm{CPA}_{3}$ & $\mathrm{MeCN}$ & $5 \AA$ & 87 & 96 & 5 \\
\hline $15^{f}$ & $\mathrm{CPA}_{3}$ & $\mathrm{MeCN}$ & $5 \AA$ & Trace & - & trace \\
\hline
\end{tabular}
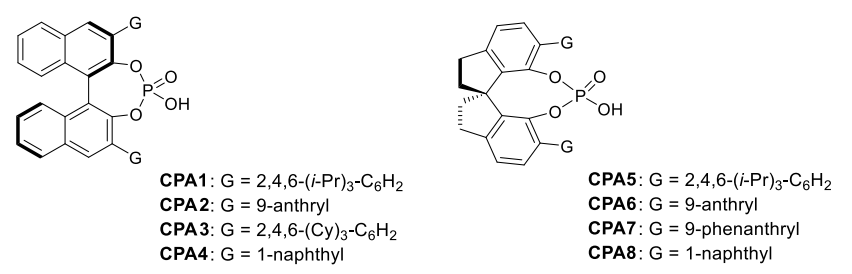

${ }^{a}$ Reaction conditions: $1 \mathbf{a}(0.12 \mathrm{mmol}), \mathbf{2 a}(0.10 \mathrm{mmol}), \mathbf{C P A}(10 \mathrm{~mol} \%)$ and MS (40 mg) in toluene (1.0 mL) at $23{ }^{\circ} \mathrm{C}$ for 24 h. ${ }^{b}$ Isolated yield. ${ }^{c}$ Determined by HPLC analysis using a chiral stationary phase. ${ }^{d} \mathrm{NMR}$ yield ${ }^{e} 72 \mathrm{~h}$. $f$ Performed at $\mathrm{o}$ ${ }^{\circ} \mathrm{C}$ 


\subsection{Test of dependence of ee product on ee of CPA catalyst}

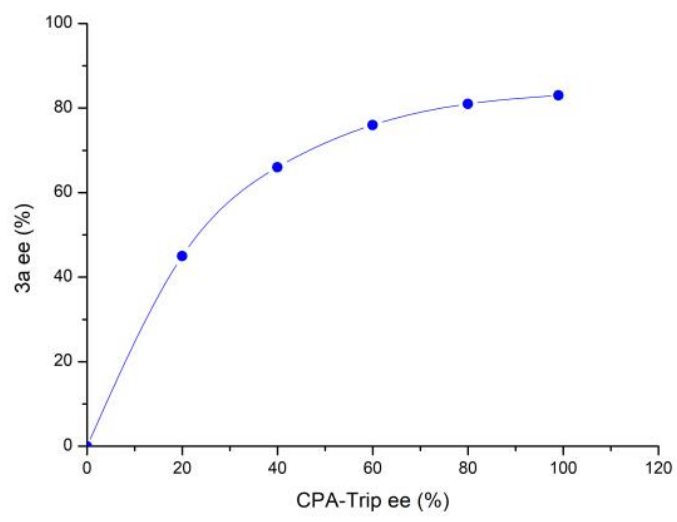

A positive non-linear effect was observed for the reaction of $\mathbf{3 a}$ formation from $\mathbf{1}$ and $\mathbf{2 a}$ under standard conditions as shown above. At this point the origin of this observation is not clear. While it is possible that a heterodimer of the acid catalyst might lead to this positive non-linear effect, we could not observe any precipitate due to the small scale reaction as well as the use of molecular sieves. A selective complexation of the product with one enantiomer of the acid catalyst could be another reason for this effect as well.

\subsection{General procedure for the synthesis of cyclic keto-hydrazone 3}

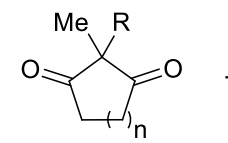<smiles>NN(P)[Te]O[Na]</smiles>

2a

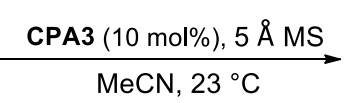

To a $4 \mathrm{~mL}$ vial was added the 1,3-dione $1(0.12 \mathrm{mmol})$, hydrazine $2 \mathrm{a}(0.10 \mathrm{mmol}), 5 \AA \mathrm{MS}$ (40 mg) and CPA3 $(0.01 \mathrm{mmol})$ under argon atmosphere. MeCN $(1.0 \mathrm{~mL})$ was then added. The reaction mixture was allowed to stir at $23{ }^{\circ} \mathrm{C}$ for $72 \mathrm{~h}$ (the reactions to prepare $\mathbf{3 m}-\mathbf{3 p}$ were performed at $-20{ }^{\circ} \mathrm{C}$ using toluene as the solvent). The crude reaction mixture was directly purified by silica gel column chromatography with hexanes/ethyl acetate $(20: 1 \mathrm{v} / \mathrm{v})$ as eluent to afford product $\mathbf{3}$ in pure form.

Note: the preparation of the corresponding racemic products for HPLC analysis used $20 \mathrm{~mol} \%$ $\mathrm{TsOH}$ as the catalyst. 


\subsection{Characterization of 3}

$(R, E)$-2-benzyl-2-methyl-3-(2-(naphthalen-1-yl)-2-phenylhydrazono)cyclopentanone (3a)

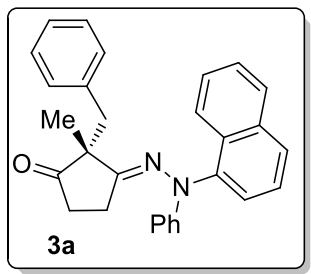

Colorless oil ( $36.3 \mathrm{mg}, 87 \%$ yield).

${ }^{1} \mathbf{H}$ NMR $\left(500 \mathrm{MHz}, \mathrm{CDCl}_{3}\right): \delta 7.91(\mathrm{~d}, J=9.0 \mathrm{~Hz}, 2 \mathrm{H}), 7.84(\mathrm{~d}, J=8.5 \mathrm{~Hz}, 1 \mathrm{H}), 7.52-7.42$ (m, 2H), 7.45-7.39 (m, 2H), 7.22-7.19 (m, 2H), 7.15-7.09 (m, 3H), 7.00 (dd, $J=7.5 \mathrm{~Hz}, J=$ $1.5 \mathrm{~Hz}, 2 \mathrm{H}), 6.94-6.89(\mathrm{~m}, 3 \mathrm{H}), 3.14(\mathrm{~d}, J=13.0 \mathrm{~Hz}, 1 \mathrm{H}), 3.09(\mathrm{~d}, J=13.0 \mathrm{~Hz}, 1 \mathrm{H})$, 2.09-2.02 (m, 1H), 1.90-1.83 (m, 1H), 1.63-1.55 (m, 2H), $1.46(\mathrm{~s}, 3 \mathrm{H}), 1.44-1.36(\mathrm{~m}, 1 \mathrm{H})$.

${ }^{13}$ C NMR $\left(125 \mathrm{MHz}, \mathrm{CDCl}_{3}\right): \delta 219.6,166.3,150.1,142.4,137.1,134.6,130.9,130.7,128.7$, $128.5,128.2,127.7,126.8,126.7,126.5,126.3,126.0$, 124.2, 120.7, 115.8, 56.4, 46.0, 36.7, 27.2, 23.5.

IR $v\left(\mathrm{~cm}^{-1}\right): 3480.27,3059.26,2964.84,2924.66,1973.48,1744.66,1594.53,1491.10$, $1452.07,1287.65,1215.83,777.54,753.55,702.03$.

HRMS (ESI): $\mathrm{m} / \mathrm{z}$ Calcd for $\left[\mathrm{C}_{29} \mathrm{H}_{27} \mathrm{~N}_{2} \mathrm{O}, \mathrm{M}+\mathrm{H}\right]^{+}: 419.2118$; Found: 419.2125 .

Optical Rotation: $[\alpha]_{\mathrm{D}}^{25}=78.4\left(0.9, \mathrm{CHCl}_{3}\right) .96 \%$ ee. The absolute configuration of 3a was assigned by conversion to 7 followed by single crystal X-ray analysis. (HPLC condition: Chiralpak IB column, $n$-hexane $/ i$ - $\mathrm{PrOH}=90: 10$, flow rate $=1.0 \mathrm{~mL} / \mathrm{min}$, wavelength $=254$ $\mathrm{nm}, \mathrm{t}_{\mathrm{R}}=5.11 \mathrm{~min}$ for major isomer, $\mathrm{t}_{\mathrm{R}}=6.10 \mathrm{~min}$ for minor isomer).

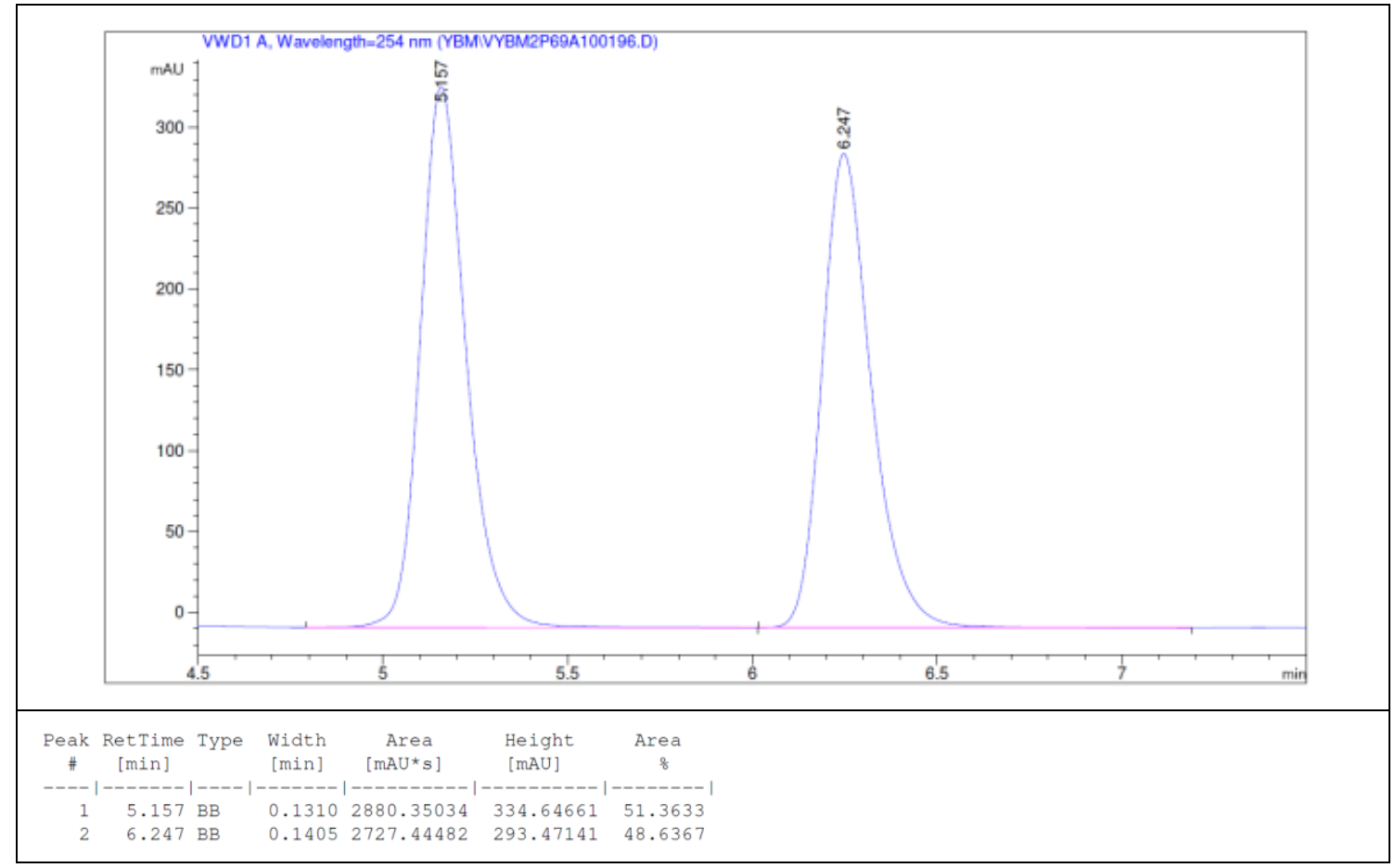




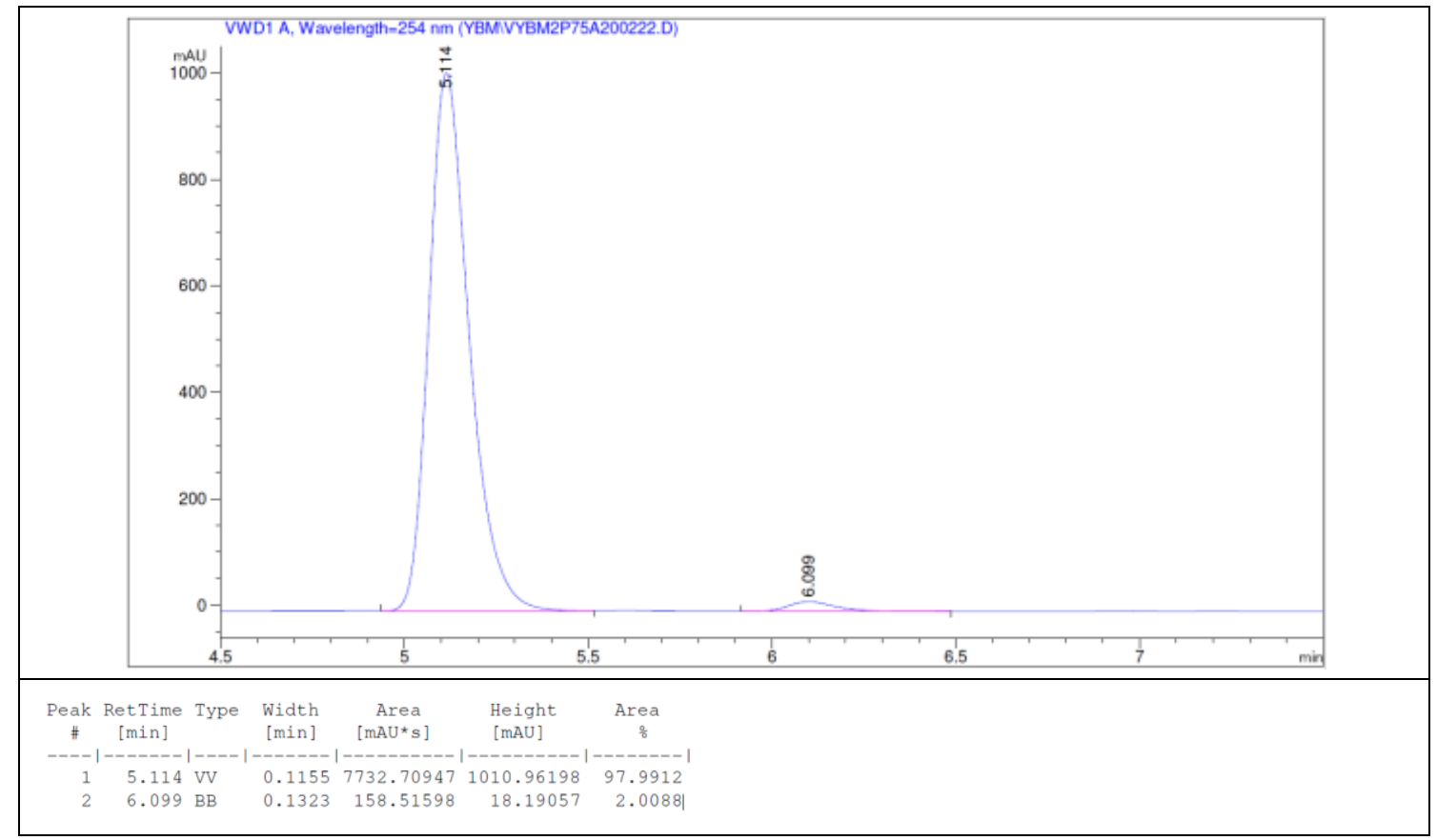

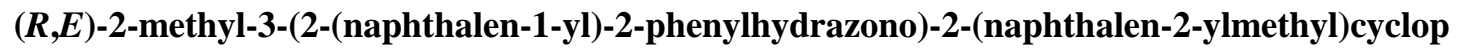
entanone (3b)

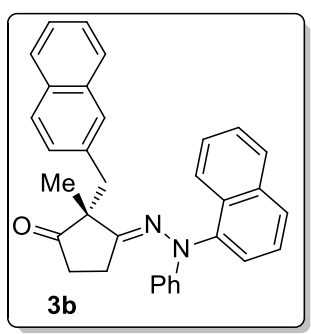

Colorless oil (33.2 $\mathrm{mg}, 71 \%$ yield).

${ }^{1} \mathbf{H}$ NMR $\left(500 \mathrm{MHz}, \mathrm{CDCl}_{3}\right): \delta 7.88(\mathrm{~d}, J=9.0 \mathrm{~Hz}, 2 \mathrm{H}), 7.78-7.76(\mathrm{~m}, 2 \mathrm{H}), 7.69(\mathrm{~d}, J=7.0 \mathrm{~Hz}$, $1 \mathrm{H}), 7.60(\mathrm{~d}, J=8.0 \mathrm{~Hz}, 1 \mathrm{H}), 7.54(\mathrm{~s} 1 \mathrm{H}), 7.49-7.42(\mathrm{~m}, 3 \mathrm{H}), 7.39-7.34(\mathrm{~m}, 2 \mathrm{H}), 7.28(\mathrm{t}, J=8.0$ $\mathrm{Hz}, 1 \mathrm{H}), 7.20(\mathrm{t}, J=8.0 \mathrm{~Hz}, 2 \mathrm{H}), 7.15(\mathrm{~d}, J=8.0 \mathrm{~Hz}, 1 \mathrm{H}), 6.95-6.89(\mathrm{~m}, 3 \mathrm{H}), 3.31(\mathrm{~s}, 2 \mathrm{H})$, 2.10-2.03 (m, 1H), 1.86-1.78 (m, 1H), 1.64-1.57 (m, 2H), $1.51(\mathrm{~s}, 3 \mathrm{H}), 1.42-1.35(\mathrm{~m}, 1 \mathrm{H})$.

${ }^{13} \mathrm{C}$ NMR $\left(125 \mathrm{MHz}, \mathrm{CDCl}_{3}\right): \delta$ 219.7, 166.0, 150.0, 142.4, 134.9, 134.6, 133.2, 132.2, 130.8, $128.7,128.5,127.9,127.8,127.7,127.6,127.5,126.8,126.5,126.0,125.9,125.6,124.2,120.8$, $115.9,56.5,45.9,36.8,27.2,23.9$.

IR $v\left(\mathrm{~cm}^{-1}\right): 3468.17,2961.88,2924.42,2854.13,1724.09,1594.46,1490.57,1451.82,1261.19$, 1031.94, 800.85, 745.71, 704.40.

HRMS (ESI): m/z Calcd for $\left[\mathrm{C}_{33} \mathrm{H}_{29} \mathrm{~N}_{2} \mathrm{O}, \mathrm{M}+\mathrm{H}\right]^{+}: 469.2274$; Found: 469.2282.

Optical Rotation: $[\alpha]_{\mathrm{D}}^{25}=49.3\left(0.6, \mathrm{CHCl}_{3}\right) .95 \%$ ee. The absolute configuration of the product was assigned by analogy to 3a. (HPLC condition: Chiralpak IB column, $n$-hexane/ $i$-PrOH $=90: 10$, flow rate $=1.0 \mathrm{~mL} / \mathrm{min}$, wavelength $=254 \mathrm{~nm}, \mathrm{t}_{\mathrm{R}}=8.54 \mathrm{~min}$ for major isomer, $\mathrm{t}_{\mathrm{R}}=8.39 \min$ for minor isomer). 


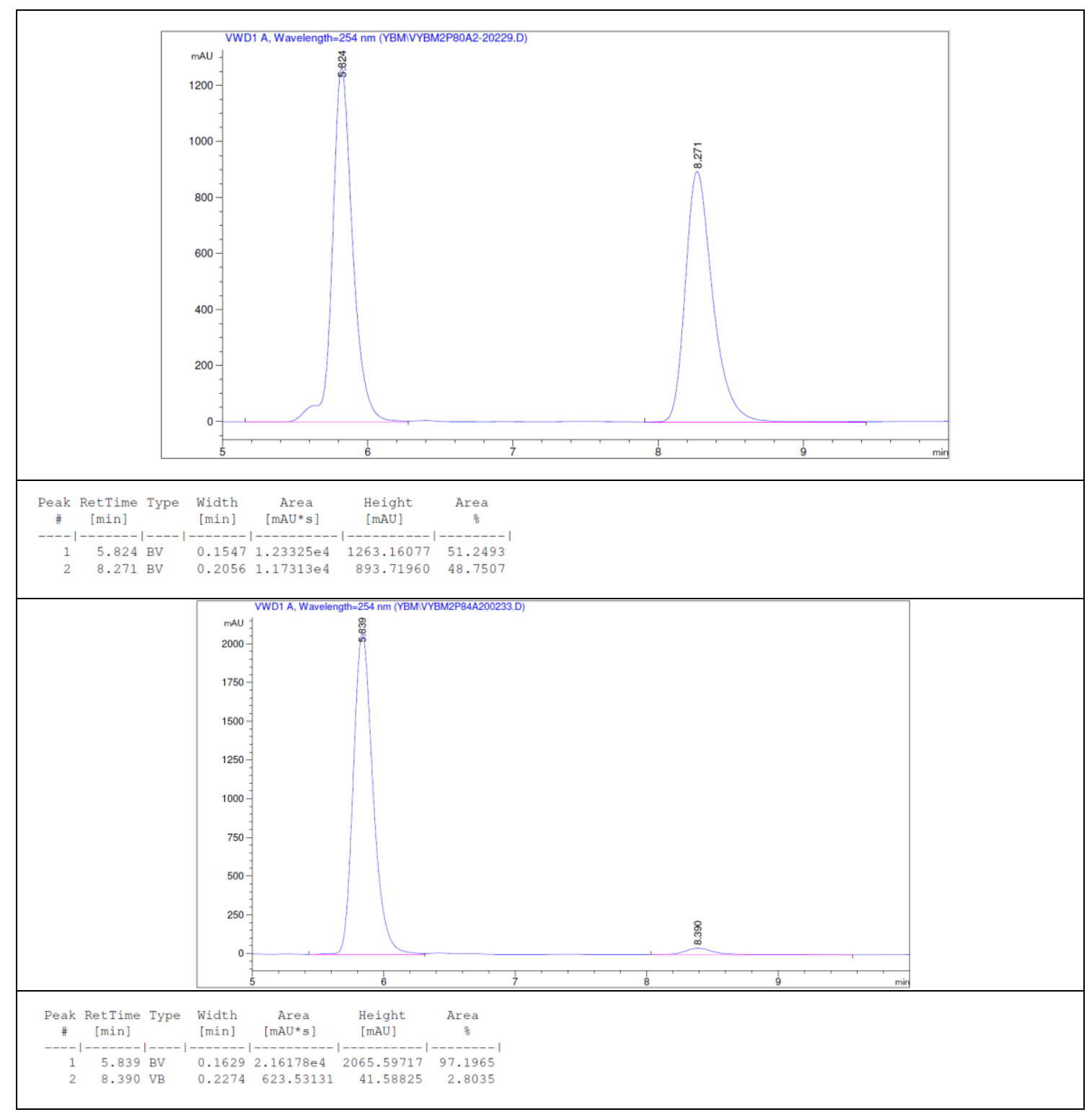

(R,E)-2-methyl-2-(2-methylbenzyl)-3-(2-(naphthalen-1-yl)-2-phenylhydrazono)cyclopentano ne (3c)

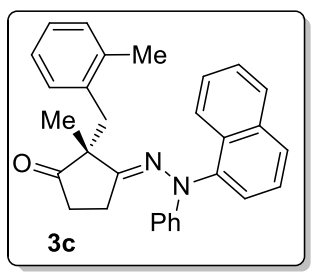

Colorless oil (32.4 mg, $75 \%$ yield).

${ }^{1} \mathbf{H}$ NMR $\left(500 \mathrm{MHz}, \mathrm{CDCl}_{3}\right): \delta 7.94(\mathrm{~d}, J=8.5 \mathrm{~Hz}, 2 \mathrm{H}), 7.86(\mathrm{~d}, J=8.5 \mathrm{~Hz}, 1 \mathrm{H})$, 7.55-7.44 (m, $3 \mathrm{H}), 7.31-7.29(\mathrm{~m}, 1 \mathrm{H}), 7.21(\mathrm{t}, J=8.0 \mathrm{~Hz}, 2 \mathrm{H}), 7.14-7.08(\mathrm{~m}, 2 \mathrm{H}), 6.95-6.92(\mathrm{~m}, 3 \mathrm{H}), 6.88(\mathrm{~d}, J$ $=8.5 \mathrm{~Hz}, 2 \mathrm{H}), 3.24(\mathrm{~d}, J=13.5 \mathrm{~Hz}, 1 \mathrm{H}), 3.20(\mathrm{~d}, J=13.5 \mathrm{~Hz}, 1 \mathrm{H}), 2.32(\mathrm{~s}, 3 \mathrm{H}), 2.14-2.07(\mathrm{~m}$, 1H), 1.90-1.80 (m, 1H), 1.78-1.70 (m, 1H), 1.66-1.59 (m, 1H), 1.50 (s, 3H).

${ }^{13} \mathrm{C}$ NMR $\left(125 \mathrm{MHz}, \mathrm{CDCl}_{3}\right): \delta$ 219.4, 165.5, 150.0, 142.3, 136.9, 135.8, 134.6, 130.9, 130.6, 129.3 , 128.6, 128.5, 127.6, 126.8, 126.7, 126.5, 126.0, 125.6, 124.2, 120.6, 115.8, 56.1 , 41.5, 36.4, 
27.2, 23.7, 20.1.

IR $v\left(\mathrm{~cm}^{-1}\right): 3475.17,3056.29,2962.50,2924.63,2854.07,1975.29,1743.77,1594.44,1490.73$, 1452.38, 1391.80, 1261.22, 1018.46, 800.76, 752.37, 691.67 .

HRMS (ESI): $\mathrm{m} / \mathrm{z}$ Calcd for $\left[\mathrm{C}_{30} \mathrm{H}_{29} \mathrm{~N}_{2} \mathrm{O}, \mathrm{M}+\mathrm{H}\right]^{+}:$: 433.2274; Found: 433.2282.

Optical Rotation: $[\alpha]_{\mathrm{D}}^{25}=85.5\left(0.5, \mathrm{CHCl}_{3}\right) .97 \%$ ee. The absolute configuration of the product was assigned by analogy to 3a. (HPLC condition: Chiralpak IB column, $n$-hexane/ $i$-PrOH $=90: 10$, flow rate $=1.0 \mathrm{~mL} / \mathrm{min}$, wavelength $=254 \mathrm{~nm}, \mathrm{t}_{\mathrm{R}}=5.03 \mathrm{~min}$ for major isomer, $\mathrm{t}_{\mathrm{R}}=5.77 \mathrm{~min}$ for minor isomer).

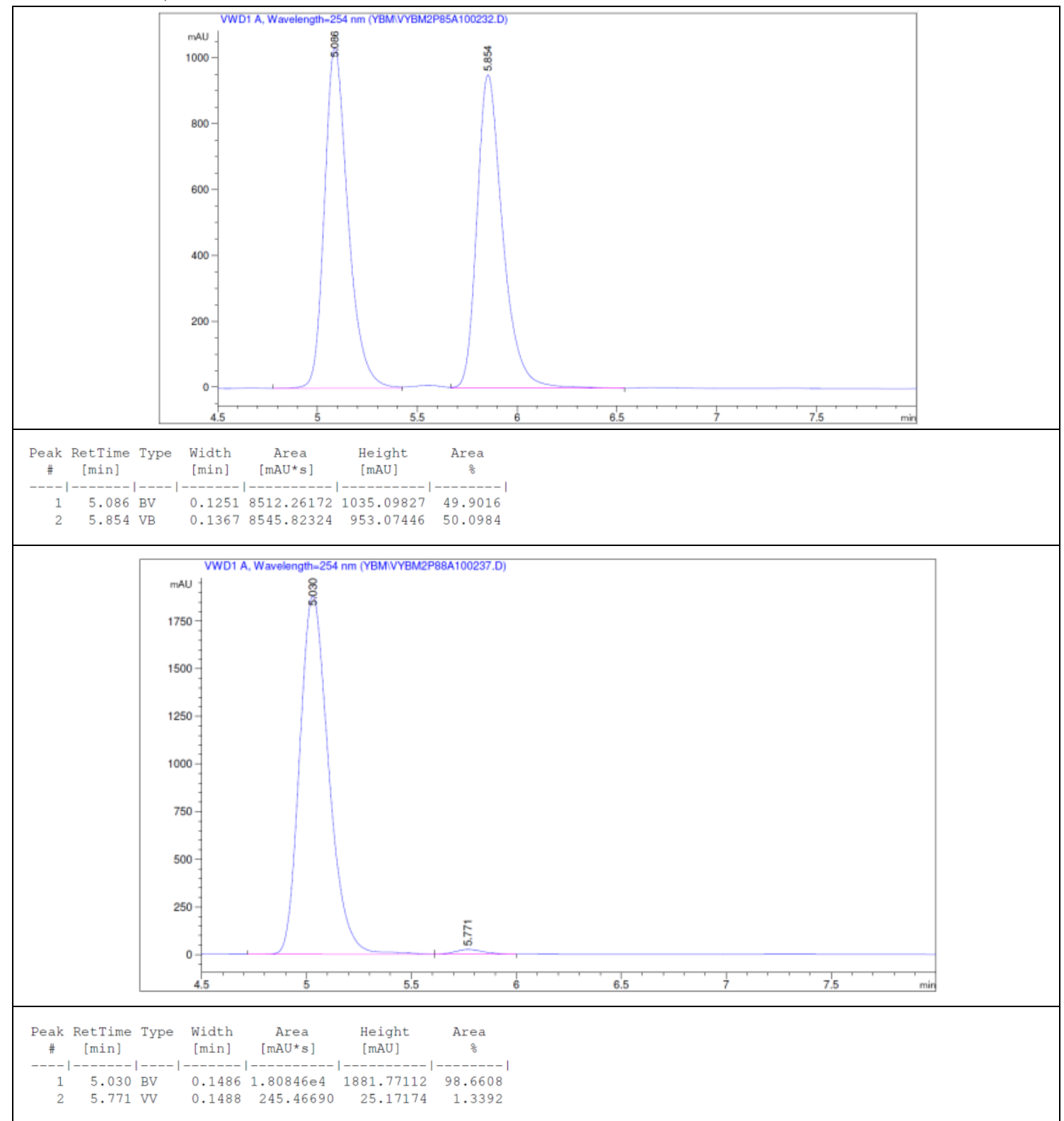

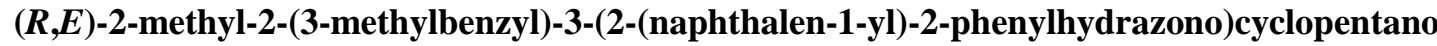
ne (3d) 


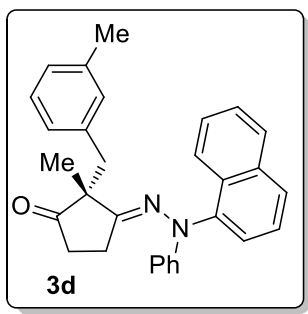

Colorless oil (30.7 mg, $71 \%$ yield).

${ }^{1} \mathbf{H}$ NMR (500 MHz, $\left.\mathrm{CDCl}_{3}\right): \delta 7.92(\mathrm{dd}, J=8.5 \mathrm{~Hz}, J=3.5 \mathrm{~Hz}, J=2 \mathrm{H}), 7.84(\mathrm{~d}, J=8.0 \mathrm{~Hz}, 1 \mathrm{H})$, 7.52-7.48 (m, 2H), 7.44-7.39 (m, 2H), $7.21(\mathrm{t}, J=8.0 \mathrm{~Hz}, 2 \mathrm{H}), 7.02$ (t $J=9.0 \mathrm{~Hz}, 1 \mathrm{H}), 6.97-6.87$ $(\mathrm{m}, 5 \mathrm{H}), 6.82(\mathrm{~d}, J=7.0 \mathrm{~Hz}, 1 \mathrm{H}), 3.13(\mathrm{~d}, J=7.5 \mathrm{~Hz}, 1 \mathrm{H}), 3.07(\mathrm{~d}, J=7.5 \mathrm{~Hz}, 1 \mathrm{H}), 2.23(\mathrm{~s}, 3 \mathrm{H})$, 2.10-2.03 (m, 1H), 1.90-1.84 (m, 1H), 1.66-1.58 (m, 1H), 1.51-1.44 (m, 4H).

${ }^{3}$ C NMR (125 MHz, $\left.\mathrm{CDCl}_{3}\right): \delta 219.6,166.5,150.0,142.5,137.7,137.0,134.6,130.8,130.4$, $128.6,128.5,128.1,127.6,127.4,126.8,126.7,126.5,126.0,125.9$, 124.2, 120.7, 115.9, 56.4, $45.9,36.8,27.2,23.5,21.3$.

IR $v\left(\mathrm{~cm}^{-1}\right): 3465.88,2923.64,2854.39,1988.36,1745.11,1594.61,1490.85,1453.72$, 1262.92, 777.56, 703.59.

HRMS (ESI): m/z Calcd fo $\left[\mathrm{C}_{30} \mathrm{H}_{29} \mathrm{~N}_{2} \mathrm{O}, \mathrm{M}+\mathrm{H}\right]^{+}: 433.2274$; Found: 433.2282.

Optical Rotation: $[\alpha]_{\mathrm{D}}^{25}=48.2\left(0.58, \mathrm{CHCl}_{3}\right) .94 \%$ ee. The absolute configuration of the product was assigned by analogy to 3a. (HPLC condition: Chiralpak IB column, $n$-hexane/ $i$-PrOH $=90: 10$, flow rate $=1.0 \mathrm{~mL} / \mathrm{min}$, wavelength $=254 \mathrm{~nm}, \mathrm{t}_{\mathrm{R}}=5.00 \mathrm{~min}$ for major isomer, $\mathrm{t}_{\mathrm{R}}=5.86 \mathrm{~min}$ for minor isomer).

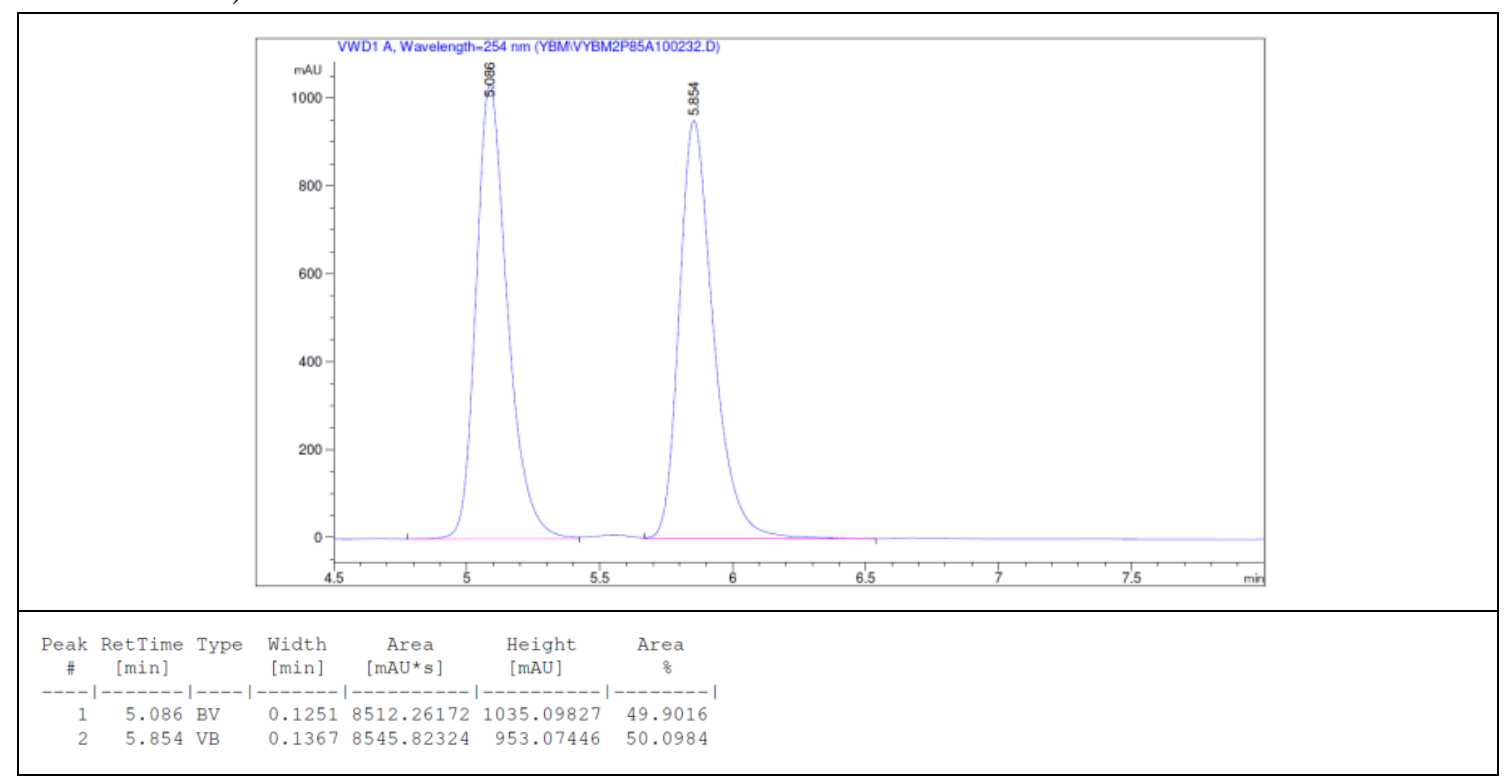




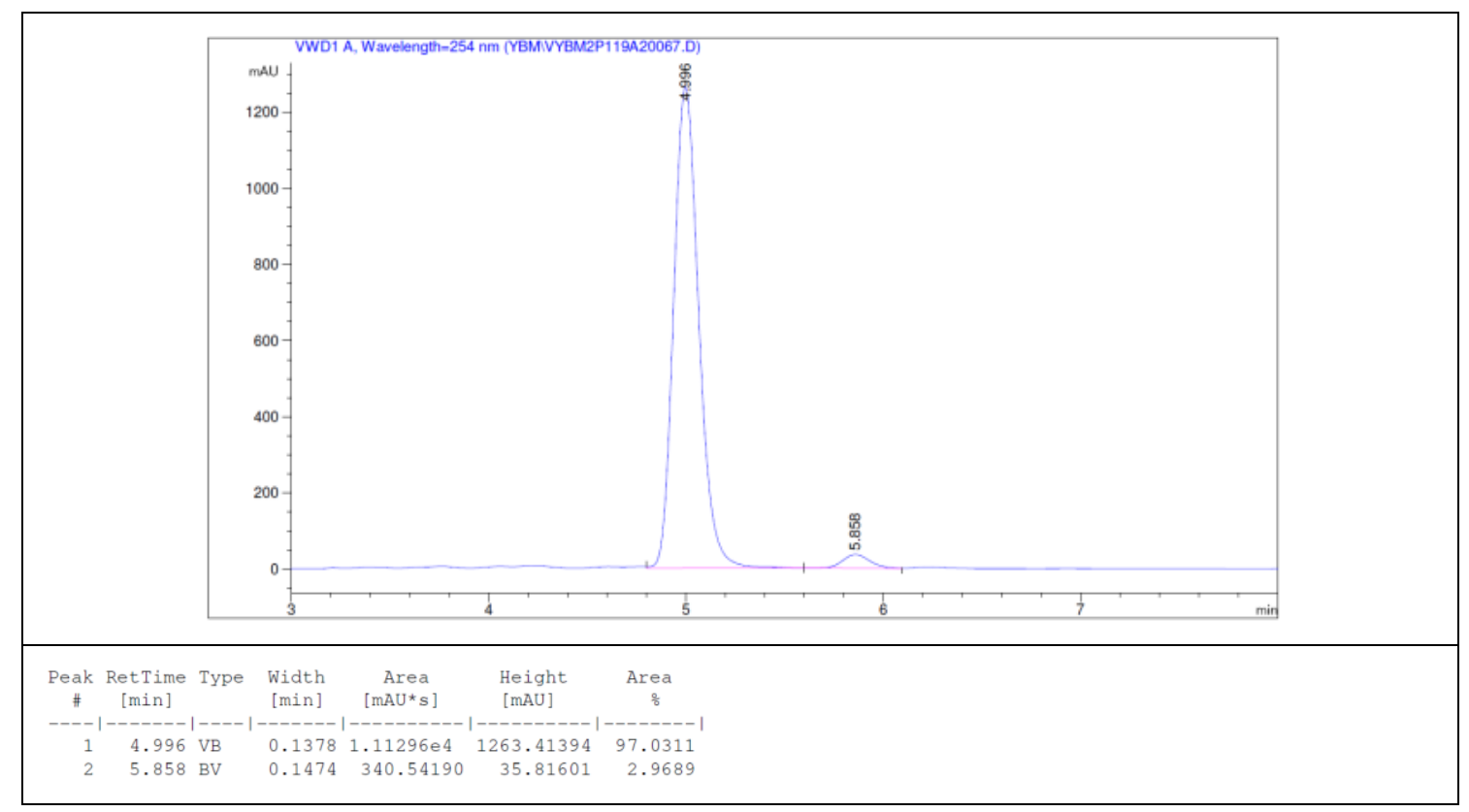

(R,E)-2-methyl-2-(4-methylbenzyl)-3-(2-(naphthalen-1-yl)-2-phenylhydrazono)cyclopentano ne (3e)

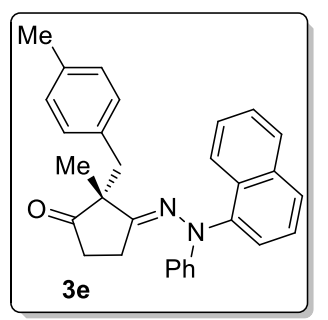

Colorless oil (33.7 $\mathrm{mg}, 75 \%$ yield).

${ }^{1} \mathbf{H}$ NMR $\left(500 \mathrm{MHz}, \mathrm{CDCl}_{3}\right): \delta 7.92(\mathrm{~d}, J=8.5 \mathrm{~Hz}, 2 \mathrm{H}), 7.84(\mathrm{~d}, J=8.5 \mathrm{~Hz}, 1 \mathrm{H}), 7.50(\mathrm{dd}, J=$ 14.0, $6.5 \mathrm{~Hz}, 2 \mathrm{H}), 7.42(\mathrm{dd}, J=14.0 \mathrm{~Hz}, J=6.5 \mathrm{~Hz}, 2 \mathrm{H}), 7.21(\mathrm{t}, J=8.5 \mathrm{~Hz}, 2 \mathrm{H}), 6.94-6.89(\mathrm{~m}$, $7 \mathrm{H}), 3.11(\mathrm{~d}, J=13.0 \mathrm{~Hz}, 1 \mathrm{H}), 3.05(\mathrm{~d}, J=13.0 \mathrm{~Hz}, 1 \mathrm{H}), 2.26(\mathrm{~s}, 3 \mathrm{H}), 2.09-2.03(\mathrm{~m}, 1 \mathrm{H})$, 1.91-1.84 (m, 1H), 1.65-1.58 (m, 1H), 1.48-1.40 (m, 1H), 1.45 (s, 3H).

${ }^{3} \mathrm{C}$ NMR $\left(125 \mathrm{MHz}, \mathrm{CDCl}_{3}\right): \delta 219.7,166.8,150.1,142.5,136.2,134.6,133.9,130.9,129.6$, $128.8,128.6,128.5,127.6,126.8,126.5,126.2$, 126.0, 124.3, 120.7, 115.8, 56.4, 45.6, 36.8, 27.2, 23.3, 21.0.

IR $v\left(\mathrm{~cm}^{-1}\right): 3470.51,3055.77,2923.40,2854.63,1967.29,1745.06,1594.53,1491.04$, 1452.87, 1391.68, 1287.39, 1217.15, 1111.47, 777.06, 691.32.

HRMS (ESI): $\mathrm{m} / \mathrm{z}$ Calcd for $\left[\mathrm{C}_{30} \mathrm{H}_{29} \mathrm{~N}_{2} \mathrm{O}, \mathrm{M}+\mathrm{H}\right]^{+}: 433.2274$; Found: 433.2283.

Optical Rotation: $[\alpha]_{\mathrm{D}}^{25}=56.4\left(0.7, \mathrm{CHCl}_{3}\right) .98 \%$ ee. The absolute configuration of the product was assigned by analogy to 3a. (HPLC condition: Chiralpak IB column, $n$-hexane/ $i$-PrOH $=90: 10$, flow rate $=1.0 \mathrm{~mL} / \mathrm{min}$, wavelength $=254 \mathrm{~nm}, \mathrm{t}_{\mathrm{R}}=4.94$ min for major isomer, $\mathrm{t}_{\mathrm{R}}=6.06 \mathrm{~min}$ for minor isomer). 


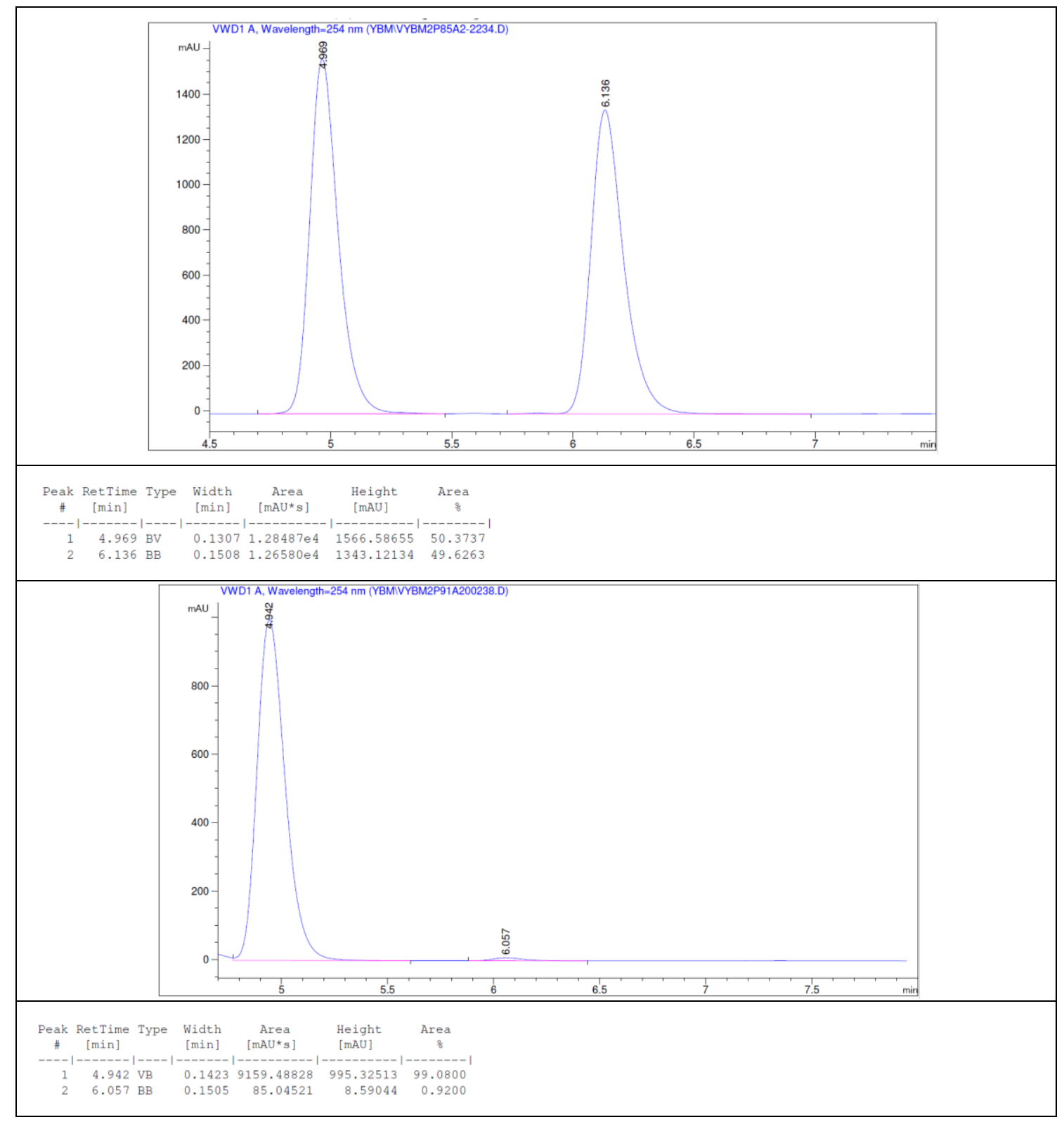

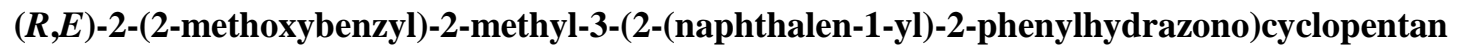
one (3f)

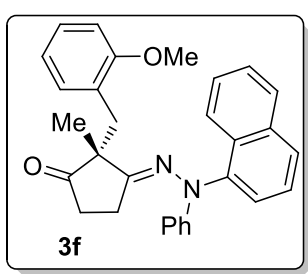

Colorless oil (30.5 mg, $68 \%$ yield).

${ }^{1}$ H NMR $\left(500 \mathrm{MHz}, \mathrm{CDCl}_{3}\right): \delta 7.90(\mathrm{t}, J=8.0 \mathrm{~Hz}, 2 \mathrm{H}), 7.80(\mathrm{~d}, J=8.5 \mathrm{~Hz}, 1 \mathrm{H}), 7.50(\mathrm{td}, J=6.5$ $\mathrm{Hz}, 1.0 \mathrm{~Hz}, 1 \mathrm{H}), 7.45-7.40(\mathrm{~m}, 2 \mathrm{H}), 7.23-7.14(\mathrm{~m}, 4 \mathrm{H}), 7.09(\mathrm{dd}, J=7.5 \mathrm{~Hz}, J=2.0 \mathrm{~Hz}, 1 \mathrm{H}), 6.90$ $(\mathrm{t}, J=7.5 \mathrm{~Hz}, 1 \mathrm{H}), 6.84(\mathrm{td}, J=7.0 \mathrm{~Hz}, J=0.5 \mathrm{~Hz}, 1 \mathrm{H}), 6.77(\mathrm{~d}, J=8.5 \mathrm{~Hz}, 3 \mathrm{H}), 3.64(\mathrm{~s}, 3 \mathrm{H})$, $3.09(\mathrm{~s}, 2 \mathrm{H}), 2.25-2.18(\mathrm{~m}, 1 \mathrm{H}), 2.16-2.09(\mathrm{~m}, 1 \mathrm{H}), 2.04-1.96(\mathrm{~m}, 1 \mathrm{H}), 1.76-1.69(\mathrm{~m}, 1 \mathrm{H}), 1.42(\mathrm{~s}$, $3 \mathrm{H})$. 
${ }^{13}$ C NMR (125 MHz, $\left.\mathrm{CDCl}_{3}\right): \delta 217.0,167.5,157.4,150.0,142.8,134.5,132.1,130.6,128.5$, $128.5,128.2,127.1,126.7,126.4,126.2,125.4,125.2,124.2,120.7,120.4,116.1,110.3,55.0$, 54.6, 40.0, 35.6, 26.9, 21.6.

IR $v\left(\mathrm{~cm}^{-1}\right): 3471.71,3058.95,2923.07,2853.17,1933.63,1746.78,1633.72,1594.27$, 1454.98, 1259.98, 1024.09, 800.35, 691.75.

HRMS (ESI): $\mathrm{m} / \mathrm{z}$ Calcd for $\left[\mathrm{C}_{30} \mathrm{H}_{29} \mathrm{~N}_{2} \mathrm{O}_{2}, \mathrm{M}+\mathrm{H}\right]^{+}:$449.2224; Found: 449.2232.

Optical Rotation: $[\alpha]_{\mathrm{D}}^{25}=-6.0\left(1.7, \mathrm{CHCl}_{3}\right) .94 \%$ ee. The absolute configuration of the product was assigned by analogy to 3a. (HPLC condition: Chiralpak IB column, $n$-hexane $/ i$-PrOH $=95: 5$, flow rate $=1.0 \mathrm{~mL} / \mathrm{min}$, wavelength $=254 \mathrm{~nm}, \mathrm{t}_{\mathrm{R}}=7.52 \mathrm{~min}$ for major isomer, $\mathrm{t}_{\mathrm{R}}=11.98 \mathrm{~min}$ for minor isomer).

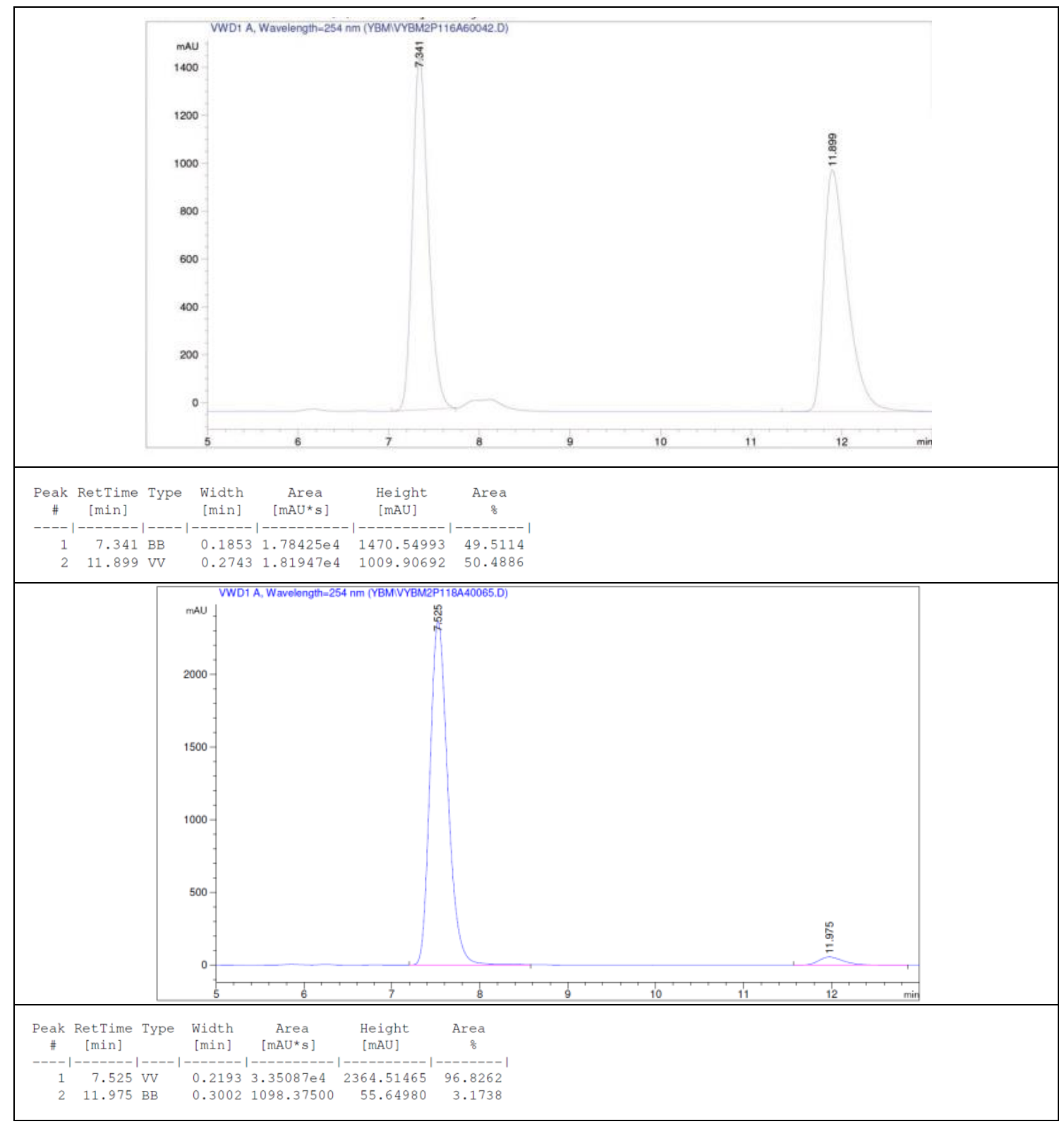

$(R, E)$-2-(3-chlorobenzyl)-2-methyl-3-(2-(naphthalen-1-yl)-2-phenylhydrazono)cyclopentanon e (3g) 


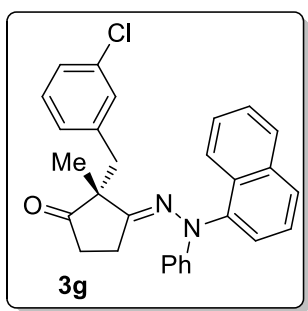

Colorless oil (31.7 mg, $70 \%$ yield).

${ }^{1} \mathbf{H}$ NMR $\left(500 \mathrm{MHz}, \mathrm{CDCl}_{3}\right): \delta 7.90(\mathrm{t}, J=7.5 \mathrm{~Hz}, 2 \mathrm{H}), 7.84(\mathrm{~d}, J=8.5 \mathrm{~Hz}, 1 \mathrm{H}), 7.52(\mathrm{~d}, J=8.0$ $\mathrm{Hz}, 1 \mathrm{H}), 7.49$ (d, $J=8.0 \mathrm{~Hz}, 1 \mathrm{H}), 7.43(\mathrm{t}, J=7.5 \mathrm{~Hz}, 1 \mathrm{H}), 7.39$ (d, $J=7.0 \mathrm{~Hz}, 1 \mathrm{H}), 7.21$ (t, $J=$ $8.0 \mathrm{~Hz}, 2 \mathrm{H}), 7.16-7.15(\mathrm{~m}, 2 \mathrm{H}), 7.07(\mathrm{t}, J=8.0 \mathrm{~Hz}, 1 \mathrm{H}), 6.95-6.88(\mathrm{~m}, 4 \mathrm{H}), 3.13(\mathrm{~d}, J=12.5 \mathrm{~Hz}$, $1 \mathrm{H}), 3.10(\mathrm{~d}, J=12.5 \mathrm{~Hz}, 1 \mathrm{H}), 2.15-2.08(\mathrm{~m}, 1 \mathrm{H}), 1.86-1.78(\mathrm{~m}, 1 \mathrm{H}), 1.73-1.66(\mathrm{~m}, 1 \mathrm{H})$, $1.51-1.47(\mathrm{~m}, 1 \mathrm{H}), 1.44(\mathrm{~s}, 3 \mathrm{H})$.

${ }^{13} \mathrm{C}$ NMR $\left(125 \mathrm{MHz}, \mathrm{CDCl}_{3}\right): \delta 219.2,164.2,149.9,142.3,139.4,134.6,134.0,130.9,129.9$, 129.5, 128.7, 128.5, 128.0, 127.8, 126.9, 126.5, 126.1, 126.0, 124.1, 120.9, 116.0, 56.2, 44.7, 36.7, 26.9, 24.0.

IR $v\left(\mathrm{~cm}^{-1}\right): 3469.19,2961.64,2924.26,2853.94,1937.32,1744.98,1594.87,1490.89$, 1391.61, 1261.20, 1083.42, 799.70, 690.27.

HRMS (ESI): $\mathrm{m} / \mathrm{z}$ Calcd for $\left[\mathrm{C}_{29} \mathrm{H}_{26} \mathrm{ClN}_{2} \mathrm{O}, \mathrm{M}+\mathrm{H}\right]^{+}: 453.1728$; Found: 453.1741.

Optical Rotation: $[\alpha]_{\mathrm{D}}^{25}=86.0\left(0.4, \mathrm{CHCl}_{3}\right) .97 .5 \% \mathrm{ee}$. The absolute configuration of the product was assigned by analogy to 3a. (HPLC condition: Chiralpak IB column, $n$-hexane $/ i-\mathrm{PrOH}=95: 5$, flow rate $=1.0 \mathrm{~mL} / \mathrm{min}$, wavelength $=254 \mathrm{~nm}, \mathrm{t}_{\mathrm{R}}=5.95 \mathrm{~min}$ for major isomer, $\mathrm{t}_{\mathrm{R}}=8.18 \mathrm{~min}$ for minor isomer).

\begin{tabular}{|l|l|l|l|}
\hline & \\
\hline & & & \\
\hline
\end{tabular}




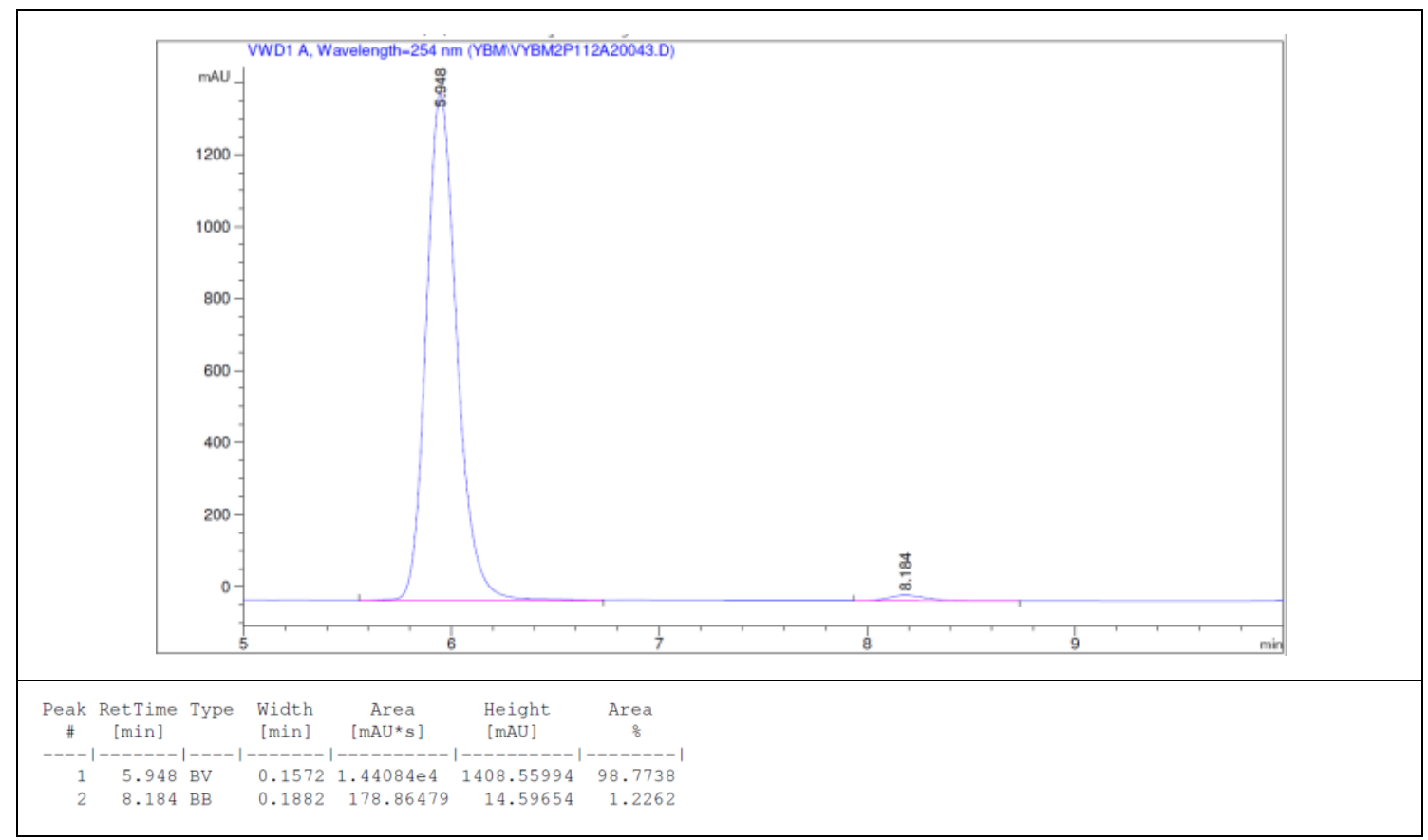

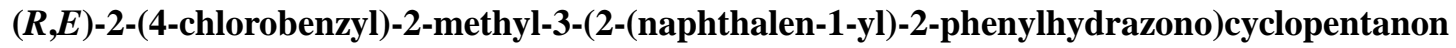
e (3h)

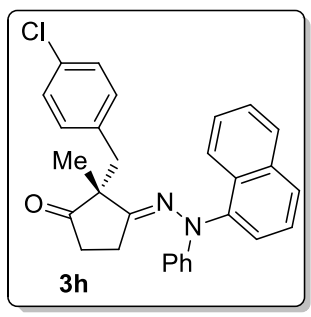

Colorless oil (33.5 mg, 74\% yield).

${ }^{1} \mathbf{H}$ NMR $\left(500 \mathrm{MHz}, \mathrm{CDCl}_{3}\right): \delta$ 7.94-7.85 (m, 3H), 7.53-7.49 (m, 2H), 7.46-7.42 (m, 1H), $7.38(\mathrm{dd}$, $J=8.0 \mathrm{~Hz}, J=1.0 \mathrm{~Hz}, 1 \mathrm{H}), 7.23-7.20(\mathrm{~m}, 2 \mathrm{H}), 7.09-7.06(\mathrm{~m}, 2 \mathrm{H}), 6.94-6.88(\mathrm{~m}, 5 \mathrm{H}), 3.11(\mathrm{~d}, J=$ $7.5 \mathrm{~Hz}, 1 \mathrm{H}), 3.07(\mathrm{~d}, J=7.5 \mathrm{~Hz}, 1 \mathrm{H}), 2.14-2.07(\mathrm{~m}, 1 \mathrm{H}), 1.93-1.86(\mathrm{~m}, 1 \mathrm{H}), 1.69-1.61(\mathrm{~m}, 1 \mathrm{H})$, $1.49-1.40(\mathrm{~m}, 1 \mathrm{H}), 1.42(\mathrm{~s}, 3 \mathrm{H})$.

${ }^{13} \mathrm{C}$ NMR $\left(125 \mathrm{MHz}, \mathrm{CDCl}_{3}\right): \delta 219.3,165.5,150.0,142.3,135.7,134.7,132.6,131.1,130.9$, $128.7,128.6,128.3,127.9,126.9,126.6,126.4,125.9,124.2$, 120.8, 115.7, 56.3, 44.6, 36.7, 27.1, 23.8 .

IR $v\left(\mathrm{~cm}^{-1}\right): 3469.26,3057.68,2966.68,2923.86,2864.49,1930.82,1744.28,1594.05$, $1490.50,1391.52,1287.57,1102.12,753.36,691.59$.

HRMS (ESI): $\mathrm{m} / \mathrm{z}$ Calcd for $\left[\mathrm{C}_{29} \mathrm{H}_{26} \mathrm{ClN}_{2} \mathrm{O}, \mathrm{M}+\mathrm{H}\right]^{+}: 453.1728$; Found: 453.1745.

Optical Rotation: $[\alpha]_{\mathrm{D}}^{25}=55.3\left(0.5, \mathrm{CHCl}_{3}\right) .93 \%$ ee. The absolute configuration of the product was assigned by analogy to 3a. (HPLC condition: Chiralpak IB column, $n$-hexane/ $i$-PrOH $=90: 10$, flow rate $=1.0 \mathrm{~mL} / \mathrm{min}$, wavelength $=254 \mathrm{~nm}, \mathrm{t}_{\mathrm{R}}=5.44$ min for major isomer, $\mathrm{t}_{\mathrm{R}}=7.88 \mathrm{~min}$ for minor isomer). 


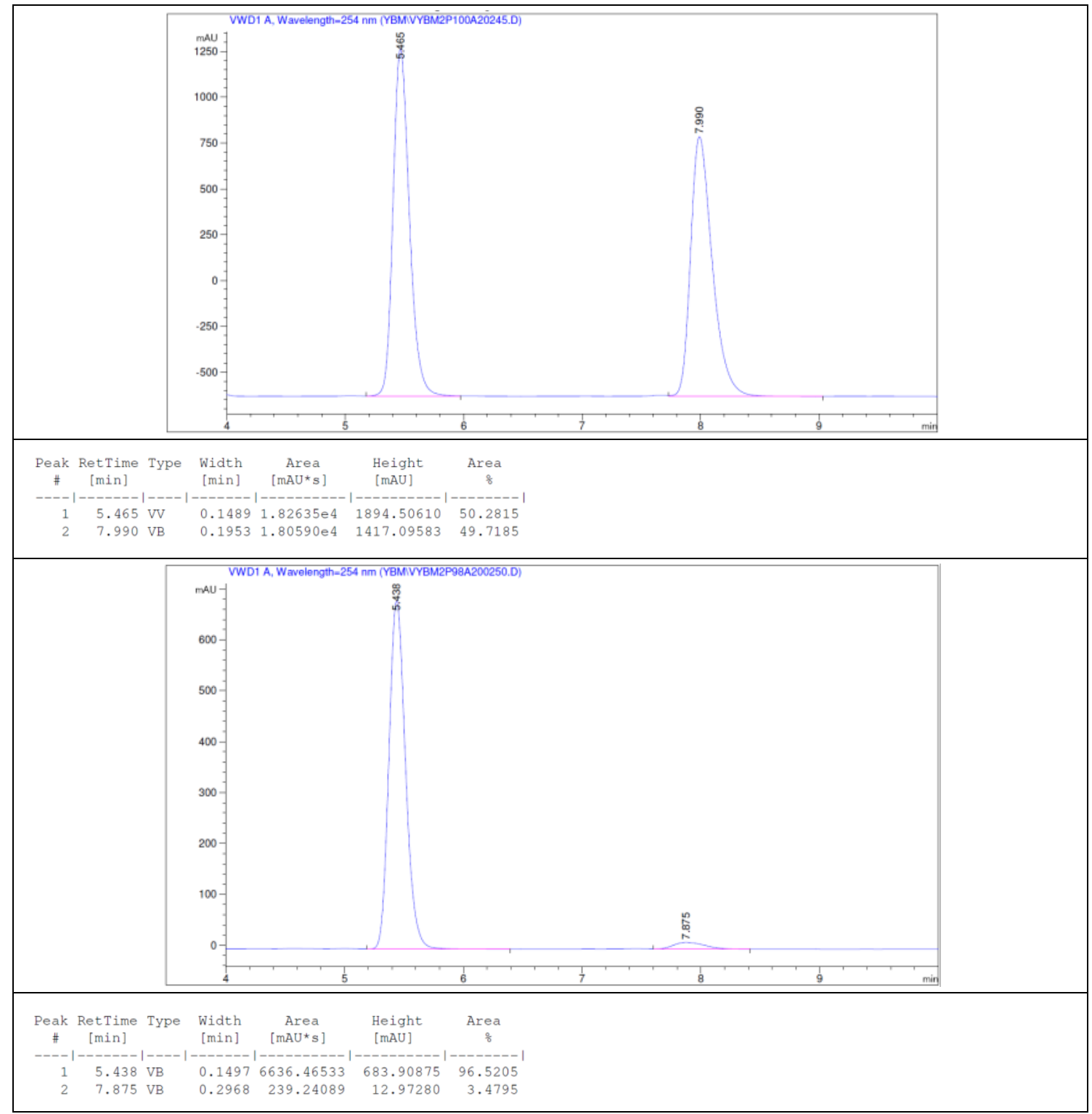

(R,E)-2-methyl-3-(2-(naphthalen-1-yl)-2-phenylhydrazono)-2-(4-nitrobenzyl)cyclopentanone

(3i)

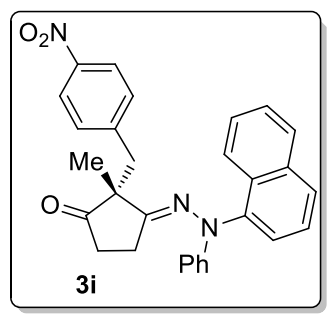

Amorphous solid (32.4 mg, $70 \%$ yield).

${ }^{1} \mathbf{H}$ NMR $\left(500 \mathrm{MHz}, \mathrm{CDCl}_{3}\right): \delta 7.97(\mathrm{~d}, J=8.0 \mathrm{~Hz}, 2 \mathrm{H}), 7.93(\mathrm{~d}, J=8.0 \mathrm{~Hz}, 1 \mathrm{H}), 7.87(\mathrm{~d}, J=4.0$ $\mathrm{Hz}, 1 \mathrm{H}), 7.86(\mathrm{~d}, J=4.0 \mathrm{~Hz}, 1 \mathrm{H}), 7.53-7.47(\mathrm{~m}, 2 \mathrm{H}), 7.43(\mathrm{t}, J=6.0 \mathrm{~Hz}, 1 \mathrm{H}), 7.38(\mathrm{~d}, J=7.5 \mathrm{~Hz}$, 1H), 7.23-7.18 (m, 4H), $6.94(\mathrm{t}, J=7.5 \mathrm{~Hz}, 1 \mathrm{H}), 6.88(\mathrm{~d}, J=8.0 \mathrm{~Hz}, 2 \mathrm{H}), 3.25$ (d, $J=12.5 \mathrm{~Hz}$, $1 \mathrm{H}), 3.20(\mathrm{~d}, J=12.5 \mathrm{~Hz}, 1 \mathrm{H}), 2.21-2.14(\mathrm{~m}, 1 \mathrm{H}), 1.92-1.85(\mathrm{~m}, 1 \mathrm{H}), 1.74-1.67(\mathrm{~m}, 1 \mathrm{H})$, 1.53-1.47 (m, 1H). $1.45(\mathrm{~s}, 3 \mathrm{H})$.

${ }^{13} \mathrm{C}$ NMR $\left(125 \mathrm{MHz}, \mathrm{CDCl}_{3}\right): \delta 218.5,163.7,149.8,146.8,145.2,142.2,134.7,130.8,128.8$, 
128.6, 128.1, 127.0, 126.7, 126.5, 125.9, 124.6, 123.2, 121.0, 115.6, 56.2, 44.0, 36.5, 26.8, 24.4.

IR $v\left(\mathrm{~cm}^{-1}\right): 3463.92,2958.88,2923.79,2854.03,1937.67,1745.23,1595.32,1490.92$, $1260.31,1017.83,800.45,700.91$.

HRMS (ESI): $\mathrm{m} / \mathrm{z}$ Calcd for $\left[\mathrm{C}_{29} \mathrm{H}_{26} \mathrm{~N}_{3} \mathrm{O}_{3}, \mathrm{M}+\mathrm{H}\right]^{+}: 464.1969$; Found: 464.1973.

Optical Rotation: $[\alpha]_{\mathrm{D}}^{25}=83.2(0.5, \mathrm{CHCl} 3) .98 \% e e$. The absolute configuration of the product was assigned by analogy to 3a. (HPLC condition: Chiralpak IB column, $n$-hexane/i-PrOH $=90: 10$, flow rate $=1.0 \mathrm{~mL} / \mathrm{min}$, wavelength $=254 \mathrm{~nm}, \mathrm{t}_{\mathrm{R}}=11.02 \min$ for major isomer, $\mathrm{t}_{\mathrm{R}}=13.31 \mathrm{~min}$ for minor isomer).

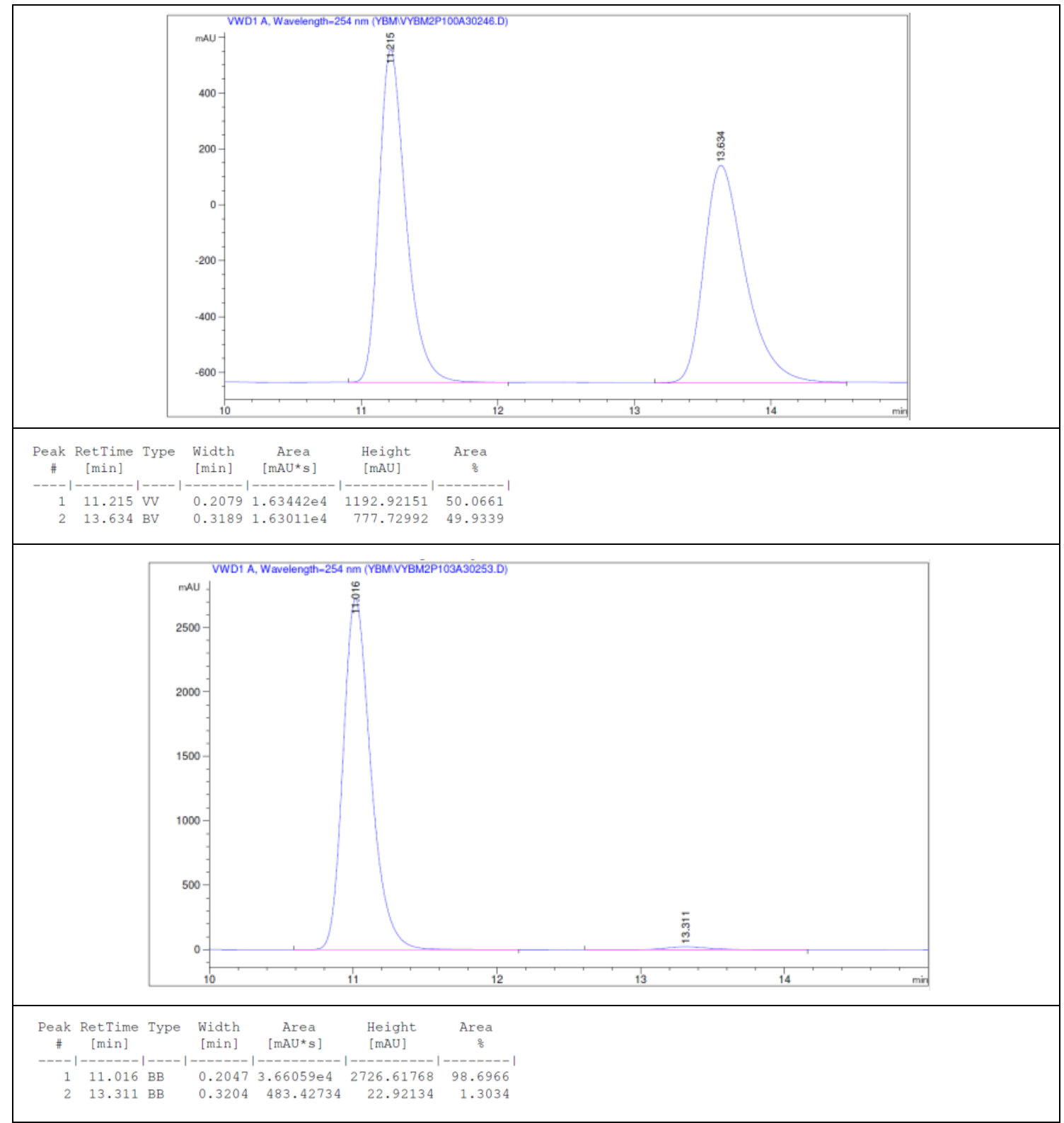

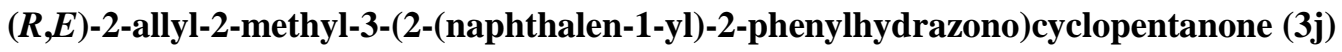

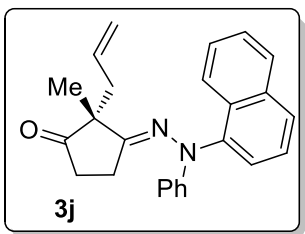


Colorless oil (32.0 mg, $87 \%$ yield).

${ }^{1}$ H NMR $\left(500 \mathrm{MHz}, \mathrm{CDCl}_{3}\right): \delta 7.93(\mathrm{~d}, J=5.0 \mathrm{~Hz}, 1 \mathrm{H}), 7.91(\mathrm{~d}, J=5.5 \mathrm{~Hz}, 1 \mathrm{H}), 7.83(\mathrm{~d}, J=8.0$ $\mathrm{Hz}, 1 \mathrm{H}), 7.52(\mathrm{t}, J=7.5 \mathrm{~Hz}, 2 \mathrm{H}), 7.43(\mathrm{t}, J=9.0 \mathrm{~Hz}, 2 \mathrm{H}), 7.19(\mathrm{t}, J=8.5 \mathrm{~Hz}, 21 \mathrm{H}), 6.92(\mathrm{t}, J=$ $7.0 \mathrm{~Hz}, 1 \mathrm{H}), 6.84(\mathrm{~d}, J=9.0 \mathrm{~Hz}, 2 \mathrm{H}), 5.71-5.62(\mathrm{~m}, 1 \mathrm{H}), 5.09(\mathrm{dd}, J=17.5 \mathrm{~Hz}, J=1.0 \mathrm{~Hz}, 1 \mathrm{H})$, $5.03(\mathrm{~d}, J=10.0 \mathrm{~Hz}, 1 \mathrm{H}), 2.58(\mathrm{dd}, J=13.5 \mathrm{~Hz}, J=7.0 \mathrm{~Hz}, 1 \mathrm{H}), 2.47(\mathrm{dd}, J=13.5 \mathrm{~Hz}, J=7.5 \mathrm{~Hz}$, $1 \mathrm{H}), 2.32-2.16(\mathrm{~m}, 2 \mathrm{H}), 2.10-2.03(\mathrm{~m}, 1 \mathrm{H}), 1.94-1.87(\mathrm{~m}, 1 \mathrm{H}) .1 .34(\mathrm{~s}, 3 \mathrm{H})$.

${ }^{13} \mathbf{C}$ NMR $\left(125 \mathrm{MHz}, \mathrm{CDCl}_{3}\right): \delta 218.6,167.6,150.0,143.0,134.6,133.1,130.5,128.6,128.5$, 127.3, 126.7, 126.5, 126.1, 125.2, 124.1, 120.9, 118.8, 116.2, 54.7, 43.0, 36.4, 27.0, 22.0.

IR $v\left(\mathrm{~cm}^{-1}\right): 3471.79,3059.17,2958.62,2925.04,2853.07,1931.50,1745.06,1594.29$, 1491.23, 1450.79, 1259.97, 921.77, 777.24, 691.59.

HRMS (ESI): $\mathrm{m} / \mathrm{z}$ Calcd for $\left[\mathrm{C}_{25} \mathrm{H}_{25} \mathrm{~N}_{2} \mathrm{O}, \mathrm{M}+\mathrm{H}\right]^{+}: 369.1961$; Found: 369.1965.

Optical Rotation: $[\alpha]_{\mathrm{D}}^{25}=-10.2(0.86, \mathrm{CHCl} 3) .82 \% e e$. The absolute configuration of the product was assigned by analogy to 3a. (HPLC condition: Chiralpak IB column, $n$-hexane $/ i$-PrOH $=90: 10$, flow rate $=1.0 \mathrm{~mL} / \mathrm{min}$, wavelength $=254 \mathrm{~nm}, \mathrm{t}_{\mathrm{R}}=6.27 \mathrm{~min}$ for major isomer, $\mathrm{t}_{\mathrm{R}}=8.43 \mathrm{~min}$ for minor isomer).

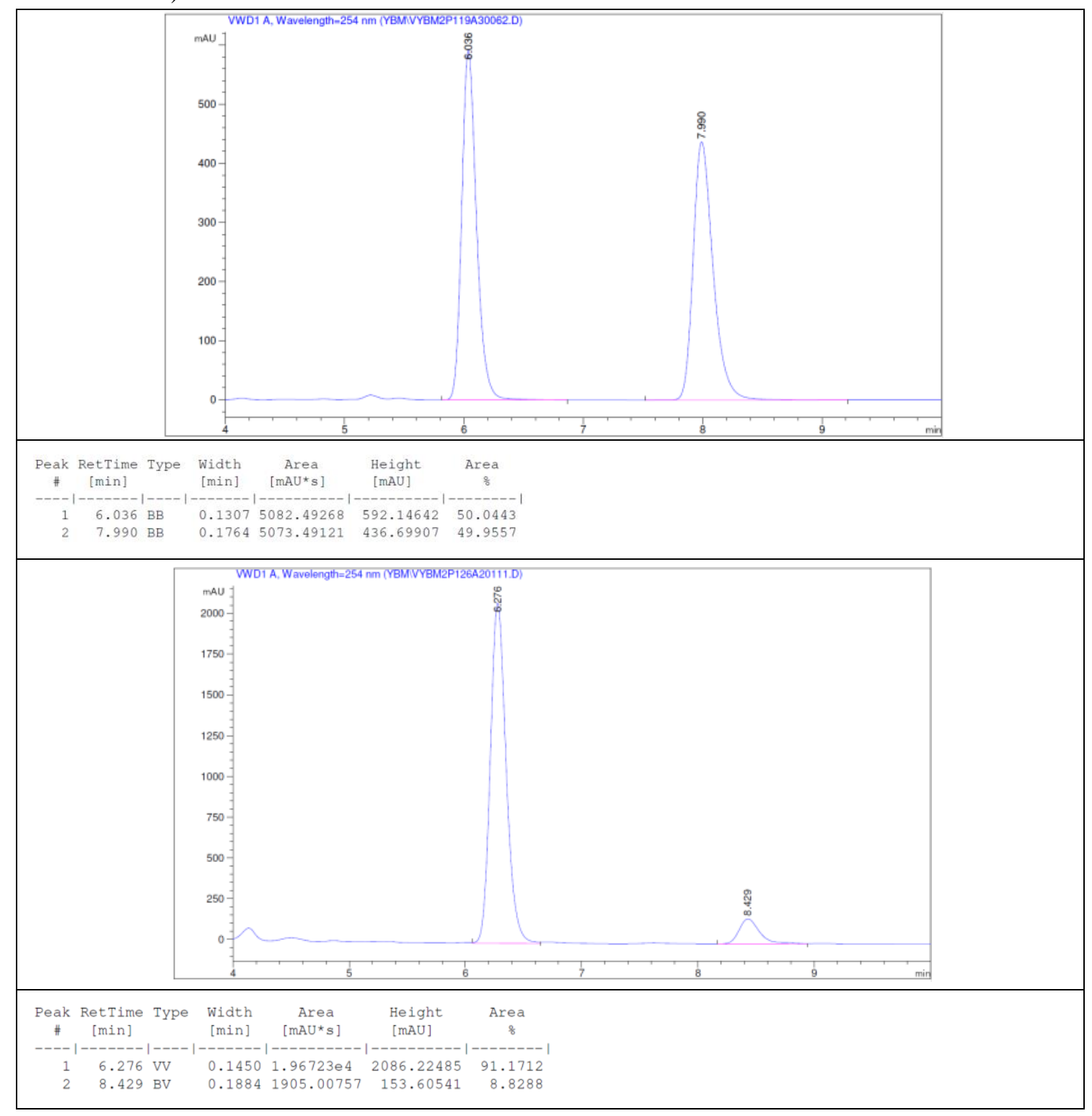




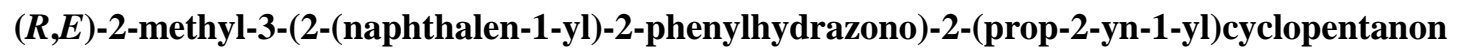
e (3k)

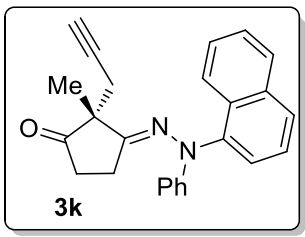

Colorless oil (24.2 mg, 66\% yield).

${ }^{1} \mathbf{H}$ NMR $\left(500 \mathrm{MHz} \mathrm{CDCl}_{3}\right): \delta$ 7.96-7.91 (m, 2H), $7.82(\mathrm{~d}, J=8.5 \mathrm{~Hz}, 1 \mathrm{H}), 7.51(\mathrm{t}, J=5.0 \mathrm{~Hz}$, 2H), 7.50-7.42 (m, 2H), 7.21-7.17 (m, 2H), $6.93(\mathrm{t}, J=5.0 \mathrm{~Hz}, 1 \mathrm{H}), 6.88(\mathrm{~d}, J=8.5 \mathrm{~Hz}, 2 \mathrm{H}), 2.75$ $(\mathrm{dd}, J=16.0 \mathrm{~Hz}, J=2.5 \mathrm{~Hz}, 1 \mathrm{H}), 2.61(\mathrm{dd}, J=16.0 \mathrm{~Hz}, J=1.5 \mathrm{~Hz}, 1 \mathrm{H}), 2.36-2.31(\mathrm{~m}, 2 \mathrm{H})$, 2.12-1.97 (m, 2H), 1.87 (s, 1H), 1.32 (s, 3H).

${ }^{13} \mathrm{C} \mathrm{NMR}\left(125 \mathrm{MHz}, \mathrm{CDCl}_{3}\right): \delta 217.7,166.0,149.8,142.7,134.5,130.5,128.6,128.5,127.3$, $126.7,126.5,126.1,125.2,124.2,121.1,80.6,70.0,53.8,36.6,27.5,22.4$.

IR $v\left(\mathrm{~cm}^{-1}\right): 3467.14,3292.36,2962.19,2927.74,2852.42,1748.49,1594.49,1594.56$, $1491.05,1260.96,1095.54,1018.81,800.81,691.62$.

HRMS (ESI): m/z Calcd for $\left[\mathrm{C}_{25} \mathrm{H}_{23} \mathrm{~N}_{2} \mathrm{O}, \mathrm{M}+\mathrm{H}\right]^{+}: 367.1805$; Found: 367.1809.

Optical Rotation: $[\alpha]_{\mathrm{D}}^{25}=84.2\left(0.4, \mathrm{CHCl}_{3}\right)$. $79 \%$ ee. The absolute configuration of the product was assigned by analogy to 3a. (HPLC condition: Chiralpak IE column, $n$-hexane/ $i$-PrOH $=98: 2$, flow rate $=1.0 \mathrm{~mL} / \mathrm{min}$, wavelength $=254 \mathrm{~nm}, \mathrm{t}_{\mathrm{R}}=8.36 \mathrm{~min}$ for major isomer, $\mathrm{t}_{\mathrm{R}}=8.86$ min for minor isomer).

\begin{tabular}{|c|c|c|c|c|}
\hline & \\
\hline
\end{tabular}




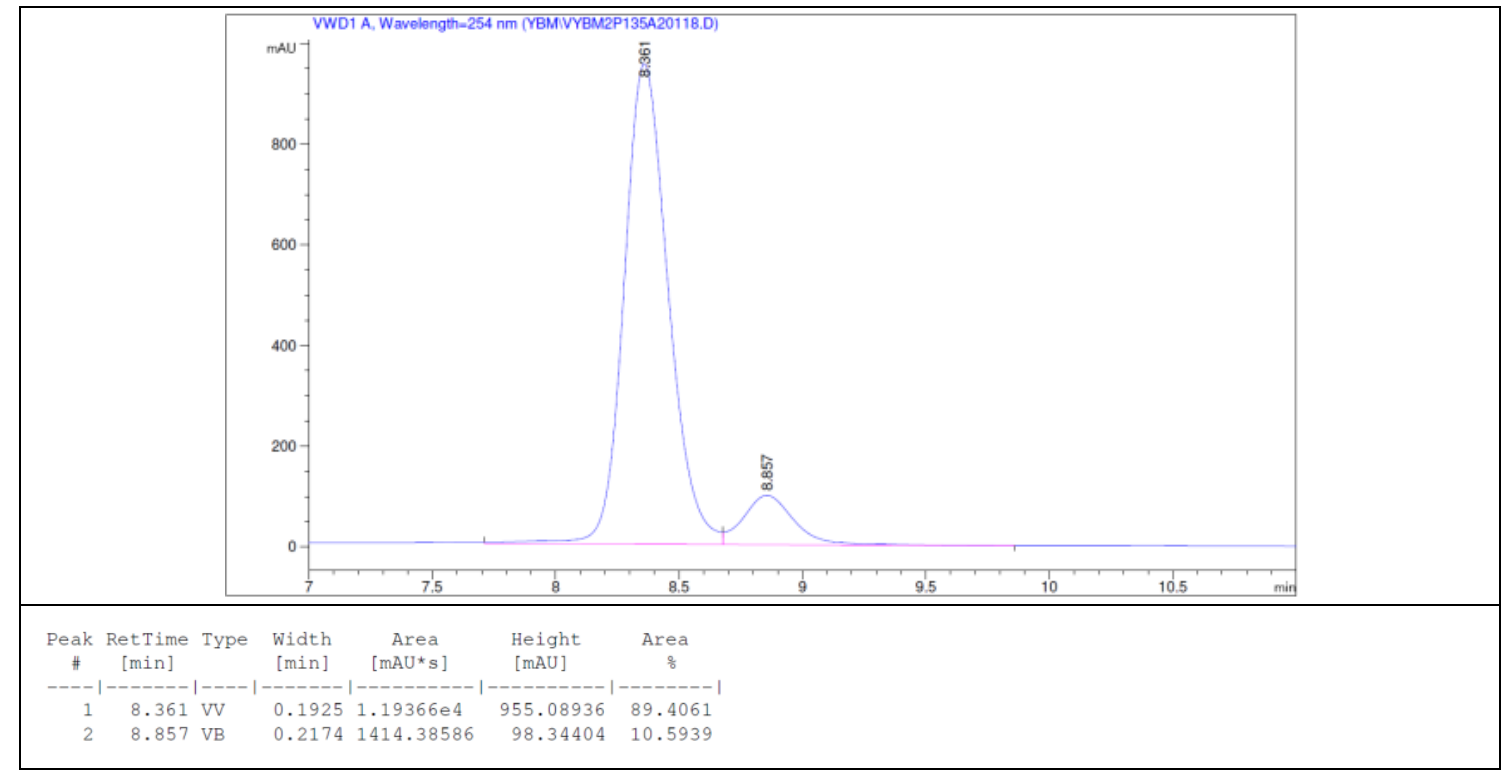

(R,E)-2-methyl-3-(2-(naphthalen-1-yl)-2-phenylhydrazono)-2-(3-phenylprop-2-yn-1-yl)cyclo pentanone (3l)

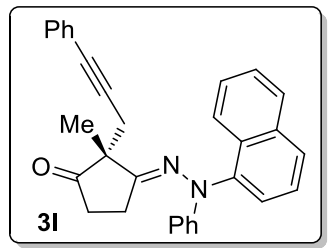

Colorless oil $(35.8 \mathrm{mg}, 81 \%$ yield $)$.

${ }^{1} \mathbf{H}$ NMR $\left(500 \mathrm{MHz}, \mathrm{CDCl}_{3}\right): \delta 7.96(\mathrm{~d}, J=8.5 \mathrm{~Hz}, 1 \mathrm{H}), 7.91(\mathrm{~d}, J=8.5 \mathrm{~Hz}, 1 \mathrm{H}), 7.77(\mathrm{~d}, J=8.5$ $\mathrm{Hz}, 1 \mathrm{H}), 7.52-7.49$ (m, 2H), 7.45-7.41 (m, 1H), 7.35-7.26 (m, 4H), 7.24-7.19 (m, 4H), 6.97-6.92 (m, 3H), $2.97(\mathrm{~d}, J=16.0 \mathrm{~Hz}, 1 \mathrm{H}), 2.81(\mathrm{~d}, J=16.0 \mathrm{~Hz}, 1 \mathrm{H}), 2.40(\mathrm{t}, J=8.5 \mathrm{~Hz}, 2 \mathrm{H}), 2.16-2.12$ $(\mathrm{m}, 2 \mathrm{H}), 1.43$ (s, 3H).

${ }^{13} \mathrm{C}$ NMR $\left(125 \mathrm{MHz}, \mathrm{CDCl}_{3}\right): \delta 218.0,166.3,149.9,142.6,134.5,131.6,130.4,128.6,128.4$, $128.1,127.9,127.1,126.7,126.4,126.2,125.0,124.1,123.0,121.0,116.5,86.0,82.1,54.1,36.6$, 29.1, 27.6, 22.1.

IR $v\left(\mathrm{~cm}^{-1}\right): 3476.84,3057.13,2968.04,2926.69,1948.16,1747.98,1594.67,1490.28$, 1391.92, 1287.58, 912.61, 776.93, 691.90.

HRMS (ESI): m/z Calcd for $\left[\mathrm{C}_{31} \mathrm{H}_{27} \mathrm{~N}_{2} \mathrm{O}, \mathrm{M}+\mathrm{H}\right]^{+}: 443.2118$; Found: 443.2122.

Optical Rotation: $[\alpha]_{\mathrm{D}}^{25}=101.6\left(0.5, \mathrm{CHCl}_{3}\right) .87 \%$ ee. The absolute configuration of the product was assigned by analogy to 3a. (HPLC condition: Chiralpak IB column, $n$-hexane/i-PrOH = 98:2, flow rate $=1.0 \mathrm{~mL} / \mathrm{min}$, wavelength $=254 \mathrm{~nm}, \mathrm{t}_{\mathrm{R}}=12.09$ min for major isomer, $\mathrm{t}_{\mathrm{R}}=11.51 \mathrm{~min}$ for minor isomer). 


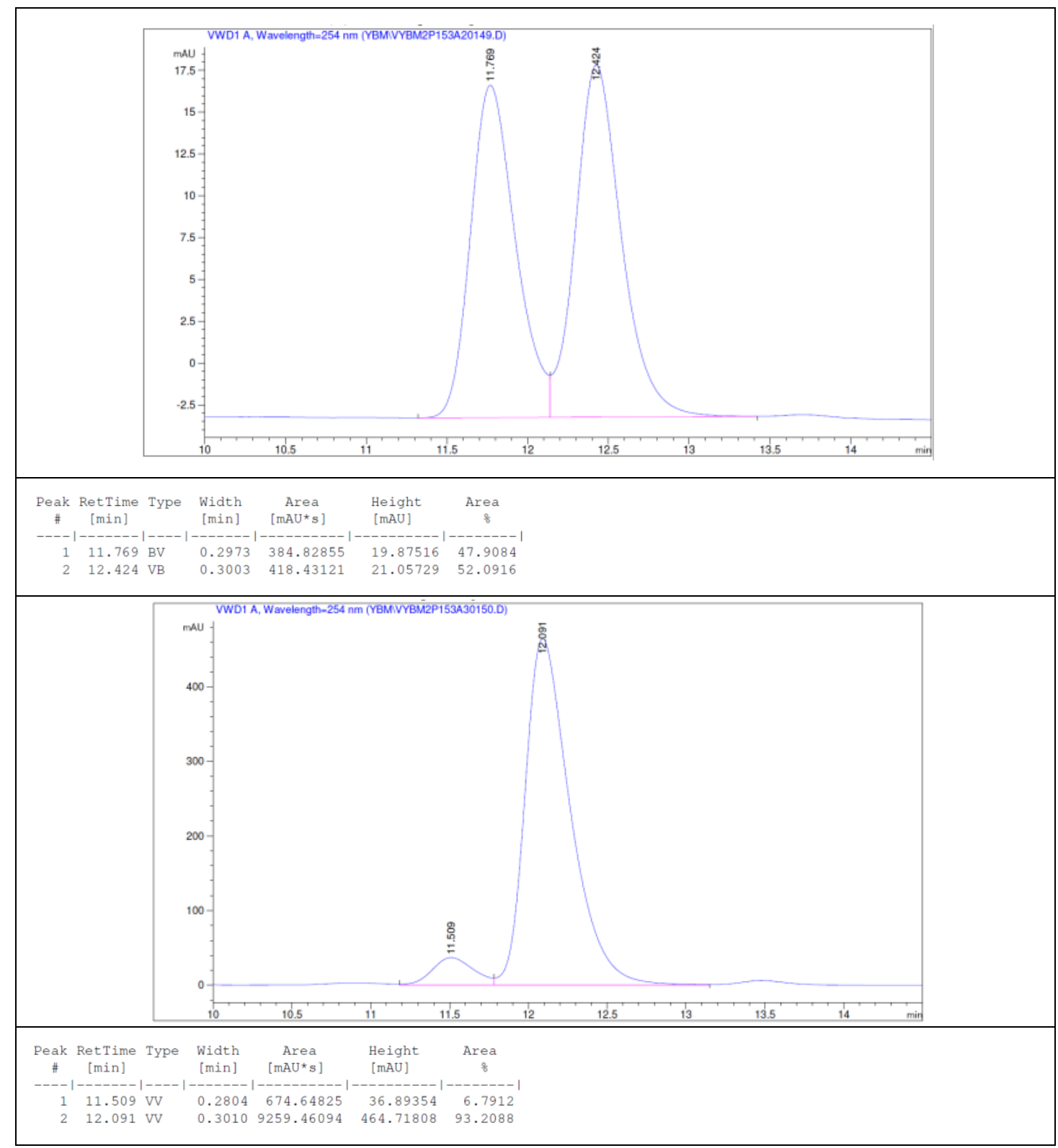

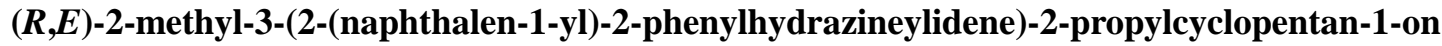
e (3m)

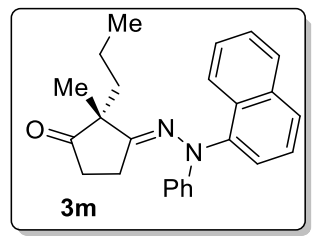

Colorless oil (25.2 mg, 68\% yield).

${ }^{1} \mathbf{H}$ NMR $\left(500 \mathrm{MHz}, \mathrm{CDCl}_{3}\right): \delta 7.91(\mathrm{t}, J=7.5 \mathrm{~Hz}, 2 \mathrm{H}), 7.81(\mathrm{~d}, J=8.5 \mathrm{~Hz}, 1 \mathrm{H}), 7.54-7.49(\mathrm{~m}$, 2H), 7.44-7.40 (m, 2H), 7.20-7.17 (m, 2H), $6.91(\mathrm{t}, J=7.5 \mathrm{~Hz}, 1 \mathrm{H}), 6.84-6.82(\mathrm{~m}, 2 \mathrm{H}), 2.34-2.21$ $(\mathrm{m}, 2 \mathrm{H}), 2.16-2.09(\mathrm{~m}, 1 \mathrm{H}), 1.95-1.88(\mathrm{~m}, 1 \mathrm{H}), 1.82-1.76(\mathrm{~m}, 1 \mathrm{H}), 1.74-1.68(\mathrm{~m}, 1 \mathrm{H}), 1.31(\mathrm{~s}, 3 \mathrm{H})$, $1.21-1.12(\mathrm{~m}, 2 \mathrm{H}), 0.88(\mathrm{t}, J=7.5 \mathrm{~Hz}, 3 \mathrm{H})$.

${ }^{13} \mathrm{C}$ NMR $\left(125 \mathrm{MHz}, \mathrm{CDCl}_{3}\right): \delta 219.6,168.8,150.0,143.1,134.6,130.5,128.6,128.5,127.3$, $126.7,126.5,126.1,125.1,124.2,120.9,116.1,54.6,41.0,36.3,27.1,22.4,18.4,14.5$. 
IR $v\left(\mathrm{~cm}^{-1}\right) 3469.84 .3058 .14,2924.82,2851.80,1930.57,1743.43,1594.14,1489.05,1391.57$, 1286.16, 1214.06, 1100.37, 776.76, 691.91 .

HRMS (ESI): $\mathrm{m} / \mathrm{z}$ Calcd for $\left[\mathrm{C}_{25} \mathrm{H}_{27} \mathrm{~N}_{2} \mathrm{O}, \mathrm{M}+\mathrm{H}\right]^{+}: 371.2118$; Found: 371.2118 .

Optical rotation: $[\alpha]_{\mathrm{D}}^{25}=-35.0(0.2, \mathrm{CHCl} 3) .92 \% e e$. The absolute configuration of the product was assigned by analogy to 3a. (HPLC condition: Chiralpak IB column, $n$-hexane/ $i$-PrOH $=90: 10$, flow rate $=1.0 \mathrm{~mL} / \mathrm{min}$, wavelength $=254 \mathrm{~nm}, \mathrm{t}_{\mathrm{R}}=5.77 \mathrm{~min}$ for major isomer, $\mathrm{t}_{\mathrm{R}}=7.66 \mathrm{~min}$ for minor isomer).

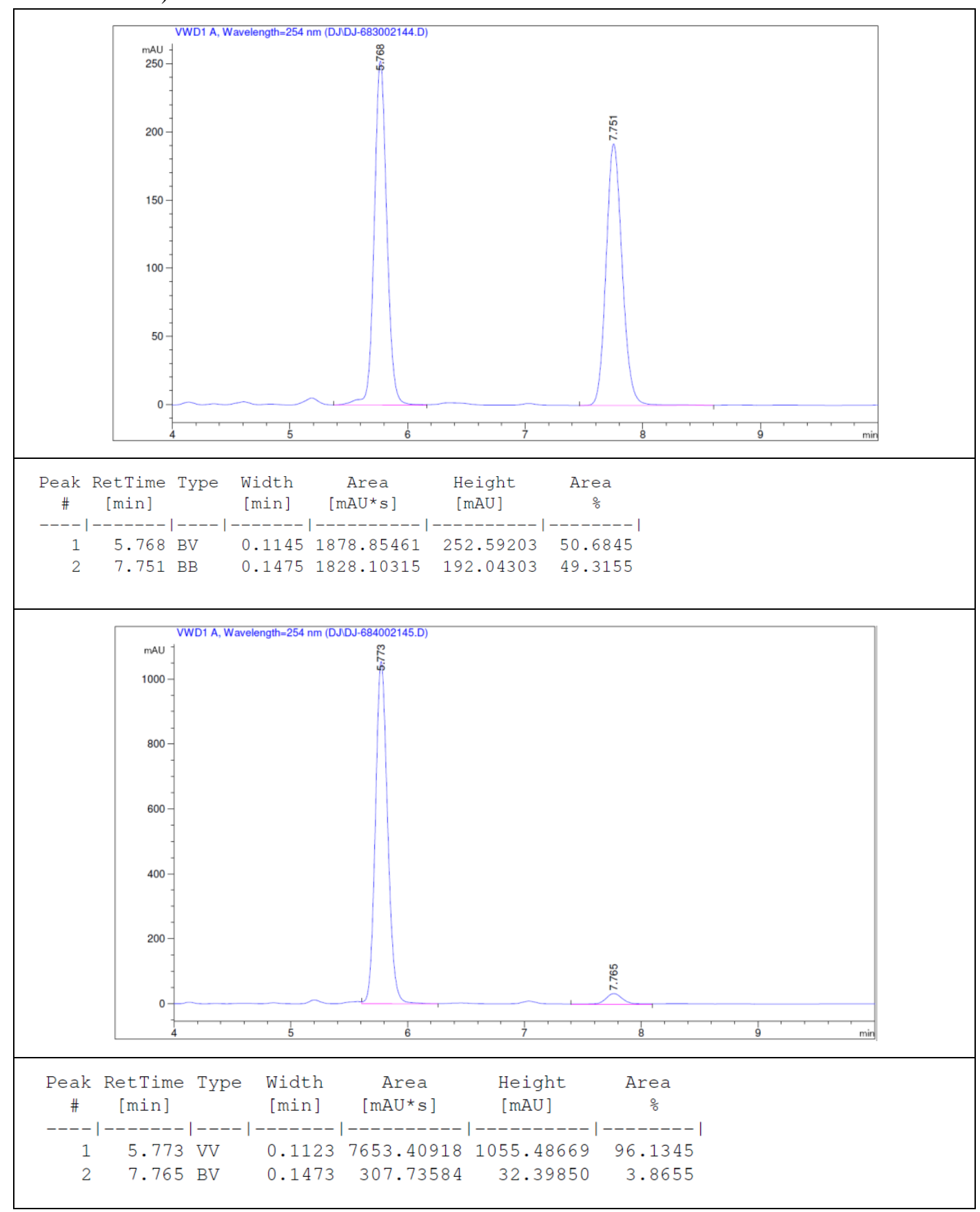

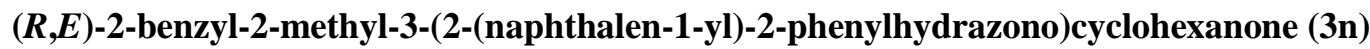




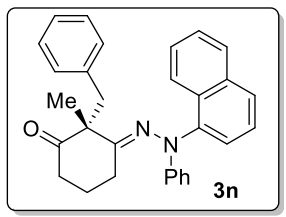

Colorless oil (31.5 mg, $73 \%$ yield).

${ }^{1} \mathbf{H}$ NMR $\left(500 \mathrm{MHz}, \mathrm{CDCl}_{3}\right): \delta 7.99(\mathrm{dd}, J=8.5 \mathrm{~Hz}, J=1.0 \mathrm{~Hz}, 1 \mathrm{H}), 7.92(\mathrm{~d}, J=8.5 \mathrm{~Hz}, 1 \mathrm{H})$, $7.81(\mathrm{~d}, J=8.5 \mathrm{~Hz}, 1 \mathrm{H}), 7.52-7.47(\mathrm{~m}, 2 \mathrm{H}), 7.45-7.41(\mathrm{~m}, 1 \mathrm{H}), 7.28(\mathrm{dd}, J=7.5 \mathrm{~Hz}, J=1.0 \mathrm{~Hz}$, $1 \mathrm{H}), 7.17-7.12(\mathrm{~m}, 5 \mathrm{H}), 6.96-6.95(\mathrm{~m}, 2 \mathrm{H}), 6.92-6.88(\mathrm{~m}, 1 \mathrm{H}), 6.68-6.65(\mathrm{~m}, 2 \mathrm{H}), 3.32(\mathrm{~d}, J=$ $13.5 \mathrm{~Hz}, 1 \mathrm{H}), 3.23(\mathrm{~d}, J=13.5 \mathrm{~Hz}, 1 \mathrm{H}), 2.42-2.36(\mathrm{~m}, 1 \mathrm{H}), 2.26-2.23(\mathrm{~m}, 2 \mathrm{H}), 2.06-1.99(\mathrm{~m}, 1 \mathrm{H})$, $1.60(\mathrm{~s}, 3 \mathrm{H}), 1.41-1.33(\mathrm{~m}, 1 \mathrm{H}), 1.23-1.15(\mathrm{~m}, 1 \mathrm{H})$.

${ }^{13} \mathrm{C}$ NMR $\left(125 \mathrm{MHz}, \mathrm{CDCl}_{3}\right): \delta 212.4,170.2,150.5,144.0,137.5,134.6,130.3,130.0,128.6$, 128.5, 128.1, 126.8, 126.6, 126.5, 126.3, 126.2, 124.7, 124.4, 120.8, 116.5, 59.0, 45.6, 38.9, 28.7, 24.5, 17.9 .

IR $v\left(\mathrm{~cm}^{-1}\right): 3338.66,3056.46,2924.60,2853.00,1932.43,1709.89,1593.54,1491.61$, 1392.47, 1285.53, 775.99, 695.07.

HRMS (ESI): $\mathrm{m} / \mathrm{z}$ Calcd for $\left[\mathrm{C}_{30} \mathrm{H}_{29} \mathrm{~N}_{2} \mathrm{O}, \mathrm{M}+\mathrm{H}\right]^{+}:$: 433.2274; Found: 433.2283.

Optical Rotation: $[\alpha]_{\mathrm{D}}^{25}=24.7\left(1.0, \mathrm{CHCl}_{3}\right) .94 \%$ ee. The absolute configuration of the product was assigned by analogy to 3a. (HPLC condition: Chiralpak IB column, $n$-hexane $/ i-\mathrm{PrOH}=95: 5$, flow rate $=1.0 \mathrm{~mL} / \mathrm{min}$, wavelength $=254 \mathrm{~nm}, \mathrm{t}_{\mathrm{R}}=6.68 \mathrm{~min}$ for major isomer, $\mathrm{t}_{\mathrm{R}}=7.17 \mathrm{~min}$ for minor isomer).

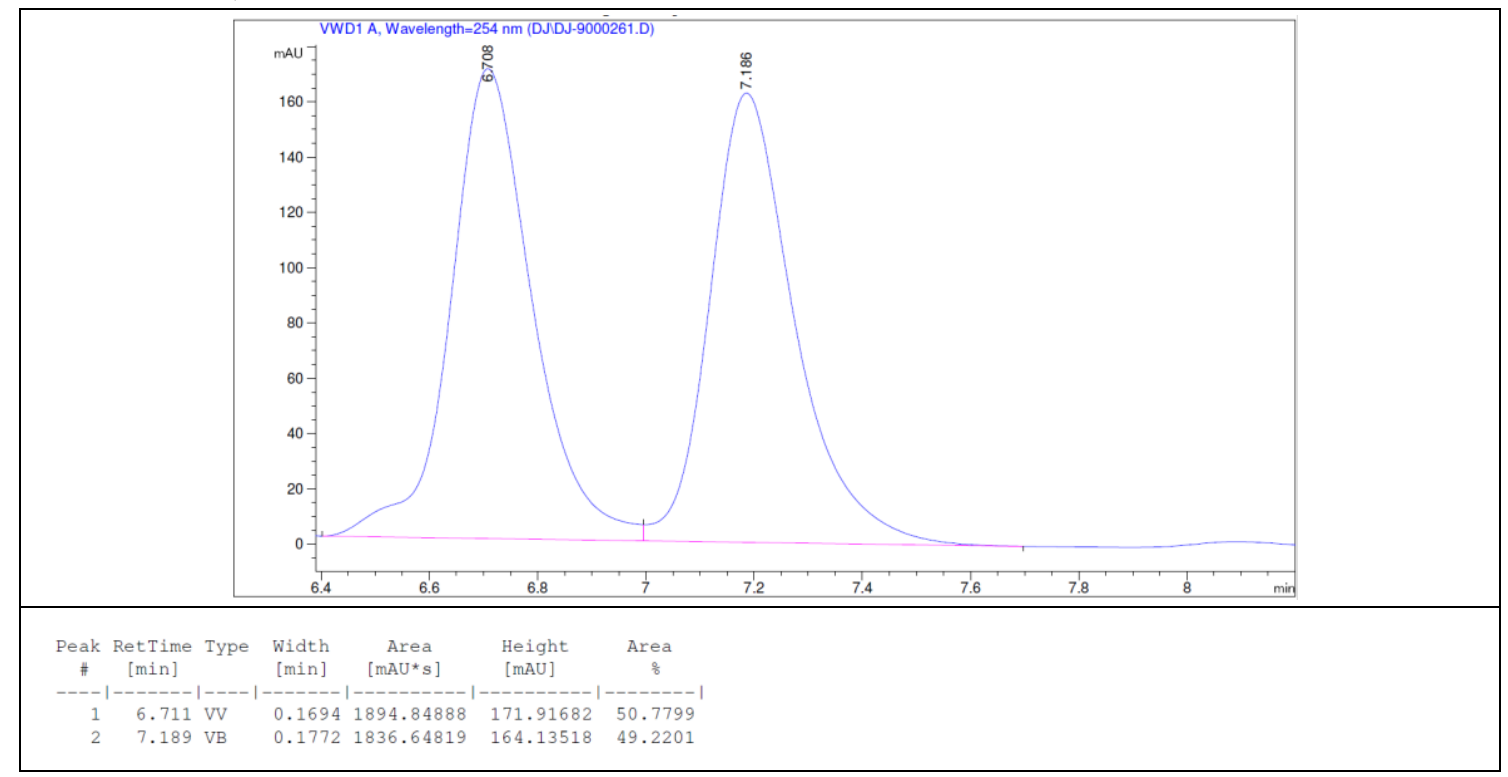




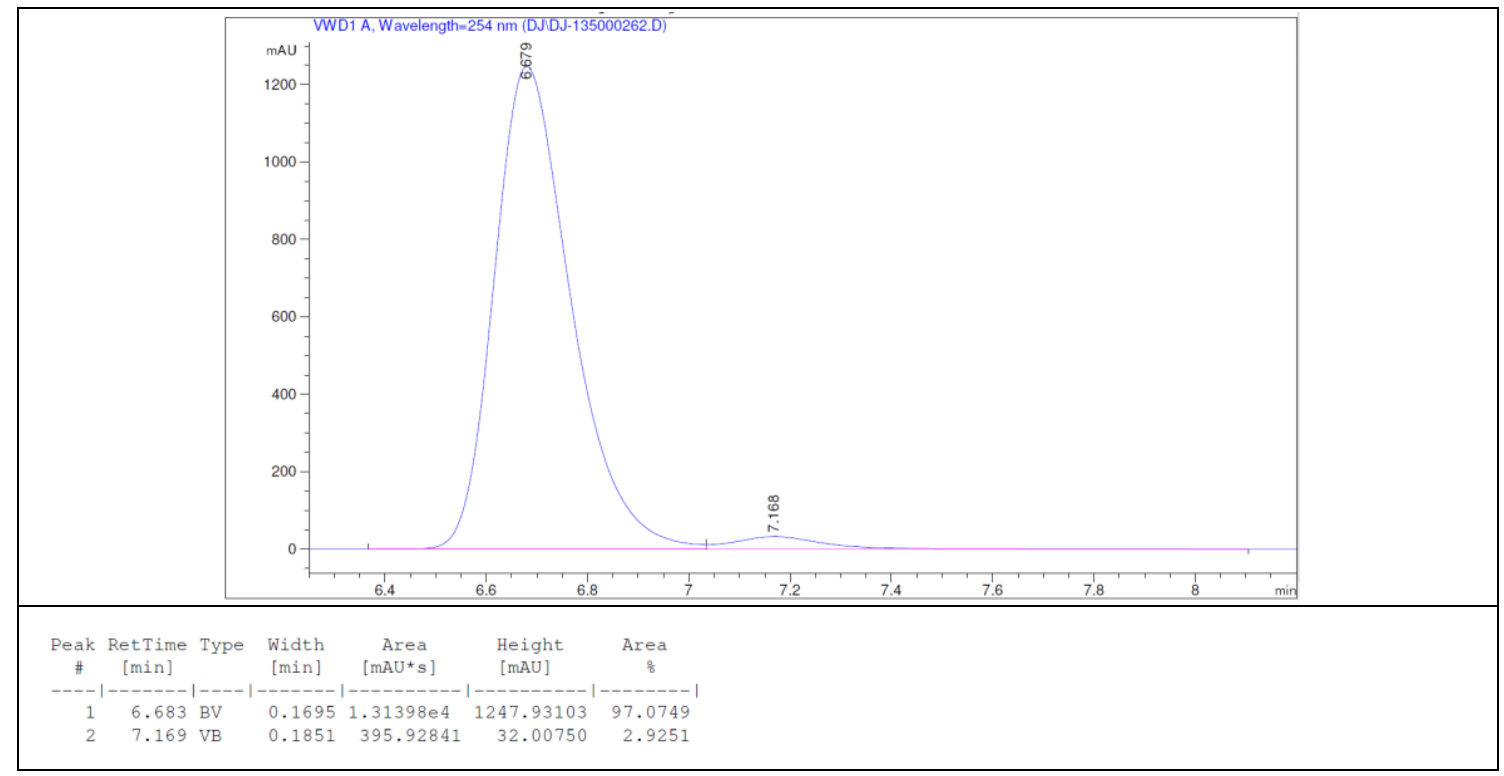

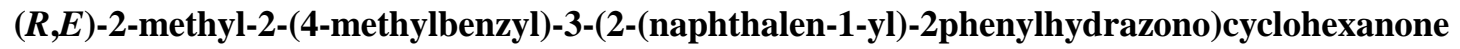
(3o)

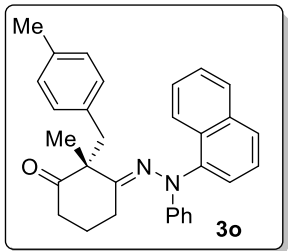

Colorless oil (36.1 mg, $81 \%$ yield).

${ }^{1} \mathbf{H}$ NMR $\left(500 \mathrm{MHz}, \mathrm{CDCl}_{3}\right): \delta 8.01(\mathrm{~d}, J=8.5 \mathrm{~Hz}, 1 \mathrm{H}), 7.93(\mathrm{~d}, J=8.5 \mathrm{~Hz}, 1 \mathrm{H}), 7.82(\mathrm{~d}, J=8.5$ $\mathrm{Hz}, 1 \mathrm{H}), 7.53-7.49$ (m, 2H), 7.45-7.42 (m, 1H), 7.30 (dd, $J=7.5 \mathrm{~Hz}, J=1.0 \mathrm{~Hz}, 1 \mathrm{H}), 7.17-7.13$ $(\mathrm{m}, 2 \mathrm{H}), 6.96(\mathrm{~d}, J=8.0 \mathrm{~Hz}, 2 \mathrm{H}), 6.91(\mathrm{t}, J=7.5 \mathrm{~Hz}, 1 \mathrm{H}), 6.84(\mathrm{~d}, J=8.0 \mathrm{~Hz}, 2 \mathrm{H}), 6.67(\mathrm{dd}, J=$ $9.0 \mathrm{~Hz}, J=1.5 \mathrm{~Hz}, 2 \mathrm{H}), 3.26(\mathrm{~d}, J=13.0 \mathrm{~Hz}, 1 \mathrm{H}), 3.18(\mathrm{~d}, J=13.0 \mathrm{~Hz}, 1 \mathrm{H}), 2.46-2.40(\mathrm{~m}, 1 \mathrm{H})$, 2.30 (s, 3H), 2.26-2.24 (m, 2H), 2.05-1.99 (m, 1H), 1.60 (s, 3H), 1.40-1.34 (m, 1H), 1.26-1.21 (m, $1 \mathrm{H})$.

${ }^{13} \mathrm{C}$ NMR $\left(125 \mathrm{MHz}, \mathrm{CDCl}_{3}\right): \delta 212.5,170.4,150.4,144.0,136.1,134.6,134.2,130.3,129.8$, $128.8,128.5,128.4,126.8,126.5,126.3,126.2,124.7,124.3,120.7,116.5,59.1,45.4,38.9,28.7$, 24.0, 21.0, 18.0 .

IR $v\left(\mathrm{~cm}^{-1}\right): 3403.78,3053.09,2928.58,1931.08,1711.99,1594.00,1489.44,1391.47$, 1276.40, 1212.21, 776.73, 755.05, 692.78.

HRMS (ESI): m/z Calcd for $\left[\mathrm{C}_{31} \mathrm{H}_{31} \mathrm{~N}_{2} \mathrm{O}, \mathrm{M}+\mathrm{H}\right]^{+}: 447.2431$; Found: 447.2438.

Optical Rotation: $[\alpha]_{\mathrm{D}}^{25}=69.5\left(1.0, \mathrm{CHCl}_{3}\right) .96 \%$ ee. The absolute configuration of the product was assigned by analogy to 3a. (HPLC condition: Chiralpak AD-H column, $n$-hexane $i-\mathrm{PrOH}=$ $99: 1$, flow rate $=1.0 \mathrm{~mL} / \mathrm{min}$, wavelength $=254 \mathrm{~nm}, \mathrm{t}_{\mathrm{R}}=6.5 \mathrm{~min}$ for minor isomer, $\mathrm{t}_{\mathrm{R}}=7.34 \mathrm{~min}$ for major isomer). 


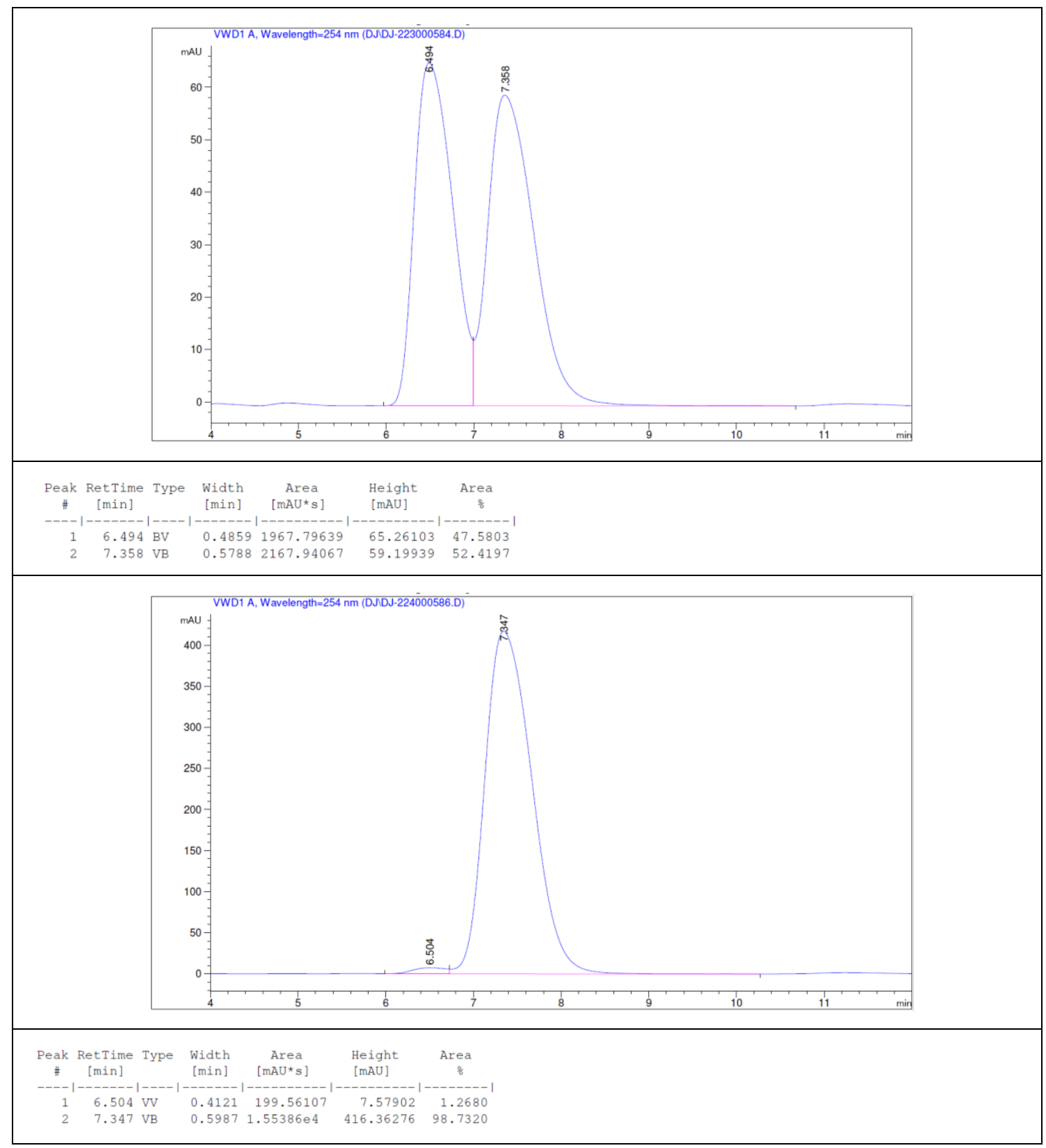

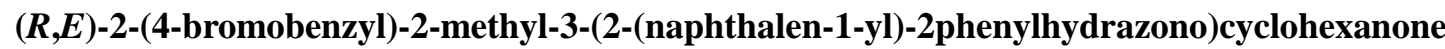
(3p)

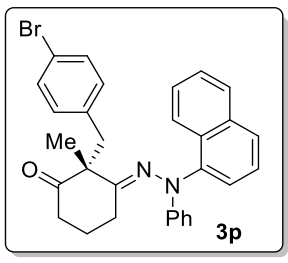

Colorless oil (39.8 mg, $78 \%$ yield).

${ }^{1} \mathrm{H}$ NMR $\left(400 \mathrm{MHz}, \mathrm{CDCl}_{3}\right): \delta 7.96(\mathrm{~d}, J=8.6 \mathrm{~Hz}, 1 \mathrm{H}), 7.93(\mathrm{~d}, J=8.2 \mathrm{~Hz}, 1 \mathrm{H}), 7.82(\mathrm{~d}, J=8.2$ $\mathrm{Hz}, 1 \mathrm{H}), 7.53-7.47(\mathrm{~m}, 2 \mathrm{H}), 7.44-7.40(\mathrm{~m}, 1 \mathrm{H}), 7.27-7.22(\mathrm{~m}, 3 \mathrm{H})$, 7.17-7.13 (m, 2H), 6.93-6.89 (m, 1H), 6.82-6.79 (m, 2H), 6.65-6.62 (m, 2H), $3.29(\mathrm{~d}, J=13.4 \mathrm{~Hz}, 1 \mathrm{H}), 3.18(\mathrm{~d}, J=13.4 \mathrm{~Hz}$, $1 \mathrm{H}), 2.41-2.35(\mathrm{~m}, 1 \mathrm{H}), 2.33-2.26(\mathrm{~m}, 1 \mathrm{H}), 2.24-2.17(\mathrm{~m}, 1 \mathrm{H}), 2.09-2.02(\mathrm{~m}, 1 \mathrm{H}), 1.58(\mathrm{~s}, 3 \mathrm{H})$, $1.47-1.41(\mathrm{~m}, 1 \mathrm{H}), 1.29-1.22(\mathrm{~m}, 1 \mathrm{H})$. 
${ }^{13} \mathrm{C}$ NMR $\left(125 \mathrm{MHz}, \mathrm{CDCl}_{3}\right): \delta 212.2,169.8,150.4,143.9,136.7,134.7,131.7,131.1,130.3$, 128.5, 127.0, 126.6, 126.4, 126.2, 124.8, 124.4, 120.9, 120.4, 116.5, 58.7, 44.3, 38.9, 28.7, 25.1, 18.0 .

IR $v\left(\mathrm{~cm}^{-1}\right): 3399.11,3056.97,2929.46,1930.92,1712.55,1593.47,1488.33,1391.42$, 1280.06, 1212.70, 776.29, 754.97, 692.87.

HRMS (ESI): $\mathrm{m} / \mathrm{z}$ Calcd for $\left[\mathrm{C}_{30} \mathrm{H}_{28} \mathrm{BrN}_{2} \mathrm{O}, \mathrm{M}+\mathrm{H}\right]^{+}:$511.1380; Found: 511.1380.

Optical Rotation: $[\alpha]_{\mathrm{D}}^{25}=54.1\left(1.0, \mathrm{CHCl}_{3}\right) .97 \%$ ee. The absolute configuration of the product was assigned by analogy to 3a. (HPLC condition: Chiralpak IC column, $n$-hexane $/ i$-PrOH $=98: 2$, flow rate $=1.0 \mathrm{~mL} / \mathrm{min}$, wavelength $=254 \mathrm{~nm}, \mathrm{t}_{\mathrm{R}}=6.99 \min$ for minor isomer, $\mathrm{t}_{\mathrm{R}}=7,54 \mathrm{~min}$ for major isomer).

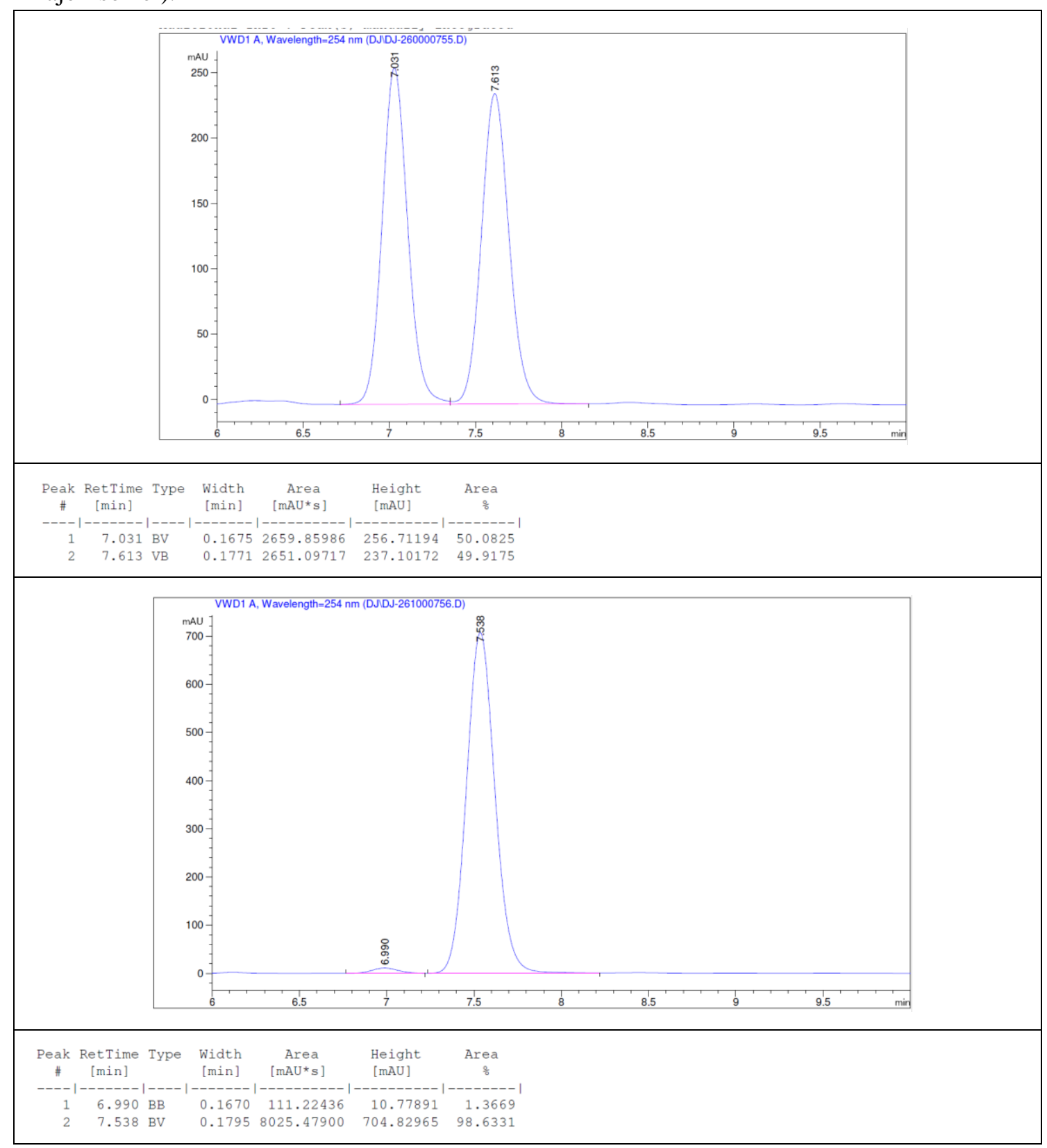

$(R, E)-2$-(4-(bromomethyl)benzyl)-2-methyl-3-(2-(naphthalen-1-yl)-2-phenylhydrazono)cyclo hexanone (3q) 


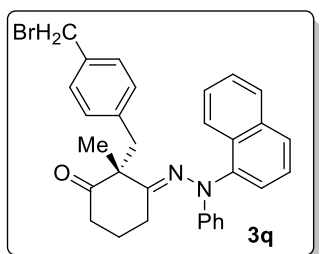

Colorless oil (43.5 mg, $83 \%$ yield).

${ }^{1} \mathbf{H}$ NMR $\left(500 \mathrm{MHz}, \mathrm{CDCl}_{3}\right): \delta 7.98(\mathrm{~d}, J=8.5 \mathrm{~Hz}, 1 \mathrm{H}), 7.93(\mathrm{~d}, J=8.0 \mathrm{~Hz}, 1 \mathrm{H}), 7.82(\mathrm{~d}, J=8.5$ $\mathrm{Hz}, 1 \mathrm{H}), 7.53-7.49(\mathrm{~m}, 2 \mathrm{H}), 7.44-7.41(\mathrm{~m}, 1 \mathrm{H}), 7.27-2.25(\mathrm{~m}, 1 \mathrm{H}), 7.18-7.13(\mathrm{~m}, 4 \mathrm{H}), 6.95-6.89$ $(\mathrm{m}, 3 \mathrm{H}), 6.64(\mathrm{~d}, J=8.5 \mathrm{~Hz}, 2 \mathrm{H}), 4.45(\mathrm{~s}, 2 \mathrm{H}), 3.33(\mathrm{~d}, J=13.5 \mathrm{~Hz}, 1 \mathrm{H}), 3.25(\mathrm{~d}, J=13.5 \mathrm{~Hz}$, $1 \mathrm{H}), 2.41-2.34(\mathrm{~m}, 1 \mathrm{H}), 2.32-2.21(\mathrm{~m}, 2 \mathrm{H}), 2.10-2.04(\mathrm{~m}, 1 \mathrm{H}), 1.60(\mathrm{~s}, 3 \mathrm{H}), 1.45-1.38(\mathrm{~m}, 1 \mathrm{H})$, $1.29-1.22(\mathrm{~m}, 1 \mathrm{H})$.

${ }^{13} \mathrm{C}$ NMR $\left(125 \mathrm{MHz}, \mathrm{CDCl}_{3}\right): \delta 212.2,169.7,150.4,143.9,138.1,136.0,134.6,130.4,130.3$, $128.8,128.5,126.9,126.6,126.3,126.2,124.7,124.4,120.9,116.5,58.9,44.7,38.8,33.3,28.6$, $24.9,18.0$.

IR $v\left(\mathrm{~cm}^{-1}\right): 3401.68,3055.77,2929.49,1929.49,1711.48,1593.62,1488.64,1391.35$, $1250.73,1097.11,1018.33,776.35,692.92,604.64$.

HRMS (ESI): $\mathrm{m} / \mathrm{z}$ Calcd for $\left[\mathrm{C}_{31} \mathrm{H}_{30} \mathrm{BrN}_{2} \mathrm{O}, \mathrm{M}+\mathrm{H}\right]^{+}:$525.1536; Found: 525.1524.

Optical Rotation: $[\alpha]_{\mathrm{D}}^{25}=62.2\left(1.0, \mathrm{CHCl}_{3}\right) .96 \%$ ee. The absolute configuration of the product was assigned by analogy to 3a. (HPLC condition: Chiralpak IB column, $n$-hexane $/ i$-PrOH $=95: 5$, flow rate $=1.0 \mathrm{~mL} / \mathrm{min}$, wavelength $=254 \mathrm{~nm}, \mathrm{t}_{\mathrm{R}}=8.62 \mathrm{~min}$ for major isomer, $\mathrm{t}_{\mathrm{R}}=9.32 \mathrm{~min}$ for minor isomer).

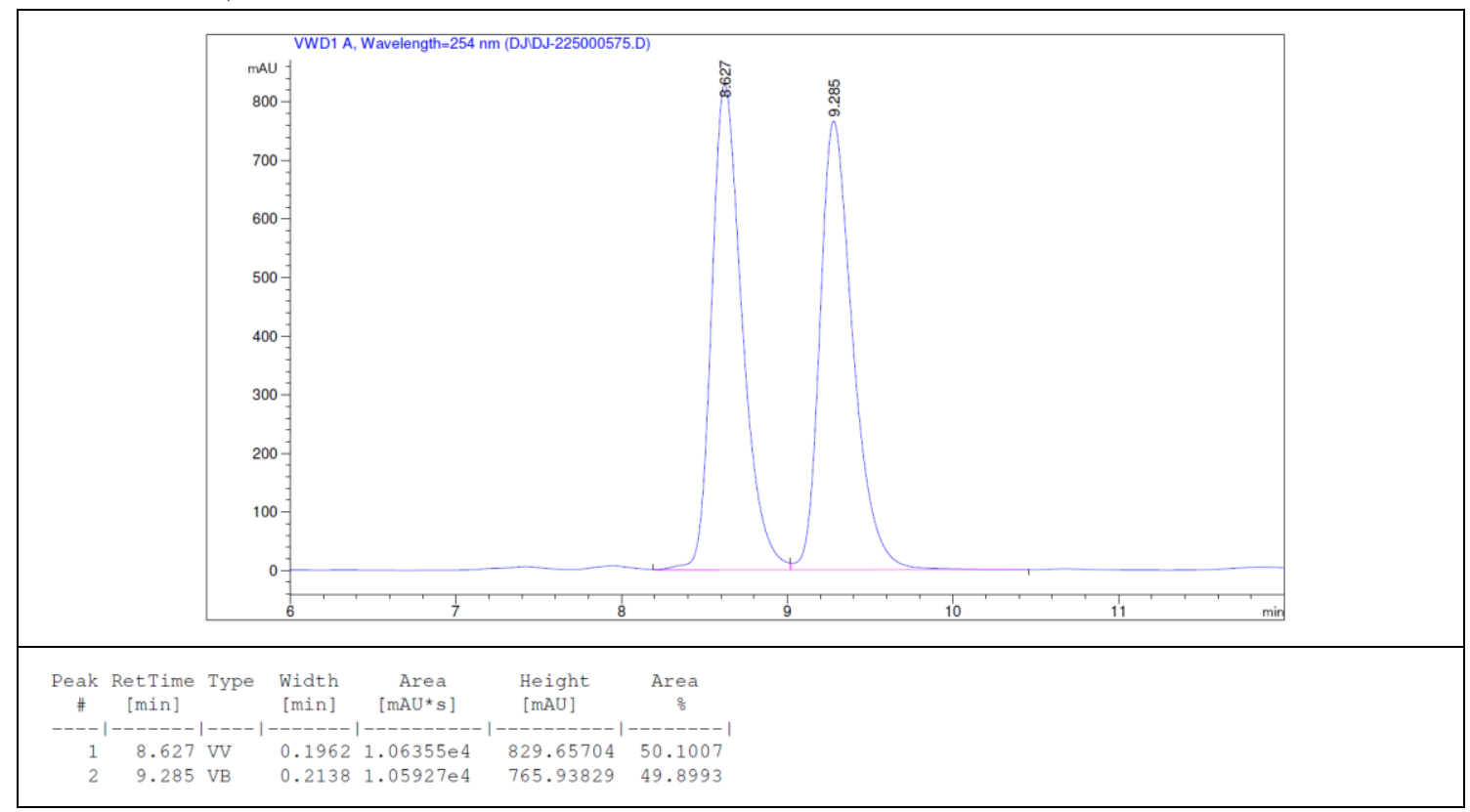




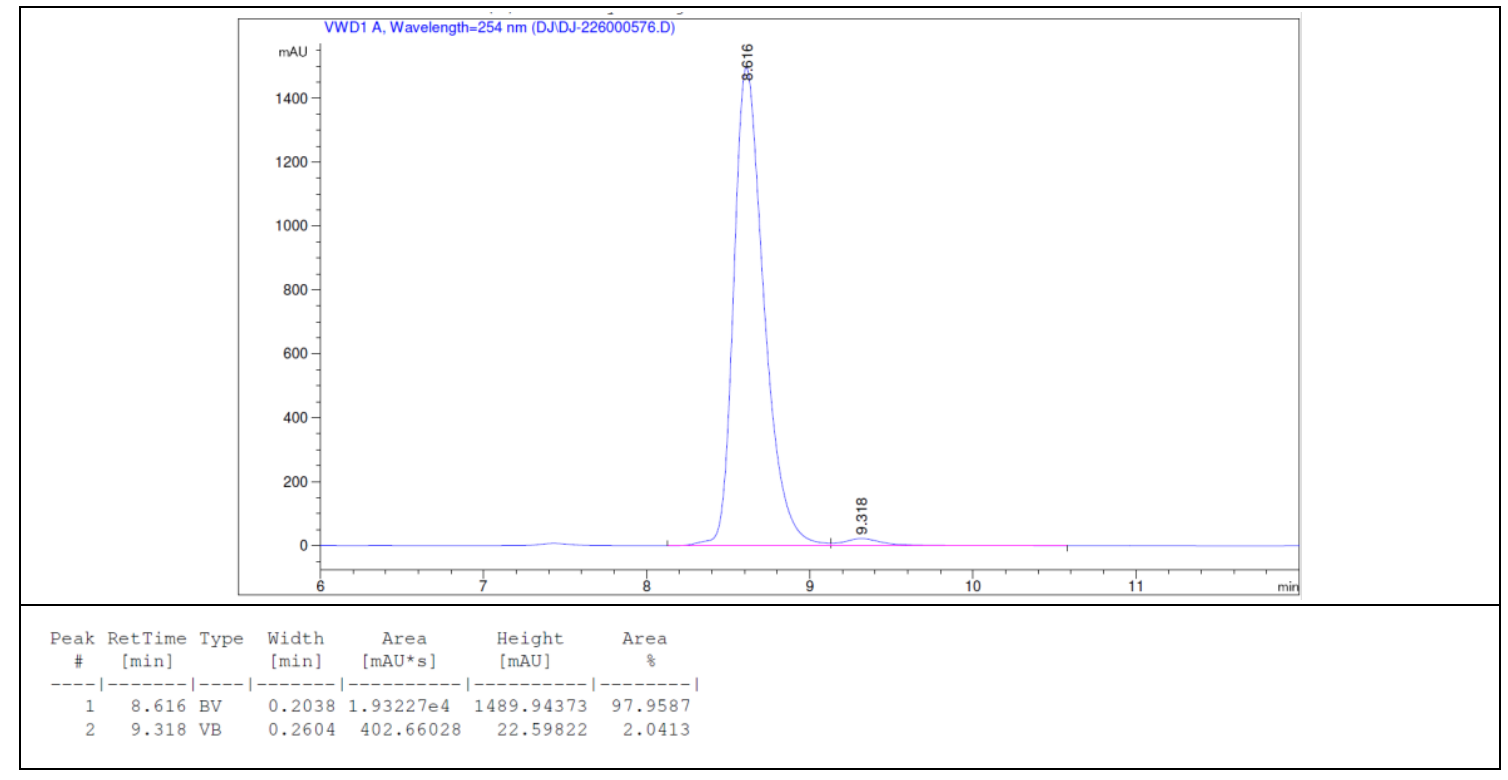

(2E,2'E)-2,2'-(2-benzyl-2-methylcyclopentane-1,3-diylidene)bis(1-(naphthalen-1-yl)-1-phenyl hydrazine) (4a)

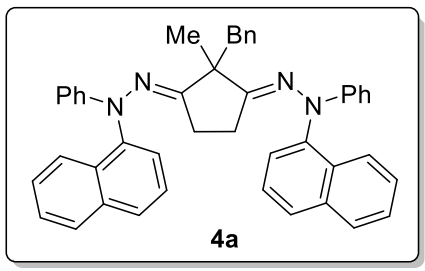

${ }^{1} \mathbf{H}$ NMR $\left(500 \mathrm{MHz}, \mathrm{CDCl}_{3}\right): \delta 7.82(\mathrm{~d}, J=8.5 \mathrm{~Hz}, 4 \mathrm{H}), 7.71(\mathrm{~d}, J=8.5 \mathrm{~Hz}, 2 \mathrm{H}), 7.43$ (t, $J=8.0$ $\mathrm{Hz}, 2 \mathrm{H}), 7.39-7.32(\mathrm{~m}, 4 \mathrm{H}), 7.22(\mathrm{~d}, J=8.5 \mathrm{~Hz}, 2 \mathrm{H}), 7.15(\mathrm{t}, J=8.0 \mathrm{~Hz}, 4 \mathrm{H}), 7.09$ (t, $J=7.0 \mathrm{~Hz}$, 1H), 7.04-6.98 (m, 4H), $6.88(\mathrm{t}, J=7.5 \mathrm{~Hz}, 2 \mathrm{H}), 6.79(\mathrm{~d}, J=8.5 \mathrm{~Hz}, 4 \mathrm{H}), 3.36(\mathrm{~s}, 2 \mathrm{H}), 1.76(\mathrm{~s}$, $3 \mathrm{H}), 1.54-1.50(\mathrm{~m}, 2 \mathrm{H}), 1.01-1.05(\mathrm{~m}, 2 \mathrm{H})$.

${ }^{13} \mathrm{C}$ NMR $\left(125 \mathrm{MHz}, \mathrm{CDCl}_{3}\right): \delta 169.7,150.1,142.4,138.7,134.4,130.9,129.7,128.5,128.3$, 127.9, 127.2, 126.6, 126.3, 126.1, 126.0, 125.9, 124.2, 120.4, 115.7, 54.5, 48.0, 29.2, 27.0.

IR (KBr) 3423.80, 3055.93, 2922.36, 2851.99, 1948.88, 1593.28, 1489.39, 1391.39, 1285.12, 1209.87, 1091.57, 799.78, 774.05, 692.30.

HRMS (ESI): $\mathrm{m} / \mathrm{z}$ Calcd for $\left[\mathrm{C}_{45} \mathrm{H}_{39} \mathrm{~N}_{4}, \mathrm{M}+\mathrm{H}\right]^{+}:$635.3169; Found: 635.3168. 


\section{Synthesis and characterization of keto-hydrazones 6.}

\subsection{General procedure for the synthesis of acyclic keto-hydrazone 6}

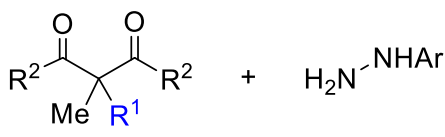

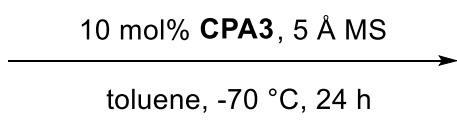

2

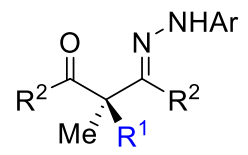

6

To a $4 \mathrm{~mL}$ vial was added the 1,3-dione 5 (0.15 mmol), $5 \AA$ MS (40 mg) and CPA3 (0.01 mmol) under argon atmosphere. Anhydrous toluene $(1.0 \mathrm{~mL})$ was then added. The reaction mixture was cooled to $-70^{\circ} \mathrm{C}$ and hydrazine $2(0.10 \mathrm{mmol})$ was added. The reaction mixture was then allowed to stir at $-70{ }^{\circ} \mathrm{C}$ for $24 \mathrm{~h}$. The crude reaction mixture was directly purified by silica gel column chromatography with hexanes/ethyl acetate $(10: 1 \mathrm{v} / \mathrm{v})$ as eluent to afford product $\mathbf{6}$ in pure form. 


\subsection{Characterization of 6}

( $R, E)$-3-methyl-3-phenyl-4-(2-phenylhydrazono)pentan-2-one (6a)

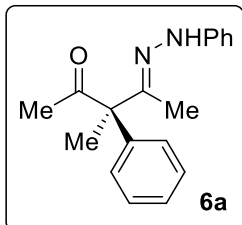

Reaction temperature of $-60^{\circ} \mathrm{C}$ was used with $36 \mathrm{~h}$ reaction time.

Yellow oil (22.7 mg, 81\% yield).

${ }^{1} \mathbf{H}$ NMR $\left(500 \mathrm{MHz}, \mathrm{CD}_{3} \mathrm{COCD}_{3}\right): \delta 8.45(\mathrm{~s}, 1 \mathrm{H}), 7.40-7.36(\mathrm{~m}, 2 \mathrm{H}), 7.33-7.28(\mathrm{~m}, 3 \mathrm{H})$, 7.19-7.15 (m, 2H), 7.09-7.06 (m, 2H), $6.75(\mathrm{tt}, J=7.5 \mathrm{~Hz}, J=1.0 \mathrm{~Hz}, 1 \mathrm{H}), 2.08(\mathrm{~s}, 3 \mathrm{H}), 1.89(\mathrm{~s}$, $3 \mathrm{H}), 1.72(\mathrm{~s}, 3 \mathrm{H})$.

${ }^{13}$ C NMR $\left(125 \mathrm{MHz}, \mathrm{CD}_{3} \mathrm{COCD}_{3}\right): \delta 207.1,147.9,147.2,142.5,129.6,129.1,128.9,127.7,119.8$, $113.5,64.9,28.0,21.8,13.7$.

IR $v\left(\mathrm{~cm}^{-1}\right): 3331.77,3060.40,2982.69,2928.21,2854.02,1957.03,1715.30,1598.50$, $1494.92,1447.49,1229.62,1050.44,763.73,699.36$.

HRMS (ESI): $\mathrm{m} / \mathrm{z}$ Calcd for $\left[\mathrm{C}_{18} \mathrm{H}_{21} \mathrm{~N}_{2} \mathrm{O}, \mathrm{M}+\mathrm{H}\right]^{+}: 281.1648$; Found: 281.1655.

Optical Rotation: $[\alpha]_{\mathrm{D}}^{25}=9.4\left(0.5, \mathrm{CHCl}_{3}\right) .90 \%$ ee. The absolute configuration of the product was assigned by analogy to 6b. (HPLC condition: Chiralpak OD-H column, $n$-hexane/ $i$-PrOH $=$ $90: 10$, flow rate $=1.0 \mathrm{~mL} / \mathrm{min}$, wavelength $=254 \mathrm{~nm}, \mathrm{t}_{\mathrm{R}}=11.87 \mathrm{~min}$ for minor isomer, $\mathrm{t}_{\mathrm{R}}=12.94$ min for major isomer).

\begin{tabular}{|c|c|c|c|}
\hline & \\
\hline
\end{tabular}




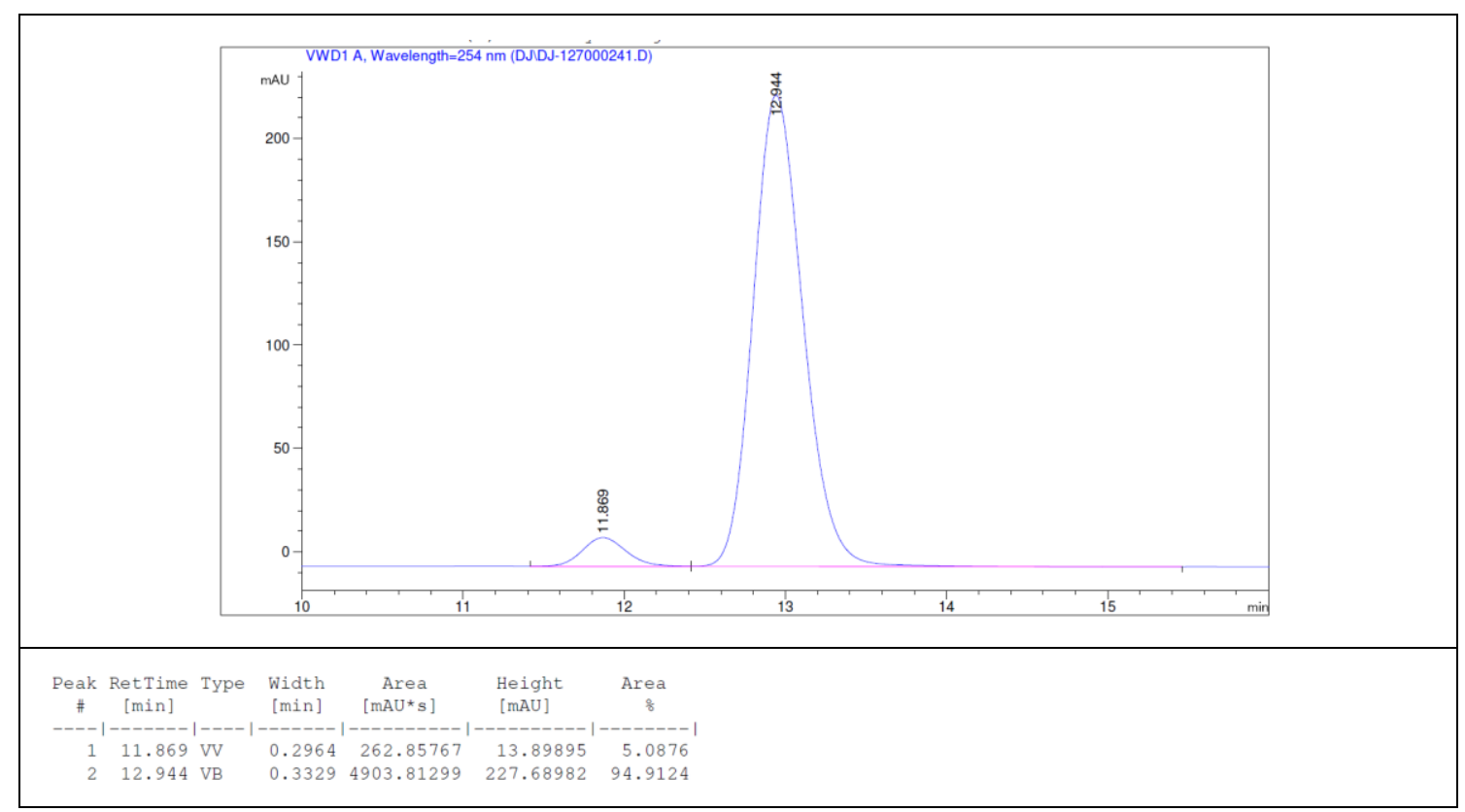

(R,E)-3-benzyl-3-methyl-4-(2-phenylhydrazono)pentan-2-one (6b)

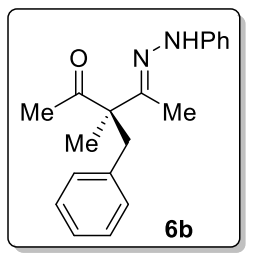

Crystalline solid (24.4 mg, $83 \%$ yield).

${ }^{1} \mathbf{H}$ NMR $\left(500 \mathrm{MHz}, \mathrm{CD}_{3} \mathrm{COCD}_{3}\right): \delta 8.42(\mathrm{~s}, 1 \mathrm{H}), 7.22-7.16(\mathrm{~m}, 5 \mathrm{H}), 7.13-7.09(\mathrm{~m}, 4 \mathrm{H}), 6.74(\mathrm{tt}$, $J=7.0 \mathrm{~Hz}, J=1.0 \mathrm{~Hz}, 1 \mathrm{H}), 3.21(\mathrm{~d}, J=14.0 \mathrm{~Hz}, 1 \mathrm{H}), 3.16(\mathrm{~d}, J=14.0 \mathrm{~Hz}, 1 \mathrm{H}), 2.11(\mathrm{~s}, 3 \mathrm{H}), 1.85$ (s, 3H), $1.26(\mathrm{~s}, 3 \mathrm{H})$.

${ }^{13} \mathrm{C}$ NMR (125 MHz, $\left.\mathrm{CD}_{3} \mathrm{COCD}_{3}\right): \delta 209.2,147.3,145.1,139.0,131.1,129.5,128.5,126.8,119.7$, $113.5,61.0,41.1,26.1,19.5,13.3$.

IR $v\left(\mathrm{~cm}^{-1}\right): 3335.47,3059.98,3028.79,2992.95,2932.46,1952.83,1699.12,1602.14$, 1496.00, 1354.82, 1254.00, 1089.19, 752.31, 696.81.

HRMS (ESI): m/z Calcd for $\left[\mathrm{C}_{19} \mathrm{H}_{23} \mathrm{~N}_{2} \mathrm{O}, \mathrm{M}+\mathrm{H}\right]^{+}: 295.1805$; Found: 295.1805.

Optical Rotation: $[\alpha]_{\mathrm{D}}^{25}=-7.4\left(1.0, \mathrm{CHCl}_{3}\right) .94 \%$ ee. The absolute configuration $\mathbf{6 b}$ was assigned based on single crystal x-ray analysis. (HPLC condition: Chiralpak OD-H column, $n$-hexane $/ i$-PrOH $=90: 10$, flow rate $=1.0 \mathrm{~mL} / \mathrm{min}$, wavelength $=254 \mathrm{~nm}, \mathrm{t}_{\mathrm{R}}=10.58 \mathrm{~min}$ for major isomer, $\mathrm{t}_{\mathrm{R}}=11.4 \mathrm{~min}$ for minor isomer). 


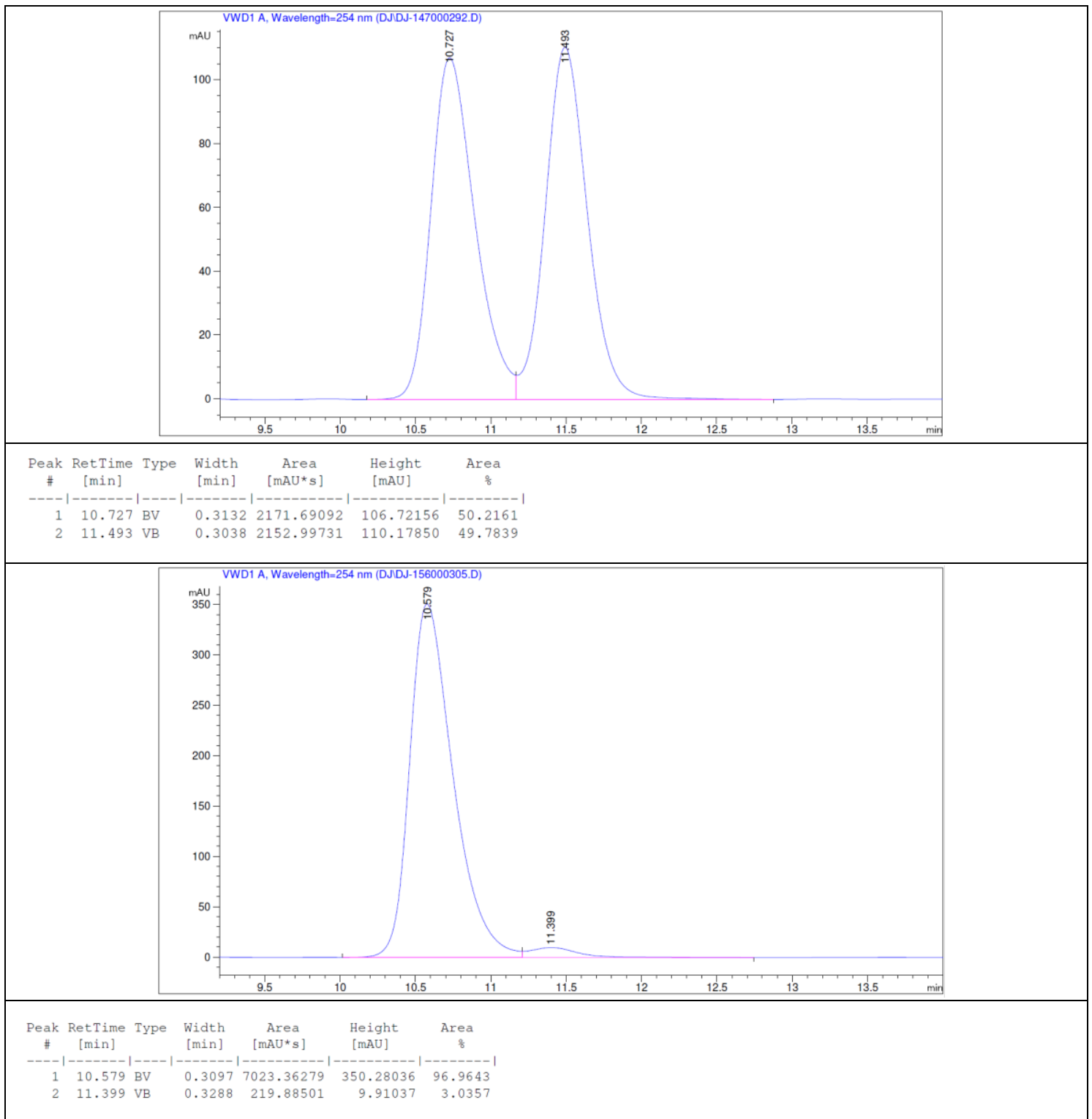

(R,E)-3-methyl-3-(4-methylbenzyl)-4-(2-phenylhydrazono)pentan-2-one (6c)

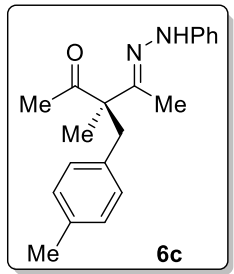

Colorless oil (24 mg, 78\% yield).

${ }^{1} \mathbf{H}$ NMR $\left(400 \mathrm{MHz}, \mathrm{CD}_{3} \mathrm{COCD}_{3}\right): \delta 8.41(\mathrm{~s}, 1 \mathrm{H}), 7.18-7.14(\mathrm{~m}, 2 \mathrm{H}), 7.11-7.08(\mathrm{~m}, 2 \mathrm{H})$, 7.03-6.98 (m, 4H), $6.74(\mathrm{tt}, J=7.0 \mathrm{~Hz}, J=1.0 \mathrm{~Hz}, 1 \mathrm{H}), 3.16(\mathrm{~d}, J=14.0 \mathrm{~Hz}, 1 \mathrm{H}), 3.12(\mathrm{~d}, J=$ $14.0 \mathrm{~Hz}, 1 \mathrm{H}), 2.24$ (s, 3H), 2.10 (s, 3H), 1.85 (s, 3H), 1.25 (s, 3H).

${ }^{13} \mathrm{C}$ NMR $\left(125 \mathrm{MHz}, \mathrm{CD}_{3} \mathrm{COCD}_{3}\right): \delta 209.2,147.3,145.3,136.1,135.8,130.9,129.5,129.2,119.7$, $113.5,61.0,40.7,26.1,20.8,19.6,13.3$.

IR $v\left(\mathrm{~cm}^{-1}\right): 3381.66,2961.57,2924.94,2855.53,1906.32,1699.23,1600.93,1513.99$, $1260.99,1095.83,807.47,765.83,690.46$.

HRMS (ESI): $\mathrm{m} / \mathrm{z}$ Calcd for $\left[\mathrm{C}_{20} \mathrm{H}_{25} \mathrm{~N}_{2} \mathrm{O}, \mathrm{M}+\mathrm{H}\right]^{+}: 309.1961$; Found: 309.1965. 
Optical Rotation: $[\alpha]_{\mathrm{D}}^{25}=-6.8\left(0.5, \mathrm{CHCl}_{3}\right) .91 \%$ ee. The absolute configuration of the product was assigned by analogy to $\mathbf{6 b}$. (HPLC condition: Chiralpak OD-H column, $n$-hexane/ $i-\mathrm{PrOH}=$ $90: 10$, flow rate $=1.0 \mathrm{~mL} / \mathrm{min}$, wavelength $=254 \mathrm{~nm}, \mathrm{t}_{\mathrm{R}}=9.15 \mathrm{~min}$ for major isomer, $\mathrm{t}_{\mathrm{R}}=11.0$ min for minor isomer).

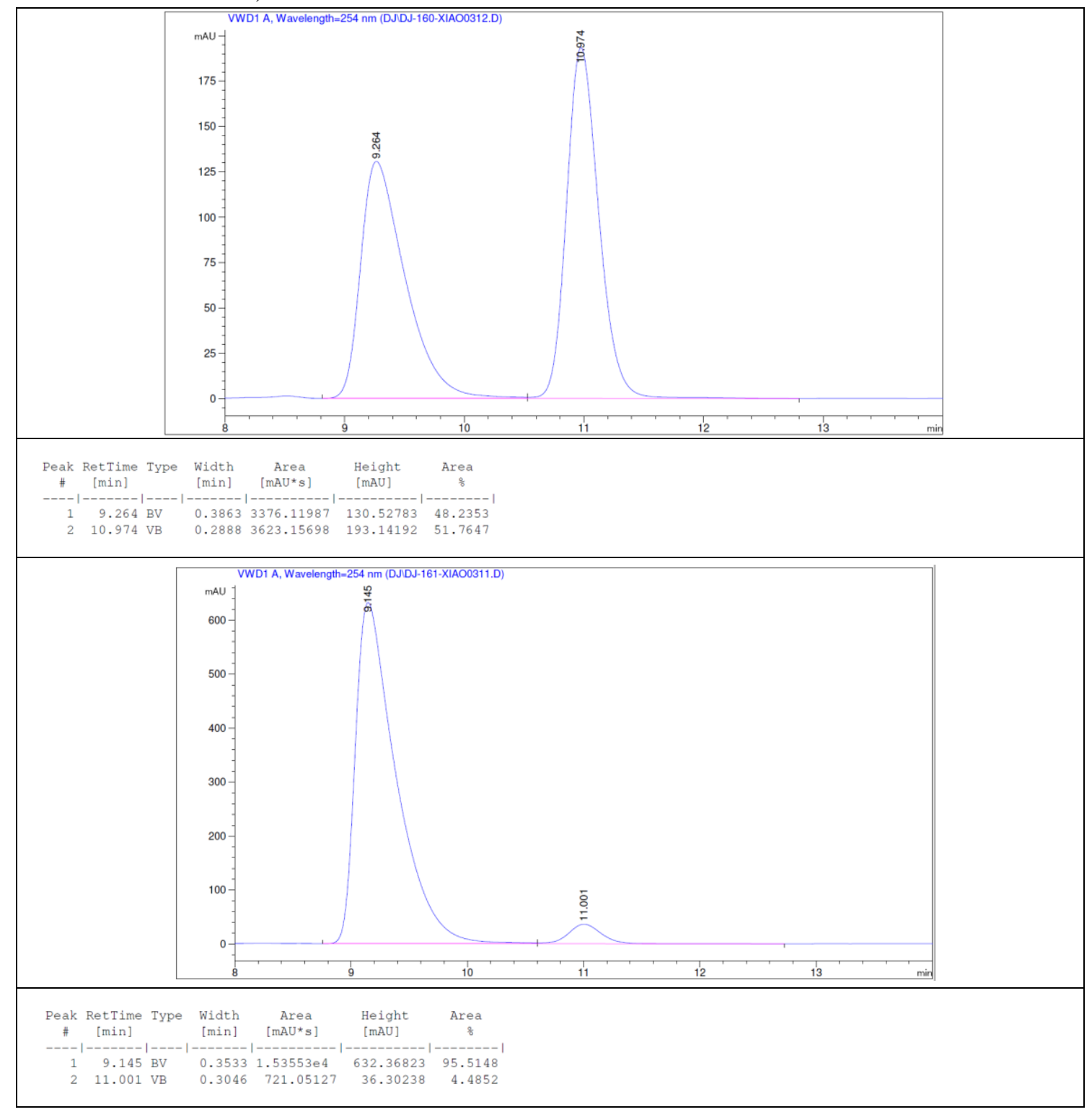

(R,E)-3-(3-methoxybenzyl)-3-methyl-4-(2-phenylhydrazono)pentan-2-one (6d)

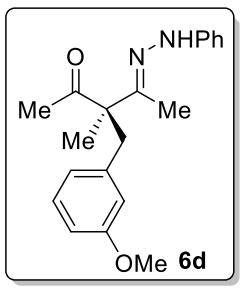

Colorless oil (27.8 mg, $83 \%$ yield).

${ }^{1} \mathbf{H}$ NMR $\left(400 \mathrm{MHz}, \mathrm{CD}_{3} \mathrm{COCD}_{3}\right): \delta 8.43(\mathrm{~s}, 1 \mathrm{H}), 7.19-7.14(\mathrm{~m}, 2 \mathrm{H}), 7.12-7.09(\mathrm{~m}, 3 \mathrm{H})$, 6.76-6.68 (m, 4H), $3.70(\mathrm{~s}, 3 \mathrm{H}), 3.16(\mathrm{~s}, 2 \mathrm{H}), 2.11(\mathrm{~s}, 3 \mathrm{H}), 1.86(\mathrm{~s}, 3 \mathrm{H}), 1.27(\mathrm{~s}, 3 \mathrm{H})$.

${ }^{13} \mathrm{C}$ NMR (125 MHz, $\left.\mathrm{CD}_{3} \mathrm{COCD}_{3}\right): \delta 209.2,160.1,147.3,145.1,140.5,129.5,129.4,123.4,119.7$, 
116.7, 113.5, 112.3, 61.0, 55.1, 41.2, 26.1, 19.6, 13.3.

IR $v\left(\mathrm{~cm}^{-1}\right): 3400.66,2935.37,2836.52,1930.88,1698.24,1600.53,1490.42,1455.05$, 1356.88, 1264.98, 1047.24, 781.72, 695.14.

HRMS (ESI): $\mathrm{m} / \mathrm{z}$ Calcd for $\left[\mathrm{C}_{20} \mathrm{H}_{25} \mathrm{~N}_{2} \mathrm{O}_{2}, \mathrm{M}+\mathrm{H}\right]^{+}: 325.1911$; Found: 325.1917.

Optical Rotation: $[\alpha]_{\mathrm{D}}^{25}=-6.1\left(0.4, \mathrm{CHCl}_{3}\right) .94 \% e e$. The absolute configuration of the product was assigned by analogy to $\mathbf{6 b}$. (HPLC condition: Chiralpak OD-H column, $n$-hexane/ $i-\mathrm{PrOH}=$ 95:5, flow rate $=1.0 \mathrm{~mL} / \mathrm{min}$, wavelength $=254 \mathrm{~nm}, \mathrm{t}_{\mathrm{R}}=24.04 \mathrm{~min}$ for major isomer, $\mathrm{t}_{\mathrm{R}}=27.04$ min for minor isomer).

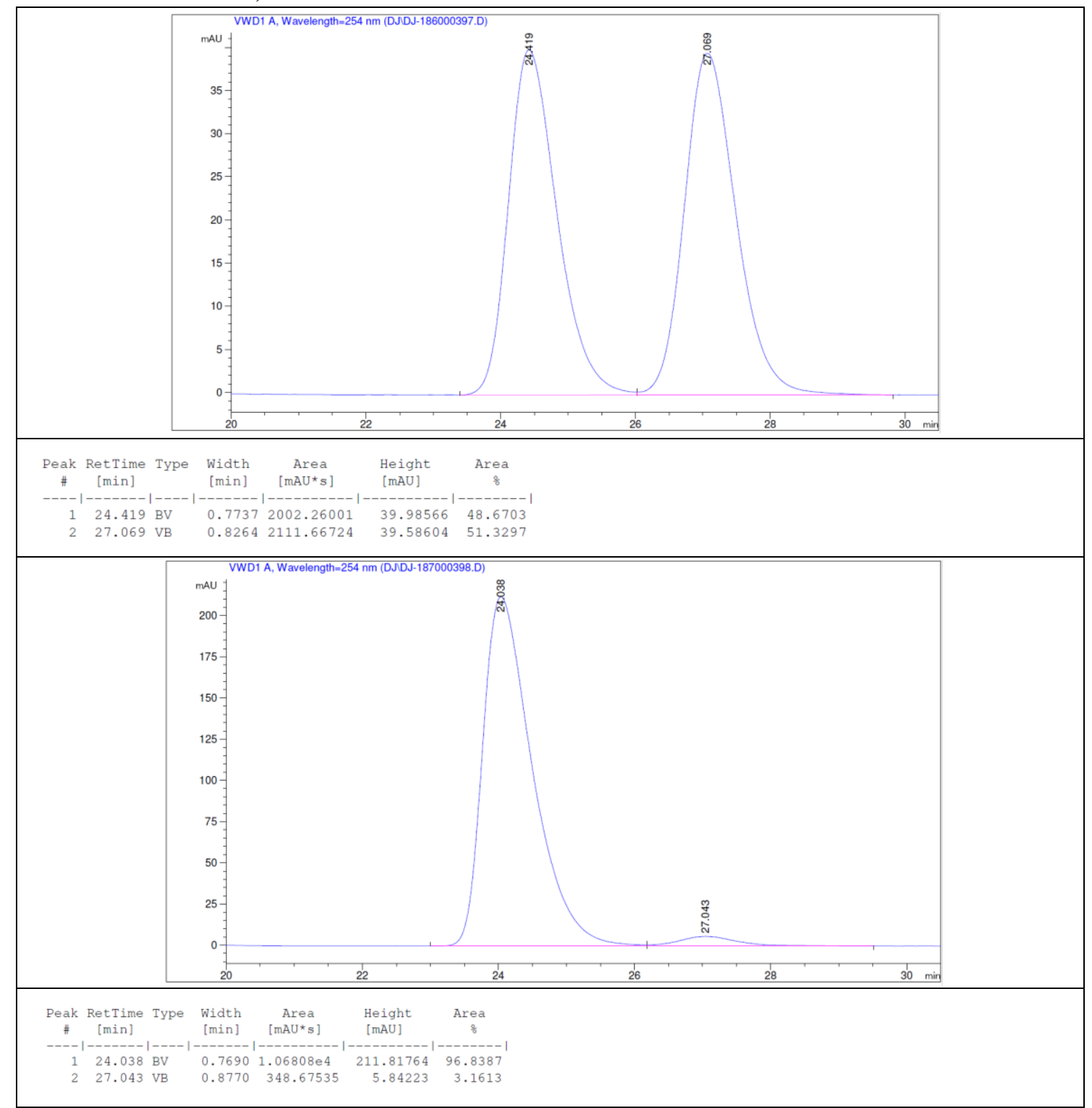

(R,E)-3-(4-chlorobenzyl)-3-methyl-4-(2-phenylhydrazono)pentan-2-one (6e)

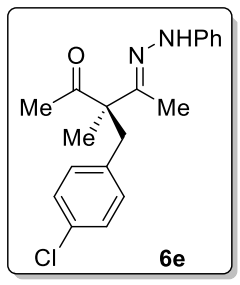

Colorless oil (25.9 mg, $79 \%$ yield). 
${ }^{1} \mathbf{H}$ NMR $\left(400 \mathrm{MHz}, \mathrm{CD}_{3} \mathrm{COCD}_{3}\right): \delta 8.43(\mathrm{~s}, 1 \mathrm{H}), 7.25-7.22(\mathrm{~m}, 2 \mathrm{H}), 7.18-7.13(\mathrm{~m}, 4 \mathrm{H})$, 7.10-7.07 (m, 2H), 6.74 (tt, $J=7.0 \mathrm{~Hz}, J=1.0 \mathrm{~Hz}, 1 \mathrm{H}), 3.19$ (d, $J=14.0 \mathrm{~Hz}, 1 \mathrm{H}), 3.15$ (d, $J=$ $14.0 \mathrm{~Hz}, 1 \mathrm{H}), 2.11(\mathrm{~s}, 3 \mathrm{H}), 1.86(\mathrm{~s}, 3 \mathrm{H}), 1.26(\mathrm{~s}, 3 \mathrm{H})$.

${ }^{13} \mathrm{C}$ NMR (125 MHz, $\left.\mathrm{CD}_{3} \mathrm{COCD}_{3}\right): \delta 209.0,147.2,144.8,138.0,132.8,132.3,129.5,128.5,119.8$, 113.5, 60.9, 40.4, 26.1, 19.5, 13.3 .

IR $v\left(\mathrm{~cm}^{-1}\right): 3385.04,2988.71,2928.33,2854.78,1904.61,1699.64,1595.63,1491.77$, 1356.16, 1094.96, 1016.17, 766.05, 689.70.

HRMS (ESI): $\mathrm{m} / \mathrm{z}$ Calcd for $\left[\mathrm{C}_{19} \mathrm{H}_{22} \mathrm{ClN}_{2} \mathrm{O}, \mathrm{M}+\mathrm{H}\right]^{+}:$329.1415; Found: 329.1403.

Optical Rotation: $[\alpha]_{\mathrm{D}}^{25}=-5.2\left(0.7, \mathrm{CHCl}_{3}\right)$. $97 \%$ ee. The absolute configuration of the product was assigned by analogy to $\mathbf{6 b}$. (HPLC condition: Chiralpak AD-H column, $n$-hexane $/ i-\mathrm{PrOH}=$ $95: 5$, flow rate $=1.0 \mathrm{~mL} / \mathrm{min}$, wavelength $=254 \mathrm{~nm}, \mathrm{t}_{\mathrm{R}}=9.31$ min for major isomer, $\mathrm{t}_{\mathrm{R}}=10.18$ min for minor isomer).

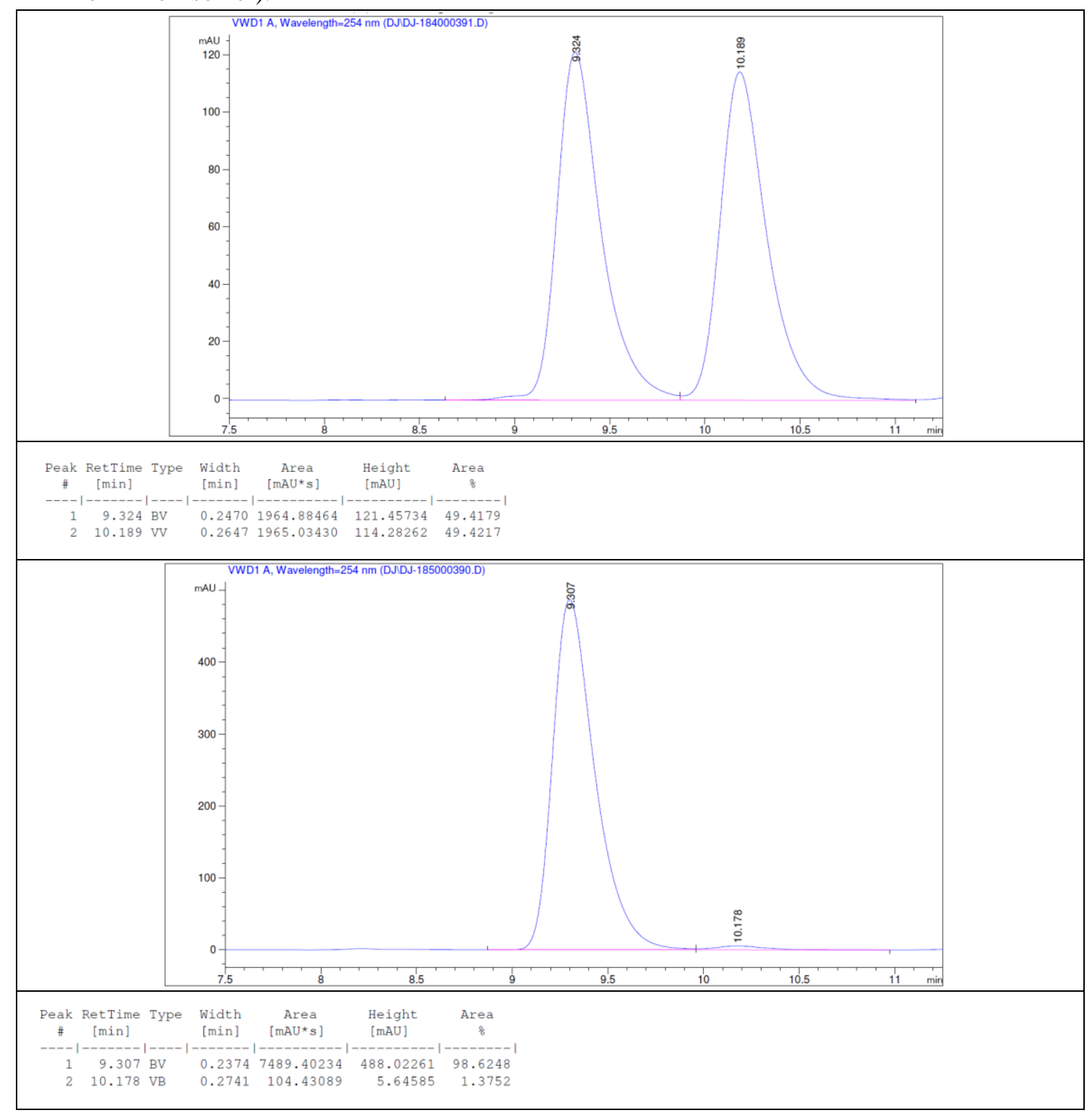

(R,E)-3-(3-chlorobenzyl)-3-methyl-4-(2-phenylhydrazono)pentan-2-one (6f) 


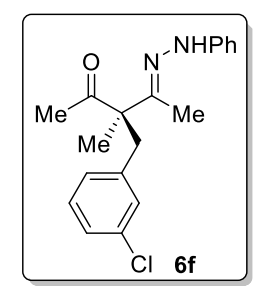

Colorless oil (27.5 mg, 84\% yield).

${ }^{1} \mathbf{H}$ NMR $\left(500 \mathrm{MHz}, \mathrm{CD}_{3} \mathrm{COCD}_{3}\right): \delta 8.45(\mathrm{~s}, 1 \mathrm{H}), 7.23-7.15(\mathrm{~m}, 5 \mathrm{H}), 7.10-7.07(\mathrm{~m}, 3 \mathrm{H}), 6.75(\mathrm{tt}$, $J=7.5 \mathrm{~Hz}, J=1.0 \mathrm{~Hz}, 1 \mathrm{H}), 3.21(\mathrm{~d}, J=14.0 \mathrm{~Hz}, 1 \mathrm{H}), 3.16(\mathrm{~d}, J=14.0 \mathrm{~Hz}, 1 \mathrm{H}), 2.12(\mathrm{~s}, 3 \mathrm{H})$, $1.87(\mathrm{~s}, 3 \mathrm{H}), 1.28(\mathrm{~s}, 3 \mathrm{H})$

${ }^{13} \mathrm{C}$ NMR (125 MHz, $\left.\mathrm{CD}_{3} \mathrm{COCD}_{3}\right): \delta$ 209.0, 147.2, 144.7, 141.6, 133.8, 131.0, 130.1, 129.7,129.5, $126.9,119.8,113.5,60.9,40.6,26.0,19.5,13.3$.

IR $v\left(\mathrm{~cm}^{-1}\right): 3385.70,3063.61,2988.18,2930.19,2855.70,1951.52,1700.21,1596.98$, 1453.98, 1207.60, 1083.57, 766.00, 688.77.

HRMS (ESI): m/z Calcd for $\left[\mathrm{C}_{19} \mathrm{H}_{22} \mathrm{ClN}_{2} \mathrm{O}, \mathrm{M}+\mathrm{H}\right]^{+}: 329.1415$; Found: 329.1403.

Optical Rotation: $[\alpha]_{\mathrm{D}}^{25}=-5.9\left(0.5, \mathrm{CHCl}_{3}\right) .94 \%$ ee. The absolute configuration of the product was assigned by analogy to 6b. (HPLC condition: Chiralpak AD-H column, $n$-hexane $/ i-\mathrm{PrOH}=$ $98: 2$, flow rate $=1.0 \mathrm{~mL} / \mathrm{min}$, wavelength $=254 \mathrm{~nm}, \mathrm{t}_{\mathrm{R}}=13.2 \mathrm{~min}$ for major isomer, $\mathrm{t}_{\mathrm{R}}=14.0 \mathrm{~min}$ for minor isomer).

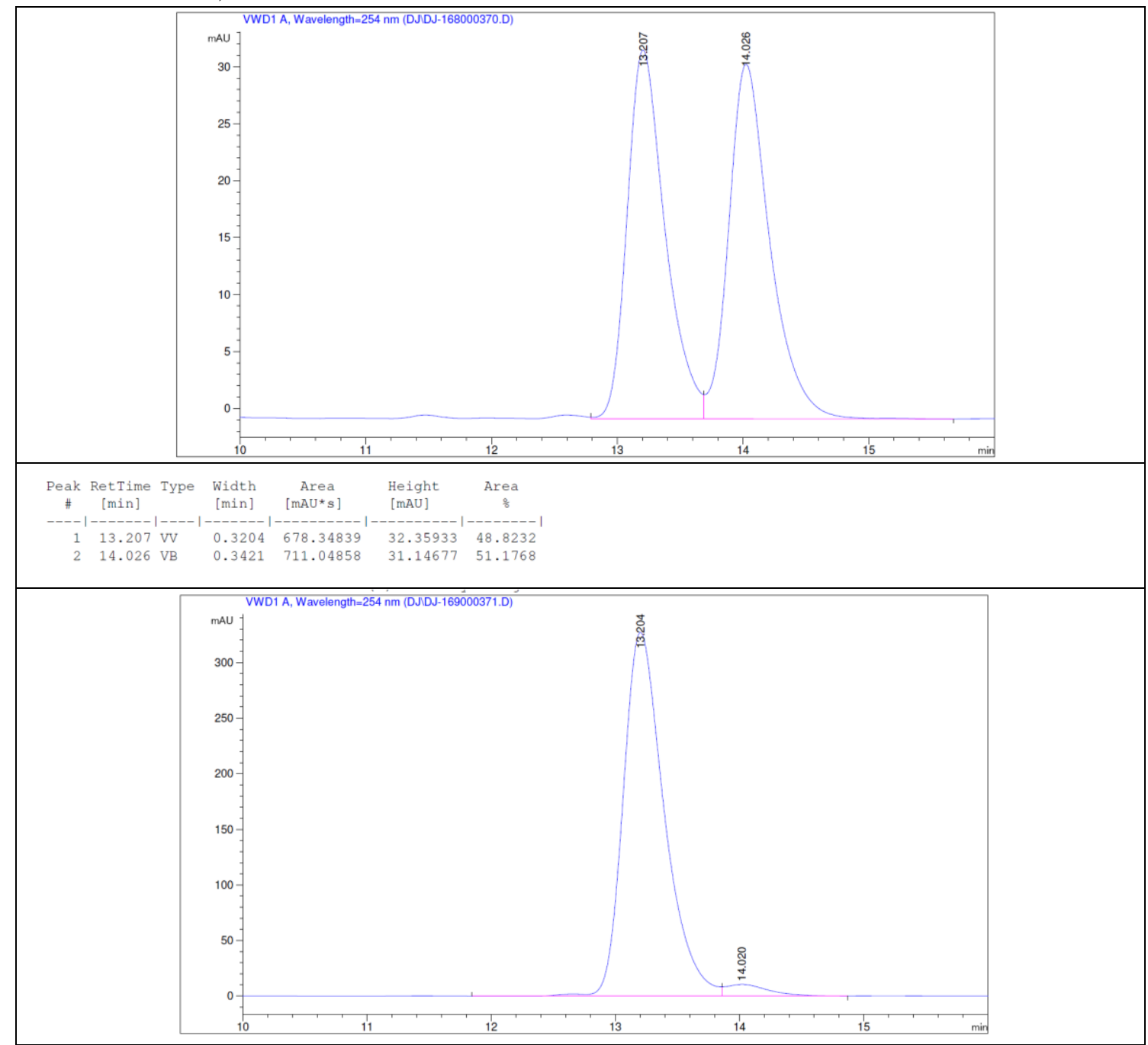




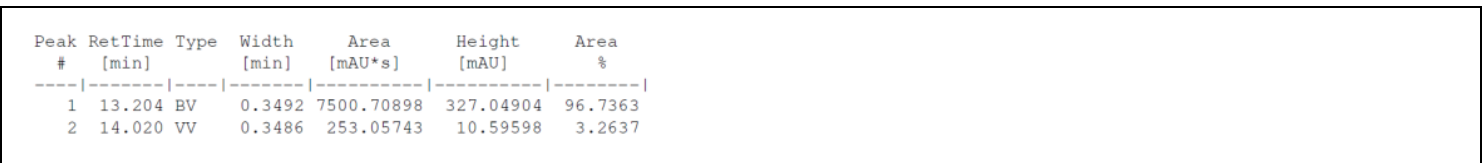

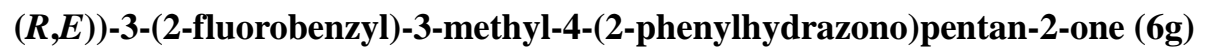

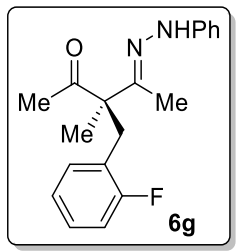

Colorless oil (25.6 mg, $82 \%$ yield).

${ }^{1} \mathbf{H}$ NMR $\left(500 \mathrm{MHz}, \mathrm{CD}_{3} \mathrm{COCD}_{3}\right): \delta 8.44(\mathrm{~s}, 1 \mathrm{H}), 7.22-7.14(\mathrm{~m}, 4 \mathrm{H}), 7.09-7.07(\mathrm{~m}, 2 \mathrm{H})$, 7.05-6.99 (m, 2H), $6.74(\mathrm{tt}, J=7.5 \mathrm{~Hz}, J=1.0 \mathrm{~Hz}, 1 \mathrm{H}), 3.28(\mathrm{~d}, J=14.0 \mathrm{~Hz}, 1 \mathrm{H}), 3.20(\mathrm{~d}, J=$ $14.0 \mathrm{~Hz}, 1 \mathrm{H}), 2.12$ (s, 3H), 1.88 (s, 3H), 1.25 (d, $J=1.5 \mathrm{~Hz}, 3 \mathrm{H})$.

${ }^{13}$ C NMR (125 MHz, $\left.\mathrm{CD}_{3} \mathrm{COCD}_{3}\right): \delta 209.0,162.3\left(\mathrm{~d},{ }^{1} J_{\mathrm{CF}}=243.5 \mathrm{~Hz}\right), 147.2,144.7,133.5(\mathrm{~d}$, $\left.{ }^{4} J_{\mathrm{CF}}=4.5 \mathrm{~Hz}\right), 129.5,129.0\left(\mathrm{~d},{ }^{3} J_{\mathrm{CF}}=8.5 \mathrm{~Hz}\right), 125.7\left(\mathrm{~d},{ }^{2} J_{\mathrm{CF}}=17.5 \mathrm{~Hz}\right), 124.4\left(\mathrm{~d},{ }^{4} J_{\mathrm{CF}}=3.5 \mathrm{~Hz}\right)$, $119.7,115.5\left(\mathrm{~d},{ }^{2} J_{\mathrm{CF}}=24.5 \mathrm{~Hz}\right), 113.5,60.9,33.4,25.9,19.1,13.3$.

${ }^{19}$ F NMR $\left(469 \mathrm{MHz}, \mathrm{CD}_{3} \mathrm{COCD}_{3}\right): \delta-116.9(\mathrm{~s})$.

IR $v\left(\mathrm{~cm}^{-1}\right): 3400.71,2989.92,2932.37,1955.07,1700.78,1585.22,1492.42,1356.54$, $1229.96,1113.37,759.39,690.89$.

HRMS (ESI): $\mathrm{m} / \mathrm{z}$ Calcd for $\left[\mathrm{C}_{19} \mathrm{H}_{22} \mathrm{FN} 2 \mathrm{O}, \mathrm{M}+\mathrm{H}\right]^{+}: 313.1711$; Found: 313.1700 .

Optical Rotation: $[\alpha]_{\mathrm{D}}^{25}=-4.3\left(0.6, \mathrm{CHCl}_{3}\right) .95 \% e e$. The absolute configuration of the product was assigned by analogy to $6 \mathbf{b}$. (HPLC condition: Chiralpak AS-H column, $n$-hexane/ $i$-PrOH $=$ $90: 10$, flow rate $=1.0 \mathrm{~mL} / \mathrm{min}$, wavelength $=254 \mathrm{~nm}, \mathrm{t}_{\mathrm{R}}=11.29 \mathrm{~min}$ for major isomer, $\mathrm{t}_{\mathrm{R}}=14.02$ min for minor isomer).

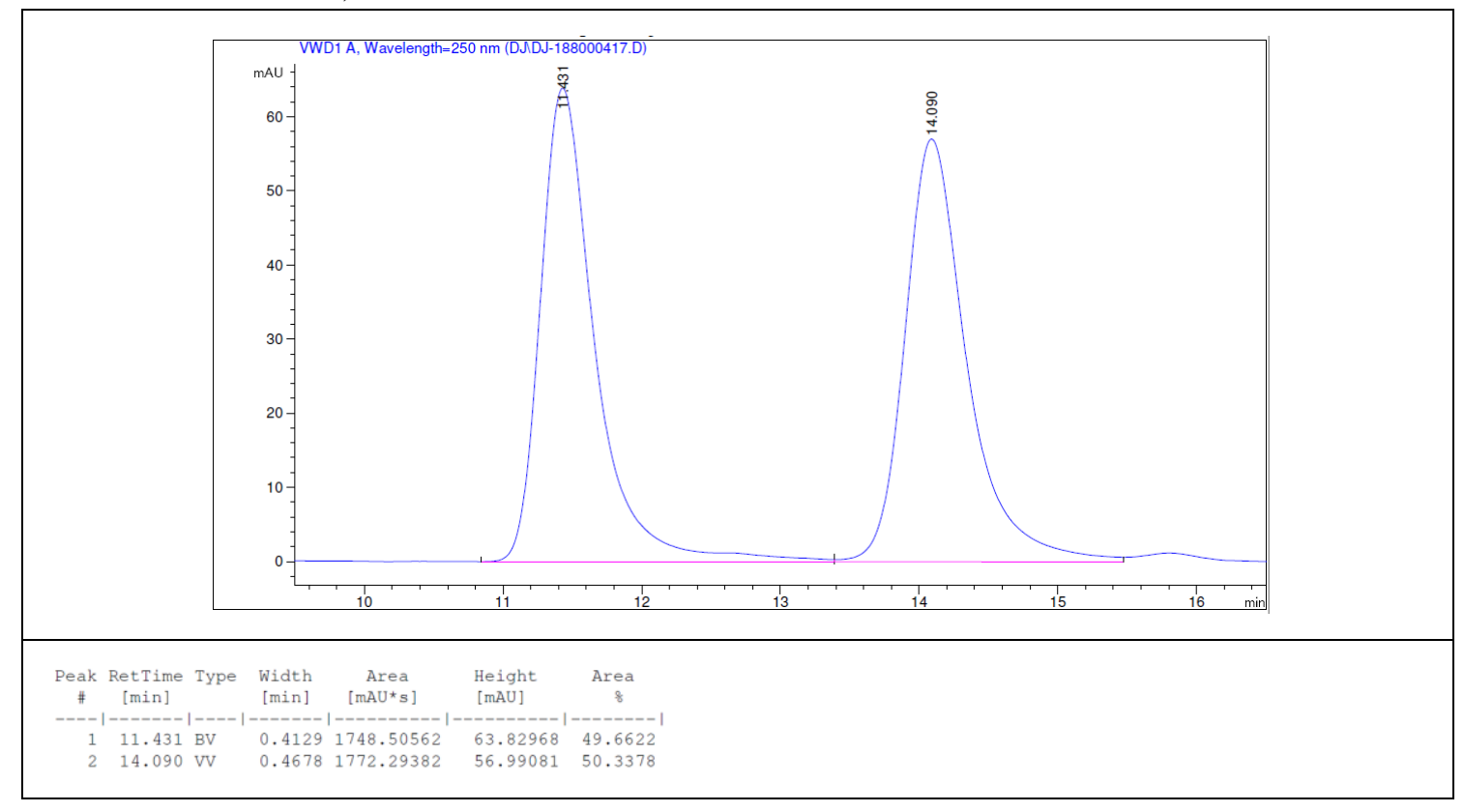




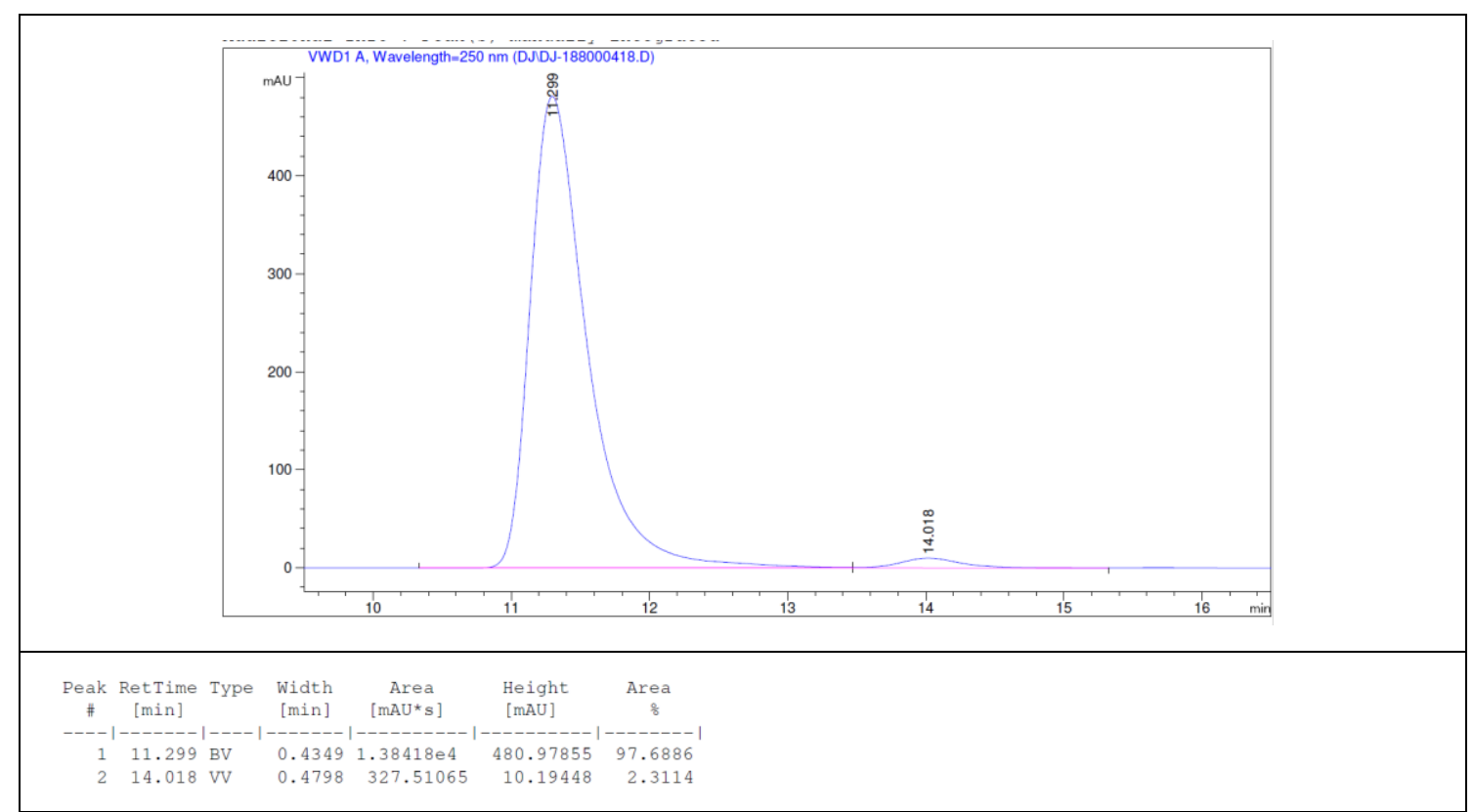

(R,E)-3-methyl-3-(4-nitrobenzyl)-4-(2-phenylhydrazono)pentan-2-one (6h)

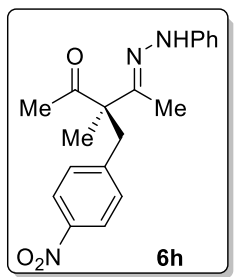

Colorless oil (24.7 $\mathrm{mg}, 73 \%$ yield).

${ }^{1} \mathbf{H}$ NMR $\left(500 \mathrm{MHz}, \mathrm{CD}_{3} \mathrm{COCD}_{3}\right): \delta 8.46(\mathrm{~s}, 1 \mathrm{H}), 8.10-8.08(\mathrm{~m}, 2 \mathrm{H}), 7.44-7.41(\mathrm{~m}, 2 \mathrm{H})$, 7.17-7.14 (m, 2H), 7.08-7.05 (m, 2H), $6.74(\mathrm{tt}, J=7.5 \mathrm{~Hz}, J=1.0 \mathrm{~Hz}, 1 \mathrm{H}), 3.32$ (s, 2H), $2.14(\mathrm{~s}$, $3 \mathrm{H}), 1.90$ (s, 3H), 1.30 (s, 3H).

${ }^{13}$ C NMR $\left(125 \mathrm{MHz}, \mathrm{CD}_{3} \mathrm{COCD}_{3}\right): \delta 208.8,147.6,147.4,147.1,144.3,132.3,129.5,123.4,119.9$, $113.5,61.0,40.8,26.0,19.6,13.3$.

IR $v\left(\mathrm{~cm}^{-1}\right): 3404.45,2925.34,2850.23,1934.42,1698.94,1603.58,1519.10,1346.84$, 1268.94, 854.70, 753.40, 694.30.

HRMS (ESI): $\mathrm{m} / \mathrm{z}$ Calcd for $\left[\mathrm{C}_{19} \mathrm{H}_{22} \mathrm{~N}_{3} \mathrm{O}_{3}, \mathrm{M}+\mathrm{H}\right]^{+}:$340.1656; Found: 340.1656.

Optical Rotation: $[\alpha]_{\mathrm{D}}^{25}=-5.2\left(0.7, \mathrm{CHCl}_{3}\right) .92 \%$ ee. The absolute configuration of the product was assigned by analogy to 6b. (HPLC condition: Chiralpak OD-H column, $n$-hexane/ $i$-PrOH = $80: 20$, flow rate $=1.0 \mathrm{~mL} / \mathrm{min}$, wavelength $=254 \mathrm{~nm}, \mathrm{t}_{\mathrm{R}}=14.22 \mathrm{~min}$ for major isomer, $\mathrm{t}_{\mathrm{R}}=16.98$ min for minor isomer). 


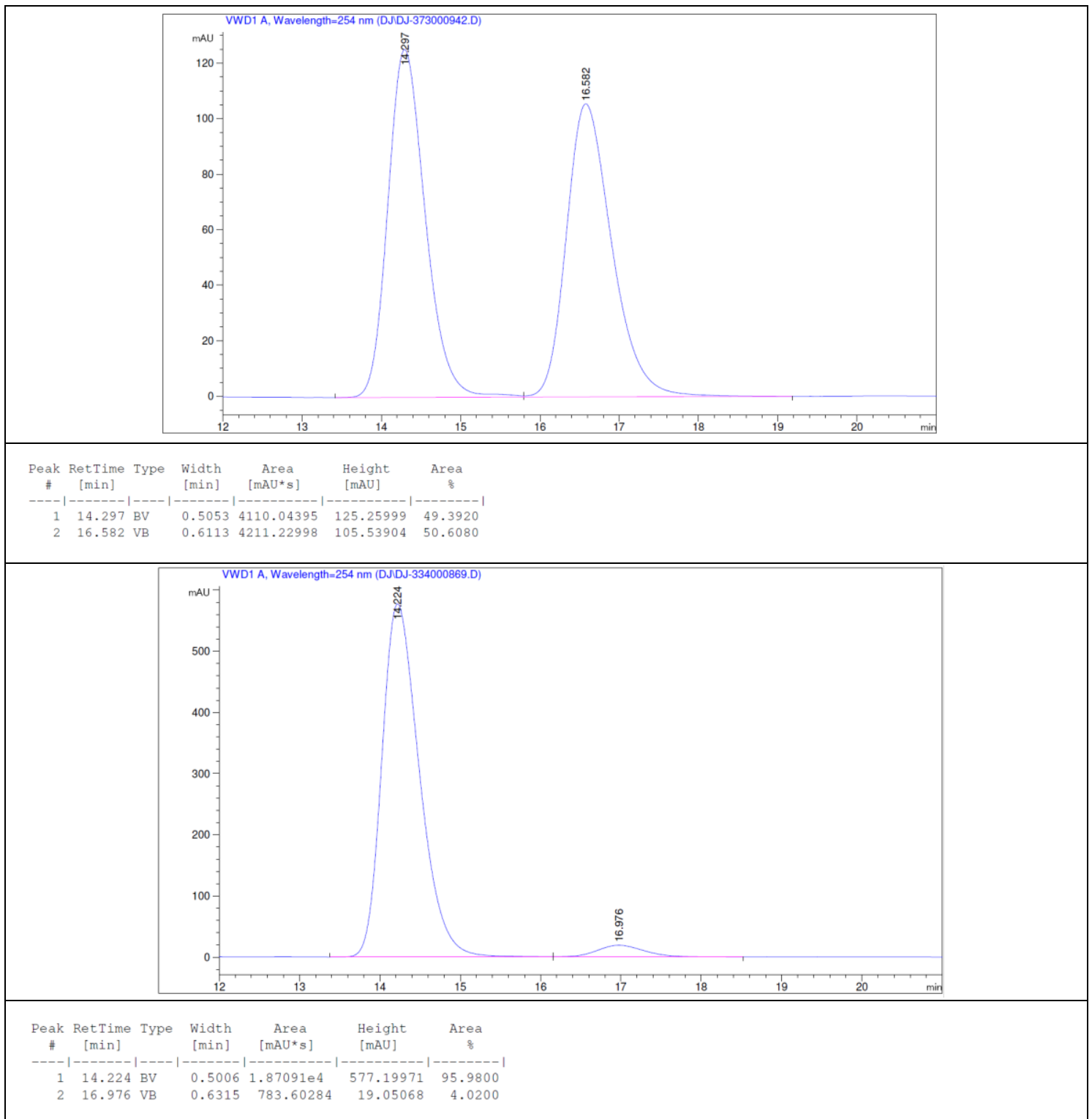

$(R, E)$-4-benzyl-4-methyl-5-(2-phenylhydrazono)heptan-3-one (6i)

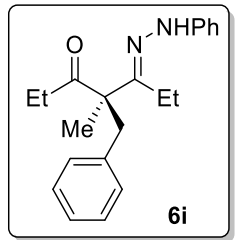

Reaction temperature of $-40{ }^{\circ} \mathrm{C}$ was used with $48 \mathrm{~h}$ reaction time.

Yellow oil (22.8 $\mathrm{mg}, 71 \%$ yield).

${ }^{1} \mathbf{H}$ NMR $\left(500 \mathrm{MHz}, \mathrm{CD}_{3} \mathrm{COCD}_{3}\right): \delta 8.53(\mathrm{~s}, 1 \mathrm{H}), 7.23-7.21(\mathrm{~m}, 2 \mathrm{H}), 7.19-7.15(\mathrm{~m}, 3 \mathrm{H})$, 7.14-7.12 (m, 4H), 6.75 (tt, $J=7.0 \mathrm{~Hz}, J=1.0 \mathrm{~Hz}, 1 \mathrm{H}), 3.26(\mathrm{~d}, J=14.0 \mathrm{~Hz}, 1 \mathrm{H}), 3.18(\mathrm{~d}, J=$ $14.0 \mathrm{~Hz}, 1 \mathrm{H}), 2.68-2.60(\mathrm{~m}, 1 \mathrm{H}), 2.44-2.37(\mathrm{~m}, 1 \mathrm{H}), 2.29-2.21(\mathrm{~m}, 2 \mathrm{H}), 1.30(\mathrm{~s}, 3 \mathrm{H}), 1.05(\mathrm{t}, J=$ $7.5 \mathrm{~Hz}, 3 \mathrm{H}), 0.99(\mathrm{t}, J=7.5 \mathrm{~Hz}, 3 \mathrm{H})$.

${ }^{13} \mathrm{C}$ NMR $\left(125 \mathrm{MHz}, \mathrm{CD}_{3} \mathrm{COCD}_{3}\right): \delta 211.5,149.6,147.2,139.2,131.2,129.6,128.5,126.8,119.7$, $113.5,60.6,42.2,31.8,20.5,19.3,10.0,8.6$.

IR $v\left(\mathrm{~cm}^{-1}\right): 3401.65,3029.43,2977.43,2938.17,2879.77,1952.73,1714.85,1600.63$, 1496.92, 1454.66, 1377.57, 1089.53, 754.56, 701.87 . 
HRMS (ESI): $\mathrm{m} / \mathrm{z}$ Calcd for $\left[\mathrm{C}_{21} \mathrm{H}_{27} \mathrm{~N}_{2} \mathrm{O}, \mathrm{M}+\mathrm{H}\right]^{+}:$323.2118; Found: 323.2121.

Optical Rotation: $[\alpha]_{\mathrm{D}}^{25}=-5.9\left(0.6, \mathrm{CHCl}_{3}\right) .81 \%$ ee. The absolute configuration of the product was assigned by analogy to $\mathbf{6 b}$. (HPLC condition: Chiralpak OD-H column, $n$-hexane $/ i-\mathrm{PrOH}=$ 95:5, flow rate $=1.0 \mathrm{~mL} / \mathrm{min}$, wavelength $=254 \mathrm{~nm}, \mathrm{t}_{\mathrm{R}}=8.16 \mathrm{~min}$ for major isomer, $\mathrm{t}_{\mathrm{R}}=10.82$ min for minor isomer).

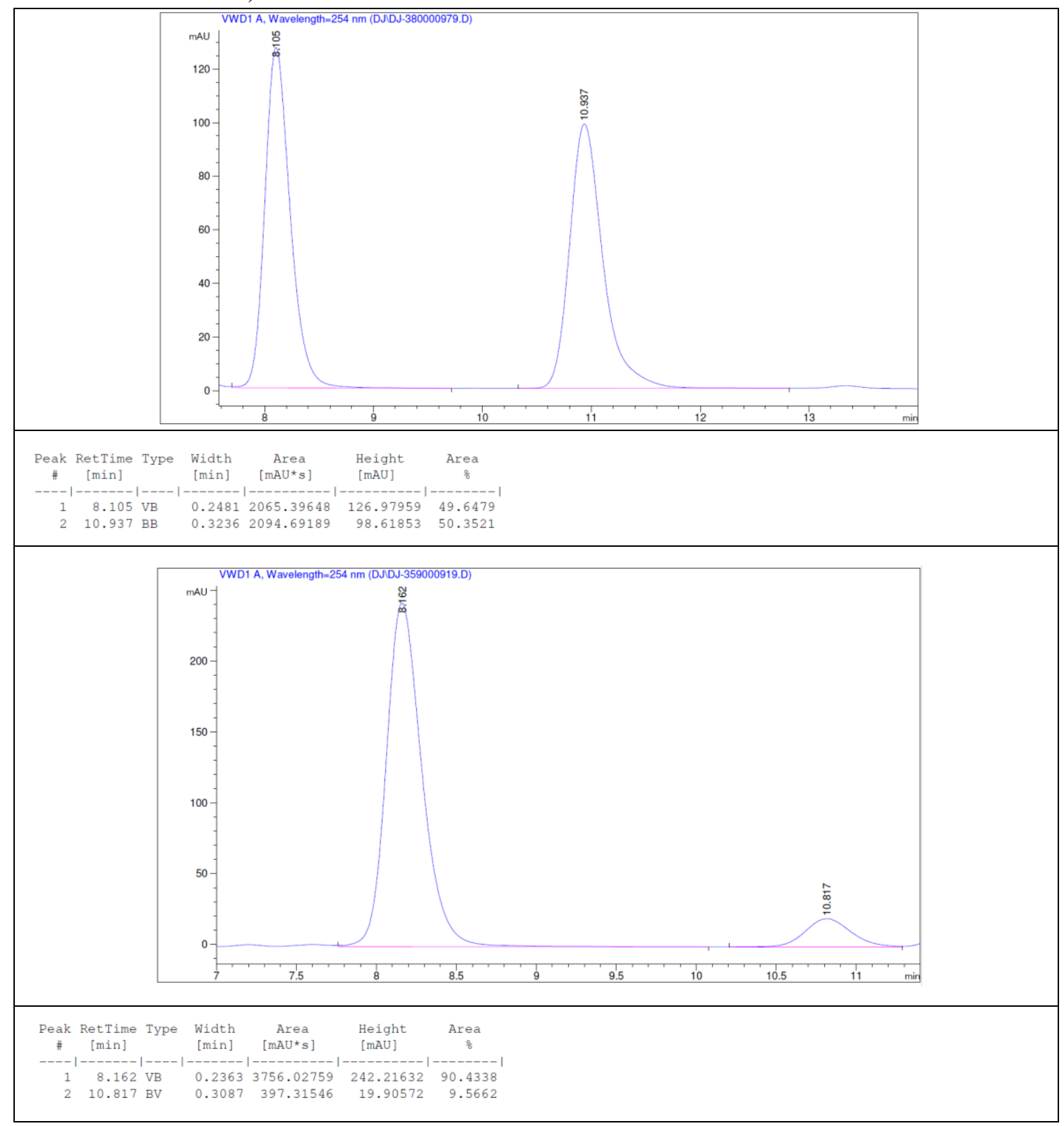

(R,E)-4-(3-chlorobenzyl)-4-methyl-5-(2-phenylhydrazono)heptan-3-one (6j)

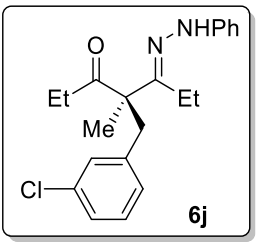

Reaction temperature of $-40{ }^{\circ} \mathrm{C}$ was used with $48 \mathrm{~h}$ reaction time.

Yellow oil (24.2 mg, $68 \%$ yield).

${ }^{1}$ H NMR $\left(500 \mathrm{MHz}, \mathrm{CD}_{3} \mathrm{COCD}_{3}\right): \delta 8.56(\mathrm{~s}, 1 \mathrm{H}), 7.25-7.16(\mathrm{~m}, 5 \mathrm{H}), 7.14-7.10(\mathrm{~m}, 3 \mathrm{H}), 6.75$ (tt, 
$J=7.0 \mathrm{~Hz}, J=1.0 \mathrm{~Hz}, 1 \mathrm{H}), 3.27(\mathrm{~d}, J=13.5 \mathrm{~Hz}, 1 \mathrm{H}), 3.18(\mathrm{~d}, J=13.5 \mathrm{~Hz}, 1 \mathrm{H}), 2.69-2.60(\mathrm{~m}$, $1 \mathrm{H}), 2.44-2.36(\mathrm{~m}, 1 \mathrm{H}), 2.33-2.21(\mathrm{~m}, 2 \mathrm{H}), 1.32(\mathrm{~s}, 3 \mathrm{H}), 1.06(\mathrm{t}, J=7.5 \mathrm{~Hz}, 3 \mathrm{H}), 0.99$ (t, $J=7.5$ $\mathrm{Hz}, 3 \mathrm{H})$.

${ }^{13}$ C NMR (125 MHz, $\left.\mathrm{CD}_{3} \mathrm{COCD}_{3}\right): \delta 211.4,149.1,147.1,141.8,133.9,131.2,130.1,129.9,129.6$, $126.9,119.8,113.5,60.5,41.7,31.8,20.4,19.3,10.0,8.5$.

IR $v\left(\mathrm{~cm}^{-1}\right): 3388.64,2978.06,2938.97,1949.36,1715.98,1597.18,1500.32,1460.25$, $1207.15,1083.42,763.55,689.34$.

HRMS (ESI): $\mathrm{m} / \mathrm{z}$ Calcd for $\left[\mathrm{C}_{21} \mathrm{H}_{26} \mathrm{ClN}_{2} \mathrm{O}, \mathrm{M}+\mathrm{H}\right]^{+}:$: 357.1728; Found: 357.1732.

Optical Rotation: $[\alpha]_{\mathrm{D}}^{25}=-7.0\left(0.8, \mathrm{CHCl}_{3}\right) .78 \%$ ee. The absolute configuration of the product was assigned by analogy to $\mathbf{6 b}$. (HPLC condition: Chiralpak OD-H column, $n$-hexane/ $i-\mathrm{PrOH}=$ $95: 5$, flow rate $=1.0 \mathrm{~mL} / \mathrm{min}$, wavelength $=254 \mathrm{~nm}, \mathrm{t}_{\mathrm{R}}=8.49 \mathrm{~min}$ for major isomer, $\mathrm{t}_{\mathrm{R}}=11.76$ min for minor isomer).

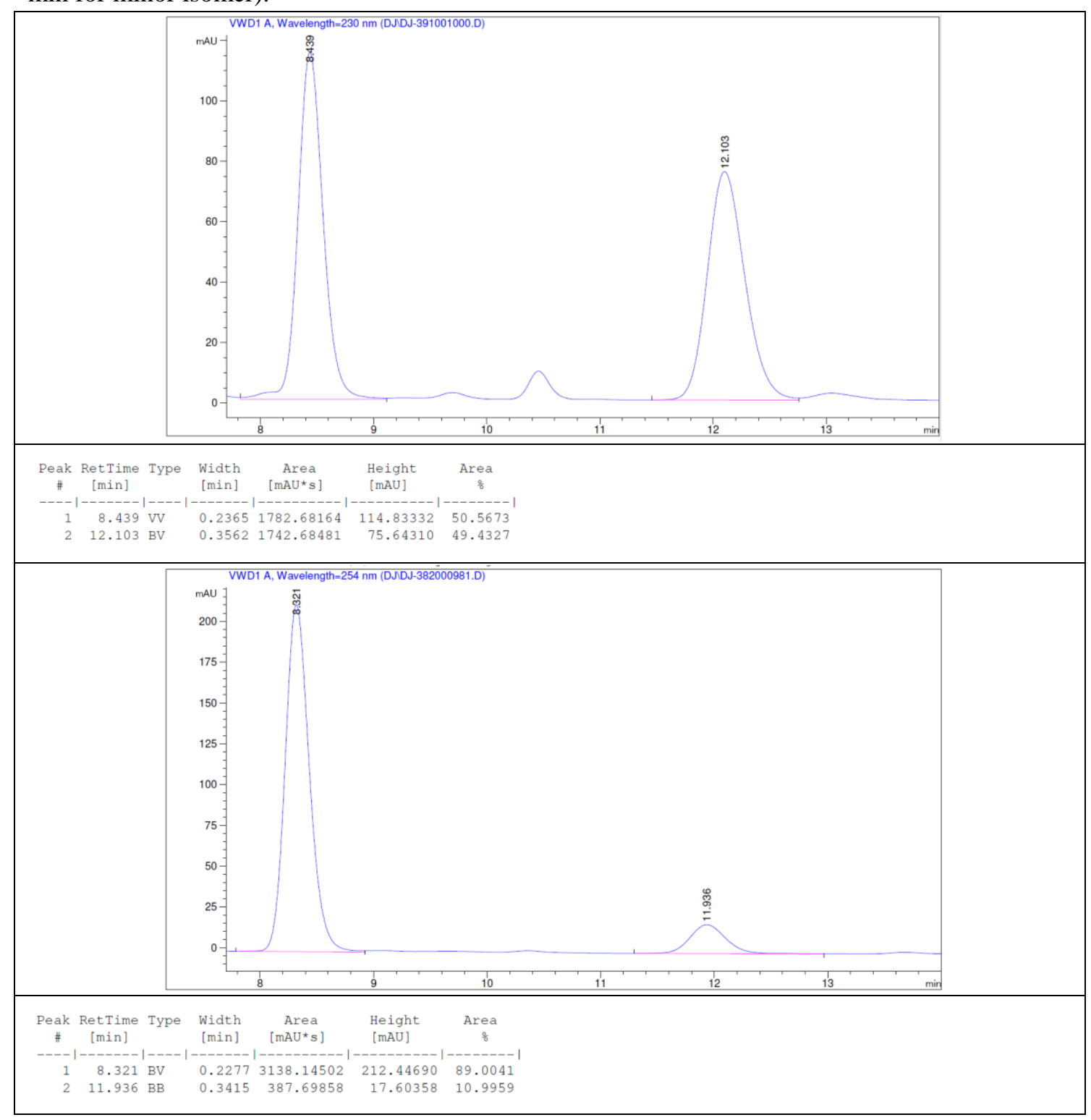

(R,E)-3-benzyl-3-methyl-4-(2-(4-(trifluoromethyl)phenyl)hydrazono)pentan-2-one (6k) 


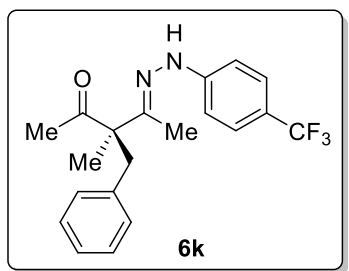

Reaction temperature of $-78{ }^{\circ} \mathrm{C}$ was used with $48 \mathrm{~h}$ reaction time.

Yellow oil (29.3 mg, $81 \%$ yield).

${ }^{1} \mathbf{H}$ NMR $\left(500 \mathrm{MHz}, \mathrm{CD}_{3} \mathrm{COCD}_{3}\right): \delta 8.92(\mathrm{~s}, 1 \mathrm{H}), 7.47(\mathrm{~d}, J=8.5 \mathrm{~Hz}, 2 \mathrm{H}), 7.23-7.19(\mathrm{~m}, 4 \mathrm{H})$, 7.17-7.11 (m, 3H), $3.22(\mathrm{~d}, J=14.0 \mathrm{~Hz}, 1 \mathrm{H}), 3.18(\mathrm{~d}, J=14.0 \mathrm{~Hz}, 1 \mathrm{H}), 2.12(\mathrm{~s}, 3 \mathrm{H}), 1.90(\mathrm{~s}, 3 \mathrm{H})$, $1.28(\mathrm{~s}, 3 \mathrm{H})$.

${ }^{13} \mathrm{C}$ NMR $\left(125 \mathrm{MHz}, \mathrm{CD}_{3} \mathrm{COCD}_{3}\right): \delta 208.9,150.1,147.9,138.8,131.1,128.5,126.9\left(\mathrm{q},{ }^{4} J_{\mathrm{CF}}=\right.$ $4.5 \mathrm{~Hz}), 126.9,124.9\left(\mathrm{q},{ }^{1} J_{\mathrm{CF}}=269.5 \mathrm{~Hz}\right), 120.7\left(\mathrm{q},{ }^{2} J_{\mathrm{CF}}=32.5 \mathrm{~Hz}\right), 113.1,61.2,41.1,26.2,19.6$, 13.7.

${ }^{19} \mathbf{F}$ NMR $\left(376 \mathrm{MHz}, \mathrm{CD}_{3} \mathrm{COCD}_{3}\right): \delta-61.5(\mathrm{~s})$.

IR $v\left(\mathrm{~cm}^{-1}\right): 3340.81,3030.06,2928.46,2850.92,1949.12,1706.67,1614.79,1528.73$, 1496.07, 1324.43, 1162.99, 1112.54, 1063.91, 836.86, 754.94, 702.71 .

HRMS (ESI): $\mathrm{m} / \mathrm{z}$ Calcd for $\left[\mathrm{C}_{20} \mathrm{H}_{22} \mathrm{~F}_{3} \mathrm{~N}_{2} \mathrm{O}, \mathrm{M}+\mathrm{H}\right]^{+}: 363.1679$; Found: 363.1683 .

Optical Rotation: $[\alpha]_{\mathrm{D}}^{25}=-18.7\left(1.0, \mathrm{CHCl}_{3}\right) .90 \% e e$. The absolute configuration of the product was assigned by analogy to $\mathbf{6 b}$. (HPLC condition: Chiralpak OD-H column, $n$-hexane/i-PrOH $=$ $90: 10$, flow rate $=1.0 \mathrm{~mL} / \mathrm{min}$, wavelength $=254 \mathrm{~nm}, \mathrm{t}_{\mathrm{R}}=8.58 \mathrm{~min}$ for major isomer, $\mathrm{t}_{\mathrm{R}}=11.16$ $\min$ for minor isomer).

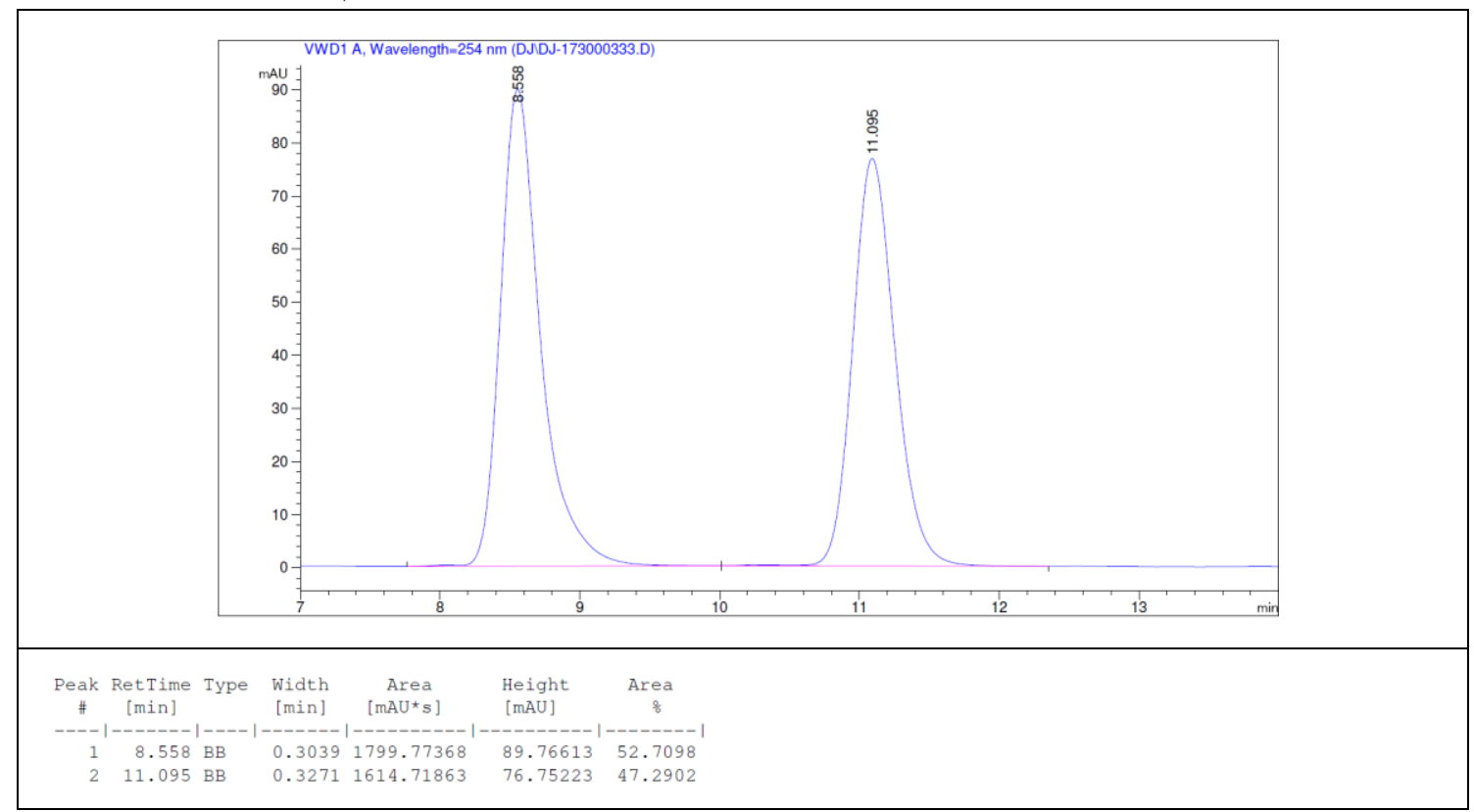




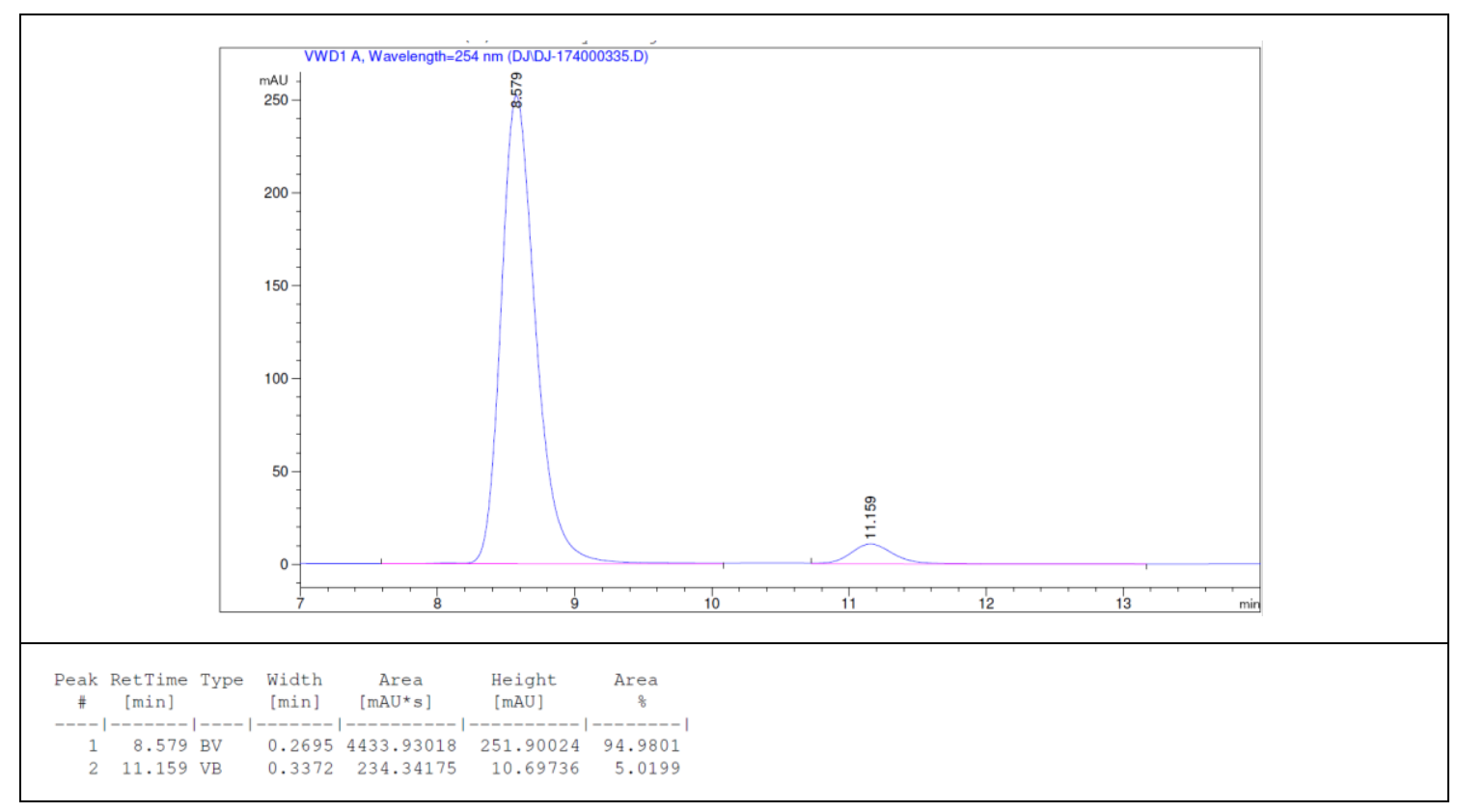




\section{Procedure for reduction of $3 a$ to 7}

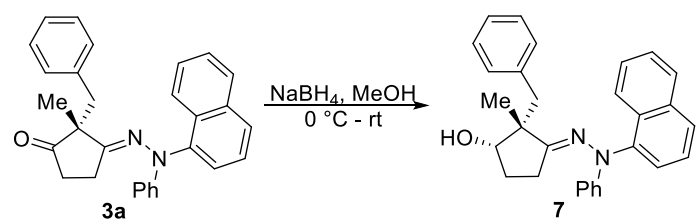

Compound 3a (41.8 mg, $0.1 \mathrm{mmol})$ was dissolved in AR $\mathrm{MeOH}(1.0 \mathrm{~mL}) . \mathrm{NaBH}_{4}(11.4 \mathrm{mg}, 0.3$ mmol) was added slowly at $0{ }^{\circ} \mathrm{C}$. The reaction mixture was then allowed to stir at ambient temperature for $6 \mathrm{~h}$. The reaction system was monitored by TLC until 3a disappeared completely. After that, the reaction mixture was quenched by a drop of water and directly purified by column chromatography on silica gel (eluting with ethyl acetate/hexanes 1:20-1:8) to provide compound 7 as a white solid (37.4 mg, $89 \%$ yield, $>20: 1 \mathrm{dr}$ ).

(1S,2R,E)-2-benzyl-2-methyl-3-(2-(naphthalen-1-yl)-2-phenylhydrazono)cyclopentanol (7)

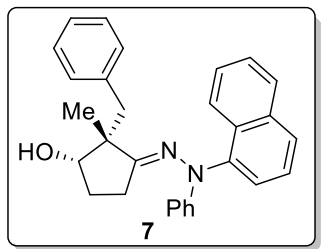

${ }^{1}$ H NMR $\left(500 \mathrm{MHz}, \mathrm{CD}_{3} \mathrm{COCD}_{3}\right): \delta 8.00(\mathrm{dd}, J=8.0 \mathrm{~Hz}, J=1.0 \mathrm{~Hz}, 1 \mathrm{H}), 7.91(\mathrm{~d}, J=8.5 \mathrm{~Hz}$, 2H), 7.61 (t, $J=7.5 \mathrm{~Hz}, 1 \mathrm{H}), 7.53$ (td, $J=7.5 \mathrm{~Hz}, J=1.0 \mathrm{~Hz}, 1 \mathrm{H}), 7.48-7.39(\mathrm{~m}, 4 \mathrm{H}), 7.26-7.23$ $(\mathrm{m}, 2 \mathrm{H}), 7.18(\mathrm{tt}, J=7.5 \mathrm{~Hz}, J=1.5 \mathrm{~Hz}, 1 \mathrm{H}), 7.14-7.10(\mathrm{~m}, 2 \mathrm{H}), 6.82(\mathrm{tt}, J=7.0 \mathrm{~Hz}, J=1.0 \mathrm{~Hz}$, $1 \mathrm{H}), 6.80-6.77(\mathrm{~m}, 2 \mathrm{H}), 3.96(\mathrm{~d}, J=3.5 \mathrm{~Hz}, 1 \mathrm{H}), 3.76-3.74(\mathrm{~m}, 1 \mathrm{H}), 3.31(\mathrm{~d}, J=13.5 \mathrm{~Hz}, 1 \mathrm{H})$, $3.02(\mathrm{~d}, J=13.5 \mathrm{~Hz}, 1 \mathrm{H}), 2.01-1.95(\mathrm{~m}, 1 \mathrm{H}), 1.77-1.67(\mathrm{~m}, 2 \mathrm{H}), 1.56-1.51(\mathrm{~m}, 1 \mathrm{H}), 0.97(\mathrm{~s}, 3 \mathrm{H})$.

${ }^{13} \mathrm{C}$ NMR $\left(125 \mathrm{MHz}, \mathrm{CD}_{3} \mathrm{COCD}_{3}\right): \delta 174.5,151.4,144.1,140.3,135.5,131.7,131.6,129.2$, 129.0, 128.4, 127.7, 127.1, 127.0, 126.4, 126.3, 125.0, 120.7, 116.6, 77.0, 53.4, 48.8, 30.4, 28.3, 22.7.

IR $v\left(\mathrm{~cm}^{-1}\right): 3318.74,3025.64,2961.28,2917.98,2872.37,1939.66,1598.75,1488.56$, 1390.08, 1284.42, 1083.56, 777.44, 752.45, 705.81.

HRMS (ESI): $\mathrm{m} / \mathrm{z}$ Calcd for $\left[\mathrm{C}_{29} \mathrm{H}_{29} \mathrm{~N}_{2} \mathrm{O}, \mathrm{M}+\mathrm{H}\right]^{+}: 421.2274$; Found: 421.2284.

Optical Rotation: $[\alpha]_{\mathrm{D}}^{25}=87.9\left(1.0, \mathrm{CHCl}_{3}\right) .95 \%$ ee. The relative and absolute configuration of 7 was assigned based on single crystal $\mathrm{x}$-ray analysis. (HPLC condition: Chiralpak AD-H column, $n$-hexane $/ i-\mathrm{PrOH}=95: 5$, flow rate $=1.0 \mathrm{~mL} / \mathrm{min}$, wavelength $=254 \mathrm{~nm}, \mathrm{t}_{\mathrm{R}}=9.52 \mathrm{~min}$ for minor isomer, $\mathrm{t}_{\mathrm{R}}=12.44 \mathrm{~min}$ for major isomer). 


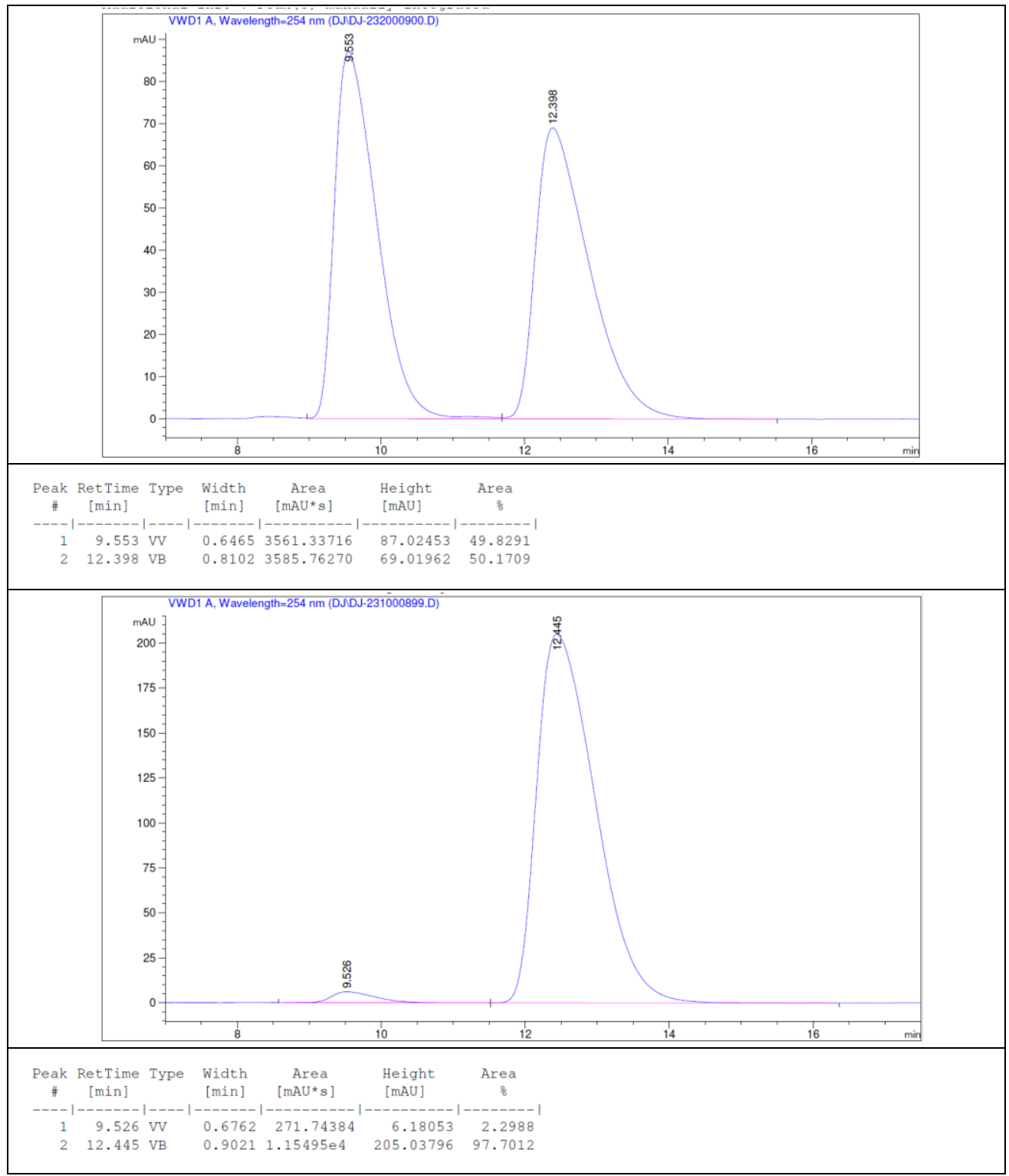

\section{Procedure for the synthesis of indole 8}

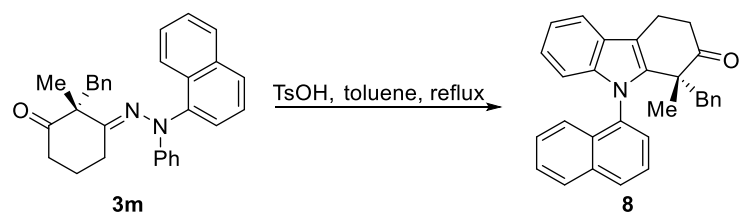

Compound 3m (21.6 mg, $0.05 \mathrm{mmol})$ and $\mathrm{TsOH}(17.8 \mathrm{mg}, 0.1 \mathrm{mmol})$ were dissolved in toluene $(0.5 \mathrm{~mL})$. The reaction mixture was refluxed for $12 \mathrm{~h}$ and cooled to room temperature. The mixture was then concentrated under vacuum. Purification of the residue by column chromatography on silica gel (eluting with ethyl acetate/hexanes 1:50 -1:20) provided compound 8 as a colorless oil (12.9 $\mathrm{mg}, 62 \%$ yield).

(R)-1-benzyl-1-methyl-9-(naphthalen-1-yl)-3,4-dihydro-1H-carbazol-2(9H)-one (8) 


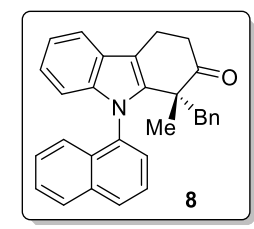

${ }^{1}$ H NMR $\left(500 \mathrm{MHz}, \mathrm{CDCl}_{3}\right): \delta 7.90(\mathrm{dd}, J=8.0 \mathrm{~Hz}, J=1.0 \mathrm{~Hz}, 1 \mathrm{H}), 7.78(\mathrm{dt}, J=7.5 \mathrm{~Hz}, J=1.5$ $\mathrm{Hz}, 1 \mathrm{H}), 7.69-7.64(\mathrm{~m}, 2 \mathrm{H}), 7.63-7.60(\mathrm{~m}, 1 \mathrm{H}), 7.58(\mathrm{~s}, 2 \mathrm{H}), 7.46(\mathrm{~d}, J=7.5 \mathrm{~Hz}, 1 \mathrm{H}), 7.30(\mathrm{tt}, J=$ $7.5 \mathrm{~Hz}, J=1.0 \mathrm{~Hz}, 1 \mathrm{H}), 7.11-7.07(\mathrm{~m}, 2 \mathrm{H}), 7.04-7.01(\mathrm{~m}, 2 \mathrm{H}), 6.74(\mathrm{~d}, J=8.5 \mathrm{~Hz}, 1 \mathrm{H}), 6.69-6.66$ (m, 2H), 3.09 (s, 2H), 2.95-2.90 (m, 1H), 2.63-2.57 (m, 1H), 2.33-2.22 (m, 2H), $1.26(\mathrm{~s}, 3 \mathrm{H})$.

${ }^{13} \mathrm{C}$ NMR $\left(125 \mathrm{MHz}, \mathrm{CDCl}_{3}\right): \delta 213.8,141.8,137.3,136.8,132.6,131.8,131.6,130.2,129.9$, $129.8,129.7,129.2,127.9,126.4,125.0,123.3,122.7,122.3,121,6,120.6,118.0,114.2,52.7$, 45.9, 38.3, 25.4, 17.9 .

IR $v\left(\mathrm{~cm}^{-1}\right): 3399.29,2924.94,2853.96,1947.61,1713.69,1495.67,1456.87,1400.70$, 1278.01, 1080.29, 967.92, 806.00, 743.93, 701.18.

HRMS (ESI): $\mathrm{m} / \mathrm{z}$ Calcd for $\left[\mathrm{C}_{30} \mathrm{H}_{26} \mathrm{NO}, \mathrm{M}+\mathrm{H}\right]^{+}:$416.2009; Found: 416.2011.

Optical Rotation: $[\alpha]_{\mathrm{D}}^{25}=17.3\left(0.4, \mathrm{CHCl}_{3}\right) .93 \%$ ee. The absolute configuration of the product was assigned by that of $\mathbf{3 m}$. (HPLC condition: Chiralpak OD-H column, $n$-hexane/ $i$-PrOH $=95: 5$, flow rate $=1.0 \mathrm{~mL} / \mathrm{min}$, wavelength $=254 \mathrm{~nm}, \mathrm{t}_{\mathrm{R}}=6.62 \mathrm{~min}$ for major isomer, $\mathrm{t}_{\mathrm{R}}=7.71 \mathrm{~min}$ for minor isomer).

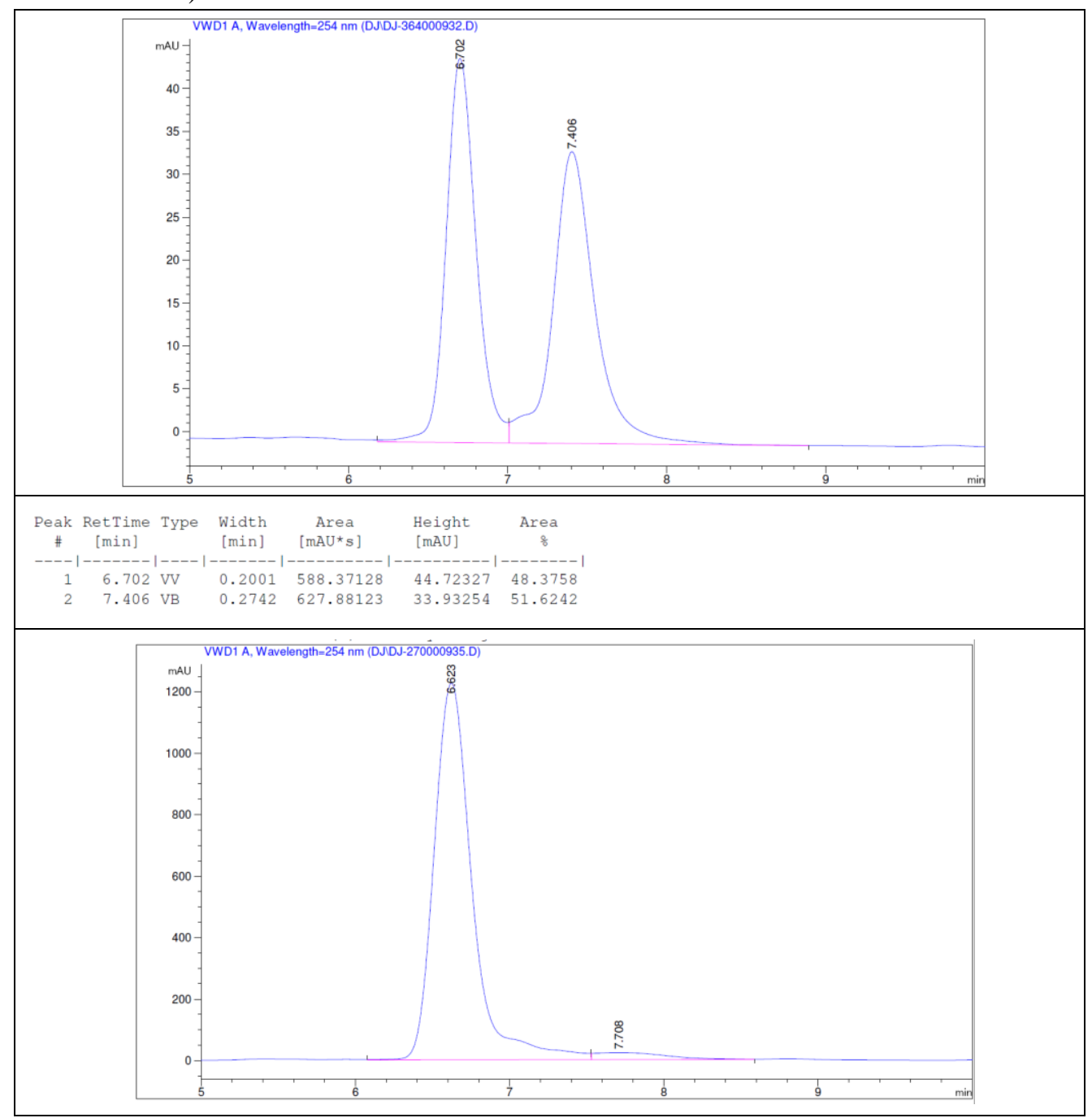




\begin{tabular}{|c|c|c|c|c|c|c|}
\hline $\begin{array}{c}\text { Peak } \\
\#\end{array}$ & $\begin{array}{c}\text { RetTime } \\
{[\mathrm{min}]}\end{array}$ & Type & $\begin{array}{l}\text { Width } \\
\text { [min] }\end{array}$ & $\begin{array}{c}\text { Area } \\
{\left[m A U^{\star} s\right]}\end{array}$ & $\begin{array}{l}\text { Height } \\
{[\mathrm{mAU}]}\end{array}$ & $\begin{array}{c}\text { Area } \\
\frac{8}{8}\end{array}$ \\
\hline 1 & 6.623 & vV & 0.2643 & $2.10040 \mathrm{e} 4$ & 1224.19812 & 96.7164 \\
\hline 2 & 7.708 & VB & 0.4810 & 713.11163 & 22.83423 & 3.2836 \\
\hline
\end{tabular}

\section{Procedure for the synthesis of 9 and 10}

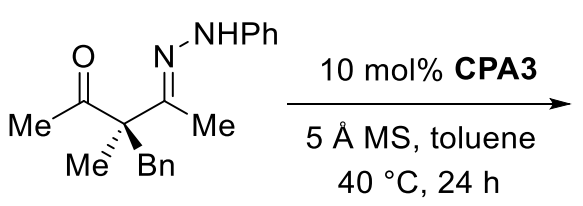

$6 b$<smiles>C=C1N(c2ccccc2)N=C(C)[C@@]1(C)Br</smiles>

9

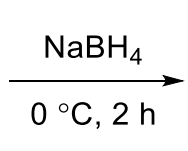<smiles>CC1=NN(c2ccccc2)C(C)[C@@]1(C)Br</smiles>

10

Compound 6b (29.4 mg, $0.1 \mathrm{mmol}), \mathbf{C P A 3}(9.9 \mathrm{mg}, 0.01 \mathrm{mmol})$ and $5 \AA \mathrm{MS}(40 \mathrm{mg})$ were dissolved in toluene $(0.8 \mathrm{~mL})$. The reaction mixture was allowed to stir at $40{ }^{\circ} \mathrm{C}$ for $24 \mathrm{~h}$. The mixture was then concentrated under vacuum. Purification of the residue by column chromatography on silica gel (eluting with ethyl acetate/hexanes 1:50 -1:30) provided compound 9 as a yellow oil ( $18.8 \mathrm{mg}, 68 \%$ yield).

Compound 9 (13.8 mg, $0.05 \mathrm{mmol})$ was dissolved in $\mathrm{MeOH}(0.5 \mathrm{~mL}), \mathrm{NaBH}_{4}(9.5 \mathrm{mg}, 0.25 \mathrm{mmol})$ was added at $0{ }^{\circ} \mathrm{C}$, and the resulting mixture was stirred at $0{ }^{\circ} \mathrm{C}$ for $2 \mathrm{~h}$. The reaction mixture was quenched with water, and extracted with EtOAc $(3 \times 5 \mathrm{~mL})$. The combined organic layers were washed with brine, dried over $\mathrm{Na}_{2} \mathrm{SO}_{4}$, and filtered. The solvent was removed under reduced pressure, and the residue was purified by silica gel column chromatography (ethyl acetate/petroleum ether 1:50 -1:30) to afford compound $\mathbf{1 0}$ as colorless oil (12.3 mg, 88\% yield, 19:1 dr).

\section{(S)-4-benzyl-3,4-dimethyl-5-methylene-1-phenyl-4,5-dihydro-1H-pyrazole (9)}

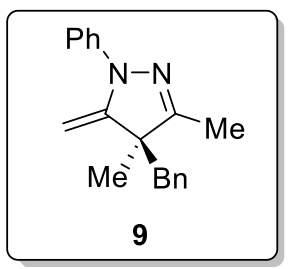

${ }^{1}$ H NMR $\left(500 \mathrm{MHz}, \mathrm{CD}_{3} \mathrm{COCD}_{3}\right): \delta$ 7.32-7.28 (m, 2H), 7.25-7.16 (m, 7H), $7.03(\mathrm{tt}, J=7.5 \mathrm{~Hz}, J$ $=1.0 \mathrm{~Hz}, 1 \mathrm{H}), 4.67(\mathrm{~d}, J=2.0 \mathrm{~Hz}, 1 \mathrm{H}), 3.85(\mathrm{~d}, J=2.0 \mathrm{~Hz}, 1 \mathrm{H}), 2.97(\mathrm{~d}, J=14.0 \mathrm{~Hz}, 1 \mathrm{H}), 2.83$ (d, $J=14.0 \mathrm{~Hz}, 1 \mathrm{H}), 2.01$ (s, 3H), 1.39 (s, 3H).

${ }^{13} \mathrm{C}$ NMR $\left(125 \mathrm{MHz}, \mathrm{CD}_{3} \mathrm{COCD}_{3}\right): \delta 156.6,153.2,141.6,136.3,129.7,128.6,127.8,126.7$, 123.1, 120.5, 77.6, 57.1, 45.8, 25.0, 12.5.

IR $v\left(\mathrm{~cm}^{-1}\right): 3388.82,3061.45,3029.13,2923.03,2852.84,1947.90,1595.47,1498.44$, 1453.87, 1364.75, 1097.54, 1030.02, 754.73, 697.81.

HRMS (ESI): $\mathrm{m} / \mathrm{z}$ Calcd for $\left[\mathrm{C}_{19} \mathrm{H}_{21} \mathrm{~N}_{2}, \mathrm{M}+\mathrm{H}\right]^{+}: 277.1699$; Found: 277.1703.

Optical Rotation: $[\alpha]_{\mathrm{D}}^{25}=-102.1\left(0.1, \mathrm{CHCl}_{3}\right) .90 \%$ ee. The absolute configuration of the product was assigned by that of $\mathbf{6 b}$. (HPLC condition: Chiralpak OD-H column, $n$-hexane $/ i-\mathrm{PrOH}=95: 5$, flow rate $=1.0 \mathrm{~mL} / \mathrm{min}$, wavelength $=254 \mathrm{~nm}, \mathrm{t}_{\mathrm{R}}=4.95 \mathrm{~min}$ for major isomer, $\mathrm{t}_{\mathrm{R}}=5.28 \mathrm{~min}$ for minor isomer). 


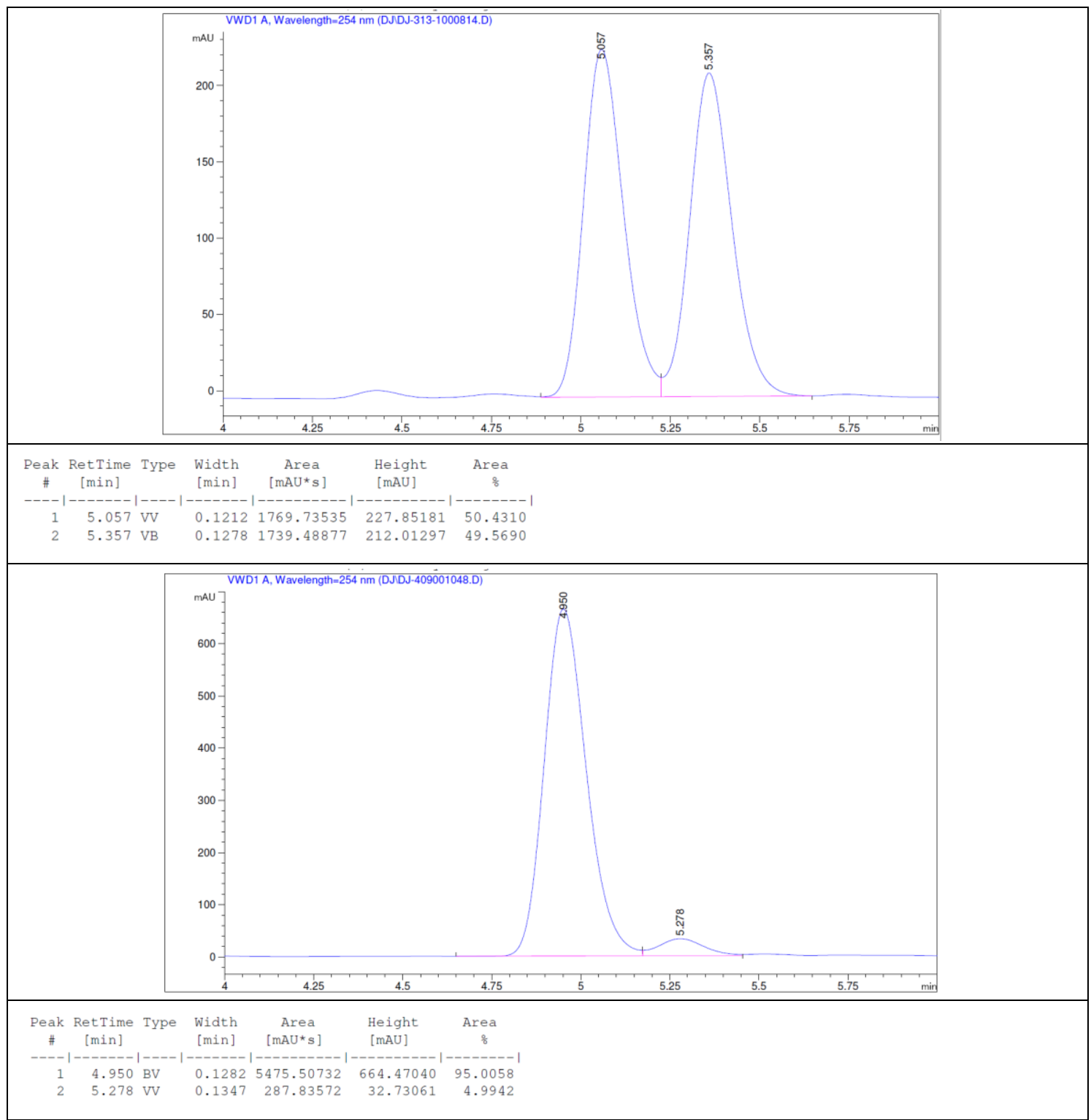

(4S,5R)-4-benzyl-3,4,5-trimethyl-1-phenyl-4,5-dihydro-1H-pyrazole (10)

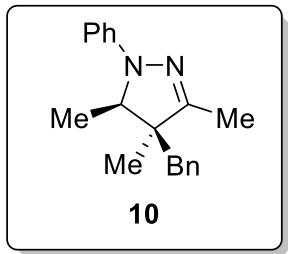

${ }^{1} \mathbf{H}$ NMR $\left(500 \mathrm{MHz}, \mathrm{CD}_{3} \mathrm{COCD}_{3}\right): \delta$ 7.32-7.28 (m, 4H), 7.27-7.23 (m, 3H), 7.15-7.13 (m, 2H), 6.93 (tt, $J=7.5 \mathrm{~Hz}, J=1.0 \mathrm{~Hz}, 1 \mathrm{H}), 3.60(\mathrm{q}, J=6.5 \mathrm{~Hz}, 1 \mathrm{H}), 3.05(\mathrm{~d}, J=14.0 \mathrm{~Hz}, 1 \mathrm{H}), 2.75(\mathrm{~d}, J$ $=14.0 \mathrm{~Hz}, 1 \mathrm{H}), 1.66(\mathrm{~s}, 3 \mathrm{H}), 1.32(\mathrm{~d}, J=6.5 \mathrm{~Hz}, 3 \mathrm{H}), 1.18(\mathrm{~s}, 3 \mathrm{H})$.

${ }^{13}$ C NMR (125 MHz, $\left.\mathrm{CD}_{3} \mathrm{COCD}_{3}\right): \delta 157.7,146.6,137.7,130.3,128.9,128.2,126.4,120.8,117.3$, 68.2, 54.0, 37.6, 20.7, 13.8, 11.8 .

IR $v\left(\mathrm{~cm}^{-1}\right): 3395.43,3027.99,2924.45,2853.46,1944.68,1597.82,1497.13,1454.87$, 1360.70, 1082.25, 969.05, 747.85, 702.29.

HRMS (ESI): $\mathrm{m} / \mathrm{z}$ Calcd for $\left[\mathrm{C}_{19} \mathrm{H}_{23} \mathrm{~N}_{2}, \mathrm{M}+\mathrm{H}\right]^{+}: 279.1856$ Found: 279.1859.

Optical Rotation: $[\alpha]_{\mathrm{D}}^{25}=-128.7\left(0.3, \mathrm{CHCl}_{3}\right) .90 \%$ ee. The absolute configuration of the product 
was assigned by that of $\mathbf{9}$, relative configuration was determined by 1D NMR-NOESY . (HPLC condition: Chiralpak OD-H column, $n$-hexane $/ i-\mathrm{PrOH}=95: 5$, flow rate $=1.0 \mathrm{~mL} / \mathrm{min}$, wavelength $=254 \mathrm{~nm}, \mathrm{t}_{\mathrm{R}}=6.22 \mathrm{~min}$ for major isomer, $\mathrm{t}_{\mathrm{R}}=8.07 \mathrm{~min}$ for minor isomer).

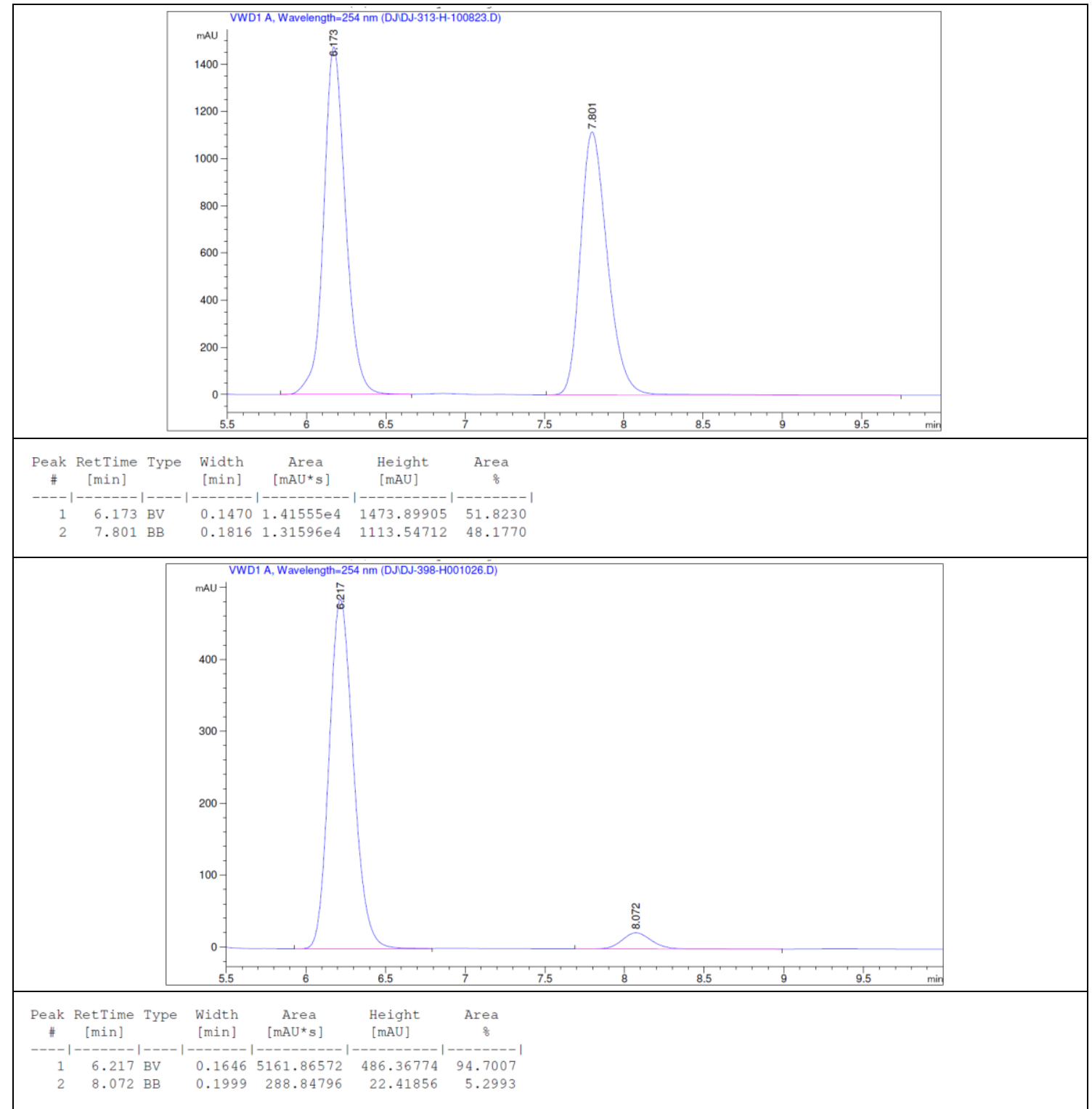

\section{(S)-2-benzyl-2-methylcyclopentan-1-one (11)}

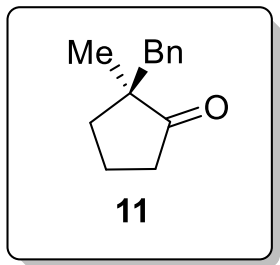

Yellow oil (12.1 mg, 32\% yield).

${ }^{1} \mathbf{H}$ NMR $\left(500 \mathrm{MHz}, \mathrm{CDCl}_{3}\right): \delta$ 7.25-7.22 (m, 2H), 7.19-7.16 (m, 1H), 7.10-7.08 (m, 2H), $2.83(\mathrm{~d}$, $J=13.6 \mathrm{~Hz}, 1 \mathrm{H}), 2.47(\mathrm{~d}, J=13.6 \mathrm{~Hz}, 1 \mathrm{H}), 2.28-2.23(\mathrm{~m}, 1 \mathrm{H}), 2.05-1.90(\mathrm{~m}, 2 \mathrm{H}), 1.79-1.73(\mathrm{~m}$, $1 \mathrm{H}), 1.71-1.64(\mathrm{~m}, 1 \mathrm{H}), 1.62-1.57(\mathrm{~m}, 1 \mathrm{H}), 1.01(\mathrm{~s}, 3 \mathrm{H})$.

${ }^{13}$ C NMR (125 MHz, $\left.\mathrm{CDCl}_{3}\right): \delta 222.7,137.7,129.9,127.9,126.1,49.4,42.4,37.7,34.3,22.4$, 
18.3.

IR $v\left(\mathrm{~cm}^{-1}\right): 3452.37,3061.98,3028.17,2961.56,2869.59,1950.83,1735.34,1602.82,1494.97$, 1454.22, 1405.35, 1160.64, 1060.61, 758.59, 704.70.

Optical rotation: $[\alpha]_{\mathrm{D}}^{25}=-75.1(0.7, \mathrm{CHCl} 3) .90 \%$ ee. (HPLC condition: Chiralpak IA column, $n$-hexane $/ i-\mathrm{PrOH}=98: 2$, flow rate $=1.0 \mathrm{~mL} / \mathrm{min}$, wavelength $=220 \mathrm{~nm}, \mathrm{t}_{\mathrm{R}}=4.89 \mathrm{~min}$ for minor isomer, $\mathrm{t}_{\mathrm{R}}=5.75 \mathrm{~min}$ for major isomer).

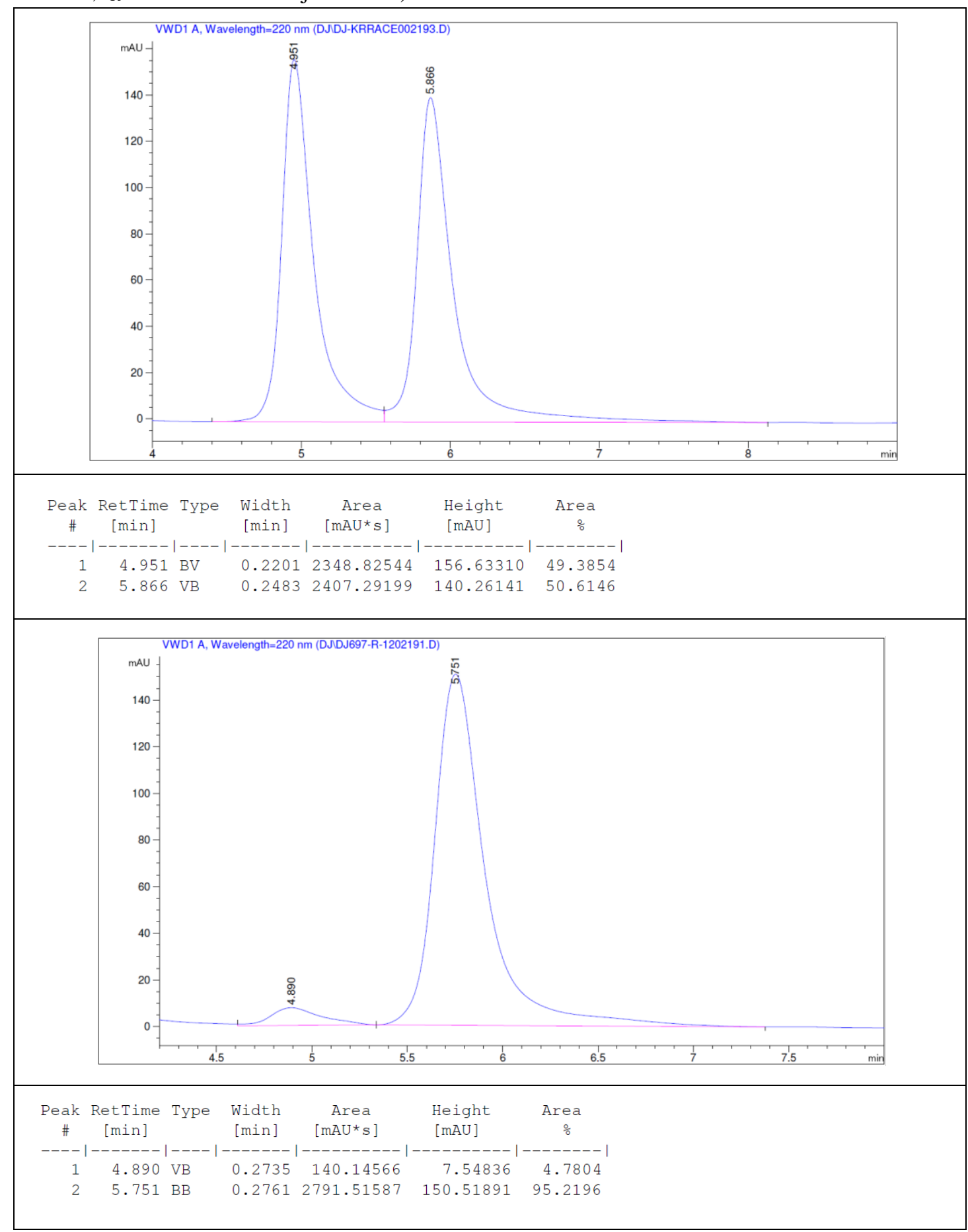

(R,E)-2-(2-benzyl-2-methylcyclopentylidene)-1-(naphthalen-1-yl)-1-phenylhydrazine $(R, E)$-2-(2-benzyl-2-methylcyclopentylidene)-1-(naphthalen-1-yl)-1-phenylhydrazine (12) 


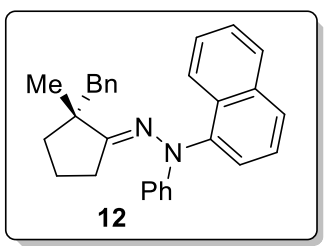

Yellow oil (50.12 mg, 62\% yield).

${ }^{1}$ H NMR $\left(400 \mathrm{MHz}, \mathrm{CDCl}_{3}\right): \delta 7.86(\mathrm{~d}, J=8.4 \mathrm{~Hz}, 1 \mathrm{H}), 7.81(\mathrm{~d}, J=8.4 \mathrm{~Hz}, 1 \mathrm{H}), 7.70(\mathrm{~d}, J=8.4$ $\mathrm{Hz}, 1 \mathrm{H}), 7.44-7.38(\mathrm{~m}, 2 \mathrm{H}), 7.35-7.29(\mathrm{~m}, 2 \mathrm{H}), 7.18-7.05(\mathrm{~m}, 7 \mathrm{H}), 6.81-6.74(\mathrm{~m}, 3 \mathrm{H}), 2.91(\mathrm{~d}, J=$ $13.6 \mathrm{~Hz}, 1 \mathrm{H}), 2.82(\mathrm{~d}, J=13.6 \mathrm{~Hz}, 1 \mathrm{H}), 1.69-1.61(\mathrm{~m}, 3 \mathrm{H}), 1.36-1.19(\mathrm{~m}, 3 \mathrm{H}), 1.14(\mathrm{~s}, 3 \mathrm{H})$.

${ }^{13} \mathrm{C}$ NMR (100 MHz, $\left.\mathrm{CDCl}_{3}\right): \delta 176.9,150.3,143.3,139.0,134.5,130.8,130.5,128.5,128.4$, 127.9, 126.7, 126.3, 126.2, 126.0, 125.1, 124.5, 120.2, 116.0, 48.1, 45.2, 36.3, 31.2, 24.9, 21.1.

IR $v\left(\mathrm{~cm}^{-1}\right): 3083.93,3059.31,3027.78,2958.92,2867.16,2603.16,2245.47,1944.80,1594.49$, 1491.06, 1391.25, 1287.11, 1218.09, 1168.24, 908.78, 776.72, 702.79, 846.43.

HRMS (ESI): $\mathrm{m} / \mathrm{z}$ Calcd for $\left[\mathrm{C}_{29} \mathrm{H}_{29} \mathrm{~N}_{2}, \mathrm{M}+\mathrm{H}\right]^{+}: 405.2325$; Found: 405.2326.

Optical rotation: $[\alpha]_{\mathrm{D}}^{25}=26.8(0.5, \mathrm{CHCl} 3) .48 \%$ ee. (HPLC condition: Chiralpak OD-H column, $n$-hexane $/ i-\mathrm{PrOH}=98: 2$, flow rate $=1.0 \mathrm{~mL} / \mathrm{min}$, wavelength $=254 \mathrm{~nm}, \mathrm{t}_{\mathrm{R}}=4.59 \mathrm{~min}$ for major isomer, $\mathrm{t}_{\mathrm{R}}=5.34 \mathrm{~min}$ for minor isomer).

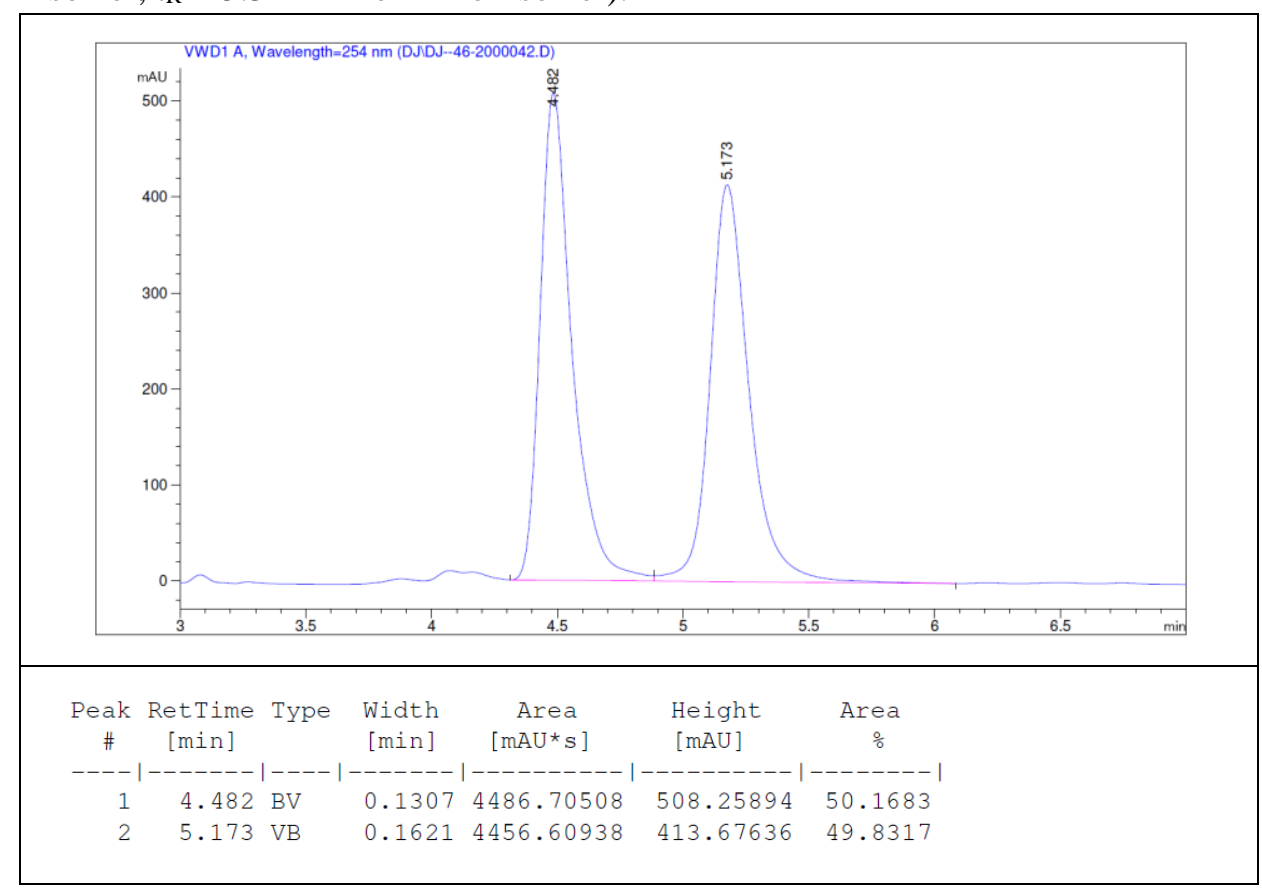




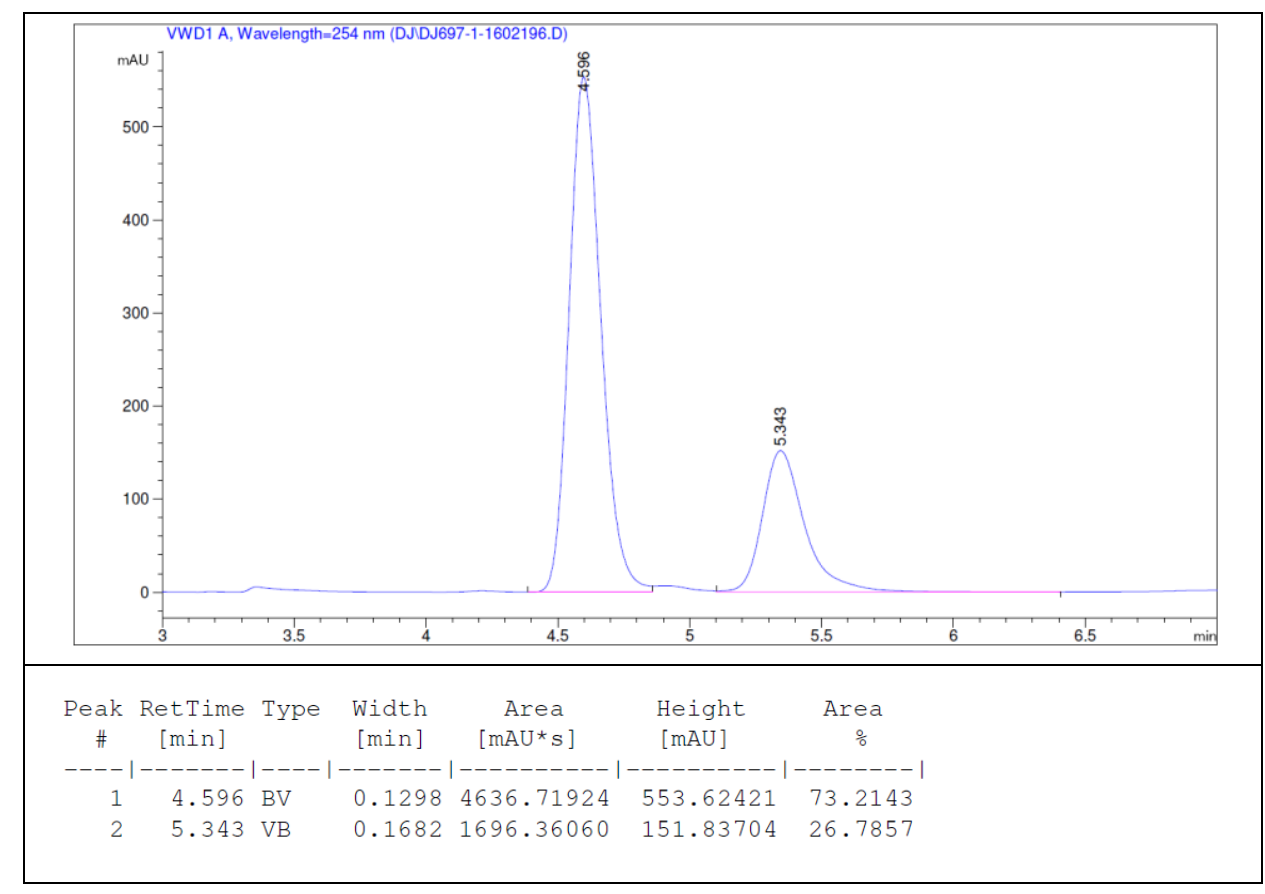




\section{Crystal structures of $6 \mathrm{~b}$ and 7}

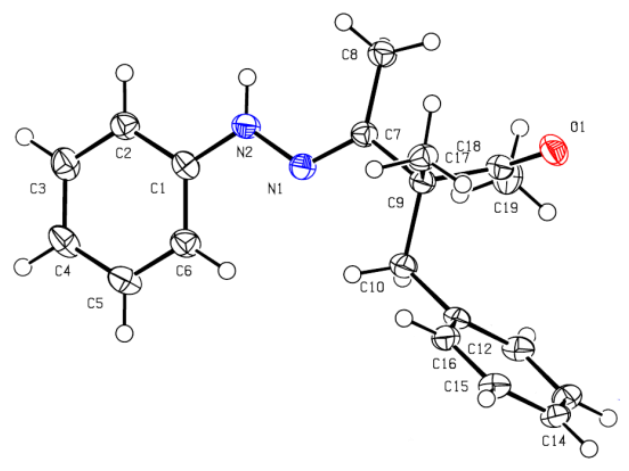

6b (CCDC No.: 1999211)

Identification code

Chemical formula

Formula weight

Temperature

Wavelength

Crystal size

Crystal system

Space group

Unit cell dimensions

Volume

$\mathrm{Z}$

Density (calculated)

Absorption coefficient

$\mathrm{F}(000)$

Theta range for data collection

Index ranges

Reflections collected

Independent reflections

Coverage of independent reflections

Absorption correction

Max. and min. transmission
K156

$\mathrm{C}_{19} \mathrm{H}_{22} \mathrm{~N}_{2} \mathrm{O}$

$294.38 \mathrm{~g} / \mathrm{mol}$

100(2) K

$1.54178 \AA$

$0.094 \times 0.121 \times 0.418 \mathrm{~mm}$

orthorhombic

P 212121

$a=13.1406(7)$

$\AA$

$\alpha=90^{\circ}$

$\mathrm{b}=13.2896(8)$

$\AA$

$\mathrm{c}=$

$19.2525(11) \AA \gamma=90^{\circ}$

3362.1(3) $\AA^{3}$

8

$1.163 \mathrm{~g} / \mathrm{cm}^{3}$

$0.565 \mathrm{~mm}^{-1}$

1264

4.04 to $70.09^{\circ}$

$-16<=\mathrm{h}<=15,-16<=\mathrm{k}<=16,-23<=1<=23$

110625

$6375[\mathrm{R}(\mathrm{int})=0.0522]$

$99.9 \%$

Multi-Scan

0.7533 and 0.6302 
Structure solution technique

Structure solution program

Refinement method

Refinement program

Function minimized

Data / restraints / parameters

Goodness-of-fit on F2

Final $\mathrm{R}$ indices

Weighting scheme

Absolute structure parameter

Largest diff. peak and hole

R.M.S. deviation from mean direct methods

SHELXT-(Sheldrick 2015)

Full-matrix least-squares on F2

SHELXL-2017/1 (Sheldrick, 2017)

$\Sigma \mathrm{w}(\mathrm{Fo} 2-\mathrm{Fc} 2) 2$

6375 / 0 / 411

1.055

6314 data;

$\mathrm{I}>2 \sigma(\mathrm{I})$

$\mathrm{R} 1=0.0271, \mathrm{wR} 2=0.0722$

all data

$\mathrm{R} 1=0.0273, \mathrm{wR} 2=0.0725$

$\mathrm{w}=1 /\left[\sigma^{2}\left(\mathrm{~F}_{\mathrm{o}}^{2}\right)+(0.0368 \mathrm{P})^{2}+0.6531 \mathrm{P}\right]$

where $\mathrm{P}=\left(\mathrm{F}_{\mathrm{o}}^{2}+2 \mathrm{~F}_{\mathrm{c}}^{2}\right) / 3$

$0.08(4)$

0.177 and $-0.198 \mathrm{e}^{-3}$

$0.031 \mathrm{e}^{-3}$ 


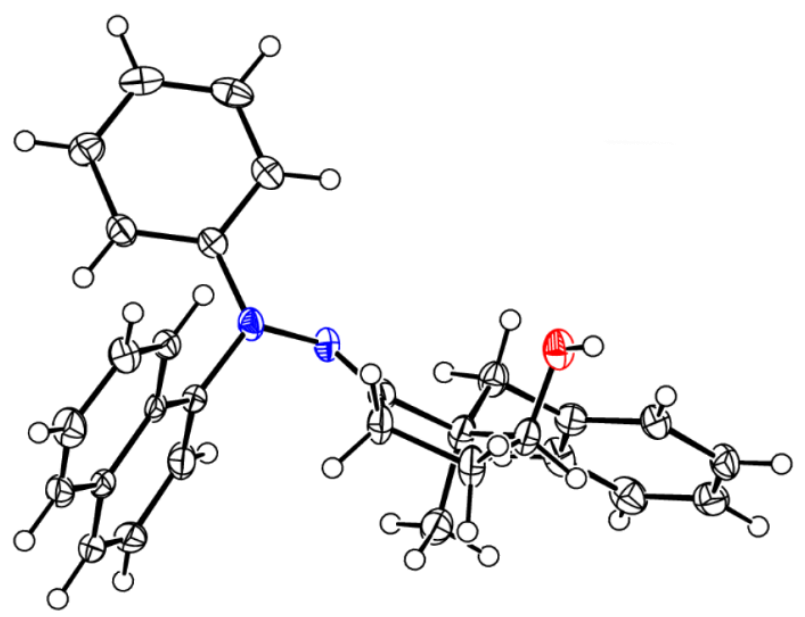

7 (CCDC No.: 1999210)

Identification code

Chemical formula

Formula weight

Temperature

Wavelength

Crystal size

Crystal system

Unit cell dimensions

Volume

$\mathrm{Z}$

Density (calculated)

Absorption coefficient

$\mathrm{F}(000)$

Theta range for data collection

Index ranges

Reflections collected

Independent reflections

Coverage of independent reflections

Absorption correction

Structure solution technique

Structure solution program

Refinement method

Refinement program
K088

$\mathrm{C}_{29.50} \mathrm{H}_{29} \mathrm{ClN}_{2} \mathrm{O}$

$463.00 \mathrm{~g} / \mathrm{mol}$

100(2) K

$1.54178 \AA$

$0.068 \times 0.132 \times 0.307 \mathrm{~mm}$

monoclinic

$\mathrm{a}=15.3252 \AA$

$\alpha=90.000^{\circ}$

$\mathrm{b}=10.5177 \AA$

$\beta=96.183^{\circ}$

$\mathrm{c}=15.5455 \AA$

$\gamma=90.000^{\circ}$

$2497.8 \AA^{3}$

4

$1.234 \mathrm{~g} / \mathrm{cm}^{3}$

$1.535 \mathrm{~mm}^{-1}$

980

2.90 to $66.59^{\circ}$

$-18<=\mathrm{h}<=18,-12<=\mathrm{k}<=12,-18<=\mathrm{l}<=18$

81824

$8790[\mathrm{R}(\mathrm{int})=0.0314]$

$100.0 \%$

Multi-Scan

direct methods

XT, VERSION 2014/5

Full-matrix least-squares on F2

SHELXL-2017/1 (Sheldrick, 2017) 
Function minimized

Data / restraints / parameters

Goodness-of-fit on F2

Final $\mathrm{R}$ indices

Weighting scheme

Absolute structure parameter

Largest diff. peak and hole

R.M.S. deviation from mean
$\Sigma \mathrm{w}(\mathrm{Fo} 2-\mathrm{Fc} 2) 2$

8790 / 247 / 660

1.040

8713 data; $\mathrm{I}>2 \sigma(\mathrm{I}) \quad \mathrm{R} 1=0.0540, \mathrm{wR} 2=0.1584$

all data

$\mathrm{R} 1=0.0543, \mathrm{wR} 2=0.1589$

$\mathrm{w}=1 /\left[\sigma^{2}\left(\mathrm{~F}_{\mathrm{o}}{ }^{2}\right)+(0.1103 \mathrm{P})^{2}+1.9458 \mathrm{P}\right]$

where $\mathrm{P}=\left(\mathrm{F}_{0}{ }^{2}+2 \mathrm{~F}_{\mathrm{c}}{ }^{2}\right) / 3$

$0.045(3)$

1.358 and $-0.851 \mathrm{e}^{-3}$

$0.070 \mathrm{e}^{-3}$ 


\section{References}

(1) (a) Gong, Q.; Wen, J.; Zhang, X. Chem. Sci., 2019, 10, 6350-6353.

(2) (a) Martínez, A.; Webber, M. J.; Müller, S.; List, B. Angew. Chem. Int. Ed. 2013, 52, 9486-9490. (b) Chaudhary, P.; Gupta, S.; Muniyappan, N.; Sabiah, S.; Kandasamy, J. Green Chem., 2016, 18, 2323-2330. (c) Chaudhary, P.; Gupta, S.; Sabiah, S.; Kandasamy, J. Green Chem., 2016, 18, 6215-6221. 


\section{NMR spectra}

${ }^{1} \mathrm{H}$ NMR spectrum of compound 3a $\left(500 \mathrm{MHz}, \mathrm{CDCl}_{3}\right)$

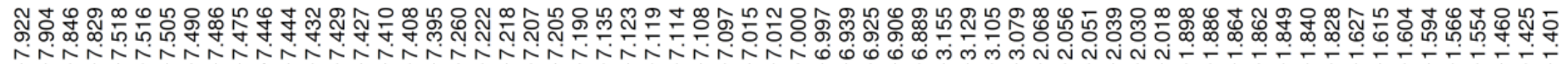
作

$1 \mathrm{H}$ AMX500

vybm2p80a1-2(2nd)

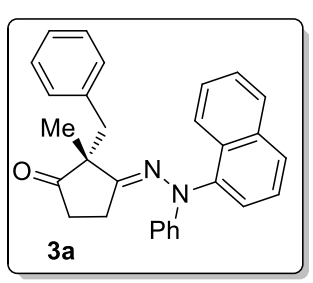

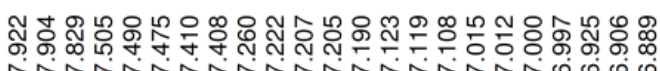

ininanininaninanomo
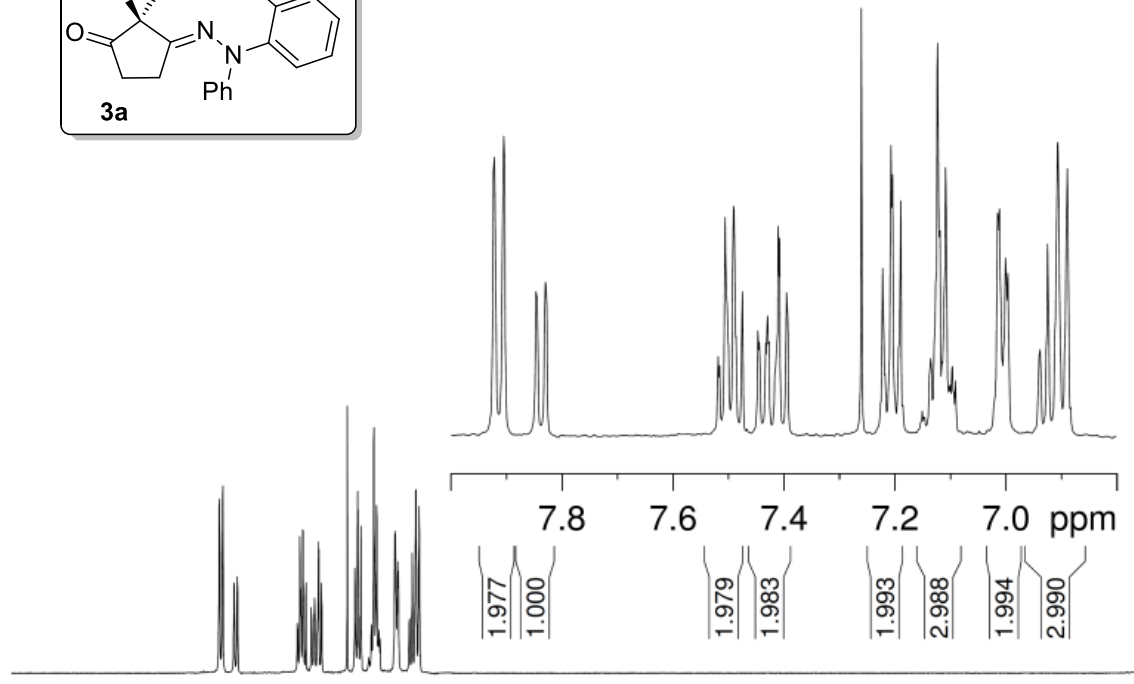

Nownew

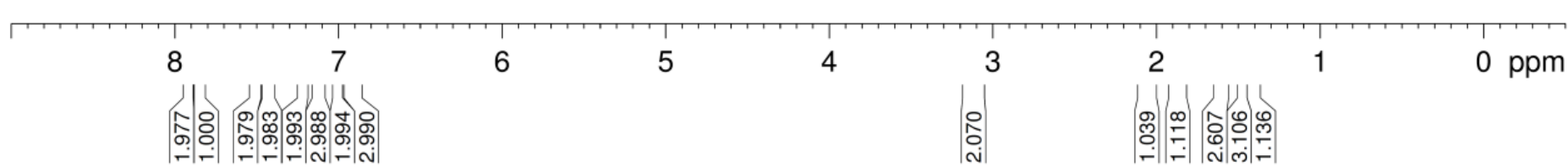


${ }^{13} \mathrm{C}$ NMR spectrum of compound $\mathbf{3 a}\left(125 \mathrm{MHz}, \mathrm{CDCl}_{3}\right)$

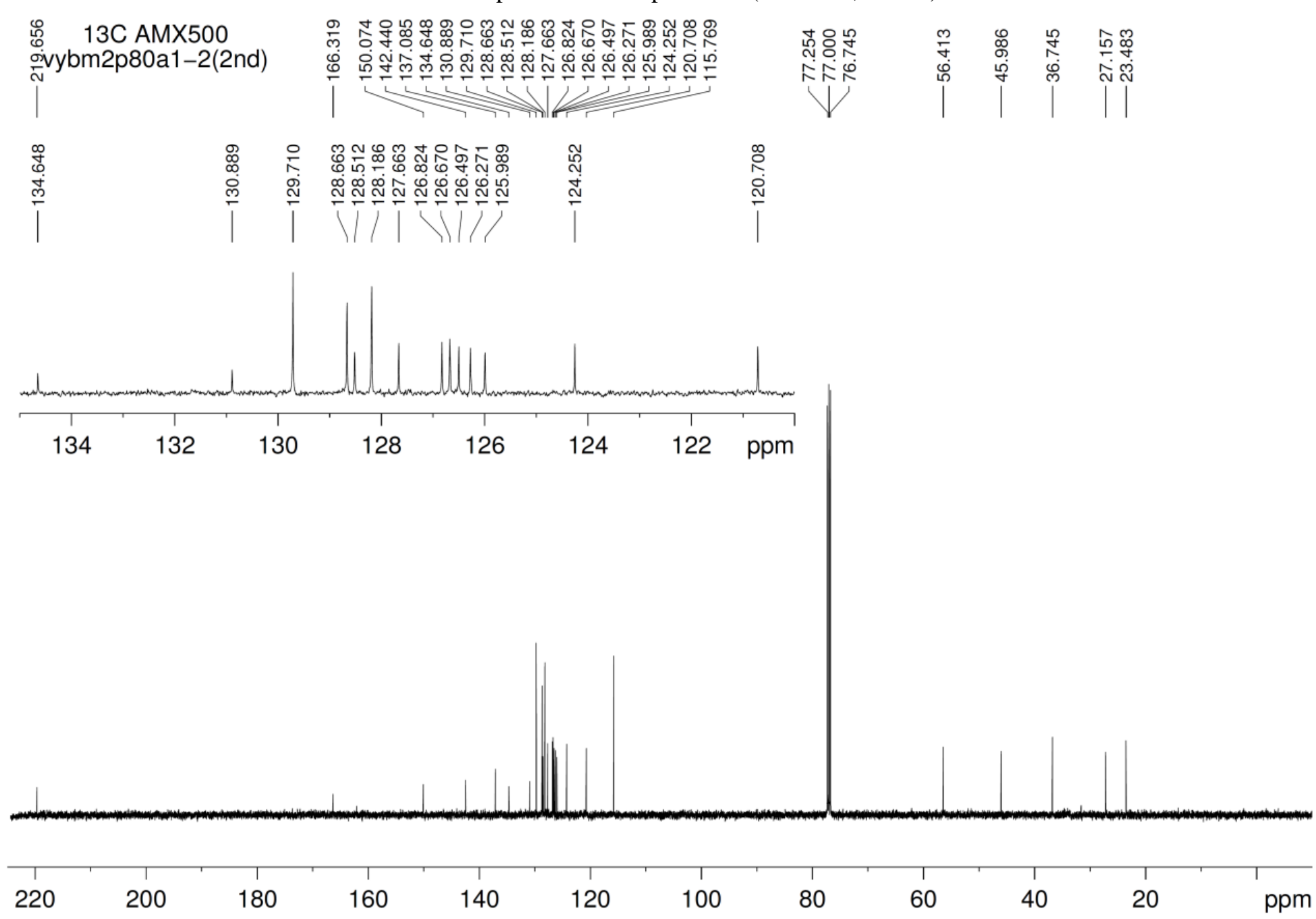


${ }^{1} \mathrm{H}$ NMR spectrum of compound $\mathbf{3 b}\left(500 \mathrm{MHz}, \mathrm{CDCl}_{3}\right)$

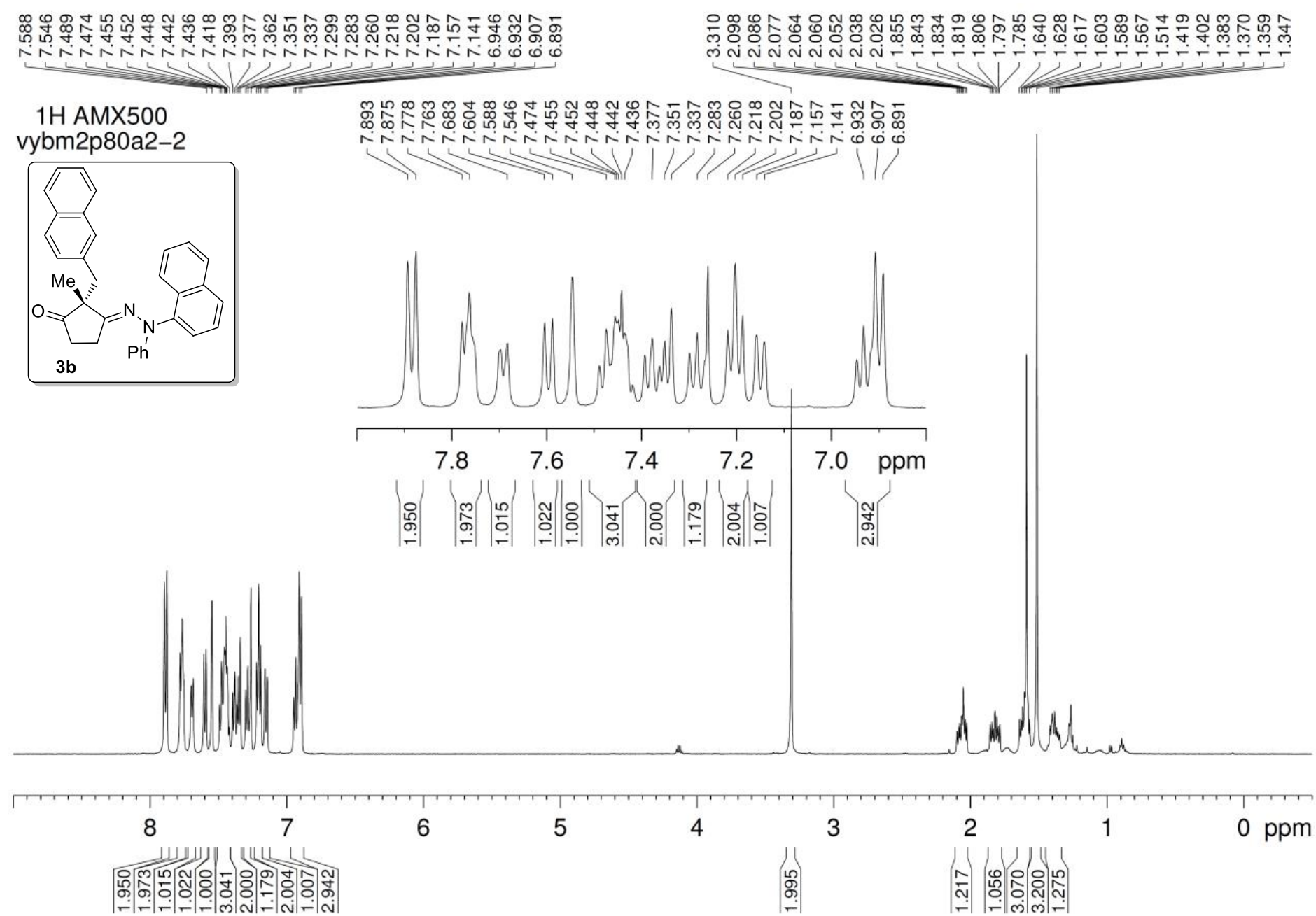


${ }^{13} \mathrm{C}$ NMR spectrum of compound $\mathbf{3 b}\left(125 \mathrm{MHz}, \mathrm{CDCl}_{3}\right)$
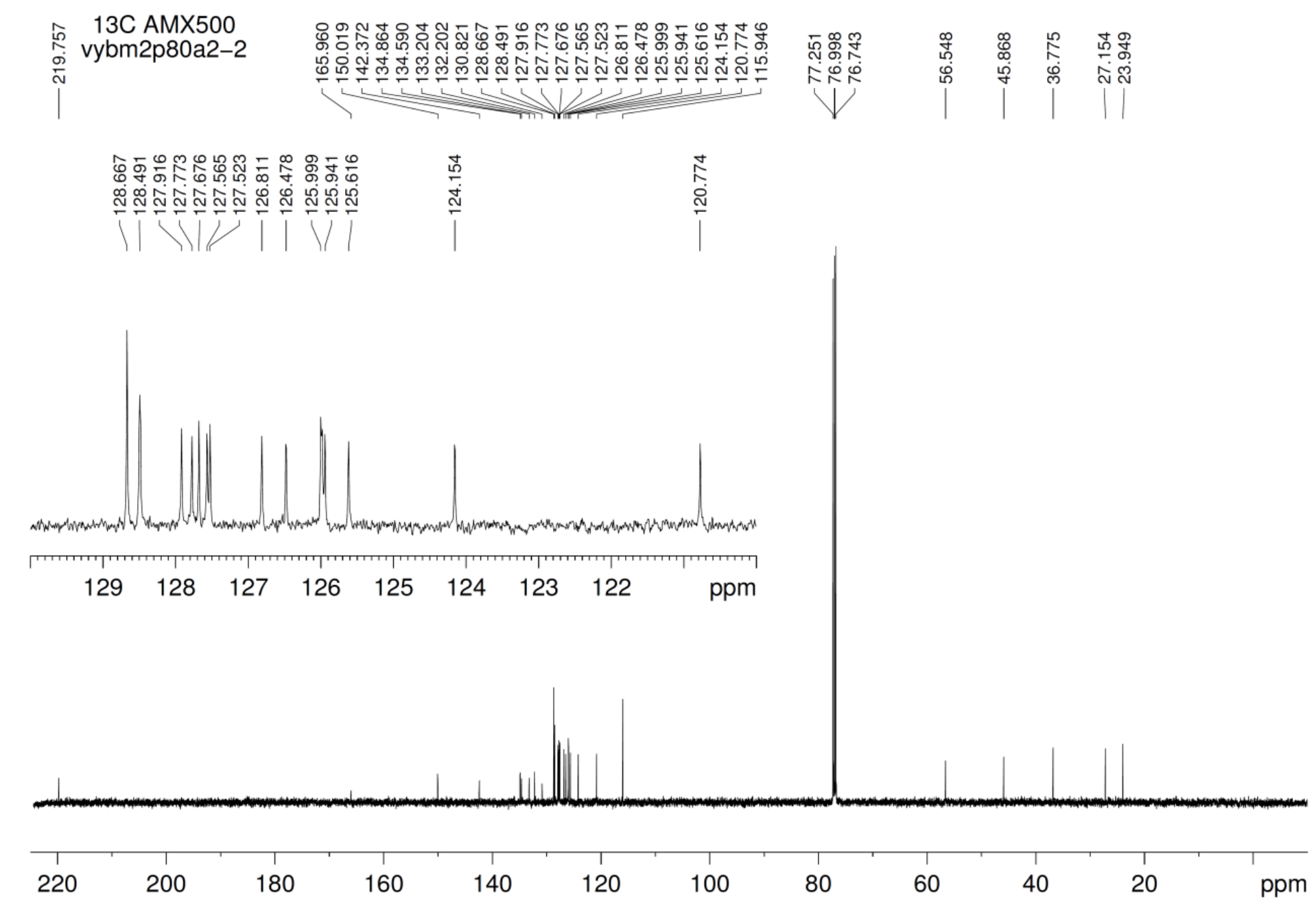
${ }^{1} \mathrm{H}$ NMR spectrum of compound $3 \mathbf{c}\left(500 \mathrm{MHz}, \mathrm{CDCl}_{3}\right)$

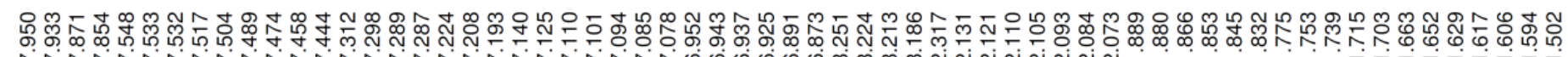

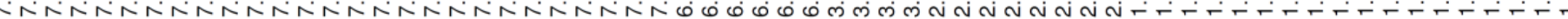

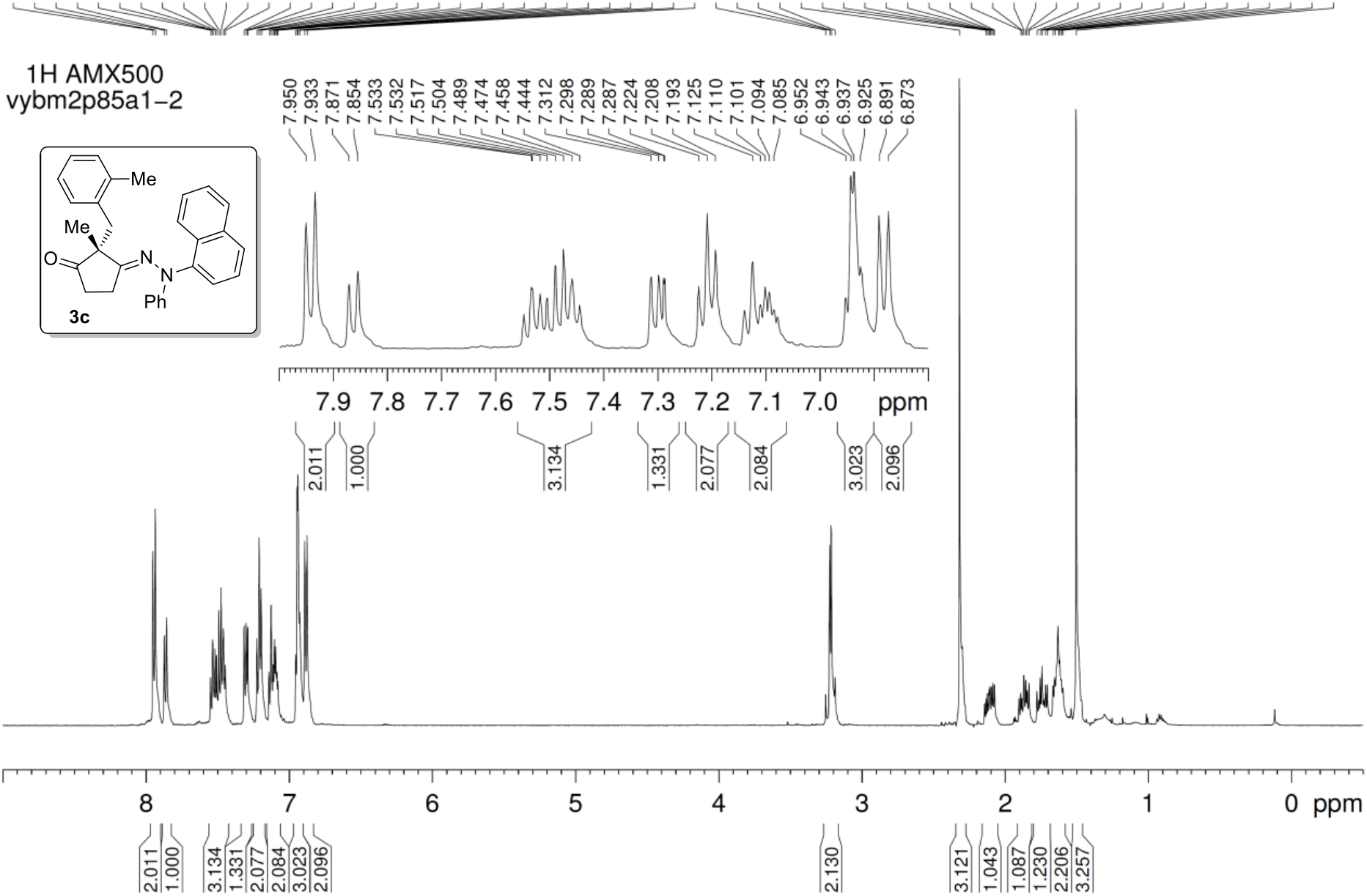


${ }^{13} \mathrm{C}$ NMR spectrum of compound $3 \mathbf{c}\left(125 \mathrm{MHz}, \mathrm{CDCl}_{3}\right)$

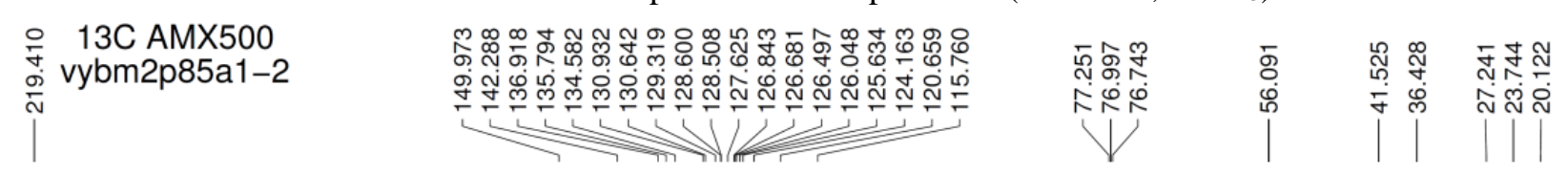

|

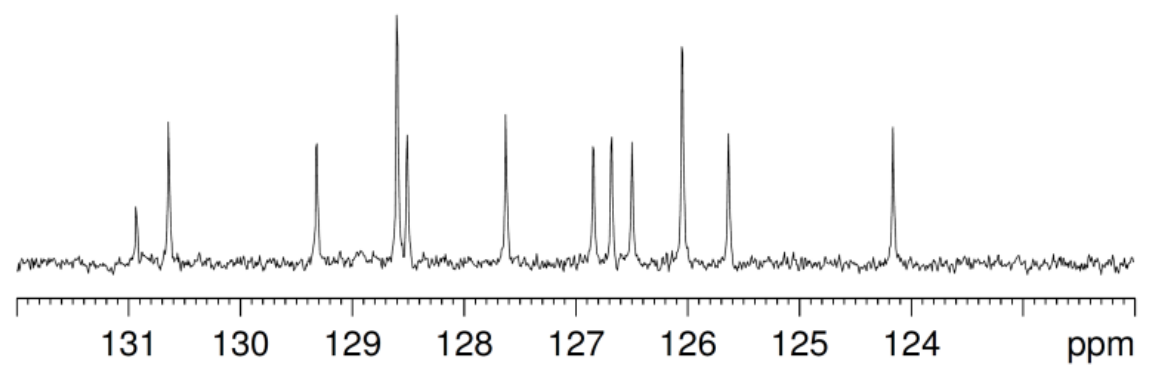

$\begin{array}{lllllllll}131 & 130 & 129 & 128 & 127 & 126 & 125 & 124 & \text { ppm }\end{array}$

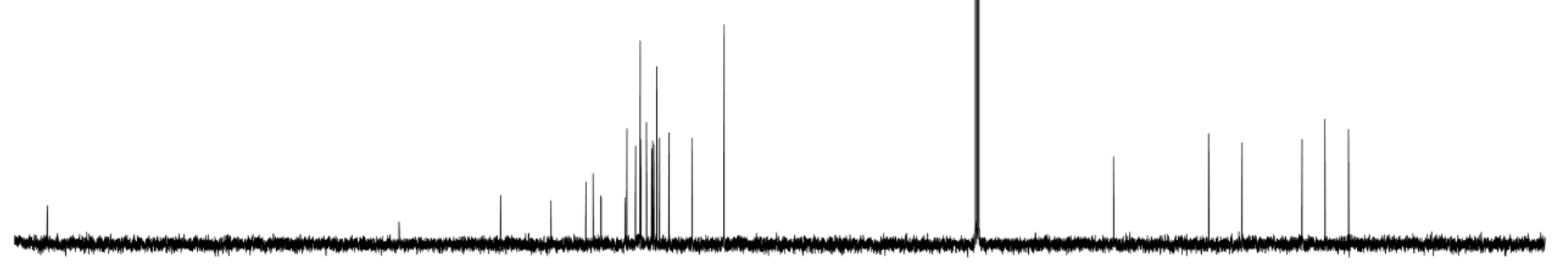

\begin{tabular}{rrrrrrrrrrr}
\hline 220 & 200 & 180 & 160 & 140 & 120 & 100 & 80 & 60 & 40 & 20 \\
${ }^{1} \mathrm{H}$ NMR spectrum of compound $\mathbf{3 d}\left(500 \mathrm{MHz}, \mathrm{CDCl}_{3}\right)$ & &
\end{tabular}




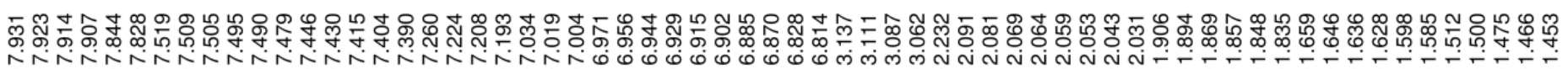

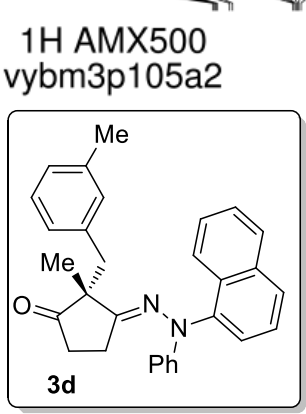

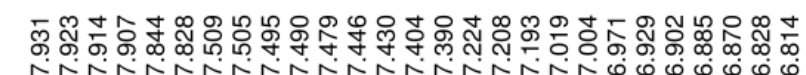

vybm3p105a

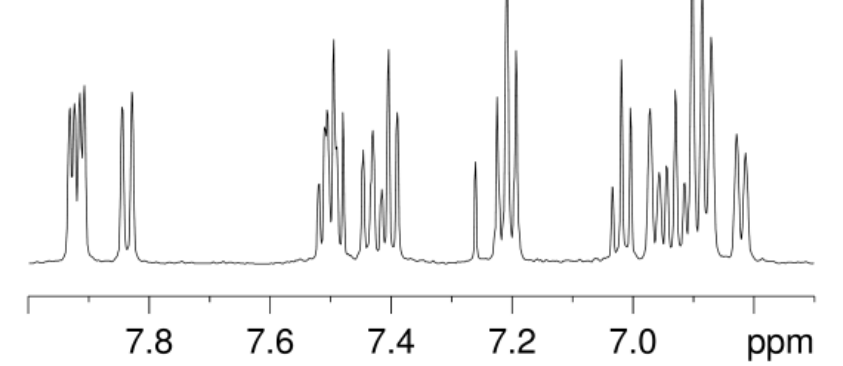

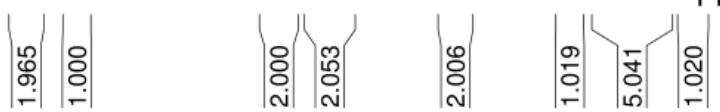

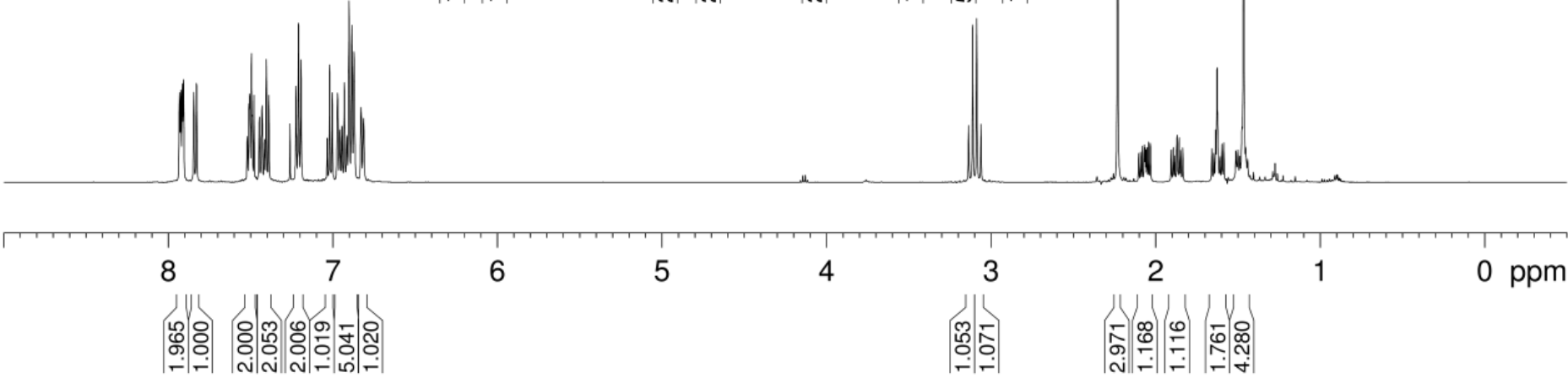


${ }^{13} \mathrm{C}$ NMR spectrum of compound $\mathbf{3 d}\left(125 \mathrm{MHz}, \mathrm{CDCl}_{3}\right)$

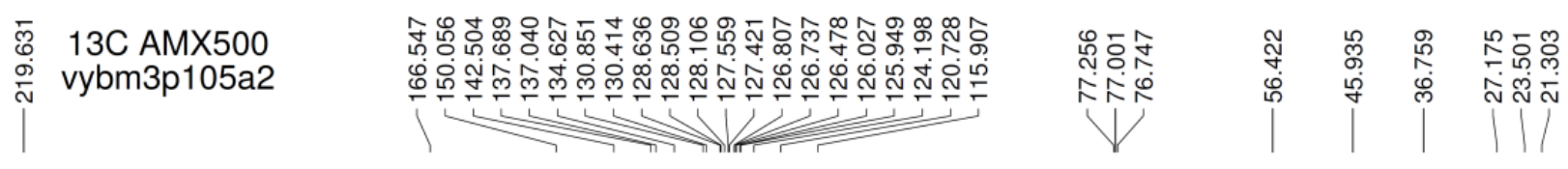

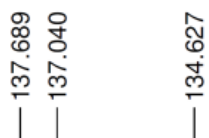

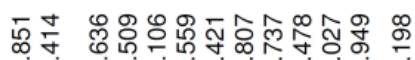

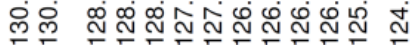
$\stackrel{\text { N }}{N}$
। jujiji
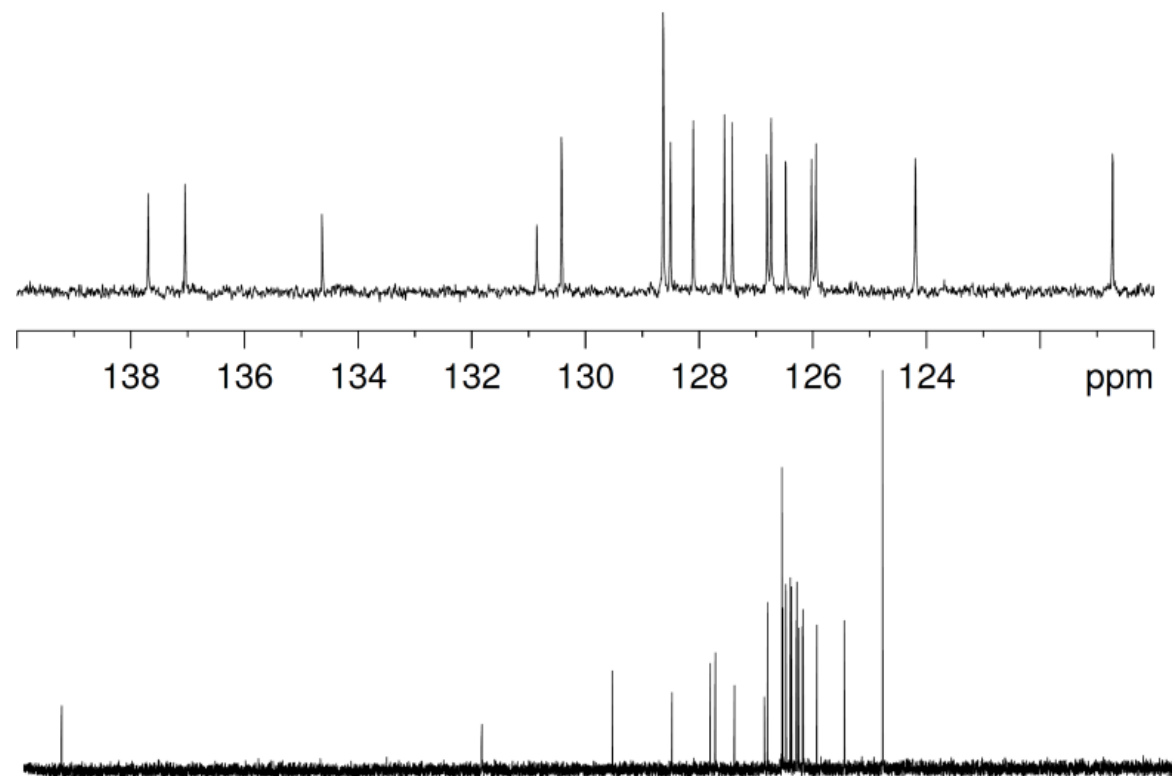

220

200

180

160

140

120

100

80

60

40

20 
${ }^{1} \mathrm{H}$ NMR spectrum of compound $\mathbf{3 e}\left(500 \mathrm{MHz}, \mathrm{CDCl}_{3}\right)$

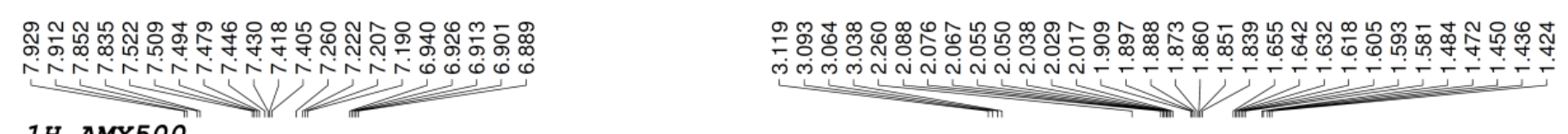

1H AMX500

vybm2p85a2-2
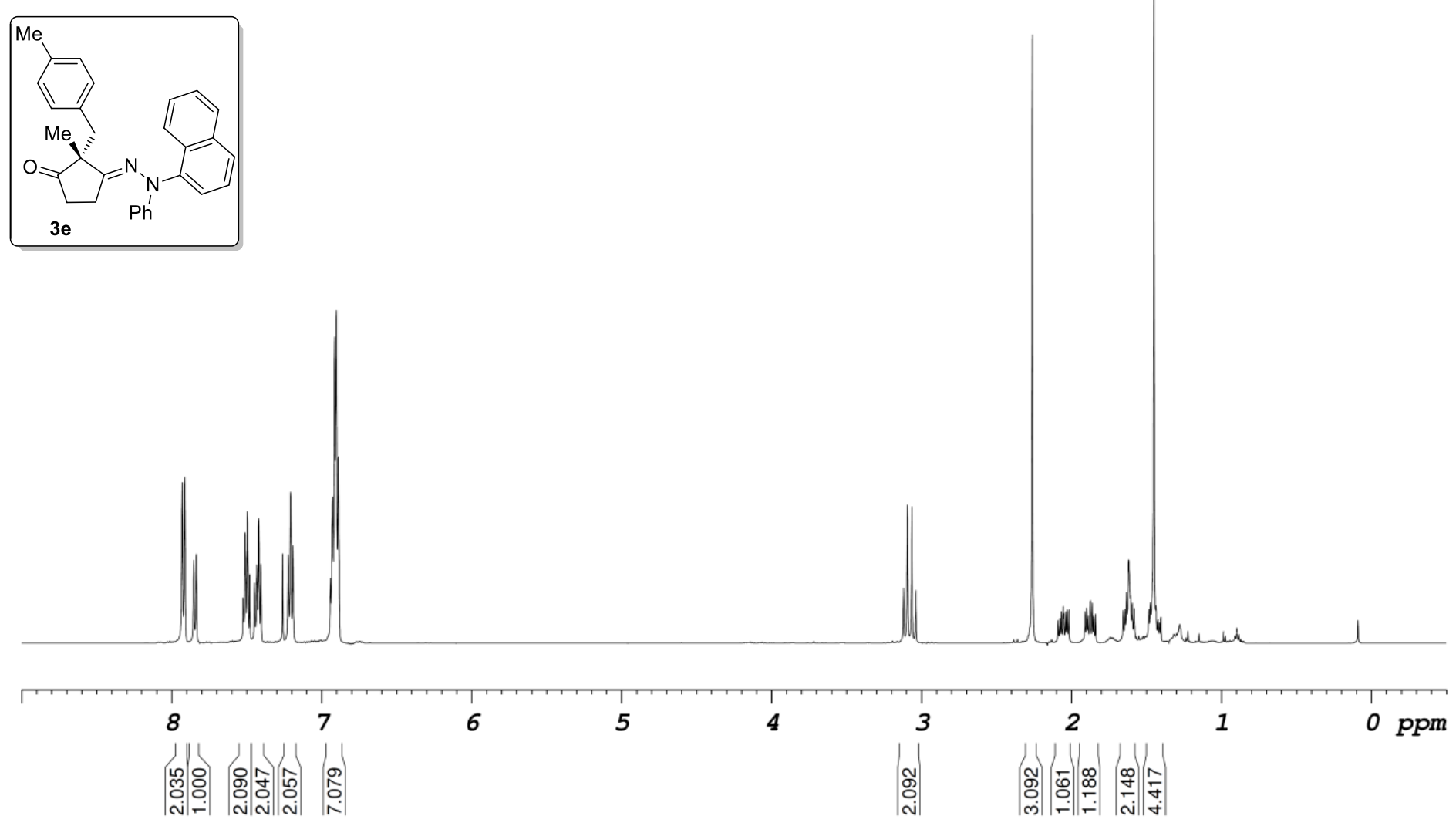
${ }^{13} \mathrm{C}$ NMR spectrum of compound $\mathbf{3 e}\left(125 \mathrm{MHz}, \mathrm{CDCl}_{3}\right)$

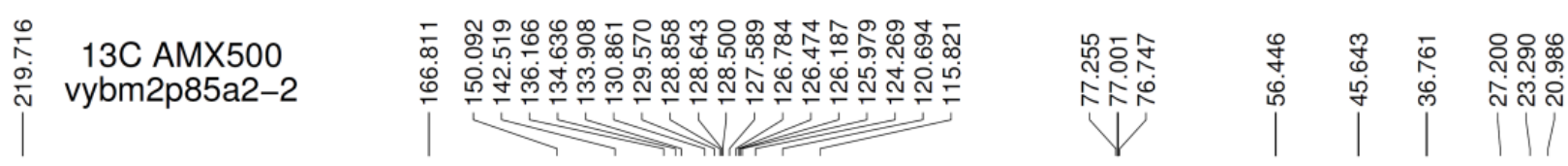

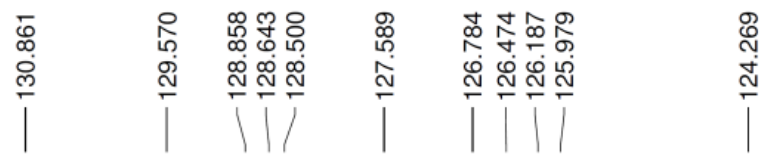
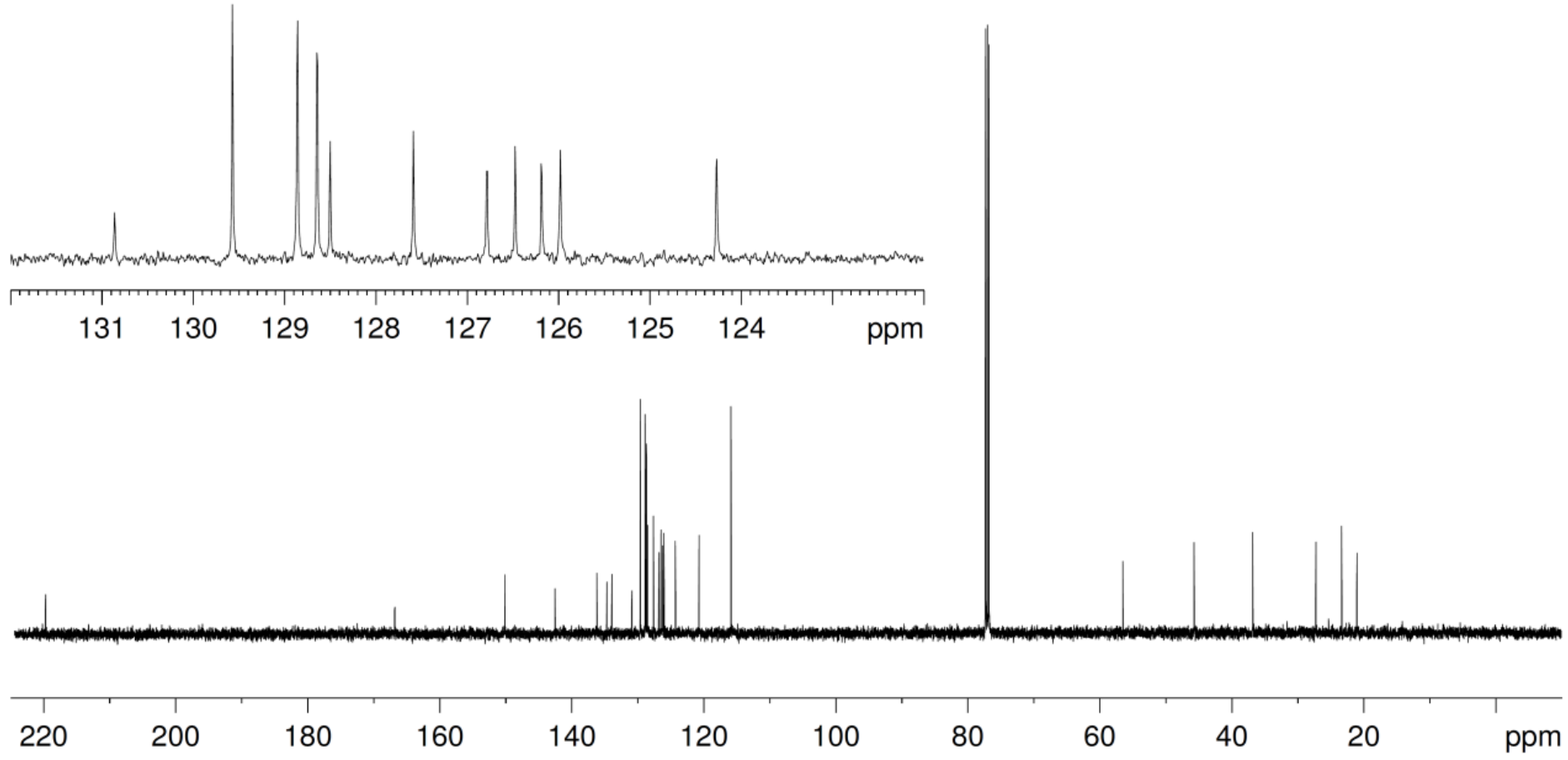
${ }^{1} \mathrm{H}$ NMR spectrum of compound $3 \mathbf{f}\left(500 \mathrm{MHz}, \mathrm{CDCl}_{3}\right)$

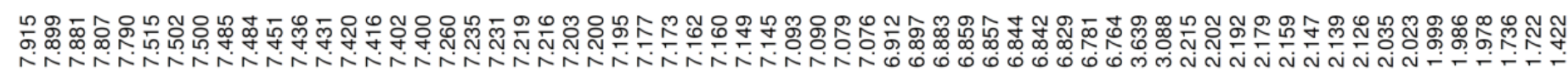

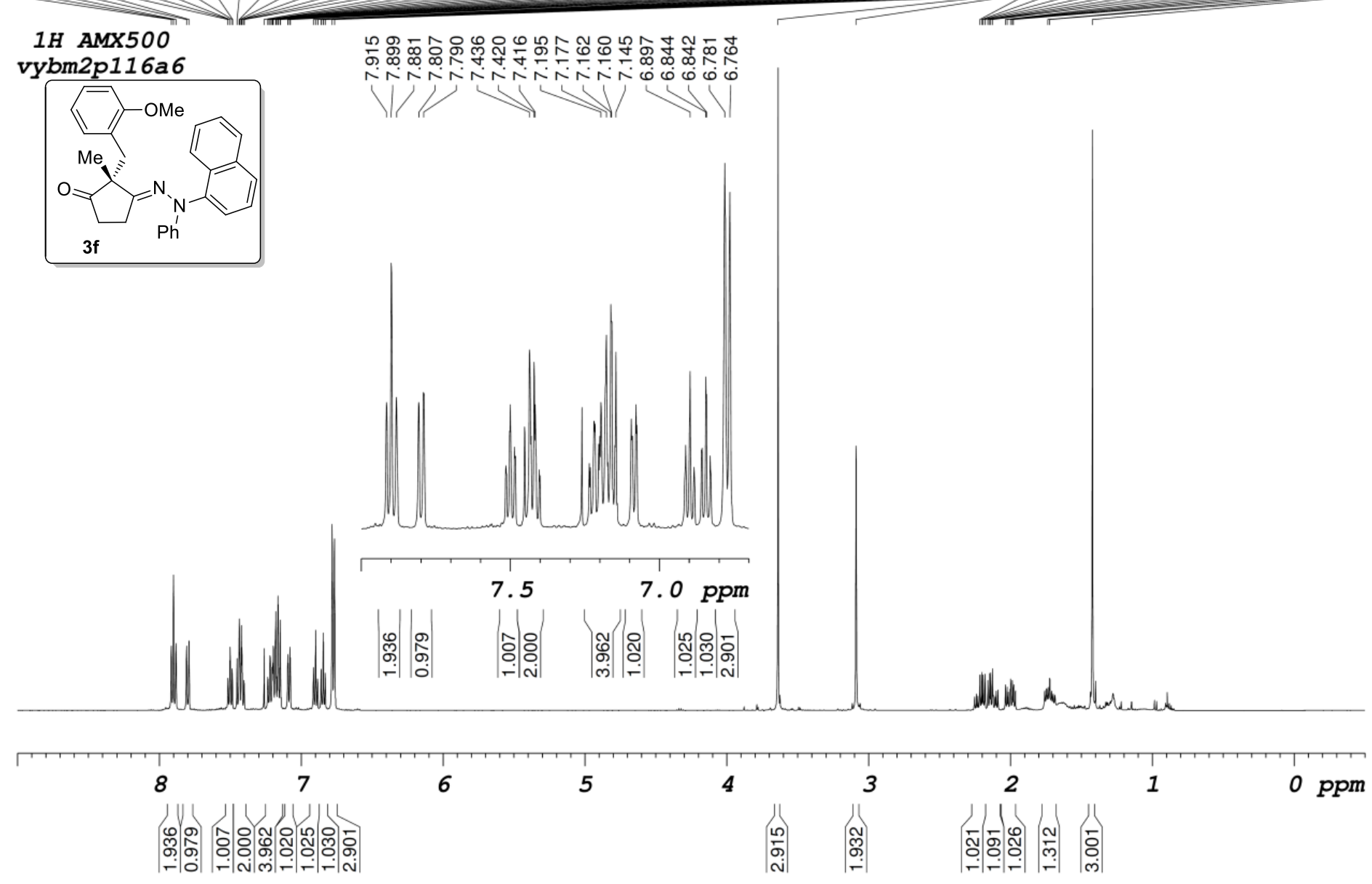


${ }^{13} \mathrm{C}$ NMR spectrum of compound $3 \mathbf{f}\left(125 \mathrm{MHz}, \mathrm{CDCl}_{3}\right)$

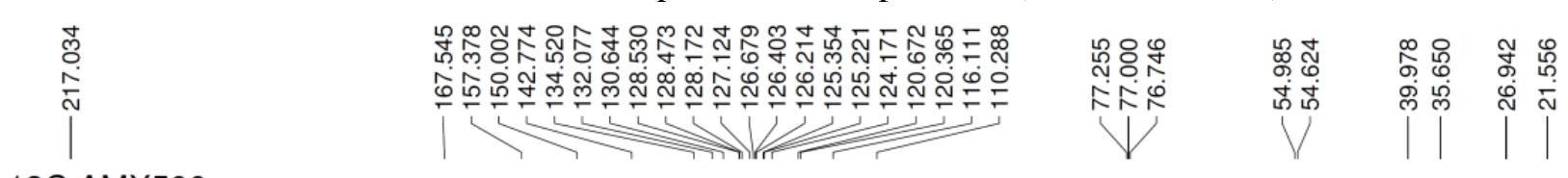

13C AMX500

vybm2p116a6
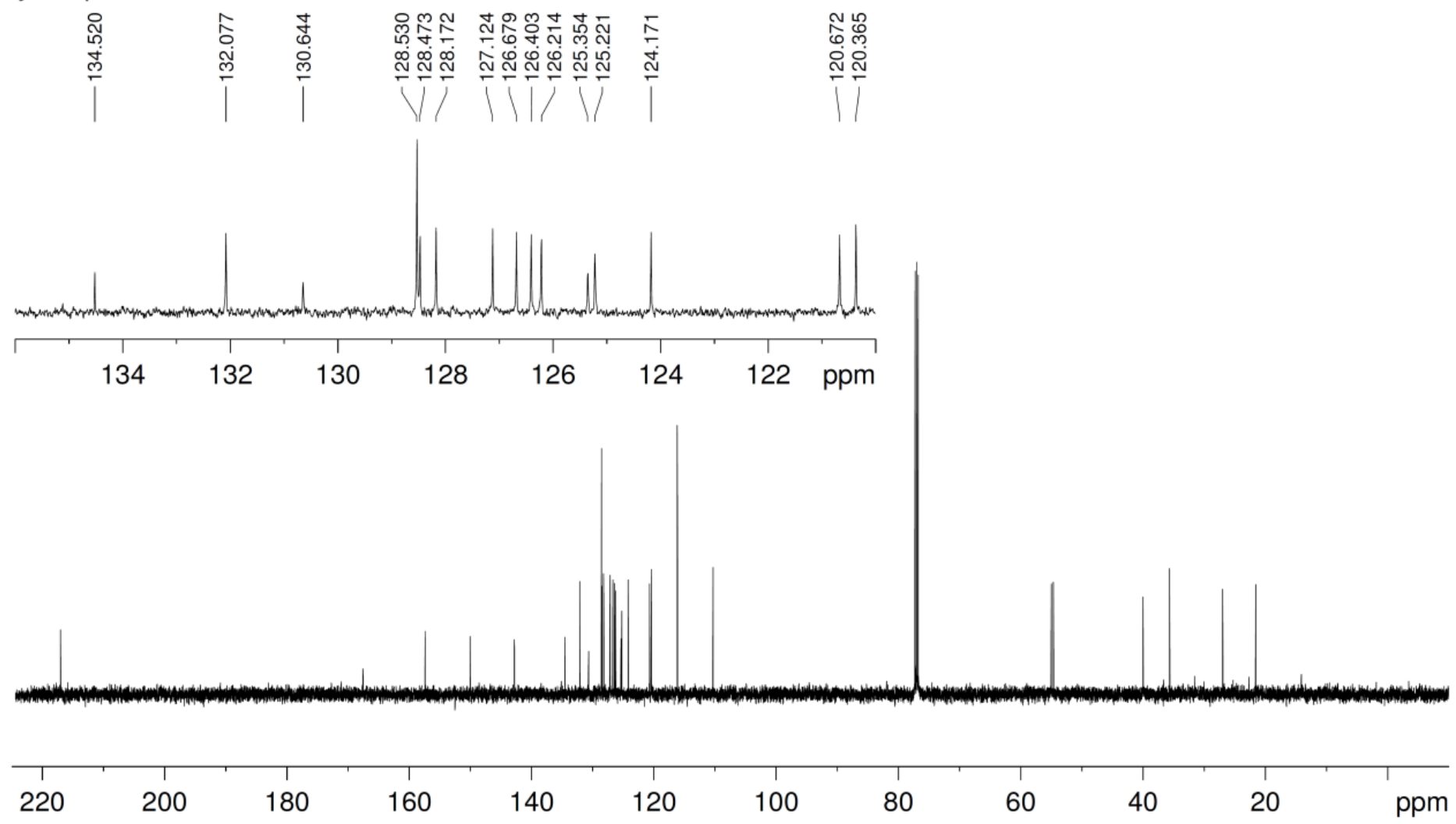
${ }^{1} \mathrm{H}$ NMR spectrum of compound $\mathbf{3 g}\left(500 \mathrm{MHz}, \mathrm{CDCl}_{3}\right)$

న్心

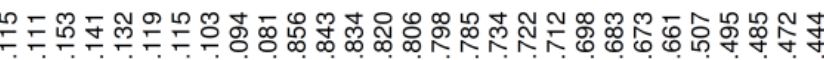

1H AMX500

vybm2p110a1

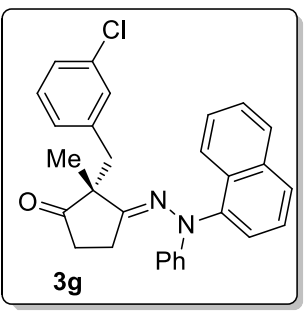

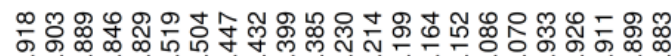

$1 / 1$

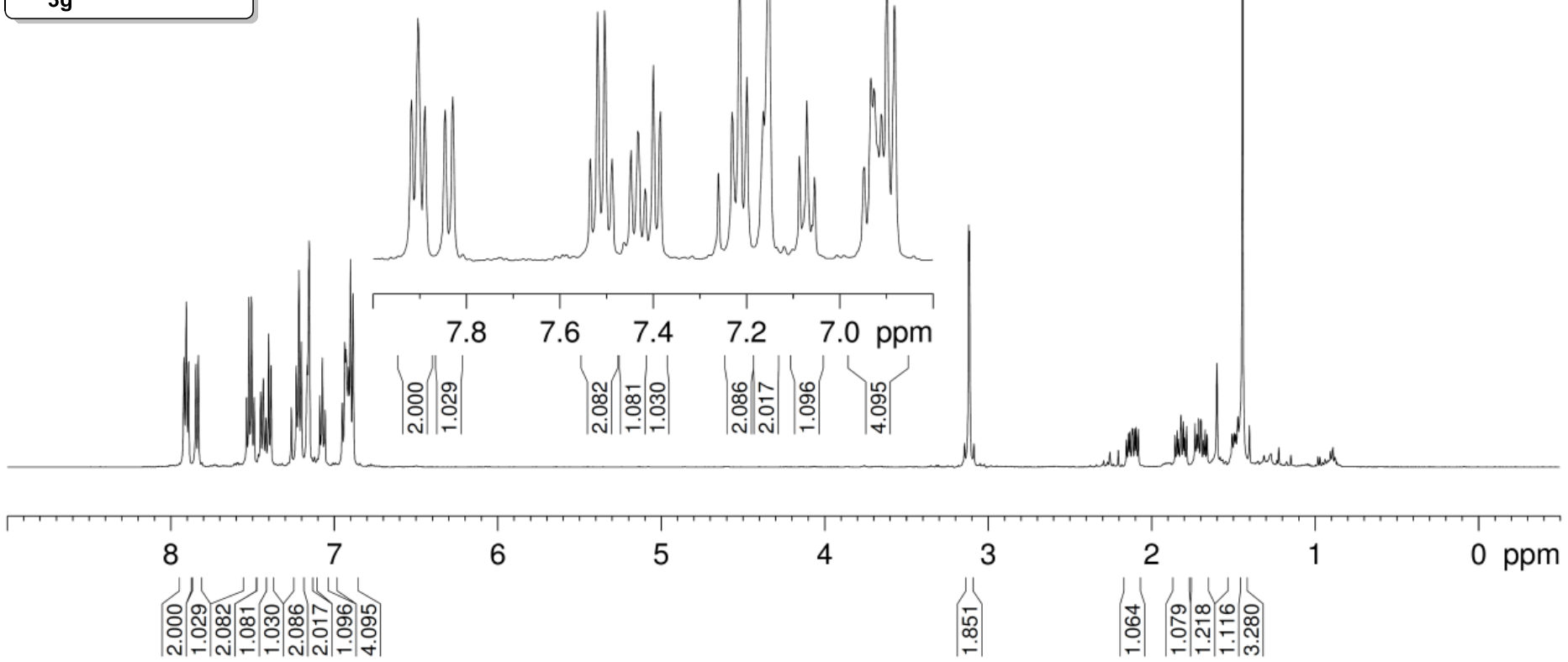


${ }^{13} \mathrm{C}$ NMR spectrum of compound $\mathbf{3 g}\left(125 \mathrm{MHz}, \mathrm{CDCl}_{3}\right)$

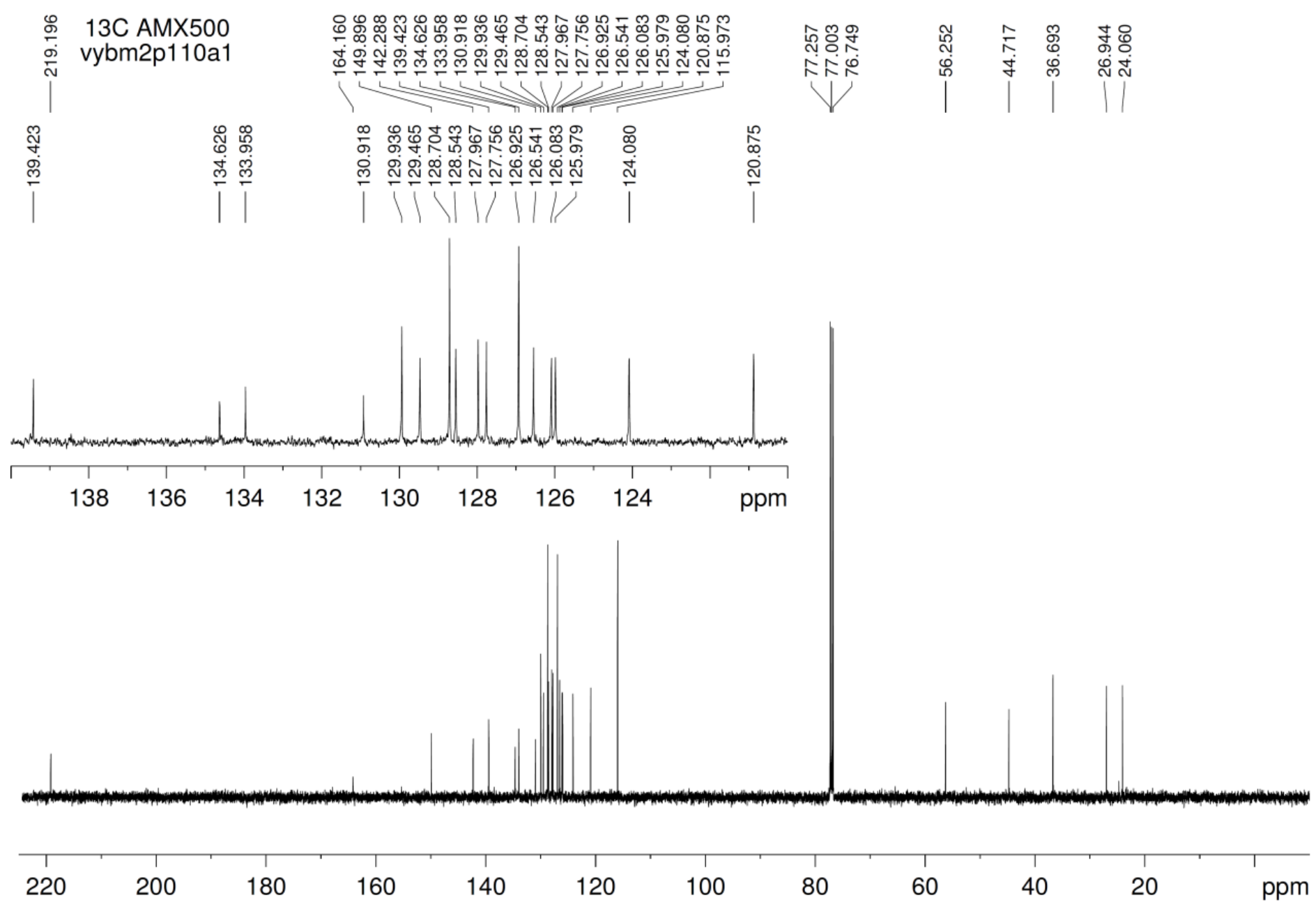


${ }^{1} \mathrm{H}$ NMR spectrum of compound $3 \mathbf{h}\left(500 \mathrm{MHz}, \mathrm{CDCl}_{3}\right)$

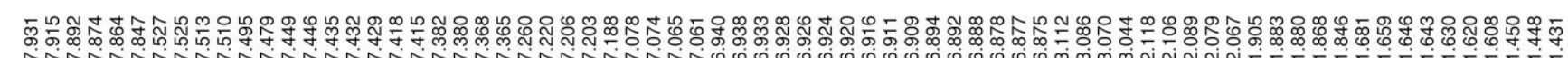
(n)

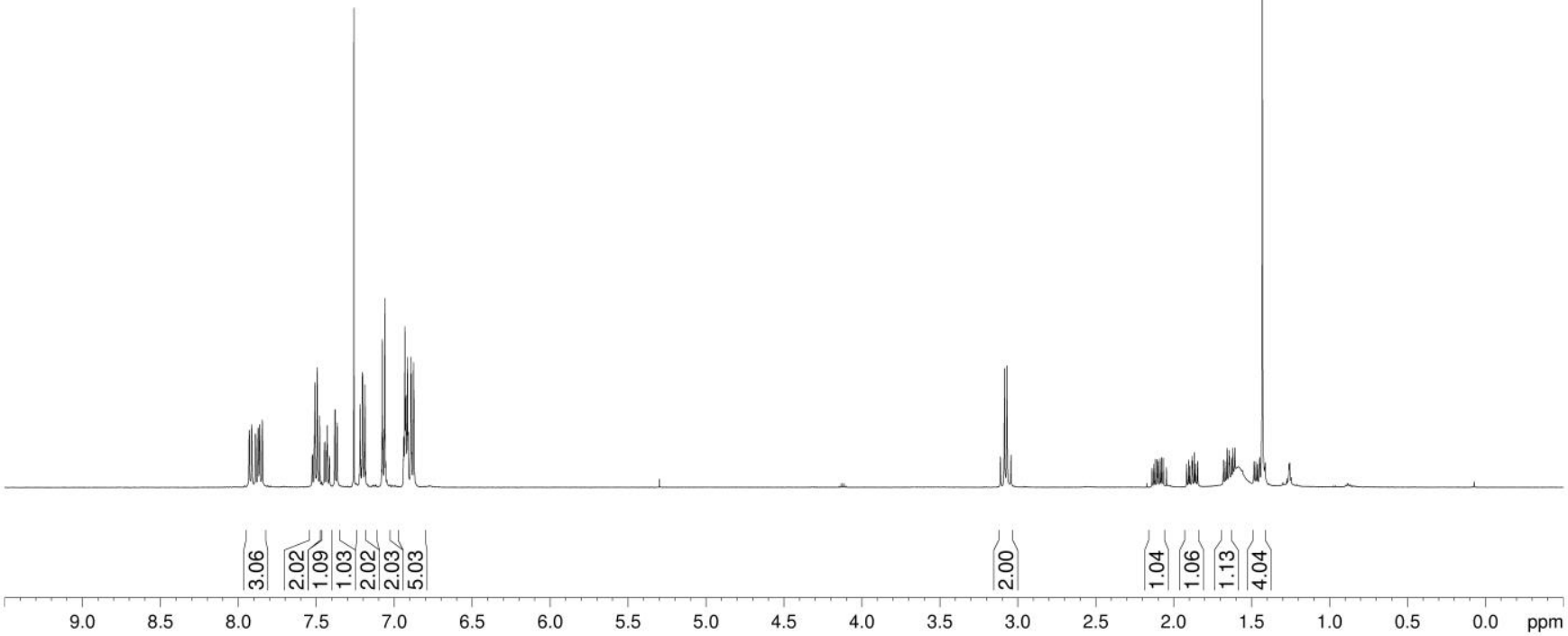


${ }^{13} \mathrm{C}$ NMR spectrum of compound $\mathbf{3 h}\left(125 \mathrm{MHz}, \mathrm{CDCl}_{3}\right)$

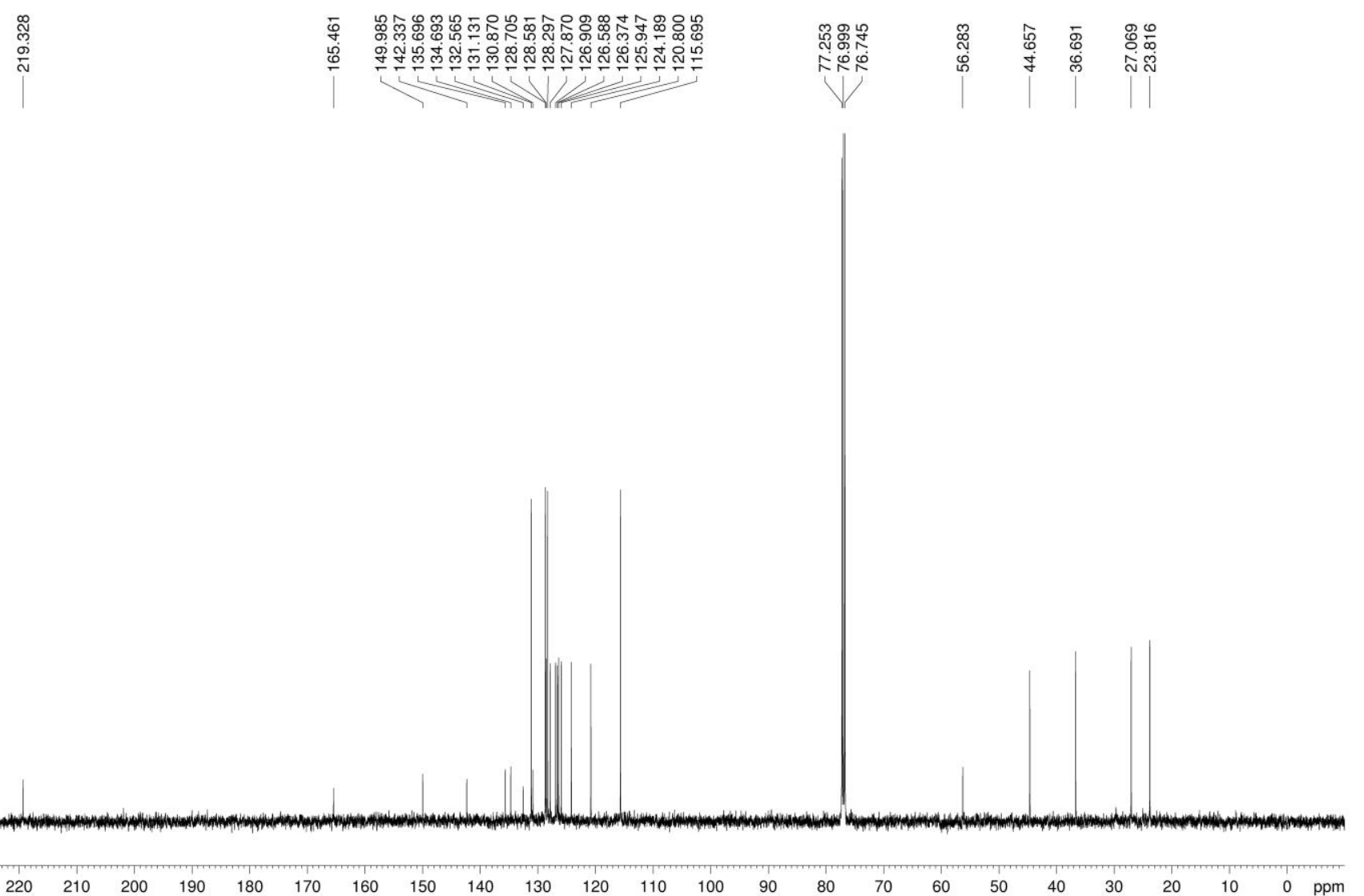


${ }^{1} \mathrm{H}$ NMR spectrum of compound $3 \mathbf{i}\left(500 \mathrm{MHz}, \mathrm{CDCl}_{3}\right)$

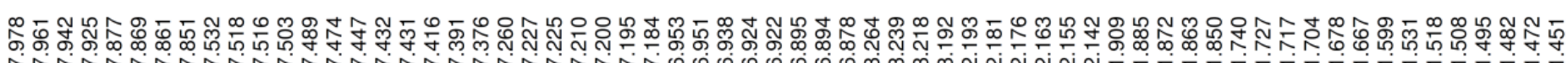

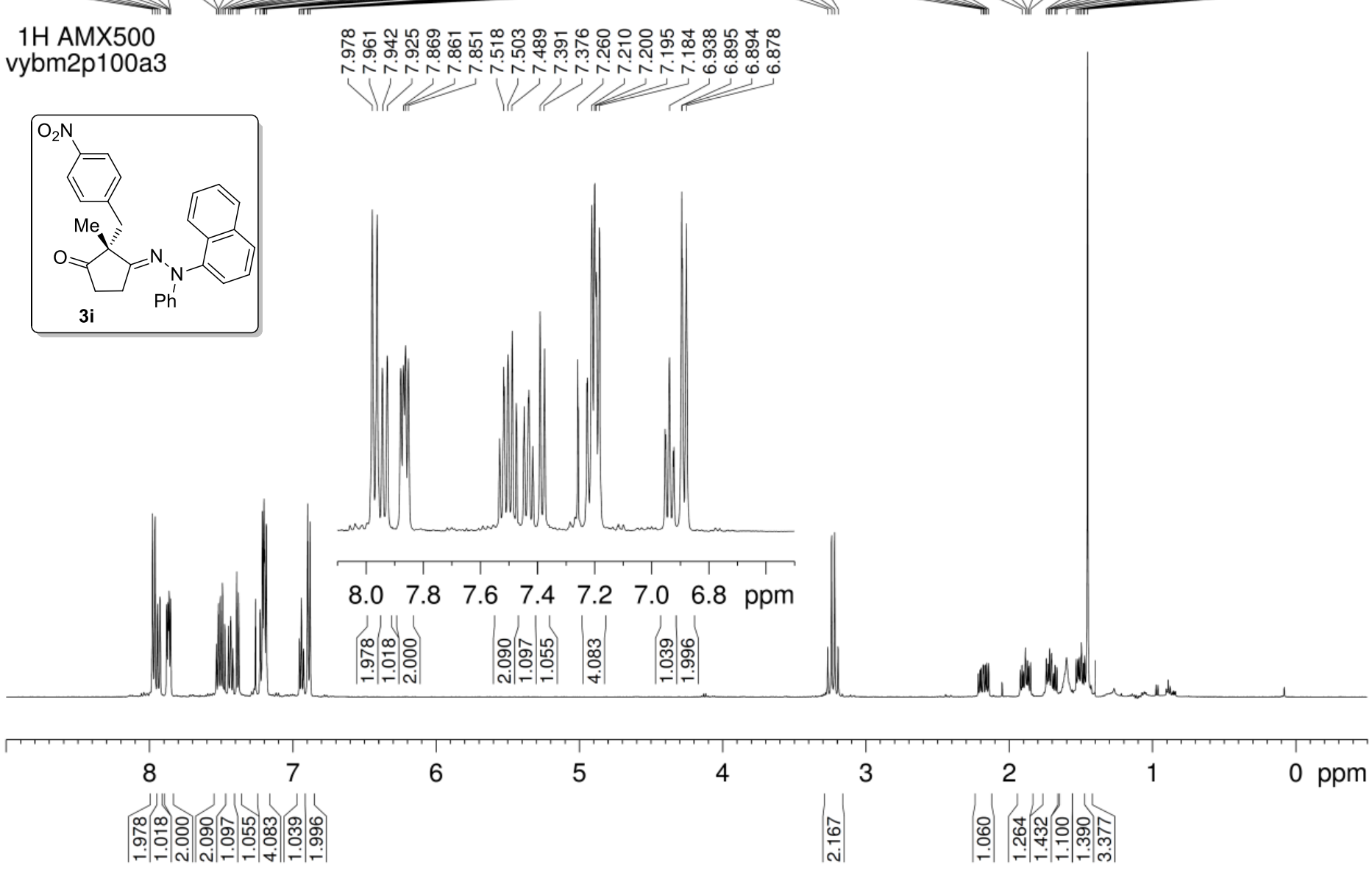


${ }^{13} \mathrm{C}$ NMR spectrum of compound $3 \mathbf{i}\left(125 \mathrm{MHz}, \mathrm{CDCl}_{3}\right)$

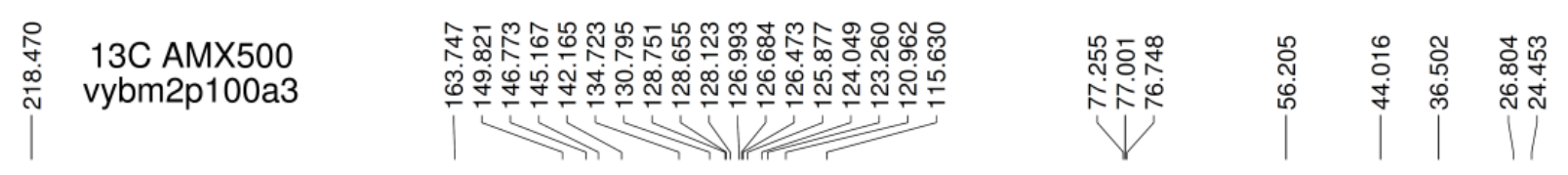

|lll

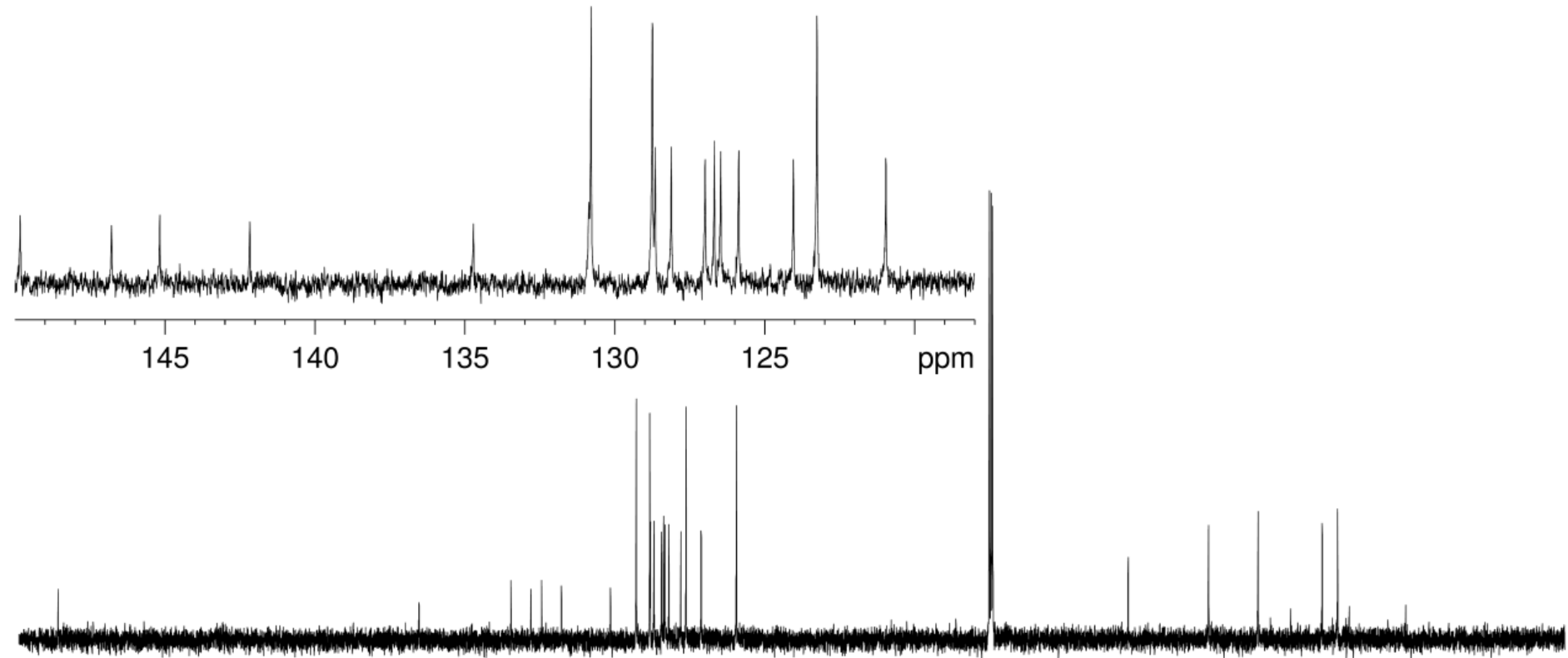

220

200

180

160

140

120

100

80

60

40

20

ppm 
${ }^{1} \mathrm{H}$ NMR spectrum of compound $\mathbf{3 j}\left(500 \mathrm{MHz}, \mathrm{CDCl}_{3}\right)$

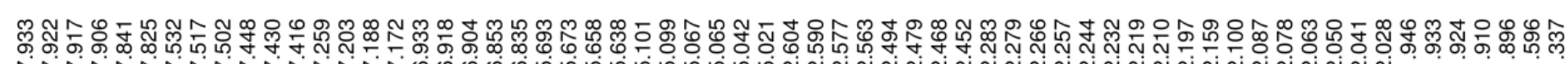

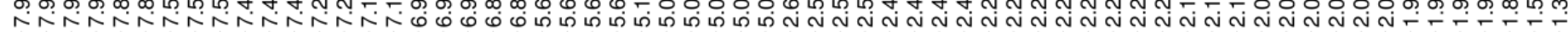

\section{$1 \mathrm{H}$ AMX500}

vybm2p119a3
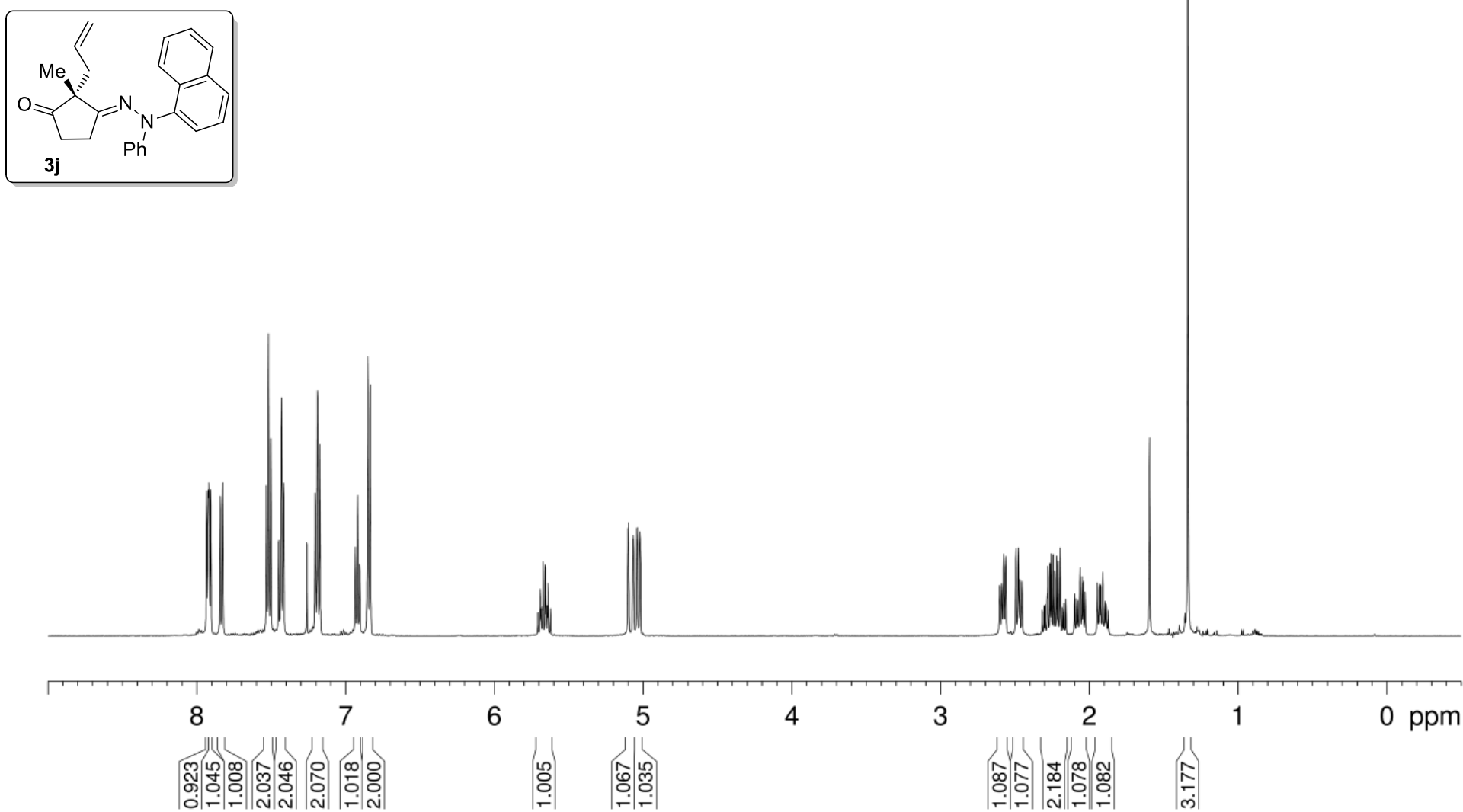
${ }^{13} \mathrm{C}$ NMR spectrum of compound $3 \mathbf{j}\left(125 \mathrm{MHz}, \mathrm{CDCl}_{3}\right)$
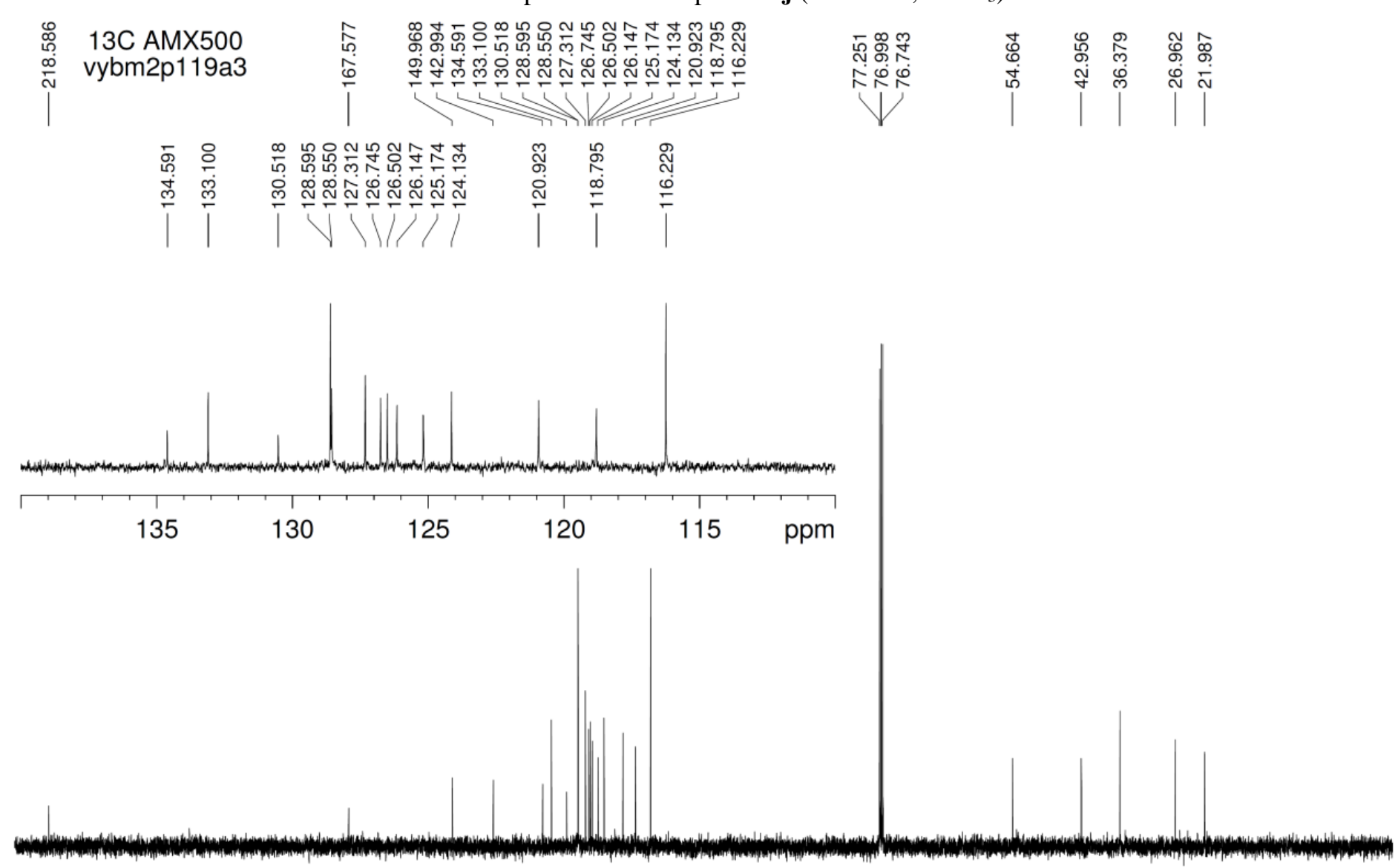

\begin{tabular}{|c|c|c|c|c|c|c|c|c|c|c|}
\hline 220 & 200 & $\begin{array}{c}1 \\
180\end{array}$ & 160 & $\begin{array}{c}1 \\
140\end{array}$ & $\begin{array}{c}1 \\
120\end{array}$ & $\begin{array}{c}1 \\
100\end{array}$ & 80 & 60 & 40 & 20 \\
\hline
\end{tabular}


${ }^{1} \mathrm{H}$ NMR spectrum of compound $3 \mathbf{k}\left(500 \mathrm{MHz}, \mathrm{CDCl}_{3}\right)$

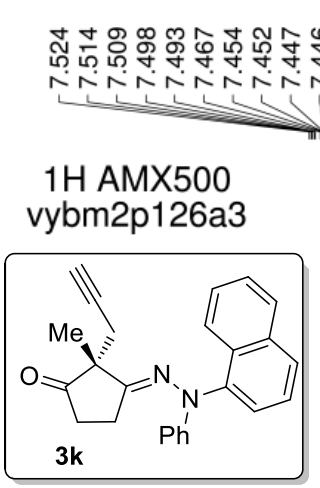

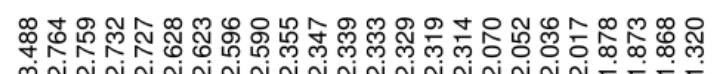

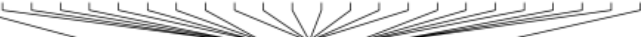

vybm2p126a3

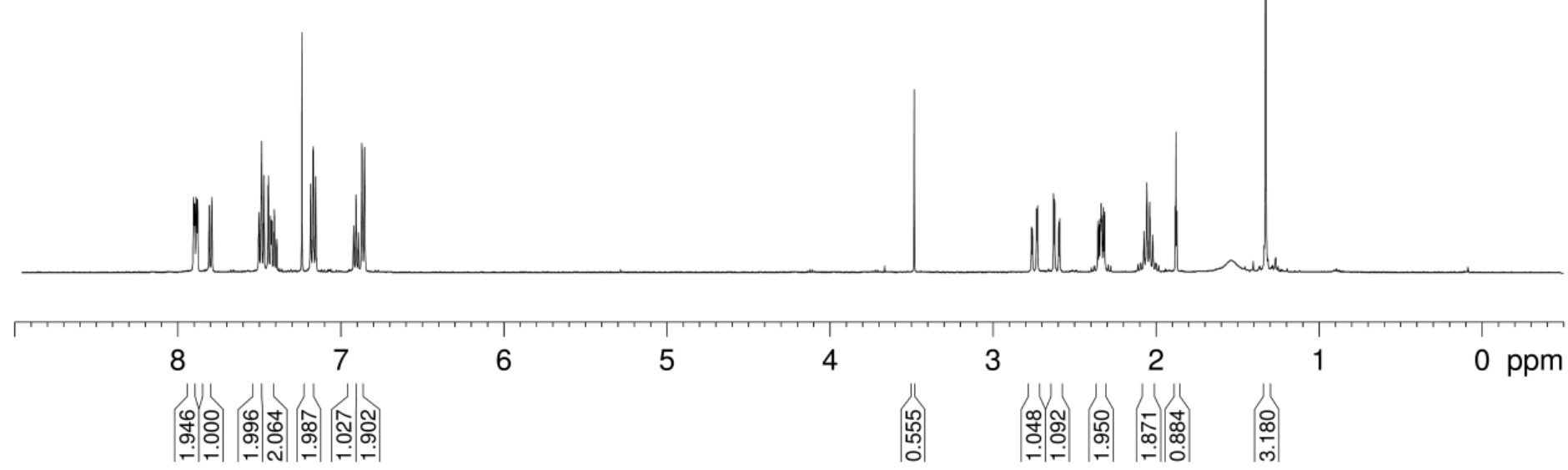


${ }^{13} \mathrm{C}$ NMR spectrum of compound $3 \mathbf{k}\left(125 \mathrm{MHz}, \mathrm{CDCl}_{3}\right)$

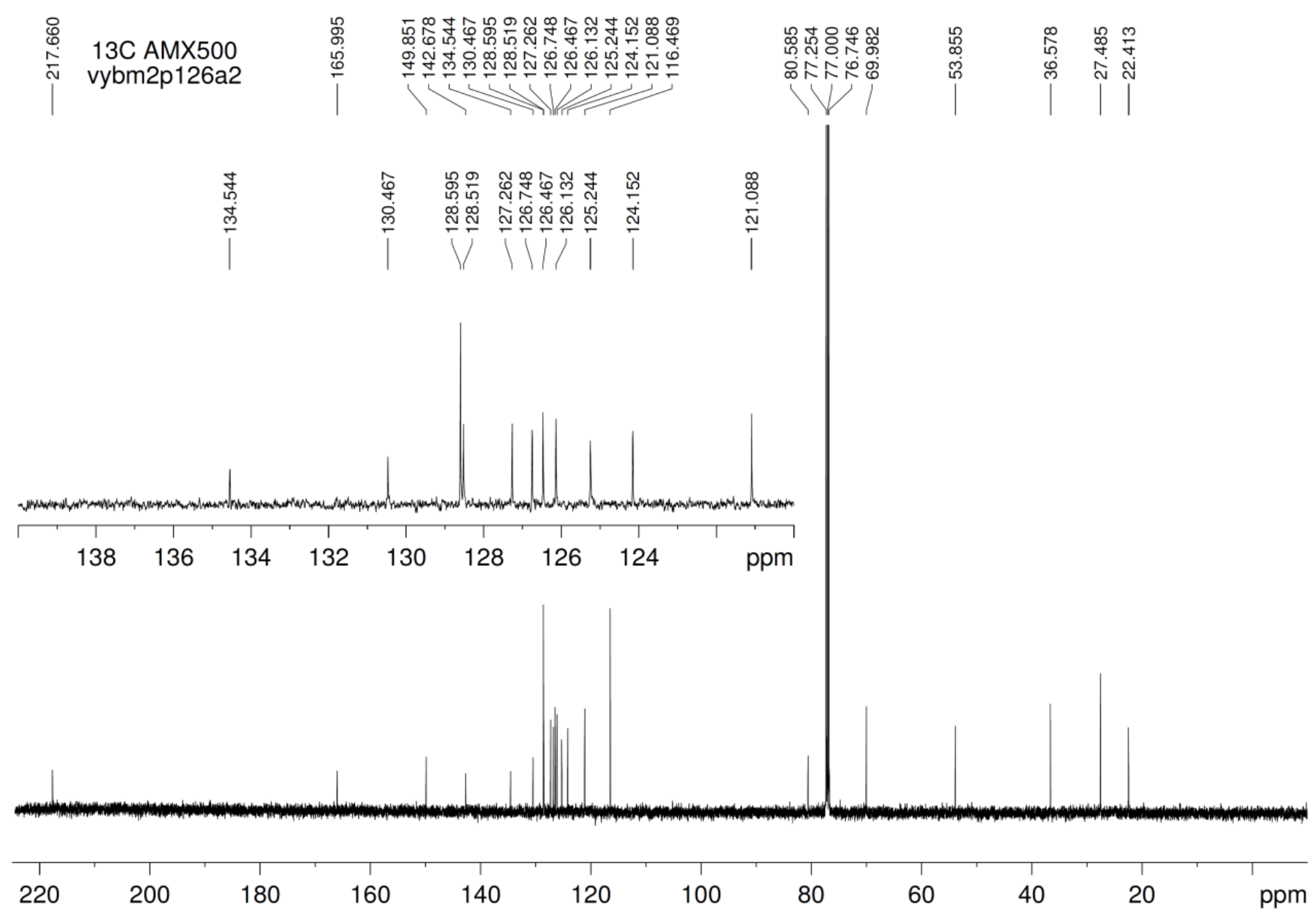


${ }^{1} \mathrm{H}$ NMR spectrum of compound $31\left(500 \mathrm{MHz}, \mathrm{CDCl}_{3}\right)$

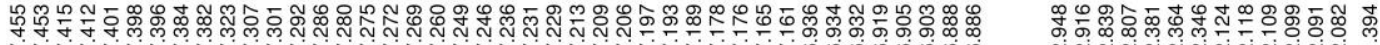
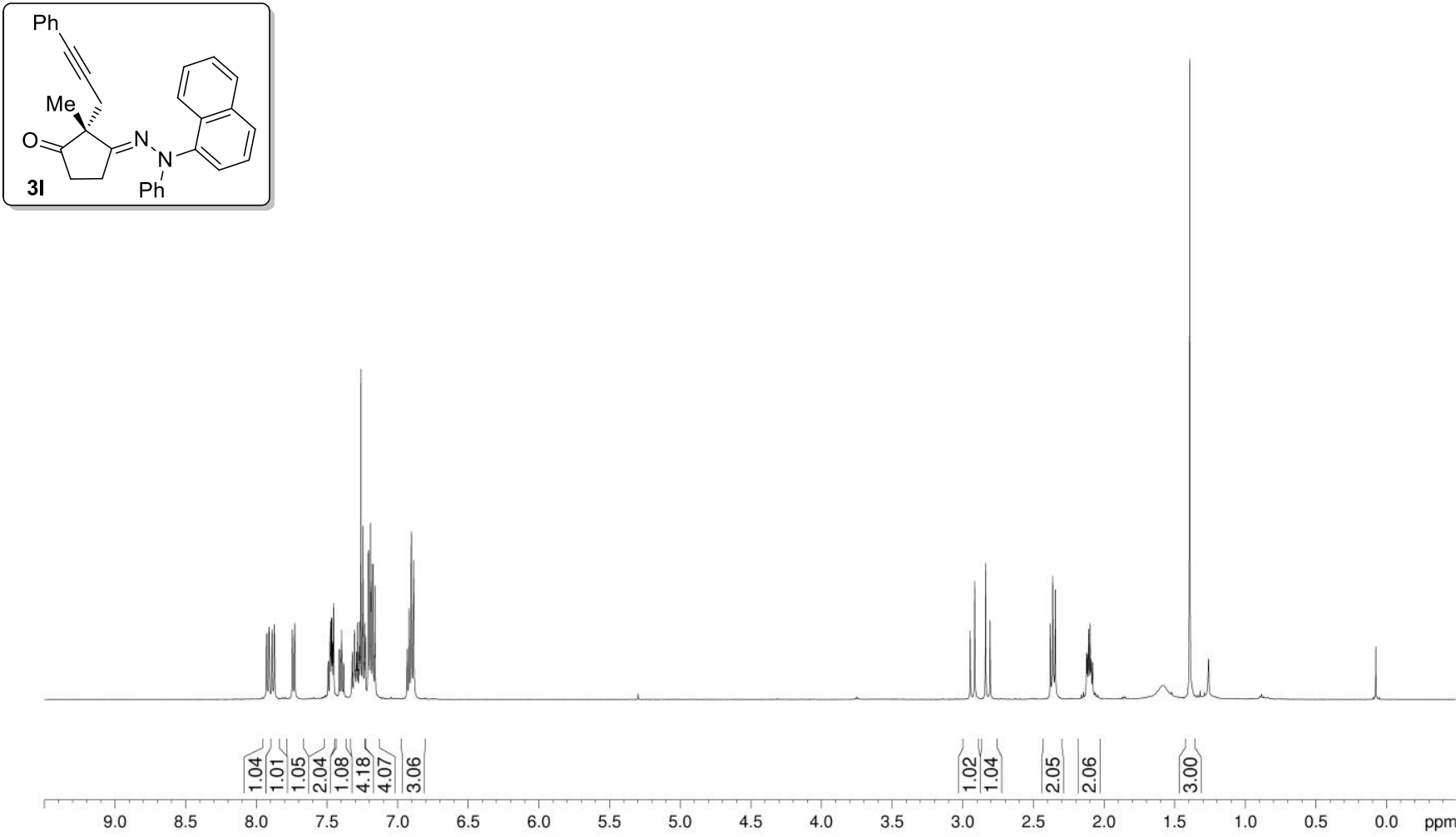
${ }^{13} \mathrm{C}$ NMR spectrum of compound 31 (125 MHz, $\mathrm{CDCl}_{3}$ )
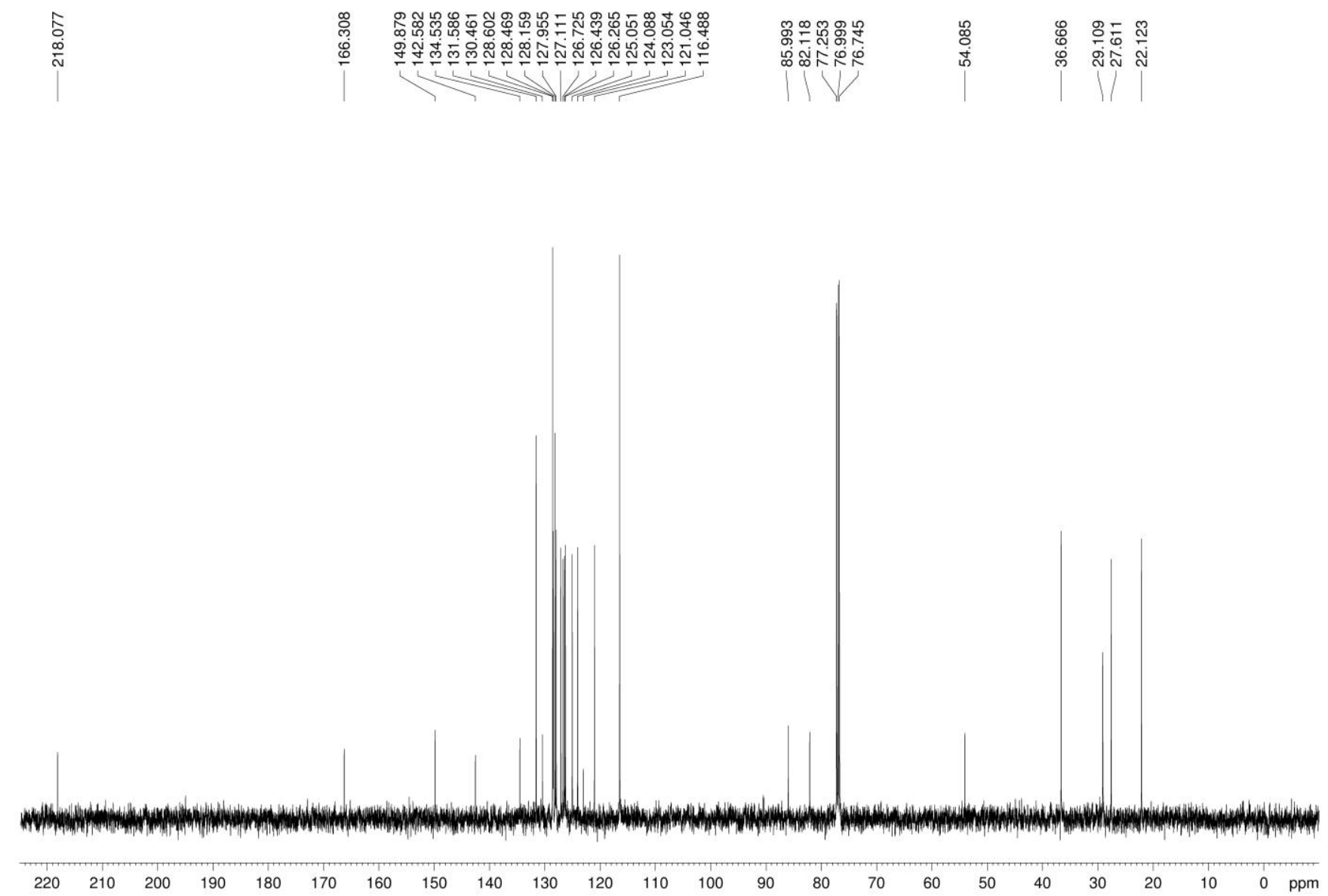
${ }^{1} \mathrm{H}$ NMR spectrum of compound $\mathbf{3 m}\left(500 \mathrm{MHz}, \mathrm{CDCl}_{3}\right)$

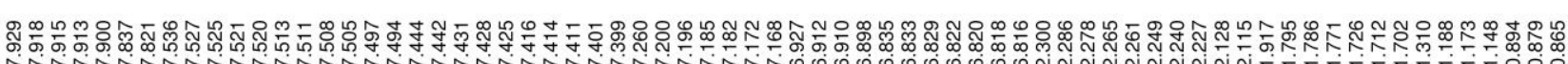
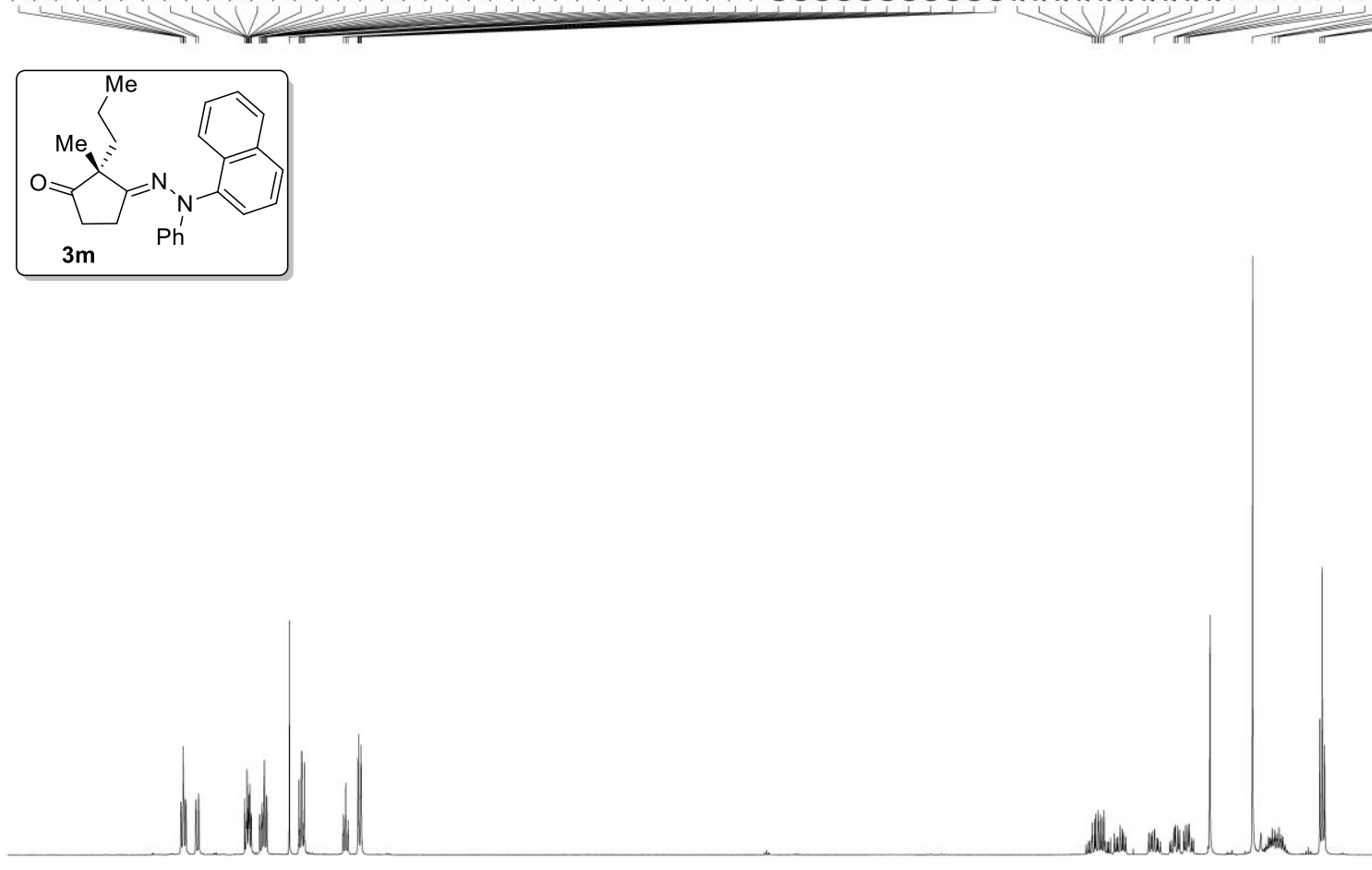

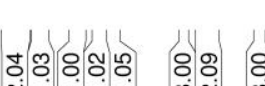

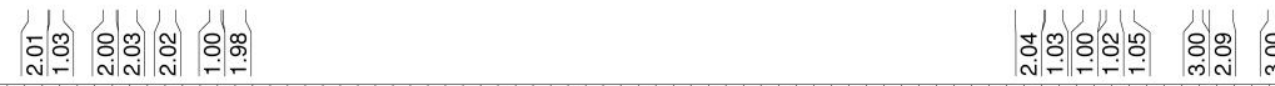

$\begin{array}{lllllllllllllllllll}8.5 & 8.0 & 7.5 & 7.0 & 6.5 & 6.0 & 5.5 & 5.0 & 4.5 & 4.0 & 3.5 & 3.0 & 2.5 & 2.0 & 1.5 & 1.0 & 0.5 & 0.0 & \mathrm{ppm}\end{array}$ 
${ }^{13} \mathrm{C}$ NMR spectrum of compound $3 \mathbf{m}\left(125 \mathrm{MHz}, \mathrm{CDCl}_{3}\right)$
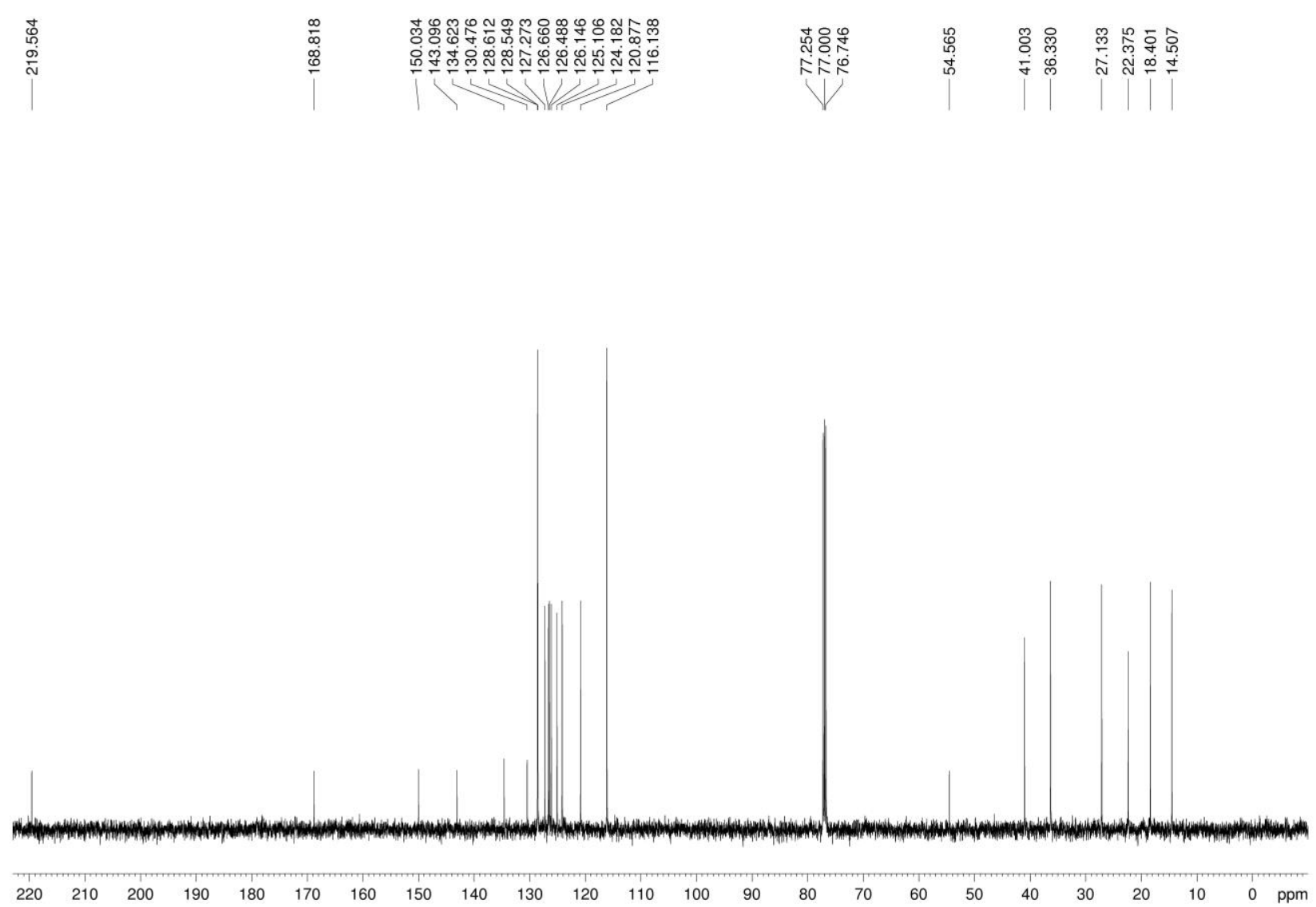
${ }^{1} \mathrm{H}$ NMR spectrum of compound $3 \mathbf{n}\left(500 \mathrm{MHz}, \mathrm{CDCl}_{3}\right)$

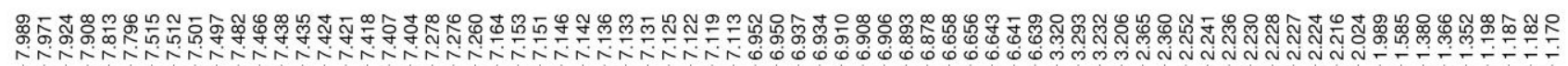
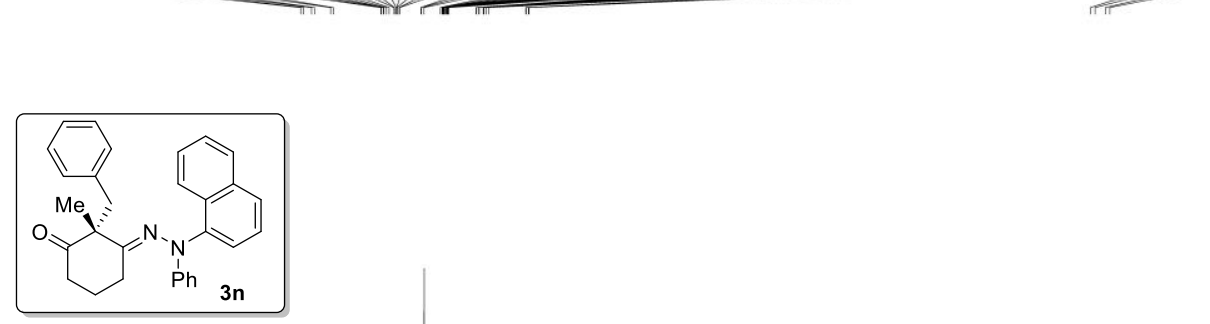

$3 n$

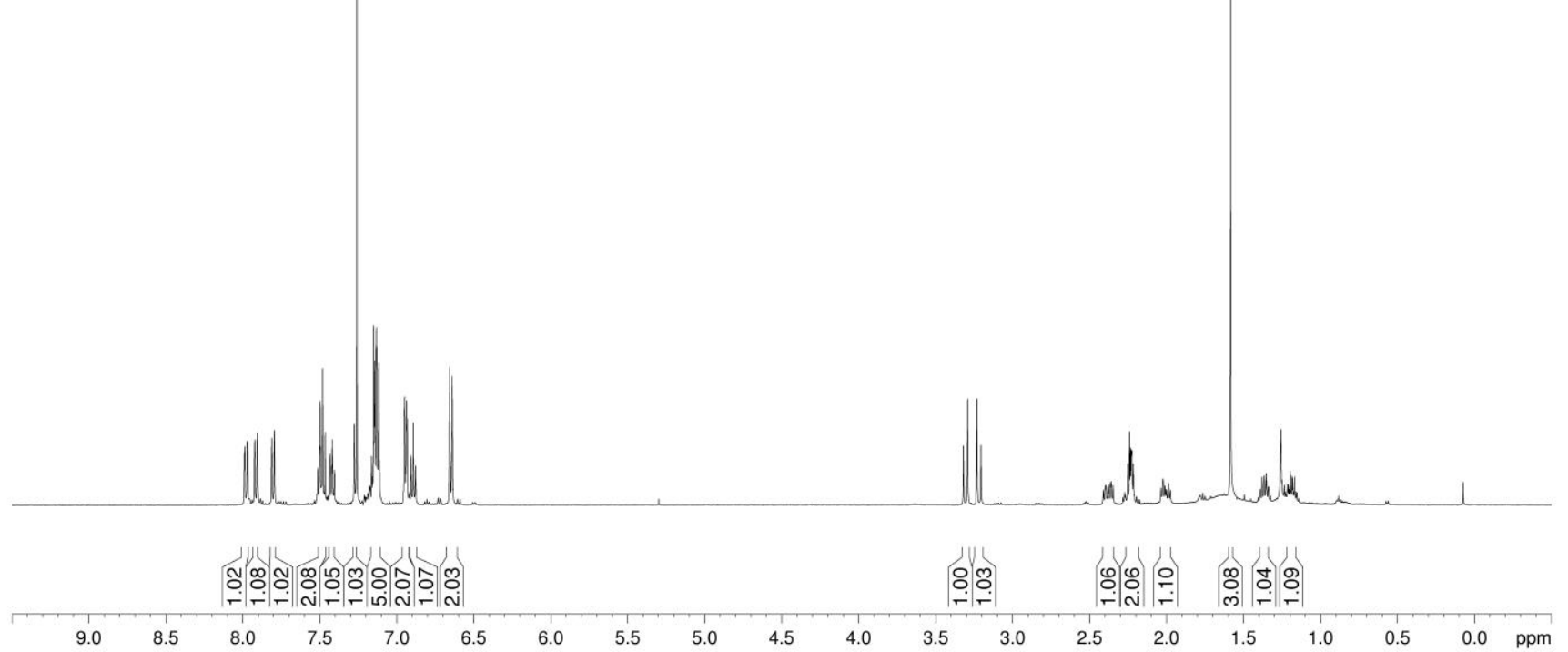


${ }^{13} \mathrm{C}$ NMR spectrum of compound $3 n\left(125 \mathrm{MHz}, \mathrm{CDCl}_{3}\right)$
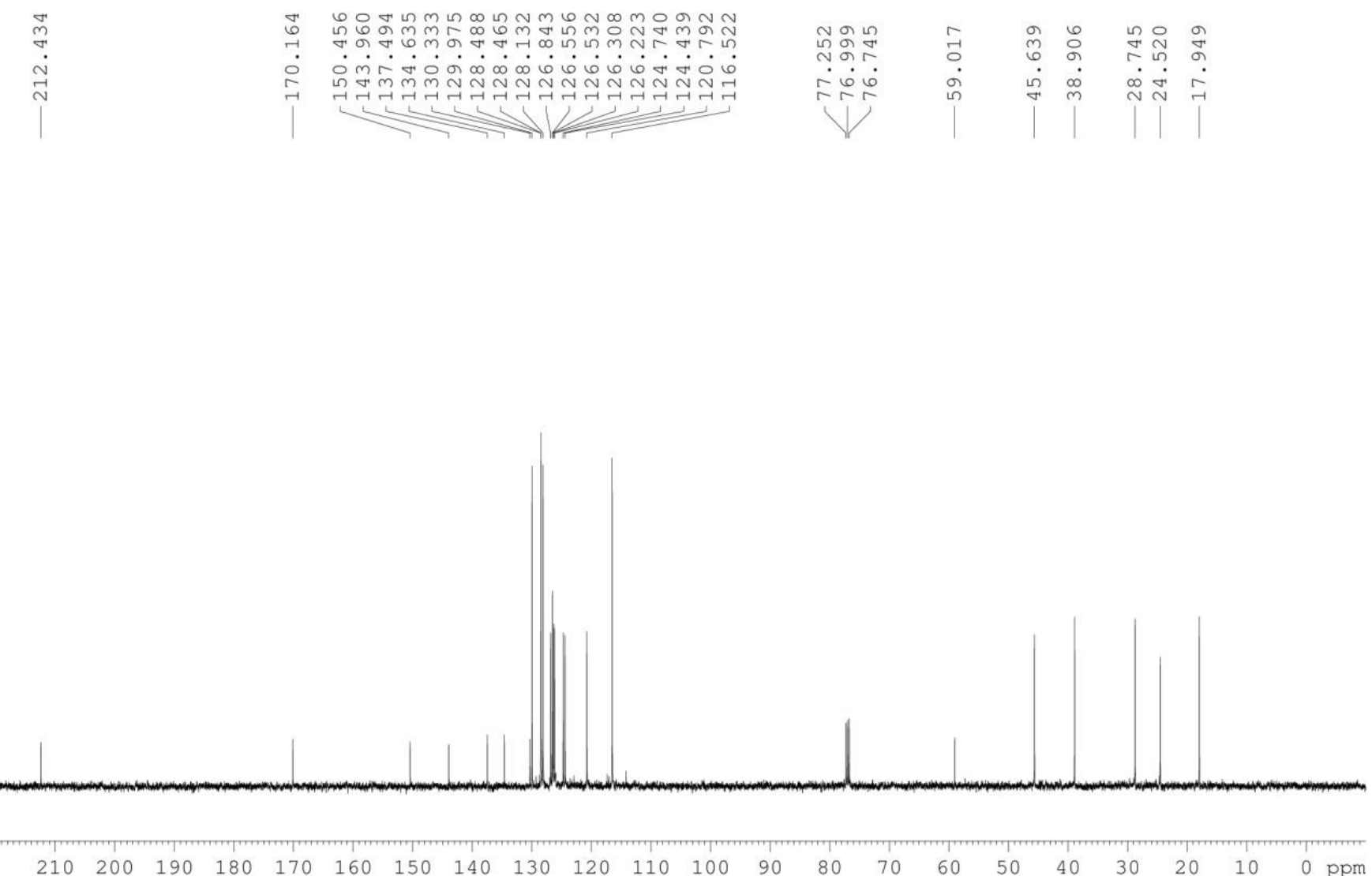
${ }^{1} \mathrm{H}$ NMR spectrum of compound $30\left(500 \mathrm{MHz}, \mathrm{CDCl}_{3}\right)$

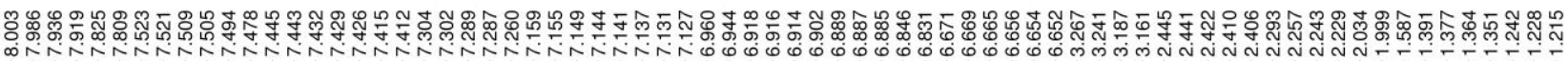

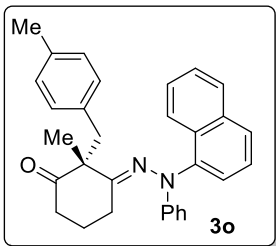

3o

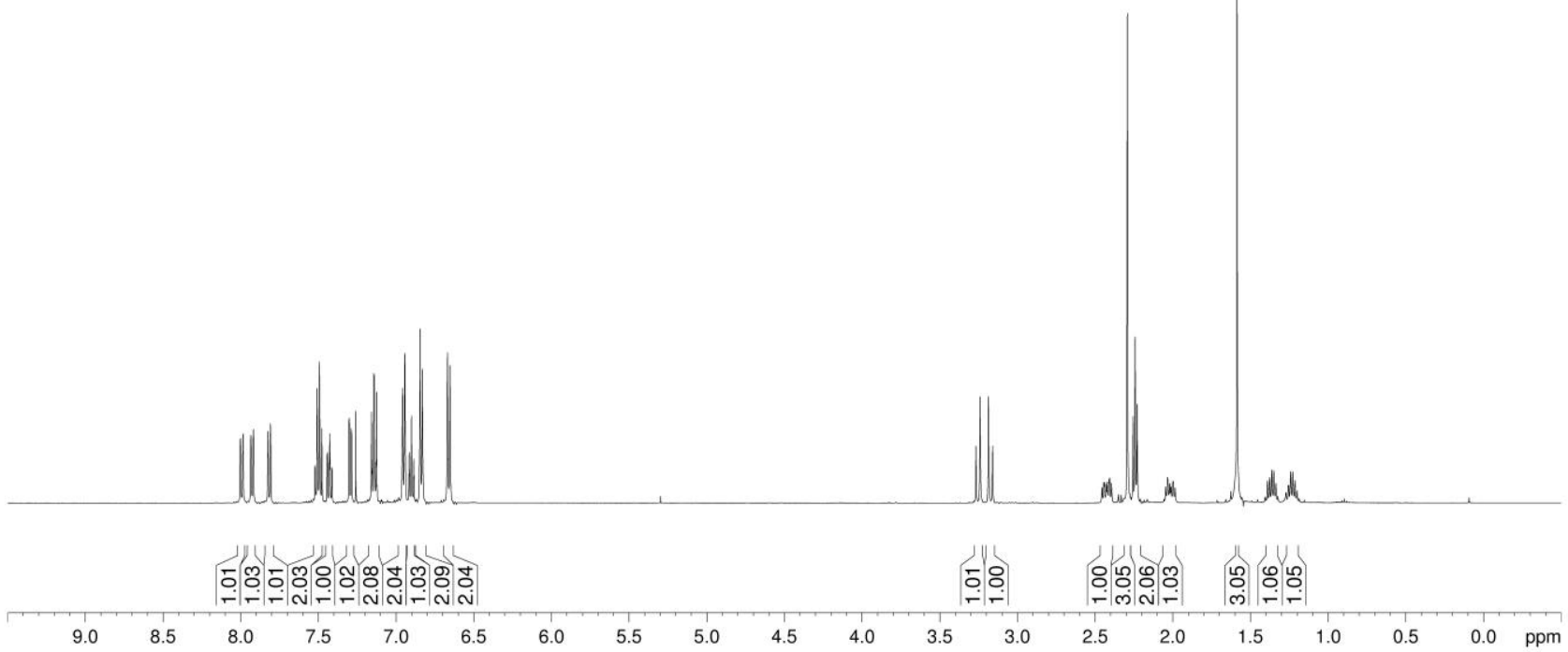


${ }^{13} \mathrm{C}$ NMR spectrum of compound $30\left(125 \mathrm{MHz}, \mathrm{CDCl}_{3}\right)$

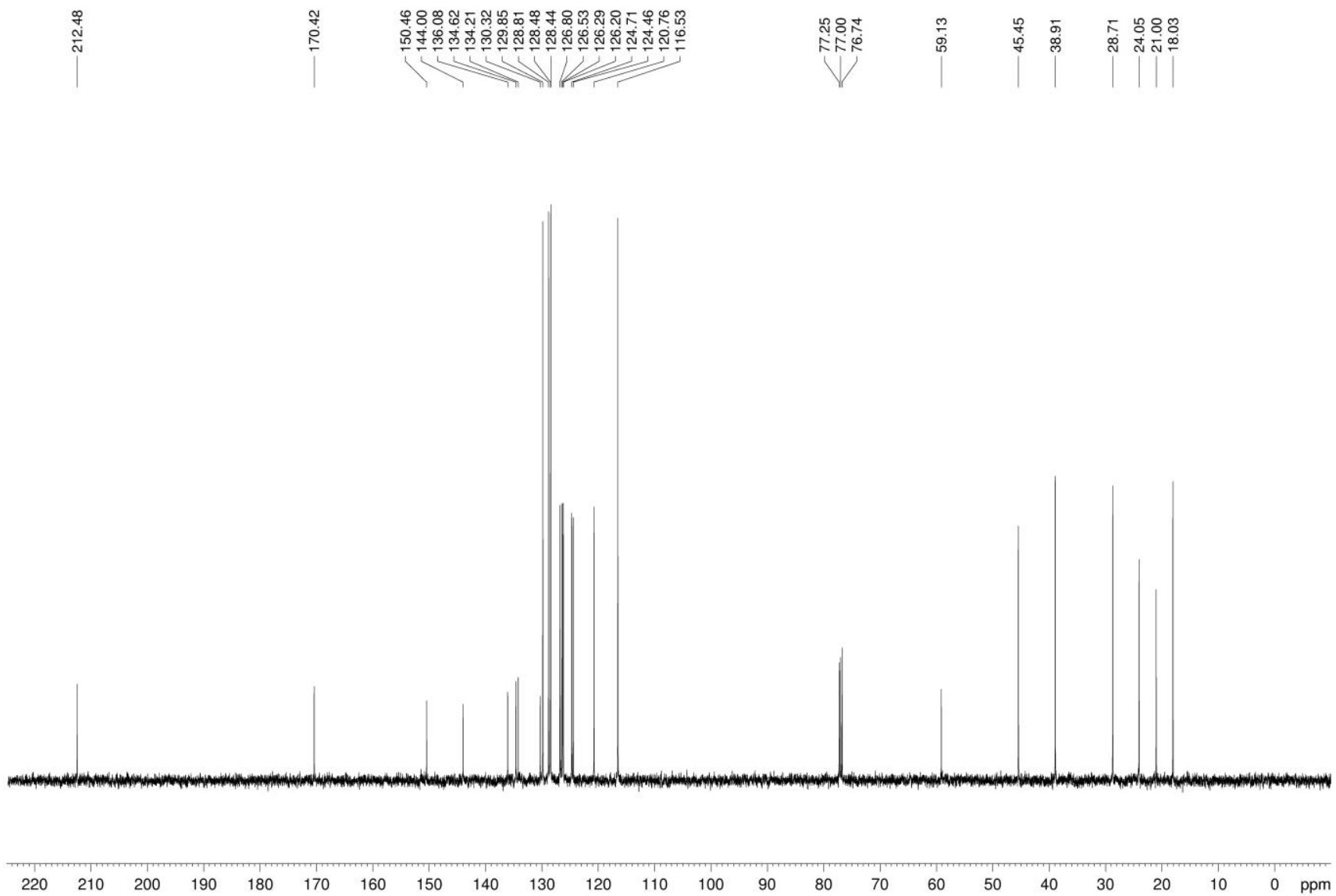


${ }^{1} \mathrm{H}$ NMR spectrum of compound $3 \mathbf{p}\left(400 \mathrm{MHz}, \mathrm{CDCl}_{3}\right)$

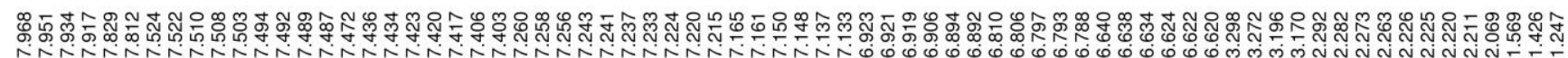
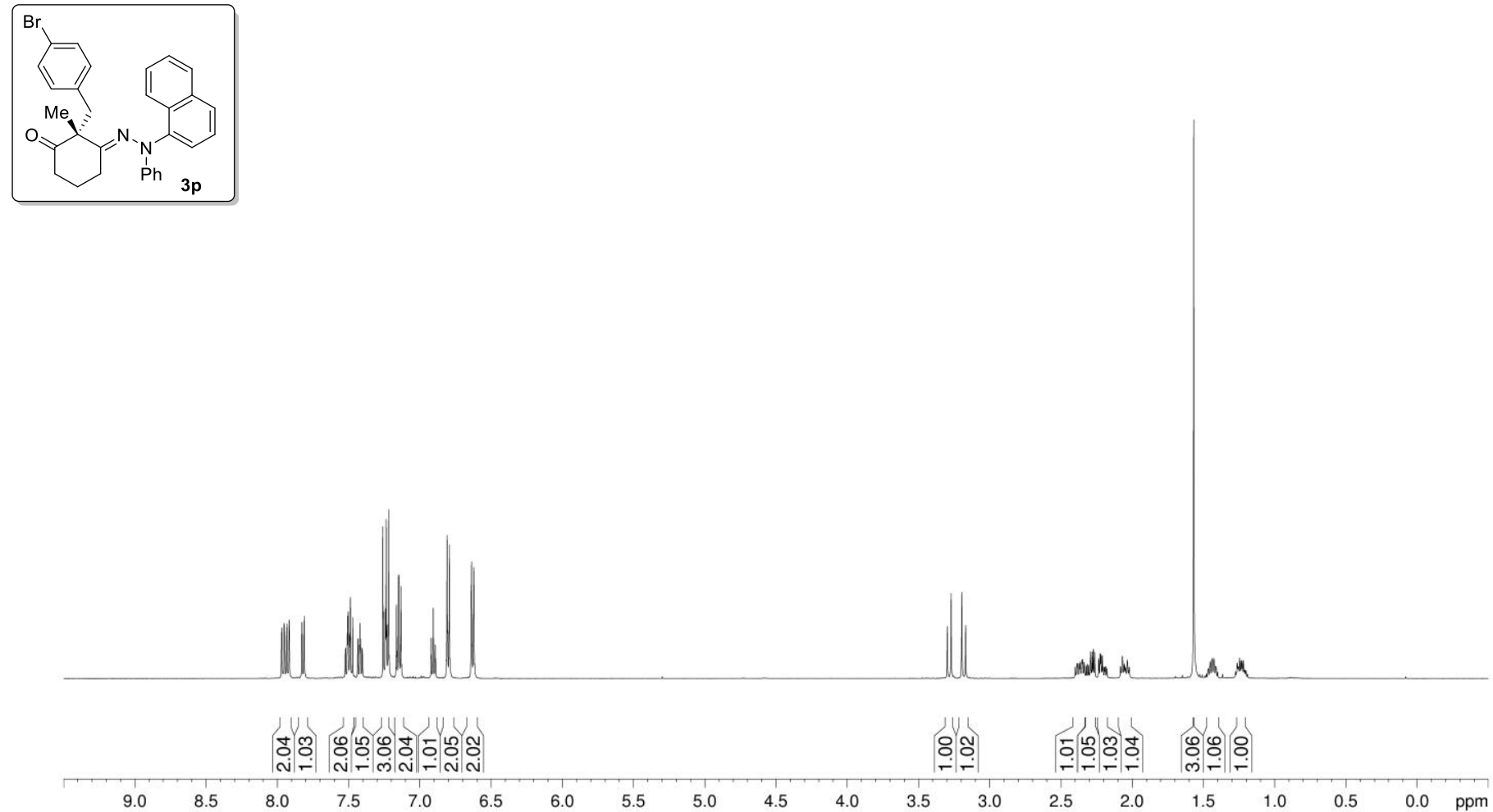
${ }^{13} \mathrm{C}$ NMR spectrum of compound $3 \mathbf{p}\left(100 \mathrm{MHz}, \mathrm{CDCl}_{3}\right)$

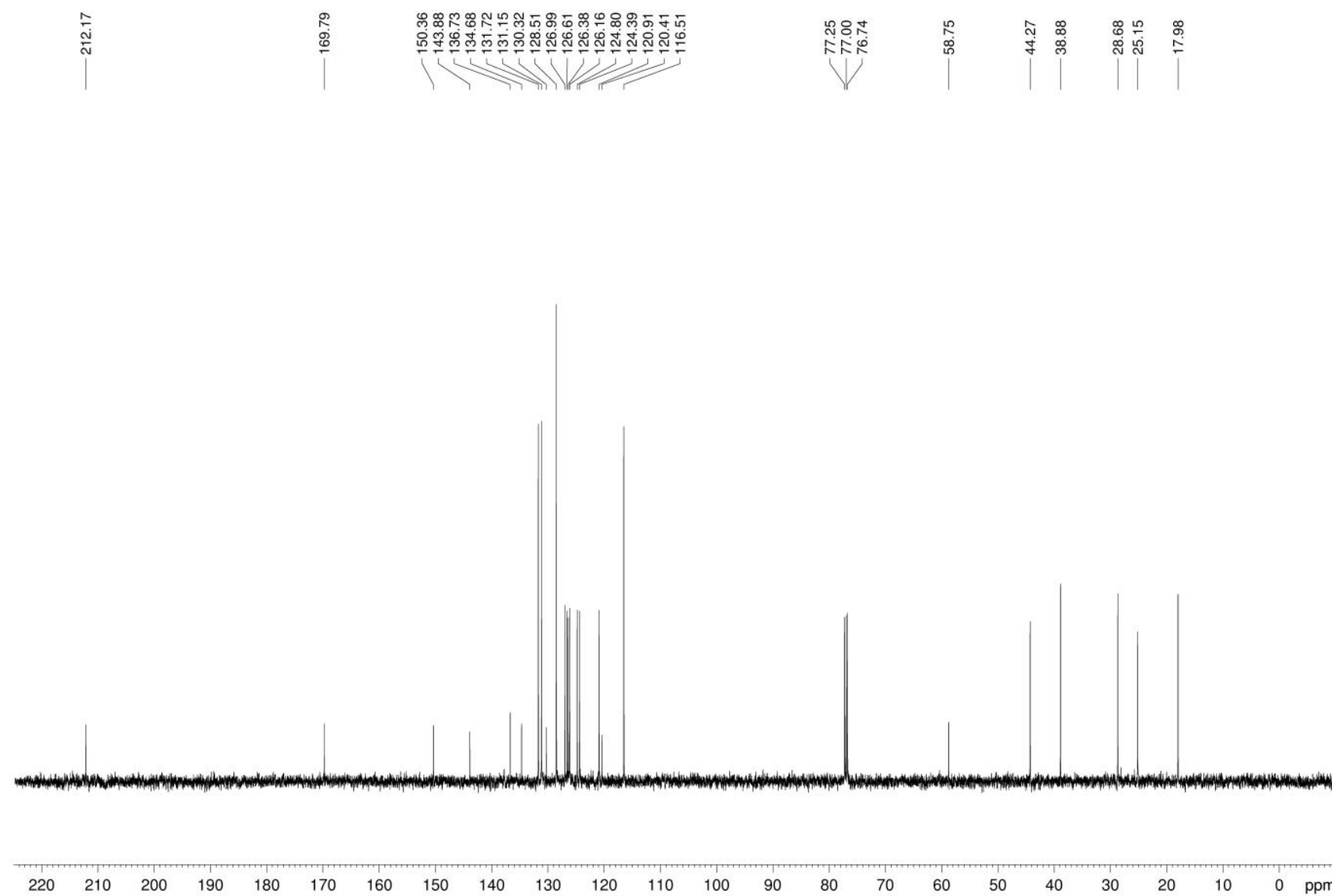


${ }^{1} \mathrm{H}$ NMR spectrum of compound $\mathbf{3 q}\left(500 \mathrm{MHz}, \mathrm{CDCl}_{3}\right)$

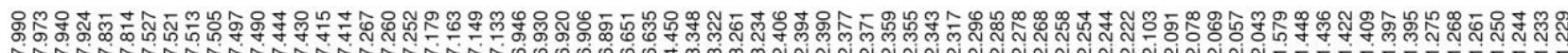

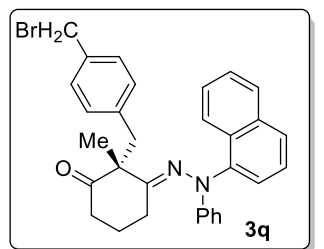

$3 q$

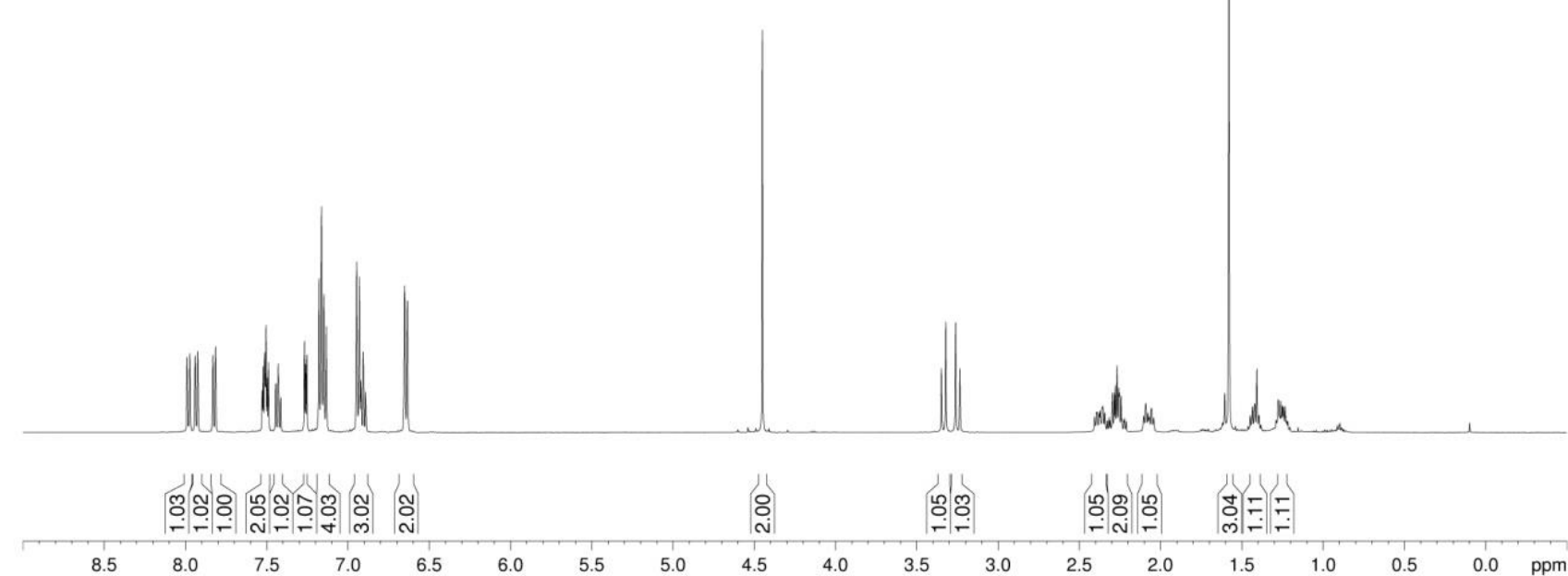


${ }^{13} \mathrm{C}$ NMR spectrum of compound $\mathbf{3 q}\left(125 \mathrm{MHz}, \mathrm{CDCl}_{3}\right)$

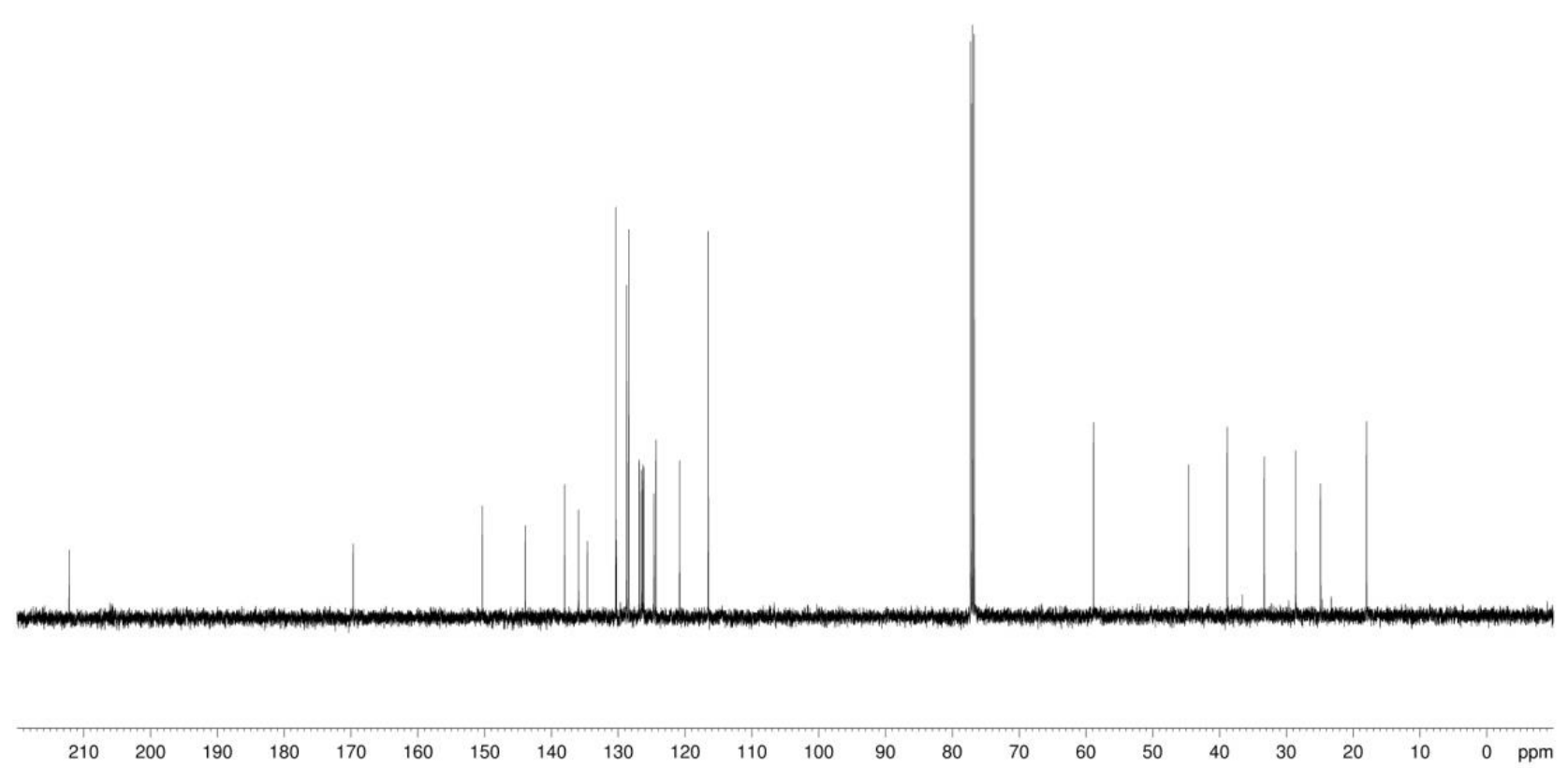


${ }^{1} \mathrm{H}$ NMR spectrum of compound $4\left(500 \mathrm{MHz}, \mathrm{CDCl}_{3}\right)$

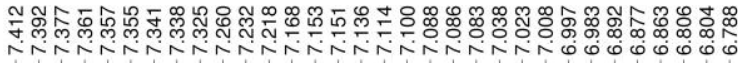
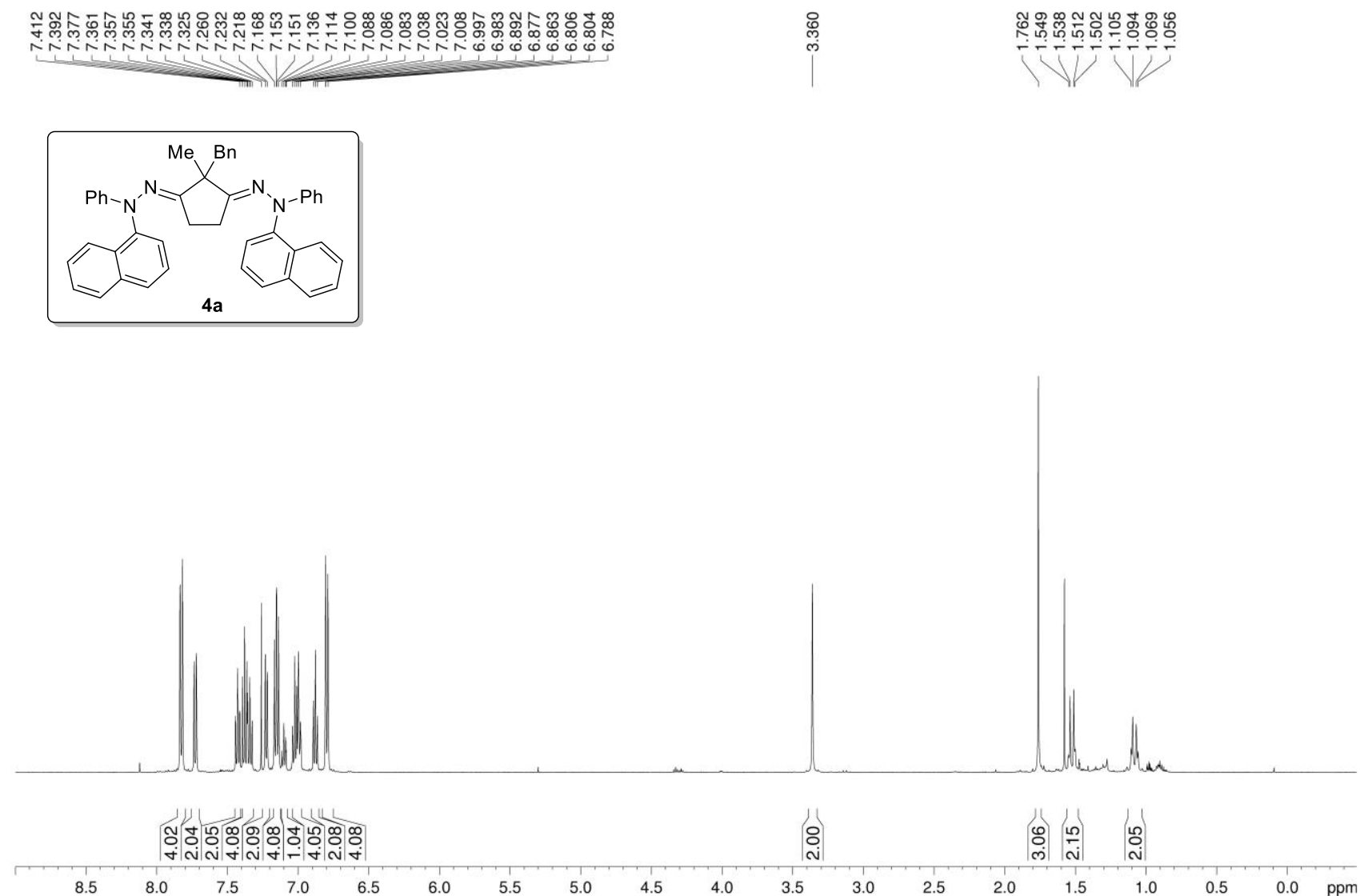
${ }^{13} \mathrm{C}$ NMR spectrum of compound $4\left(500 \mathrm{MHz}, \mathrm{CD}_{3} \mathrm{COCD}_{3}\right)$

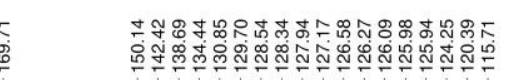

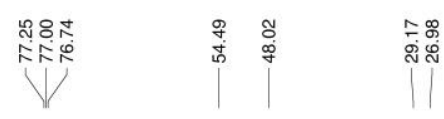

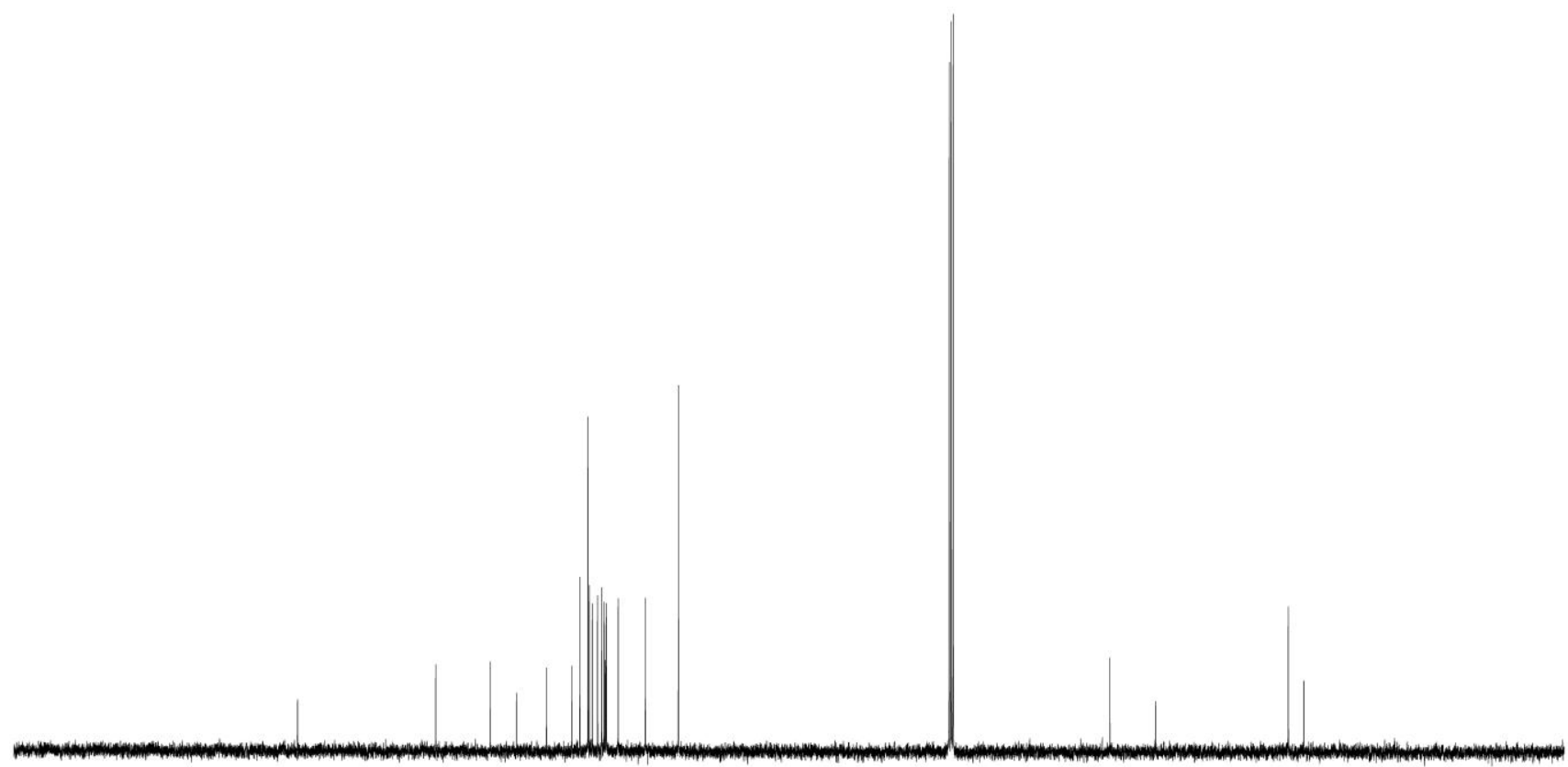

$\begin{array}{llllllllllllllllllllll}200 & 190 & 180 & 170 & 160 & 150 & 140 & 130 & 120 & 110 & 100 & 90 & 80 & 70 & 60 & 50 & 40 & 30 & 20 & 10 & 0 & \mathrm{ppm}\end{array}$ 
${ }^{1} \mathrm{H}$ NMR spectrum of compound $\mathbf{6 a}\left(500 \mathrm{MHz}, \mathrm{CD}_{3} \mathrm{COCD}_{3}\right)$

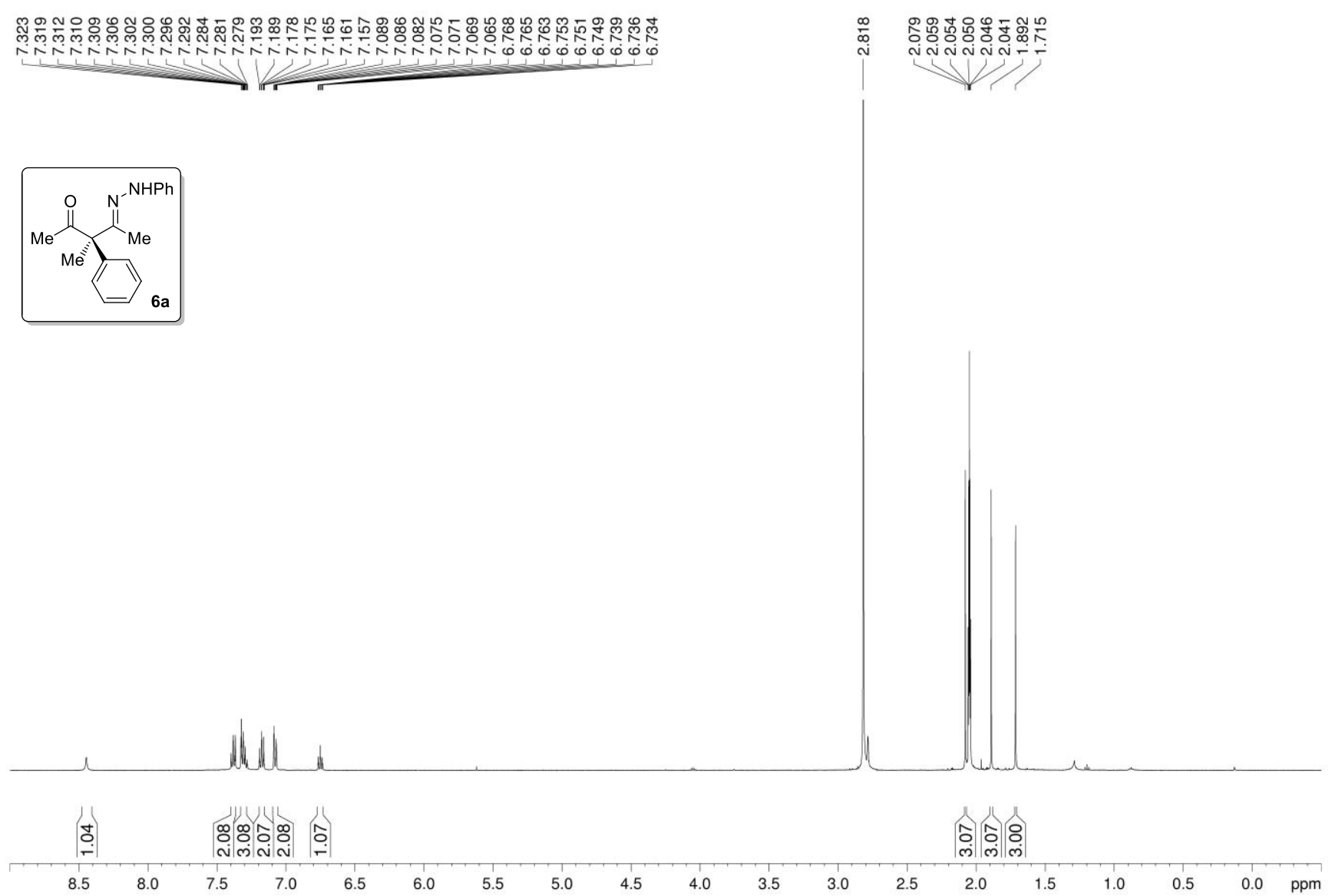


${ }^{13} \mathrm{C}$ NMR spectrum of compound $\mathbf{6 a}\left(125 \mathrm{MHz}, \mathrm{CD}_{3} \mathrm{COCD}_{3}\right)$

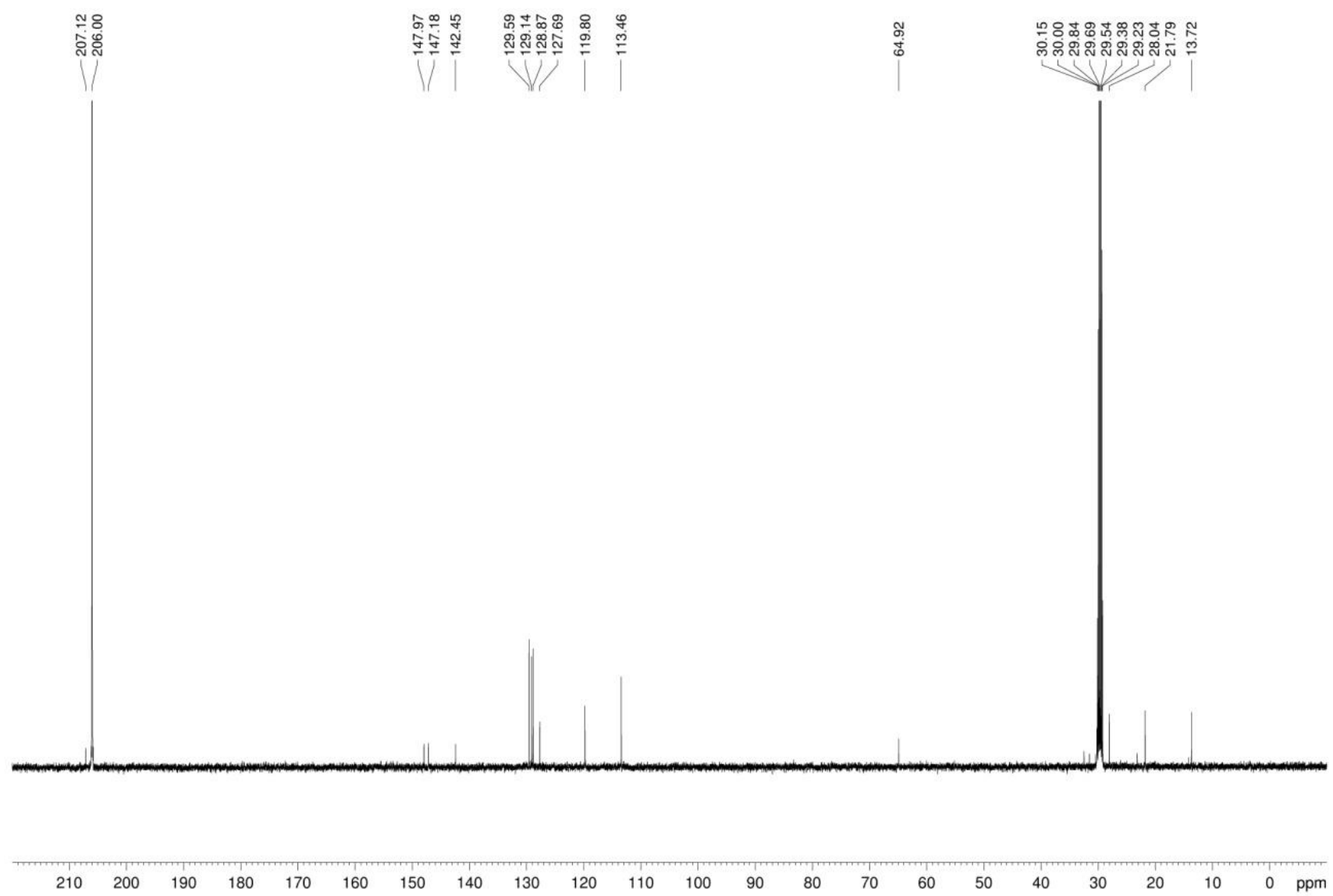


${ }^{1} \mathrm{H}$ NMR spectrum of compound $\mathbf{6 b}\left(500 \mathrm{MHz}, \mathrm{CD}_{3} \mathrm{COCD}_{3}\right)$

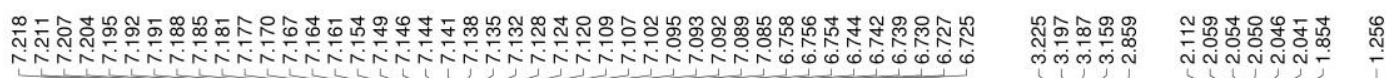

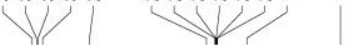

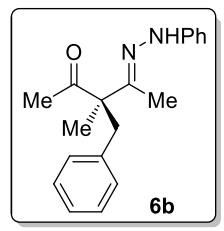

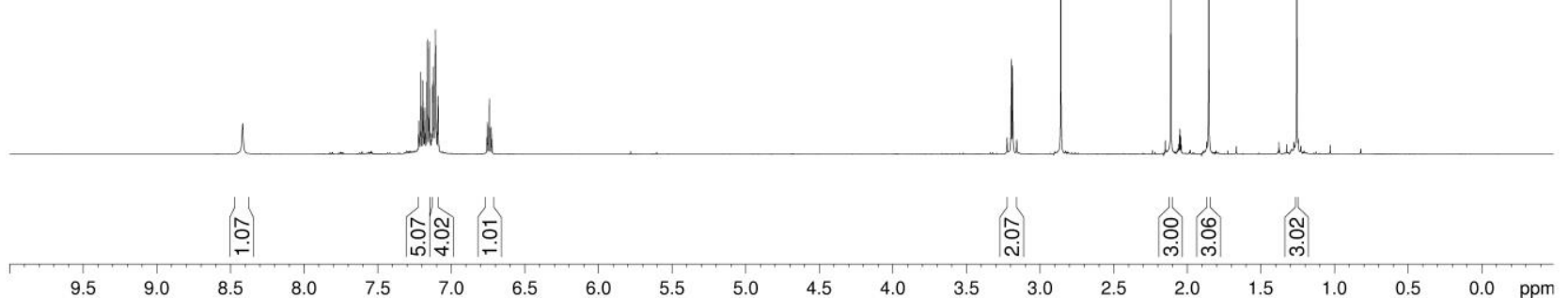


${ }^{13} \mathrm{C}$ NMR spectrum of compound $\mathbf{6 b}\left(125 \mathrm{MHz}, \mathrm{CD}_{3} \mathrm{COCD}_{3}\right)$

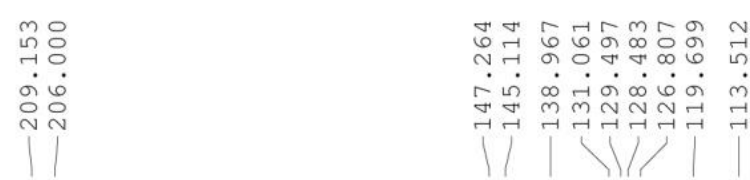

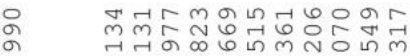

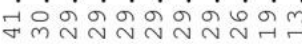

1

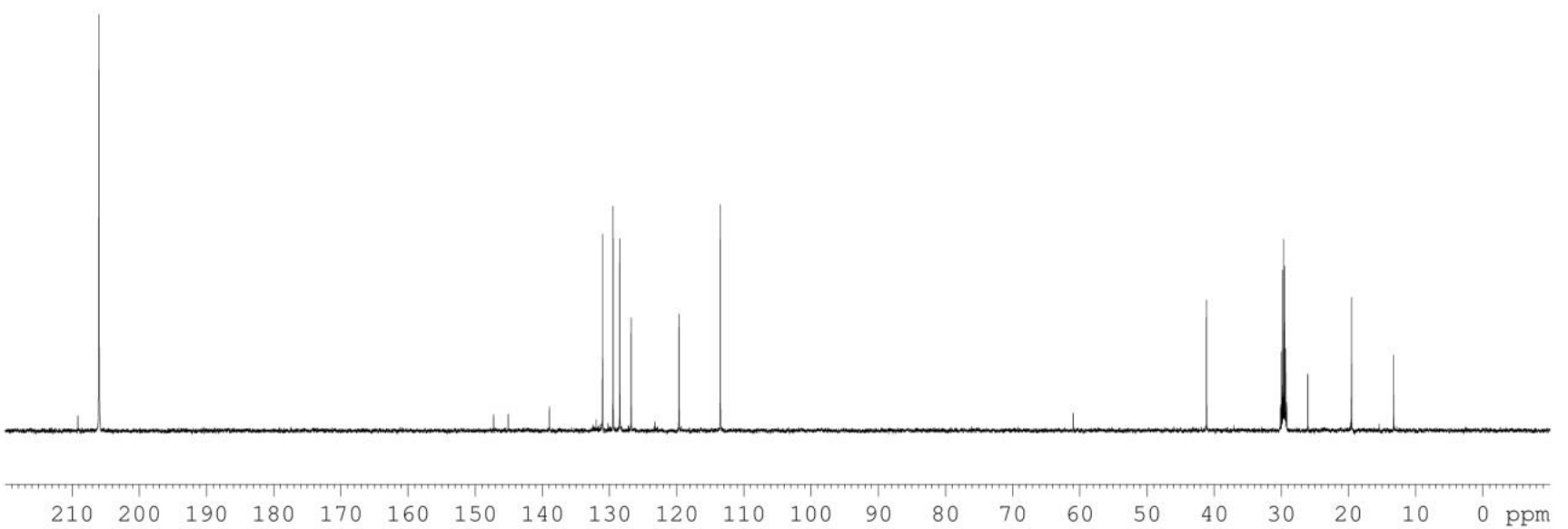


${ }^{1} \mathrm{H}$ NMR spectrum of compound $\mathbf{6 c}\left(400 \mathrm{MHz}, \mathrm{CD}_{3} \mathrm{COCD}_{3}\right)$

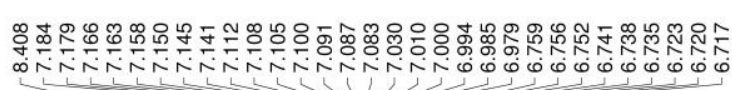

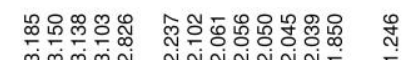

(Ic)
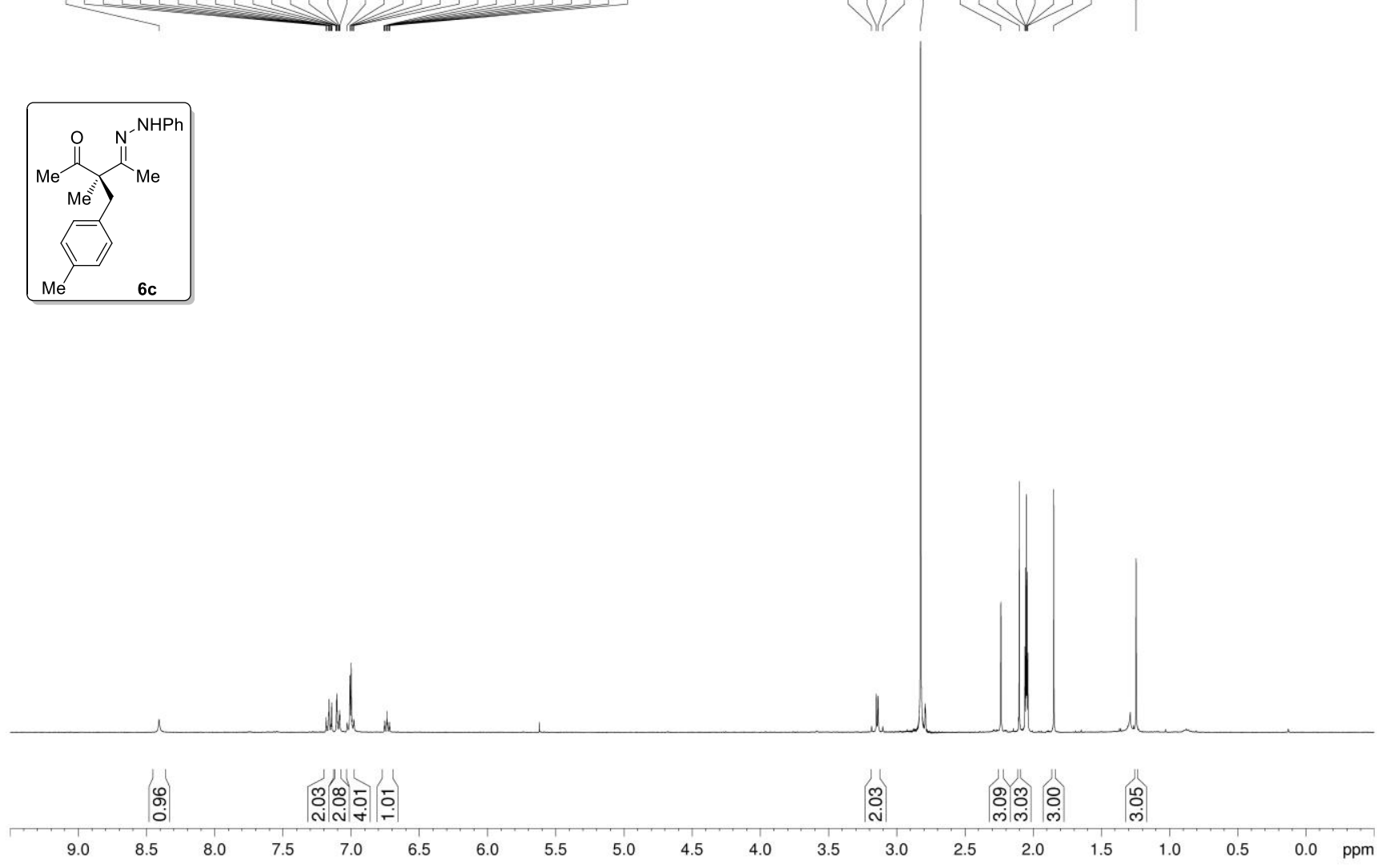
${ }^{13} \mathrm{C}$ NMR spectrum of compound $\mathbf{6 c}\left(125 \mathrm{MHz}, \mathrm{CD}_{3} \mathrm{COCD}_{3}\right)$

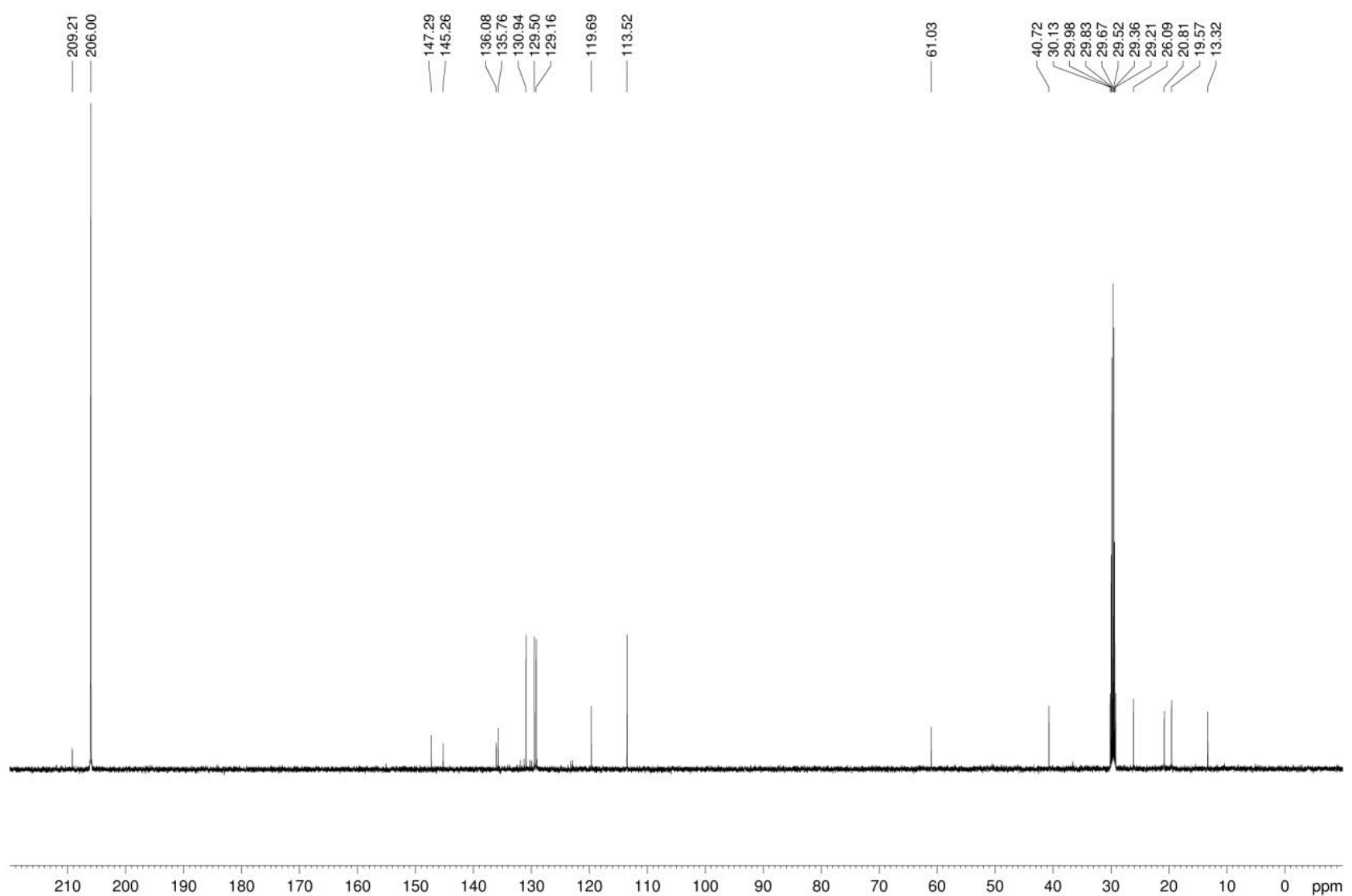


${ }^{1} \mathrm{H}$ NMR spectrum of compound $\mathbf{6 d}\left(400 \mathrm{MHz}, \mathrm{CD}_{3} \mathrm{COCD}_{3}\right)$

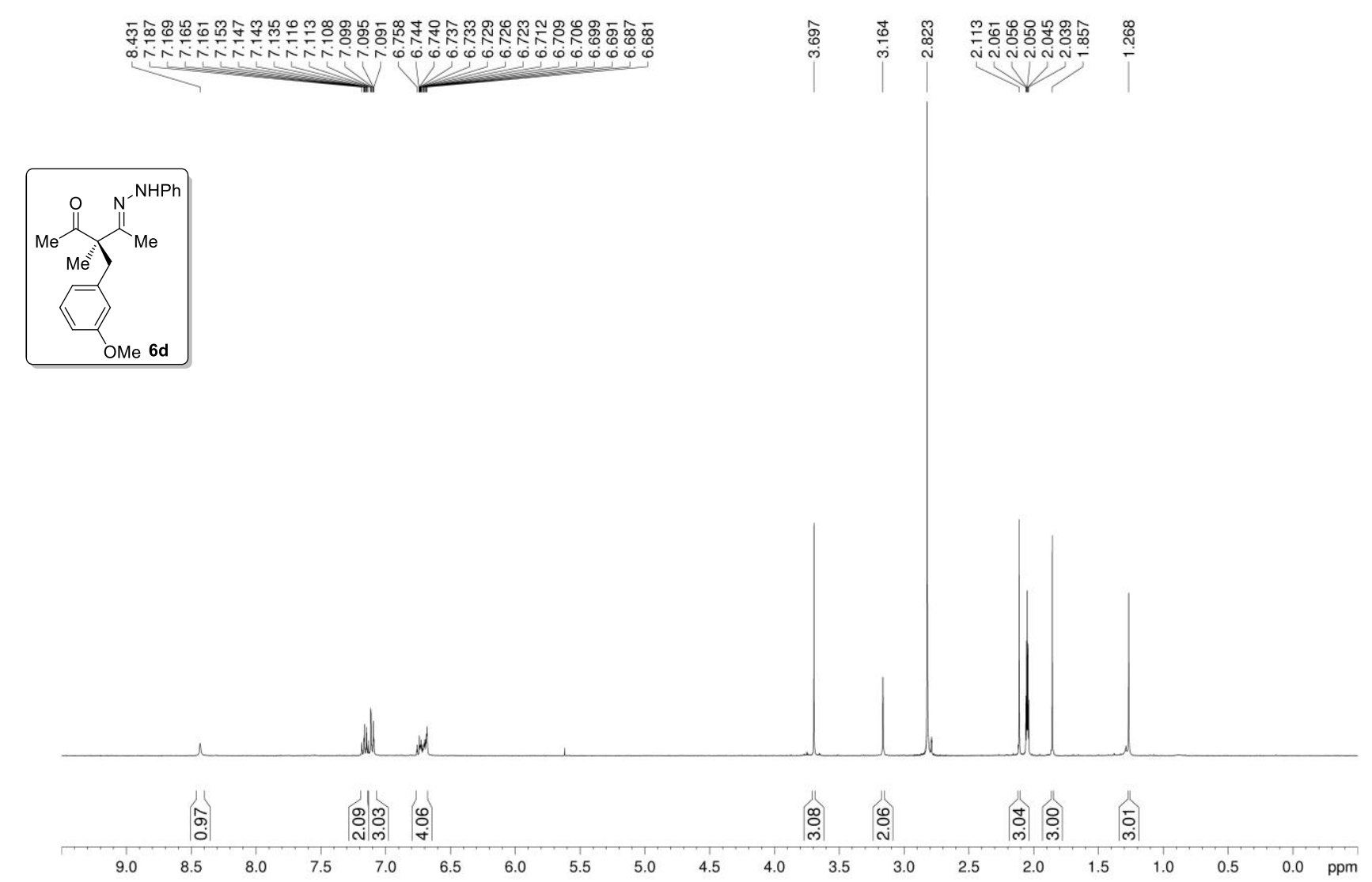


${ }^{13} \mathrm{C}$ NMR spectrum of compound $\mathbf{6 d}\left(125 \mathrm{MHz}, \mathrm{CD}_{3} \mathrm{COCD}_{3}\right)$

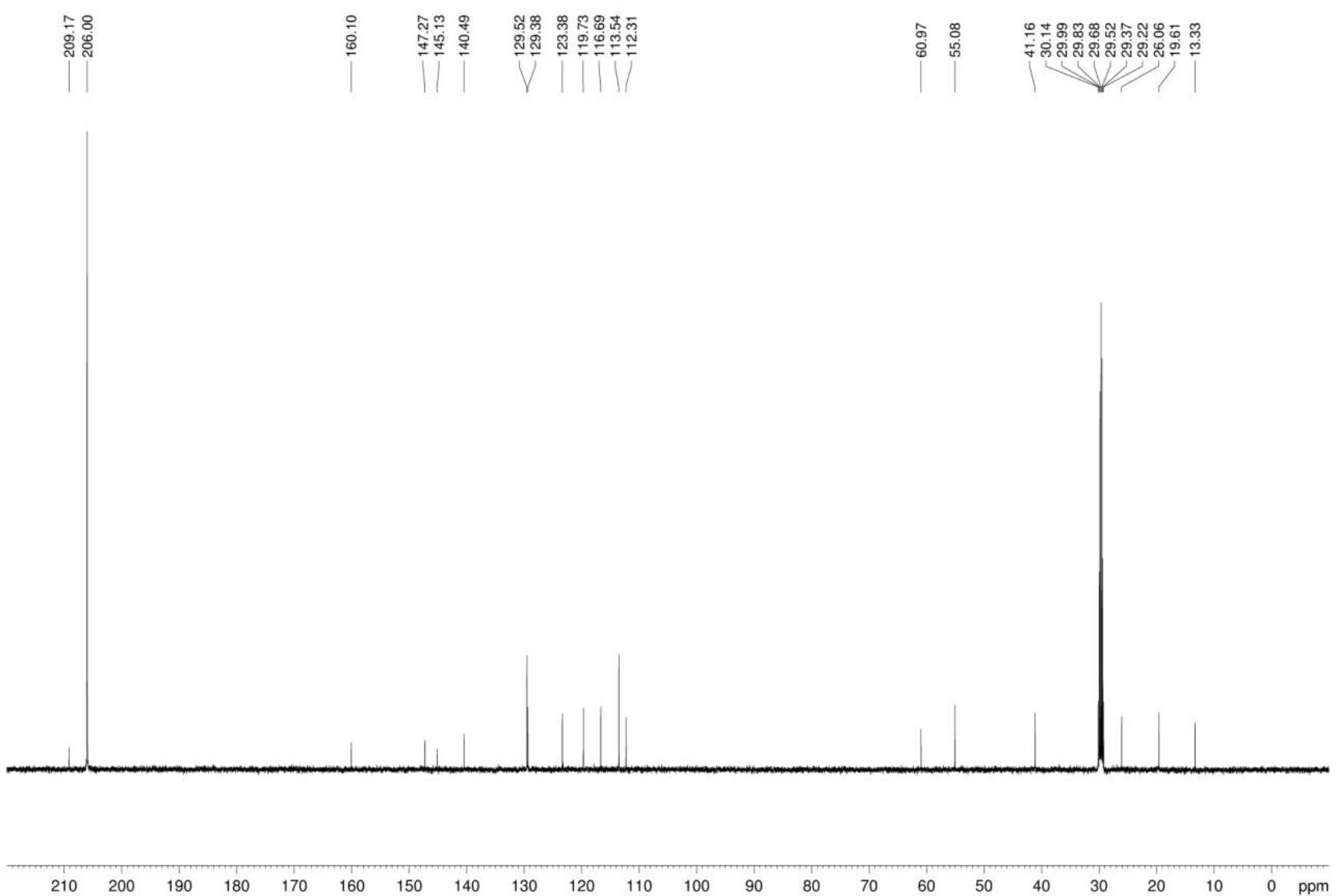


${ }^{1} \mathrm{H}$ NMR spectrum of compound $6 \mathbf{e}\left(400 \mathrm{MHz}, \mathrm{CD}_{3} \mathrm{COCD}_{3}\right)$

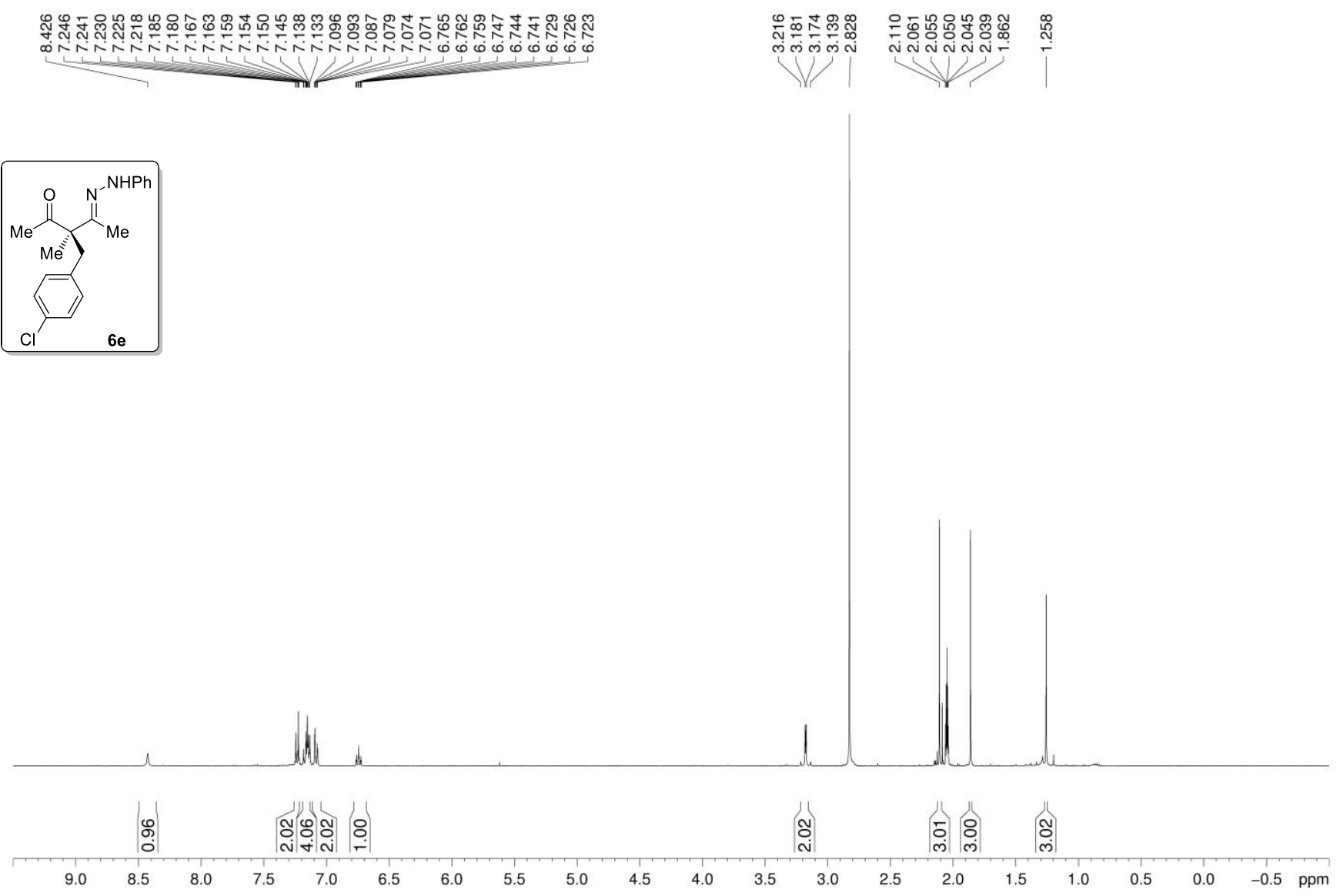


${ }^{13} \mathrm{C}$ NMR spectrum of compound $\mathbf{6 e}\left(125 \mathrm{MHz}, \mathrm{CD}_{3} \mathrm{COCD}_{3}\right)$

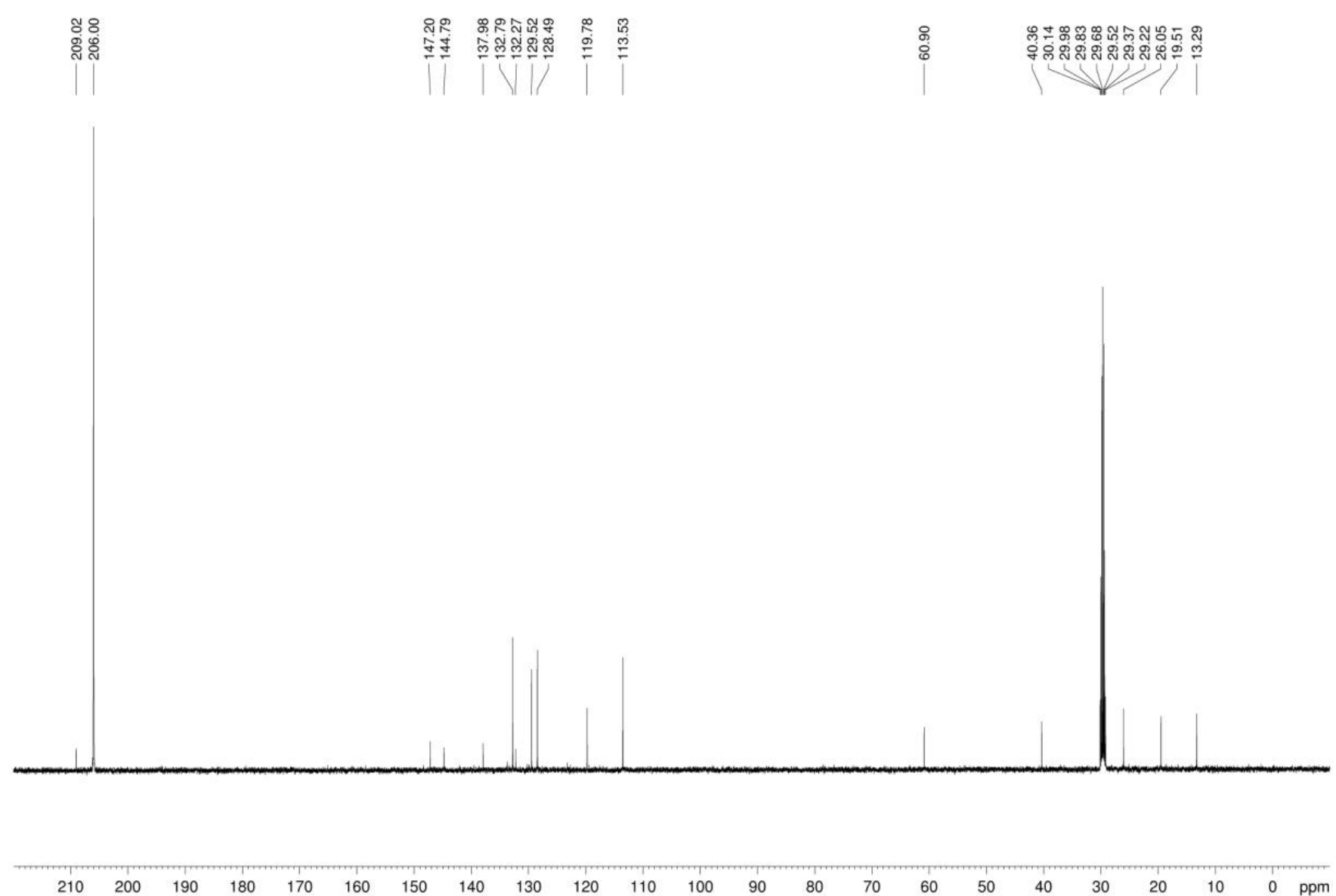


${ }^{1} \mathrm{H}$ NMR spectrum of compound $\mathbf{6 f}\left(500 \mathrm{MHz}, \mathrm{CD}_{3} \mathrm{COCD}_{3}\right)$

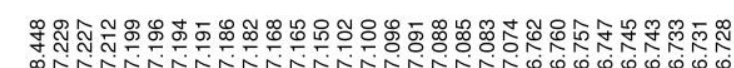

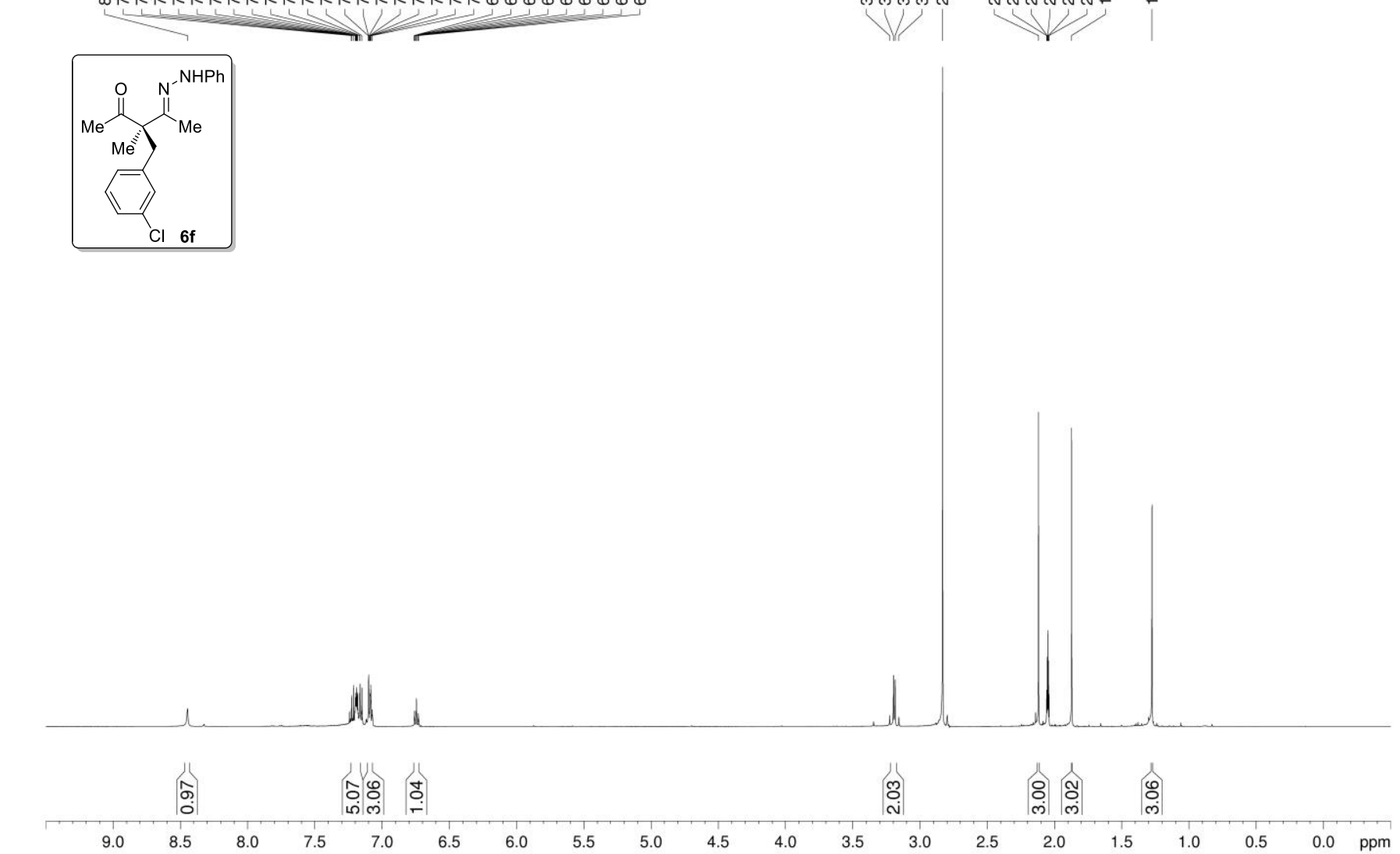

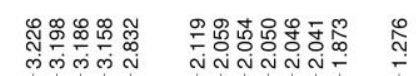


${ }^{13} \mathrm{C} \mathrm{NMR}$ spectrum of compound $\mathbf{6 f}\left(125 \mathrm{MHz}, \mathrm{CD}_{3} \mathrm{COCD}_{3}\right)$

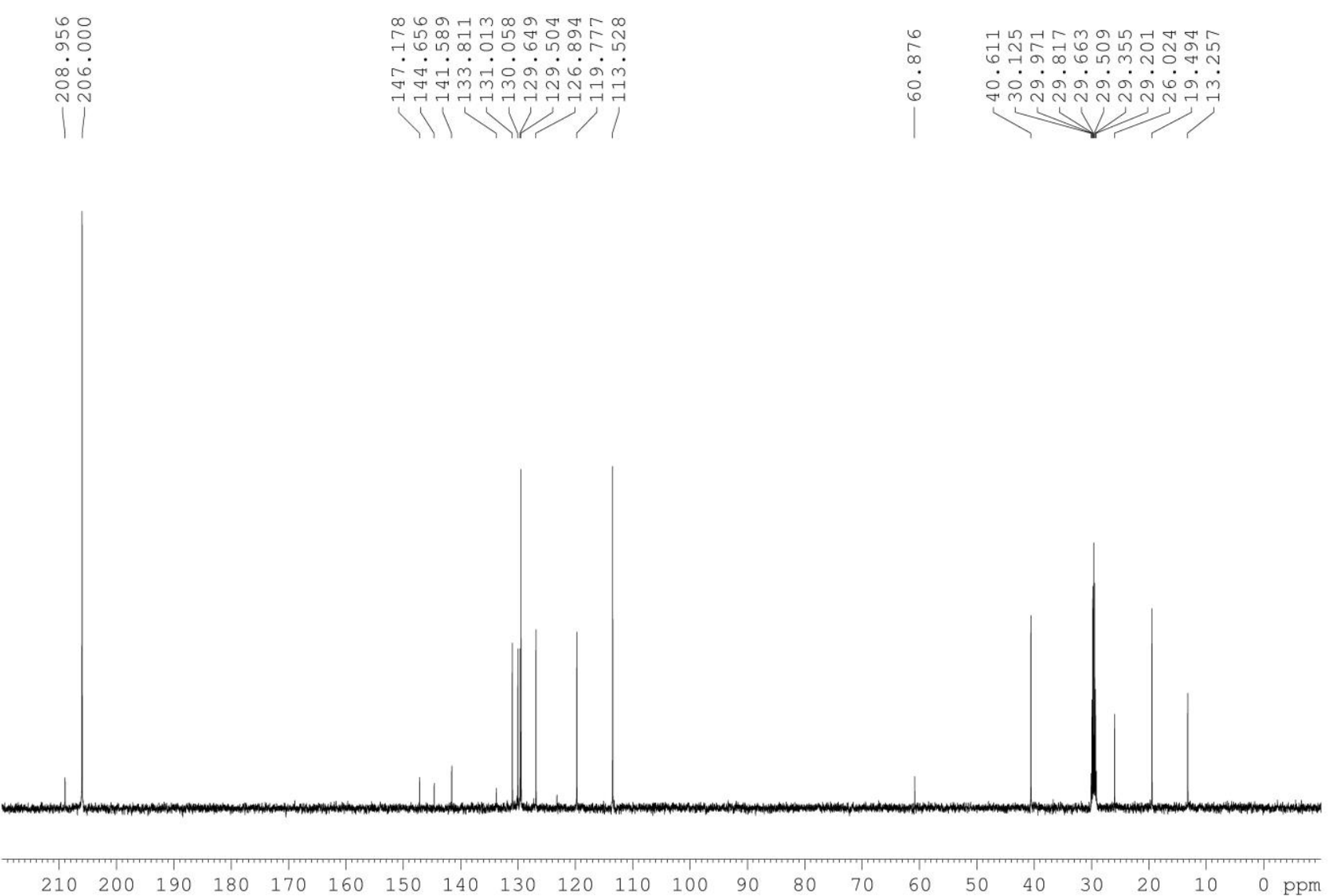


${ }^{1} \mathrm{H}$ NMR spectrum of compound $\mathbf{6 g}\left(500 \mathrm{MHz}, \mathrm{CD}_{3} \mathrm{COCD}_{3}\right)$

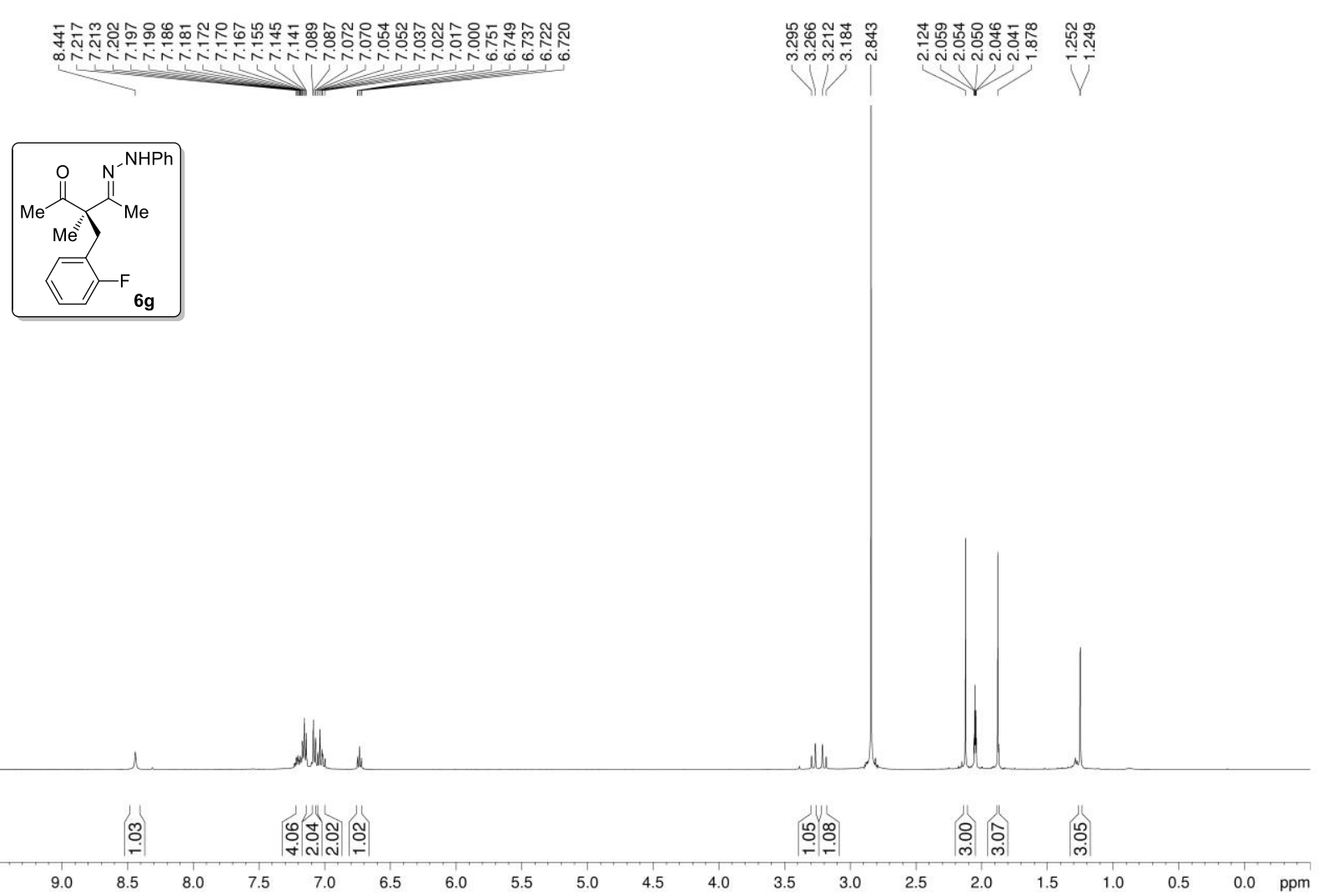


${ }^{13} \mathrm{C}$ NMR spectrum of compound $\mathbf{6 g}\left(125 \mathrm{MHz}, \mathrm{CD}_{3} \mathrm{COCD}_{3}\right)$
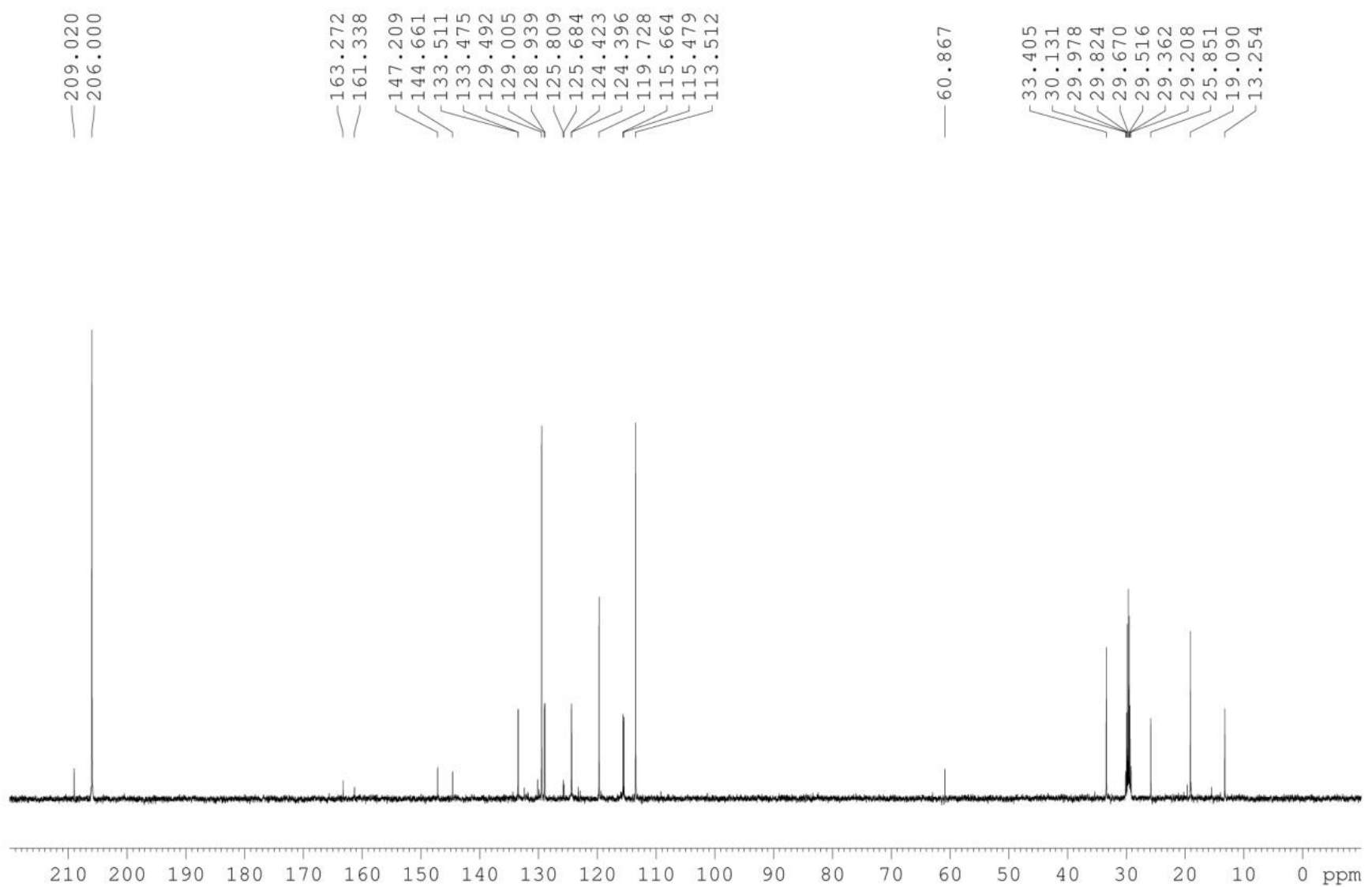
${ }^{19} \mathrm{~F}$ NMR spectrum of compound $\mathbf{6 g}\left(469 \mathrm{MHz}, \mathrm{CD}_{3} \mathrm{COCD}_{3}\right)$

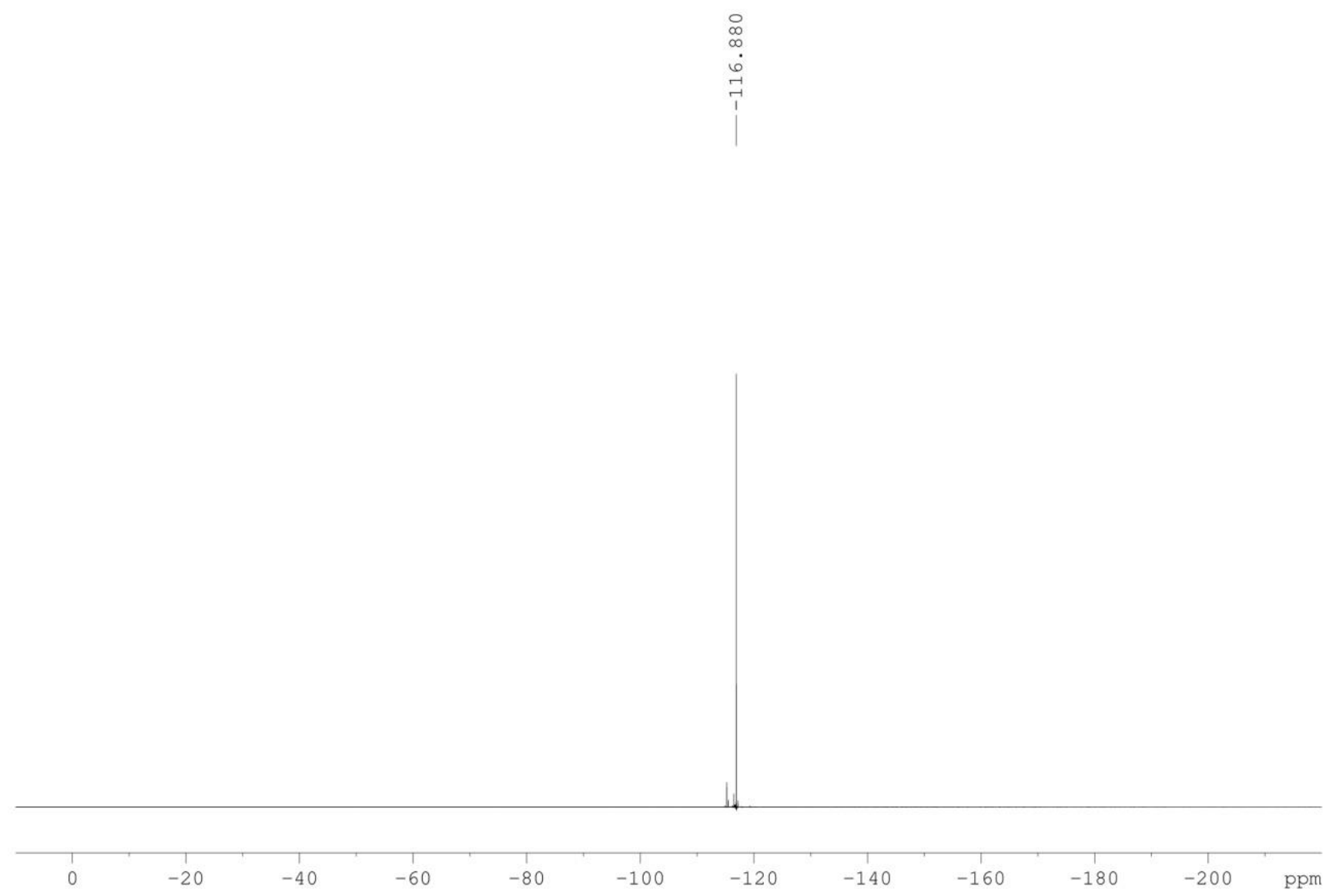


${ }^{13} \mathrm{C}$ NMR spectrum of compound $\mathbf{6 h}\left(500 \mathrm{MHz}, \mathrm{CD}_{3} \mathrm{COCD}_{3}\right)$

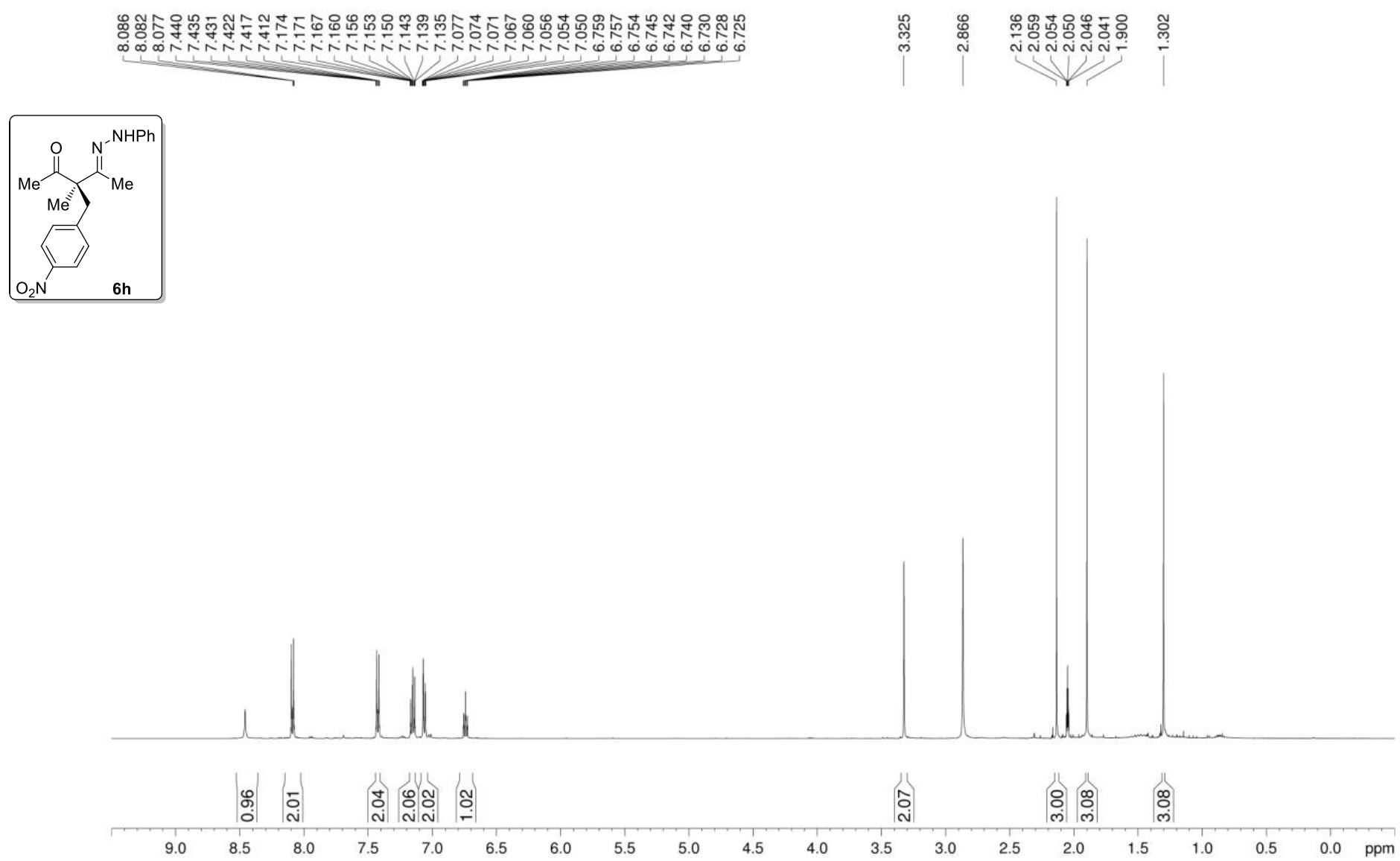


${ }^{13} \mathrm{C}$ NMR spectrum of compound $\mathbf{6 h}\left(125 \mathrm{MHz}, \mathrm{CD}_{3} \mathrm{COCD}_{3}\right)$

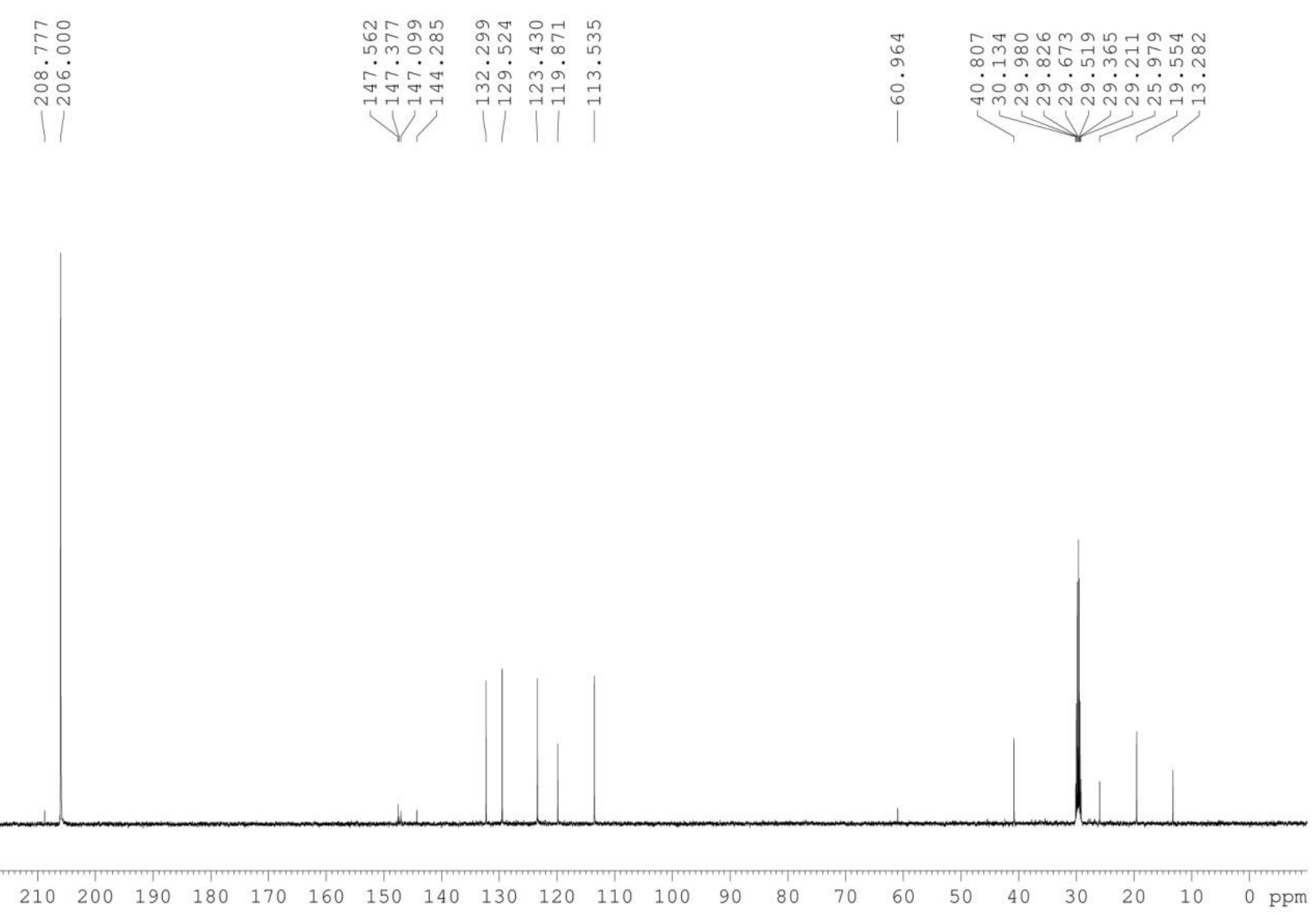


${ }^{1} \mathrm{H}$ NMR spectrum of compound $\mathbf{6 i}\left(500 \mathrm{MHz}, \mathrm{CD}_{3} \mathrm{COCD}_{3}\right)$

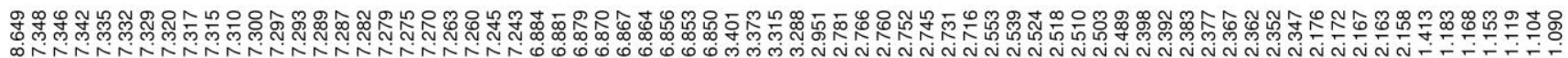

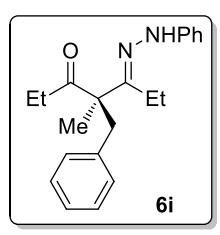


${ }^{13} \mathrm{C}$ NMR spectrum of compound $\mathbf{6 i}\left(126 \mathrm{MHz}, \mathrm{CD}_{3} \mathrm{COCD}_{3}\right)$

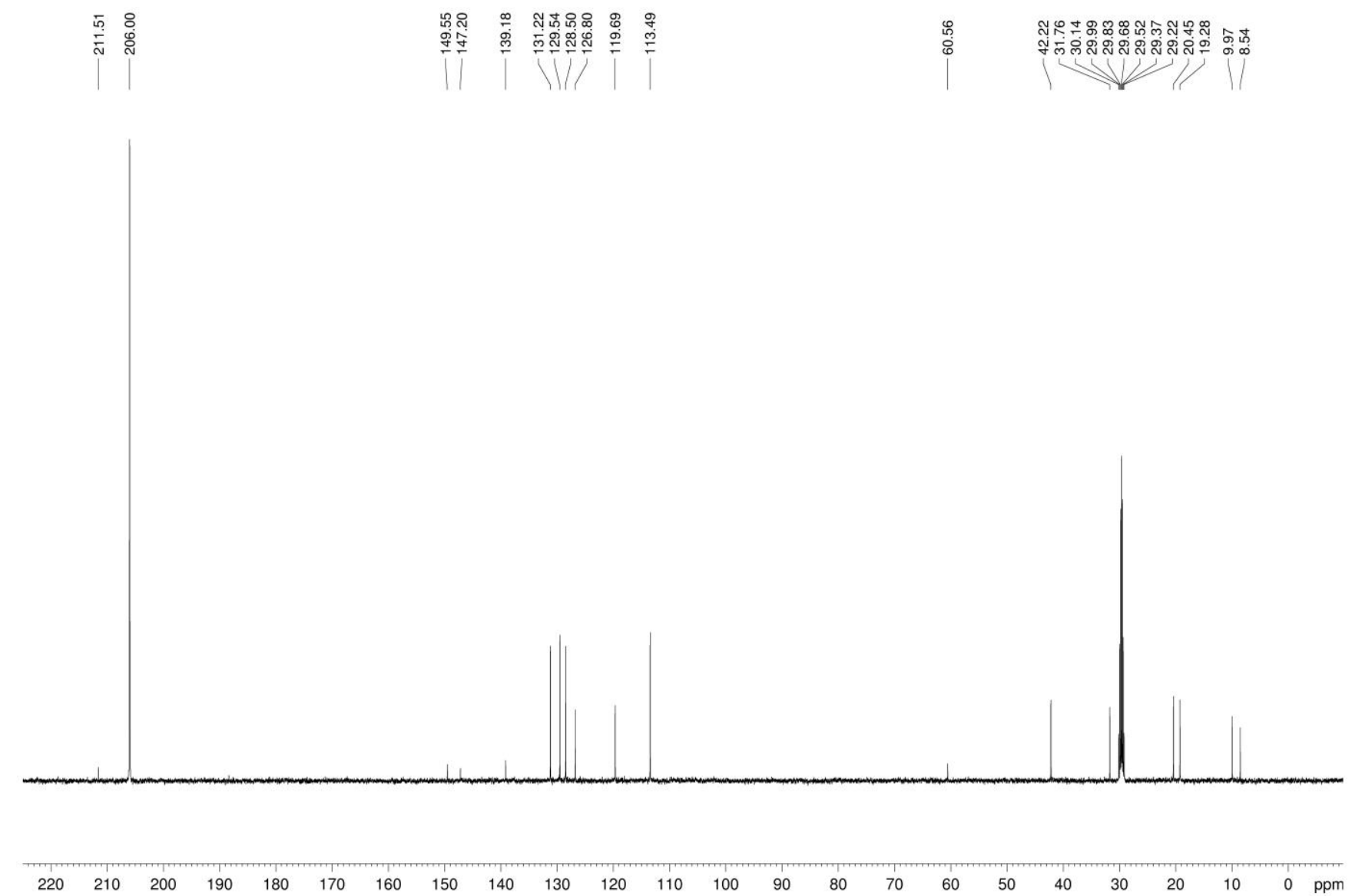


${ }^{1} \mathrm{H}$ NMR spectrum of compound $\mathbf{6 j}\left(500 \mathrm{MHz}, \mathrm{CD}_{3} \mathrm{COCD}_{3}\right)$

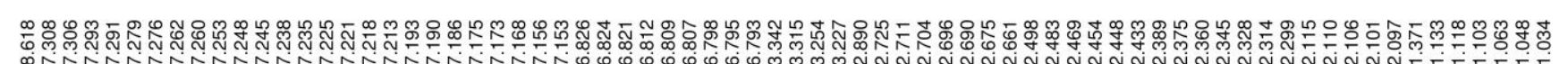

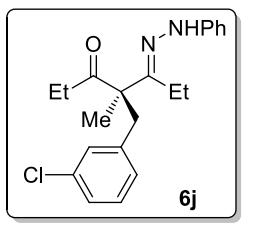


${ }^{13} \mathrm{C}$ NMR spectrum of compound $\mathbf{6 j}\left(125 \mathrm{MHz}, \mathrm{CD}_{3} \mathrm{COCD}_{3}\right)$

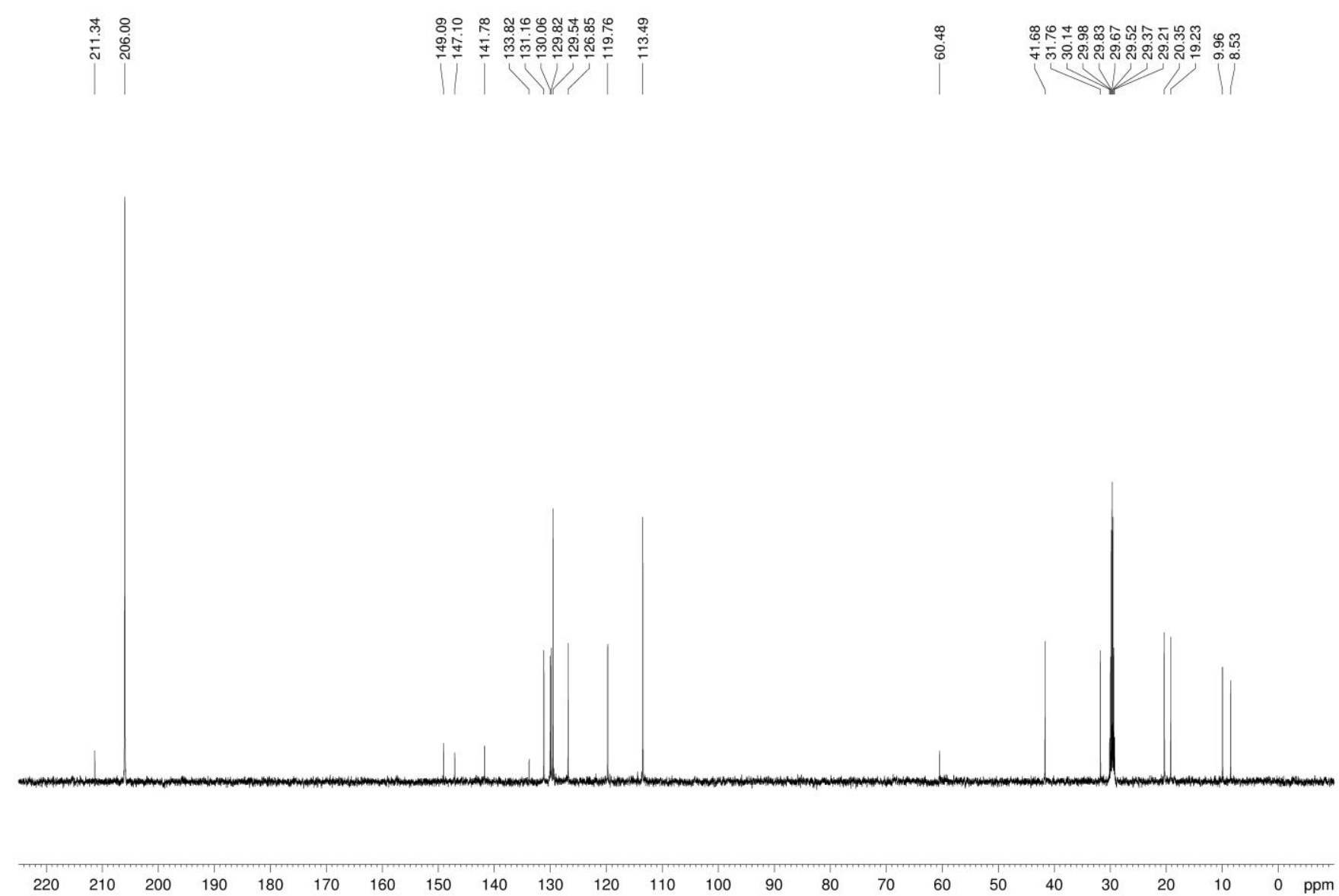


${ }^{1} \mathrm{H}$ NMR spectrum of compound $\mathbf{6 k}\left(500 \mathrm{MHz}, \mathrm{CD}_{3} \mathrm{COCD}_{3}\right)$

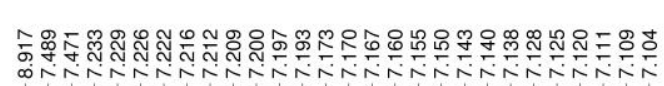

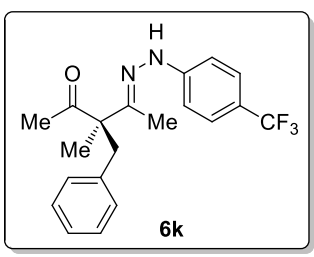

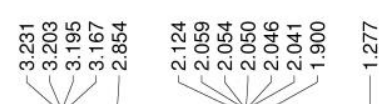

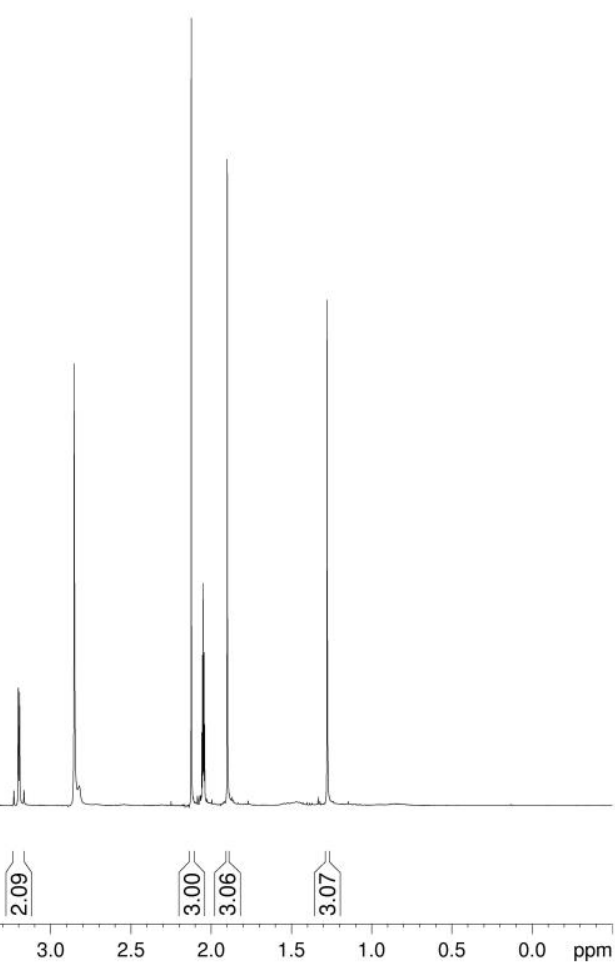


${ }^{13} \mathrm{C}$ NMR spectrum of compound $\mathbf{6 k}\left(125 \mathrm{MHz}, \mathrm{CD}_{3} \mathrm{COCD}_{3}\right)$

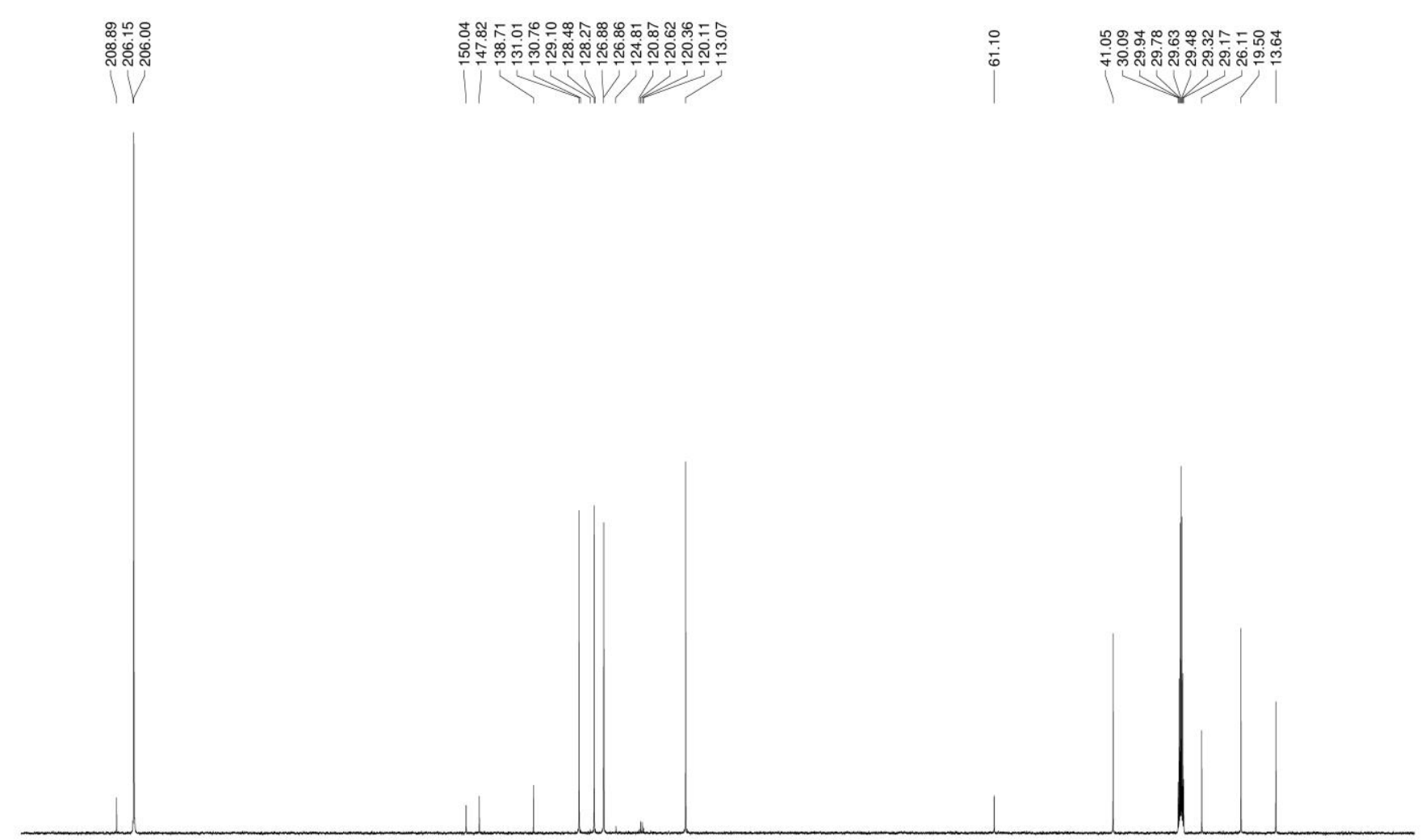


${ }^{19} \mathrm{~F}$ NMR spectrum of compound $\mathbf{6 k}\left(376 \mathrm{MHz}, \mathrm{CD}_{3} \mathrm{COCD}_{3}\right)$

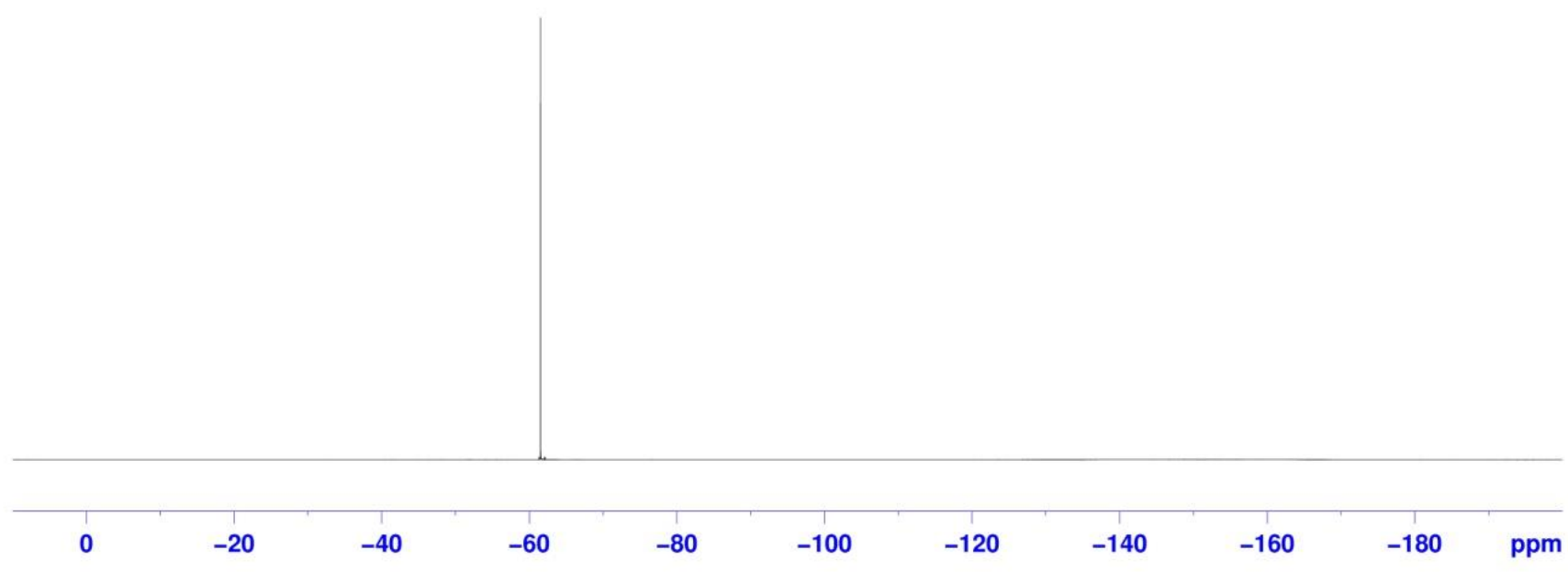


${ }^{1} \mathrm{H}$ NMR spectrum of compound $7\left(500 \mathrm{MHz}, \mathrm{CD}_{3} \mathrm{COCD}_{3}\right)$

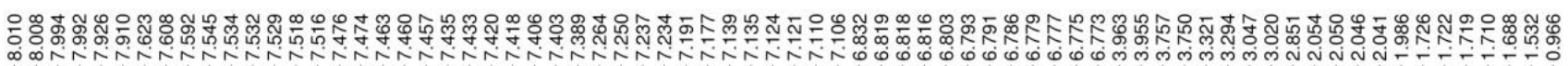
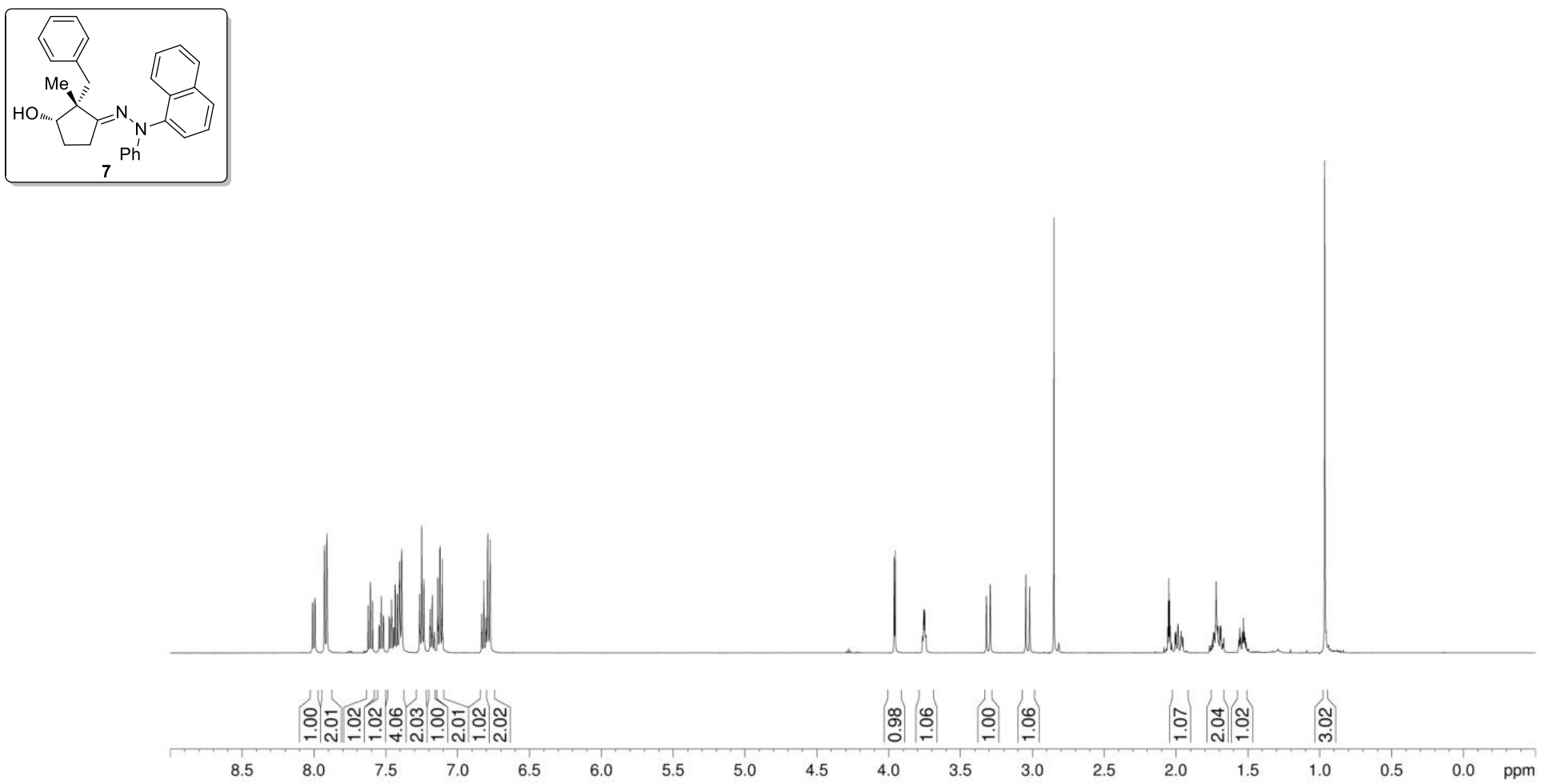
${ }^{13} \mathrm{C}$ NMR spectrum of compound $7\left(125 \mathrm{MHz}, \mathrm{CD}_{3} \mathrm{COCD}_{3}\right)$

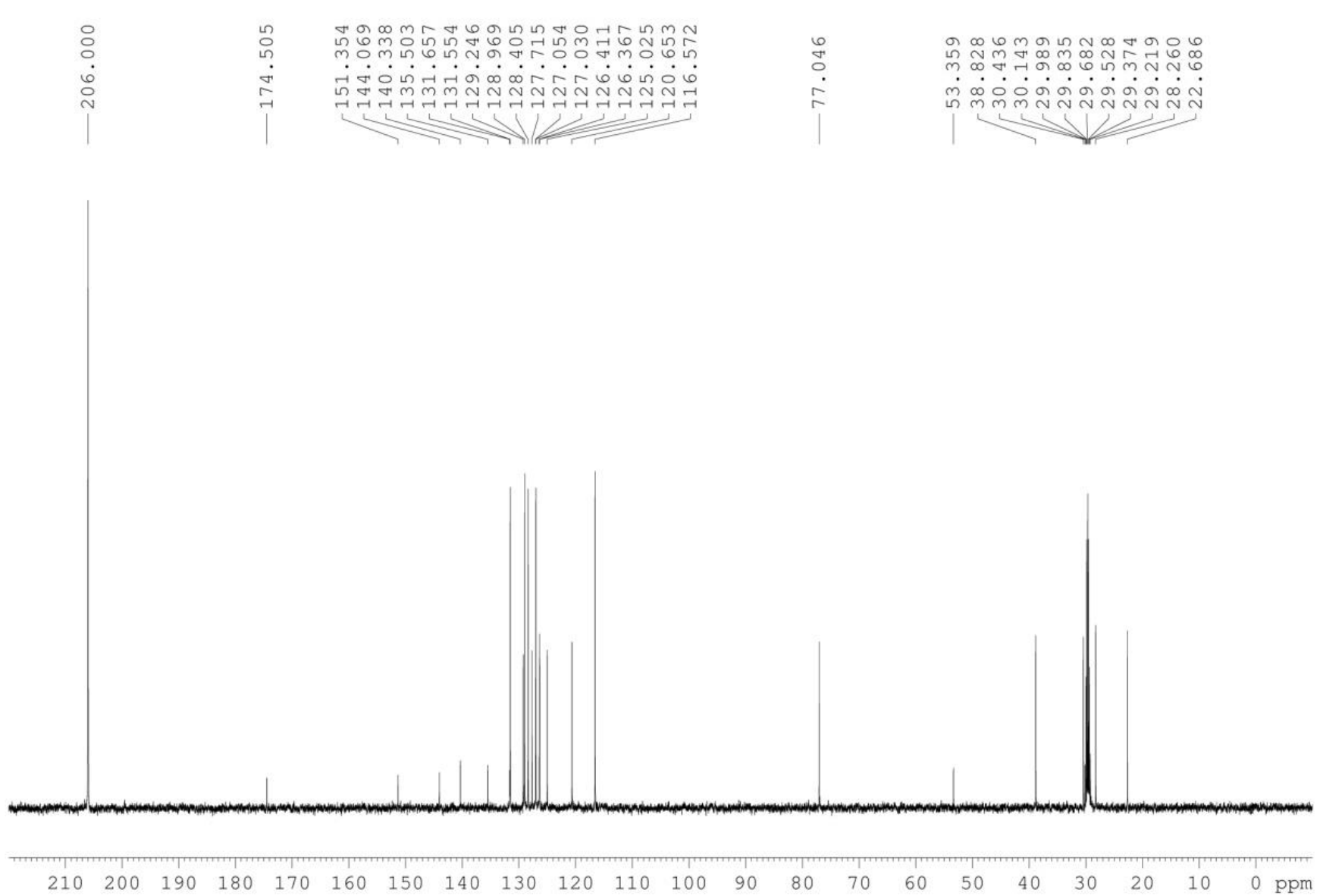


${ }^{1} \mathrm{H}$ NMR spectrum of compound $8\left(500 \mathrm{MHz}, \mathrm{CDCl}_{3}\right)$
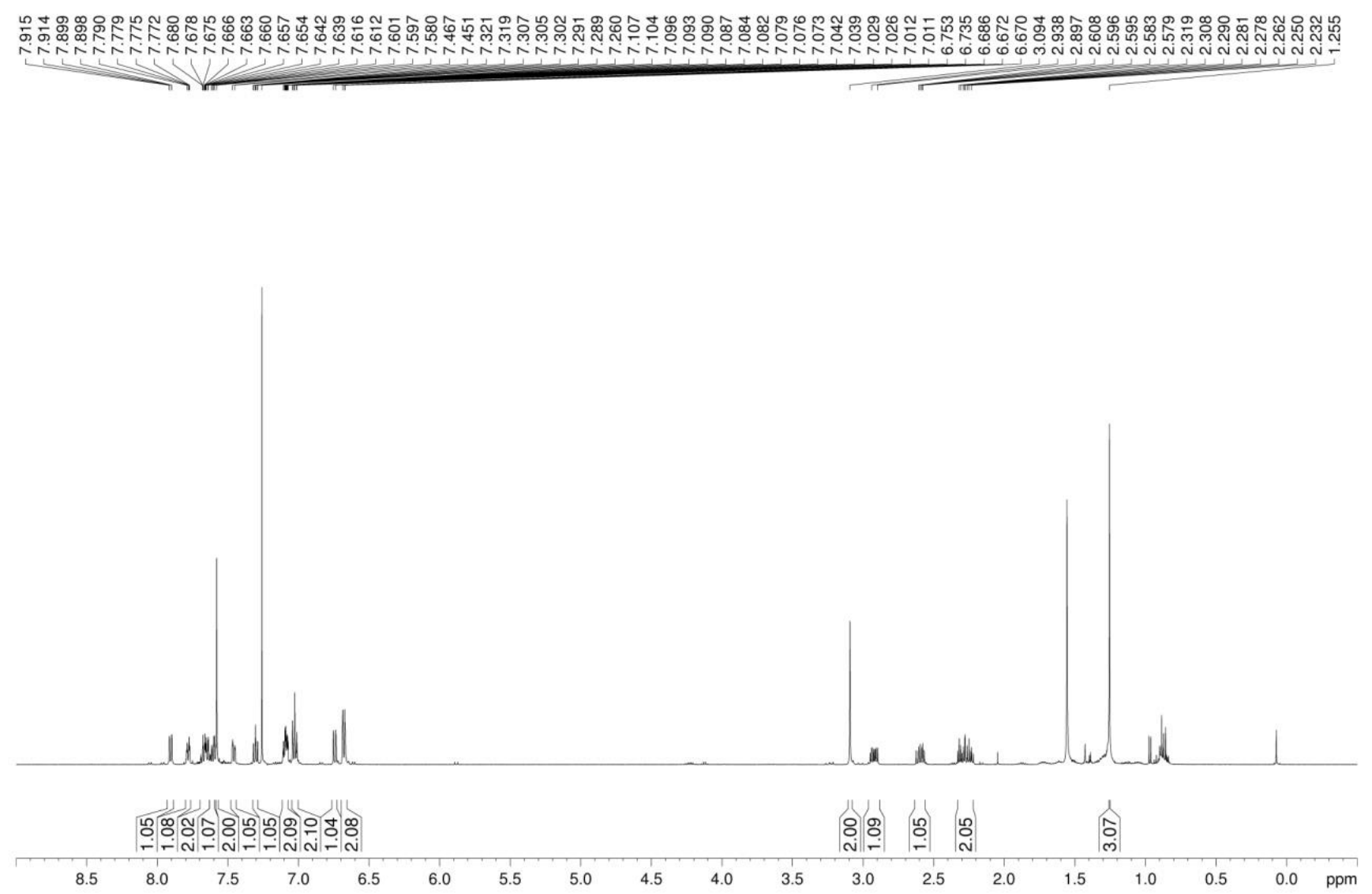
${ }^{13} \mathrm{C}$ NMR spectrum of compound $8\left(125 \mathrm{MHz}, \mathrm{CDCl}_{3}\right)$

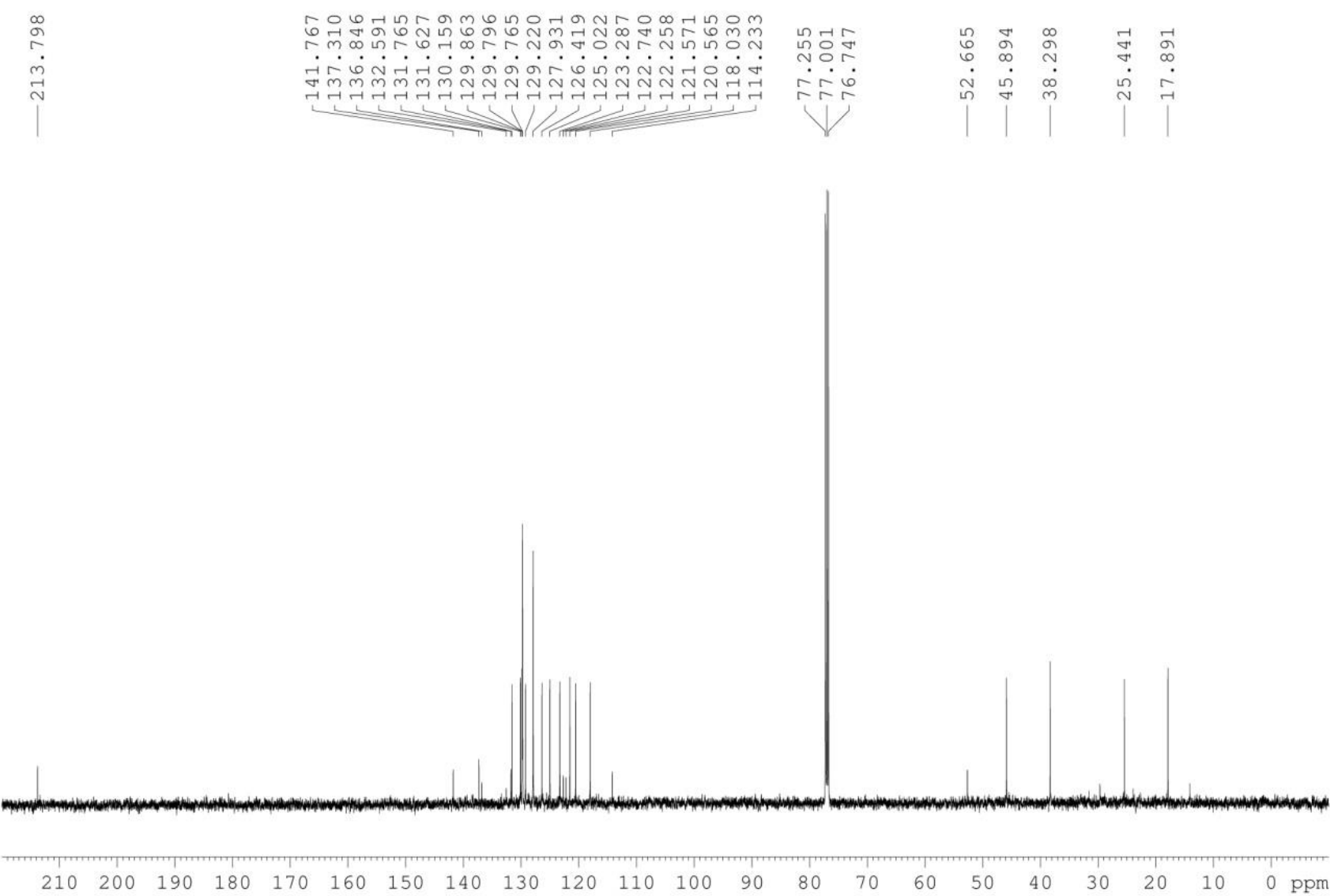


${ }^{1} \mathrm{H}$ NMR spectrum of compound $9\left(500 \mathrm{MHz}, \mathrm{CDCl}_{3}\right)$

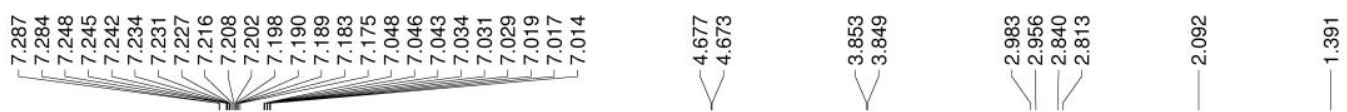
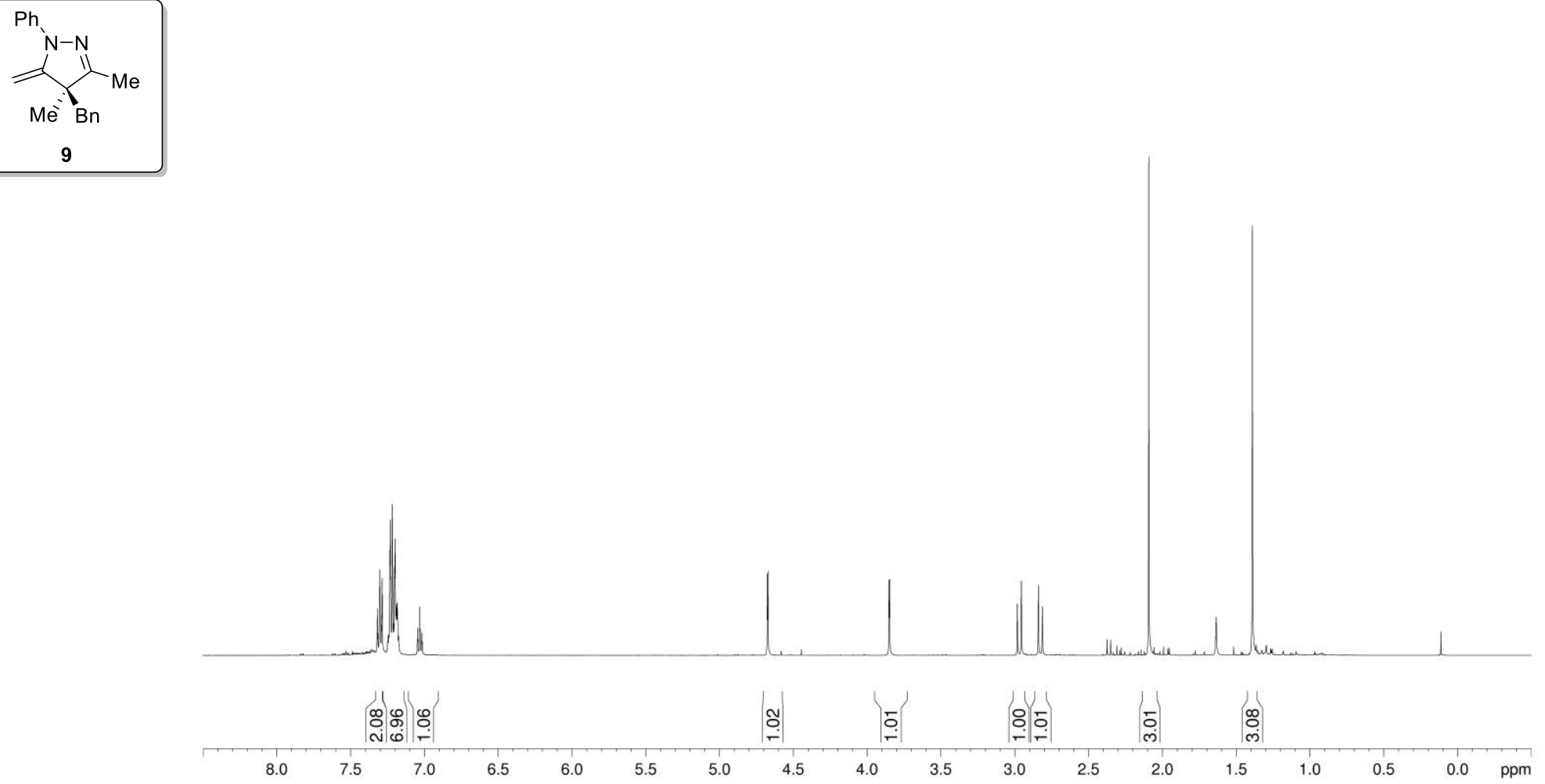
${ }^{13} \mathrm{C}$ NMR spectrum of compound $9\left(125 \mathrm{MHz}, \mathrm{CDCl}_{3}\right)$
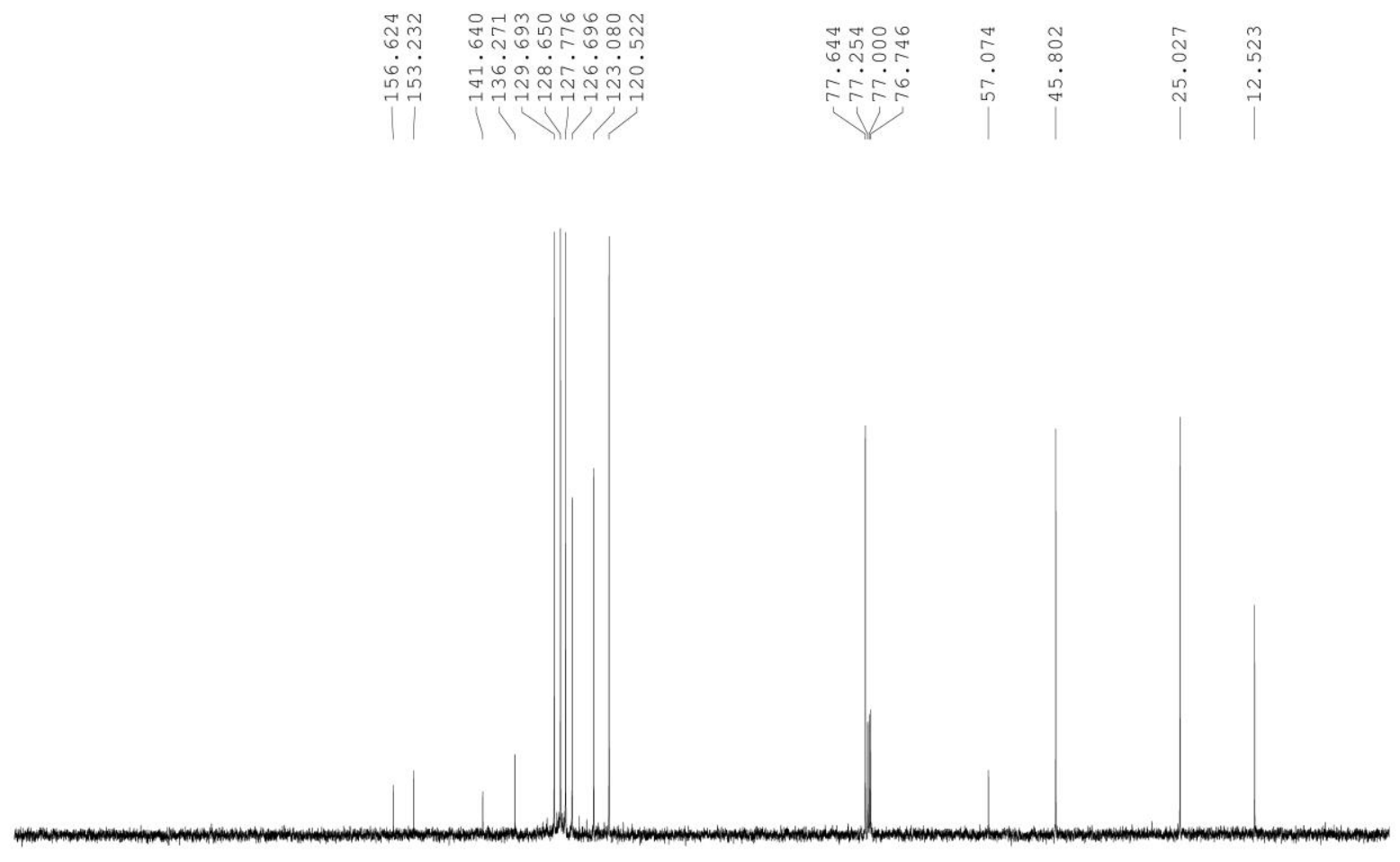

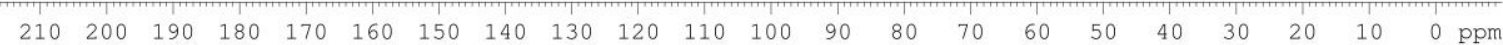


${ }^{1} \mathrm{H}$ NMR spectrum of compound $10\left(500 \mathrm{MHz}, \mathrm{CDCl}_{3}\right)$

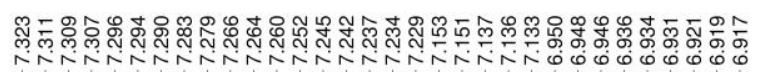

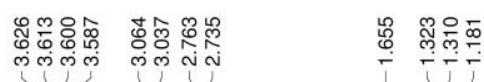

T

${ }_{10}$


${ }^{13} \mathrm{C}$ NMR spectrum of compound $10\left(125 \mathrm{MHz}, \mathrm{CDCl}_{3}\right)$

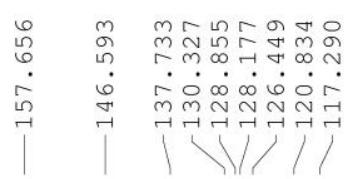

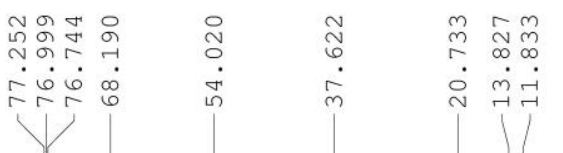

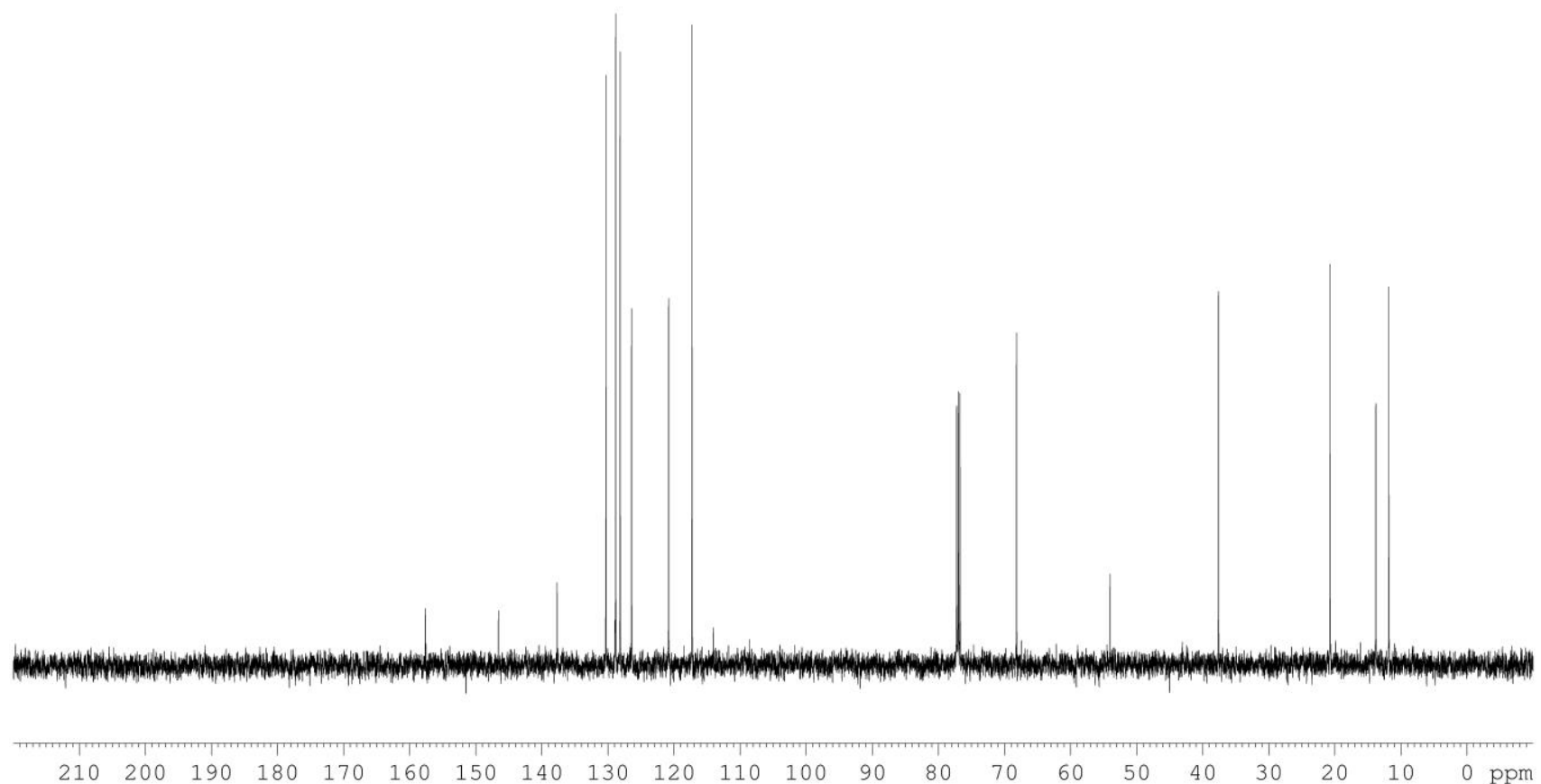


1D NOE-NMR studies for $\mathbf{1 0}$

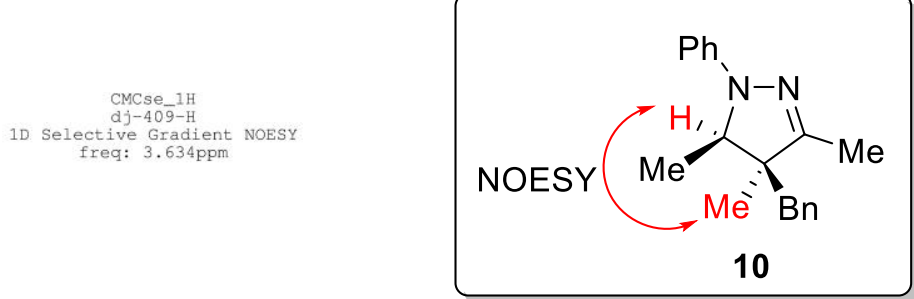

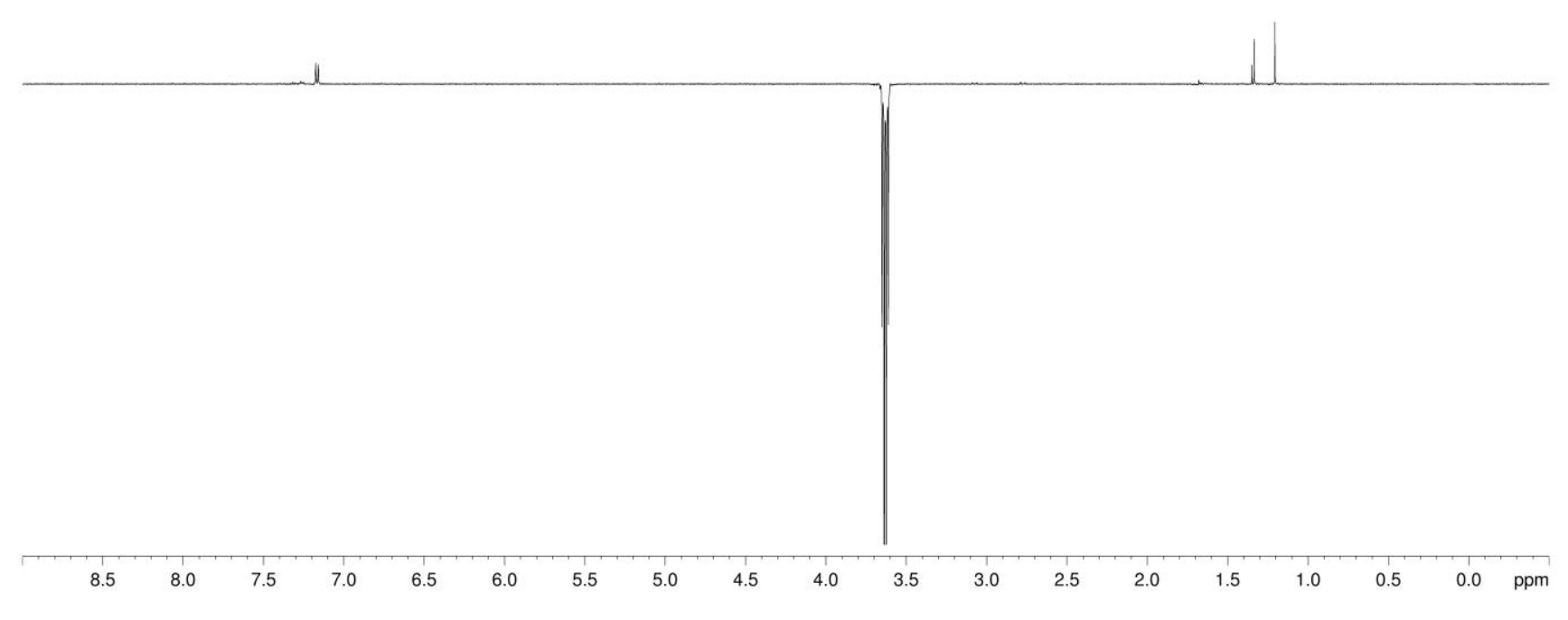


1D NOE-NMR studies for $\mathbf{1 0}$

Me

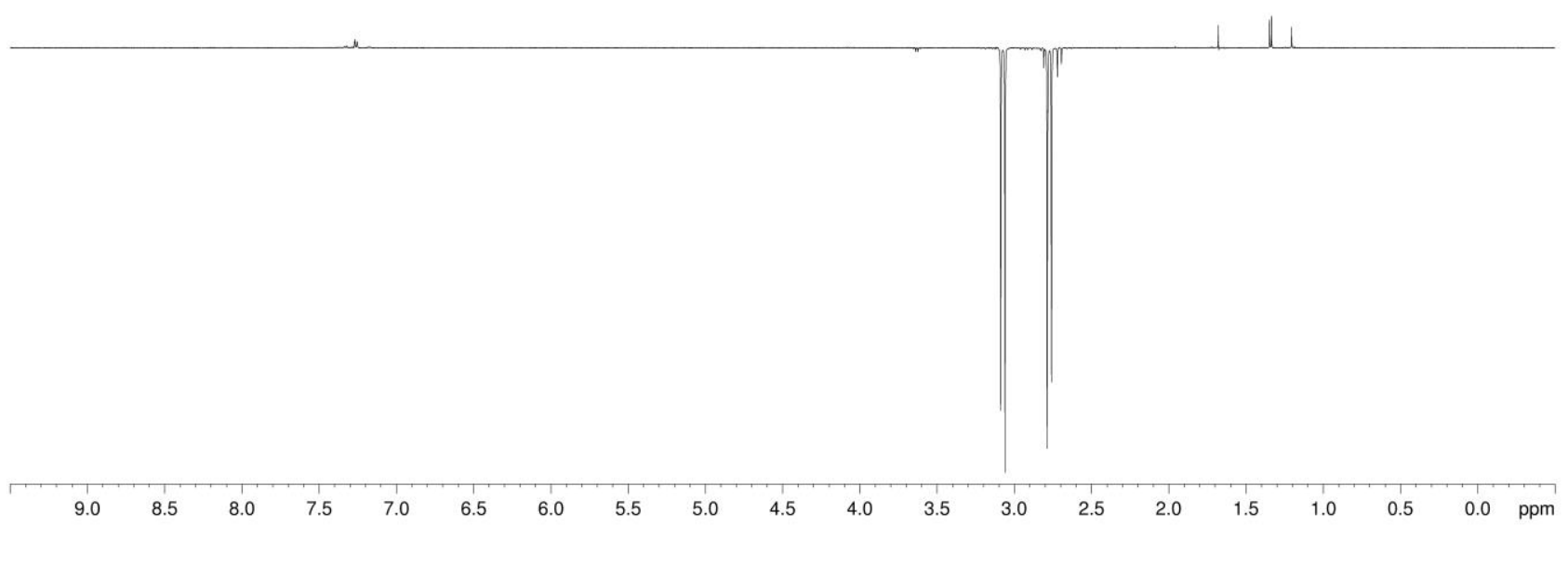


${ }^{1} \mathrm{H}$ NMR spectrum of compound $11\left(500 \mathrm{MHz}, \mathrm{CDCl}_{3}\right)$

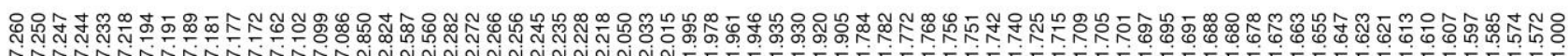
(1)
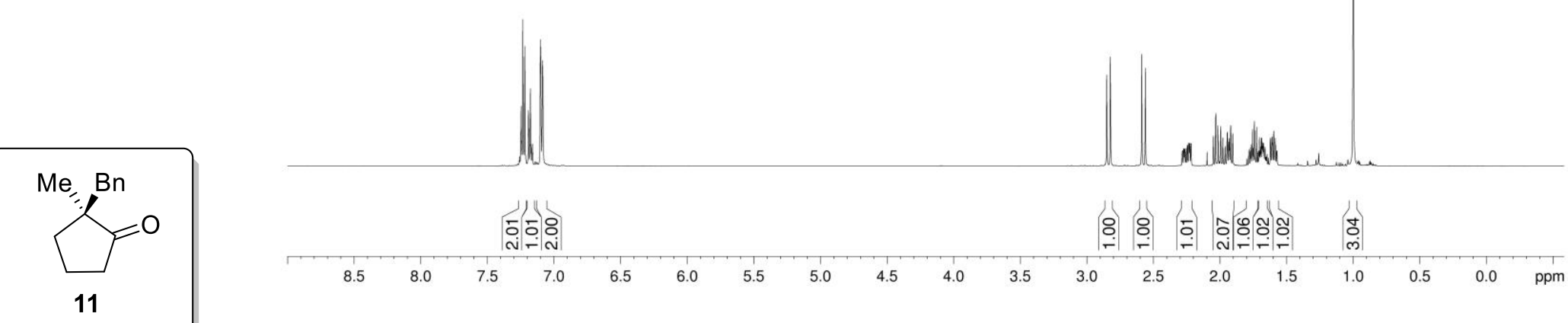
${ }^{13} \mathrm{C}$ NMR spectrum of compound $11\left(500 \mathrm{MHz}, \mathrm{CDCl}_{3}\right)$

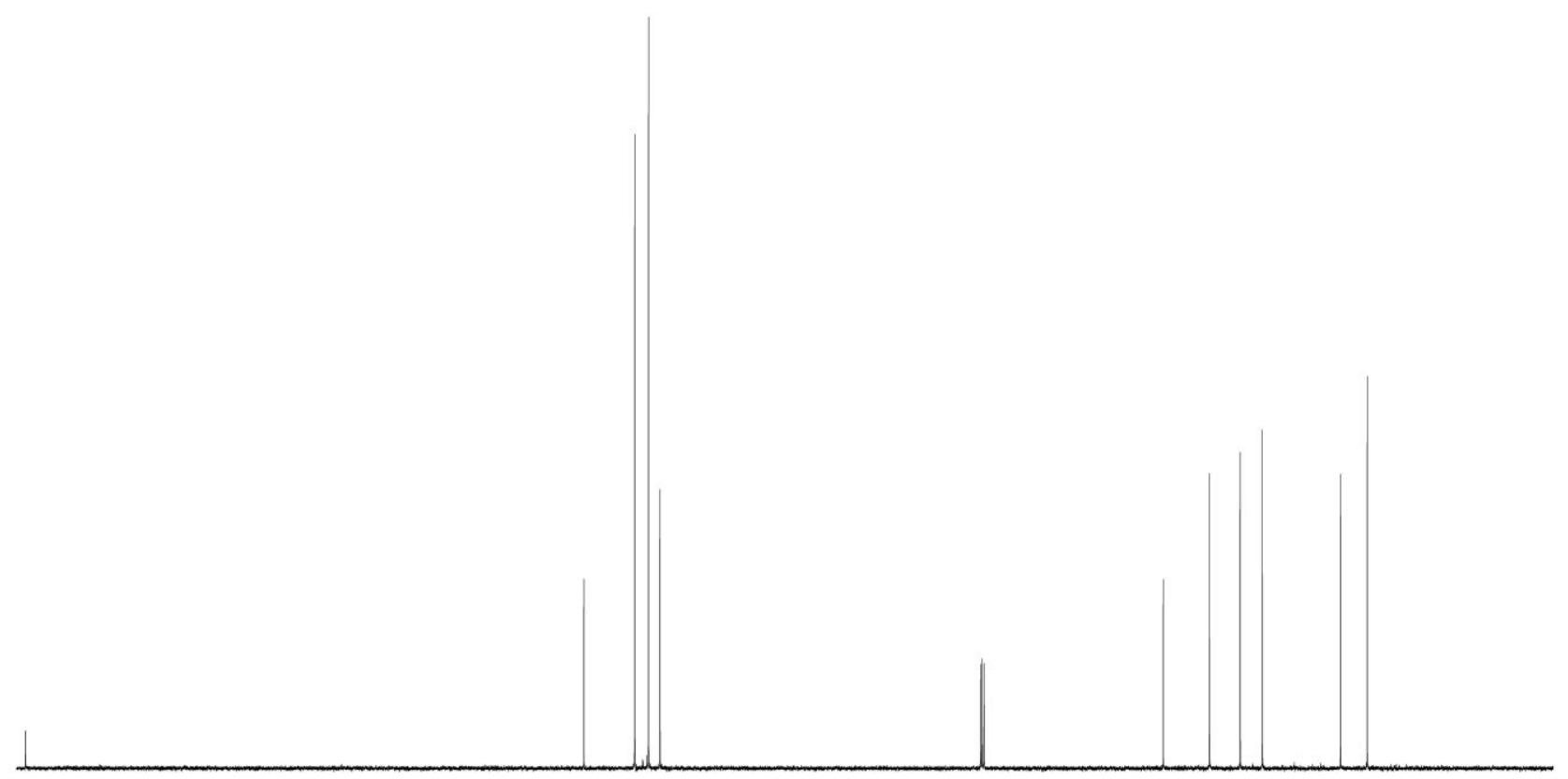


${ }^{1} \mathrm{H}$ NMR spectrum of compound $12\left(500 \mathrm{MHz}, \mathrm{CDCl}_{3}\right)$

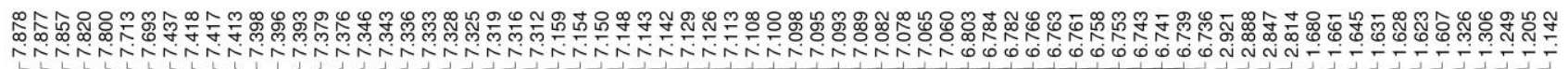 \\ re}
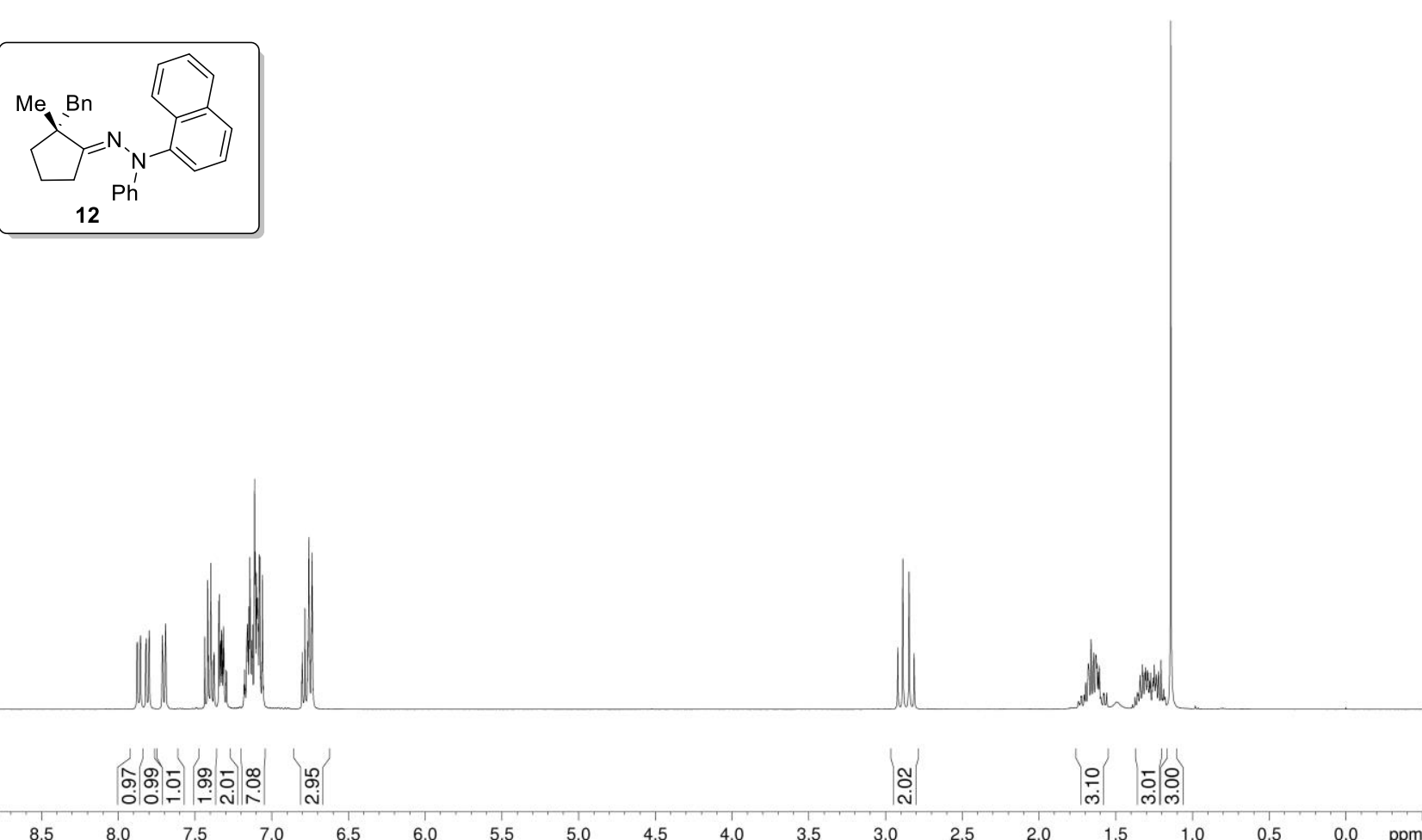
${ }^{13} \mathrm{C}$ NMR spectrum of compound $12\left(500 \mathrm{MHz}, \mathrm{CDCl}_{3}\right)$

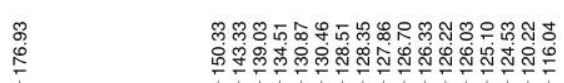

这

กำ

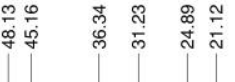

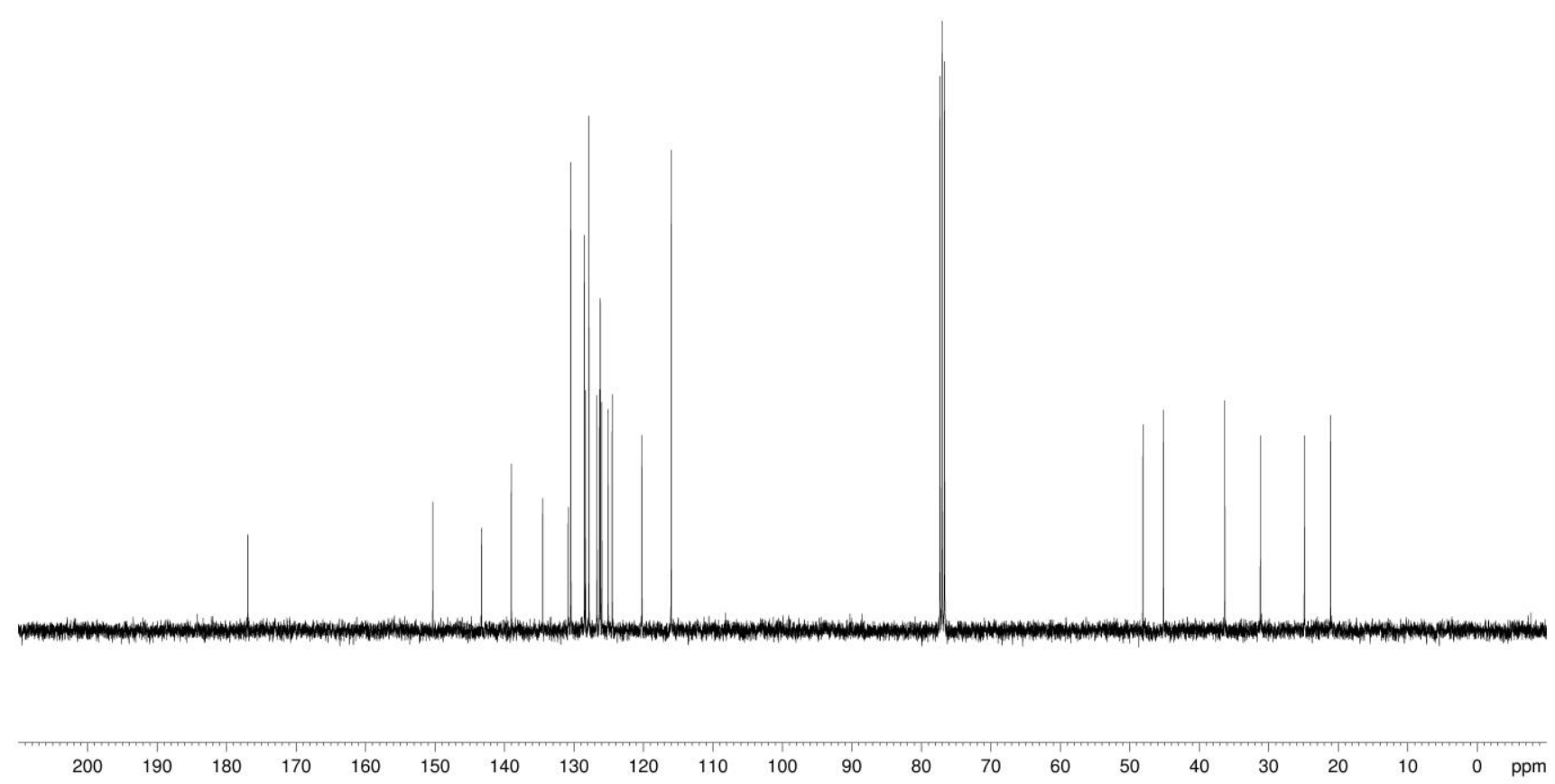




\section{DFT Calculation}

All density functional theory calculations were carried out with the Gaussian 09 programs. ${ }^{1}$ Geometries were optimized using B3LYP/def2-SVP ${ }^{2-7}$ level of theory in the gas phase. Vibrational frequency calculations were performed for all the stationary points to confirm if each optimized structure is a local minimum or a transition state structure. The solvent effects were considered by single point energy calculations on the gas-phase stationary points using M06- $2 \mathrm{X}^{8-9}$ functional with the def2-TZVPP ${ }^{10-13}$ basis set in a SMD ${ }^{14}$ continuum solvation model. The energies given in this work are M06-2X calculated Gibbs free energies in acetonitrile solvent.

\section{Absolute calculation energies, enthalpies, and free energies}

\begin{tabular}{cccccc}
\hline Geometry & $\mathrm{E}_{(\mathrm{elec}-\mathrm{B} 3 \mathrm{LYP})}{ }^{1}$ & $\mathrm{E}_{(\mathrm{solv}, \mathrm{M} 06-2 \mathrm{X})^{2}}$ & $\mathrm{G}_{(\mathrm{corr}-\mathrm{B} 3 \mathrm{LYP})^{3}}$ & $\mathrm{H}_{(\text {(corr-B3LYP) }}{ }^{4}$ & $\mathrm{IF}^{5}$ \\
\hline CPA3 & -3280.286711 & -3282.424958 & 1.239907 & 1.411425 & - \\
1a & -653.791930 & -654.261194 & 0.196901 & 0.253323 & - \\
2a & -727.091965 & -727.594065 & 0.220062 & 0.276886 & - \\
int-0-R-R & -4661.197322 & -4664.299479 & 1.697243 & 1.945886 & - \\
ts-1-R-R & -4661.181787 & -4664.296172 & 1.705160 & 1.942935 & $256.64 i$ \\
int-1-R-R & -4661.188002 & -4664.312643 & 1.716076 & 1.948549 & - \\
ts-2-R-R & -4661.180669 & -4664.302568 & 1.708979 & 1.943524 & $693.57 i$ \\
int-2a-R-R & -4661.182103 & -4664.302267 & 1.711115 & 1.947284 & - \\
int-2b-R-R & -4661.193694 & -4664.314863 & 1.711621 & 1.948055 & - \\
ts-3-R-R & -4661.177863 & -4664.301676 & 1.711887 & 1.944048 & $310.74 i$ \\
int-3-R & -4661.192826 & -4664.317570 & 1.708125 & 1.945767 & - \\
ts-4-R & -4661.189285 & -4664.312806 & 1.704387 & 1.941454 & $360.21 i$ \\
int-4 & -3356.675472 & -3358.876666 & 1.264302 & 1.439530 & - \\
R-3a & -1304.510472 & -1305.420445 & 0.415173 & 0.503789 & - \\
H2O & -76.358315 & -76.436911 & 0.003572 & 0.025014 & - \\
ts-5 & -4661.141683 & -4664.258601 & 1.709256 & 1.941314 & $1640.91 i$ \\
\hline & & & & & \\
\hline
\end{tabular}




\begin{tabular}{cccccc}
\hline ts-1-S-S & -4661.179130 & -4664.297273 & 1.708848 & 1.943579 & $201.43 i$ \\
ts-1-R-S & -4661.179539 & -4664.297006 & 1.711594 & 1.944260 & $201.13 i$ \\
ts-1-S-R & -4661.181817 & -4664.294809 & 1.708331 & 1.943572 & $252.33 i$ \\
ts-3-S-S & -4661.176845 & -4664.300567 & 1.712773 & 1.944269 & $253.54 i$ \\
ts-3-R-S & -4661.178935 & -4664.305226 & 1.712096 & 1.943664 & $323.90 i$ \\
ts-3-S-R & -4661.173121 & -4664.292080 & 1.710912 & 1.945435 & $160.95 i$ \\
\hline
\end{tabular}

${ }^{1}$ The electronic energy calculated by B3LYP in gas phase. ${ }^{2}$ The electronic energy calculated by M06-2X in toluene solvent. ${ }^{3}$ The thermal correction to Gibbs free energy calculated by B3LYP in gas phase. ${ }^{4}$ The thermal correction to enthalpy calculated by B3LYP in gas phase. ${ }^{5}$ The B3LYP calculated imaginary frequencies for the transition states.

\section{B3LYP geometries for all the compounds and transition states}

\begin{tabular}{|c|c|c|c|c|c|c|c|}
\hline CPA3 & & & & $\mathrm{O}$ & -0.46285800 & -2.06396800 & 0.42839000 \\
\hline $\mathrm{C}$ & -1.54200300 & 1.12148200 & 0.62445000 & $\mathrm{C}$ & -3.83224800 & 0.03942600 & 0.29038300 \\
\hline $\mathrm{C}$ & -0.66187700 & 2.02205300 & 1.21308100 & $\mathrm{C}$ & -4.36274500 & 0.46475400 & -0.95282600 \\
\hline $\mathrm{C}$ & -1.08614100 & 2.68701300 & 2.41920200 & $\mathrm{C}$ & -4.21906600 & -1.21183700 & 0.83627300 \\
\hline $\mathrm{C}$ & -2.43700900 & 2.51093500 & 2.86934200 & $\mathrm{C}$ & -5.27837700 & -0.36450200 & -1.61546700 \\
\hline $\mathrm{C}$ & -3.31460400 & 1.66623200 & 2.13803100 & $\mathrm{C}$ & -5.14331600 & -1.99574000 & 0.13142500 \\
\hline $\mathrm{C}$ & -2.89377700 & 0.94046600 & 1.04048400 & $\mathrm{C}$ & -5.68810700 & -1.59555000 & -1.09462400 \\
\hline $\mathrm{H}$ & 0.82648500 & 3.60158200 & 2.91136100 & $\mathrm{H}$ & -5.69491000 & -0.03825600 & -2.57260900 \\
\hline $\mathrm{C}$ & -0.21441200 & 3.48616700 & 3.21332500 & $\mathrm{H}$ & -5.44348300 & -2.95567000 & 0.55744500 \\
\hline $\mathrm{C}$ & -2.87183300 & 3.17589400 & 4.05077600 & $\mathrm{C}$ & 3.79054100 & 0.22614300 & -0.37071000 \\
\hline $\mathrm{H}$ & -4.35115800 & 1.57317400 & 2.47022200 & $\mathrm{C}$ & 4.30289400 & -0.50120800 & 0.73561600 \\
\hline $\mathrm{C}$ & -2.00658800 & 3.95862300 & 4.78395500 & $\mathrm{C}$ & 4.17684200 & -0.13128000 & -1.68877900 \\
\hline $\mathrm{C}$ & -0.66140900 & 4.10170100 & 4.36475500 & $\mathrm{C}$ & 5.19158000 & -1.55799500 & 0.50079300 \\
\hline $\mathrm{H}$ & -3.90762800 & 3.04112500 & 4.37356600 & $\mathrm{C}$ & 5.06994000 & -1.20022600 & -1.86138500 \\
\hline $\mathrm{H}$ & -2.35030200 & 4.45765900 & 5.69334500 & $\mathrm{C}$ & 5.59304400 & -1.92768000 & -0.78855400 \\
\hline $\mathrm{H}$ & 0.03100600 & 4.70143000 & 4.96082400 & $\mathrm{H}$ & 5.59029300 & -2.10453700 & 1.35880200 \\
\hline $\mathrm{C}$ & 2.87989900 & 1.39738200 & -0.14092800 & $\mathrm{H}$ & 5.37658700 & -1.47659400 & -2.87371500 \\
\hline $\mathrm{C}$ & 3.30523700 & 2.69263900 & -0.36505800 & $\mathrm{C}$ & 3.95722600 & -0.14516500 & 2.17688400 \\
\hline $\mathrm{C}$ & 2.44219600 & 3.81071000 & -0.21699300 & $\mathrm{C}$ & 5.17985600 & 0.40138700 & 2.95056000 \\
\hline $\mathrm{C}$ & 1.09935600 & 3.61017600 & 0.24601900 & $\mathrm{C}$ & 3.30739600 & -1.31366600 & 2.95118400 \\
\hline $\mathrm{C}$ & 0.67660700 & 2.27811500 & 0.60041000 & $\mathrm{H}$ & 3.21765000 & 0.66925700 & 2.16235600 \\
\hline $\mathrm{C}$ & 1.54421000 & 1.22559700 & 0.33197200 & $\mathrm{C}$ & 4.80956400 & 0.80488600 & 4.38393000 \\
\hline $\mathrm{H}$ & 3.91086600 & 5.26139000 & -0.88484900 & $\mathrm{H}$ & 5.97097500 & -0.36986400 & 2.97910500 \\
\hline $\mathrm{H}$ & 4.33171400 & 2.86603000 & -0.69567700 & $\mathrm{H}$ & 5.60775200 & 1.26130900 & 2.40810300 \\
\hline $\mathrm{C}$ & 2.87997500 & 5.12473100 & -0.54723500 & $\mathrm{C}$ & 2.93855800 & -0.90524500 & 4.38340300 \\
\hline $\mathrm{C}$ & 0.23523800 & 4.74168400 & 0.29832200 & $\mathrm{H}$ & 4.00910700 & -2.16642300 & 2.98993900 \\
\hline $\mathrm{C}$ & 0.68340900 & 5.99970800 & -0.04997600 & $\mathrm{H}$ & 2.41439100 & -1.66151100 & 2.40919600 \\
\hline $\mathrm{C}$ & 2.02291600 & 6.20039500 & -0.46323500 & $\mathrm{C}$ & 4.14743100 & -0.34918900 & 5.14700300 \\
\hline $\mathrm{H}$ & -0.80108900 & 4.60824200 & 0.60775000 & $\mathrm{H}$ & 5.70619100 & 1.15796200 & 4.92142100 \\
\hline $\mathrm{H}$ & -0.00410100 & 6.84829800 & -0.00974400 & $\mathrm{H}$ & 4.11192400 & 1.66225900 & 4.34856700 \\
\hline $\mathrm{H}$ & 2.36815200 & 7.20299200 & -0.72732200 & $\mathrm{H}$ & 2.50727600 & -1.76630800 & 4.92140800 \\
\hline $\mathrm{O}$ & 1.13630900 & -0.07234500 & 0.60002700 & $\mathrm{H}$ & 2.14488800 & -0.13625800 & 4.34669700 \\
\hline $\mathrm{O}$ & -1.10885900 & 0.38403600 & -0.47418500 & $\mathrm{H}$ & 3.84652000 & -0.01827700 & 6.15561400 \\
\hline $\mathrm{P}$ & -0.04224800 & -0.81658100 & -0.23514300 & $\mathrm{H}$ & 4.88773400 & -1.15789500 & 5.29482900 \\
\hline \multicolumn{8}{|c|}{$\mathrm{S} 13$} \\
\hline
\end{tabular}




\begin{tabular}{|c|c|c|c|}
\hline $\mathrm{C}$ & 6.58179700 & -3.05718600 & -1.02533500 \\
\hline $\mathrm{C}$ & 6.11208900 & -4.41095100 & -0.45026200 \\
\hline $\mathrm{C}$ & 7.99528200 & -2.71403800 & -0.50133700 \\
\hline $\mathrm{H}$ & 6.66847900 & -3.18543000 & -2.12021100 \\
\hline $\mathrm{C}$ & 7.12036500 & -5.53334500 & -0.73119200 \\
\hline $\mathrm{H}$ & 5.97152300 & -4.31543500 & 0.64152800 \\
\hline $\mathrm{H}$ & 5.12367900 & -4.66777100 & -0.86655400 \\
\hline $\mathrm{C}$ & 9.00176400 & -3.83747600 & -0.78051100 \\
\hline $\mathrm{H}$ & 7.94109000 & -2.53364600 & 0.58776100 \\
\hline $\mathrm{H}$ & 8.33700800 & -1.76819700 & -0.95370300 \\
\hline $\mathrm{C}$ & 8.52192000 & -5.18037700 & -0.21661100 \\
\hline $\mathrm{H}$ & 6.77083400 & -6.47664400 & -0.27847300 \\
\hline $\mathrm{H}$ & 7.16913400 & -5.71334700 & -1.82136200 \\
\hline $\mathrm{H}$ & 9.98763000 & -3.57440000 & -0.36105700 \\
\hline $\mathrm{H}$ & 9.14631900 & -3.93278500 & -1.87284600 \\
\hline $\mathrm{H}$ & 9.23683400 & -5.98144000 & -0.46980000 \\
\hline $\mathrm{H}$ & 8.49881300 & -5.12241900 & 0.88765900 \\
\hline $\mathrm{C}$ & 3.68182600 & 0.61398200 & -2.92573600 \\
\hline $\mathrm{C}$ & 3.09249400 & -0.31140100 & -4.01344100 \\
\hline $\mathrm{C}$ & 4.78735600 & 1.50366400 & -3.54296900 \\
\hline $\mathrm{H}$ & 2.86453200 & 1.28297800 & -2.61512900 \\
\hline $\mathrm{C}$ & 2.57375800 & 0.48724600 & -5.21698200 \\
\hline $\mathrm{H}$ & 3.86297400 & -1.02232200 & -4.36108400 \\
\hline $\mathrm{H}$ & 2.27875900 & -0.91066500 & -3.57827500 \\
\hline $\mathrm{C}$ & 4.27893700 & 2.29998400 & -4.75179600 \\
\hline $\mathrm{H}$ & 5.63211400 & 0.86175300 & -3.85311100 \\
\hline $\mathrm{H}$ & 5.19161000 & 2.18764800 & -2.77876200 \\
\hline $\mathrm{C}$ & 3.66444400 & 1.38351100 & -5.81627600 \\
\hline $\mathrm{H}$ & 2.17847600 & -0.20066300 & -5.98369800 \\
\hline $\mathrm{H}$ & 1.72179700 & 1.11396700 & -4.89518600 \\
\hline $\mathrm{H}$ & 5.10089200 & 2.89535600 & -5.18463800 \\
\hline $\mathrm{H}$ & 3.51595500 & 3.02452200 & -4.41205000 \\
\hline $\mathrm{H}$ & 3.25677000 & 1.98125200 & -6.64901800 \\
\hline $\mathrm{H}$ & 4.45897100 & 0.74779500 & -6.25019400 \\
\hline $\mathrm{C}$ & -3.67658100 & -1.72475500 & 2.16653600 \\
\hline $\mathrm{C}$ & -3.12074300 & -3.16445800 & 2.08958700 \\
\hline $\mathrm{C}$ & -4.72453900 & -1.61852900 & 3.29908900 \\
\hline $\mathrm{H}$ & -2.82738100 & -1.08434100 & 2.45292800 \\
\hline $\mathrm{C}$ & -2.54834700 & -3.61659900 & 3.43910700 \\
\hline $\mathrm{H}$ & -3.92521200 & -3.86160300 & 1.79325800 \\
\hline $\mathrm{H}$ & -2.34275800 & -3.20778400 & 1.31420500 \\
\hline $\mathrm{C}$ & -4.15947900 & -2.08085900 & 4.64862000 \\
\hline $\mathrm{H}$ & -5.60260100 & -2.23618100 & 3.03450000 \\
\hline $\mathrm{H}$ & -5.09409900 & -0.58231900 & 3.37656100 \\
\hline $\mathrm{C}$ & -3.58063600 & -3.49882900 & 4.56719200 \\
\hline $\mathrm{H}$ & -2.18113900 & -4.65419800 & 3.36251500 \\
\hline $\mathrm{H}$ & -1.66614800 & -2.99600000 & 3.68095100 \\
\hline $\mathrm{H}$ & -4.94259000 & -2.02784000 & 5.42452200 \\
\hline $\mathrm{H}$ & -3.36283200 & -1.38165000 & 4.96390900 \\
\hline $\mathrm{H}$ & -3.13257400 & -3.78524300 & 5.53412400 \\
\hline $\mathrm{H}$ & -4.40369900 & -4.21478200 & 4.38257900 \\
\hline $\mathrm{C}$ & -6.69050400 & -2.45852400 & -1.84325600 \\
\hline $\mathrm{C}$ & -8.00897300 & -2.65517000 & -1.06236300 \\
\hline $\mathrm{C}$ & -6.10777100 & -3.82626600 & -2.26224500 \\
\hline $\mathrm{H}$ & -6.94643800 & -1.91994900 & -2.77446200 \\
\hline $\mathrm{C}$ & -9.02299600 & -3.49675200 & -1.84875700 \\
\hline $\mathrm{H}$ & -7.78913200 & -3.15389200 & -0.10109300 \\
\hline $\mathrm{H}$ & -8.43871400 & -1.67178200 & -0.80844100 \\
\hline
\end{tabular}

$\begin{array}{lrrr}\text { C } & -7.12309700 & -4.66624800 & -3.04854000 \\ \text { H } & -5.79816900 & -4.38115700 & -1.35835200 \\ \text { H } & -5.19219000 & -3.67151500 & -2.85704600 \\ \text { C } & -8.43306500 & -4.84798700 & -2.27142900 \\ \text { H } & -9.93630300 & -3.64665400 & -1.24824300 \\ \text { H } & -9.33491000 & -2.93897200 & -2.75140600 \\ \text { H } & -6.68679100 & -5.64745600 & -3.30158900 \\ \text { H } & -7.33932400 & -4.16659500 & -4.01127200 \\ \text { H } & -9.16275900 & -5.41361300 & -2.87536000 \\ \text { H } & -8.23845300 & -5.45736500 & -1.36911600 \\ \text { C } & -3.99352500 & 1.80254500 & -1.58303300 \\ \text { C } & -5.20152900 & 2.76302400 & -1.67471000 \\ \text { C } & -3.31868000 & 1.65100200 & -2.96508100 \\ \text { H } & -3.25781000 & 2.29738800 & -0.93067400 \\ \text { C } & -4.80535000 & 4.12126100 & -2.26799900 \\ \text { H } & -5.98708700 & 2.30552100 & -2.30281600 \\ \text { H } & -5.64604400 & 2.89598400 & -0.67402100 \\ \text { C } & -2.92540200 & 3.01184800 & -3.55534800 \\ \text { H } & -4.01128600 & 1.14148100 & -3.65933400 \\ \text { H } & -2.43374500 & 1.00200400 & -2.86927800 \\ \text { C } & -4.12350400 & 3.96692900 & -3.63329300 \\ \text { H } & -5.69335600 & 4.77074100 & -2.35266600 \\ \text { H } & -4.11356500 & 4.63271400 & -1.57291900 \\ \text { H } & -2.47816600 & 2.87490200 & -4.55466000 \\ \text { H } & -2.13864700 & 3.46629700 & -2.92511400 \\ \text { H } & -3.80671200 & 4.95141400 & -4.01776400 \\ \text { H } & -4.85666500 & 3.57106200 & -4.36092300 \\ \text { O } & 0.47368800 & -0.96975600 & -1.75132500 \\ \text { H } & 0.66743000 & -1.90042400 & -1.94265600\end{array}$

$1 a$

C

C

C

$\mathrm{H}$

$\mathrm{H}$

$\mathrm{H}$

$\mathrm{H}$

C

$\mathrm{O}$

C

O

C

$\mathrm{H}$

H

H

C

$\mathrm{H}$

$\mathrm{H}$

$\mathrm{C}$
$\mathrm{C}$

C

C

C

$\mathrm{H}$

C

$\mathrm{H}$

$-1.67489400$

$-1.35405000$

$-1.44272600$

$-1.05268000$

$-2.72541800$

$-0.32460900$

$-2.02028200$

$-1.40520700$

$-1.41481400$

$-1.49110800$

$-1.41440200$

$-2.73356100$

$-2.74323400$

$-2.77893400$

$-3.63681600$

$-0.20209600$

$-0.29822100$

$-0.27518800$

1.14166900

1.76611800

1.78973100

3.00036200

1.27741700

3.02360900

1.32034700

3.63311400

3.47202100
$-0.78343700$

0.71505400

0.02376100

$-1.41477100$

$-0.98987100$

0.89960600

1.31922500

1.21261600

2.37154700

$-1.21119800$

$-2.35240200$

0.08722600

1.01811200

$-0.77709400$

0.07439700

0.00549200

$-0.88727700$

0.89100300

$-0.00560900$

$-1.21712000$

1.19733900

$-1.22577400$

$-2.16150500$

1.19001900

2.14881400

$-0.02217300$

$-2.17879300$
1.79175500

1.83954600

$-0.58043300$

2.44258800

2.06326100

2.19352700

2.47277900

0.39388600

0.05815900

0.33292000

$-0.05104600$

$-1.42375800$

$-2.01025100$

$-2.10311400$

$-0.79293200$

$-1.51619200$

$-2.15380100$

$-2.16686900$

$-0.81541300$

$-0.47164800$

$-0.48496800$

0.18486300

$-0.72408400$

0.17230300

$-0.74834300$

0.50986100

0.43873100 


\begin{tabular}{|c|c|c|c|}
\hline $\mathrm{H}$ & 3.51336800 & 2.13655600 & 0.41593900 \\
\hline $\mathrm{H}$ & 4.60018600 & -0.02874300 & 1.01933800 \\
\hline \multicolumn{4}{|l|}{$2 a$} \\
\hline $\mathrm{C}$ & 2.84035200 & -2.17464700 & -0.60378200 \\
\hline $\mathrm{C}$ & 1.65683900 & -1.59925800 & -0.18945400 \\
\hline $\mathrm{C}$ & 1.57343700 & -0.20037900 & 0.05895300 \\
\hline $\mathrm{C}$ & 2.73842900 & 0.61151000 & -0.15506700 \\
\hline $\mathrm{C}$ & 3.94395500 & -0.01703200 & -0.57839600 \\
\hline $\mathrm{C}$ & 3.99787100 & -1.37798500 & -0.79254100 \\
\hline $\mathrm{H}$ & 2.88600400 & -3.24930900 & -0.79779800 \\
\hline $\mathrm{H}$ & 0.75597000 & -2.20519800 & -0.06998000 \\
\hline $\mathrm{C}$ & 0.36333500 & 0.42818200 & 0.50502900 \\
\hline $\mathrm{C}$ & 2.65212000 & 2.01746500 & 0.04608300 \\
\hline $\mathrm{H}$ & 4.83071900 & 0.60313100 & -0.73575600 \\
\hline $\mathrm{H}$ & 4.93049700 & -1.84563100 & -1.11805900 \\
\hline $\mathrm{C}$ & 1.46705300 & 2.59932400 & 0.44397800 \\
\hline $\mathrm{C}$ & 0.32239600 & 1.80136600 & 0.68195800 \\
\hline $\mathrm{H}$ & 3.54109400 & 2.63035500 & -0.12508900 \\
\hline $\mathrm{H}$ & 1.40696200 & 3.68070500 & 0.59046900 \\
\hline $\mathrm{H}$ & -0.61059500 & 2.26773500 & 1.00720800 \\
\hline $\mathrm{N}$ & -0.76708000 & -0.38813900 & 0.81839400 \\
\hline $\mathrm{C}$ & -2.01273300 & -0.21609900 & 0.18816700 \\
\hline $\mathrm{C}$ & -3.20165700 & -0.67522000 & 0.78898400 \\
\hline $\mathrm{C}$ & -2.09113100 & 0.38502200 & -1.08626000 \\
\hline $\mathrm{C}$ & -4.42483400 & -0.52889000 & 0.13241400 \\
\hline $\mathrm{H}$ & -3.14213400 & -1.15087400 & 1.76678200 \\
\hline $\mathrm{C}$ & -3.32278100 & 0.52761000 & -1.72582200 \\
\hline $\mathrm{H}$ & -1.18282000 & 0.73327400 & -1.58024200 \\
\hline $\mathrm{C}$ & -4.50213600 & 0.07503200 & -1.12592800 \\
\hline $\mathrm{H}$ & -5.33365800 & -0.89334500 & 0.61980600 \\
\hline $\mathrm{H}$ & -3.35546500 & 0.99568100 & -2.71368100 \\
\hline $\mathrm{H}$ & -5.46339000 & 0.18810700 & -1.63243900 \\
\hline $\mathrm{N}$ & -0.75971100 & -0.99513700 & 2.08568600 \\
\hline $\mathrm{H}$ & -0.02836400 & -1.70597100 & 2.12064200 \\
\hline H & -0.55036700 & -0.30173800 & 2.80968500 \\
\hline
\end{tabular}

\section{int-0-R-R}

$\begin{array}{cccc}\mathrm{C} & 0.90475000 & -3.32016500 & -0.07716300 \\ \mathrm{C} & 2.25218000 & -3.66746700 & -0.08464500 \\ \mathrm{C} & 2.65660300 & -4.79863300 & -0.88228200 \\ \mathrm{C} & 1.64585200 & -5.60856500 & -1.49886900 \\ \mathrm{C} & 0.27990100 & -5.24976600 & -1.35496500 \\ \mathrm{C} & -0.11647600 & -4.10237200 & -0.69437300 \\ \mathrm{H} & 4.80737400 & -4.51402100 & -0.69877100 \\ \mathrm{C} & 4.01994800 & -5.13731700 & -1.12167500 \\ \mathrm{C} & 2.02992500 & -6.74261000 & -2.26956200 \\ \mathrm{H} & -0.47949800 & -5.88523300 & -1.81684200 \\ \mathrm{C} & 3.35845200 & -7.05297800 & -2.46219300 \\ \mathrm{C} & 4.36049200 & -6.23021200 & -1.89252900 \\ \mathrm{H} & 1.24518300 & -7.35576300 & -2.72087700 \\ \mathrm{H} & 3.64117700 & -7.92079000 & -3.06318600 \\ \mathrm{H} & 5.41453500 & -6.45994100 & -2.06775500 \\ \mathrm{C} & 4.42568000 & -0.72041100 & 1.07619000 \\ \mathrm{C} & 5.32925500 & -1.37227400 & 1.89335300 \\ \mathrm{C} & 5.19021500 & -2.74374600 & 2.23493900 \\ \mathrm{C} & 4.13000700 & -3.51209700 & 1.64968200 \\ \mathrm{C} & 3.25147300 & -2.87818900 & 0.69825400\end{array}$

$\begin{array}{llll}\text { C } & 3.37071400 & -1.50416200 & 0.51905800\end{array}$

$\begin{array}{llll}\mathrm{H} & 6.88935400 & -2.76950700 & 3.58388600\end{array}$

$\begin{array}{llll}\mathrm{H} & 6.16787900 & -0.81104000 & 2.31165700\end{array}$

$\begin{array}{llll}\text { C } & 6.07794100 & -3.36588600 & 3.15798100\end{array}$

$\begin{array}{llll}\mathrm{C} & 3.97652400 & -4.86561300 & 2.06663900\end{array}$

$\begin{array}{llll}\text { C } & 4.84088300 & -5.43410000 & 2.98013300\end{array}$

$\begin{array}{llll}\text { C } & 5.91261000 & -4.68432300 & 3.52306800\end{array}$

$\begin{array}{llll}\mathrm{H} & 3.15306700 & -5.45658200 & 1.66576300\end{array}$

$\begin{array}{llll}\mathrm{H} & 4.69384400 & -6.47177800 & 3.29006100\end{array}$

$\begin{array}{llll}\mathrm{H} & 6.59589000 & -5.14863900 & 4.23846000\end{array}$

$\begin{array}{llll}\text { O } & 2.46565700 & -0.84804100 & -0.30333900\end{array}$

$\begin{array}{llll}\mathrm{O} & 0.51038300 & -2.19059700 & 0.62077700\end{array}$

$\begin{array}{llll}\mathrm{P} & 0.90518900 & -0.68701000 & 0.11301900\end{array}$

$\begin{array}{llll}\text { O } & 0.09772800 & -0.14338500 & -1.00266700\end{array}$

$\begin{array}{llll}\text { C } & -1.56499400 & -3.71234800 & -0.64326600\end{array}$

$\begin{array}{llll}\text { C } & -2.30275600 & -3.85223600 & 0.55816900\end{array}$

$\begin{array}{llll}\text { C } & -2.21349200 & -3.25782000 & -1.82169300\end{array}$

$\begin{array}{llll}\text { C } & -3.67462400 & -3.56230600 & 0.54626800\end{array}$

$\begin{array}{llll}\text { C } & -3.58752900 & -2.98354300 & -1.77390600\end{array}$

$\begin{array}{llll}\text { C } & -4.34298700 & -3.13517100 & -0.60560100\end{array}$

$\begin{array}{llll}\mathrm{H} & -4.25044000 & -3.68368400 & 1.46819000\end{array}$

$\begin{array}{llll}\mathrm{H} & -4.08214500 & -2.63928000 & -2.68540300\end{array}$

$\begin{array}{llll}\text { C } & 4.58389500 & 0.73885300 & 0.75876800\end{array}$

$\begin{array}{llll}\mathrm{C} & 5.00208100 & 1.13891200 & -0.53762200\end{array}$

$\begin{array}{llll}\text { C } & 4.37285600 & 1.71844100 & 1.76273500\end{array}$

$\begin{array}{llll}\text { C } & 5.20289900 & 2.50095700 & -0.79596500\end{array}$

$\begin{array}{llll}\text { C } & 4.60157900 & 3.06681900 & 1.44846100\end{array}$

$\begin{array}{llll}\text { C } & 5.01485000 & 3.48600900 & 0.18073500\end{array}$

$\begin{array}{llll}\mathrm{H} & 5.52664600 & 2.79959400 & -1.79602600\end{array}$

$\begin{array}{llll}\mathrm{H} & 4.45002100 & 3.82487700 & 2.22163700\end{array}$

$\begin{array}{llll}\text { C } & 5.27507800 & 0.13894800 & -1.65596700\end{array}$

$\begin{array}{llll}\text { C } & 6.75552400 & 0.14402200 & -2.10163100\end{array}$

$\begin{array}{llll}\text { C } & 4.34507400 & 0.33371500 & -2.87522700\end{array}$

$\mathrm{H} \quad 5.07711700 \quad-0.87247300 \quad-1.27002200$

$\begin{array}{llll}\text { C } & 7.02462600 & -0.89747100 & -3.19596000\end{array}$

$\begin{array}{llll}\mathrm{H} & 7.02256400 & 1.14697100 & -2.48048300\end{array}$

$\mathrm{H} \quad 7.40613300 \quad-0.03865100 \quad-1.22972400$

$\begin{array}{llll}\text { C } & 4.61819900 & -0.70962600 & -3.96662500\end{array}$

$\begin{array}{llll}\mathrm{H} & 4.49687300 & 1.34497500 & -3.29445200\end{array}$

$\begin{array}{llll}\mathrm{H} & 3.29585300 & 0.28080300 & -2.54647700\end{array}$

$\begin{array}{llll}\text { C } & 6.09003900 & -0.71238300 & -4.39825700\end{array}$

$\mathrm{H} \quad 8.07937000 \quad-0.84559900 \quad-3.51607300$

$\begin{array}{llll}\mathrm{H} & 6.87823600 & -1.91019000 & -2.77620200\end{array}$

$\begin{array}{llll}\mathrm{H} & 3.96366100 & -0.52601300 & -4.83567400\end{array}$

$\mathrm{H} \quad 4.34888000 \quad-1.71179900 \quad-3.58483800$

$\mathrm{H} \quad 6.27002100 \quad-1.50082500 \quad-5.14887700$

$\begin{array}{llll}\mathrm{H} & 6.32459200 & 0.24791800 & -4.89494000\end{array}$

$\begin{array}{llll}\text { C } & 5.25010600 & 4.95816500 & -0.11407500\end{array}$

$\begin{array}{llll}\text { C } & 4.31077200 & 5.50624100 & -1.21096600\end{array}$

$\begin{array}{llll}\text { C } & 6.72250500 & 5.26711400 & -0.46644500\end{array}$

$\begin{array}{llll}\mathrm{H} & 5.01906600 & 5.51295600 & 0.81427200\end{array}$

$\begin{array}{llll}\text { C } & 4.53966400 & 7.00132600 & -1.46689400\end{array}$

$\begin{array}{llll}\mathrm{H} & 4.48210900 & 4.94673900 & -2.14839300\end{array}$

$\begin{array}{llll}\mathrm{H} & 3.26054700 & 5.32056300 & -0.93229400\end{array}$

$\begin{array}{llll}\text { C } & 6.95111800 & 6.76181300 & -0.72780700\end{array}$

$\begin{array}{llll}\mathrm{C} & 7.00711500 & 4.69258000 & -1.36662300\end{array}$

$\begin{array}{llll}\mathrm{H} & 7.37942300 & 4.91250900 & 0.34540200\end{array}$

$\begin{array}{llll}\text { C } & 6.00472300 & 7.30064800 & -1.80803500\end{array}$ 


\begin{tabular}{|c|c|c|c|c|c|c|c|}
\hline $\mathrm{H}$ & 3.87491200 & 7.35091400 & -2.27442700 & $\mathrm{C}$ & -2.19847700 & -5.72146100 & 2.29235900 \\
\hline $\mathrm{H}$ & 4.25392100 & 7.57236900 & -0.56353500 & $\mathrm{C}$ & -1.80892700 & -3.31933400 & 3.00900900 \\
\hline $\mathrm{H}$ & 8.00205900 & 6.93847400 & -1.01356900 & $\mathrm{H}$ & -0.58697800 & -4.46339900 & 1.68105400 \\
\hline $\mathrm{H}$ & 6.78798000 & 7.32391600 & 0.21073200 & $\mathrm{C}$ & -1.51770200 & -6.21421600 & 3.57666800 \\
\hline $\mathrm{H}$ & 6.15476800 & 8.38516300 & -1.94394000 & $\mathrm{H}$ & -3.28946800 & -5.65918800 & 2.45755500 \\
\hline $\mathrm{H}$ & 6.25437000 & 6.82909100 & -2.77687400 & $\mathrm{H}$ & -2.05145700 & -6.44931200 & 1.47676600 \\
\hline $\mathrm{C}$ & 3.91173100 & 1.36306500 & 3.17298000 & $\mathrm{C}$ & -1.12181300 & -3.81464000 & 4.28827600 \\
\hline $\mathrm{C}$ & 2.66786600 & 2.15898600 & 3.62714900 & $\mathrm{H}$ & -2.88120400 & -3.15136800 & 3.21865500 \\
\hline $\mathrm{C}$ & 5.03895800 & 1.51903800 & 4.22036800 & $\mathrm{H}$ & -1.39496300 & -2.34718300 & 2.69998800 \\
\hline $\mathrm{H}$ & 3.61447900 & 0.30333600 & 3.17310700 & $\mathrm{C}$ & -1.64222800 & -5.19301400 & 4.71453100 \\
\hline $\mathrm{C}$ & 2.19995800 & 1.72462200 & 5.02250000 & $\mathrm{H}$ & -1.94403600 & -7.18500700 & 3.88227300 \\
\hline $\mathrm{H}$ & 2.90086900 & 3.23864000 & 3.64889900 & $\mathrm{H}$ & -0.44668700 & -6.39775800 & 3.37107700 \\
\hline $\mathrm{H}$ & 1.86489000 & 2.01830800 & 2.88803100 & $\mathrm{H}$ & -1.26366300 & -3.08257600 & 5.10170300 \\
\hline $\mathrm{C}$ & 4.57987900 & 1.09680600 & 5.62232700 & $\mathrm{H}$ & -0.03167000 & -3.87395000 & 4.11373300 \\
\hline $\mathrm{H}$ & 5.36858200 & 2.57402300 & 4.24040600 & $\mathrm{H}$ & -1.10399000 & -5.54867500 & 5.60960800 \\
\hline $\mathrm{H}$ & 5.92078400 & 0.93147100 & 3.91636400 & $\mathrm{H}$ & -2.70561500 & -5.10448300 & 5.00644400 \\
\hline $\mathrm{C}$ & 3.32071400 & 1.85455200 & 6.06166900 & $\mathrm{O}$ & 0.94259900 & 0.11071000 & 1.47908800 \\
\hline $\mathrm{H}$ & 1.32357500 & 2.32075400 & 5.33177100 & $\mathrm{H}$ & 0.04726500 & 0.33000400 & 1.87792700 \\
\hline $\mathrm{H}$ & 1.85932700 & 0.67382700 & 4.97951200 & $\mathrm{C}$ & -5.66256000 & 3.12757800 & -1.58570900 \\
\hline $\mathrm{H}$ & 5.39430500 & 1.25188500 & 6.35048900 & $\mathrm{C}$ & -4.31663100 & 3.27026200 & -1.85453800 \\
\hline $\mathrm{H}$ & 4.36779300 & 0.01171300 & 5.62050600 & $\mathrm{C}$ & -3.77780400 & 2.90843100 & -3.12114200 \\
\hline $\mathrm{H}$ & 2.97824900 & 1.49246600 & 7.04599800 & $\mathrm{C}$ & -4.66655100 & 2.40749200 & -4.13138600 \\
\hline $\mathrm{H}$ & 3.56934800 & 2.92434900 & 6.19315700 & $\mathrm{C}$ & -6.05021200 & 2.27382000 & -3.82272000 \\
\hline $\mathrm{C}$ & -1.47363100 & -3.05401200 & -3.13992300 & $\mathrm{C}$ & -6.53795300 & 2.62149800 & -2.57973400 \\
\hline $\mathrm{C}$ & -1.58219600 & -1.60744200 & -3.67293600 & $\mathrm{H}$ & -6.04567200 & 3.41287000 & -0.60303100 \\
\hline $\mathrm{C}$ & -1.91821500 & -4.06112800 & -4.22534900 & $\mathrm{H}$ & -3.65248400 & 3.68289400 & -1.09378100 \\
\hline $\mathrm{H}$ & -0.40283000 & -3.23351900 & -2.96018300 & $\mathrm{C}$ & -2.38047700 & 3.03126500 & -3.42662900 \\
\hline $\mathrm{C}$ & -0.79581600 & -1.42904000 & -4.97834100 & $\mathrm{C}$ & -4.14102900 & 2.06948100 & -5.41037900 \\
\hline $\mathrm{H}$ & -2.64210200 & -1.35478800 & -3.85625400 & $\mathrm{H}$ & -6.72451100 & 1.89228000 & -4.59454800 \\
\hline $\mathrm{H}$ & -1.21196500 & -0.91282500 & -2.90429300 & $\mathrm{H}$ & -7.60348300 & 2.51626400 & -2.36007000 \\
\hline $\mathrm{C}$ & -1.13719100 & -3.87640100 & -5.53320400 & $\mathrm{C}$ & -2.79766400 & 2.21740300 & -5.68274400 \\
\hline $\mathrm{H}$ & -2.99810500 & -3.93020900 & -4.42150700 & $\mathrm{C}$ & -1.91373600 & 2.69552500 & -4.68399600 \\
\hline $\mathrm{H}$ & -1.79959900 & -5.09278600 & -3.85292500 & $\mathrm{H}$ & -4.82323300 & 1.69290900 & -6.17740900 \\
\hline $\mathrm{C}$ & -1.23009300 & -2.43566600 & -6.05073000 & $\mathrm{H}$ & -2.40471300 & 1.96024200 & -6.66940900 \\
\hline $\mathrm{H}$ & -0.91819100 & -0.39738100 & -5.34860900 & $\mathrm{H}$ & -0.84755400 & 2.80154500 & -4.89567500 \\
\hline $\mathrm{H}$ & 0.28303600 & -1.55812500 & -4.77259900 & $\mathrm{~N}$ & -1.47447800 & 3.46126600 & -2.40898300 \\
\hline $\mathrm{H}$ & -1.50383800 & -4.58472700 & -6.29600600 & $\mathrm{C}$ & -1.15521000 & 4.80488800 & -2.20254600 \\
\hline $\mathrm{H}$ & -0.07529100 & -4.13094500 & -5.35873600 & $\mathrm{C}$ & -1.71557800 & 5.81514000 & -3.01652400 \\
\hline $\mathrm{H}$ & -0.62100100 & -2.31673200 & -6.96323200 & $\mathrm{C}$ & -0.24549900 & 5.19114800 & -1.19314300 \\
\hline $\mathrm{H}$ & -2.27535200 & -2.22346900 & -6.34446800 & $\mathrm{C}$ & -1.38137000 & 7.15439800 & -2.81544300 \\
\hline $\mathrm{C}$ & -5.83876500 & -2.86612300 & -0.58010700 & $\mathrm{H}$ & -2.41919500 & 5.54787100 & -3.80569000 \\
\hline $\mathrm{C}$ & -6.63444100 & -3.89004100 & -1.42139300 & $\mathrm{C}$ & 0.07235300 & 6.53759900 & -1.00411900 \\
\hline $\mathrm{C}$ & -6.20455500 & -1.42774100 & -1.00510600 & $\mathrm{H}$ & 0.21402300 & 4.42150500 & -0.57485500 \\
\hline $\mathrm{H}$ & -6.16972300 & -2.98698200 & 0.46804800 & $\mathrm{C}$ & -0.48842700 & 7.53440100 & -1.80860600 \\
\hline $\mathrm{C}$ & -8.14645000 & -3.63681500 & -1.35608000 & $\mathrm{H}$ & -1.83483300 & 7.91364400 & -3.45890400 \\
\hline $\mathrm{H}$ & -6.29776000 & -3.83096200 & -2.47240600 & $\mathrm{H}$ & 0.78098000 & 6.80718700 & -0.21551100 \\
\hline $\mathrm{H}$ & -6.39923700 & -4.91172600 & -1.07943200 & $\mathrm{H}$ & -0.23287000 & 8.58539300 & -1.65637600 \\
\hline $\mathrm{C}$ & -7.71628800 & -1.17574400 & -0.93660200 & $\mathrm{~N}$ & -1.09043800 & 2.51360300 & -1.44117800 \\
\hline $\mathrm{H}$ & -5.85501800 & -1.24996200 & -2.03764400 & $\mathrm{H}$ & -1.90017700 & 1.94354100 & -1.18724500 \\
\hline $\mathrm{H}$ & -5.66544700 & -0.70443600 & -0.37146400 & $\mathrm{H}$ & -0.40049000 & 1.84973900 & -1.80527700 \\
\hline C & -8.49988500 & -2.20130100 & -1.76525500 & $\mathrm{C}$ & -2.29997900 & 4.15604200 & 2.04027900 \\
\hline $\mathrm{H}$ & -8.67932700 & -4.36150400 & -1.99496500 & $\mathrm{C}$ & -1.25570900 & 3.03519800 & 1.93444300 \\
\hline $\mathrm{H}$ & -8.49996900 & -3.81670700 & -0.32363500 & $\mathrm{C}$ & -3.40167700 & 2.03208600 & 2.76226100 \\
\hline $\mathrm{H}$ & -7.94110800 & -0.15149700 & -1.27834900 & $\mathrm{H}$ & -2.16001400 & 4.76661800 & 2.94975900 \\
\hline $\mathrm{H}$ & -8.04768400 & -1.23108100 & 0.11720500 & $\mathrm{H}$ & -2.30890200 & 4.84827900 & 1.18669800 \\
\hline $\mathrm{H}$ & -9.58507900 & -2.02945800 & -1.66466100 & $\mathrm{H}$ & -0.32613500 & 3.20168000 & 2.50053500 \\
\hline $\mathrm{H}$ & -8.26090400 & -2.06048000 & -2.83594800 & $\mathrm{H}$ & -0.95804400 & 2.86305000 & 0.88005300 \\
\hline $\mathrm{C}$ & -1.66635900 & -4.33715900 & 1.85517600 & $\mathrm{C}$ & -1.94342300 & 1.77088800 & 2.40419200 \\
\hline
\end{tabular}




$\begin{array}{cccc}\mathrm{O} & -1.41920900 & 0.67111600 & 2.47952100 \\ \mathrm{C} & -3.63969500 & 3.42909300 & 2.16057000 \\ \mathrm{O} & -4.70923200 & 3.85479500 & 1.80162600 \\ \mathrm{C} & -4.35467000 & 0.97102200 & 2.21371500 \\ \mathrm{H} & -4.11389000 & -0.01722800 & 2.63157400 \\ \mathrm{H} & -5.39141200 & 1.23309200 & 2.46380400 \\ \mathrm{H} & -4.27789500 & 0.90487300 & 1.11806300 \\ \mathrm{C} & -3.44046500 & 2.11309700 & 4.34066400 \\ \mathrm{H} & -3.07052000 & 1.13983300 & 4.69979000 \\ \mathrm{H} & -2.71294900 & 2.86970900 & 4.67889100 \\ \mathrm{C} & -4.79413200 & 2.40705000 & 4.94579100 \\ \mathrm{C} & -5.63006800 & 1.35923400 & 5.36581000 \\ \mathrm{C} & -5.24360700 & 3.72680900 & 5.11462300 \\ \mathrm{C} & -6.88056600 & 1.62117300 & 5.93199400 \\ \mathrm{H} & -5.29263500 & 0.32486400 & 5.25629700 \\ \mathrm{C} & -6.49277100 & 3.99247600 & 5.67998900 \\ \mathrm{H} & -4.60840300 & 4.56099700 & 4.80504500 \\ \mathrm{C} & -7.31665400 & 2.93970300 & 6.08905500 \\ \mathrm{H} & -7.51394900 & 0.79142000 & 6.25615400 \\ \mathrm{H} & -6.82278600 & 5.02703000 & 5.80329200 \\ \mathrm{H} & -8.29343700 & 3.14688100 & 6.53339000\end{array}$

\section{ts-1-R-R}

$\begin{array}{cccc}\mathrm{C} & 2.10693800 & -2.98752500 & 0.16766100 \\ \mathrm{C} & 3.44158300 & -2.71961300 & -0.12397700 \\ \mathrm{C} & 4.17339200 & -3.67884300 & -0.91310700 \\ \mathrm{C} & 3.56478700 & -4.94274900 & -1.21071000 \\ \mathrm{C} & 2.24231700 & -5.19910100 & -0.76312700 \\ \mathrm{C} & 1.48393400 & -4.24130600 & -0.11766300 \\ \mathrm{H} & 5.93793400 & -2.45404500 & -1.26446900 \\ \mathrm{C} & 5.47076400 & -3.42237900 & -1.44374300 \\ \mathrm{C} & 4.28777600 & -5.90842500 & -1.96700500 \\ \mathrm{H} & 1.79952300 & -6.17667500 & -0.96957100 \\ \mathrm{C} & 5.54978100 & -5.63377000 & -2.44745800 \\ \mathrm{C} & 6.13748600 & -4.37056900 & -2.19294700 \\ \mathrm{H} & 3.81298400 & -6.87174600 & -2.17243900 \\ \mathrm{H} & 6.09174400 & -6.37981000 & -3.03397100 \\ \mathrm{H} & 7.12719500 & -4.14223500 & -2.59659900 \\ \mathrm{C} & 4.20501700 & 1.00024800 & 0.43449900 \\ \mathrm{C} & 5.42871000 & 0.91872400 & 1.07047500 \\ \mathrm{C} & 5.98931800 & -0.31881600 & 1.48151300 \\ \mathrm{C} & 5.30961700 & -1.53881700 & 1.15769700 \\ \mathrm{C} & 4.09555700 & -1.47670700 & 0.38157700 \\ \mathrm{C} & 3.54197500 & -0.22663200 & 0.12199200 \\ \mathrm{H} & 7.71344700 & 0.57273600 & 2.45127200 \\ \mathrm{H} & 5.97450700 & 1.83859300 & 1.29241500 \\ \mathrm{C} & 7.20170000 & -0.36702600 & 2.22640700 \\ \mathrm{C} & 5.85539300 & -2.75751900 & 1.65513600 \\ \mathrm{C} & 7.02062900 & -2.77014800 & 2.39468900 \\ \mathrm{C} & 7.71272500 & -1.56633100 & 2.67280600 \\ \mathrm{H} & 5.33459100 & -3.69444400 & 1.45894300 \\ \mathrm{H} & 7.41012800 & -3.71881100 & 2.77267000 \\ \mathrm{H} & 8.63959600 & -1.59053300 & 3.25126400 \\ \mathrm{O} & 2.32639000 & -0.15224200 & -0.53405500 \\ \mathrm{O} & 1.36044100 & -2.05027000 & 0.84900400 \\ \mathrm{P} & 0.93583900 & -0.59106300 & 0.22482300 \\ \mathrm{O} & -0.16650500 & -0.64459200 & -0.79342800 \\ \mathrm{C} & 0.06330200 & -4.52108800 & 0.27206300\end{array}$

\begin{tabular}{|c|c|c|c|}
\hline $\mathrm{C}$ & -0.28442100 & -4.68563500 & 1.63622600 \\
\hline $\mathrm{C}$ & -0.92941500 & -4.66646900 & -0.73240600 \\
\hline $\mathrm{C}$ & -1.61289000 & -4.99194700 & 1.96206300 \\
\hline $\mathrm{C}$ & -2.24263600 & -4.97159800 & -0.34632200 \\
\hline $\mathrm{C}$ & -2.61033600 & -5.14230100 & 0.99367100 \\
\hline $\mathrm{H}$ & -1.88264000 & -5.12894400 & 3.01296800 \\
\hline $\mathrm{H}$ & -3.00271900 & -5.08241900 & -1.12388700 \\
\hline $\mathrm{C}$ & 3.62914600 & 2.33481300 & 0.06076700 \\
\hline $\mathrm{C}$ & 3.61727500 & 2.74159100 & -1.29865400 \\
\hline $\mathrm{C}$ & 3.15902900 & 3.21915100 & 1.06563300 \\
\hline $\mathrm{C}$ & 3.13217500 & 4.01532600 & -1.62353200 \\
\hline $\mathrm{C}$ & 2.68742900 & 4.48434500 & 0.68073500 \\
\hline $\mathrm{C}$ & 2.66335100 & 4.90812600 & -0.65216500 \\
\hline $\mathrm{H}$ & 3.13792300 & 4.32468100 & -2.67151300 \\
\hline $\mathrm{H}$ & 2.33523900 & 5.17508700 & 1.45215500 \\
\hline $\mathrm{C}$ & 4.16740300 & 1.86052100 & -2.41493400 \\
\hline $\mathrm{C}$ & 5.41865300 & 2.47704900 & -3.08269200 \\
\hline $\mathrm{C}$ & 3.10938900 & 1.50051100 & -3.48119300 \\
\hline $\mathrm{H}$ & 4.49567300 & 0.90964100 & -1.96942100 \\
\hline $\mathrm{C}$ & 5.99248100 & 1.56168100 & -4.17247800 \\
\hline $\mathrm{H}$ & 5.15500000 & 3.45322700 & -3.52854900 \\
\hline $\mathrm{H}$ & 6.18274700 & 2.68536200 & -2.31501400 \\
\hline $\mathrm{C}$ & 3.68847500 & 0.58741000 & -4.57030600 \\
\hline $\mathrm{H}$ & 2.72863900 & 2.42702300 & -3.94895400 \\
\hline $\mathrm{H}$ & 2.25352500 & 1.01285600 & -2.98916100 \\
\hline $\mathrm{C}$ & 4.93545100 & 1.19735100 & -5.22242500 \\
\hline $\mathrm{H}$ & 6.86147600 & 2.04385800 & -4.65199700 \\
\hline $\mathrm{H}$ & 6.37241700 & 0.63478500 & -3.70414500 \\
\hline $\mathrm{H}$ & 2.92022700 & 0.37600100 & -5.33356900 \\
\hline $\mathrm{H}$ & 3.95183700 & -0.38762400 & -4.12079700 \\
\hline $\mathrm{H}$ & 5.35853300 & 0.50302900 & -5.96818900 \\
\hline $\mathrm{H}$ & 4.64639000 & 2.10996000 & -5.77703400 \\
\hline $\mathrm{C}$ & 2.19086000 & 6.30760500 & -1.01279400 \\
\hline $\mathrm{C}$ & 1.09644000 & 6.33096600 & -2.10086200 \\
\hline $\mathrm{C}$ & 3.36514900 & 7.22767500 & -1.42059400 \\
\hline $\mathrm{H}$ & 1.74712300 & 6.74352000 & -0.09819600 \\
\hline $\mathrm{C}$ & 0.62565900 & 7.75834700 & -2.41027200 \\
\hline $\mathrm{H}$ & 1.48969800 & 5.87380000 & -3.02638900 \\
\hline $\mathrm{H}$ & 0.24518800 & 5.70344600 & -1.78915100 \\
\hline $\mathrm{C}$ & 2.89765700 & 8.65429800 & -1.73723700 \\
\hline $\mathrm{H}$ & 3.86404800 & 6.79782200 & -2.30836800 \\
\hline $\mathrm{H}$ & 4.12103600 & 7.23888000 & -0.61788200 \\
\hline $\mathrm{C}$ & 1.79615700 & 8.66794100 & -2.80442600 \\
\hline $\mathrm{H}$ & -0.13394000 & 7.74045200 & -3.20998800 \\
\hline $\mathrm{H}$ & 0.12464400 & 8.17820400 & -1.51792900 \\
\hline $\mathrm{H}$ & 3.75442800 & 9.26945600 & -2.06073600 \\
\hline $\mathrm{H}$ & 2.51256500 & 9.12330400 & -0.81256400 \\
\hline $\mathrm{H}$ & 1.44110100 & 9.69799300 & -2.97727900 \\
\hline $\mathrm{H}$ & 2.21773100 & 8.31948800 & -3.76575700 \\
\hline $\mathrm{C}$ & 3.18397100 & 2.86336000 & 2.54989700 \\
\hline $\mathrm{C}$ & 1.82282600 & 3.04247200 & 3.25724500 \\
\hline $\mathrm{C}$ & 4.27874200 & 3.64924400 & 3.31036100 \\
\hline $\mathrm{H}$ & 3.43179500 & 1.79484400 & 2.63510900 \\
\hline $\mathrm{C}$ & 1.89531600 & 2.63920300 & 4.73638900 \\
\hline $\mathrm{H}$ & 1.49924700 & 4.09743500 & 3.18968600 \\
\hline $\mathrm{H}$ & 1.06880100 & 2.43152600 & 2.74143900 \\
\hline $\mathrm{C}$ & 4.35028600 & 3.25172900 & 4.79052400 \\
\hline $\mathrm{H}$ & 4.06711900 & 4.73133900 & 3.22962800 \\
\hline
\end{tabular}




\begin{tabular}{|c|c|c|c|}
\hline $\mathrm{H}$ & 5.25773500 & 3.49513200 & 2.82729900 \\
\hline$C$ & 2.99194600 & 3.40586700 & 5.48506100 \\
\hline $\mathrm{H}$ & 0.91628600 & 2.80097200 & 5.21902000 \\
\hline $\mathrm{H}$ & 2.09603000 & 1.55441800 & 4.80240800 \\
\hline $\mathrm{H}$ & 5.11747800 & 3.85397100 & 5.30674000 \\
\hline $\mathrm{H}$ & 4.67910400 & 2.19885000 & 4.86593600 \\
\hline $\mathrm{H}$ & 3.05501400 & 3.06434400 & 6.53227400 \\
\hline $\mathrm{H}$ & 2.72431200 & 4.47863000 & 5.52388200 \\
\hline $\mathrm{C}$ & -0.62386900 & -4.49375000 & -2.21748400 \\
\hline $\mathrm{C}$ & -1.39295300 & -3.31393600 & -2.85087300 \\
\hline $\mathrm{C}$ & -0.85727600 & -5.78738500 & -3.02999400 \\
\hline $\mathrm{H}$ & 0.44284900 & -4.24740100 & -2.31877700 \\
\hline $\mathrm{C}$ & -1.03833000 & -3.13326400 & -4.33275800 \\
\hline $\mathrm{H}$ & -2.47943300 & -3.49693600 & -2.76038700 \\
\hline $\mathrm{H}$ & -1.16435700 & -2.39640700 & -2.28604900 \\
\hline $\mathrm{C}$ & -0.50401200 & -5.60263200 & -4.51202900 \\
\hline $\mathrm{H}$ & -1.91647800 & -6.09090900 & -2.94310100 \\
\hline $\mathrm{H}$ & -0.26791200 & -6.61317400 & -2.59634900 \\
\hline $\mathrm{C}$ & -1.26084700 & -4.42207700 & -5.13360500 \\
\hline $\mathrm{H}$ & -1.62633300 & -2.30491100 & -4.76303500 \\
\hline $\mathrm{H}$ & 0.02218700 & -2.83202100 & -4.41471600 \\
\hline $\mathrm{H}$ & -0.71436100 & -6.53139600 & -5.06960800 \\
\hline $\mathrm{H}$ & 0.58335100 & -5.42474200 & -4.60527300 \\
\hline $\mathrm{H}$ & -0.95520300 & -4.28117300 & -6.18435000 \\
\hline $\mathrm{H}$ & -2.34218800 & -4.65552600 & -5.15420900 \\
\hline $\mathrm{C}$ & -4.03704800 & -5.47443500 & 1.39982600 \\
\hline $\mathrm{C}$ & -4.55890900 & -6.78227200 & 0.76577100 \\
\hline $\mathrm{C}$ & -5.01756900 & -4.31414500 & 1.11863100 \\
\hline $\mathrm{H}$ & -4.03084800 & -5.62961500 & 2.49453600 \\
\hline $\mathrm{C}$ & -5.98677200 & -7.11091100 & 1.22139700 \\
\hline $\mathrm{H}$ & -4.54361300 & -6.68559400 & -0.33500300 \\
\hline $\mathrm{H}$ & -3.87474200 & -7.61144700 & 1.01159400 \\
\hline $\mathrm{C}$ & -6.44732200 & -4.64361600 & 1.56714100 \\
\hline $\mathrm{H}$ & -5.01586800 & -4.09244400 & 0.03623500 \\
\hline $\mathrm{H}$ & -4.65887200 & -3.39920000 & 1.61901200 \\
\hline $\mathrm{C}$ & -6.95153000 & -5.95124900 & 0.94362600 \\
\hline $\mathrm{H}$ & -6.33949800 & -8.03167100 & 0.72642400 \\
\hline $\mathrm{H}$ & -5.98146800 & -7.32611200 & 2.30620900 \\
\hline $\mathrm{H}$ & -7.12366600 & -3.80991900 & 1.31280400 \\
\hline $\mathrm{H}$ & -6.47028900 & -4.73550000 & 2.66904700 \\
\hline $\mathrm{H}$ & -7.96057900 & -6.19214200 & 1.31906400 \\
\hline $\mathrm{H}$ & -7.05126000 & -5.81719300 & -0.14995100 \\
\hline $\mathrm{C}$ & 0.74058000 & -4.58715800 & 2.76007700 \\
\hline $\mathrm{C}$ & 0.94179100 & -5.93809300 & 3.48545100 \\
\hline $\mathrm{C}$ & 0.41552000 & -3.47140800 & 3.77879000 \\
\hline $\mathrm{H}$ & 1.71357400 & -4.33045400 & 2.31472500 \\
\hline $\mathrm{C}$ & 2.00740600 & -5.84127100 & 4.58532500 \\
\hline $\mathrm{H}$ & -0.01549900 & -6.26100400 & 3.93325400 \\
\hline $\mathrm{H}$ & 1.21649100 & -6.71521000 & 2.75232900 \\
\hline $\mathrm{C}$ & 1.48311200 & -3.37997000 & 4.87722500 \\
\hline 11 & -0.56633000 & -3.67307500 & 4.24469500 \\
\hline $\mathrm{H}$ & 0.32796800 & -2.50937300 & 3.25145800 \\
\hline $\mathrm{C}$ & 1.68952500 & -4.72393400 & 5.58695800 \\
\hline $\mathrm{H}$ & 2.10269000 & -6.80977900 & 5.10564600 \\
\hline $\mathrm{H}$ & 2.99084900 & -5.64181200 & 4.12046600 \\
\hline 11 & 1.20731100 & -2.59898100 & 5.60616000 \\
\hline $\mathrm{H}$ & 2.43865900 & -3.05560800 & 4.42548000 \\
\hline $\mathrm{H}$ & 2.49276900 & -4.64342400 & 6.33920100 \\
\hline
\end{tabular}

\begin{tabular}{|c|c|c|c|}
\hline $\mathrm{H}$ & 0.76950900 & -4.98680500 & 6.14237800 \\
\hline $\mathrm{O}$ & 0.72519700 & 0.29185800 & 1.46265000 \\
\hline $\mathrm{H}$ & -0.42371600 & 0.70057700 & 1.67684200 \\
\hline $\mathrm{C}$ & -7.66797000 & 2.46480400 & -1.24092200 \\
\hline $\mathrm{C}$ & -6.34174300 & 2.08713800 & -1.29240600 \\
\hline $\mathrm{C}$ & -5.97681600 & 0.75100300 & -1.62621200 \\
\hline $\mathrm{C}$ & -7.02324100 & -0.19829100 & -1.90197400 \\
\hline $\mathrm{C}$ & -8.37944500 & 0.23129000 & -1.84173800 \\
\hline $\mathrm{C}$ & -8.69803800 & 1.53281000 & -1.52070800 \\
\hline $\mathrm{H}$ & -7.92296500 & 3.49385700 & -0.97615000 \\
\hline $\mathrm{H}$ & -5.56496800 & 2.81611300 & -1.06481200 \\
\hline $\mathrm{C}$ & -4.61818600 & 0.29882900 & -1.70428400 \\
\hline $\mathrm{C}$ & -6.68602700 & -1.54101700 & -2.22506600 \\
\hline $\mathrm{H}$ & -9.16722000 & -0.49601300 & -2.05601100 \\
\hline $\mathrm{H}$ & -9.74351100 & 1.84833200 & -1.47784700 \\
\hline $\mathrm{C}$ & -5.36913600 & -1.94401200 & -2.27938700 \\
\hline $\mathrm{C}$ & -4.33260500 & -1.01737000 & -2.02320000 \\
\hline $\mathrm{H}$ & -7.49187500 & -2.25018800 & -2.43201000 \\
\hline $\mathrm{H}$ & -5.11462900 & -2.97524600 & -2.53429600 \\
\hline $\mathrm{H}$ & -3.29298500 & -1.34414400 & -2.08554600 \\
\hline $\mathrm{N}$ & -3.52983800 & 1.22620100 & -1.45031400 \\
\hline $\mathrm{C}$ & -2.86525700 & 1.73496900 & -2.61458100 \\
\hline $\mathrm{C}$ & -3.61650900 & 2.50996000 & -3.51814200 \\
\hline $\mathrm{C}$ & -1.51306300 & 1.47991200 & -2.89580600 \\
\hline $\mathrm{C}$ & -3.02630300 & 3.01618000 & -4.67548400 \\
\hline $\mathrm{H}$ & -4.66573400 & 2.71752700 & -3.30861300 \\
\hline $\mathrm{C}$ & -0.92842600 & 2.01039000 & -4.05232000 \\
\hline $\mathrm{H}$ & -0.90221300 & 0.85420500 & -2.24309200 \\
\hline $\mathrm{C}$ & -1.67496400 & 2.77704600 & -4.94791300 \\
\hline $\mathrm{H}$ & -3.62884800 & 3.61469100 & -5.36356400 \\
\hline $\mathrm{H}$ & 0.12620700 & 1.80429700 & -4.24838900 \\
\hline $\mathrm{H}$ & -1.21163900 & 3.18291000 & -5.85023100 \\
\hline $\mathrm{N}$ & -2.68570800 & 0.84417400 & -0.35917900 \\
\hline $\mathrm{H}$ & -3.23946700 & 0.24193500 & 0.25123400 \\
\hline $\mathrm{H}$ & -1.84958300 & 0.28047200 & -0.62864600 \\
\hline $\mathrm{C}$ & -1.94983500 & 4.03150600 & -0.23272700 \\
\hline $\mathrm{C}$ & -1.02814200 & 2.93279700 & 0.32042200 \\
\hline $\mathrm{C}$ & -3.16308300 & 2.81493000 & 1.57912700 \\
\hline $\mathrm{H}$ & -1.57687000 & 5.04888200 & -0.03339100 \\
\hline $\mathrm{H}$ & -2.10620200 & 3.96544100 & -1.31927600 \\
\hline $\mathrm{H}$ & -0.28365600 & 3.33416400 & 1.02571500 \\
\hline $\mathrm{H}$ & -0.45659200 & 2.39781200 & -0.44591400 \\
\hline $\mathrm{C}$ & -1.94248800 & 2.00577100 & 1.11794900 \\
\hline $\mathrm{O}$ & -1.50419300 & 1.08169700 & 1.88446500 \\
\hline $\mathrm{C}$ & -3.30362000 & 3.84900400 & 0.45747100 \\
\hline $\mathrm{O}$ & -4.31954800 & 4.43201500 & 0.16380000 \\
\hline $\mathrm{C}$ & -4.42800000 & 2.02099200 & 1.88332200 \\
\hline $\mathrm{H}$ & -4.22400200 & 1.25895800 & 2.65139800 \\
\hline $\mathrm{H}$ & -5.21437800 & 2.69119300 & 2.25449600 \\
\hline $\mathrm{H}$ & -4.82985800 & 1.52856600 & 0.98948800 \\
\hline $\mathrm{C}$ & -2.66380200 & 3.56950000 & 2.88395700 \\
\hline $\mathrm{H}$ & -2.34243800 & 2.77847700 & 3.57763600 \\
\hline $\mathrm{H}$ & -1.76364700 & 4.15552700 & 2.64182200 \\
\hline $\mathrm{C}$ & -3.67439700 & 4.46911400 & 3.55910100 \\
\hline $\mathrm{C}$ & -4.43260400 & 3.99932400 & 4.64463500 \\
\hline $\mathrm{C}$ & -3.86606800 & 5.79655200 & 3.14141900 \\
\hline $\mathrm{C}$ & -5.35902600 & 4.82449700 & 5.28775300 \\
\hline $\mathrm{H}$ & -4.28785800 & 2.97392100 & 4.99603300 \\
\hline
\end{tabular}




$\begin{array}{llll}\mathrm{C} & -4.79127000 & 6.62473900 & 3.78115700 \\ \mathrm{H} & -3.28270800 & 6.19240300 & 2.30637300 \\ \mathrm{C} & -5.54303800 & 6.14092200 & 4.85590000 \\ \mathrm{H} & -5.93494600 & 4.43841900 & 6.13285100 \\ \mathrm{H} & -4.92330500 & 7.65452500 & 3.43938900 \\ \mathrm{H} & -6.26526100 & 6.78940800 & 5.35838700\end{array}$

int-1-R-R

\begin{tabular}{|c|c|c|c|c|c|c|c|}
\hline & \multirow{2}{*}{\multicolumn{2}{|c|}{$\mathrm{H} \quad 3.00238300$}} & \multirow{2}{*}{2.44587100} & \multirow{2}{*}{-3.91418200} \\
\hline $\mathrm{C}$ & 2.08893000 & -2.95538400 & 0.21925200 & & & & \\
\hline $\mathrm{C}$ & 3.43826200 & -2.69747000 & -0.01343400 & $\mathrm{H}$ & 2.48922200 & 1.01201300 & -3.00195400 \\
\hline $\mathrm{C}$ & 4.20125400 & -3.66797900 & -0.75904500 & $\mathrm{C}$ & 5.32140000 & 1.28088400 & -5.03211000 \\
\hline $\mathrm{C}$ & 3.60764000 & -4.93749400 & -1.06192100 & $\mathrm{H}$ & 7.18172800 & 2.16095200 & -4.31395000 \\
\hline $\mathrm{C}$ & 2.26459500 & -5.18376800 & -0.67516000 & $\mathrm{H}$ & 6.65542500 & 0.73064200 & -3.41884500 \\
\hline $\mathrm{C}$ & 1.47808700 & -4.21198100 & -0.08744100 & $\mathrm{H}$ & 3.33947800 & 0.41494900 & -5.29846500 \\
\hline $\mathrm{H}$ & 5.97717200 & -2.44637000 & -1.05807400 & $\mathrm{H}$ & 4.29812800 & -0.33873900 & -4.02101000 \\
\hline $\mathrm{C}$ & 5.51975500 & -3.41891300 & -1.23930200 & $\mathrm{H}$ & 5.81291500 & 0.60569600 & -5.75306200 \\
\hline $\mathrm{C}$ & 4.36379700 & -5.91606800 & -1.76735900 & $\mathrm{H}$ & 5.05200000 & 2.19389800 & -5.59590100 \\
\hline $\mathrm{H}$ & 1.82882800 & -6.16297100 & -0.88987600 & $\mathrm{C}$ & 2.15048600 & 6.28043200 & -0.98662600 \\
\hline $\mathrm{C}$ & 5.64569600 & -5.64869200 & -2.19659600 & $\mathrm{C}$ & 1.00776600 & 6.23412400 & -2.02373800 \\
\hline $\mathrm{C}$ & 6.21979100 & -4.37951100 & -1.94087200 & $\mathrm{C}$ & 3.28143200 & 7.21197600 & -1.47865400 \\
\hline $\mathrm{H}$ & 3.89879800 & -6.88352400 & -1.97617400 & $\mathrm{H}$ & 1.73490000 & 6.73875300 & -0.06997100 \\
\hline $\mathrm{H}$ & 6.21380600 & -6.40469600 & -2.74441800 & $\mathrm{C}$ & 0.48954800 & 7.63533100 & -2.37384400 \\
\hline $\mathrm{H}$ & 7.22571700 & -4.15636200 & -2.30553800 & $\mathrm{H}$ & 1.36967400 & 5.74122400 & -2.94383100 \\
\hline $\mathrm{C}$ & 4.21170700 & 1.01853000 & 0.55255800 & $\mathrm{H}$ & 0.18663100 & 5.60366500 & -1.64394800 \\
\hline $\mathrm{C}$ & 5.41313800 & 0.93139700 & 1.22835400 & $\mathrm{C}$ & 2.76249500 & 8.61214900 & -1.83105600 \\
\hline $\mathrm{C}$ & 5.94899100 & -0.30839900 & 1.66491300 & $\mathrm{H}$ & 3.75599700 & 6.76214000 & -2.36968800 \\
\hline $\mathrm{C}$ & 5.26792000 & -1.52381700 & 1.32753200 & $\mathrm{H}$ & 4.06876500 & 7.27501000 & -0.70924200 \\
\hline $\mathrm{C}$ & 4.08183500 & -1.45642300 & 0.50875300 & $\mathrm{C}$ & 1.62007100 & 8.55466100 & -2.85278500 \\
\hline $\mathrm{C}$ & 3.54768900 & -0.20378500 & 0.22171500 & $\mathrm{H}$ & -0.30092000 & 7.56477500 & -3.14026700 \\
\hline $\mathrm{H}$ & 7.64931700 & 0.57411700 & 2.68349300 & $\mathrm{H}$ & 0.01411700 & 8.08138100 & -1.48030200 \\
\hline $\mathrm{H}$ & 5.95769700 & 1.84848000 & 1.46510900 & $\mathrm{H}$ & 3.58824400 & 9.23638700 & -2.21265000 \\
\hline $\mathrm{C}$ & 7.13549900 & -0.36244000 & 2.44995500 & $\mathrm{H}$ & 2.40094000 & 9.10758100 & -0.91066900 \\
\hline $\mathrm{C}$ & 5.78278100 & -2.74304700 & 1.85567900 & $\mathrm{H}$ & 1.23266800 & 9.56783500 & -3.05395300 \\
\hline $\mathrm{C}$ & 6.92171900 & -2.76124700 & 2.63498600 & $\mathrm{H}$ & 2.01251200 & 8.17577300 & -3.81503500 \\
\hline $\mathrm{C}$ & 7.61824300 & -1.56273600 & 2.92439100 & $\mathrm{C}$ & 3.12213800 & 2.88495000 & 2.62927900 \\
\hline $\mathrm{H}$ & 5.25828100 & -3.67622000 & 1.65186000 & $\mathrm{C}$ & 1.73968700 & 3.05129900 & 3.29619300 \\
\hline $\mathrm{H}$ & 7.28655500 & -3.71036600 & 3.03588600 & $\mathrm{C}$ & 4.18872600 & 3.68876700 & 3.41106900 \\
\hline $\mathrm{H}$ & 8.52477700 & -1.59137300 & 3.53411500 & $\mathrm{H}$ & 3.37833100 & 1.81977400 & 2.72824900 \\
\hline $\mathrm{O}$ & 2.35006400 & -0.12645300 & -0.46676000 & $\mathrm{C}$ & 1.77620200 & 2.66933500 & 4.78206200 \\
\hline $\mathrm{O}$ & 1.31118900 & -2.02514400 & 0.86473500 & $\mathrm{H}$ & 1.39943200 & 4.09925800 & 3.20423700 \\
\hline $\mathrm{P}$ & 0.95095600 & -0.51102000 & 0.31991800 & $\mathrm{H}$ & 1.01873500 & 2.41429800 & 2.76585500 \\
\hline $\mathrm{O}$ & -0.16068700 & -0.51671600 & -0.71445300 & $\mathrm{C}$ & 4.22079100 & 3.31015100 & 4.89765100 \\
\hline $\mathrm{C}$ & 0.02996600 & -4.46600200 & 0.20327200 & $\mathrm{H}$ & 3.97098800 & 4.76814500 & 3.31111400 \\
\hline $\mathrm{C}$ & -0.42614500 & -4.58471900 & 1.53977800 & $\mathrm{H}$ & 5.18238800 & 3.53671700 & 2.95786100 \\
\hline $\mathrm{C}$ & -0.88664100 & -4.61182500 & -0.87163200 & $\mathrm{C}$ & 2.84152300 & 3.46019400 & 5.55020000 \\
\hline $\mathrm{C}$ & -1.78704200 & -4.82978200 & 1.76990900 & $\mathrm{H}$ & 0.78202300 & 2.82377500 & 5.23540900 \\
\hline $\mathrm{C}$ & -2.23725800 & -4.85190100 & -0.58047800 & $\mathrm{H}$ & 1.98932000 & 1.58833400 & 4.86823300 \\
\hline $\mathrm{C}$ & -2.71385200 & -4.96418100 & 0.73147800 & $\mathrm{H}$ & 4.96751200 & 3.92569700 & 5.42817200 \\
\hline $\mathrm{H}$ & -2.14037700 & -4.92860200 & 2.80010200 & $\mathrm{H}$ & 4.55610900 & 2.26123500 & 4.99603800 \\
\hline $\mathrm{H}$ & -2.93921300 & -4.95825000 & -1.41151300 & $\mathrm{H}$ & 2.87834200 & 3.13592600 & 6.60422100 \\
\hline $\mathrm{C}$ & 3.65228700 & 2.35308000 & 0.15810500 & $\mathrm{H}$ & 2.56159500 & 4.53058200 & 5.56420100 \\
\hline $\mathrm{C}$ & 3.69416400 & 2.75948500 & -1.20062400 & $\mathrm{C}$ & -0.46090200 & -4.49493300 & -2.33293900 \\
\hline $\mathrm{C}$ & 3.13402300 & 3.23188000 & 1.14287600 & $\mathrm{C}$ & -1.02384500 & -3.22757400 & -3.01100700 \\
\hline $\mathrm{C}$ & 3.19293300 & 4.02026000 & -1.54936200 & $\mathrm{C}$ & -0.79931200 & -5.74970300 & -3.16802800 \\
\hline $\mathrm{C}$ & 2.65227700 & 4.48606100 & 0.73561700 & $\mathrm{H}$ & 0.63235200 & -4.39012800 & -2.36174500 \\
\hline $\mathrm{C}$ & 2.66351300 & 4.90272300 & -0.59926100 & $\mathrm{C}$ & -0.54546400 & -3.09560900 & -4.46261000 \\
\hline $\mathrm{H}$ & 3.23535100 & 4.32869000 & -2.59705700 & $\mathrm{H}$ & -2.12756100 & -3.27462200 & -2.99672500 \\
\hline $\mathrm{H}$ & 2.25943300 & 5.17249400 & 1.49094500 & $\mathrm{H}$ & -0.72868600 & -2.34371900 & -2.42316200 \\
\hline
\end{tabular}




\begin{tabular}{|c|c|c|c|c|c|c|c|}
\hline $\mathrm{C}$ & -0.31562300 & -5.61363400 & -4.61820000 & $\mathrm{C}$ & -4.69493100 & -1.74328100 & -2.80182000 \\
\hline $\mathrm{H}$ & -1.89169100 & -5.91724700 & -3.16594800 & $\mathrm{C}$ & -3.79303800 & -0.80097600 & -2.25544500 \\
\hline $\mathrm{H}$ & -0.35358000 & -6.64265900 & -2.69753600 & $\mathrm{H}$ & -6.71342600 & -2.12275900 & -3.43311400 \\
\hline $\mathrm{C}$ & -0.87003100 & -4.34856700 & -5.28550800 & $\mathrm{H}$ & -4.32713300 & -2.73055500 & -3.08736000 \\
\hline $\mathrm{H}$ & -0.99389700 & -2.20163200 & -4.92872900 & $\mathrm{H}$ & -2.74191600 & -1.07655700 & -2.14609000 \\
\hline $\mathrm{H}$ & 0.54755200 & -2.93002300 & -4.46815600 & $\mathrm{~N}$ & -3.25958500 & 1.41951500 & -1.34818000 \\
\hline $\mathrm{H}$ & -0.59846400 & -6.50936900 & -5.19726400 & $\mathrm{C}$ & -2.48549100 & 2.07976100 & -2.38424600 \\
\hline $\mathrm{H}$ & 0.78930100 & -5.57372000 & -4.62965600 & $\mathrm{C}$ & -3.12032000 & 3.11544100 & -3.08913700 \\
\hline $\mathrm{H}$ & -0.47426400 & -4.25030000 & -6.31068600 & $\mathrm{C}$ & -1.18217500 & 1.70117400 & -2.73636800 \\
\hline $\mathrm{H}$ & -1.96795700 & -4.44377900 & -5.38521900 & $\mathrm{C}$ & -2.46509500 & 3.75396900 & -4.14298600 \\
\hline $\mathrm{C}$ & -4.18119500 & -5.22246400 & 1.03353600 & $\mathrm{H}$ & -4.12786000 & 3.42048300 & -2.80978300 \\
\hline $\mathrm{C}$ & -4.70898800 & -6.52783000 & 0.39929000 & $\mathrm{C}$ & -0.52704900 & 2.36592100 & -3.77940300 \\
\hline $\mathrm{C}$ & -5.08442700 & -4.03241000 & 0.64039800 & $\mathrm{H}$ & -0.65865400 & 0.90020500 & -2.21044600 \\
\hline $\mathrm{H}$ & -4.26768800 & -5.34045900 & 2.12946300 & $\mathrm{C}$ & -1.16240800 & 3.38525900 & -4.49097800 \\
\hline $\mathrm{C}$ & -6.18174600 & -6.78189400 & 0.74709000 & $\mathrm{H}$ & -2.97577000 & 4.55396600 & -4.68468800 \\
\hline $\mathrm{H}$ & -4.60193900 & -6.46873400 & -0.69902300 & $\mathrm{H}$ & 0.49234800 & 2.06921100 & -4.03294400 \\
\hline $\mathrm{H}$ & -4.08332500 & -7.37565800 & 0.72439000 & $\mathrm{H}$ & -0.64574600 & 3.89102600 & -5.31013000 \\
\hline $\mathrm{C}$ & -6.55791800 & -4.28775500 & 0.98352400 & $\mathrm{~N}$ & -2.50462200 & 0.86707600 & -0.24196400 \\
\hline $\mathrm{H}$ & -4.98839300 & -3.84719900 & -0.44463000 & $\mathrm{H}$ & -3.07386900 & 0.10799900 & 0.14801200 \\
\hline $\mathrm{H}$ & -4.72590900 & -3.11727000 & 1.14105100 & $\mathrm{H}$ & -1.58152200 & 0.38138900 & -0.50220800 \\
\hline $\mathrm{C}$ & -7.06821700 & -5.59217700 & 0.35835900 & $\mathrm{C}$ & -2.13955800 & 4.09475900 & 0.23668300 \\
\hline $\mathrm{H}$ & -6.53426100 & -7.70238500 & 0.25154200 & $\mathrm{C}$ & -1.23015300 & 2.91224200 & 0.61560100 \\
\hline $\mathrm{H}$ & -6.27286200 & -6.96220000 & 1.83444700 & $\mathrm{C}$ & -3.46247400 & 2.46543600 & 1.57260400 \\
\hline $\mathrm{H}$ & -7.17591000 & -3.43534800 & 0.65380600 & $\mathrm{H}$ & -1.85551200 & 5.03143000 & 0.74338300 \\
\hline $\mathrm{H}$ & -6.67021400 & -4.34468500 & 2.08228800 & $\mathrm{H}$ & -2.15306200 & 4.31711300 & -0.83932500 \\
\hline $\mathrm{H}$ & -8.11403800 & -5.77770500 & 0.65658900 & $\mathrm{H}$ & -0.59619200 & 3.14668300 & 1.47990200 \\
\hline $\mathrm{H}$ & -7.07309900 & -5.48901500 & -0.74311200 & $\mathrm{H}$ & -0.54670700 & 2.61000000 & -0.18699300 \\
\hline $\mathrm{C}$ & 0.51914300 & -4.51457200 & 2.73325000 & $\mathrm{C}$ & -2.17326800 & 1.76575800 & 1.04651400 \\
\hline $\mathrm{C}$ & 0.61805600 & -5.87277000 & 3.46685200 & $\mathrm{O}$ & -1.70092900 & 0.84905400 & 1.92256400 \\
\hline $\mathrm{C}$ & 0.16771300 & -3.38765700 & 3.73061100 & $\mathrm{C}$ & -3.55197100 & 3.69981400 & 0.67020000 \\
\hline $\mathrm{H}$ & 1.52941200 & -4.29483500 & 2.35634200 & $\mathrm{O}$ & -4.56560000 & 4.27965100 & 0.35895200 \\
\hline $\mathrm{C}$ & 1.60827300 & -5.81980300 & 4.63723000 & $\mathrm{C}$ & -4.71812600 & 1.60092600 & 1.62149300 \\
\hline $\mathrm{H}$ & -0.37977100 & -6.15793300 & 3.84685700 & $\mathrm{H}$ & -4.58178100 & 0.77058900 & 2.33178800 \\
\hline $\mathrm{H}$ & 0.91140800 & -6.65962100 & 2.75158500 & $\mathrm{H}$ & -5.57382200 & 2.20285700 & 1.95486300 \\
\hline $\mathrm{C}$ & 1.16089500 & -3.34022500 & 4.89944800 & $\mathrm{H}$ & -4.98964500 & 1.18806000 & 0.64258500 \\
\hline $\mathrm{H}$ & -0.85101200 & -3.55067300 & 4.12756900 & $\mathrm{C}$ & -3.11678600 & 2.98279000 & 3.02749700 \\
\hline $\mathrm{H}$ & 0.15596400 & -2.42193700 & 3.20328200 & $\mathrm{H}$ & -2.79818300 & 2.09444500 & 3.58931600 \\
\hline $\mathrm{C}$ & 1.26616200 & -4.69101300 & 5.61806800 & $\mathrm{H}$ & -2.24017200 & 3.64641000 & 2.98016000 \\
\hline $\mathrm{H}$ & 1.62862000 & -6.79177200 & 5.15948800 & $\mathrm{C}$ & -4.23226900 & 3.69080500 & 3.76534200 \\
\hline $\mathrm{H}$ & 2.62868900 & -5.65949400 & 4.24225800 & $\mathrm{C}$ & -5.02127000 & 2.99556100 & 4.69720900 \\
\hline $\mathrm{H}$ & 0.86753500 & -2.54891600 & 5.61003200 & $\mathrm{C}$ & -4.49187100 & 5.05757100 & 3.56866800 \\
\hline $\mathrm{H}$ & 2.15663600 & -3.05339600 & 4.51412000 & $\mathrm{C}$ & -6.04221500 & 3.63900000 & 5.40189700 \\
\hline $\mathrm{H}$ & 2.01837300 & -4.64154000 & 6.42385200 & $\mathrm{H}$ & -4.82520000 & 1.93538800 & 4.88014600 \\
\hline $\mathrm{H}$ & 0.30054400 & -4.91783100 & 6.10820600 & $\mathrm{C}$ & -5.51113900 & 5.70524400 & 4.27089400 \\
\hline $\mathrm{O}$ & 0.75526200 & 0.33757000 & 1.55683200 & $\mathrm{H}$ & -3.88686000 & 5.62902200 & 2.86051200 \\
\hline $\mathrm{H}$ & -0.69932100 & 0.66854900 & 1.81704900 & $\mathrm{C}$ & -6.29210100 & 4.99750300 & 5.18924100 \\
\hline $\mathrm{C}$ & -7.45743500 & 2.40927200 & -1.84646200 & $\mathrm{H}$ & -6.64000000 & 3.07767400 & 6.12482000 \\
\hline $\mathrm{C}$ & -6.12586900 & 2.10467100 & -1.64987400 & $\mathrm{H}$ & -5.69334500 & 6.76968100 & 4.10185500 \\
\hline $\mathrm{C}$ & -5.59878400 & 0.84032400 & -2.04041500 & $\mathrm{H}$ & -7.08757600 & 5.50435300 & 5.74154500 \\
\hline $\mathrm{C}$ & -6.49787600 & -0.11991100 & -2.62621900 & & & & \\
\hline $\mathrm{C}$ & -7.86331000 & 0.23400600 & -2.81872100 & ts-2-R-R & & & \\
\hline $\mathrm{C}$ & -8.33576900 & 1.47224000 & -2.44314000 & $\mathrm{C}$ & -0.60430700 & 3.35233600 & 0.27424400 \\
\hline $\mathrm{H}$ & -7.83597800 & 3.38377700 & -1.52864800 & $\mathrm{C}$ & -1.94008200 & 3.73621900 & 0.19375400 \\
\hline $\mathrm{H}$ & -5.48086000 & 2.83532900 & -1.16585600 & $\mathrm{C}$ & -2.25521400 & 4.97810600 & -0.47064000 \\
\hline $\mathrm{C}$ & -4.22379200 & 0.45965300 & -1.87939000 & $\mathrm{C}$ & -1.18774100 & 5.86268900 & -0.83282000 \\
\hline $\mathrm{C}$ & -6.01545800 & -1.40509600 & -2.99418200 & $\mathrm{C}$ & 0.15203900 & 5.45987700 & -0.59988400 \\
\hline $\mathrm{H}$ & -8.53332700 & -0.50227600 & -3.27059800 & $\mathrm{C}$ & 0.47485000 & 4.20809100 & -0.11082100 \\
\hline $\mathrm{H}$ & -9.38663100 & 1.73038200 & -2.59567400 & $\mathrm{H}$ & -4.41087600 & 4.69251800 & -0.58403500 \\
\hline \multicolumn{8}{|c|}{$\mathrm{U}$} \\
\hline
\end{tabular}




\begin{tabular}{|c|c|c|c|c|c|c|c|}
\hline $\mathrm{C}$ & -3.58401000 & 5.36567700 & -0.80958400 & $\mathrm{H}$ & -5.44678900 & 2.05106000 & -5.48976400 \\
\hline $\mathrm{C}$ & -1.48661100 & 7.10985800 & -1.45181800 & $\mathrm{H}$ & -5.75378900 & 0.31790400 & -5.32482800 \\
\hline $\mathrm{H}$ & 0.95831300 & 6.14632600 & -0.86944700 & $\mathrm{C}$ & -5.77746200 & -4.66535200 & -0.68200200 \\
\hline $\mathrm{C}$ & -2.78609900 & 7.46200800 & -1.74472700 & $\mathrm{C}$ & -5.02094000 & -5.30452100 & -1.86481300 \\
\hline $\mathrm{C}$ & -3.84096100 & 6.56989100 & -1.43296400 & $\mathrm{C}$ & -7.30182900 & -4.70069600 & -0.93941100 \\
\hline $\mathrm{H}$ & -0.65905900 & 7.77777200 & -1.70596900 & $\mathrm{H}$ & -5.58772400 & -5.30266800 & 0.20125400 \\
\hline $\mathrm{H}$ & -3.00396000 & 8.41748700 & -2.22824000 & $\mathrm{C}$ & -5.52279700 & -6.72281300 & -2.16829900 \\
\hline $\mathrm{H}$ & -4.86909900 & 6.83536900 & -1.69172300 & $\mathrm{H}$ & -5.15238100 & -4.67620100 & -2.76457800 \\
\hline $\mathrm{C}$ & -4.31640500 & 0.78971100 & 0.83585300 & $\mathrm{H}$ & -3.94048300 & -5.31758000 & -1.64881900 \\
\hline $\mathrm{C}$ & -5.27325900 & 1.41021400 & 1.61787100 & $\mathrm{C}$ & -7.80229500 & -6.11832400 & -1.24576000 \\
\hline $\mathrm{C}$ & -5.11295700 & 2.72872100 & 2.11556500 & $\mathrm{H}$ & -7.53770100 & -4.03565200 & -1.79017600 \\
\hline $\mathrm{C}$ & -3.96540200 & 3.49039500 & 1.72136200 & $\mathrm{H}$ & -7.83307500 & -4.28470600 & -0.06727800 \\
\hline $\mathrm{C}$ & -3.02884700 & 2.90857400 & 0.79084900 & $\mathrm{C}$ & -7.03639700 & -6.75048800 & -2.41445700 \\
\hline $\mathrm{C}$ & -3.17893800 & 1.56538000 & 0.46000100 & $\mathrm{H}$ & -4.98448200 & -7.13650200 & -3.03806200 \\
\hline $\mathrm{H}$ & -6.93707200 & 2.70757000 & 3.29096100 & $\mathrm{H}$ & -5.28176800 & -7.38303400 & -1.31443400 \\
\hline $\mathrm{H}$ & -6.17144500 & 0.85315500 & 1.89359800 & $\mathrm{H}$ & -8.88440100 & -6.09931300 & -1.45980700 \\
\hline $\mathrm{C}$ & -6.05982300 & 3.29831200 & 3.01370900 & $\mathrm{H}$ & -7.67596600 & -6.74923100 & -0.34622400 \\
\hline $\mathrm{C}$ & -3.78864700 & 4.77924100 & 2.30264800 & $\mathrm{H}$ & -7.37930700 & -7.78525300 & -2.58381700 \\
\hline $\mathrm{C}$ & -4.71033900 & 5.29448400 & 3.19103700 & $\mathrm{H}$ & -7.26400800 & -6.19258800 & -3.34204900 \\
\hline $\mathrm{C}$ & -5.86778000 & 4.55671200 & 3.54058600 & $\mathrm{C}$ & -4.06643000 & -1.44316600 & 2.80786500 \\
\hline $\mathrm{H}$ & -2.90110100 & 5.36052900 & 2.05359500 & $\mathrm{C}$ & -2.90060100 & -2.36304500 & 3.23199600 \\
\hline $\mathrm{H}$ & -4.54251400 & 6.28020600 & 3.63218600 & $\mathrm{C}$ & -5.23495200 & -1.57483400 & 3.81182200 \\
\hline $\mathrm{H}$ & -6.59551300 & 4.98063100 & 4.23694400 & $\mathrm{H}$ & -3.68422300 & -0.41440300 & 2.88532100 \\
\hline $\mathrm{O}$ & -2.19464700 & 0.95220800 & -0.31287700 & $\mathrm{C}$ & -2.44699300 & -2.07092500 & 4.66788700 \\
\hline $\mathrm{O}$ & -0.26390300 & 2.14493100 & 0.84832300 & $\mathrm{H}$ & -3.21131000 & -3.42110700 & 3.15913700 \\
\hline $\mathrm{P}$ & -0.76097900 & 0.65185700 & 0.41273900 & $\mathrm{H}$ & -2.06451900 & -2.21585000 & 2.53445200 \\
\hline $\mathrm{O}$ & 0.14886600 & 0.10933400 & -0.72412500 & $\mathrm{C}$ & -4.78458400 & -1.29116400 & 5.25135200 \\
\hline $\mathrm{C}$ & 1.90871300 & 3.77523700 & -0.04145700 & $\mathrm{H}$ & -5.65187900 & -2.59684900 & 3.75066200 \\
\hline $\mathrm{C}$ & 2.55715900 & 3.58218900 & 1.20373300 & $\mathrm{H}$ & -6.05533600 & -0.89404300 & 3.52991500 \\
\hline $\mathrm{C}$ & 2.63643300 & 3.60197800 & -1.25002200 & $\mathrm{C}$ & -3.60655100 & -2.18161000 & 5.66547500 \\
\hline $\mathrm{C}$ & 3.91085000 & 3.21671200 & 1.21101500 & $\mathrm{H}$ & -1.63108900 & -2.75729600 & 4.95401100 \\
\hline $\mathrm{C}$ & 3.98516200 & 3.22668500 & -1.18027900 & $\mathrm{H}$ & -2.02149400 & -1.05194300 & 4.70727600 \\
\hline $\mathrm{C}$ & 4.64684600 & 3.02766900 & 0.03706500 & $\mathrm{H}$ & -5.63146000 & -1.42593200 & 5.94597400 \\
\hline $\mathrm{H}$ & 4.41562900 & 3.07590200 & 2.17052100 & $\mathrm{H}$ & -4.48292500 & -0.23055200 & 5.33158500 \\
\hline $\mathrm{H}$ & 4.53715700 & 3.08651500 & -2.11312600 & $\mathrm{H}$ & -3.26786500 & -1.92004000 & 6.68247200 \\
\hline $\mathrm{C}$ & -4.53877300 & -0.63064200 & 0.40278400 & $\mathrm{H}$ & -3.94652100 & -3.23336600 & 5.71211300 \\
\hline $\mathrm{C}$ & -4.91254600 & -0.91802700 & -0.93541700 & $\mathrm{C}$ & 2.00588600 & 3.79086400 & -2.62719800 \\
\hline $\mathrm{C}$ & -4.50126000 & -1.67321100 & 1.36394700 & $\mathrm{C}$ & 1.97112000 & 2.48269300 & -3.44422400 \\
\hline $\mathrm{C}$ & -5.27924300 & -2.22924600 & -1.27082000 & $\mathrm{C}$ & 2.67598000 & 4.91796000 & -3.44472300 \\
\hline $\mathrm{C}$ & -4.88379800 & -2.96472700 & 0.97167800 & $\mathrm{H}$ & 0.95727100 & 4.08792500 & -2.48876400 \\
\hline $\mathrm{C}$ & -5.29325800 & -3.26748700 & -0.33019700 & $\mathrm{C}$ & 1.29721800 & 2.67920900 & -4.80879800 \\
\hline $\mathrm{H}$ & -5.58861600 & -2.43807900 & -2.29814700 & $\mathrm{H}$ & 3.00301700 & 2.11913000 & -3.59284300 \\
\hline $\mathrm{H}$ & -4.87226800 & -3.76782300 & 1.71367400 & $\mathrm{H}$ & 1.44438800 & 1.71034100 & -2.86073300 \\
\hline $\mathrm{C}$ & -4.96744900 & 0.15772200 & -2.01479800 & $\mathrm{C}$ & 1.99845700 & 5.11245200 & -4.80782800 \\
\hline $\mathrm{C}$ & -6.39351300 & 0.37422800 & -2.57065600 & $\mathrm{H}$ & 3.74325300 & 4.67757800 & -3.60002500 \\
\hline $\mathrm{C}$ & -3.97436900 & -0.11055100 & -3.16764200 & $\mathrm{H}$ & 2.65676900 & 5.85911400 & -2.86947400 \\
\hline $\mathrm{H}$ & -4.66613700 & 1.11401700 & -1.56136900 & $\mathrm{C}$ & 1.95733100 & 3.80698500 & -5.61175800 \\
\hline $\mathrm{C}$ & -6.42924200 & 1.48430600 & -3.63010800 & $\mathrm{H}$ & 1.31617800 & 1.73664300 & -5.38305900 \\
\hline $\mathrm{H}$ & -6.76153100 & -0.56662700 & -3.01817000 & $\mathrm{H}$ & 0.22944500 & 2.91980800 & -4.65357800 \\
\hline $\mathrm{H}$ & -7.08183300 & 0.61135400 & -1.74207400 & $\mathrm{H}$ & 2.51828000 & 5.89977100 & -5.37997700 \\
\hline $\mathrm{C}$ & -4.01570200 & 0.99930300 & -4.22654700 & $\mathrm{H}$ & 0.96523900 & 5.47386900 & -4.65127100 \\
\hline $\mathrm{H}$ & -4.21903000 & -1.07685000 & -3.64556100 & $\mathrm{H}$ & 1.42760100 & 3.96075200 & -6.56715200 \\
\hline $\mathrm{H}$ & -2.95714900 & -0.20710000 & -2.75678000 & $\mathrm{H}$ & 2.99052100 & 3.51053600 & -5.87379100 \\
\hline $\mathrm{C}$ & -5.43446800 & 1.21802900 & -4.76649000 & $\mathrm{C}$ & 6.11131100 & 2.62439600 & 0.09192800 \\
\hline $\mathrm{H}$ & -7.45182300 & 1.59109400 & -4.03065300 & $\mathrm{C}$ & 7.04732100 & 3.68917700 & -0.52255400 \\
\hline $\mathrm{H}$ & -6.18226300 & 2.45022000 & -3.15146100 & $\mathrm{C}$ & 6.37298100 & 1.24432600 & -0.55181900 \\
\hline $\mathrm{H}$ & -3.32289400 & 0.76012000 & -5.05122600 & $\mathrm{H}$ & 6.38249900 & 2.53717600 & 1.16023900 \\
\hline $\mathrm{H}$ & -3.64821100 & 1.94023500 & -3.77699800 & $\mathrm{C}$ & 8.52325100 & 3.27927800 & -0.43312700 \\
\hline
\end{tabular}




\begin{tabular}{|c|c|c|c|c|c|c|c|}
\hline $\mathrm{H}$ & 6.77602000 & 3.84160000 & -1.58299700 & $\mathrm{H}$ & -2.43824800 & -3.05634700 & -2.57755000 \\
\hline $\mathrm{H}$ & 6.88431100 & 4.65761000 & -0.02079600 & $\mathrm{H}$ & -2.10629300 & -5.31327200 & -3.60297300 \\
\hline $\mathrm{C}$ & 7.84987900 & 0.83782900 & -0.46537900 & $\mathrm{~N}$ & 1.78997300 & -1.79623000 & -0.30150000 \\
\hline $\mathrm{H}$ & 6.06410400 & 1.27228600 & -1.61245200 & $\mathrm{H}$ & 2.61644200 & -1.19236600 & -0.33778200 \\
\hline $\mathrm{H}$ & 5.73780300 & 0.48415000 & -0.06703100 & $\mathrm{H}$ & 0.84519600 & -0.81752800 & -0.51654500 \\
\hline $\mathrm{C}$ & 8.76888900 & 1.90619600 & -1.07077200 & $\mathrm{C}$ & 1.18820000 & -4.67130000 & 1.20757600 \\
\hline $\mathrm{H}$ & 9.15771300 & 4.04498700 & -0.91096800 & $\mathrm{C}$ & 0.57711200 & -3.27249300 & 1.38001100 \\
\hline $\mathrm{H}$ & 8.82733100 & 3.24541200 & 0.62965900 & $\mathrm{C}$ & 3.01563500 & -3.02987500 & 1.63138500 \\
\hline $\mathrm{H}$ & 8.00367800 & -0.13240700 & -0.96717000 & $\mathrm{H}$ & 0.90696400 & -5.37118700 & 2.01124000 \\
\hline $\mathrm{H}$ & 8.12292600 & 0.68384100 & 0.59525500 & $\mathrm{H}$ & 0.91059900 & -5.15906000 & 0.26200100 \\
\hline $\mathrm{H}$ & 9.82622000 & 1.61278400 & -0.95689900 & $\mathrm{H}$ & 0.20695600 & -3.12364200 & 2.40348800 \\
\hline $\mathrm{H}$ & 8.58041300 & 1.97288100 & -2.15871800 & $\mathrm{H}$ & -0.26846500 & -3.07733200 & 0.71052800 \\
\hline $\mathrm{C}$ & 1.84895900 & 3.80111700 & 2.53470400 & $\mathrm{C}$ & 1.73030900 & -2.25778300 & 1.17349000 \\
\hline $\mathrm{C}$ & 2.36173700 & 5.06042100 & 3.27226300 & $\mathrm{O}$ & 1.60642800 & -1.07733300 & 1.87443800 \\
\hline $\mathrm{C}$ & 1.91816400 & 2.57121900 & 3.46594600 & $\mathrm{C}$ & 2.70298700 & -4.47081100 & 1.21832500 \\
\hline $\mathrm{H}$ & 0.78296300 & 3.97884700 & 2.32931800 & $\mathrm{O}$ & 3.51874700 & -5.32829900 & 0.97010800 \\
\hline $\mathrm{C}$ & 1.60025100 & 5.29641900 & 4.58408400 & $\mathrm{C}$ & 4.33925900 & -2.48575700 & 1.10301400 \\
\hline $\mathrm{H}$ & 3.43965800 & 4.94467600 & 3.48790800 & $\mathrm{H}$ & 4.52709600 & -1.47715300 & 1.50415400 \\
\hline $\mathrm{H}$ & 2.27126700 & 5.94018200 & 2.61295800 & $\mathrm{H}$ & 5.16499900 & -3.13991200 & 1.41276300 \\
\hline $\mathrm{C}$ & 1.14855000 & 2.80890900 & 4.77100200 & $\mathrm{H}$ & 4.36985100 & -2.43865700 & 0.00786000 \\
\hline $\mathrm{H}$ & 2.97321000 & 2.34995900 & 3.70867500 & $\mathrm{C}$ & 3.02666800 & -3.01713900 & 3.21378200 \\
\hline $\mathrm{H}$ & 1.52627500 & 1.68678500 & 2.94201500 & $\mathrm{H}$ & 2.98240000 & -1.95930500 & 3.50394800 \\
\hline $\mathrm{C}$ & 1.64304400 & 4.06570700 & 5.49863400 & $\mathrm{H}$ & 2.09811800 & -3.47541800 & 3.58737700 \\
\hline $\mathrm{H}$ & 2.01029100 & 6.17822900 & 5.10562200 & $\mathrm{C}$ & 4.20838100 & -3.68499500 & 3.88330100 \\
\hline $\mathrm{H}$ & 0.54647900 & 5.53727500 & 4.35060400 & $\mathrm{C}$ & 5.30667600 & -2.91984900 & 4.31121600 \\
\hline $\mathrm{H}$ & 1.23595900 & 1.92618600 & 5.42688100 & $\mathrm{C}$ & 4.23406700 & -5.06865200 & 4.12600500 \\
\hline $\mathrm{H}$ & 0.07258200 & 2.91721200 & 4.54161900 & $\mathrm{C}$ & 6.39876800 & -3.51479000 & 4.94850400 \\
\hline $\mathrm{H}$ & 1.04607800 & 4.24491700 & 6.40910000 & $\mathrm{H}$ & 5.30037300 & -1.83836500 & 4.14917500 \\
\hline $\mathrm{H}$ & 2.68357300 & 3.90281900 & 5.83727600 & $\mathrm{C}$ & 5.32313300 & -5.66826600 & 4.76320200 \\
\hline $\mathrm{O}$ & -0.85779800 & -0.19127700 & 1.64846800 & $\mathrm{H}$ & 3.39053800 & -5.68949600 & 3.81506400 \\
\hline $\mathrm{H}$ & 0.65342500 & -0.79658200 & 1.93053300 & $\mathrm{C}$ & 6.41169000 & -4.89391100 & 5.17494300 \\
\hline $\mathrm{C}$ & 5.66908600 & -5.01004500 & -2.32342600 & $\mathrm{H}$ & 7.23976100 & -2.89712200 & 5.27483500 \\
\hline $\mathrm{C}$ & 4.52566300 & -4.32278600 & -1.97082600 & $\mathrm{H}$ & 5.31943200 & -6.74683600 & 4.94110400 \\
\hline $\mathrm{C}$ & 4.14084700 & -3.13530500 & -2.65572800 & $\mathrm{H}$ & 7.26294600 & -5.36239000 & 5.67569000 \\
\hline $\mathrm{C}$ & 4.98012000 & -2.67121700 & -3.73031400 & & & & \\
\hline $\mathrm{C}$ & 6.15166100 & -3.40746900 & -4.06616600 & \multicolumn{2}{|c|}{ int-2a-R-R } & & \\
\hline $\mathrm{C}$ & 6.49199500 & -4.55326400 & -3.38143900 & $\mathrm{C}$ & -1.02452600 & 3.33080600 & 0.25264600 \\
\hline $\mathrm{H}$ & 5.94129000 & -5.91267700 & -1.77107400 & $\mathrm{C}$ & -2.39113700 & 3.56934100 & 0.15208600 \\
\hline $\mathrm{H}$ & 3.92319200 & -4.69004600 & -1.14411900 & $\mathrm{C}$ & -2.82425600 & 4.75443500 & -0.54832100 \\
\hline $\mathrm{C}$ & 2.96057900 & -2.37805700 & -2.34362400 & $\mathrm{C}$ & -1.84534700 & 5.72660900 & -0.93702900 \\
\hline $\mathrm{C}$ & 4.62670900 & -1.49365200 & -4.44205300 & $\mathrm{C}$ & -0.47216000 & 5.46315300 & -0.69720000 \\
\hline $\mathrm{H}$ & 6.77824900 & -3.04303800 & -4.88472400 & $\mathrm{C}$ & -0.02998600 & 4.26689700 & -0.16471000 \\
\hline $\mathrm{H}$ & 7.39434300 & -5.10829400 & -3.64995400 & $\mathrm{H}$ & -4.94124300 & 4.25658600 & -0.65556000 \\
\hline $\mathrm{C}$ & 3.48813200 & -0.79132000 & -4.11734700 & $\mathrm{C}$ & -4.18302800 & 5.00017200 & -0.89976500 \\
\hline $\mathrm{C}$ & 2.65148200 & -1.23960900 & -3.06904700 & $\mathrm{C}$ & -2.26226100 & 6.91978200 & -1.59307800 \\
\hline $\mathrm{H}$ & 5.27152700 & -1.15840800 & -5.25867700 & $\mathrm{H}$ & 0.26421600 & 6.21355900 & -0.99481300 \\
\hline $\mathrm{H}$ & 3.21174800 & 0.10389600 & -4.67614800 & $\mathrm{C}$ & -3.58905100 & 7.13598700 & -1.89499300 \\
\hline $\mathrm{H}$ & 1.73773500 & -0.68660400 & -2.84190600 & $\mathrm{C}$ & -4.55355600 & 6.15536600 & -1.55780200 \\
\hline $\mathrm{N}$ & 2.04148800 & -2.83050900 & -1.30211100 & $\mathrm{H}$ & -1.50269700 & 7.65637200 & -1.86846800 \\
\hline $\mathrm{C}$ & 0.87252900 & -3.46217000 & -1.87280200 & $\mathrm{H}$ & -3.89700000 & 8.05127000 & -2.40640800 \\
\hline $\mathrm{C}$ & 1.05113400 & -4.73921700 & -2.43499100 & $\mathrm{H}$ & -5.60166000 & 6.31202200 & -1.82489700 \\
\hline $\mathrm{C}$ & -0.39492700 & -2.86511800 & -1.93630600 & $\mathrm{C}$ & -4.41610300 & 0.37715900 & 0.83205700 \\
\hline $\mathrm{C}$ & -0.00973100 & -5.39366100 & -3.06120800 & $\mathrm{C}$ & -5.44550800 & 0.89306400 & 1.59719000 \\
\hline $\mathrm{H}$ & 2.02984800 & -5.21608400 & -2.38495200 & $\mathrm{C}$ & -5.44947600 & 2.23213000 & 2.06607200 \\
\hline $\mathrm{C}$ & -1.45857200 & -3.53819400 & -2.54743700 & $\mathrm{C}$ & -4.39864900 & 3.11942000 & 1.66327900 \\
\hline $\mathrm{H}$ & -0.56668000 & -1.87172600 & -1.52866400 & $\mathrm{C}$ & -3.38878500 & 2.63595300 & 0.75374900 \\
\hline $\mathrm{C}$ & -1.27350400 & -4.79853400 & -3.11878700 & $\mathrm{C}$ & -3.37926300 & 1.27871100 & 0.44839900 \\
\hline $\mathrm{H}$ & 0.15446500 & -6.38234500 & -3.49725000 & $\mathrm{H}$ & -7.26815200 & 2.01512400 & 3.22956200 \\
\hline
\end{tabular}




\begin{tabular}{|c|c|c|c|}
\hline $\mathrm{H}$ & -6.27187900 & 0.23726800 & 1.87988400 \\
\hline $\mathrm{C}$ & -6.46648900 & 2.70185200 & 2.94507400 \\
\hline $\mathrm{C}$ & -4.38390700 & 4.43192500 & 2.21723700 \\
\hline $\mathrm{C}$ & -5.36998600 & 4.85041500 & 3.08707900 \\
\hline $\mathrm{C}$ & -6.43269000 & 3.98513400 & 3.44506400 \\
\hline $\mathrm{H}$ & -3.57074400 & 5.11088000 & 1.96166300 \\
\hline $\mathrm{H}$ & -5.32741200 & 5.85844800 & 3.50707500 \\
\hline $\mathrm{H}$ & -7.21278800 & 4.33241300 & 4.12696300 \\
\hline $\mathrm{O}$ & -2.32094200 & 0.76940100 & -0.30989300 \\
\hline $\mathrm{O}$ & -0.57310400 & 2.17873100 & 0.87670500 \\
\hline $\mathrm{P}$ & -0.87690000 & 0.65103000 & 0.42629300 \\
\hline $\mathrm{O}$ & 0.08432400 & 0.27979400 & -0.77140700 \\
\hline $\mathrm{C}$ & 1.43677300 & 3.96713400 & -0.08419000 \\
\hline $\mathrm{C}$ & 2.09847600 & 3.89063600 & 1.16652500 \\
\hline $\mathrm{C}$ & 2.17526400 & 3.79693000 & -1.28673400 \\
\hline $\mathrm{C}$ & 3.47893700 & 3.64598400 & 1.18578700 \\
\hline $\mathrm{C}$ & 3.55132900 & 3.54244400 & -1.20360900 \\
\hline C & 4.22847200 & 3.46550100 & 0.01914600 \\
\hline $\mathrm{H}$ & 3.99458800 & 3.59747700 & 2.14865900 \\
\hline $\mathrm{H}$ & 4.11406500 & 3.40528800 & -2.13015700 \\
\hline $\mathrm{C}$ & -4.45503400 & -1.06598900 & 0.41723900 \\
\hline $\mathrm{C}$ & -4.81100600 & -1.41152000 & -0.91190400 \\
\hline C & -4.25230200 & -2.08480600 & 1.38277400 \\
\hline $\mathrm{C}$ & -4.98713600 & -2.76388300 & -1.23709200 \\
\hline $\mathrm{C}$ & -4.44812000 & -3.42040100 & 1.00066100 \\
\hline $\mathrm{C}$ & -4.82846100 & -3.78719200 & -0.29378600 \\
\hline $\mathrm{H}$ & -5.28378900 & -3.02178000 & -2.25695000 \\
\hline $\mathrm{H}$ & -4.30967600 & -4.20970500 & 1.74419300 \\
\hline$C$ & -5.05625800 & -0.35849000 & -1.98660100 \\
\hline S & -6.51567500 & -0.36090100 & -2.49622300 \\
\hline $\mathrm{C}$ & -4.07130500 & -0.46962900 & -3.17237000 \\
\hline $\mathrm{H}$ & -4.89037200 & 0.63247000 & -1.53712600 \\
\hline$c$ & -6.75561500 & 0.73559400 & -3.54214200 \\
\hline $\mathrm{H}$ & -6.74889100 & -1.34485700 & -2.94140400 \\
\hline 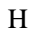 & -7.20573000 & -0.23687100 & -1.64473700 \\
\hline $\mathrm{C}$ & -4.31626100 & 0.62601600 & -4.21841100 \\
\hline$H$ & -4.18283800 & -1.45958600 & -3.65116200 \\
\hline $\mathrm{H}$ & -3.03782200 & -0.41394200 & -2.79544600 \\
\hline $\mathrm{C}$ & -5.76856700 & 0.62903300 & -4.71167700 \\
\hline $\mathrm{H}$ & -7.79491500 & 0.68718200 & -3.90942200 \\
\hline $\mathrm{H}$ & -6.64254000 & 1.72555200 & -3.06231700 \\
\hline 4 & -3.62261700 & 0.49888100 & -5.06680400 \\
\hline $\mathrm{H}$ & -4.08151700 & 1.61013300 & -3.77235000 \\
\hline 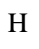 & -5.93015200 & 1.45343900 & -5.42684300 \\
\hline 11 & -5.96525600 & -0.30678500 & -5.26784000 \\
\hline$C$ & -5.10369700 & -5.24336400 & -0.63298400 \\
\hline . & -4.27018400 & -5.77328300 & -1.81839500 \\
\hline $\mathrm{C}$ & -6.60800200 & -5.50401600 & -0.87781400 \\
\hline 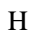 & -4.81539600 & -5.83878900 & 0.25289900 \\
\hline$C$ & -4.55931600 & -7.25295000 & -2.10470800 \\
\hline$U$ & -4.50208400 & -5.17988800 & -2.72170500 \\
\hline 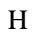 & -3.19765100 & -5.62257800 & -1.61531400 \\
\hline $\mathrm{C}$ & -6.89794100 & -6.98180500 & -1.17094200 \\
\hline 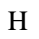 & -6.94482900 & -4.88734000 & -1.73121800 \\
\hline $\mathrm{H}$ & -7.18829800 & -5.16327600 & -0.00420400 \\
\hline $\mathrm{C}$ & -6.05384800 & -7.50530900 & -2.33964000 \\
\hline $\mathrm{H}$ & -3.97104400 & -7.59132100 & -2.97458700 \\
\hline & -4.21809600 & -7.86185600 & -1.24687700 \\
\hline
\end{tabular}

\begin{tabular}{|c|c|c|c|}
\hline $\mathrm{H}$ & -7.97231300 & -7.12171200 & -1.37915600 \\
\hline $\mathrm{H}$ & -6.67802500 & -7.58102700 & -0.26780100 \\
\hline $\mathrm{H}$ & -6.24213300 & -8.58059500 & -2.49860100 \\
\hline $\mathrm{H}$ & -6.36571300 & -6.99458600 & -3.26992100 \\
\hline $\mathrm{C}$ & -3.85085500 & -1.78079100 & 2.82283000 \\
\hline $\mathrm{C}$ & -2.59923600 & -2.56074600 & 3.28110600 \\
\hline $\mathrm{C}$ & -5.00777400 & -2.01461900 & 3.82227800 \\
\hline $\mathrm{H}$ & -3.58242200 & -0.71484600 & 2.87938000 \\
\hline $\mathrm{C}$ & -2.19275800 & -2.18002000 & 4.71054700 \\
\hline $\mathrm{H}$ & -2.79857600 & -3.64688800 & 3.24113600 \\
\hline $\mathrm{H}$ & -1.77525400 & -2.35230100 & 2.58462800 \\
\hline $\mathrm{C}$ & -4.60701500 & -1.64346900 & 5.25633900 \\
\hline $\mathrm{H}$ & -5.30643500 & -3.07839900 & 3.78593500 \\
\hline $\mathrm{H}$ & -5.89642800 & -1.43858600 & 3.51597100 \\
\hline $\mathrm{C}$ & -3.34222600 & -2.38634900 & 5.70461000 \\
\hline $\mathrm{H}$ & -1.31037600 & -2.76597100 & 5.02076900 \\
\hline $\mathrm{H}$ & -1.87901100 & -1.12052400 & 4.72304200 \\
\hline $\mathrm{H}$ & -5.44129100 & -1.85201300 & 5.94795800 \\
\hline $\mathrm{H}$ & -4.42548800 & -0.55410500 & 5.30855800 \\
\hline $\mathrm{H}$ & -3.04311200 & -2.05885700 & 6.71487500 \\
\hline $\mathrm{H}$ & -3.56572400 & -3.46699200 & 5.78196800 \\
\hline $\mathrm{C}$ & 1.53457900 & 3.87943400 & -2.67035500 \\
\hline $\mathrm{C}$ & 1.61885900 & 2.55104500 & -3.45180200 \\
\hline $\mathrm{C}$ & 2.10747900 & 5.03799900 & -3.51792700 \\
\hline $\mathrm{H}$ & 0.46275000 & 4.08621300 & -2.54326300 \\
\hline $\mathrm{C}$ & 0.94072300 & 2.65184600 & -4.82488200 \\
\hline $\mathrm{H}$ & 2.67932000 & 2.27501900 & -3.58648800 \\
\hline $\mathrm{H}$ & 1.15507400 & 1.75112700 & -2.85277700 \\
\hline $\mathrm{C}$ & 1.42439700 & 5.13704700 & -4.88801500 \\
\hline $\mathrm{H}$ & 3.19249900 & 4.88723600 & -3.66238500 \\
\hline $\mathrm{H}$ & 2.00389800 & 5.98905200 & -2.96848700 \\
\hline $\mathrm{C}$ & 1.50251900 & 3.81177100 & -5.65631000 \\
\hline $\mathrm{H}$ & 1.04985000 & 1.69907500 & -5.37088300 \\
\hline $\mathrm{H}$ & -0.14551200 & 2.80013600 & -4.68191000 \\
\hline $\mathrm{H}$ & 1.87743200 & 5.95147200 & -5.47862800 \\
\hline $\mathrm{H}$ & 0.36256500 & 5.41077000 & -4.74538300 \\
\hline $\mathrm{H}$ & 0.96569500 & 3.89169800 & -6.61674100 \\
\hline $\mathrm{H}$ & 2.55890500 & 3.60106000 & -5.90853400 \\
\hline $\mathrm{C}$ & 5.72253100 & 3.19653200 & 0.08866600 \\
\hline $\mathrm{C}$ & 6.56121600 & 4.27490300 & -0.63249400 \\
\hline $\mathrm{C}$ & 6.09762000 & 1.79069900 & -0.43093400 \\
\hline $\mathrm{H}$ & 6.00582300 & 3.23069100 & 1.15681800 \\
\hline $\mathrm{C}$ & 8.06678100 & 4.00151300 & -0.51924900 \\
\hline $\mathrm{H}$ & 6.27703700 & 4.30245500 & -1.70007100 \\
\hline $\mathrm{H}$ & 6.31742600 & 5.26916200 & -0.22231300 \\
\hline $\mathrm{C}$ & 7.60344300 & 1.51845400 & -0.32008200 \\
\hline $\mathrm{H}$ & 5.78670300 & 1.69822400 & -1.48735000 \\
\hline $\mathrm{H}$ & 5.52859400 & 1.02705500 & 0.12483300 \\
\hline $\mathrm{C}$ & 8.42900200 & 2.60034300 & -1.02755800 \\
\hline $\mathrm{H}$ & 8.63282600 & 4.77003400 & -1.07261300 \\
\hline $\mathrm{H}$ & 8.37376100 & 4.09436000 & 0.53921100 \\
\hline $\mathrm{H}$ & 7.83741300 & 0.52305800 & -0.73337900 \\
\hline $\mathrm{H}$ & 7.88878600 & 1.48506000 & 0.74786100 \\
\hline $\mathrm{H}$ & 9.50733200 & 2.41004100 & -0.89343300 \\
\hline $\mathrm{H}$ & 8.23793300 & 2.54878000 & -2.11586900 \\
\hline $\mathrm{C}$ & 1.37298000 & 4.10779400 & 2.48852700 \\
\hline $\mathrm{C}$ & 1.79388300 & 5.42566500 & 3.18046500 \\
\hline $\mathrm{C}$ & 1.52763600 & 2.91934000 & 3.46293700 \\
\hline
\end{tabular}




\begin{tabular}{|c|c|c|c|c|c|c|c|}
\hline $\mathrm{H}$ & 0.29726400 & 4.20309100 & 2.27696400 & $\mathrm{O}$ & 4.06206100 & -4.99641500 & 1.04813900 \\
\hline $\mathrm{C}$ & 1.01699600 & 5.65348800 & 4.48455900 & $\mathrm{C}$ & 4.63922500 & -2.09468600 & 1.14055900 \\
\hline $\mathrm{H}$ & 2.87686800 & 5.39421000 & 3.39875100 & $\mathrm{H}$ & 4.73749100 & -1.07021700 & 1.53370100 \\
\hline $\mathrm{H}$ & 1.64235600 & 6.27321800 & 2.49089800 & $\mathrm{H}$ & 5.50547500 & -2.67907000 & 1.47850200 \\
\hline $\mathrm{C}$ & 0.74427500 & 3.14860900 & 4.76145600 & $\mathrm{H}$ & 4.69143200 & -2.05418900 & 0.04660900 \\
\hline $\mathrm{H}$ & 2.59579100 & 2.78005500 & 3.70862900 & $\mathrm{C}$ & 3.31307300 & -2.70101500 & 3.22173500 \\
\hline $\mathrm{H}$ & 1.19677100 & 1.99220600 & 2.97135000 & $\mathrm{H}$ & 3.17140700 & -1.64723000 & 3.49502800 \\
\hline $\mathrm{C}$ & 1.14824100 & 4.46249300 & 5.44231000 & $\mathrm{H}$ & 2.41703200 & -3.23274600 & 3.57717100 \\
\hline $\mathrm{H}$ & 1.36289300 & 6.58049500 & 4.97304300 & $\mathrm{C}$ & 4.52609700 & -3.25698700 & 3.93612600 \\
\hline $\mathrm{H}$ & -0.05135800 & 5.81005600 & 4.24532500 & $\mathrm{C}$ & 5.54994400 & -2.39854100 & 4.37159700 \\
\hline $\mathrm{H}$ & 0.89470000 & 2.29823200 & 5.44780300 & $\mathrm{C}$ & 4.65323000 & -4.62816400 & 4.21511700 \\
\hline $\mathrm{H}$ & -0.33710900 & 3.17268100 & 4.53236000 & $\mathrm{C}$ & 6.66732000 & -2.89045500 & 5.05151600 \\
\hline $\mathrm{H}$ & 0.54056500 & 4.63084000 & 6.34768500 & $\mathrm{H}$ & 5.46426500 & -1.32517600 & 4.18066300 \\
\hline $\mathrm{H}$ & 2.19788100 & 4.38638800 & 5.78310800 & $\mathrm{C}$ & 5.76765900 & -5.12515400 & 4.89545000 \\
\hline $\mathrm{O}$ & -0.81167900 & -0.24688200 & 1.61470600 & $\mathrm{H}$ & 3.86963200 & -5.32017000 & 3.89753300 \\
\hline $\mathrm{H}$ & 0.80638900 & -0.73003200 & 1.88239700 & $\mathrm{C}$ & 6.78114600 & -4.25842100 & 5.31457100 \\
\hline $\mathrm{C}$ & 6.23284300 & -4.60033900 & -2.18374100 & $\mathrm{H}$ & 7.44879600 & -2.20103700 & 5.38221900 \\
\hline $\mathrm{C}$ & 5.03464900 & -3.98717100 & -1.88095500 & $\mathrm{H}$ & 5.84308900 & -6.19621600 & 5.10094900 \\
\hline $\mathrm{C}$ & 4.62254300 & -2.80358200 & -2.55640800 & $\mathrm{H}$ & 7.65223000 & -4.64662900 & 5.84880000 \\
\hline $\mathrm{C}$ & 5.49438600 & -2.26142900 & -3.56666400 & & & & \\
\hline $\mathrm{C}$ & 6.72250200 & -2.92287300 & -3.85316800 & \multicolumn{2}{|c|}{ int-2b-R-R } & & \\
\hline $\mathrm{C}$ & 7.08703200 & -4.06826500 & -3.18029200 & $\mathrm{C}$ & -2.31566300 & -2.71675800 & -0.10631300 \\
\hline $\mathrm{H}$ & 6.52475800 & -5.50128000 & -1.63876300 & $\mathrm{C}$ & -1.64083800 & -3.92131700 & -0.26282000 \\
\hline $\mathrm{H}$ & 4.40645100 & -4.40709200 & -1.09995000 & $\mathrm{C}$ & -2.23558400 & -4.93145800 & -1.10056500 \\
\hline $\mathrm{C}$ & 3.38451100 & -2.12501200 & -2.29129900 & $\mathrm{C}$ & -3.55840700 & -4.71589600 & -1.61037800 \\
\hline $\mathrm{C}$ & 5.11859200 & -1.08142900 & -4.26293600 & $\mathrm{C}$ & -4.23549100 & -3.50652200 & -1.30209500 \\
\hline $\mathrm{H}$ & 7.37347400 & -2.50058800 & -4.62360500 & $\mathrm{C}$ & -3.63820000 & -2.48506200 & -0.58780100 \\
\hline $\mathrm{H}$ & 8.03305300 & -4.56492900 & -3.41028400 & $\mathrm{H}$ & -0.53772500 & -6.29391300 & -1.13513500 \\
\hline $\mathrm{C}$ & 3.92460700 & -0.45444400 & -3.98604500 & $\mathrm{C}$ & -1.55756400 & -6.12541800 & -1.48097400 \\
\hline $\mathrm{C}$ & 3.05490700 & -0.98422800 & -3.00493100 & $\mathrm{C}$ & -4.16402800 & -5.71189200 & -2.42802400 \\
\hline $\mathrm{H}$ & 5.79071700 & -0.68442400 & -5.02831700 & $\mathrm{H}$ & -5.25743100 & -3.37153000 & -1.66396200 \\
\hline $\mathrm{H}$ & 3.63061800 & 0.44315700 & -4.53334700 & $\mathrm{C}$ & -3.48566500 & -6.86385300 & -2.76185200 \\
\hline $\mathrm{H}$ & 2.09884100 & -0.49137700 & -2.81994400 & $\mathrm{C}$ & -2.16434900 & -7.06263600 & -2.29208800 \\
\hline $\mathrm{N}$ & 2.43800300 & -2.64439300 & -1.30959200 & $\mathrm{H}$ & -5.17777400 & -5.53730000 & -2.79836900 \\
\hline $\mathrm{C}$ & 1.34965900 & -3.34529300 & -1.94641400 & $\mathrm{H}$ & -3.95780100 & -7.61757900 & -3.39676900 \\
\hline $\mathrm{C}$ & 1.64740600 & -4.56323200 & -2.58730300 & $\mathrm{H}$ & -1.61812300 & -7.96515500 & -2.57757700 \\
\hline $\mathrm{C}$ & 0.02795100 & -2.87635900 & -1.98051300 & $\mathrm{C}$ & 2.04957500 & -3.52427700 & 0.70625700 \\
\hline $\mathrm{C}$ & 0.65349900 & -5.27858600 & -3.25488000 & $\mathrm{C}$ & 2.24137000 & -4.67090600 & 1.45396600 \\
\hline $\mathrm{H}$ & 2.66658400 & -4.94817300 & -2.56407600 & $\mathrm{C}$ & 1.17522000 & -5.54042900 & 1.80289600 \\
\hline $\mathrm{C}$ & -0.96644200 & -3.60760900 & -2.63905900 & $\mathrm{C}$ & -0.13963700 & -5.27527300 & 1.29795900 \\
\hline $\mathrm{H}$ & -0.23944600 & -1.93603200 & -1.50806700 & $\mathrm{C}$ & -0.33400300 & -4.14873400 & 0.42129800 \\
\hline $\mathrm{C}$ & -0.66307100 & -4.80799200 & -3.28368300 & $\mathrm{C}$ & 0.72902400 & -3.27313500 & 0.22570500 \\
\hline $\mathrm{H}$ & 0.91303900 & -6.21894100 & -3.74803500 & $\mathrm{H}$ & 2.39844300 & -6.85865700 & 3.01742800 \\
\hline $\mathrm{H}$ & -1.98815900 & -3.22082500 & -2.64449200 & $\mathrm{H}$ & 3.24711000 & -4.91187300 & 1.80498000 \\
\hline $\mathrm{H}$ & -1.44310300 & -5.37225800 & -3.79979500 & $\mathrm{C}$ & 1.38655900 & -6.66328300 & 2.65242500 \\
\hline $\mathrm{N}$ & 2.04333100 & -1.63426900 & -0.33298600 & $\mathrm{C}$ & -1.20180600 & -6.12757000 & 1.71692400 \\
\hline $\mathrm{H}$ & 2.77426700 & -0.92047500 & -0.37187800 & $\mathrm{C}$ & -0.96904100 & -7.19576700 & 2.55912500 \\
\hline $\mathrm{H}$ & 0.77172400 & -0.46266700 & -0.57802100 & $\mathrm{C}$ & 0.33912500 & -7.47834100 & 3.02281400 \\
\hline $\mathrm{C}$ & 1.67842600 & -4.52716000 & 1.17406700 & $\mathrm{H}$ & -2.21645600 & -5.92383300 & 1.37503500 \\
\hline $\mathrm{C}$ & 0.95066800 & -3.18468500 & 1.33639200 & $\mathrm{H}$ & -1.80326200 & -7.82800800 & 2.87341900 \\
\hline $\mathrm{C}$ & 3.34852700 & -2.73465800 & 1.64049400 & $\mathrm{H}$ & 0.51120800 & -8.33297300 & 3.68170100 \\
\hline $\mathrm{H}$ & 1.42497600 & -5.26137600 & 1.95596800 & $\mathrm{O}$ & 0.52929800 & -2.13389600 & -0.54281000 \\
\hline $\mathrm{H}$ & 1.47907200 & -5.01972100 & 0.21064900 & $\mathrm{O}$ & -1.69663900 & -1.69756500 & 0.61201200 \\
\hline $\mathrm{H}$ & 0.56236100 & -3.06642400 & 2.35738700 & $\mathrm{P}$ & -0.43291700 & -0.91325000 & -0.04743300 \\
\hline $\mathrm{H}$ & 0.09785100 & -3.06363700 & 0.65851500 & $\mathrm{O}$ & -0.71678700 & 0.00976100 & -1.17632400 \\
\hline $\mathrm{C}$ & 2.01680100 & -2.07857700 & 1.12389200 & $\mathrm{C}$ & -4.38396300 & -1.21403400 & -0.30269500 \\
\hline $\mathrm{O}$ & 1.77547900 & -0.89900600 & 1.81505200 & $\mathrm{C}$ & -4.90568800 & -0.98435500 & 0.99528400 \\
\hline $\mathrm{C}$ & 3.17041100 & -4.20262500 & 1.24356900 & $\mathrm{C}$ & -4.63214400 & -0.27953600 & -1.34174100 \\
\hline & & & & & & & \\
\hline
\end{tabular}




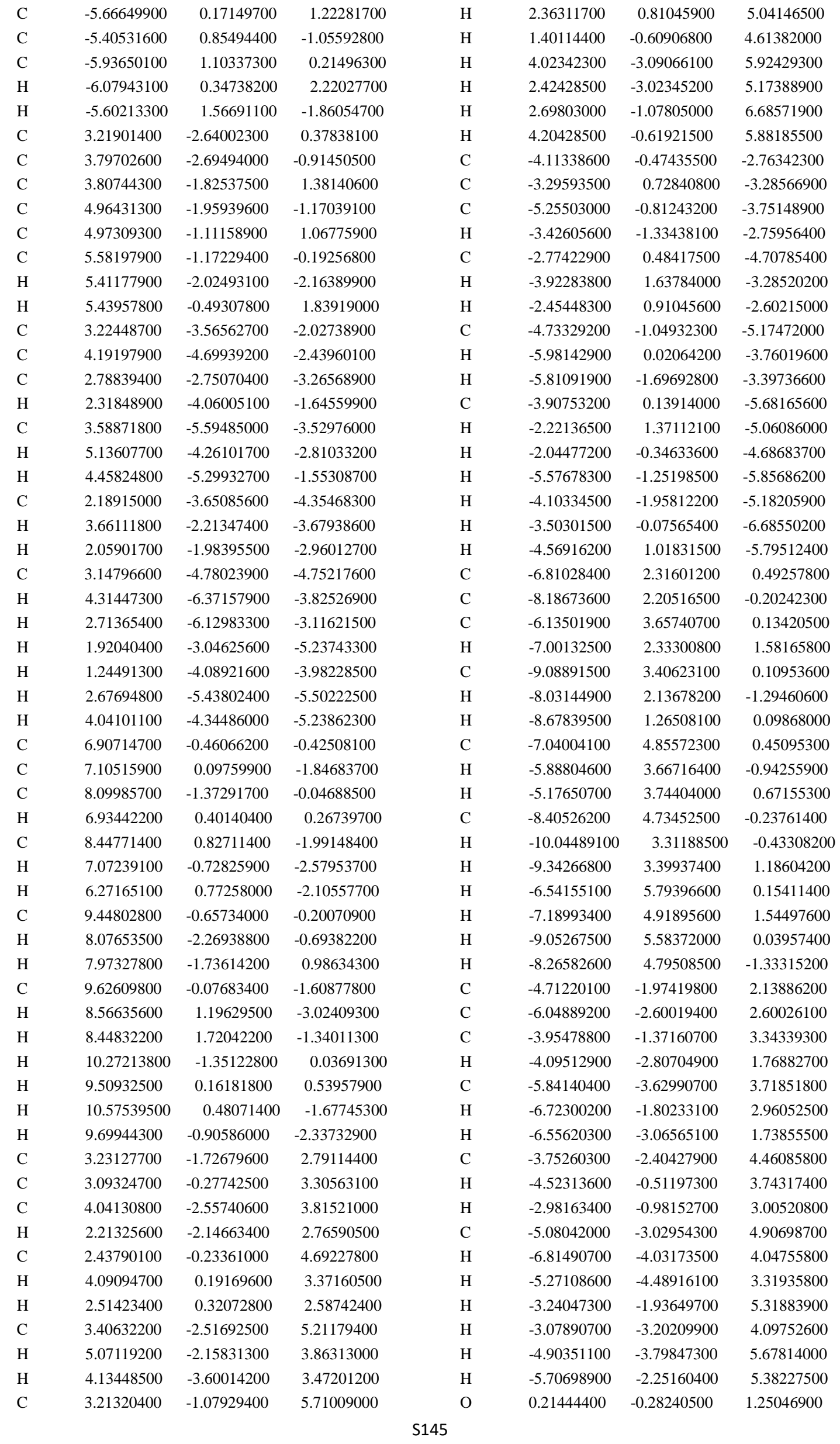




\begin{tabular}{|c|c|c|c|c|c|c|c|}
\hline $\mathrm{H}$ & 0.63320000 & 0.61952800 & 1.10311300 & $\mathrm{C}$ & 7.50285600 & 5.62523600 & 1.12484800 \\
\hline $\mathrm{C}$ & -0.68596400 & 7.09823200 & 2.49441300 & $\mathrm{H}$ & 8.18491200 & 4.72435700 & -0.72059100 \\
\hline $\mathrm{C}$ & -0.57215600 & 6.16118100 & 1.48788900 & $\mathrm{H}$ & 6.55474200 & 6.33343500 & 2.93636400 \\
\hline $\mathrm{C}$ & -1.20091500 & 4.88751700 & 1.59416600 & $\mathrm{H}$ & 8.39625600 & 6.24213700 & 1.25129000 \\
\hline $\mathrm{C}$ & -1.96872900 & 4.60373100 & 2.77707800 & & & & \\
\hline $\mathrm{C}$ & -2.06070500 & 5.59398100 & 3.79665300 & ts-3-R-R & & & \\
\hline $\mathrm{C}$ & -1.43516500 & 6.81428200 & 3.66272200 & $\mathrm{C}$ & 5.35122800 & 5.45717600 & -0.70308000 \\
\hline $\mathrm{H}$ & -0.19548300 & 8.06901900 & 2.38771700 & $\mathrm{C}$ & 4.41114700 & 4.61388300 & -0.14746200 \\
\hline $\mathrm{H}$ & -0.00288900 & 6.39740200 & 0.59110300 & $\mathrm{C}$ & 4.04302600 & 3.39771400 & -0.79001400 \\
\hline $\mathrm{C}$ & -1.11435400 & 3.87712600 & 0.56990400 & $\mathrm{C}$ & 4.67163800 & 3.07014000 & -2.04053800 \\
\hline $\mathrm{C}$ & -2.61782300 & 3.34491000 & 2.90925400 & $\mathrm{C}$ & 5.63540400 & 3.96593100 & -2.58519700 \\
\hline $\mathrm{H}$ & -2.64546600 & 5.36622400 & 4.69207900 & $\mathrm{C}$ & 5.97267200 & 5.13201000 & -1.93340200 \\
\hline $\mathrm{H}$ & -1.51756900 & 7.56517900 & 4.45254100 & $\mathrm{H}$ & 5.61691200 & 6.38534100 & -0.19137400 \\
\hline $\mathrm{C}$ & -2.52915200 & 2.40397400 & 1.90710300 & $\mathrm{H}$ & 3.94651400 & 4.87384900 & 0.80329300 \\
\hline $\mathrm{C}$ & -1.78511800 & 2.67541300 & 0.73468100 & $\mathrm{C}$ & 3.07585100 & 2.47937600 & -0.25036400 \\
\hline $\mathrm{H}$ & -3.19201400 & 3.13960900 & 3.81648700 & $\mathrm{C}$ & 4.31868000 & 1.86697700 & -2.71311400 \\
\hline $\mathrm{H}$ & -3.04176800 & 1.44457600 & 1.99752600 & $\mathrm{H}$ & 6.10678300 & 3.70725500 & -3.53712800 \\
\hline $\mathrm{H}$ & -1.73426600 & 1.91343600 & -0.04391900 & $\mathrm{H}$ & 6.71546100 & 5.80838600 & -2.36326800 \\
\hline $\mathrm{N}$ & -0.31482700 & 4.11676500 & -0.60261800 & $\mathrm{C}$ & 3.40321100 & 0.99970700 & -2.16023600 \\
\hline $\mathrm{C}$ & -0.80475200 & 4.99400100 & -1.61058600 & $\mathrm{C}$ & 2.79293200 & 1.29984100 & -0.91850500 \\
\hline $\mathrm{C}$ & -0.02793700 & 5.27519000 & -2.75250800 & $\mathrm{H}$ & 4.79588400 & 1.63732200 & -3.66916000 \\
\hline $\mathrm{C}$ & -2.06786600 & 5.60625200 & -1.49384600 & $\mathrm{H}$ & 3.15024000 & 0.06221700 & -2.65747400 \\
\hline $\mathrm{C}$ & -0.49702200 & 6.15435300 & -3.72899600 & $\mathrm{H}$ & 2.10294600 & 0.58334600 & -0.47361100 \\
\hline $\mathrm{H}$ & 0.94167500 & 4.79693000 & -2.87570900 & $\mathrm{~N}$ & 2.37951200 & 2.81580200 & 0.96461700 \\
\hline $\mathrm{C}$ & -2.52258200 & 6.48844100 & -2.47690200 & $\mathrm{C}$ & 3.06088500 & 2.75212900 & 2.21982400 \\
\hline $\mathrm{H}$ & -2.70422000 & 5.38965500 & -0.63611400 & $\mathrm{C}$ & 2.37301000 & 3.03759000 & 3.41457600 \\
\hline $\mathrm{C}$ & -1.74447500 & 6.77296000 & -3.60103900 & $\mathrm{C}$ & 4.42372700 & 2.41183300 & 2.29508000 \\
\hline $\mathrm{H}$ & 0.12894100 & 6.35647100 & -4.60221200 & $\mathrm{C}$ & 3.03919200 & 3.00993800 & 4.64063100 \\
\hline $\mathrm{H}$ & -3.50739400 & 6.94895900 & -2.36031000 & $\mathrm{H}$ & 1.30970800 & 3.26796100 & 3.38479300 \\
\hline $\mathrm{H}$ & -2.10587700 & 7.46121500 & -4.36857700 & $\mathrm{C}$ & 5.08108000 & 2.39765200 & 3.52744100 \\
\hline $\mathrm{N}$ & 0.49419900 & 3.07429000 & -1.08762100 & $\mathrm{H}$ & 4.97411500 & 2.14564500 & 1.39400600 \\
\hline $\mathrm{H}$ & -0.06004700 & 2.26526300 & -1.38261200 & $\mathrm{C}$ & 4.40017400 & 2.69948500 & 4.70901000 \\
\hline $\mathrm{C}$ & 3.10620800 & 2.15693100 & -2.29588500 & $\mathrm{H}$ & 2.48082600 & 3.23490100 & 5.55305200 \\
\hline $\mathrm{C}$ & 2.25837300 & 1.47504300 & -1.21680600 & $\mathrm{H}$ & 6.14110400 & 2.13241500 & 3.55766700 \\
\hline $\mathrm{C}$ & 2.84077000 & 3.68914400 & -0.33234500 & $\mathrm{H}$ & 4.91886000 & 2.68191100 & 5.67004400 \\
\hline $\mathrm{H}$ & 4.05992900 & 1.64361300 & -2.49680100 & $\mathrm{~N}$ & 1.03461200 & 2.42604400 & 1.05002600 \\
\hline $\mathrm{H}$ & 2.58609800 & 2.24397900 & -3.26213600 & $\mathrm{H}$ & 0.85579200 & 1.42180200 & 1.26069300 \\
\hline $\mathrm{H}$ & 2.86549100 & 0.83081100 & -0.56656300 & $\mathrm{C}$ & -2.38319300 & 3.59220700 & 0.38154100 \\
\hline $\mathrm{H}$ & 1.45433600 & 0.85375000 & -1.62988900 & $\mathrm{C}$ & -1.37433900 & 2.63531500 & 1.00792300 \\
\hline $\mathrm{C}$ & 1.67275100 & 2.63353700 & -0.37231000 & $\mathrm{C}$ & -0.07272200 & 4.60876600 & 0.24579500 \\
\hline $\mathrm{O}$ & 1.35903200 & 2.10257800 & 0.94363800 & $\mathrm{H}$ & -2.85485300 & 3.17024400 & -0.51886600 \\
\hline $\mathrm{C}$ & 3.37292800 & 3.56667100 & -1.77277700 & $\mathrm{H}$ & -3.20295500 & 3.88315200 & 1.05328500 \\
\hline $\mathrm{O}$ & 3.90556600 & 4.45287500 & -2.39408000 & $\mathrm{H}$ & -1.57241400 & 1.57976000 & 0.79990900 \\
\hline $\mathrm{H}$ & 0.85743400 & 2.76999400 & 1.43888500 & $\mathrm{H}$ & -1.34717500 & 2.73864000 & 2.10375200 \\
\hline $\mathrm{C}$ & 3.96089000 & 3.16197400 & 0.64547500 & $\mathrm{C}$ & -0.00446000 & 3.07992800 & 0.48603100 \\
\hline $\mathrm{H}$ & 3.48549500 & 3.04204100 & 1.62908600 & $\mathrm{O}$ & -0.22066200 & 2.47031200 & -1.21629200 \\
\hline $\mathrm{H}$ & 4.27098700 & 2.15149100 & 0.34033800 & $\mathrm{C}$ & -1.58804100 & 4.81792400 & -0.03223200 \\
\hline $\mathrm{C}$ & 2.49701300 & 5.13607900 & 0.00863700 & $\mathrm{O}$ & -2.06034400 & 5.83049400 & -0.48179500 \\
\hline $\mathrm{H}$ & 1.75905300 & 5.55540600 & -0.68279500 & $\mathrm{C}$ & 0.21942100 & 5.33053500 & 1.58365500 \\
\hline $\mathrm{H}$ & 2.11263500 & 5.22792800 & 1.03556300 & $\mathrm{H}$ & -0.01910700 & 6.39656100 & 1.47517000 \\
\hline $\mathrm{H}$ & 3.40568600 & 5.74780200 & -0.07291000 & $\mathrm{H}$ & -0.39025900 & 4.92991100 & 2.40770700 \\
\hline $\mathrm{C}$ & 5.19344000 & 4.02908600 & 0.79530900 & $\mathrm{C}$ & 0.80301700 & 5.16278100 & -0.92020200 \\
\hline $\mathrm{C}$ & 6.24252100 & 3.98495900 & -0.13853600 & $\mathrm{H}$ & 1.82139300 & 4.77960700 & -0.78183600 \\
\hline $\mathrm{C}$ & 5.33109600 & 4.88581800 & 1.90023500 & $\mathrm{H}$ & 0.41855700 & 4.73845800 & -1.85781500 \\
\hline $\mathrm{C}$ & 7.38410800 & 4.77462800 & 0.02180000 & $\mathrm{H}$ & 1.27712400 & 5.23018700 & 1.86141500 \\
\hline $\mathrm{H}$ & 6.16895800 & 3.32588500 & -1.00649600 & $\mathrm{C}$ & 0.88913500 & 6.67122700 & -1.08551400 \\
\hline $\mathrm{C}$ & 6.47140400 & 5.67682000 & 2.06623600 & $\mathrm{C}$ & 0.07955100 & 7.34508700 & -2.01430200 \\
\hline $\mathrm{H}$ & 4.53252600 & 4.92899100 & 2.64645600 & $\mathrm{C}$ & 1.82516600 & 7.42597700 & -0.35853800 \\
\hline
\end{tabular}




\begin{tabular}{|c|c|c|c|c|c|c|c|}
\hline $\mathrm{C}$ & 0.19153300 & 8.72449400 & -2.20093800 & $\mathrm{C}$ & -4.75542200 & -1.37886600 & 2.10510300 \\
\hline $\mathrm{H}$ & -0.65667000 & 6.78212200 & -2.59106700 & $\mathrm{C}$ & -6.10400800 & -1.89555900 & 2.65678300 \\
\hline $\mathrm{C}$ & 1.93657300 & 8.80812300 & -0.53659000 & $\mathrm{C}$ & -3.83943200 & -0.91464400 & 3.26067400 \\
\hline $\mathrm{H}$ & 2.48839600 & 6.92546600 & 0.35016500 & $\mathrm{H}$ & -4.26012000 & -2.24706200 & 1.64576300 \\
\hline $\mathrm{C}$ & 1.11801800 & 9.46379700 & -1.45942200 & $\mathrm{C}$ & -5.90452900 & -2.99801000 & 3.70463100 \\
\hline $\mathrm{H}$ & -0.45113300 & 9.22497100 & -2.92994800 & $\mathrm{H}$ & -6.66310000 & -1.05891600 & 3.11337500 \\
\hline $\mathrm{H}$ & 2.67305300 & 9.37192100 & 0.04227200 & $\mathrm{H}$ & -6.72731000 & -2.26517500 & 1.82514800 \\
\hline $\mathrm{H}$ & 1.20547500 & 10.54364900 & -1.60490900 & $\mathrm{C}$ & -3.65037000 & -2.01616700 & 4.31221700 \\
\hline $\mathrm{C}$ & 0.25108400 & -3.41253600 & -0.11178500 & $\mathrm{H}$ & -4.28022900 & -0.02253100 & 3.74259600 \\
\hline $\mathrm{C}$ & -0.94551500 & -4.10997000 & 0.02362900 & $\mathrm{H}$ & -2.86385000 & -0.61022300 & 2.85037600 \\
\hline $\mathrm{C}$ & -0.94455700 & -5.33811600 & 0.77738700 & $\mathrm{C}$ & -4.99153000 & -2.53443600 & 4.84660000 \\
\hline $\mathrm{C}$ & 0.30748300 & -5.87935700 & 1.21843300 & $\mathrm{H}$ & -6.88138200 & -3.32474700 & 4.10042500 \\
\hline $\mathrm{C}$ & 1.50585900 & -5.17515700 & 0.93337700 & $\mathrm{H}$ & -5.45508300 & -3.88378100 & 3.21871600 \\
\hline $\mathrm{C}$ & 1.50916100 & -3.94173200 & 0.30948900 & $\mathrm{H}$ & -3.02515400 & -1.64208400 & 5.14077500 \\
\hline $\mathrm{H}$ & -3.10179300 & -5.62139600 & 0.84665900 & $\mathrm{H}$ & -3.09069300 & -2.85451200 & 3.85866000 \\
\hline $\mathrm{C}$ & -2.13550100 & -6.03330300 & 1.13725200 & $\mathrm{H}$ & -4.82793700 & -3.35548300 & 5.56522300 \\
\hline $\mathrm{C}$ & 0.32564000 & -7.10438500 & 1.94405500 & $\mathrm{H}$ & -5.49687600 & -1.72609100 & 5.40810700 \\
\hline $\mathrm{H}$ & 2.45699200 & -5.61477200 & 1.24328400 & $\mathrm{C}$ & -6.64920600 & 3.13044000 & 0.76270800 \\
\hline $\mathrm{C}$ & -0.84466400 & -7.76104200 & 2.25676400 & $\mathrm{C}$ & -6.29523600 & 3.78109100 & 2.11510300 \\
\hline $\mathrm{C}$ & -2.08678300 & -7.20881300 & 1.85873500 & $\mathrm{C}$ & -8.16984700 & 2.85148300 & 0.69516000 \\
\hline $\mathrm{H}$ & 1.29092800 & -7.50858500 & 2.26105600 & $\mathrm{H}$ & -6.42719600 & 3.87750100 & -0.02196900 \\
\hline $\mathrm{H}$ & -0.81896100 & -8.69674300 & 2.82081200 & $\mathrm{C}$ & -7.11734000 & 5.05195700 & 2.36849200 \\
\hline $\mathrm{H}$ & -3.01657800 & -7.71575500 & 2.12910100 & $\mathrm{H}$ & -6.48715400 & 3.06421900 & 2.93325100 \\
\hline $\mathrm{C}$ & -3.93496900 & -1.83339900 & -0.72617900 & $\mathrm{H}$ & -5.21665300 & 4.00925000 & 2.15177000 \\
\hline $\mathrm{C}$ & -4.70842400 & -2.67567200 & -1.50298300 & $\mathrm{C}$ & -9.00155200 & 4.11323800 & 0.95932100 \\
\hline $\mathrm{C}$ & -4.24409100 & -3.93960500 & -1.95022500 & $\mathrm{H}$ & -8.42067300 & 2.07675000 & 1.44289600 \\
\hline $\mathrm{C}$ & -2.96209800 & -4.40729100 & -1.51275100 & $\mathrm{H}$ & -8.42283900 & 2.42330300 & -0.28876900 \\
\hline $\mathrm{C}$ & -2.19657100 & -3.59302000 & -0.60362500 & $\mathrm{C}$ & -8.62392700 & 4.77492900 & 2.29052000 \\
\hline $\mathrm{C}$ & -2.65205700 & -2.30945600 & -0.31509500 & $\mathrm{H}$ & -6.85646100 & 5.48096100 & 3.35076300 \\
\hline $\mathrm{H}$ & -6.00398400 & -4.37684000 & -3.14193300 & $\mathrm{H}$ & -6.84739400 & 5.81552900 & 1.61574700 \\
\hline $\mathrm{H}$ & -5.70749400 & -2.35115100 & -1.80215700 & $\mathrm{H}$ & -10.07682500 & 3.86692800 & 0.94355800 \\
\hline $\mathrm{C}$ & -5.02231400 & -4.74396200 & -2.83022800 & $\mathrm{H}$ & -8.83724800 & 4.83391700 & 0.13680100 \\
\hline $\mathrm{C}$ & -2.49422000 & -5.65076700 & -2.02852900 & $\mathrm{H}$ & -9.19387100 & 5.70884400 & 2.43125600 \\
\hline $\mathrm{C}$ & -3.26279200 & -6.39845900 & -2.89746000 & $\mathrm{H}$ & -8.91246700 & 4.10618700 & 3.12284800 \\
\hline $\mathrm{C}$ & -4.54665800 & -5.95097700 & -3.29455200 & $\mathrm{C}$ & -4.20529100 & 0.39328500 & -2.70740400 \\
\hline $\mathrm{H}$ & -1.50625300 & -6.01022500 & -1.74071200 & $\mathrm{C}$ & -3.34539600 & 1.58795200 & -3.17558900 \\
\hline $\mathrm{H}$ & -2.87471800 & -7.34371800 & -3.28543600 & $\mathrm{C}$ & -5.36963700 & 0.16958500 & -3.70094700 \\
\hline $\mathrm{H}$ & -5.14922700 & -6.55669500 & -3.97598000 & $\mathrm{H}$ & -3.55357200 & -0.49223600 & -2.76142400 \\
\hline $\mathrm{O}$ & -1.87475500 & -1.48308400 & 0.47196500 & $\mathrm{C}$ & -2.83560000 & 1.39029500 & -4.60948900 \\
\hline $\mathrm{O}$ & 0.24411400 & -2.19180300 & -0.75769800 & $\mathrm{H}$ & -3.93995200 & 2.51844500 & -3.13336200 \\
\hline $\mathrm{P}$ & -0.45226400 & -0.85930000 & -0.08604000 & $\mathrm{H}$ & -2.49426700 & 1.71804000 & -2.49203500 \\
\hline $\mathrm{O}$ & 0.30175600 & -0.31479900 & 1.09440800 & $\mathrm{C}$ & -4.86835400 & -0.02289900 & -5.13822600 \\
\hline $\mathrm{C}$ & 2.80611800 & -3.22750500 & 0.06743500 & $\mathrm{H}$ & -6.04941400 & 1.04063800 & -3.66093300 \\
\hline $\mathrm{C}$ & 3.35032600 & -3.17175300 & -1.24039400 & $\mathrm{H}$ & -5.97086100 & -0.70021100 & -3.39070600 \\
\hline $\mathrm{C}$ & 3.53070200 & -2.68133600 & 1.15881200 & $\mathrm{C}$ & -3.98584000 & 1.14496600 & -5.59383400 \\
\hline $\mathrm{C}$ & 4.60858800 & -2.58054800 & -1.42381100 & $\mathrm{H}$ & -2.24401100 & 2.26782600 & -4.92220200 \\
\hline $\mathrm{C}$ & 4.78667900 & -2.10640100 & 0.91541400 & $\mathrm{H}$ & -2.14470100 & 0.52773900 & -4.62844200 \\
\hline $\mathrm{C}$ & 5.35103000 & -2.04840000 & -0.36397500 & $\mathrm{H}$ & -5.72448200 & -0.14791800 & -5.82318400 \\
\hline $\mathrm{H}$ & 5.03794600 & -2.54549300 & -2.42928200 & $\mathrm{H}$ & -4.28648900 & -0.96122400 & -5.19397100 \\
\hline $\mathrm{H}$ & 5.34246900 & -1.69445900 & 1.76121500 & $\mathrm{H}$ & -3.59294200 & 0.95654500 & -6.60751800 \\
\hline $\mathrm{C}$ & -4.49148500 & -0.50371400 & -0.30477400 & $\mathrm{H}$ & -4.60347700 & 2.05992400 & -5.66520600 \\
\hline $\mathrm{C}$ & -4.93394600 & -0.31398800 & 1.02935300 & $\mathrm{C}$ & 3.00074200 & -2.70580600 & 2.58951300 \\
\hline $\mathrm{C}$ & -4.68853700 & 0.52287800 & -1.26522000 & $\mathrm{C}$ & 2.86700300 & -1.29604200 & 3.20629000 \\
\hline $\mathrm{C}$ & -5.59597100 & 0.87660600 & 1.36426300 & $\mathrm{C}$ & 3.84320200 & -3.61817200 & 3.51087400 \\
\hline $\mathrm{C}$ & -5.36560000 & 1.68811400 & -0.87532000 & $\mathrm{H}$ & 1.98444900 & -3.12650600 & 2.56693700 \\
\hline $\mathrm{C}$ & -5.84168800 & 1.88578200 & 0.42640100 & $\mathrm{C}$ & 2.30398000 & -1.35423400 & 4.63246700 \\
\hline $\mathrm{H}$ & -5.95070700 & 1.00400200 & 2.38937500 & $\mathrm{H}$ & 3.85322900 & -0.79932100 & 3.22780900 \\
\hline $\mathrm{H}$ & -5.53844300 & 2.47383500 & -1.61628900 & $\mathrm{H}$ & 2.20970300 & -0.69040700 & 2.56590300 \\
\hline
\end{tabular}




\begin{tabular}{|c|c|c|c|c|c|c|c|}
\hline $\mathrm{C}$ & 3.27866900 & -3.67109400 & 4.93663600 & $\mathrm{C}$ & 4.20681700 & 3.03111300 & -2.53737500 \\
\hline $\mathrm{H}$ & 4.88211700 & -3.24230100 & 3.54370600 & $\mathrm{H}$ & 5.78094500 & 5.20011800 & -2.82896600 \\
\hline $\mathrm{H}$ & 3.90016800 & -4.63431700 & 3.08513300 & $\mathrm{H}$ & 5.97901500 & 7.10851900 & -1.25374700 \\
\hline $\mathrm{C}$ & 3.13516300 & -2.26870500 & 5.54055600 & $\mathrm{C}$ & 3.36984300 & 1.97591500 & -2.25390100 \\
\hline $\mathrm{H}$ & 2.25339300 & -0.33653000 & 5.05476700 & $\mathrm{C}$ & 2.61548900 & 1.96239600 & -1.05732600 \\
\hline $\mathrm{H}$ & 1.26368500 & -1.72683300 & 4.59406900 & $\mathrm{H}$ & 4.79343200 & 3.04449900 & -3.45953300 \\
\hline $\mathrm{H}$ & 3.92107000 & -4.30249200 & 5.57445800 & $\mathrm{H}$ & 3.28448600 & 1.12985600 & -2.93865500 \\
\hline $\mathrm{H}$ & 2.28693400 & -4.15975800 & 4.91491000 & $\mathrm{H}$ & 1.97533900 & 1.10876600 & -0.83814400 \\
\hline $\mathrm{H}$ & 2.68475100 & -2.32886300 & 6.54629100 & $\mathrm{~N}$ & 1.85504700 & 3.02807800 & 1.01382000 \\
\hline $\mathrm{H}$ & 4.14096700 & -1.82838000 & 5.67636100 & $\mathrm{C}$ & 2.40321600 & 2.77770300 & 2.30902100 \\
\hline $\mathrm{C}$ & 6.73360200 & -1.46266200 & -0.60361300 & $\mathrm{C}$ & 1.56334500 & 2.76854200 & 3.43731700 \\
\hline $\mathrm{C}$ & 7.85450000 & -2.34684300 & -0.01031500 & $\mathrm{C}$ & 3.78428600 & 2.59042800 & 2.48744500 \\
\hline $\mathrm{C}$ & 6.87875300 & -0.01038600 & -0.10218500 & $\mathrm{C}$ & 2.09945200 & 2.59371800 & 4.71416600 \\
\hline $\mathrm{H}$ & 6.88885400 & -1.44100600 & -1.69828500 & $\mathrm{H}$ & 0.48669800 & 2.89365000 & 3.32291900 \\
\hline $\mathrm{C}$ & 9.25042400 & -1.77406500 & -0.29124500 & $\mathrm{C}$ & 4.30925200 & 2.43405300 & 3.77161700 \\
\hline $\mathrm{H}$ & 7.70417000 & -2.43181100 & 1.08144700 & $\mathrm{H}$ & 4.44891900 & 2.56094000 & 1.62518300 \\
\hline $\mathrm{H}$ & 7.77004800 & -3.36997100 & -0.41295800 & $\mathrm{C}$ & 3.47625100 & 2.43631500 & 4.89338400 \\
\hline $\mathrm{C}$ & 8.27383400 & 0.56055100 & -0.38968200 & $\mathrm{H}$ & 1.42817100 & 2.58615100 & 5.57646700 \\
\hline $\mathrm{H}$ & 6.69585400 & 0.01823900 & 0.98712000 & $\mathrm{H}$ & 5.38698100 & 2.29592700 & 3.88959800 \\
\hline $\mathrm{H}$ & 6.10256900 & 0.61876300 & -0.56652700 & $\mathrm{H}$ & 3.89368900 & 2.30815200 & 5.89443100 \\
\hline $\mathrm{C}$ & 9.38050200 & -0.32556400 & 0.19534800 & $\mathrm{~N}$ & 0.58059400 & 2.46228400 & 0.82270300 \\
\hline $\mathrm{H}$ & 10.02093900 & -2.40852500 & 0.17888100 & $\mathrm{H}$ & 0.50493800 & 1.37117900 & 0.84836600 \\
\hline $\mathrm{H}$ & 9.44367400 & -1.80911000 & -1.37972000 & $\mathrm{C}$ & -2.75314400 & 3.45929300 & -0.22202500 \\
\hline $\mathrm{H}$ & 8.35162700 & 1.58710800 & 0.00691700 & $\mathrm{C}$ & -1.78276800 & 2.41338700 & 0.32695900 \\
\hline $\mathrm{H}$ & 8.41440300 & 0.64242900 & -1.48366700 & $\mathrm{C}$ & -0.64638700 & 4.64633500 & 0.40483700 \\
\hline $\mathrm{H}$ & 10.37424300 & 0.07841200 & -0.06245000 & $\mathrm{H}$ & -2.70255100 & 3.46025200 & -1.32422600 \\
\hline $\mathrm{H}$ & 9.31477400 & -0.30667100 & 1.29939100 & $\mathrm{H}$ & -3.79710800 & 3.31360700 & 0.07605900 \\
\hline $\mathrm{C}$ & 2.63320800 & -3.76982600 & -2.44579700 & $\mathrm{H}$ & -1.65237500 & 1.53885400 & -0.32314200 \\
\hline $\mathrm{C}$ & 3.44156300 & -4.90475500 & -3.11514400 & $\mathrm{H}$ & -2.11400900 & 2.03260500 & 1.31045300 \\
\hline $\mathrm{C}$ & 2.22769600 & -2.70877700 & -3.49395900 & $\mathrm{C}$ & -0.48040900 & 3.14634600 & 0.53610500 \\
\hline $\mathrm{H}$ & 1.69811800 & -4.22871800 & -2.09057000 & $\mathrm{O}$ & -0.57018200 & 2.26901900 & -2.49964000 \\
\hline $\mathrm{C}$ & 2.67901000 & -5.52840900 & -4.29164500 & $\mathrm{C}$ & -2.19353300 & 4.77063500 & 0.28625700 \\
\hline $\mathrm{H}$ & 4.40669600 & -4.50773700 & -3.47809200 & $\mathrm{O}$ & -2.81334300 & 5.75585300 & 0.59158300 \\
\hline $\mathrm{H}$ & 3.68751200 & -5.67562700 & -2.36562500 & $\mathrm{C}$ & -0.14201500 & 5.47025500 & 1.59658900 \\
\hline $\mathrm{C}$ & 1.46855200 & -3.33490900 & -4.67168800 & $\mathrm{H}$ & -0.52501500 & 6.49392100 & 1.48751700 \\
\hline $\mathrm{H}$ & 3.13654500 & -2.20670600 & -3.87503600 & $\mathrm{H}$ & -0.52665700 & 5.07510700 & 2.54873300 \\
\hline $\mathrm{H}$ & 1.60778200 & -1.93812900 & -3.01001700 & $\mathrm{C}$ & -0.00732000 & 5.11754500 & -0.97063600 \\
\hline $\mathrm{C}$ & 2.26421900 & -4.47224800 & -5.32399500 & $\mathrm{H}$ & 1.07947300 & 5.02168500 & -0.85351700 \\
\hline $\mathrm{H}$ & 3.29343300 & -6.31159200 & -4.76777600 & $\mathrm{H}$ & -0.30372200 & 4.39850300 & -1.74697400 \\
\hline $\mathrm{H}$ & 1.77404000 & -6.03544500 & -3.90869300 & $\mathrm{H}$ & 0.95253700 & 5.49282700 & 1.64232900 \\
\hline $\mathrm{H}$ & 1.22384400 & -2.56002500 & -5.41805200 & $\mathrm{C}$ & -0.34410900 & 6.53100700 & -1.38750400 \\
\hline $\mathrm{H}$ & 0.50169900 & -3.72804700 & -4.30793500 & $\mathrm{C}$ & -1.45642200 & 6.79838400 & -2.20242400 \\
\hline $\mathrm{H}$ & 1.67667600 & -4.93725500 & -6.13386200 & $\mathrm{C}$ & 0.46944700 & 7.60914800 & -0.99987500 \\
\hline $\mathrm{H}$ & 3.17188000 & -4.05528200 & -5.79975300 & $\mathrm{C}$ & -1.75743600 & 8.10150500 & -2.60453100 \\
\hline $\mathrm{O}$ & -0.71667100 & 0.07750400 & -1.26578200 & $\mathrm{H}$ & -2.09360700 & 5.97384900 & -2.53356700 \\
\hline $\mathrm{H}$ & -0.44762200 & 1.38251800 & -1.21771300 & $\mathrm{C}$ & 0.17150100 & 8.91459400 & -1.39959100 \\
\hline \multirow[t]{2}{*}{$\mathrm{H}$} & 0.63616000 & 2.54639300 & -1.66644400 & $\mathrm{H}$ & 1.35767800 & 7.42067000 & -0.39120300 \\
\hline & & & & C & -0.94575400 & 9.16564000 & -2.20095300 \\
\hline int-3-R & & & & $\mathrm{H}$ & -2.62835100 & 8.28584800 & -3.23870800 \\
\hline $\mathrm{C}$ & 4.54302400 & 6.28718700 & 0.16237400 & $\mathrm{H}$ & 0.81979200 & 9.73831300 & -1.08935700 \\
\hline $\mathrm{C}$ & 3.69034900 & 5.24284100 & 0.45916800 & $\mathrm{H}$ & -1.17917800 & 10.18586700 & -2.51603400 \\
\hline $\mathrm{C}$ & 3.55506900 & 4.13134900 & -0.42059500 & $\mathrm{C}$ & 0.75193300 & -3.40585000 & -0.25944900 \\
\hline $\mathrm{C}$ & 4.32875800 & 4.12247200 & -1.63187700 & $\mathrm{C}$ & -0.34167100 & -4.25171500 & -0.08134300 \\
\hline $\mathrm{C}$ & 5.19726200 & 5.21709500 & -1.90470900 & $\mathrm{C}$ & -0.14368400 & -5.48724800 & 0.63750400 \\
\hline $\mathrm{C}$ & 5.30647500 & 6.27680500 & -1.03040200 & $\mathrm{C}$ & 1.18995300 & -5.89083000 & 0.97493400 \\
\hline $\mathrm{H}$ & 4.63254400 & 7.12734700 & 0.85564100 & $\mathrm{C}$ & 2.27337700 & -5.03373400 & 0.65278500 \\
\hline $\mathrm{H}$ & 3.12176300 & 5.26156000 & 1.38876700 & $\mathrm{C}$ & 2.08530900 & -3.78586700 & 0.09081700 \\
\hline $\mathrm{C}$ & 2.68741900 & 3.01547900 & -0.16434800 & $\mathrm{H}$ & -2.24332600 & -6.01996700 & 0.85977300 \\
\hline
\end{tabular}




\begin{tabular}{|c|c|c|c|c|c|c|c|}
\hline $\mathrm{C}$ & -1.21673200 & -6.32394100 & 1.06274800 & $\mathrm{H}$ & -3.53277700 & -3.55583000 & 5.86550900 \\
\hline $\mathrm{C}$ & 1.40203200 & -7.12330500 & 1.65547900 & $\mathrm{H}$ & -4.40636400 & -2.02560800 & 5.71746200 \\
\hline $\mathrm{H}$ & 3.28796600 & -5.35490900 & 0.90175200 & $\mathrm{C}$ & -6.68608000 & 2.36705600 & 1.04915900 \\
\hline $\mathrm{C}$ & 0.34114600 & -7.91870100 & 2.03013100 & $\mathrm{C}$ & -6.14090800 & 3.21638500 & 2.21705800 \\
\hline $\mathrm{C}$ & -0.98137400 & -7.50201300 & 1.74218600 & $\mathrm{C}$ & -8.13029600 & 1.90592100 & 1.35904000 \\
\hline $\mathrm{H}$ & 2.42825300 & -7.41881000 & 1.89061200 & $\mathrm{H}$ & -6.74733400 & 3.03294400 & 0.16821000 \\
\hline $\mathrm{H}$ & 0.51492500 & -8.85845100 & 2.56025600 & $\mathrm{C}$ & -7.06483600 & 4.39727800 & 2.54594000 \\
\hline $\mathrm{H}$ & -1.82556100 & -8.11656500 & 2.06528800 & $\mathrm{H}$ & -6.03425900 & 2.58274000 & 3.11578000 \\
\hline $\mathrm{C}$ & -3.64409800 & -2.36735300 & -0.57559100 & $\mathrm{H}$ & -5.13088700 & 3.58786300 & 1.97618700 \\
\hline $\mathrm{C}$ & -4.37433300 & -3.31503300 & -1.26813900 & $\mathrm{C}$ & -9.05476800 & 3.08347900 & 1.69413400 \\
\hline $\mathrm{C}$ & -3.79334900 & -4.51239100 & -1.76076500 & $\mathrm{H}$ & -8.10358000 & 1.20213900 & 2.21104500 \\
\hline $\mathrm{C}$ & -2.42339900 & -4.80268000 & -1.45580700 & $\mathrm{H}$ & -8.52679800 & 1.33684000 & 0.50197700 \\
\hline $\mathrm{C}$ & -1.69042900 & -3.89369100 & -0.61087700 & $\mathrm{C}$ & -8.49661500 & 3.93446900 & 2.84128200 \\
\hline $\mathrm{C}$ & -2.28080500 & -2.67746400 & -0.27810800 & $\mathrm{H}$ & -6.65969200 & 4.96512100 & 3.40037000 \\
\hline $\mathrm{H}$ & -5.58834400 & -5.18744400 & -2.77642100 & $\mathrm{H}$ & -7.07603700 & 5.09654600 & 1.68971800 \\
\hline $\mathrm{H}$ & -5.42993800 & -3.12551700 & -1.47432700 & $\mathrm{H}$ & -10.06303400 & 2.71232300 & 1.94480000 \\
\hline $\mathrm{C}$ & -4.54007700 & -5.41774100 & -2.56711900 & $\mathrm{H}$ & -9.17312900 & 3.71796100 & 0.79609400 \\
\hline $\mathrm{C}$ & -1.84470600 & -5.96965500 & -2.03344400 & $\mathrm{H}$ & -9.14986900 & 4.80347100 & 3.02844500 \\
\hline $\mathrm{C}$ & -2.58743600 & -6.81592900 & -2.83146200 & $\mathrm{H}$ & -8.50076000 & 3.33675600 & 3.77192200 \\
\hline $\mathrm{C}$ & -3.95423200 & -6.54981300 & -3.09071600 & $\mathrm{C}$ & -4.47507300 & -0.32460400 & -2.60558700 \\
\hline $\mathrm{H}$ & -0.79246500 & -6.18841000 & -1.85147700 & $\mathrm{C}$ & -3.80573800 & 0.90625300 & -3.25626700 \\
\hline $\mathrm{H}$ & -2.11455900 & -7.69812300 & -3.27054200 & $\mathrm{C}$ & -5.75514500 & -0.69576400 & -3.39198300 \\
\hline $\mathrm{H}$ & -4.53455800 & -7.23333400 & -3.71554600 & $\mathrm{H}$ & -3.76302800 & -1.15505500 & -2.72210900 \\
\hline $\mathrm{O}$ & -1.54365400 & -1.75240000 & 0.43092300 & $\mathrm{C}$ & -3.51625300 & 0.66837500 & -4.74516900 \\
\hline $\mathrm{O}$ & 0.59150100 & -2.19313200 & -0.88614400 & $\mathrm{H}$ & -4.46454500 & 1.78878000 & -3.15882100 \\
\hline $\mathrm{P}$ & -0.32765700 & -0.93687500 & -0.33789000 & $\mathrm{H}$ & -2.86738600 & 1.14589400 & -2.73769100 \\
\hline $\mathrm{O}$ & 0.39215300 & -0.15125400 & 0.74626500 & $\mathrm{C}$ & -5.47207800 & -0.92842200 & -4.88191700 \\
\hline $\mathrm{C}$ & 3.25829700 & -2.87474200 & -0.11610000 & $\mathrm{H}$ & -6.49355100 & 0.11969500 & -3.28248300 \\
\hline $\mathrm{C}$ & 3.74932100 & -2.61064200 & -1.41864100 & $\mathrm{H}$ & -6.22495900 & -1.59038600 & -2.95216200 \\
\hline $\mathrm{C}$ & 3.92037700 & -2.32476400 & 1.01319300 & $\mathrm{C}$ & -4.77915700 & 0.27995100 & -5.52245500 \\
\hline $\mathrm{C}$ & 4.89425900 & -1.81312300 & -1.56124700 & $\mathrm{H}$ & -3.05743200 & 1.57087600 & -5.18567200 \\
\hline $\mathrm{C}$ & 5.05787500 & -1.53049800 & 0.80957800 & $\mathrm{H}$ & -2.76669200 & -0.13878600 & -4.83995800 \\
\hline $\mathrm{C}$ & 5.56932900 & -1.26413400 & -0.46566000 & $\mathrm{H}$ & -6.41148600 & -1.15956200 & -5.41296700 \\
\hline $\mathrm{H}$ & 5.28499900 & -1.61984200 & -2.56457700 & $\mathrm{H}$ & -4.82618000 & -1.81891600 & -4.99214400 \\
\hline $\mathrm{H}$ & 5.56132100 & -1.10925900 & 1.68321200 & $\mathrm{H}$ & -4.53524200 & 0.06881500 & -6.57767600 \\
\hline $\mathrm{C}$ & -4.30519700 & -1.09093800 & -0.14052200 & $\mathrm{H}$ & -5.47867800 & 1.13693600 & -5.53007700 \\
\hline $\mathrm{C}$ & -4.58057800 & -0.85893600 & 1.23274800 & $\mathrm{C}$ & 3.43526200 & -2.55302000 & 2.44200300 \\
\hline $\mathrm{C}$ & -4.74944000 & -0.15506100 & -1.11280200 & $\mathrm{C}$ & 2.93684400 & -1.25378300 & 3.11226000 \\
\hline $\mathrm{C}$ & -5.31735200 & 0.27750100 & 1.59812600 & $\mathrm{C}$ & 4.49207600 & -3.25204300 & 3.32642800 \\
\hline $\mathrm{C}$ & -5.49163100 & 0.95772100 & -0.68801900 & $\mathrm{H}$ & 2.56729900 & -3.22632100 & 2.40406700 \\
\hline $\mathrm{C}$ & -5.80062700 & 1.19364600 & 0.65620700 & $\mathrm{C}$ & 2.41954100 & -1.51498600 & 4.53327900 \\
\hline $\mathrm{H}$ & -5.54354100 & 0.43366700 & 2.65552000 & $\mathrm{H}$ & 3.75902400 & -0.51746300 & 3.15410700 \\
\hline $\mathrm{H}$ & -5.85408800 & 1.66763400 & -1.43671300 & $\mathrm{H}$ & 2.14383800 & -0.80910600 & 2.49108000 \\
\hline $\mathrm{C}$ & -4.14268400 & -1.82404300 & 2.32872900 & $\mathrm{C}$ & 3.96959100 & -3.50668800 & 4.74659800 \\
\hline $\mathrm{C}$ & -5.33840400 & -2.44907500 & 3.08327200 & $\mathrm{H}$ & 5.40075700 & -2.62587700 & 3.38295100 \\
\hline $\mathrm{C}$ & -3.15091100 & -1.18129000 & 3.32465100 & $\mathrm{H}$ & 4.80409000 & -4.20105300 & 2.85752600 \\
\hline $\mathrm{H}$ & -3.60922400 & -2.66091700 & 1.85493200 & $\mathrm{C}$ & 3.46946100 & -2.21490200 & 5.40492100 \\
\hline $\mathrm{C}$ & -4.87540700 & -3.44689600 & 4.15332400 & $\mathrm{H}$ & 2.10714400 & -0.56453100 & 4.99693500 \\
\hline $\mathrm{H}$ & -5.93162300 & -1.65049600 & 3.56424000 & $\mathrm{H}$ & 1.51349100 & -2.14677900 & 4.47929700 \\
\hline $\mathrm{H}$ & -6.01284800 & -2.94340400 & 2.36391800 & $\mathrm{H}$ & 4.75834900 & -3.97283400 & 5.36197600 \\
\hline $\mathrm{C}$ & -2.69546600 & -2.17970400 & 4.39695100 & $\mathrm{H}$ & 3.13956500 & -4.23596400 & 4.70294300 \\
\hline $\mathrm{H}$ & -3.63339400 & -0.31617500 & 3.81625000 & $\mathrm{H}$ & 3.06045000 & -2.42928500 & 6.40731200 \\
\hline $\mathrm{H}$ & -2.28098100 & -0.79702900 & 2.77025800 & $\mathrm{H}$ & 4.32645100 & -1.53208800 & 5.55792000 \\
\hline $\mathrm{C}$ & -3.88455400 & -2.80799500 & 5.13451900 & $\mathrm{C}$ & 6.83109700 & -0.43883100 & -0.66495500 \\
\hline $\mathrm{H}$ & -5.74731900 & -3.85152600 & 4.69523800 & $\mathrm{C}$ & 8.09729800 & -1.17958100 & -0.17671500 \\
\hline $\mathrm{H}$ & -4.39012200 & -4.30915000 & 3.65951600 & $\mathrm{C}$ & 6.75249900 & 0.96304200 & -0.02371300 \\
\hline $\mathrm{H}$ & -2.01870900 & -1.68198400 & 5.11235800 & $\mathrm{H}$ & 6.94852000 & -0.28786700 & -1.75415000 \\
\hline $\mathrm{H}$ & -2.10120600 & -2.97771300 & 3.91563100 & $\mathrm{C}$ & 9.37324000 & -0.36120700 & -0.41546600 \\
\hline
\end{tabular}




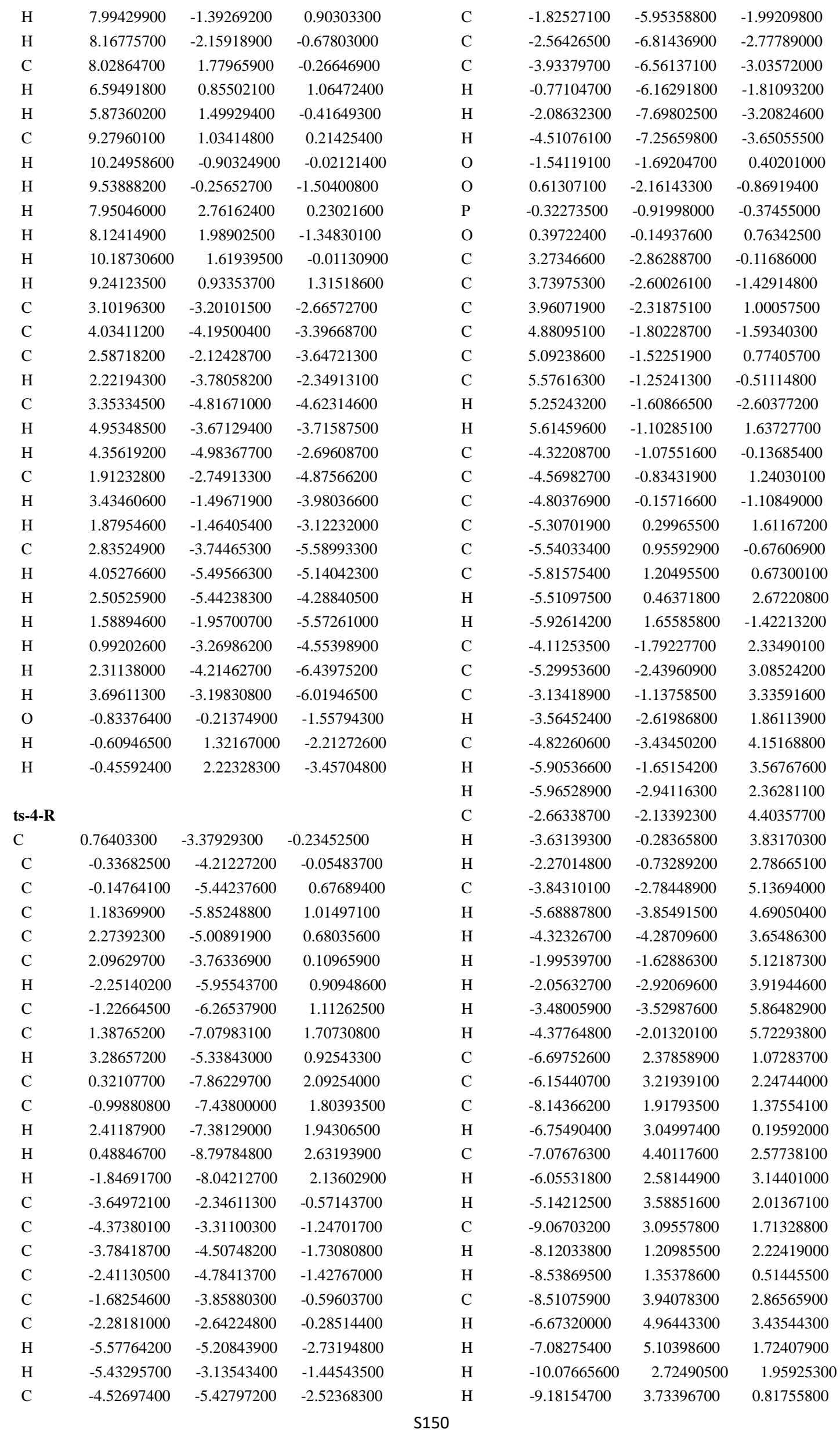




\begin{tabular}{|c|c|c|c|c|c|c|c|}
\hline $\mathrm{H}$ & -9.16271800 & 4.81055600 & 3.05364800 & $\mathrm{H}$ & 4.91652400 & -3.70059000 & -3.71897700 \\
\hline $\mathrm{H}$ & -8.51990500 & 3.33955700 & 3.79402900 & $\mathrm{H}$ & 4.31577400 & -4.99293900 & -2.67605800 \\
\hline $\mathrm{C}$ & -4.58350200 & -0.34900100 & -2.60755200 & $\mathrm{C}$ & 1.87693100 & -2.76978100 & -4.87264200 \\
\hline $\mathrm{C}$ & -3.96856400 & 0.88142200 & -3.30968500 & $\mathrm{H}$ & 3.41896100 & -1.52037900 & -4.00899700 \\
\hline $\mathrm{C}$ & -5.88704500 & -0.76065000 & -3.33445200 & $\mathrm{H}$ & 1.87203200 & -1.45333800 & -3.14203400 \\
\hline $\mathrm{H}$ & -3.86204400 & -1.16950400 & -2.73957900 & $\mathrm{C}$ & 2.78487400 & -3.78520200 & -5.57803300 \\
\hline $\mathrm{C}$ & -3.73097800 & 0.61783800 & -4.80354300 & $\mathrm{H}$ & 3.99046600 & -5.53963300 & -5.10988400 \\
\hline $\mathrm{H}$ & -4.64431000 & 1.74980200 & -3.20637900 & $\mathrm{H}$ & 2.44953200 & -5.45981500 & -4.24806200 \\
\hline $\mathrm{H}$ & -3.02011500 & 1.16140700 & -2.83055800 & $\mathrm{H}$ & 1.55607400 & -1.98549900 & -5.57938900 \\
\hline $\mathrm{C}$ & -5.65634800 & -1.02217400 & -4.82838200 & $\mathrm{H}$ & 0.95407900 & -3.27698800 & -4.53643800 \\
\hline $\mathrm{H}$ & -6.63395800 & 0.04530800 & -3.21428300 & $\mathrm{H}$ & 2.24993100 & -4.26355000 & -6.41614100 \\
\hline $\mathrm{H}$ & -6.32549300 & -1.65199800 & -2.85699200 & $\mathrm{H}$ & 3.64720500 & -3.25391300 & -6.02294300 \\
\hline $\mathrm{C}$ & -5.01444300 & 0.18477400 & -5.52215900 & $\mathrm{O}$ & -0.75104400 & -0.14741800 & -1.58012300 \\
\hline $\mathrm{H}$ & -3.31132700 & 1.52075300 & -5.28042300 & $\mathrm{H}$ & -0.74174900 & 1.43712100 & -2.30473800 \\
\hline $\mathrm{H}$ & -2.96733900 & -0.17448700 & -4.91306400 & $\mathrm{C}$ & 4.59262500 & 6.20885500 & 0.21025500 \\
\hline $\mathrm{H}$ & -6.61019000 & -1.28430600 & -5.31748400 & $\mathrm{C}$ & 3.71722300 & 5.17269300 & 0.46572500 \\
\hline $\mathrm{H}$ & -4.99643100 & -1.90177100 & -4.94367900 & $\mathrm{C}$ & 3.56245100 & 4.09391900 & -0.45124300 \\
\hline $\mathrm{H}$ & -4.80683600 & -0.04559000 & -6.58103600 & $\mathrm{C}$ & 4.33755800 & 4.11641900 & -1.66181200 \\
\hline $\mathrm{H}$ & -5.73175700 & 1.02675500 & -5.52149500 & $\mathrm{C}$ & 5.23323300 & 5.19968800 & -1.89033600 \\
\hline $\mathrm{C}$ & 3.51585900 & -2.56291600 & 2.44003000 & $\mathrm{C}$ & 5.36339900 & 6.22378300 & -0.97772900 \\
\hline $\mathrm{C}$ & 3.05308300 & -1.27253900 & 3.15078200 & $\mathrm{H}$ & 4.69321300 & 7.02323700 & 0.93218700 \\
\hline $\mathrm{C}$ & 4.59401900 & -3.28636600 & 3.27867100 & $\mathrm{H}$ & 3.14225300 & 5.17338700 & 1.39098300 \\
\hline $\mathrm{H}$ & 2.64088100 & -3.22798900 & 2.41945700 & $\mathrm{C}$ & 2.66700700 & 2.98826900 & -0.23224100 \\
\hline $\mathrm{C}$ & 2.57563600 & -1.55714000 & 4.58115000 & $\mathrm{C}$ & 4.18941600 & 3.06993500 & -2.61424400 \\
\hline $\mathrm{H}$ & 3.88251600 & -0.54477100 & 3.18223400 & $\mathrm{H}$ & 5.81883800 & 5.20357300 & -2.81359400 \\
\hline $\mathrm{H}$ & 2.24777100 & -0.80280200 & 2.56518500 & $\mathrm{H}$ & 6.05532500 & 7.04780500 & -1.16833200 \\
\hline $\mathrm{C}$ & 4.11456900 & -3.56432000 & 4.70958800 & $\mathrm{C}$ & 3.31888500 & 2.03089300 & -2.37655500 \\
\hline $\mathrm{H}$ & 5.50839400 & -2.66736200 & 3.31751100 & $\mathrm{C}$ & 2.56520400 & 1.98526500 & -1.18103600 \\
\hline $\mathrm{H}$ & 4.88472100 & -4.22827800 & 2.78243100 & $\mathrm{H}$ & 4.77739600 & 3.10683200 & -3.53484100 \\
\hline $\mathrm{C}$ & 3.64547500 & -2.28147700 & 5.40702700 & $\mathrm{H}$ & 3.20693400 & 1.22064700 & -3.10006600 \\
\hline $\mathrm{H}$ & 2.28585300 & -0.61306400 & 5.07089400 & $\mathrm{H}$ & 1.89577500 & 1.14508100 & -1.01116800 \\
\hline $\mathrm{H}$ & 1.66305500 & -2.18091700 & 4.54303500 & $\mathrm{~N}$ & 1.84889800 & 2.96432600 & 0.94779000 \\
\hline $\mathrm{H}$ & 4.91887300 & -4.04778900 & 5.29043100 & $\mathrm{C}$ & 2.41215900 & 2.70905900 & 2.22845500 \\
\hline $\mathrm{H}$ & 3.27787200 & -4.28674600 & 4.67864200 & $\mathrm{C}$ & 1.57892800 & 2.65804900 & 3.36304900 \\
\hline $\mathrm{H}$ & 3.26519500 & -2.51135200 & 6.41714900 & $\mathrm{C}$ & 3.79953700 & 2.55179400 & 2.40623500 \\
\hline $\mathrm{H}$ & 4.51222200 & -1.60838800 & 5.54638800 & $\mathrm{C}$ & 2.12399000 & 2.48428300 & 4.63617400 \\
\hline $\mathrm{C}$ & 6.82926000 & -0.42085500 & -0.73518600 & $\mathrm{H}$ & 0.49952200 & 2.75436600 & 3.25243700 \\
\hline $\mathrm{C}$ & 8.10645300 & -1.14288500 & -0.24776900 & $\mathrm{C}$ & 4.33271600 & 2.39549400 & 3.68670500 \\
\hline $\mathrm{C}$ & 6.74246800 & 0.99000500 & -0.11495500 & $\mathrm{H}$ & 4.46496200 & 2.54945000 & 1.54455600 \\
\hline $\mathrm{H}$ & 6.93482400 & -0.28577300 & -1.82757600 & $\mathrm{C}$ & 3.50501900 & 2.36418700 & 4.81229300 \\
\hline $\mathrm{C}$ & 9.37217100 & -0.31621400 & -0.51101400 & $\mathrm{H}$ & 1.45499500 & 2.44938000 & 5.49988700 \\
\hline $\mathrm{H}$ & 8.01644600 & -1.34016100 & 0.83613700 & $\mathrm{H}$ & 5.41451500 & 2.28525700 & 3.79895400 \\
\hline $\mathrm{H}$ & 8.18171100 & -2.12942600 & -0.73479000 & $\mathrm{H}$ & 3.92912100 & 2.23938300 & 5.81105100 \\
\hline $\mathrm{C}$ & 8.00897200 & 1.81409400 & -0.38127600 & $\mathrm{~N}$ & 0.56562800 & 2.37309000 & 0.76501200 \\
\hline $\mathrm{H}$ & 6.59425300 & 0.89729100 & 0.97615100 & $\mathrm{H}$ & 0.46185800 & 0.99541100 & 0.76589100 \\
\hline $\mathrm{H}$ & 5.85568300 & 1.51203400 & -0.50874700 & $\mathrm{C}$ & -2.75316600 & 3.50438600 & -0.17406600 \\
\hline $\mathrm{C}$ & 9.27098700 & 1.08723800 & 0.09918000 & $\mathrm{C}$ & -1.80970300 & 2.42624600 & 0.35918900 \\
\hline $\mathrm{H}$ & 10.25723500 & -0.84437400 & -0.11737100 & $\mathrm{C}$ & -0.61048900 & 4.62773900 & 0.44400200 \\
\hline $\mathrm{H}$ & 9.52629600 & -0.22612100 & -1.60254200 & $\mathrm{H}$ & -2.69866700 & 3.51881900 & -1.27617000 \\
\hline $\mathrm{H}$ & 7.92546800 & 2.80252700 & 0.10112100 & $\mathrm{H}$ & -3.80127600 & 3.38769800 & 0.12327400 \\
\hline $\mathrm{H}$ & 8.09280400 & 2.00801100 & -1.46691800 & $\mathrm{H}$ & -1.73096400 & 1.55204000 & -0.29616100 \\
\hline $\mathrm{H}$ & 10.17126800 & 1.67730200 & -0.14288700 & $\mathrm{H}$ & -2.13925600 & 2.06452700 & 1.34980900 \\
\hline $\mathrm{H}$ & 9.24319400 & 1.00222600 & 1.20172200 & $\mathrm{C}$ & -0.46659800 & 3.11034100 & 0.54000000 \\
\hline $\mathrm{C}$ & 3.07666700 & -3.19901700 & -2.66419300 & $\mathrm{O}$ & -0.83486700 & 2.36495400 & -2.61820500 \\
\hline $\mathrm{C}$ & 3.99528300 & -4.21237500 & -3.38636100 & $\mathrm{C}$ & -2.15331100 & 4.78974500 & 0.35254500 \\
\hline $\mathrm{C}$ & 2.56733400 & -2.13240400 & -3.65939300 & $\mathrm{O}$ & -2.75079600 & 5.77955100 & 0.69126800 \\
\hline $\mathrm{H}$ & 2.19323800 & -3.76679300 & -2.33562300 & $\mathrm{H}$ & -0.76910800 & 2.29916200 & -3.57910300 \\
\hline $\mathrm{C}$ & 3.30062200 & -4.84674200 & -4.59840900 & $\mathrm{C}$ & 0.01980600 & 5.12075700 & -0.92254700 \\
\hline
\end{tabular}




\begin{tabular}{|c|c|c|c|c|c|c|c|}
\hline $\mathrm{H}$ & 1.10665400 & 5.01560600 & -0.81858900 & $\mathrm{C}$ & -5.82237900 & -1.46026000 & -0.90740700 \\
\hline $\mathrm{H}$ & -0.28898900 & 4.42194000 & -1.71346800 & $\mathrm{H}$ & -5.76147500 & -0.00205600 & -2.48073900 \\
\hline $\mathrm{C}$ & -0.08206800 & 5.41257900 & 1.65113000 & $\mathrm{H}$ & -5.60664500 & -2.74317300 & 0.80795900 \\
\hline $\mathrm{H}$ & -0.47158300 & 5.00313700 & 2.59531700 & $\mathrm{C}$ & 3.84775400 & 0.28511300 & -0.41022200 \\
\hline $\mathrm{H}$ & 1.01268500 & 5.40328100 & 1.69401400 & $\mathrm{C}$ & 4.43563100 & -0.31820100 & 0.73180400 \\
\hline $\mathrm{H}$ & -0.43737400 & 6.44909500 & 1.57159300 & $\mathrm{C}$ & 4.17808300 & -0.18381700 & -1.70661900 \\
\hline $\mathrm{C}$ & -0.30427100 & 6.54492100 & -1.31599300 & $\mathrm{C}$ & 5.35571500 & -1.35867900 & 0.54970500 \\
\hline $\mathrm{C}$ & -1.41948000 & 6.83802400 & -2.11802800 & $\mathrm{C}$ & 5.11183000 & -1.22436800 & -1.82735600 \\
\hline $\mathrm{C}$ & 0.52246900 & 7.60876100 & -0.91711000 & $\mathrm{C}$ & 5.71799900 & -1.82472200 & -0.72051400 \\
\hline $\mathrm{C}$ & -1.71015600 & 8.15063400 & -2.49657200 & $\mathrm{H}$ & 5.81303100 & -1.81288100 & 1.43228600 \\
\hline $\mathrm{H}$ & -2.06772700 & 6.02533400 & -2.45696800 & $\mathrm{H}$ & 5.37693400 & -1.58345400 & -2.82546500 \\
\hline $\mathrm{C}$ & 0.23508500 & 8.92383800 & -1.29267200 & $\mathrm{C}$ & 4.11866400 & 0.14163700 & 2.15045600 \\
\hline $\mathrm{H}$ & 1.41231200 & 7.40112000 & -0.31703900 & $\mathrm{C}$ & 5.35366800 & 0.71867300 & 2.87959500 \\
\hline $\mathrm{C}$ & -0.88496800 & 9.19994500 & -2.08178400 & $\mathrm{C}$ & 3.46067600 & -0.96770600 & 3.00269500 \\
\hline $\mathrm{H}$ & -2.58404500 & 8.35424400 & -3.12091400 & $\mathrm{H}$ & 3.38853500 & 0.96322700 & 2.08923500 \\
\hline $\mathrm{H}$ & 0.89379900 & 9.73565100 & -0.97281700 & $\mathrm{C}$ & 5.00130000 & 1.21367000 & 4.28888000 \\
\hline $\mathrm{H}$ & -1.11022100 & 10.22768400 & -2.37812800 & $\mathrm{H}$ & 6.13644500 & -0.05816800 & 2.95070700 \\
\hline & & & & $\mathrm{H}$ & 5.78751400 & 1.53839000 & 2.28258900 \\
\hline int-4 & & & & $\mathrm{C}$ & 3.11264600 & -0.47097300 & 4.41196000 \\
\hline $\mathrm{C}$ & -1.49826300 & 1.17698400 & 0.59550400 & $\mathrm{H}$ & 4.15199500 & -1.82628200 & 3.08265300 \\
\hline $\mathrm{C}$ & -0.61331900 & 2.11672500 & 1.11269800 & $\mathrm{H}$ & 2.55633600 & -1.33290100 & 2.49195100 \\
\hline $\mathrm{C}$ & -1.02088300 & 2.85410100 & 2.28206800 & $\mathrm{C}$ & 4.33587600 & 0.11505700 & 5.12764500 \\
\hline $\mathrm{C}$ & -2.36563500 & 2.70856600 & 2.75952000 & $\mathrm{H}$ & 5.90660800 & 1.58988800 & 4.79517200 \\
\hline $\mathrm{C}$ & -3.25563900 & 1.83051400 & 2.08486100 & $\mathrm{H}$ & 4.31166500 & 2.07440800 & 4.20752600 \\
\hline $\mathrm{C}$ & -2.85046600 & 1.03736000 & 1.02851900 & $\mathrm{H}$ & 2.67876000 & -1.29386800 & 5.00514700 \\
\hline $\mathrm{H}$ & 0.89864100 & 3.79490800 & 2.69127600 & $\mathrm{H}$ & 2.32649600 & 0.30312100 & 4.33930400 \\
\hline $\mathrm{C}$ & -0.13766500 & 3.69788000 & 3.01502400 & $\mathrm{H}$ & 4.05072000 & 0.50875300 & 6.11810300 \\
\hline $\mathrm{C}$ & -2.78335600 & 3.44169400 & 3.90616200 & $\mathrm{H}$ & 5.06942800 & -0.69173700 & 5.31437800 \\
\hline $\mathrm{H}$ & -4.29220100 & 1.77283300 & 2.42463900 & $\mathrm{C}$ & 6.73656800 & -2.93781700 & -0.90261400 \\
\hline $\mathrm{C}$ & -1.90665800 & 4.26279700 & 4.58158700 & $\mathrm{C}$ & 6.28483700 & -4.27659300 & -0.27837200 \\
\hline $\mathrm{C}$ & -0.56758200 & 4.37930000 & 4.13552700 & $\mathrm{C}$ & 8.13834000 & -2.54972000 & -0.38129400 \\
\hline $\mathrm{H}$ & -3.81512300 & 3.32898700 & 4.24979100 & $\mathrm{H}$ & 6.83478000 & -3.10893900 & -1.99050800 \\
\hline $\mathrm{H}$ & -2.23690900 & 4.81390900 & 5.46555000 & $\mathrm{C}$ & 7.31453700 & -5.39159300 & -0.50580000 \\
\hline $\mathrm{H}$ & 0.13370000 & 5.01169300 & 4.68571500 & $\mathrm{H}$ & 6.13106600 & -4.13832400 & 0.80732000 \\
\hline $\mathrm{C}$ & 2.91740200 & 1.45228100 & -0.25023700 & $\mathrm{H}$ & 5.30531500 & -4.56598700 & -0.69460400 \\
\hline $\mathrm{C}$ & 3.31976000 & 2.73396400 & -0.57262300 & $\mathrm{C}$ & 9.16628500 & -3.66558300 & -0.60876400 \\
\hline $\mathrm{C}$ & 2.44215700 & 3.84714900 & -0.48611300 & $\mathrm{H}$ & 8.07435900 & -2.32891300 & 0.69969800 \\
\hline $\mathrm{C}$ & 1.11123700 & 3.66123700 & 0.01681000 & $\mathrm{H}$ & 8.46724400 & -1.61632700 & -0.86764100 \\
\hline $\mathrm{C}$ & 0.71205800 & 2.34939200 & 0.46265800 & $\mathrm{C}$ & 8.70516900 & -4.99348700 & 0.00478400 \\
\hline $\mathrm{C}$ & 1.59106600 & 1.29458600 & 0.24844800 & $\mathrm{H}$ & 6.97827400 & -6.32258600 & -0.01856800 \\
\hline $\mathrm{H}$ & 3.87619100 & 5.26772000 & -1.28098100 & $\mathrm{H}$ & 7.37571300 & -5.61335900 & -1.58757500 \\
\hline $\mathrm{H}$ & 4.33728600 & 2.89531400 & -0.93636500 & $\mathrm{H}$ & 10.14441900 & -3.36928000 & -0.19337700 \\
\hline $\mathrm{C}$ & 2.85452600 & 5.14147300 & -0.91278900 & $\mathrm{H}$ & 9.32060700 & -3.80165400 & -1.69541100 \\
\hline $\mathrm{C}$ & 0.23375500 & 4.78376600 & 0.01459500 & $\mathrm{H}$ & 9.43600400 & -5.79120600 & -0.21084700 \\
\hline $\mathrm{C}$ & 0.65772300 & 6.02128400 & -0.42533200 & $\mathrm{H}$ & 8.67206000 & -4.89212800 & 1.10571400 \\
\hline $\mathrm{C}$ & 1.98499200 & 6.20982200 & -0.88147600 & $\mathrm{C}$ & 3.55667000 & 0.39354300 & -2.97386900 \\
\hline $\mathrm{H}$ & -0.79408000 & 4.65877300 & 0.35455100 & $\mathrm{C}$ & 2.84654600 & -0.67420100 & -3.83780600 \\
\hline $\mathrm{H}$ & -0.03997700 & 6.86249100 & -0.42549300 & $\mathrm{C}$ & 4.57839300 & 1.17923800 & -3.82783400 \\
\hline $\mathrm{H}$ & 2.31078600 & 7.19663200 & -1.21943500 & $\mathrm{H}$ & 2.77755200 & 1.11039000 & -2.67235100 \\
\hline $\mathrm{O}$ & 1.19547200 & 0.01059100 & 0.59638200 & $\mathrm{C}$ & 2.19427600 & -0.04918500 & -5.07798200 \\
\hline $\mathrm{O}$ & -1.08420100 & 0.36428300 & -0.45196900 & $\mathrm{H}$ & 3.57768900 & -1.43712300 & -4.16202200 \\
\hline $\mathrm{P}$ & 0.04466000 & -0.78266100 & -0.22847900 & $\mathrm{H}$ & 2.08914700 & -1.18631600 & -3.22619800 \\
\hline $\mathrm{O}$ & -0.45889600 & -1.79229400 & 0.88472000 & $\mathrm{C}$ & 3.92698100 & 1.79819000 & -5.07154200 \\
\hline $\mathrm{C}$ & -3.82492800 & 0.12957000 & 0.33091100 & $\mathrm{H}$ & 5.39082800 & 0.49782100 & -4.14000700 \\
\hline $\mathrm{C}$ & -4.35892500 & 0.51135500 & -0.92563600 & $\mathrm{H}$ & 5.05604100 & 1.96406100 & -3.21710400 \\
\hline $\mathrm{C}$ & -4.26628500 & -1.06856400 & 0.95116200 & $\mathrm{C}$ & 3.20222900 & 0.74434300 & -5.91832200 \\
\hline $\mathrm{C}$ & -5.34418700 & -0.29524600 & -1.51344900 & $\mathrm{H}$ & 1.71905100 & -0.83501200 & -5.68946500 \\
\hline $\mathrm{C}$ & -5.26213300 & -1.82802300 & 0.32174000 & $\mathrm{H}$ & 1.37855700 & 0.62376100 & -4.75500700 \\
\hline
\end{tabular}




\begin{tabular}{|c|c|c|c|}
\hline $\mathrm{H}$ & 4.68753900 & 2.32180700 & -5.67588900 \\
\hline $\mathrm{H}$ & 3.20064100 & 2.56848900 & -4.75223100 \\
\hline $\mathrm{H}$ & 2.69896500 & 1.22278000 & -6.77590800 \\
\hline $\mathrm{H}$ & 3.94782200 & 0.04761700 & -6.34589300 \\
\hline$C$ & -3.68577900 & -1.55986000 & 2.27333500 \\
\hline $\mathrm{C}$ & -3.27984700 & -3.05032500 & 2.24139600 \\
\hline$C$ & -4.62857000 & -1.30875300 & 3.47296000 \\
\hline 11 & -2.76220600 & -0.99060300 & 2.46294600 \\
\hline $\mathrm{C}$ & -2.61820000 & -3.47930200 & 3.55692400 \\
\hline $\mathrm{H}$ & -4.17347000 & -3.67804600 & 2.07586300 \\
\hline $\mathrm{H}$ & -2.61051500 & -3.23558900 & 1.38796400 \\
\hline $\mathrm{C}$ & -3.99261200 & -1.74834100 & 4.79894900 \\
\hline $\mathrm{H}$ & -5.57219300 & -1.86158200 & 3.30979500 \\
\hline $\mathrm{H}$ & -4.90403100 & -0.24285300 & 3.52153100 \\
\hline $\mathrm{C}$ & -3.53402800 & -3.21154600 & 4.75817400 \\
\hline $\mathrm{H}$ & -2.34849200 & -4.54850700 & 3.51368800 \\
\hline $\mathrm{H}$ & -1.67091400 & -2.92384200 & 3.68293600 \\
\hline $\mathrm{H}$ & -4.70230100 & -1.59169000 & 5.62936700 \\
\hline $\mathrm{H}$ & -3.12111700 & -1.10150400 & 5.00991900 \\
\hline $\mathrm{H}$ & -3.02483200 & -3.47925300 & 5.69966800 \\
\hline $\mathrm{H}$ & -4.42206300 & -3.86743600 & 4.68841500 \\
\hline $\mathrm{C}$ & -6.90783000 & -2.29152100 & -1.57102900 \\
\hline $\mathrm{C}$ & -8.20375700 & -2.35497800 & -0.73203100 \\
\hline $\mathrm{C}$ & -6.43468300 & -3.71643200 & -1.93493100 \\
\hline $\mathrm{H}$ & -7.16507200 & -1.78634800 & -2.52024500 \\
\hline $\mathrm{C}$ & -9.30254700 & -3.16487600 & -1.43245700 \\
\hline $\mathrm{H}$ & -7.97903000 & -2.81588000 & 0.24690800 \\
\hline $\mathrm{H}$ & -8.55564500 & -1.33199800 & -0.51772600 \\
\hline $\mathrm{C}$ & -7.53503300 & -4.52466500 & -2.63513700 \\
\hline $\mathrm{H}$ & -6.12728500 & -4.24305800 & -1.01334000 \\
\hline $\mathrm{H}$ & -5.53540800 & -3.65655700 & -2.57013600 \\
\hline $\mathrm{C}$ & -8.82114900 & -4.57403500 & -1.80052800 \\
\hline $\mathrm{H}$ & -10.19898200 & -3.21954800 & -0.79163000 \\
\hline $\mathrm{H}$ & -9.61227100 & -2.63563600 & -2.35285000 \\
\hline $\mathrm{H}$ & -7.17596500 & -5.54574300 & -2.84867000 \\
\hline $\mathrm{H}$ & -7.75644900 & -4.06375900 & -3.61578100 \\
\hline $\mathrm{H}$ & -9.61107700 & -5.12015700 & -2.34356300 \\
\hline $\mathrm{H}$ & -8.62983400 & -5.14595100 & -0.87331600 \\
\hline $\mathrm{C}$ & -3.90461200 & 1.76489700 & -1.66483300 \\
\hline $\mathrm{C}$ & -5.05301200 & 2.76882000 & -1.91247500 \\
\hline $\mathrm{C}$ & -3.18530000 & 1.42919400 & -2.99214400 \\
\hline $\mathrm{H}$ & -3.17101400 & 2.28873600 & -1.03302300 \\
\hline $\mathrm{C}$ & -4.56033300 & 4.03147200 & -2.63313000 \\
\hline $\mathrm{H}$ & -5.83913700 & 2.28905500 & -2.52264000 \\
\hline $\mathrm{H}$ & -5.52729700 & 3.03435800 & -0.95258900 \\
\hline $\mathrm{C}$ & -2.69775700 & 2.69399000 & -3.71068900 \\
\hline U & -3.87991000 & 0.87986000 & -3.65340000 \\
\hline $\mathrm{H}$ & -2.34120800 & 0.75186300 & -2.79036600 \\
\hline $\mathrm{C}$ & -3.83840100 & 3.69214100 & -3.94333900 \\
\hline $\mathrm{H}$ & -5.40808400 & 4.71115300 & -2.82532300 \\
\hline $\mathrm{H}$ & -3.86738500 & 4.58184900 & -1.96966900 \\
\hline $\mathrm{H}$ & -2.22280800 & 2.42326500 & -4.66900000 \\
\hline $\mathrm{H}$ & -1.91043500 & 3.17623300 & -3.10223500 \\
\hline $\mathrm{H}$ & -3.45558800 & 4.61105300 & -4.41919000 \\
\hline $\mathrm{H}$ & -4.56466800 & 3.25441600 & -4.65375700 \\
\hline $\mathrm{O}$ & 0.41285900 & -1.41232000 & -1.52513300 \\
\hline $\mathrm{H}$ & -0.14112700 & -3.24649500 & -1.32568300 \\
\hline $\mathrm{H}$ & -0.51808000 & -2.71408600 & 0.47689400 \\
\hline
\end{tabular}

$\begin{array}{cccc}\mathrm{H} & 0.31908600 & -4.49395000 & -0.54162500 \\ \mathrm{O} & -0.43685700 & -3.90005900 & -0.65365800\end{array}$

R-3a

C $\quad 1.40260700 \quad-2.40247800 \quad-1.32443100$

$\begin{array}{llll}\text { C } & 0.23029500 & -1.72587300 & -0.59078100\end{array}$

$\begin{array}{llll}\text { C } & 2.11878300 & -0.07666900 & -0.71493000\end{array}$

$\mathrm{H} \quad 1.84792800 \quad-3.23336700 \quad-0.75264600$

$\begin{array}{llll}\mathrm{H} & 1.12837800 & -2.81876300 & -2.30609800\end{array}$

$\mathrm{H} \quad 0.04795500 \quad-2.17765900 \quad 0.39745400$

$\mathrm{H} \quad-0.71642800 \quad-1.81832500 \quad-1.14201800$

C $\quad 0.62252800 \quad-0.25790200 \quad-0.45831400$

$\begin{array}{llll}\text { C } & 2.46858800 & -1.32644600 & -1.53645800\end{array}$

$\begin{array}{llll}\text { O } & 3.43068300 & -1.44273200 & -2.25463800\end{array}$

$\begin{array}{llll}\text { C } & 2.48402400 & 1.20791500 & -1.45729400\end{array}$

$\begin{array}{llll}\mathrm{H} & 2.26549400 & 2.08938500 & -0.83777100\end{array}$

$\mathrm{H} \quad 3.55062700 \quad 1.20166400 \quad-1.72024600$

$\mathrm{H} \quad 1.90169000 \quad 1.29514700 \quad-2.38706500$

$\begin{array}{llll}\text { C } & 2.84808500 & -0.16515800 & 0.68420200\end{array}$

$\begin{array}{llll}\mathrm{H} & 2.40454400 & 0.63745600 & 1.29453800\end{array}$

$\mathrm{H} \quad 2.56759200 \quad-1.11647800 \quad 1.16727900$

$\begin{array}{llll}\text { C } & 4.35414800 & -0.02802500 & 0.67996000\end{array}$

$\begin{array}{llll}\mathrm{C} & 4.95597000 & 1.22447400 & 0.88760300\end{array}$

$\begin{array}{llll}\mathrm{C} & 5.18976600 & -1.13974000 & 0.48362200\end{array}$

$\begin{array}{llll}\text { C } & 6.34625500 & 1.36469300 & 0.89204500\end{array}$

$\mathrm{H} \quad 4.32482700 \quad 2.10157000 \quad 1.05572600$

$\begin{array}{llll}\text { C } & 6.57999200 & -1.00462500 & 0.48744300\end{array}$

$\mathrm{H} \quad 4.74860300 \quad-2.12726100 \quad 0.32614300$

$\begin{array}{llll}\text { C } & 7.16388600 & 0.24924700 & 0.68996900\end{array}$

$\begin{array}{llll}\mathrm{H} & 6.79214700 & 2.34883400 & 1.05879600\end{array}$

$\begin{array}{llll}\mathrm{H} & 7.21031100 & -1.88409200 & 0.33222800\end{array}$

$\begin{array}{llll}\mathrm{H} & 8.25174200 & 0.35557100 & 0.69477400\end{array}$

$\begin{array}{llll}\mathrm{N} & -0.08132800 & 0.78251700 & -0.21207300\end{array}$

$\mathrm{N} \quad-1.42444700 \quad 0.76846900 \quad 0.05177100$

$\begin{array}{llll}\mathrm{C} & -2.04864900 & 2.04346400 & 0.03821800\end{array}$

$\begin{array}{llll}\mathrm{C} & -3.25519200 & 2.27082100 & 0.72656600\end{array}$

$\begin{array}{llll}\text { C } & -1.47207900 & 3.10417900 & -0.68754700\end{array}$

$\begin{array}{llll}\text { C } & -3.86539400 & 3.52650500 & 0.68267900\end{array}$

$\mathrm{H} \quad-3.71770200 \quad 1.47060500 \quad 1.30421400$

$\begin{array}{llll}\text { C } & -2.08788000 & 4.35533900 & -0.71184100\end{array}$

$\mathrm{H} \quad-0.53984500 \quad 2.93355800 \quad-1.22304900$

$\begin{array}{llll}\text { C } & -3.28962200 & 4.57949000 & -0.03192200\end{array}$

$\mathrm{H} \quad-4.80175200 \quad 3.67966000 \quad 1.22596700$

$\mathrm{H} \quad-1.62124300 \quad 5.16533000 \quad-1.27917100$

$\mathrm{H} \quad-3.77002400 \quad 5.56023600 \quad-0.05998100$

$\begin{array}{llll}\text { C } & -2.06683300 & -0.32603400 & 0.72145000\end{array}$

$\begin{array}{llll}\mathrm{C} & -3.13823700 & -1.03112200 & 0.08112400\end{array}$

$\begin{array}{llll}\text { C } & -1.65864900 & -0.68228100 & 1.99422200\end{array}$

$\begin{array}{llll}\text { C } & -3.58888000 & -0.71548600 & -1.23108400\end{array}$

$\begin{array}{llll}\text { C } & -3.77269300 & -2.10650800 & 0.79133400\end{array}$

$\begin{array}{llll}\text { C } & -2.27596900 & -1.75621000 & 2.67994200\end{array}$

$\mathrm{H} \quad-0.85028000 \quad-0.12115800 \quad 2.46806400$

$\begin{array}{llll}\text { C } & -4.62302600 & -1.42139600 & -1.81049300\end{array}$

$\mathrm{H} \quad-3.10208500 \quad 0.09643400 \quad-1.77358200$

$\begin{array}{llll}\text { C } & -4.83961600 & -2.80960100 & 0.16322900\end{array}$

C $\quad-3.31368500 \quad-2.44910500 \quad 2.09435100$

$\mathrm{H} \quad-1.93241700 \quad-2.02102900 \quad 3.68288900$

$\begin{array}{llll}\text { C } & -5.25817900 & -2.47547700 & -1.10695600\end{array}$ 


$\begin{array}{llll}\mathrm{H} & -4.95688500 & -1.16676900 & -2.81947700 \\ \mathrm{H} & -5.32193700 & -3.62536700 & 0.70864700 \\ \mathrm{H} & -3.79987000 & -3.27215500 & 2.62472100 \\ \mathrm{H} & -6.07847900 & -3.02401600 & -1.57657500\end{array}$

\section{$\mathrm{H}_{2} \mathrm{O}$}

$\begin{array}{lccc}\mathrm{O} & 0.00000000 & 0.00000000 & 0.12020700 \\ \mathrm{H} & 0.00000000 & 0.75703900 & -0.48082800 \\ \mathrm{H} & 0.00000000 & -0.75703900 & -0.48082800\end{array}$

\section{ts-5}

C

C

$\mathrm{H}$

$\mathrm{H}$

$\mathrm{H}$

$\mathrm{H}$

C

C

$\mathrm{H}$

C

C

$\mathrm{H}$

C

C

H

$\mathrm{H}$

C

$$
\begin{array}{ccc}
-2.62168600 & -2.47913200 & -0.07401100 \\
-2.10146200 & -3.70975100 & -0.46069100 \\
-2.86984500 & -4.51520400 & -1.37571600 \\
-4.20303100 & -4.10535900 & -1.70856700 \\
-4.72162200 & -2.90661000 & -1.15212200 \\
-3.95591700 & -2.06461600 & -0.36769900 \\
-1.33255300 & -5.99994200 & -1.78739500 \\
-2.35660700 & -5.69031000 & -1.99671900 \\
-4.97936300 & -4.90284500 & -2.59644400 \\
-5.75557100 & -2.63105600 & -1.37368900 \\
-4.45698700 & -6.04381900 & -3.16589300 \\
-3.12677800 & -6.43040700 & -2.87073600 \\
-5.99882100 & -4.58309700 & -2.82873900 \\
-5.05977500 & -6.64348200 & -3.85240400 \\
-2.70437400 & -7.32128200 & -3.34233600 \\
1.65648300 & -3.88240500 & 0.31158600 \\
1.74670700 & -5.14859400 & 0.85737800 \\
0.60164800 & -5.94055400 & 1.13361700 \\
-0.69491000 & -5.45369800 & 0.76293300 \\
-0.79482600 & -4.18607600 & 0.08264200 \\
0.35484300 & -3.41298900 & -0.04168600 \\
1.71538300 & -7.56249100 & 2.04891000 \\
2.73151300 & -5.54982000 & 1.10716200 \\
0.71757400 & -7.20068200 & 1.78626000 \\
-1.82938200 & -6.23804200 & 1.12134200 \\
-1.68594000 & -7.44577400 & 1.77372500 \\
-0.40037700 & -7.94307900 & 2.09859100 \\
-2.82819000 & -5.87003200 & 0.88793700 \\
-2.57426800 & -8.02123600 & 2.04605000 \\
-0.29972700 & -8.90497000 & 2.60735800 \\
0.25895900 & -2.15238200 & -0.60793200 \\
-1.85238100 & -1.64168000 & 0.71499900 \\
-0.49990700 & -0.91866900 & 0.15231800 \\
-0.70756900 & 0.19402900 & -0.82705600 \\
-4.53421000 & -0.79527900 & 0.18405000 \\
-4.80834600 & -0.68789400 & 1.57150200 \\
-4.87002700 & 0.27483200 & -0.68528800 \\
-5.41790800 & 0.47923800 & 2.05099700 \\
-5.48271600 & 1.41715400 & -0.14849400 \\
-5.77337500 & 1.54242900 & 1.21471800 \\
-5.64098400 & 0.55955600 & 3.11857200 \\
4.824770050900 & 2.23230800 & -0.82613000 \\
& -3.07835100 & 0.06285700 \\
\hline & -1.87075600 & 0.88853400
\end{array}
$$

\begin{tabular}{|c|c|c|c|}
\hline $\mathrm{C}$ & 5.36798700 & -1.75072900 & -0.40216100 \\
\hline $\mathrm{H}$ & 5.00989500 & -2.20995500 & -2.47665900 \\
\hline $\mathrm{H}$ & 5.40389800 & -1.45168100 & 1.72487700 \\
\hline $\mathrm{C}$ & 2.68769800 & -3.54824900 & -2.46144800 \\
\hline $\mathrm{C}$ & 3.52497400 & -4.64022900 & -3.16632500 \\
\hline $\mathrm{C}$ & 2.23006000 & -2.48007300 & -3.48005300 \\
\hline $\mathrm{H}$ & 1.77538100 & -4.04810200 & -2.10267700 \\
\hline $\mathrm{C}$ & 2.76521500 & -5.26589000 & -4.34342900 \\
\hline $\mathrm{H}$ & 4.46927900 & -4.20124900 & -3.53576700 \\
\hline $\mathrm{H}$ & 3.80992700 & -5.41729200 & -2.43727700 \\
\hline $\mathrm{C}$ & 1.47532200 & -3.10824900 & -4.65957800 \\
\hline $\mathrm{H}$ & 3.11186300 & -1.93399000 & -3.86181700 \\
\hline $\mathrm{H}$ & 1.59304000 & -1.74064900 & -2.96993100 \\
\hline $\mathrm{C}$ & 2.29937000 & -4.20386600 & -5.34753100 \\
\hline $\mathrm{H}$ & 3.39770100 & -6.01831600 & -4.84466100 \\
\hline $\mathrm{H}$ & 1.88383200 & -5.81047200 & -3.95680100 \\
\hline $\mathrm{H}$ & 1.19404900 & -2.32708700 & -5.38651600 \\
\hline $\mathrm{H}$ & 0.52749500 & -3.54257900 & -4.29188100 \\
\hline $\mathrm{H}$ & 1.71562300 & -4.67220900 & -6.15817600 \\
\hline $\mathrm{H}$ & 3.18477400 & -3.74628100 & -5.82752700 \\
\hline $\mathrm{C}$ & 6.73871500 & -1.11909100 & -0.60035400 \\
\hline $\mathrm{C}$ & 6.90473000 & -0.32411800 & -1.90967800 \\
\hline $\mathrm{C}$ & 7.86473000 & -2.17463100 & -0.48112200 \\
\hline $\mathrm{H}$ & 6.88523100 & -0.40541700 & 0.23261000 \\
\hline $\mathrm{C}$ & 8.29437700 & 0.31866600 & -2.01326000 \\
\hline $\mathrm{H}$ & 6.76506200 & -0.99896900 & -2.77273300 \\
\hline $\mathrm{H}$ & 6.11745100 & 0.44390000 & -1.98951500 \\
\hline $\mathrm{C}$ & 9.26014800 & -1.54798700 & -0.59962000 \\
\hline $\mathrm{H}$ & 7.72471500 & -2.92958100 & -1.27671300 \\
\hline $\mathrm{H}$ & 7.76614400 & -2.71375700 & 0.47541400 \\
\hline $\mathrm{C}$ & 9.40927000 & -0.72677800 & -1.88636400 \\
\hline $\mathrm{H}$ & 8.38713700 & 0.86530800 & -2.96674800 \\
\hline $\mathrm{H}$ & 8.40872200 & 1.07283100 & -1.21249500 \\
\hline $\mathrm{H}$ & 10.03205800 & -2.33481800 & -0.55340300 \\
\hline $\mathrm{H}$ & 9.43620300 & -0.89078900 & 0.27227200 \\
\hline $\mathrm{H}$ & 10.39868900 & -0.24020700 & -1.91949300 \\
\hline $\mathrm{H}$ & 9.36807800 & -1.40691800 & -2.75754900 \\
\hline $\mathrm{C}$ & 3.13558000 & -2.62932700 & 2.59326400 \\
\hline $\mathrm{C}$ & 3.09635400 & -1.27825500 & 3.34128500 \\
\hline $\mathrm{C}$ & 3.96283900 & -3.65213700 & 3.40788300 \\
\hline $\mathrm{H}$ & 2.09847200 & -2.99710100 & 2.56877600 \\
\hline $\mathrm{C}$ & 2.55176600 & -1.43846100 & 4.76681100 \\
\hline $\mathrm{H}$ & 4.11267200 & -0.84800400 & 3.39420100 \\
\hline $\mathrm{H}$ & 2.47269300 & -0.56761200 & 2.78013200 \\
\hline $\mathrm{C}$ & 3.42999700 & -3.81201000 & 4.83767800 \\
\hline $\mathrm{H}$ & 5.01577700 & -3.31695300 & 3.44145800 \\
\hline $\mathrm{H}$ & 3.96978800 & -4.62768900 & 2.89470800 \\
\hline $\mathrm{C}$ & 3.35544000 & -2.46718000 & 5.57116400 \\
\hline $\mathrm{H}$ & 2.55407800 & -0.46261500 & 5.28140600 \\
\hline $\mathrm{H}$ & 1.49487700 & -1.75733800 & 4.71337100 \\
\hline $\mathrm{H}$ & 4.06153000 & -4.52207700 & 5.39870800 \\
\hline $\mathrm{H}$ & 2.41996100 & -4.25978000 & 4.79732300 \\
\hline $\mathrm{H}$ & 2.91600000 & -2.60153300 & 6.57422900 \\
\hline $\mathrm{H}$ & 4.38063900 & -2.08331000 & 5.73034100 \\
\hline $\mathrm{C}$ & -4.58573400 & 0.23570500 & -2.18350400 \\
\hline $\mathrm{C}$ & -3.60740100 & 1.34469400 & -2.62990000 \\
\hline $\mathrm{C}$ & -5.87471700 & 0.27578500 & -3.03481100 \\
\hline $\mathrm{H}$ & -4.08598900 & -0.71774100 & -2.41023800 \\
\hline
\end{tabular}




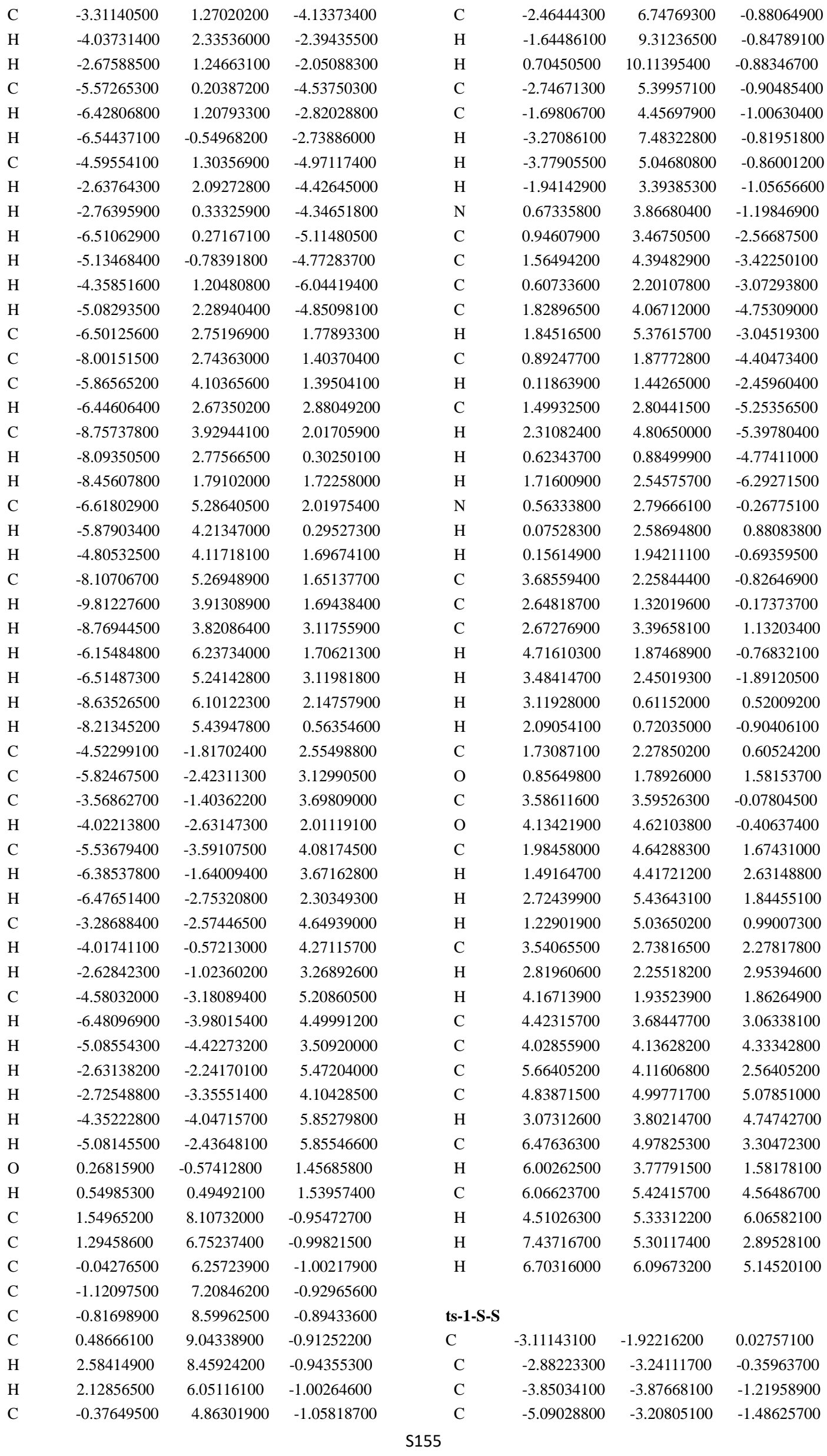




\begin{tabular}{|c|c|c|c|c|c|c|c|}
\hline $\mathrm{C}$ & -5.31031200 & -1.91528900 & -0.94513700 & $\mathrm{H}$ & 0.49911300 & -5.53612100 & -4.26375300 \\
\hline $\mathrm{C}$ & -4.33791600 & -1.23740100 & -0.23481200 & $\mathrm{H}$ & 0.49380900 & -1.84871800 & -5.29681100 \\
\hline $\mathrm{H}$ & -2.67229600 & -5.64293300 & -1.70026500 & $\mathrm{H}$ & -0.37856200 & -3.01952700 & -4.30250700 \\
\hline $\mathrm{C}$ & -3.62432200 & -5.13425900 & -1.85073000 & $\mathrm{H}$ & 0.51326700 & -4.15420600 & -6.32738700 \\
\hline $\mathrm{C}$ & -6.06645900 & -3.83898200 & -2.30871500 & $\mathrm{H}$ & 2.14611300 & -3.57862600 & -5.96866300 \\
\hline $\mathrm{H}$ & -6.26858000 & -1.42457800 & -1.13307000 & $\mathrm{C}$ & 6.23855200 & -2.17901100 & -0.69710900 \\
\hline $\mathrm{C}$ & -5.82256200 & -5.06680200 & -2.88437300 & $\mathrm{C}$ & 6.35260000 & -0.83662600 & -1.45038300 \\
\hline $\mathrm{C}$ & -4.58025400 & -5.70986600 & -2.66283600 & $\mathrm{C}$ & 7.08852400 & -3.26033800 & -1.40245900 \\
\hline $\mathrm{H}$ & -7.01173700 & -3.31942100 & -2.48784300 & $\mathrm{H}$ & 6.68460000 & -2.02354400 & 0.30266000 \\
\hline $\mathrm{H}$ & -6.57551200 & -5.53780800 & -3.52117600 & $\mathrm{C}$ & 7.81166200 & -0.39764300 & -1.63494400 \\
\hline $\mathrm{H}$ & -4.37366600 & -6.66970400 & -3.14308900 & $\mathrm{H}$ & 5.87372200 & -0.93452900 & -2.44168400 \\
\hline $\mathrm{C}$ & 0.80132700 & -4.16649900 & 0.19995900 & $\mathrm{H}$ & 5.78712700 & -0.06140600 & -0.90604200 \\
\hline $\mathrm{C}$ & 0.66922000 & -5.44661700 & 0.70347700 & $\mathrm{C}$ & 8.54699400 & -2.82063000 & -1.58457000 \\
\hline $\mathrm{C}$ & -0.59246900 & -6.00754600 & 1.03240300 & $\mathrm{H}$ & 6.64566700 & -3.47933900 & -2.39105200 \\
\hline $\mathrm{C}$ & -1.78554200 & -5.25870500 & 0.76578100 & $\mathrm{H}$ & 7.03800400 & -4.20006700 & -0.82800600 \\
\hline $\mathrm{C}$ & -1.67213500 & -3.98068800 & 0.10728100 & $\mathrm{C}$ & 8.64782200 & -1.48195400 & -2.32594300 \\
\hline $\mathrm{C}$ & -0.40097600 & -3.44800000 & -0.08366900 & $\mathrm{H}$ & 7.85393900 & 0.54454900 & -2.20741000 \\
\hline $\mathrm{H}$ & 0.22951900 & -7.84869900 & 1.83422500 & $\mathrm{H}$ & 8.25151600 & -0.17684200 & -0.64464200 \\
\hline $\mathrm{H}$ & 1.56760100 & -6.03796900 & 0.89344600 & $\mathrm{H}$ & 9.11285200 & -3.60079800 & -2.12134300 \\
\hline $\mathrm{C}$ & -0.69009000 & -7.28592100 & 1.65195600 & $\mathrm{H}$ & 9.02321200 & -2.72204800 & -0.59123200 \\
\hline $\mathrm{C}$ & -3.02726900 & -5.80814300 & 1.19754400 & $\mathrm{H}$ & 9.70084900 & -1.16268500 & -2.40353800 \\
\hline $\mathrm{C}$ & -3.08765500 & -7.03999800 & 1.81718900 & $\mathrm{H}$ & 8.28633200 & -1.61332300 & -3.36301800 \\
\hline $\mathrm{C}$ & -1.91100800 & -7.79749400 & 2.03452300 & $\mathrm{C}$ & 2.60394600 & -3.55663800 & 2.51959500 \\
\hline $\mathrm{H}$ & -3.94384700 & -5.23846100 & 1.04612200 & $\mathrm{C}$ & 2.79191000 & -2.32406100 & 3.43165300 \\
\hline $\mathrm{H}$ & -4.05311600 & -7.43200600 & 2.14703200 & $\mathrm{C}$ & 3.33341900 & -4.77587100 & 3.13307700 \\
\hline $\mathrm{H}$ & -1.97292600 & -8.77598200 & 2.51730100 & $\mathrm{H}$ & 1.52739100 & -3.78238500 & 2.53032300 \\
\hline $\mathrm{O}$ & -0.28286200 & -2.18880700 & -0.63760100 & $\mathrm{C}$ & 2.32459300 & -2.60701100 & 4.86596300 \\
\hline $\mathrm{O}$ & -2.16916300 & -1.25408100 & 0.78246300 & $\mathrm{H}$ & 3.85748300 & -2.03219700 & 3.45328000 \\
\hline $\mathrm{P}$ & -0.67548400 & -0.85253100 & 0.23788800 & $\mathrm{H}$ & 2.23718800 & -1.47296900 & 3.01476600 \\
\hline $\mathrm{O}$ & -0.63903300 & 0.32471700 & -0.69895600 & $\mathrm{C}$ & 2.87526000 & -5.05948600 & 4.56984500 \\
\hline $\mathrm{C}$ & -4.59020400 & 0.16305500 & 0.23888800 & $\mathrm{H}$ & 4.42218000 & -4.58460300 & 3.12604600 \\
\hline $\mathrm{C}$ & -4.76971100 & 0.43032100 & 1.61949700 & $\mathrm{H}$ & 3.17899700 & -5.66629700 & 2.50157900 \\
\hline $\mathrm{C}$ & -4.72095200 & 1.21481300 & -0.70730200 & $\mathrm{C}$ & 3.03185500 & -3.82670300 & 5.46811700 \\
\hline $\mathrm{C}$ & -5.08680200 & 1.73617800 & 2.01888000 & $\mathrm{H}$ & 2.49069300 & -1.71770500 & 5.49794100 \\
\hline $\mathrm{C}$ & -5.03753800 & 2.50205000 & -0.24763000 & $\mathrm{H}$ & 1.23319900 & -2.78265800 & 4.86127500 \\
\hline $\mathrm{C}$ & -5.23518600 & 2.78742000 & 1.10889100 & $\mathrm{H}$ & 3.43910800 & -5.91260200 & 4.98473600 \\
\hline $\mathrm{H}$ & -5.24073300 & 1.94238500 & 3.08183500 & $\mathrm{H}$ & 1.81312600 & -5.36622200 & 4.55613800 \\
\hline $\mathrm{H}$ & -5.14419100 & 3.30587500 & -0.98063800 & $\mathrm{H}$ & 2.64494800 & -4.03729900 & 6.47974900 \\
\hline $\mathrm{C}$ & 2.16306000 & -3.57947400 & -0.03341700 & $\mathrm{H}$ & 4.10796900 & -3.60010800 & 5.58836500 \\
\hline $\mathrm{C}$ & 2.62736800 & -3.34884700 & -1.35541200 & $\mathrm{C}$ & -4.51488300 & 1.00424400 & -2.20562700 \\
\hline $\mathrm{C}$ & 3.01919500 & -3.31688200 & 1.06919900 & $\mathrm{C}$ & -3.24601500 & 1.71273800 & -2.72980800 \\
\hline $\mathrm{C}$ & 3.93352100 & -2.87763400 & -1.54492700 & $\mathrm{C}$ & -5.74796200 & 1.40364700 & -3.04665300 \\
\hline $\mathrm{C}$ & 4.31692800 & -2.84656700 & 0.81619000 & $\mathrm{H}$ & -4.35380000 & -0.06898400 & -2.37985200 \\
\hline $\mathrm{C}$ & 4.80303600 & -2.62845100 & -0.47568100 & $\mathrm{C}$ & -3.03226600 & 1.46428700 & -4.22893200 \\
\hline $\mathrm{H}$ & 4.28804300 & -2.72218700 & -2.56710200 & $\mathrm{H}$ & -3.33098200 & 2.80006200 & -2.55018000 \\
\hline $\mathrm{H}$ & 4.98253600 & -2.65238100 & 1.66161400 & $\mathrm{H}$ & -2.37547700 & 1.35493300 & -2.15889300 \\
\hline $\mathrm{C}$ & 1.77581800 & -3.65230200 & -2.58274900 & $\mathrm{C}$ & -5.52413800 & 1.14712900 & -4.54301000 \\
\hline $\mathrm{C}$ & 2.35956700 & -4.80657900 & -3.43067700 & $\mathrm{H}$ & -5.97081400 & 2.47518000 & -2.89518400 \\
\hline $\mathrm{C}$ & 1.52147200 & -2.41061400 & -3.46628200 & $\mathrm{H}$ & -6.63671500 & 0.85395700 & -2.69225200 \\
\hline $\mathrm{H}$ & 0.78998400 & -3.99674900 & -2.23787100 & $\mathrm{C}$ & -4.26328800 & 1.85407200 & -5.05599200 \\
\hline $\mathrm{C}$ & 1.46301100 & -5.13759200 & -4.63089800 & $\mathrm{H}$ & -2.14213000 & 2.01523100 & -4.57751000 \\
\hline $\mathrm{H}$ & 3.36507600 & -4.52625900 & -3.79379700 & $\mathrm{H}$ & -2.81171300 & 0.39281500 & -4.38908400 \\
\hline $\mathrm{H}$ & 2.49794400 & -5.69893400 & -2.79725000 & $\mathrm{H}$ & -6.40853500 & 1.47065400 & -5.11830000 \\
\hline $\mathrm{C}$ & 0.62805300 & -2.74492000 & -4.66777100 & $\mathrm{H}$ & -5.42413000 & 0.05916200 & -4.71385800 \\
\hline $\mathrm{H}$ & 2.48524900 & -2.01223200 & -3.83219400 & $\mathrm{H}$ & -4.10138000 & 1.62263900 & -6.12267700 \\
\hline $\mathrm{H}$ & 1.05864500 & -1.61844800 & -2.85832900 & $\mathrm{H}$ & -4.41168000 & 2.94875100 & -4.99474200 \\
\hline $\mathrm{C}$ & 1.19746300 & -3.90135600 & -5.49947200 & $\mathrm{C}$ & -5.63594200 & 4.17245200 & 1.59139500 \\
\hline $\mathrm{H}$ & 1.91973900 & -5.94114400 & -5.23380500 & $\mathrm{C}$ & -7.06724000 & 4.55572700 & 1.15018100 \\
\hline
\end{tabular}




\begin{tabular}{|c|c|c|c|}
\hline $\mathrm{C}$ & -4.63991100 & 5.27835900 & 1.18377700 \\
\hline $\mathrm{H}$ & -5.64082900 & 4.13731300 & 2.69645400 \\
\hline $\mathrm{C}$ & -7.48480600 & 5.93287400 & 1.68270300 \\
\hline $\mathrm{H}$ & -7.11482300 & 4.55910500 & 0.04585400 \\
\hline $\mathrm{H}$ & -7.77652900 & 3.78138800 & 1.48641400 \\
\hline $\mathrm{C}$ & -5.05628300 & 6.65349100 & 1.72260500 \\
\hline $\mathrm{H}$ & -4.58385700 & 5.32705900 & 0.08099000 \\
\hline $\mathrm{H}$ & -3.62873600 & 5.01573600 & 1.53609500 \\
\hline $\mathrm{C}$ & -6.48030500 & 7.02322600 & 1.28911300 \\
\hline $\mathrm{H}$ & -8.49355200 & 6.18958000 & 1.31721000 \\
\hline $\mathrm{H}$ & -7.55774300 & 5.88856100 & 2.78532100 \\
\hline $\mathrm{H}$ & -4.34032800 & 7.42402300 & 1.38916800 \\
\hline $\mathrm{H}$ & -5.00384400 & 6.64131100 & 2.82719600 \\
\hline $\mathrm{H}$ & -6.77500900 & 7.99294600 & 1.72470300 \\
\hline $\mathrm{H}$ & -6.50144600 & 7.15675500 & 0.19129900 \\
\hline $\mathrm{C}$ & -4.68128800 & -0.65674700 & 2.68460400 \\
\hline $\mathrm{C}$ & -6.03550400 & -0.89430200 & 3.39218700 \\
\hline $\mathrm{C}$ & -3.56871900 & -0.39065700 & 3.72403500 \\
\hline $\mathrm{H}$ & -4.42534100 & -1.60430800 & 2.18766000 \\
\hline $\mathrm{C}$ & -5.94449500 & -2.01974400 & 4.43150500 \\
\hline $\mathrm{H}$ & -6.35849400 & 0.03704800 & 3.89159300 \\
\hline $\mathrm{H}$ & -6.81018600 & -1.12626400 & 2.64197400 \\
\hline $\mathrm{C}$ & -3.48433200 & -1.51593500 & 4.76393200 \\
\hline $\mathrm{H}$ & -3.76998700 & 0.56526200 & 4.24160400 \\
\hline $\mathrm{H}$ & -2.60398000 & -0.27967600 & 3.20613700 \\
\hline $\mathrm{C}$ & -4.83207600 & -1.75807500 & 5.45452600 \\
\hline $\mathrm{H}$ & -6.91549800 & -2.14369900 & 4.94094800 \\
\hline $\mathrm{H}$ & -5.74206300 & -2.97554300 & 3.91341000 \\
\hline $\mathrm{H}$ & -2.70654500 & -1.28135500 & 5.51054300 \\
\hline $\mathrm{H}$ & -3.15892200 & -2.44579800 & 4.26242300 \\
\hline $\mathrm{H}$ & -4.75654400 & -2.60100900 & 6.16252000 \\
\hline $\mathrm{H}$ & -5.09684100 & -0.86922700 & 6.05784200 \\
\hline $\mathrm{O}$ & 0.17147900 & -0.77585800 & 1.51218700 \\
\hline $\mathrm{H}$ & 1.24667000 & -0.07749100 & 1.44094600 \\
\hline $\mathrm{C}$ & 3.16792500 & 6.99414700 & -2.08035600 \\
\hline $\mathrm{C}$ & 2.76553100 & 5.68501300 & -1.91802000 \\
\hline $\mathrm{C}$ & 1.40403200 & 5.36620800 & -1.65838400 \\
\hline $\mathrm{C}$ & 0.45572500 & 6.43965900 & -1.55135400 \\
\hline $\mathrm{C}$ & 0.90634700 & 7.77858500 & -1.72638600 \\
\hline $\mathrm{C}$ & 2.23093000 & 8.05244700 & -1.98973900 \\
\hline $\mathrm{H}$ & 4.22006000 & 7.21800200 & -2.27235100 \\
\hline $\mathrm{H}$ & 3.49642400 & 4.87946200 & -1.98174100 \\
\hline $\mathrm{C}$ & 0.93096900 & 4.01869200 & -1.49586300 \\
\hline $\mathrm{C}$ & -0.90538100 & 6.14480100 & -1.25898400 \\
\hline $\mathrm{H}$ & 0.17772100 & 8.58976500 & -1.64532000 \\
\hline $\mathrm{H}$ & 2.56336200 & 9.08516800 & -2.12121000 \\
\hline $\mathrm{C}$ & -1.32292000 & 4.84188300 & -1.09813800 \\
\hline $\mathrm{C}$ & -0.40555200 & 3.77147600 & -1.23463000 \\
\hline $\mathrm{H}$ & -1.61511100 & 6.97101700 & -1.16565800 \\
\hline $\mathrm{H}$ & -2.36749200 & 4.61770200 & -0.87499300 \\
\hline $\mathrm{H}$ & -0.76864200 & 2.74692200 & -1.13489100 \\
\hline $\mathrm{N}$ & 1.87394600 & 2.94972700 & -1.61233000 \\
\hline $\mathrm{C}$ & 2.32075800 & 2.54657700 & -2.90859500 \\
\hline $\mathrm{C}$ & 3.40351700 & 1.65647800 & -3.04399100 \\
\hline $\mathrm{C}$ & 1.71540900 & 3.05207100 & -4.07249500 \\
\hline $\mathrm{C}$ & 3.85233400 & 1.27378500 & -4.30886000 \\
\hline $\mathrm{H}$ & 3.92044100 & 1.26175300 & -2.16670700 \\
\hline $\mathrm{C}$ & 2.18491000 & 2.67596400 & -5.33296400 \\
\hline
\end{tabular}

$\begin{array}{lccc}\mathrm{H} & 0.87128100 & 3.73670200 & -3.99153300 \\ \mathrm{C} & 3.25052100 & 1.78240500 & -5.46322500 \\ \mathrm{H} & 4.69298200 & 0.57990500 & -4.38618600 \\ \mathrm{H} & 1.69713800 & 3.08099100 & -6.22331200 \\ \mathrm{H} & 3.60888900 & 1.48590800 & -6.45142700 \\ \mathrm{~N} & 1.73160200 & 1.89795100 & -0.65487700 \\ \mathrm{H} & 0.82206900 & 1.38277000 & -0.75594500 \\ \mathrm{C} & 3.20068300 & 2.73184500 & 1.60633900 \\ \mathrm{C} & 2.00978500 & 1.81270500 & 1.29867700 \\ \mathrm{O} & 2.19335700 & 0.54035700 & 1.36583700 \\ \mathrm{H} & 2.45242600 & 1.20363200 & -0.85545900 \\ \mathrm{C} & 0.73933300 & 2.45863400 & 1.85903100 \\ \mathrm{H} & 0.55869000 & 1.98103700 & 2.83521700 \\ \mathrm{H} & -0.13928900 & 2.23105100 & 1.24409100 \\ \mathrm{C} & 1.05190000 & 3.95667300 & 1.99460000 \\ \mathrm{H} & 0.85564600 & 4.34431900 & 3.00759600 \\ \mathrm{H} & 0.48031000 & 4.59196900 & 1.30387600 \\ \mathrm{C} & 2.54330600 & 4.11665200 & 1.68398400 \\ \mathrm{O} & 3.11772400 & 5.16503800 & 1.53324500 \\ \mathrm{C} & 3.64740100 & 2.31983000 & 3.07192800 \\ \mathrm{H} & 3.89637900 & 1.25005100 & 3.01704700 \\ \mathrm{C} & 4.38140400 & 2.67016400 & 0.64438300 \\ \mathrm{H} & 5.18532600 & 3.32888700 & 0.99816000 \\ \mathrm{H} & 4.77345300 & 1.64302400 & 0.57858300 \\ \mathrm{H} & 8.20567700 & 2.86111000 & 4.03808800 \\ \mathrm{H} & 4.09381600 & 3.01528100 & -0.35669600 \\ \mathrm{C} & 4.80876300 & 3.09111200 & 3.65673500 \\ \mathrm{C} & 4.61559200 & 4.31176800 & 4.32448300 \\ \mathrm{C} & 6.11608200 & 2.58420900 & 3.56639000 \\ \mathrm{C} & 5.69380000 & 5.00729400 & 4.87650500 \\ \mathrm{H} & 3.60843900 & 4.72553800 & 4.41801600 \\ \mathrm{H} & 7.19769500 & 3.27667700 & 4.11712300 \\ \mathrm{H} & 6.28652300 & 1.62753400 & 3.06473300 \\ \mathrm{H} & 6.98961100 & 4.49314100 & 4.77304600 \\ \mathrm{H} & 5.51987500 & 5.95514400 & 5.39222500 \\ \mathrm{H} & & & \\ \mathrm{H} & & & \\ \mathrm{H} & .78117000 & 2.40168300 & 3.74769600 \\ \mathrm{H} & & & \\ \mathrm{H} & & & \end{array}$

\section{ts-1-R-S}

$\begin{array}{llll}\text { C } & 3.19463300 & -1.84216000 & 0.17002500\end{array}$

$\begin{array}{llll}\text { C } & 2.95490500 & -3.12602600 & 0.65583800\end{array}$

$\begin{array}{llll}\mathrm{C} & 3.86878000 & -3.66687700 & 1.63214900\end{array}$

$\begin{array}{llll}\text { C } & 5.07901300 & -2.95531800 & 1.92313400\end{array}$

$\begin{array}{llll}\text { C } & 5.31953100 & -1.70961500 & 1.28831000\end{array}$

$\begin{array}{llll}\text { C } & 4.39009200 & -1.11361700 & 0.45735600\end{array}$

$\begin{array}{llll}\mathrm{H} & 2.68175200 & -5.40624400 & 2.18583600\end{array}$

$\begin{array}{llll}\text { C } & 3.61457300 & -4.86863800 & 2.35491800\end{array}$

$\begin{array}{llll}\text { C } & 6.00306500 & -3.49453900 & 2.86280800\end{array}$

$\begin{array}{llll}\mathrm{H} & 6.25580600 & -1.18643900 & 1.49831400\end{array}$

$\begin{array}{llll}\text { C } & 5.73433500 & -4.67164900 & 3.52654600\end{array}$

$\begin{array}{llll}\text { C } & 4.51844800 & -5.35401300 & 3.27797300\end{array}$

$\begin{array}{llll}\mathrm{H} & 6.92717700 & -2.94425100 & 3.05973000\end{array}$

$\mathrm{H} \quad 6.44665300 \quad-5.07161300 \quad 4.25248000$

$\begin{array}{llll}\mathrm{H} & 4.29065700 & -6.27212500 & 3.82551100\end{array}$

$\begin{array}{llll}\text { C } & -0.67258700 & -4.16370100 & -0.05318000\end{array}$

$\begin{array}{llll}\mathrm{C} & -0.67258700 & -4.16370100 & -0.05318000 \\ \mathrm{C} & 0.49172400 & -5.48149000 & -0.42801100\end{array}$

$\begin{array}{llll}\text { C } & 0.79564000 & -6.04579500 & -0.62500700\end{array}$

$\begin{array}{llll}\text { C } & 1.95947500 & -5.25433500 & -0.35244800\end{array}$ 


\begin{tabular}{|c|c|c|c|c|c|c|c|}
\hline $\mathrm{C}$ & 1.78739800 & -3.92441500 & 0.17796100 & $\mathrm{C}$ & -8.73303100 & -1.55331500 & 1.74627200 \\
\hline C & 0.49983000 & -3.39968800 & 0.23637600 & $\mathrm{H}$ & -7.96969300 & 0.48760800 & 1.69268100 \\
\hline & 0.05071600 & -7.96668800 & -1.30630100 & $\mathrm{H}$ & -8.21840400 & -0.23724400 & 0.10356500 \\
\hline $\mathrm{H}$ & -1.36772000 & -6.10536300 & -0.61826100 & $\mathrm{H}$ & -9.14013900 & -3.68071400 & 1.50493100 \\
\hline C & 0.94931900 & -7.37267800 & -1.11826000 & $\mathrm{H}$ & -8.93806500 & -2.79893800 & -0.01282000 \\
\hline C & 3.23341000 & -5.81786200 & -0.65245400 & $\mathrm{H}$ & -9.79453600 & -1.25346200 & 1.73436200 \\
\hline C & 3.34918800 & -7.09874400 & -1.15332700 & $\mathrm{H}$ & -8.45817000 & -1.67913600 & 2.81030200 \\
\hline C & 2.19865000 & -7.89423500 & -1.37485500 & $\mathrm{C}$ & -2.33351000 & -3.79063400 & -2.52299600 \\
\hline H & 4.13116000 & -5.22026300 & -0.49562100 & $\mathrm{C}$ & -2.48503100 & -2.65511300 & -3.55990900 \\
\hline $\mathrm{H}$ & 4.33880200 & -7.50089200 & -1.38469400 & $\mathrm{C}$ & -3.00608300 & -5.07783200 & -3.05790300 \\
\hline $\mathrm{F}$ & 2.30375500 & -8.91085500 & -1.76185000 & $\mathrm{H}$ & -1.25488000 & -3.99368900 & -2.44903900 \\
\hline $\mathrm{O}$ & 0.32935600 & -2.09824900 & 0.66233200 & $\mathrm{C}$ & -1.92799700 & -3.06551100 & -4.93029400 \\
\hline C & 2.29927500 & -1.25988000 & -0.70367300 & $\mathrm{H}$ & -3.55158500 & -2.38856100 & -3.67211500 \\
\hline $\mathrm{P}$ & 0.76912900 & -0.83472400 & -0.29540200 & $\mathrm{H}$ & -1.96968500 & -1.75566700 & -3.19626700 \\
\hline C & 0.66201400 & 0.41214800 & 0.54163400 & $\mathrm{C}$ & -2.45659200 & -5.48909600 & -4.42980600 \\
\hline$C$ & 4.64951100 & 0.24993300 & -0.11029700 & $\mathrm{H}$ & -4.09585300 & -4.90848000 & -3.13368800 \\
\hline C & 4.91783700 & 0.41266200 & -1.49261000 & $\mathrm{H}$ & -2.87829000 & -5.90077400 & -2.33558300 \\
\hline$C$ & 4.69037600 & 1.37623900 & 0.75429100 & $\mathrm{C}$ & -2.57805800 & -4.35258900 & -5.45175100 \\
\hline C & 5.22821000 & 1.69187000 & -1.97477500 & $\mathrm{H}$ & -2.06919900 & -2.24470900 & -5.65434900 \\
\hline $\mathrm{C}$ & 5.00759000 & 2.63179400 & 0.21465100 & $\mathrm{H}$ & -0.83623500 & -3.21672500 & -4.84508000 \\
\hline C & 5.28914900 & 2.81572300 & -1.14463000 & $\mathrm{H}$ & -2.98001000 & -6.39061100 & -4.79196900 \\
\hline $\mathrm{H}$ & 5.45179600 & 1.81699700 & -3.03798800 & $\mathrm{H}$ & -1.39245100 & -5.76942500 & -4.32298900 \\
\hline $\mathrm{H}$ & 5.04829600 & 3.49207700 & 0.88762800 & $\mathrm{H}$ & -2.12708500 & -4.65063600 & -6.41363200 \\
\hline $\mathrm{C}$ & -2.05309400 & -3.58197300 & 0.04330700 & $\mathrm{H}$ & -3.64856600 & -4.16229200 & -5.65614500 \\
\hline C & -2.60206700 & -3.24273300 & 1.30802900 & $\mathrm{C}$ & 4.39867600 & 1.27630800 & 2.24967700 \\
\hline $\mathrm{c}$ & -2.84070200 & -3.42798100 & -1.12906300 & $\mathrm{C}$ & 3.10370200 & 2.01528200 & 2.65377300 \\
\hline C & -3.92281600 & -2.77837600 & 1.37383100 & $\mathrm{C}$ & 5.58508100 & 1.74065500 & 3.12384000 \\
\hline c & -4.15603700 & -2.95672400 & -0.99972300 & $\mathrm{H}$ & 4.22761800 & 0.21818000 & 2.49287500 \\
\hline C & -4.72583400 & -2.63578200 & 0.23563400 & $\mathrm{C}$ & 2.81221900 & 1.87271700 & 4.15385100 \\
\hline $\mathrm{H}$ & -4.34283700 & -2.54246600 & 2.35465500 & $\mathrm{H}$ & 3.19478100 & 3.08751700 & 2.40134200 \\
\hline $\mathrm{H}$ & -4.76838700 & -2.84762000 & -1.89899200 & $\mathrm{H}$ & 2.26501700 & 1.61213300 & 2.06560800 \\
\hline C & -1.82768100 & -3.41437800 & 2.61050000 & $\mathrm{C}$ & 5.28372400 & 1.59043200 & 4.62095400 \\
\hline C & -2.46340400 & -4.47945000 & 3.53412400 & $\mathrm{H}$ & 5.81446000 & 2.79955500 & 2.90706100 \\
\hline C & -1.63551300 & -2.08485900 & 3.37377900 & $\mathrm{H}$ & 6.49201500 & 1.17157300 & 2.85664200 \\
\hline $\mathrm{H}$ & -0.82092500 & -3.78382400 & 2.36575800 & $\mathrm{C}$ & 3.99688300 & 2.32585300 & 5.01543400 \\
\hline 0 & -1.65319100 & -4.67161000 & 4.82336100 & $\mathrm{H}$ & 1.90418700 & 2.44250000 & 4.41538900 \\
\hline $\mathrm{H}$ & -3.49332000 & -4.17457500 & 3.79404800 & $\mathrm{H}$ & 2.58447700 & 0.81421200 & 4.37744400 \\
\hline H & -2.55294000 & -5.43552100 & 2.99128100 & $\mathrm{H}$ & 6.13631400 & 1.95867100 & 5.21699800 \\
\hline C & -0.82997400 & -2.28122100 & 4.66490000 & $\mathrm{H}$ & 5.17635700 & 0.51695700 & 4.86366700 \\
\hline $\mathrm{H}$ & -2.62273300 & -1.65732700 & 3.62686500 & $\mathrm{H}$ & 3.77838400 & 2.16934900 & 6.08563600 \\
\hline $\mathrm{H}$ & -1.13064300 & -1.35825100 & 2.71946500 & $\mathrm{H}$ & 4.14726800 & 3.41418100 & 4.88503800 \\
\hline C & -1.45588700 & -3.34772100 & 5.57212400 & $\mathrm{C}$ & 5.69987500 & 4.16758100 & -1.70677500 \\
\hline H & -2.14857300 & -5.41261700 & 5.47397100 & $\mathrm{C}$ & 7.09612500 & 4.60452000 & -1.20673800 \\
\hline H & -0.66376300 & -5.09510700 & 4.56926100 & $\mathrm{C}$ & 4.66812000 & 5.28519600 & -1.44743100 \\
\hline $\mathrm{H}$ & -0.74366600 & -1.32142000 & 5.20195500 & $\mathrm{H}$ & 5.77530700 & 4.04965200 & -2.80352700 \\
\hline $\mathrm{H}$ & 0.20109200 & -2.58323200 & 4.40464900 & $\mathrm{C}$ & 7.53298000 & 5.94559800 & -1.81091900 \\
\hline $\mathrm{H}$ & -0.83377500 & -3.50392800 & 6.46993900 & $\mathrm{H}$ & 7.07305000 & 4.68883200 & -0.10485000 \\
\hline 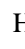 & -2.43743000 & -2.98705500 & 5.93361700 & $\mathrm{H}$ & 7.83312400 & 3.81824100 & -1.43978000 \\
\hline C & -6.18086900 & -2.20370400 & 0.32949600 & $\mathrm{C}$ & 5.10523600 & 6.62367200 & -2.05794700 \\
\hline C & -6.38456000 & -0.86477600 & 1.06901400 & $\mathrm{H}$ & 4.53889300 & 5.41376100 & -0.35726800 \\
\hline C & -7.06923200 & -3.30148300 & 0.96003000 & $\mathrm{H}$ & 3.68494500 & 4.98304400 & -1.84465500 \\
\hline 1 & -6.54118300 & -2.05722600 & -0.70577800 & $\mathrm{C}$ & 6.49367600 & 7.04567300 & -1.56162500 \\
\hline 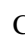 & -7.86170400 & -0.45342000 & 1.12733100 & $\mathrm{H}$ & 8.51349400 & 6.24291000 & -1.40194000 \\
\hline $\mathrm{H}$ & -5.99226700 & -0.95368800 & 2.09823400 & $\mathrm{H}$ & 7.67691300 & 5.82343300 & -2.90061100 \\
\hline H & -5.79029900 & -0.07728600 & 0.57554100 & $\mathrm{H}$ & 4.36082600 & 7.40536600 & -1.82938500 \\
\hline C & -8.54578800 & -2.88950000 & 1.01730300 & $\mathrm{H}$ & 5.12611800 & 6.53039700 & -3.15968700 \\
\hline H & -6.70668300 & -3.51180800 & 1.98275700 & $\mathrm{H}$ & 6.80523900 & 7.98648700 & -2.04609000 \\
\hline $\mathrm{H}$ & -6.95139300 & -4.23971300 & 0.39311300 & $\mathrm{H}$ & 6.44201200 & 7.25700000 & -0.47709400 \\
\hline \multicolumn{8}{|c|}{ S158 } \\
\hline
\end{tabular}




\begin{tabular}{|c|c|c|c|}
\hline $\mathrm{C}$ & 4.94393600 & -0.76200000 & -2.46371700 \\
\hline $\mathrm{C}$ & 6.36146400 & -1.01695200 & -3.02729700 \\
\hline $\mathrm{C}$ & 3.92251600 & -0.62370000 & -3.61492500 \\
\hline $\mathrm{H}$ & 4.66773200 & -1.67057000 & -1.90819800 \\
\hline $\mathrm{C}$ & 6.39183500 & -2.23086600 & -3.96487300 \\
\hline $\mathrm{H}$ & 6.70531400 & -0.12233700 & -3.57756800 \\
\hline $\mathrm{H}$ & 7.07089300 & -1.15774600 & -2.19445200 \\
\hline $\mathrm{C}$ & 3.95913400 & -1.83806200 & -4.55226100 \\
\hline $\mathrm{H}$ & 4.14543700 & 0.28922700 & -4.19714700 \\
\hline $\mathrm{H}$ & 2.91306500 & -0.49884100 & -3.19429600 \\
\hline $\mathrm{C}$ & 5.36848700 & -2.09658300 & -5.09960400 \\
\hline $\mathrm{H}$ & 7.40713500 & -2.36662200 & -4.37538100 \\
\hline $\mathrm{H}$ & 6.16866100 & -3.14396600 & -3.38237500 \\
\hline $\mathrm{H}$ & 3.24567700 & -1.69489800 & -5.38167500 \\
\hline $\mathrm{H}$ & 3.61237100 & -2.73044600 & -3.99955200 \\
\hline $\mathrm{H}$ & 5.37635500 & -3.00030400 & -5.73265100 \\
\hline $\mathrm{H}$ & 5.66367400 & -1.25574900 & -5.75528300 \\
\hline $\mathrm{O}$ & 0.01124500 & -0.87082600 & -1.62490900 \\
\hline $\mathrm{H}$ & -1.07377500 & -0.17575400 & -1.69477400 \\
\hline $\mathrm{C}$ & -3.29039300 & 7.08588800 & 1.33376900 \\
\hline $\mathrm{C}$ & -2.87021000 & 5.77526500 & 1.24235400 \\
\hline $\mathrm{C}$ & -1.49481100 & 5.45965400 & 1.06456900 \\
\hline $\mathrm{C}$ & -0.55013200 & 6.53690700 & 0.96699200 \\
\hline $\mathrm{C}$ & -1.01937900 & 7.87716900 & 1.06637100 \\
\hline $\mathrm{C}$ & -2.35792000 & 8.14869900 & 1.24959200 \\
\hline $\mathrm{H}$ & -4.35244900 & 7.30676500 & 1.46541400 \\
\hline $\mathrm{H}$ & -3.59733000 & 4.96588200 & 1.30273200 \\
\hline $\mathrm{C}$ & -1.00464300 & 4.11130500 & 0.97615900 \\
\hline $\mathrm{C}$ & 0.82707900 & 6.24411400 & 0.75943100 \\
\hline $\mathrm{H}$ & -0.29349800 & 8.69148300 & 0.99244700 \\
\hline $\mathrm{H}$ & -2.70412400 & 9.18260400 & 1.32378500 \\
\hline $\mathrm{C}$ & 1.26161700 & 4.93997900 & 0.67102200 \\
\hline $\mathrm{C}$ & 0.34588500 & 3.86701300 & 0.79827500 \\
\hline $\mathrm{H}$ & 1.53475400 & 7.07281000 & 0.67295500 \\
\hline $\mathrm{H}$ & 2.31807600 & 4.71607900 & 0.51387900 \\
\hline $\mathrm{H}$ & 0.72237200 & 2.84334000 & 0.75974500 \\
\hline $\mathrm{N}$ & -1.94865600 & 3.04257600 & 1.08449300 \\
\hline $\mathrm{C}$ & -2.45762900 & 2.70114600 & 2.37995100 \\
\hline $\mathrm{C}$ & -3.58074300 & 1.86131100 & 2.49679200 \\
\hline $\mathrm{C}$ & -1.87110700 & 3.21255800 & 3.54966500 \\
\hline $\mathrm{C}$ & -4.08821100 & 1.52880300 & 3.75348300 \\
\hline $\mathrm{H}$ & -4.07924900 & 1.47027100 & 1.60749000 \\
\hline $\mathrm{C}$ & -2.39967800 & 2.89004300 & 4.80195200 \\
\hline $\mathrm{H}$ & -0.99726200 & 3.86038200 & 3.48049600 \\
\hline $\mathrm{C}$ & -3.50505400 & 2.04374800 & 4.91503600 \\
\hline $\mathrm{H}$ & -4.95932600 & 0.87243100 & 3.81825100 \\
\hline $\mathrm{H}$ & -1.92803800 & 3.29872500 & 5.69925200 \\
\hline $\mathrm{H}$ & -3.90996300 & 1.78907700 & 5.89696500 \\
\hline $\mathrm{N}$ & -1.71746100 & 1.92565400 & 0.21708400 \\
\hline $\mathrm{H}$ & -0.80727500 & 1.44010900 & 0.41938400 \\
\hline $\mathrm{C}$ & -3.03696300 & 2.58531700 & -2.17654300 \\
\hline $\mathrm{C}$ & -1.85648200 & 1.70908400 & -1.72648600 \\
\hline $\mathrm{O}$ & -2.02288100 & 0.43187900 & -1.73503800 \\
\hline $\mathrm{H}$ & -2.43677900 & 1.23199200 & 0.42661300 \\
\hline $\mathrm{C}$ & -0.56236900 & 2.34440200 & -2.24261900 \\
\hline $\mathrm{H}$ & -0.31282400 & 1.81564000 & -3.17580900 \\
\hline $\mathrm{H}$ & 0.27858800 & 2.17002100 & -1.56148200 \\
\hline $\mathrm{C}$ & -0.89105000 & 3.82693600 & -2.48266000 \\
\hline
\end{tabular}

$\begin{array}{llll}\mathrm{H} & -0.66832400 & 4.15089600 & -3.51250600 \\ \mathrm{H} & -0.35531200 & 4.51732100 & -1.81679800 \\ \mathrm{C} & -2.39494400 & 3.98050200 & -2.24154700 \\ \mathrm{O} & -2.97299100 & 5.03309300 & -2.13147300 \\ \mathrm{C} & -4.29986400 & 2.45909300 & -1.28717800 \\ \mathrm{H} & -4.14916100 & 3.08343900 & -0.39465000 \\ \mathrm{C} & -3.33494800 & 2.16001900 & -3.64329000 \\ \mathrm{H} & -3.72290700 & 1.13172900 & -3.65415800 \\ \mathrm{H} & -4.08713200 & 2.83200500 & -4.07720600 \\ \mathrm{H} & -2.43650200 & 2.18979500 & -4.27726500 \\ \mathrm{C} & -5.63859400 & 2.79871800 & -1.91762600 \\ \mathrm{C} & -6.52201100 & 1.77235800 & -2.29017200 \\ \mathrm{C} & -6.03928600 & 4.13011000 & -2.12479600 \\ \mathrm{C} & -7.76833500 & 2.06096100 & -2.85567200 \\ \mathrm{H} & -6.23101800 & 0.72949400 & -2.13326600 \\ \mathrm{C} & -7.28472500 & 4.42036200 & -2.68809500 \\ \mathrm{H} & -5.35442300 & 4.93698700 & -1.85824700 \\ \mathrm{C} & -8.15439300 & 3.38848300 & -3.05625000 \\ \mathrm{H} & -8.43892600 & 1.24485100 & -3.13760500 \\ \mathrm{H} & -7.57799800 & 5.46255700 & -2.84060100 \\ \mathrm{H} & -9.12844300 & 3.61866400 & -3.49572200 \\ \mathrm{H} & -4.34360300 & 1.40849500 & -0.95766000\end{array}$

ts-1-S-R

$\begin{array}{llll}\text { C } & 1.64179000 & -3.20731000 & 0.04388400\end{array}$

$\begin{array}{llll}\text { C } & 3.01306300 & -3.16939400 & -0.19503400\end{array}$

$\begin{array}{llll}\text { C } & 3.58944900 & -4.19476800 & -1.02852800\end{array}$

$\begin{array}{llll}\text { C } & 2.77600100 & -5.30781700 & -1.42282600\end{array}$

$\begin{array}{llll}\mathrm{C} & 1.41451300 & -5.34804500 & -1.02395100\end{array}$

$\begin{array}{llll}\text { C } & 0.81542900 & -4.30875700 & -0.33778100\end{array}$

$\begin{array}{llll}\mathrm{H} & 5.55479300 & -3.28784400 & -1.25857700\end{array}$

$\begin{array}{llll}\text { C } & 4.92866600 & -4.14318100 & -1.51259200\end{array}$

$\begin{array}{llll}\text { C } & 3.34010100 & -6.33888500 & -2.22666100\end{array}$

$\mathrm{H} \quad 0.81250000 \quad-6.21585200 \quad-1.30478600$

$\begin{array}{llll}\text { C } & 4.64564700 & -6.26557100 & -2.66136800\end{array}$

$\begin{array}{llll}\text { C } & 5.44005500 & -5.14683100 & -2.31010900\end{array}$

$\begin{array}{llll}\mathrm{H} & 2.70860200 & -7.18668300 & -2.50611400\end{array}$

$\begin{array}{llll}\mathrm{H} & 5.06511400 & -7.05858600 & -3.28545500\end{array}$

$\begin{array}{llll}\mathrm{H} & 6.46726900 & -5.07528400 & -2.67661100\end{array}$

$\begin{array}{llll}\text { C } & 4.41425400 & 0.30801800 & 0.62799700\end{array}$

$\begin{array}{llll}\text { C } & 5.58105100 & -0.03408600 & 1.28373000\end{array}$

$\begin{array}{llll}\text { C } & 5.89546000 & -1.37611800 & 1.62290800\end{array}$

$\begin{array}{llll}\text { C } & 5.01752900 & -2.42949600 & 1.20489800\end{array}$

$\begin{array}{llll}\text { C } & 3.86167000 & -2.09957400 & 0.40758900\end{array}$

$\begin{array}{llll}\mathrm{C} & 3.55264600 & -0.75622800 & 0.21824800\end{array}$

$\begin{array}{llll}\mathrm{H} & 7.72001600 & -0.87570800 & 2.68541900\end{array}$

$\begin{array}{llll}\mathrm{H} & 6.27596200 & 0.75518200 & 1.57965100\end{array}$

$\begin{array}{llll}\text { C } & 7.05441600 & -1.69051900 & 2.38765700\end{array}$

$\begin{array}{llll}\text { C } & 5.31784500 & -3.75560400 & 1.63116100\end{array}$

$\begin{array}{llll}\text { C } & 6.43760400 & -4.02632500 & 2.39126400\end{array}$

$\begin{array}{llll}\mathrm{C} & 7.32630600 & -2.98824000 & 2.76284600\end{array}$

$\begin{array}{llll}\mathrm{H} & 4.64264700 & -4.56780900 & 1.36285000\end{array}$

$\begin{array}{llll}\mathrm{H} & 6.63698100 & -5.05158600 & 2.71315100\end{array}$

$\begin{array}{llll}\mathrm{H} & 8.21469600 & -3.21654900 & 3.35695200\end{array}$

$\begin{array}{llll}\mathrm{O} & 2.39567000 & -0.42179800 & -0.46292900\end{array}$

$\begin{array}{llll}\mathrm{O} & 1.04974500 & -2.19170400 & 0.76318900\end{array}$

$\begin{array}{llll}\mathrm{P} & 0.92328800 & -0.64188000 & 0.23344400\end{array}$

$\begin{array}{llll}\text { O } & -0.13737300 & -0.42453700 & -0.80796600\end{array}$ 


\begin{tabular}{|c|c|c|c|}
\hline $\mathrm{C}$ & -0.64767400 & -4.35241500 & -0.01217100 \\
\hline $\mathrm{C}$ & -1.08069900 & -4.51776700 & 1.32749500 \\
\hline $\mathrm{C}$ & -1.60463000 & -4.27888000 & -1.05882100 \\
\hline $\mathrm{C}$ & -2.45521800 & -4.61235400 & 1.58649200 \\
\hline $\mathrm{C}$ & -2.96619000 & -4.37918600 & -0.73890000 \\
\hline $\mathrm{C}$ & -3.41833000 & -4.55059800 & 0.57487100 \\
\hline $\mathrm{H}$ & -2.79125800 & -4.74985200 & 2.61799800 \\
\hline $\mathrm{H}$ & -3.69812500 & -4.32561200 & -1.54892800 \\
\hline $\mathrm{C}$ & 4.09800500 & 1.74561400 & 0.33552000 \\
\hline $\mathrm{C}$ & 4.19816400 & 2.23396200 & -0.99310100 \\
\hline $\mathrm{C}$ & 3.75942700 & 2.63308700 & 1.38933900 \\
\hline $\mathrm{C}$ & 3.95248400 & 3.59118600 & -1.23954700 \\
\hline $\mathrm{C}$ & 3.52606200 & 3.98311500 & 1.08233700 \\
\hline $\mathrm{C}$ & 3.61482100 & 4.48836800 & -0.21898100 \\
\hline $\mathrm{H}$ & 4.04216700 & 3.96070800 & -2.26378700 \\
\hline $\mathrm{H}$ & 3.27284200 & 4.67315700 & 1.89226700 \\
\hline $\mathrm{C}$ & 4.61177600 & 1.34031800 & -2.15703000 \\
\hline $\mathrm{C}$ & 5.97903600 & 1.74780700 & -2.75431900 \\
\hline $\mathrm{C}$ & 3.54163500 & 1.26070000 & -3.26765900 \\
\hline $\mathrm{H}$ & 4.73798100 & 0.31705900 & -1.77340900 \\
\hline $\mathrm{C}$ & 6.40380700 & 0.81148600 & -3.89358200 \\
\hline $\mathrm{H}$ & 5.92051500 & 2.78335400 & -3.13593000 \\
\hline $\mathrm{H}$ & 6.74314500 & 1.75516500 & -1.95902200 \\
\hline $\mathrm{C}$ & 3.97126000 & 0.32622600 & -4.40645600 \\
\hline $\mathrm{H}$ & 3.36030300 & 2.27140300 & -3.67699000 \\
\hline $\mathrm{H}$ & 2.59262900 & 0.91575500 & -2.82869900 \\
\hline $\mathrm{C}$ & 5.33313100 & 0.72528800 & -4.98827300 \\
\hline $\mathrm{H}$ & 7.36424200 & 1.14780200 & -4.32022000 \\
\hline $\mathrm{H}$ & 6.58482200 & -0.19927500 & -3.48336300 \\
\hline $\mathrm{H}$ & 3.20288000 & 0.31821200 & -5.19830600 \\
\hline $\mathrm{H}$ & 4.02737900 & -0.70865600 & -4.02200000 \\
\hline $\mathrm{H}$ & 5.64155400 & 0.01176200 & -5.77117500 \\
\hline $\mathrm{H}$ & 5.24209600 & 1.71000700 & -5.48431100 \\
\hline $\mathrm{C}$ & 3.39611300 & 5.96778200 & -0.49302500 \\
\hline $\mathrm{C}$ & 2.37142400 & 6.24420300 & -1.61354100 \\
\hline $\mathrm{C}$ & 4.72268000 & 6.70449300 & -0.79178600 \\
\hline $\mathrm{H}$ & 2.98733300 & 6.41030500 & 0.43467400 \\
\hline $\mathrm{C}$ & 2.15417600 & 7.74700400 & -1.83347800 \\
\hline $\mathrm{H}$ & 2.72682000 & 5.79076200 & -2.55610000 \\
\hline $\mathrm{H}$ & 1.41601400 & 5.74683200 & -1.37777400 \\
\hline $\mathrm{C}$ & 4.50928500 & 8.20642600 & -1.02106500 \\
\hline $\mathrm{H}$ & 5.18531900 & 6.25620200 & -1.69007700 \\
\hline $\mathrm{H}$ & 5.43187500 & 6.53746500 & 0.03581500 \\
\hline $\mathrm{C}$ & 3.47462000 & 8.47244800 & -2.12162500 \\
\hline $\mathrm{H}$ & 1.43822600 & 7.90894200 & -2.65690700 \\
\hline $\mathrm{H}$ & 1.68983100 & 8.18433900 & -0.92982300 \\
\hline $\mathrm{H}$ & 5.46884100 & 8.69050300 & -1.27014200 \\
\hline $\mathrm{H}$ & 4.16436000 & 8.67171100 & -0.07882700 \\
\hline $\mathrm{H}$ & 3.30177400 & 9.55626500 & -2.23292100 \\
\hline $\mathrm{H}$ & 3.87651400 & 8.12080700 & -3.09018800 \\
\hline $\mathrm{C}$ & 3.67282600 & 2.18469600 & 2.84610900 \\
\hline $\mathrm{C}$ & 2.33798400 & 2.55113700 & 3.53090500 \\
\hline $\mathrm{C}$ & 4.85846400 & 2.72001000 & 3.68385100 \\
\hline $\mathrm{H}$ & 3.73124800 & 1.08619900 & 2.86392500 \\
\hline $\mathrm{C}$ & 2.28837100 & 2.05017100 & 4.98060500 \\
\hline $\mathrm{H}$ & 2.20202400 & 3.64828800 & 3.52694100 \\
\hline $\mathrm{H}$ & 1.50944700 & 2.11310900 & 2.95733200 \\
\hline $\mathrm{C}$ & 4.80864600 & 2.22611600 & 5.13570300 \\
\hline
\end{tabular}

$\begin{array}{llll}\mathrm{H} & 4.83882500 & 3.82530400 & 3.67098800 \\ \mathrm{H} & 5.81257800 & 2.42848900 & 3.21465300 \\ \mathrm{C} & 3.47233100 & 2.56856800 & 5.80534300 \\ \mathrm{H} & 1.33462600 & 2.34762000 & 5.44905700 \\ \mathrm{H} & 2.29890300 & 0.94501000 & 4.97838500 \\ \mathrm{H} & 5.64804400 & 2.65507200 & 5.70940300 \\ \mathrm{H} & 4.95115900 & 1.12978000 & 5.14968700 \\ \mathrm{H} & 3.43929800 & 2.15705200 & 6.82850100 \\ \mathrm{H} & 3.39011100 & 3.66691900 & 5.90974100 \\ \mathrm{C} & -1.21095500 & -4.07770900 & -2.51948600 \\ \mathrm{C} & -1.72369400 & -2.73795400 & -3.09242300\end{array}$

C $\quad-1.64375000 \quad-5.25362100 \quad-3.42350900$

$\mathrm{H} \quad-0.11413400 \quad-4.02803900 \quad-2.57433800$

$\begin{array}{llll}\text { C } & -1.28582700 & -2.53652500 & -4.54913600\end{array}$

$\mathrm{H} \quad-2.82787800 \quad-2.71886600 \quad-3.04165200$

$\mathrm{H} \quad-1.35063700 \quad-1.91575000 \quad-2.46122500$

C $\quad-1.20367100 \quad-5.04649000 \quad-4.87889900$

$\mathrm{H} \quad-2.74303200 \quad-5.36190700 \quad-3.38804400$

$\begin{array}{llll}\mathrm{H} & -1.23251600 & -6.19865100 & -3.02949600\end{array}$

$\begin{array}{llll}\text { C } & -1.70936500 & -3.71125000 & -5.43942100\end{array}$

$\mathrm{H} \quad-1.69590400 \quad-1.58933200 \quad-4.93879300$

$\mathrm{H} \quad-0.18579700 \quad-2.43199400 \quad-4.58335900$

$\mathrm{H} \quad-1.55633300 \quad-5.88474300 \quad-5.50393800$

$\mathrm{H} \quad-0.09922800 \quad-5.06533400 \quad-4.92966700$

$\mathrm{H} \quad-1.34465000 \quad-3.56508600 \quad-6.47041000$

$\begin{array}{llll}\mathrm{H} & -2.81362700 & -3.74029500 & -5.50250800\end{array}$

$\begin{array}{llll}\text { C } & -4.89664900 & -4.68037100 & 0.90344900\end{array}$

$\begin{array}{llll}\text { C } & -5.53763900 & -5.93479300 & 0.26862200\end{array}$

$\begin{array}{llll}\text { C } & -5.70799400 & -3.41922000 & 0.53457600\end{array}$

$\mathrm{H} \quad-4.97314300 \quad-4.80176800 \quad 1.99974300$

$\begin{array}{llll}\text { C } & -7.02016700 & -6.07267500 & 0.63835100\end{array}$

$\begin{array}{llll}\mathrm{H} & -5.44031800 & -5.87484900 & -0.83074900\end{array}$

$\begin{array}{llll}\mathrm{H} & -4.97729300 & -6.83258100 & 0.57847500\end{array}$

$\begin{array}{llll}\text { C } & -7.19152400 & -3.55893600 & 0.89994000\end{array}$

$\mathrm{H} \quad-5.61693300 \quad-3.23312100 \quad-0.55056400$

$\begin{array}{llll}\mathrm{H} & -5.27030800 & -2.53836800 & 1.03326200\end{array}$

$\begin{array}{llll}\text { C } & -7.81484300 & -4.81254500 & 0.27334200\end{array}$

$\begin{array}{llll}\mathrm{H} & -7.45329800 & -6.95786600 & 0.14228400\end{array}$

$\begin{array}{llll}\mathrm{H} & -7.10788500 & -6.25415300 & 1.72578900\end{array}$

$\begin{array}{llll}\mathrm{H} & -7.74318600 & -2.65625600 & 0.58684300\end{array}$

$\begin{array}{llll}\mathrm{H} & -7.29130300 & -3.61568400 & 1.99992200\end{array}$

$\begin{array}{llll}\mathrm{H} & -8.86723700 & -4.91694000 & 0.58748100\end{array}$

$\begin{array}{llll}H & -8.86723700 & -4.91694000 & 0.58748100 \\ & -7.82909100 & -4.69964500 & -0.82708300\end{array}$

$\begin{array}{llll}\mathrm{H} & -7.82909100 & -4.69964500 & -0.82708300 \\ \mathrm{C} & -0.10822100 & -4.63708700 & 2.49516000\end{array}$

$\begin{array}{llll}\text { C } & -0.15681400 & -6.03295200 & 3.15951000\end{array}$

$\begin{array}{llll}\text { C } & -0.30507000 & -3.52900300 & 3.55410800\end{array}$

$\mathrm{H} \quad 0.91323200 \quad-4.51943000 \quad 2.10376500$

$\begin{array}{llll}\text { C } & 0.85472000 & -6.15150400 & 4.30771600\end{array}$

$\mathrm{H} \quad-1.17409700 \quad-6.21995600 \quad 3.54882600$

$\mathrm{H} \quad 0.03068800 \quad-6.81158500 \quad 2.40091600$

C $\quad 0.70595100 \quad-3.65396700 \quad 4.70146500$

$\mathrm{H} \quad-1.32849900 \quad-3.59210500 \quad 3.96664000$

$\begin{array}{llll}\mathrm{H} & -0.21424000 & -2.54361900 & 3.07264600\end{array}$

$\begin{array}{llll}\text { C } & 0.66347600 & -5.04307900 & 5.35018400\end{array}$

$\begin{array}{llll}\mathrm{C} & 0.66347600 & -5.04307900 & 5.35018400 \\ \mathrm{H} & 0.77340800 & -7.14471900 & 4.78179600\end{array}$

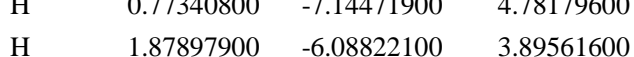

$\begin{array}{llll}\mathrm{H} & 0.51762900 & -2.87131900 & 5.45604700\end{array}$

$\begin{array}{llll}\mathrm{H} & 1.72226500 & -3.46528500 & 4.30934800\end{array}$ 


\begin{tabular}{|c|c|c|c|c|c|c|c|}
\hline $\mathrm{H}$ & 1.42956900 & -5.12379400 & 6.14020200 & $\mathrm{H}$ & -5.26505400 & 5.10292300 & 1.86997700 \\
\hline $\mathrm{H}$ & -0.31369300 & -5.18142600 & 5.85017200 & $\mathrm{C}$ & -5.98875600 & 3.19283800 & 5.17442400 \\
\hline $\mathrm{O}$ & 0.83737100 & 0.18466600 & 1.52338300 & $\mathrm{H}$ & -4.65534600 & 1.67840300 & 4.40901500 \\
\hline $\mathrm{H}$ & -0.23033100 & 0.78132500 & 1.74215700 & $\mathrm{C}$ & -6.60006700 & 4.42693300 & 4.94176300 \\
\hline $\mathrm{C}$ & -6.95741900 & 3.97034500 & -1.28397600 & $\mathrm{H}$ & -6.81414300 & 6.07472000 & 3.55617200 \\
\hline $\mathrm{C}$ & -5.70991100 & 3.38082500 & -1.30783500 & $\mathrm{H}$ & -6.18452400 & 2.64823000 & 6.10193900 \\
\hline $\mathrm{C}$ & -5.55281900 & 2.02237500 & -1.70745900 & $\mathrm{H}$ & -7.27686700 & 4.85577300 & 5.68538300 \\
\hline $\mathrm{C}$ & -6.72578400 & 1.27694100 & -2.08146000 & & & & \\
\hline $\mathrm{C}$ & -7.99480600 & 1.92147100 & -2.04560400 & ts-3-S-S & & & \\
\hline $\mathrm{C}$ & -8.11153400 & 3.23834000 & -1.65769900 & $\mathrm{C}$ & -0.91298500 & 2.79258900 & 0.44222000 \\
\hline $\mathrm{H}$ & -7.05465000 & 5.01229000 & -0.96946500 & $\mathrm{C}$ & 0.19296200 & 3.60639200 & 0.66454200 \\
\hline $\mathrm{H}$ & -4.83396000 & 3.95671400 & -1.01106500 & $\mathrm{C}$ & 0.11376600 & 4.61343500 & 1.68957800 \\
\hline $\mathrm{C}$ & -4.28468900 & 1.35566300 & -1.75885800 & $\mathrm{C}$ & -1.14629300 & 4.85422400 & 2.32852500 \\
\hline $\mathrm{C}$ & -6.59746400 & -0.08295700 & -2.47512300 & $\mathrm{C}$ & -2.27793000 & 4.09457000 & 1.93165100 \\
\hline $\mathrm{H}$ & -8.87980800 & 1.34748900 & -2.33322200 & $\mathrm{C}$ & -2.19191100 & 3.05810000 & 1.02102100 \\
\hline $\mathrm{H}$ & -9.09223000 & 3.71990500 & -1.63408100 & $\mathrm{H}$ & 2.21460500 & 5.17932800 & 1.66940200 \\
\hline $\mathrm{C}$ & -5.36306300 & -0.69493700 & -2.50440500 & $\mathrm{C}$ & 1.24142200 & 5.36830500 & 2.12341900 \\
\hline $\mathrm{C}$ & -4.20261300 & 0.03032000 & -2.14967600 & $\mathrm{C}$ & -1.23906000 & 5.85370000 & 3.33817700 \\
\hline $\mathrm{H}$ & -7.49736500 & -0.63623600 & -2.75645900 & $\mathrm{H}$ & -3.24920800 & 4.33517600 & 2.37026500 \\
\hline $\mathrm{H}$ & -5.26846900 & -1.73820600 & -2.81355100 & $\mathrm{C}$ & -0.12988700 & 6.57359700 & 3.72662900 \\
\hline $\mathrm{H}$ & -3.22933100 & -0.46241000 & -2.19150100 & $\mathrm{C}$ & 1.12343500 & 6.31813000 & 3.11758700 \\
\hline $\mathrm{N}$ & -3.07405700 & 2.07347700 & -1.39716400 & $\mathrm{H}$ & -2.20977700 & 6.03246600 & 3.80877200 \\
\hline $\mathrm{C}$ & -2.27912600 & 2.53265000 & -2.50161200 & $\mathrm{H}$ & -0.21124800 & 7.33306300 & 4.50830800 \\
\hline $\mathrm{C}$ & -2.83171600 & 3.50969400 & -3.35040500 & $\mathrm{H}$ & 2.00636600 & 6.87525800 & 3.44105100 \\
\hline $\mathrm{C}$ & -0.99380800 & 2.03842200 & -2.77577700 & $\mathrm{C}$ & 3.30645400 & 2.07394200 & -0.96894200 \\
\hline $\mathrm{C}$ & -2.11306800 & 3.97908500 & -4.44941500 & $\mathrm{C}$ & 3.81148900 & 3.18178900 & -1.62464900 \\
\hline $\mathrm{H}$ & -3.82705500 & 3.90294200 & -3.14505300 & $\mathrm{C}$ & 3.12699500 & 4.42270200 & -1.67712500 \\
\hline $\mathrm{C}$ & -0.27549300 & 2.53283200 & -3.87126900 & $\mathrm{C}$ & 1.89416100 & 4.56711000 & -0.96274500 \\
\hline $\mathrm{H}$ & -0.54094400 & 1.25536300 & -2.16525200 & $\mathrm{C}$ & 1.40945000 & 3.45582800 & -0.18486000 \\
\hline $\mathrm{C}$ & -0.82557000 & 3.49992700 & -4.71357900 & $\mathrm{C}$ & 2.07123400 & 2.23327900 & -0.26408800 \\
\hline $\mathrm{H}$ & -2.56185300 & 4.73725800 & -5.09629900 & $\mathrm{H}$ & 4.58298800 & 5.38981200 & -2.96300000 \\
\hline $\mathrm{H}$ & 0.72524800 & 2.13968300 & -4.06282700 & $\mathrm{H}$ & 4.77333300 & 3.09716200 & -2.13533800 \\
\hline $\mathrm{H}$ & -0.25934800 & 3.87646500 & -5.56868300 & $\mathrm{C}$ & 3.63386500 & 5.51617500 & -2.43487900 \\
\hline $\mathrm{N}$ & -2.35555700 & 1.47268000 & -0.31266300 & $\mathrm{C}$ & 1.19266100 & 5.80214700 & -1.08769800 \\
\hline $\mathrm{H}$ & -3.03475700 & 0.93931100 & 0.23171000 & $\mathrm{C}$ & 1.69933600 & 6.83807900 & -1.84616500 \\
\hline $\mathrm{H}$ & -1.62401400 & 0.78453800 & -0.59929500 & $\mathrm{C}$ & 2.93918300 & 6.70316300 & -2.51783200 \\
\hline $\mathrm{C}$ & -1.09053700 & 4.45800500 & 0.06235300 & $\mathrm{H}$ & 0.23471500 & 5.92595400 & -0.58341400 \\
\hline $\mathrm{C}$ & -0.38894000 & 3.17737700 & 0.54242700 & $\mathrm{H}$ & 1.13606200 & 7.77091700 & -1.93115200 \\
\hline $\mathrm{C}$ & -2.54914000 & 3.34588600 & 1.75429500 & $\mathrm{H}$ & 3.33347700 & 7.53400900 & -3.10824000 \\
\hline $\mathrm{H}$ & -0.56070900 & 5.37785800 & 0.35716100 & $\mathrm{O}$ & 1.54993700 & 1.15657300 & 0.42914000 \\
\hline $\mathrm{H}$ & -1.21462700 & 4.50647400 & -1.02918200 & $\mathrm{O}$ & -0.79344400 & 1.73265400 & -0.43966300 \\
\hline $\mathrm{H}$ & 0.39082100 & 3.38750000 & 1.29029600 & $\mathrm{P}$ & 0.13932100 & 0.43184800 & -0.03049400 \\
\hline $\mathrm{H}$ & 0.10434500 & 2.61136300 & -0.25558600 & $\mathrm{O}$ & -0.41711200 & -0.32251100 & 1.15060900 \\
\hline $\mathrm{C}$ & -1.47607800 & 2.36761100 & 1.24502000 & $\mathrm{C}$ & -3.43112700 & 2.32055200 & 0.60440000 \\
\hline $\mathrm{O}$ & -1.22926000 & 1.33093300 & 1.95242400 & $\mathrm{C}$ & -4.02986700 & 2.62066200 & -0.64534000 \\
\hline $\mathrm{C}$ & -2.47957800 & 4.46046600 & 0.70198300 & $\mathrm{C}$ & -4.05380600 & 1.39855700 & 1.48441500 \\
\hline $\mathrm{O}$ & -3.36759300 & 5.22908600 & 0.41551500 & $\mathrm{C}$ & -5.23677800 & 1.99154100 & -0.98284700 \\
\hline $\mathrm{C}$ & -3.93600900 & 2.69145800 & 1.98505200 & $\mathrm{C}$ & -5.26609800 & 0.80765600 & 1.09805000 \\
\hline $\mathrm{H}$ & -3.73804500 & 1.65582100 & 2.30554600 & $\mathrm{C}$ & -5.88064700 & 1.09111800 & -0.12800000 \\
\hline $\mathrm{H}$ & -4.46586100 & 2.64887000 & 1.02230100 & $\mathrm{H}$ & -5.70644700 & 2.22480600 & -1.94245800 \\
\hline $\mathrm{C}$ & -1.97972400 & 3.93143400 & 3.07954100 & $\mathrm{H}$ & -5.74680900 & 0.10748400 & 1.78544500 \\
\hline $\mathrm{H}$ & -2.63961000 & 4.72806900 & 3.44714800 & $\mathrm{C}$ & 4.12211200 & 0.80991400 & -0.97690200 \\
\hline $\mathrm{H}$ & -1.92823900 & 3.13431600 & 3.83476400 & $\mathrm{C}$ & 5.04504800 & 0.57870600 & 0.07403700 \\
\hline $\mathrm{H}$ & -0.96968900 & 4.34789700 & 2.95424900 & $\mathrm{C}$ & 4.08426600 & -0.06581700 & -2.09184900 \\
\hline $\mathrm{C}$ & -4.85455100 & 3.32116200 & 3.01675800 & $\mathrm{C}$ & 5.91321700 & -0.51900000 & -0.01360000 \\
\hline $\mathrm{C}$ & -5.47690100 & 4.56233900 & 2.79440500 & $\mathrm{C}$ & 4.98117300 & -1.14409100 & -2.12867500 \\
\hline $\mathrm{C}$ & -5.12482800 & 2.64822600 & 4.21879500 & $\mathrm{C}$ & 5.91008500 & -1.38800900 & -1.11112700 \\
\hline $\mathrm{C}$ & -6.33967600 & 5.10845800 & 3.74808000 & $\mathrm{H}$ & 6.62991400 & -0.68493900 & 0.79420000 \\
\hline
\end{tabular}




\begin{tabular}{|c|c|c|c|c|c|c|c|}
\hline $\mathrm{H}$ & 4.97220500 & -1.81624600 & -2.989966000 & $\mathrm{H}$ & -2.69140200 & -0.88572100 & 2.29927000 \\
\hline $\mathrm{C}$ & 5.18036900 & 1.54184400 & 1.24920900 & $\mathrm{C}$ & -3.63434400 & 1.42116800 & 5.37283600 \\
\hline $\mathrm{C}$ & 6.45327700 & 2.41238300 & 1.12228900 & $\mathrm{H}$ & -5.29875000 & 1.31785400 & 3.98491300 \\
\hline $\mathrm{C}$ & 5.13956300 & 0.85544900 & 2.62965400 & $\mathrm{H}$ & -4.34622400 & 2.79601500 & 3.84416200 \\
\hline $\mathrm{H}$ & 4.32384500 & 2.23334800 & 1.22172700 & $\mathrm{C}$ & -3.45320500 & -0.08304600 & 5.60915700 \\
\hline $\mathrm{C}$ & 6.57519500 & 3.42506100 & 2.26860800 & $\mathrm{H}$ & -2.59197500 & -1.82410000 & 4.62383500 \\
\hline $\mathrm{H}$ & 7.33985100 & 1.75213100 & 1.11740700 & $\mathrm{H}$ & -1.63184000 & -0.34667400 & 4.46454500 \\
\hline $\mathrm{H}$ & 6.45313400 & 2.93443900 & 0.15093300 & $\mathrm{H}$ & -4.25035800 & 1.86583600 & 6.17336200 \\
\hline $\mathrm{C}$ & 5.24988600 & 1.87565100 & 3.77037600 & $\mathrm{H}$ & -2.64854700 & 1.91882400 & 5.42702900 \\
\hline $\mathrm{H}$ & 5.97077100 & 0.13348300 & 2.71578000 & $\mathrm{H}$ & -2.95084200 & -0.26211700 & 6.57525300 \\
\hline $\mathrm{H}$ & 4.21193900 & 0.27180100 & 2.72662700 & $\mathrm{H}$ & -4.44837500 & -0.56057000 & 5.68293600 \\
\hline $\mathrm{C}$ & 6.50891100 & 2.74174100 & 3.63998300 & $\mathrm{C}$ & -7.21636400 & 0.47958600 & -0.52012600 \\
\hline $\mathrm{H}$ & 7.51256400 & 3.99879600 & 2.16814400 & $\mathrm{C}$ & -8.37013500 & 0.94817600 & 0.39531300 \\
\hline $\mathrm{H}$ & 5.75283200 & 4.16032900 & 2.18975300 & $\mathrm{C}$ & -7.18528400 & -1.06185100 & -0.59256800 \\
\hline $\mathrm{H}$ & 5.23995200 & 1.35377000 & 4.74209100 & $\mathrm{H}$ & -7.44828800 & 0.84295700 & -1.53839800 \\
\hline $\mathrm{H}$ & 4.35736000 & 2.52865100 & 3.76137100 & $\mathrm{C}$ & -9.72163000 & 0.36469800 & -0.03736200 \\
\hline $\mathrm{H}$ & 6.54540500 & 3.49447400 & 4.44612900 & $\mathrm{H}$ & -8.15439300 & 0.63963400 & 1.43444400 \\
\hline $\mathrm{H}$ & 7.40333900 & 2.10381400 & 3.77048100 & $\mathrm{H}$ & -8.41089100 & 2.05008600 & 0.40326500 \\
\hline $\mathrm{C}$ & 6.90017800 & -2.53709800 & -1.22508200 & $\mathrm{C}$ & -8.53761000 & -1.64413100 & -1.02563200 \\
\hline $\mathrm{C}$ & 6.74783100 & -3.58392600 & -0.10068300 & $\mathrm{H}$ & -6.91690000 & -1.46487100 & 0.40041900 \\
\hline $\mathrm{C}$ & 8.36231300 & -2.04296400 & -1.30907600 & $\mathrm{H}$ & -6.38885100 & -1.38188100 & -1.28474000 \\
\hline $\mathrm{H}$ & 6.68196100 & -3.05575700 & -2.17699400 & $\mathrm{C}$ & -9.67859800 & -1.16625700 & -0.11864300 \\
\hline $\mathrm{C}$ & 7.74914500 & -4.73760800 & -0.24952400 & $\mathrm{H}$ & -10.51442400 & 0.69090600 & 0.65708300 \\
\hline $\mathrm{H}$ & 6.90509600 & -3.09035100 & 0.87574800 & $\mathrm{H}$ & -9.99234400 & 0.77286700 & -1.02898600 \\
\hline $\mathrm{H}$ & 5.71834400 & -3.97385100 & -0.09537900 & $\mathrm{H}$ & -8.48967100 & -2.74649000 & -1.03550400 \\
\hline $\mathrm{C}$ & 9.35712500 & -3.20187600 & -1.45810000 & $\mathrm{H}$ & -8.74858400 & -1.33604700 & -2.06667400 \\
\hline $\mathrm{H}$ & 8.60377600 & -1.47244500 & -0.39380200 & $\mathrm{H}$ & -10.64509400 & -1.56022200 & -0.47618800 \\
\hline $\mathrm{H}$ & 8.46568700 & -1.33532800 & -2.14879900 & $\mathrm{H}$ & -9.53187100 & -1.57874700 & 0.89719400 \\
\hline $\mathrm{C}$ & 9.19507000 & -4.23436000 & -0.33602300 & $\mathrm{C}$ & -3.42595700 & 3.62580700 & -1.62127500 \\
\hline $\mathrm{H}$ & 7.63687900 & -5.44466400 & 0.59007400 & $\mathrm{C}$ & -4.38653400 & 4.79051600 & -1.95429800 \\
\hline $\mathrm{H}$ & 7.50945600 & -5.30882700 & -1.16591900 & $\mathrm{C}$ & -2.91672500 & 2.95911600 & -2.91950800 \\
\hline $\mathrm{H}$ & 10.38970900 & -2.81339900 & -1.47893200 & $\mathrm{H}$ & -2.54757600 & 4.08219400 & -1.14034700 \\
\hline $\mathrm{H}$ & 9.19386100 & -3.69798200 & -2.43316800 & $\mathrm{C}$ & -3.74289100 & 5.80536900 & -2.90870600 \\
\hline $\mathrm{H}$ & 9.88914700 & -5.07871700 & -0.48747900 & $\mathrm{H}$ & -5.30598100 & 4.39292000 & -2.41999600 \\
\hline $\mathrm{H}$ & 9.47697300 & -3.76971300 & 0.62752100 & $\mathrm{H}$ & -4.70325800 & 5.28686200 & -1.02160400 \\
\hline $\mathrm{C}$ & 3.13854100 & 0.17143400 & -3.26664800 & $\mathrm{C}$ & -2.28337800 & 3.97760000 & -3.87668700 \\
\hline $\mathrm{C}$ & 2.52781700 & -1.11743300 & -3.85390900 & $\mathrm{H}$ & -3.76284000 & 2.45864800 & -3.42621200 \\
\hline $\mathrm{C}$ & 3.80488200 & 0.97283700 & -4.41081000 & $\mathrm{H}$ & -2.18531600 & 2.17794000 & -2.66005000 \\
\hline $\mathrm{H}$ & 2.29394600 & 0.77009100 & -2.89090200 & $\mathrm{C}$ & -3.23903500 & 5.13472800 & -4.19263400 \\
\hline $\mathrm{C}$ & 1.50158200 & -0.79793300 & -4.94860100 & $\mathrm{H}$ & -4.46279600 & 6.60632700 & -3.14887300 \\
\hline $\mathrm{H}$ & 3.32491700 & -1.74310600 & -4.29427600 & $\mathrm{H}$ & -2.89260900 & 6.29572600 & -2.39933500 \\
\hline $\mathrm{H}$ & 2.06865000 & -1.71150200 & -3.05172200 & $\mathrm{H}$ & -1.96675200 & 3.47521600 & -4.80662200 \\
\hline $\mathrm{C}$ & 2.80839300 & 1.29419700 & -5.53392400 & $\mathrm{H}$ & -1.36339300 & 4.38192600 & -3.41641200 \\
\hline $\mathrm{H}$ & 4.64770400 & 0.38108900 & -4.81377800 & $\mathrm{H}$ & -2.74450800 & 5.87508300 & -4.84417900 \\
\hline $\mathrm{H}$ & 4.24096600 & 1.90504300 & -4.02173300 & $\mathrm{H}$ & -4.10445700 & 4.74764200 & -4.76302100 \\
\hline $\mathrm{C}$ & 2.12605200 & 0.03248300 & -6.07689800 & $\mathrm{O}$ & 0.35549300 & -0.33812400 & -1.33072000 \\
\hline $\mathrm{H}$ & 1.07747300 & -1.73231300 & -5.35698700 & $\mathrm{H}$ & 0.51096000 & -1.69479000 & -1.29276800 \\
\hline $\mathrm{H}$ & 0.65942200 & -0.24287900 & -4.49735400 & $\mathrm{C}$ & -4.05083100 & -6.06587700 & -2.78998400 \\
\hline $\mathrm{H}$ & 3.31830200 & 1.83497500 & -6.34978200 & $\mathrm{C}$ & -3.52158900 & -5.24400700 & -1.81557000 \\
\hline $\mathrm{H}$ & 2.03818000 & 1.98222100 & -5.13978100 & $\mathrm{C}$ & -3.34993600 & -3.84870300 & -2.04526500 \\
\hline $\mathrm{H}$ & 1.36287200 & 0.30258400 & -6.82695200 & $\mathrm{C}$ & -3.74806500 & -3.31239300 & -3.31834800 \\
\hline $\mathrm{H}$ & 2.87569400 & -0.58530400 & -6.60624700 & $\mathrm{C}$ & -4.28772200 & -4.19128100 & -4.30015900 \\
\hline $\mathrm{C}$ & -3.46167000 & 1.06255100 & 2.84953100 & $\mathrm{C}$ & -4.43784100 & -5.53750400 & -4.04616500 \\
\hline $\mathrm{C}$ & -3.30682200 & -0.45177700 & 3.10009100 & $\mathrm{H}$ & -4.17715300 & -7.13283800 & -2.58957200 \\
\hline $\mathrm{C}$ & -4.26448400 & 1.70825200 & 4.00367100 & $\mathrm{H}$ & -3.24165600 & -5.66112100 & -0.84826800 \\
\hline $\mathrm{H}$ & -2.44502400 & 1.48318900 & 2.88391600 & $\mathrm{C}$ & -2.81004300 & -2.94521300 & -1.06385300 \\
\hline $\mathrm{C}$ & -2.66677400 & -0.73504400 & 4.46531700 & $\mathrm{C}$ & -3.59108000 & -1.92092600 & -3.57300000 \\
\hline $\mathrm{H}$ & -4.29176600 & -0.94914700 & 3.05616500 & $\mathrm{H}$ & -4.58590700 & -3.77349200 & -5.26552700 \\
\hline
\end{tabular}




\begin{tabular}{|c|c|c|c|c|c|c|c|}
\hline $\mathrm{H}$ & -4.85690300 & -6.19851800 & -4.80874500 & $\mathrm{H}$ & -1.24145900 & 5.54920100 & -2.10564800 \\
\hline $\mathrm{C}$ & -3.08327200 & -1.08174700 & -2.60634600 & $\mathrm{C}$ & -0.25707300 & 5.52815000 & -2.57364200 \\
\hline $\mathrm{C}$ & -2.70697900 & -1.59482000 & -1.34221200 & $\mathrm{C}$ & 2.24904000 & 5.47509100 & -3.82913900 \\
\hline $\mathrm{H}$ & -3.88667200 & -1.52783500 & -4.54920300 & $\mathrm{H}$ & 3.97160800 & 3.69231400 & -2.76641200 \\
\hline $\mathrm{H}$ & -2.97387600 & -0.01343300 & -2.79515400 & $\mathrm{C}$ & 1.27769800 & 6.34772000 & -4.26981100 \\
\hline $\mathrm{H}$ & -2.33372600 & -0.91693500 & -0.57578300 & $\mathrm{C}$ & 0.00896800 & 6.36283300 & -3.64001900 \\
\hline $\mathrm{N}$ & -2.36077700 & -3.46257600 & 0.19927700 & $\mathrm{H}$ & 3.22760100 & 5.44143200 & -4.31586300 \\
\hline $\mathrm{C}$ & -3.27330400 & -3.85031700 & 1.21308200 & $\mathrm{H}$ & 1.47818500 & 7.01867300 & -5.10884700 \\
\hline $\mathrm{C}$ & -2.81745200 & -4.40524400 & 2.42409700 & $\mathrm{H}$ & -0.76849600 & 7.03917400 & -4.00437000 \\
\hline $\mathrm{C}$ & -4.66033800 & -3.71576900 & 1.01428300 & $\mathrm{C}$ & -2.82310300 & 2.84628100 & 0.73055700 \\
\hline $\mathrm{C}$ & -3.72728200 & -4.81946500 & 3.39902300 & $\mathrm{C}$ & -3.16282800 & 4.06869000 & 1.27793400 \\
\hline $\mathrm{H}$ & -1.74956900 & -4.50430800 & 2.60691700 & $\mathrm{C}$ & -2.29218800 & 5.18874100 & 1.23877700 \\
\hline $\mathrm{C}$ & -5.55794600 & -4.14438200 & 1.99368400 & $\mathrm{C}$ & -1.04310300 & 5.07814800 & 0.54465200 \\
\hline $\mathrm{H}$ & -5.03921200 & -3.26756800 & 0.09669500 & $\mathrm{C}$ & -0.72972600 & 3.84599000 & -0.13284300 \\
\hline $\mathrm{C}$ & -5.10414700 & -4.69925000 & 3.19339200 & $\mathrm{C}$ & -1.57626800 & 2.75506500 & 0.03581100 \\
\hline $\mathrm{H}$ & -3.34681000 & -5.24382600 & 4.33187500 & $\mathrm{H}$ & -3.58963900 & 6.47799000 & 2.40609900 \\
\hline $\mathrm{H}$ & -6.63011900 & -4.03055800 & 1.81326000 & $\mathrm{H}$ & -4.12756800 & 4.17544100 & 1.77916300 \\
\hline $\mathrm{H}$ & -5.81179800 & -5.02761300 & 3.95772700 & $\mathrm{C}$ & -2.62676800 & 6.40836000 & 1.89261700 \\
\hline $\mathrm{N}$ & -1.06787000 & -3.13356800 & 0.62434000 & $\mathrm{C}$ & -0.15562300 & 6.19260000 & 0.58801200 \\
\hline $\mathrm{H}$ & -0.92293000 & -2.18561200 & 1.02691300 & $\mathrm{C}$ & -0.49935700 & 7.35498500 & 1.24823300 \\
\hline $\mathrm{C}$ & 1.22438000 & -5.60704500 & -0.90077300 & $\mathrm{C}$ & -1.75289400 & 7.47379300 & 1.89680900 \\
\hline $\mathrm{C}$ & -0.19243100 & -5.10182300 & -0.60280700 & $\mathrm{H}$ & 0.81667000 & 6.11989700 & 0.10107200 \\
\hline $\mathrm{C}$ & 1.32973900 & -3.83494500 & 0.87972500 & $\mathrm{H}$ & 0.20485300 & 8.19050100 & 1.27305700 \\
\hline $\mathrm{H}$ & 1.52387100 & -5.43332000 & -1.94547800 & $\mathrm{H}$ & -2.01691900 & 8.40267400 & 2.40846900 \\
\hline $\mathrm{H}$ & 1.36428100 & -6.68046300 & -0.70261300 & $\mathrm{O}$ & -1.23924800 & 1.55404900 & -0.55765500 \\
\hline $\mathrm{H}$ & -0.82623300 & -5.00512100 & -1.49182800 & $\mathrm{O}$ & 1.16395600 & 1.80738600 & 0.27350400 \\
\hline $\mathrm{H}$ & -0.73595100 & -5.77047400 & 0.08193600 & $\mathrm{P}$ & 0.02590000 & 0.64400900 & -0.00771700 \\
\hline $\mathrm{C}$ & -0.00224700 & -3.77011600 & 0.11096000 & $\mathrm{O}$ & 0.42372700 & -0.31516600 & -1.09685500 \\
\hline $\mathrm{O}$ & 0.57725000 & -2.78618100 & -1.38306200 & $\mathrm{C}$ & 3.83029000 & 1.81053600 & -0.86291100 \\
\hline $\mathrm{C}$ & 2.15595900 & -4.78674200 & -0.01065800 & $\mathrm{C}$ & 4.57670700 & 2.13574900 & 0.29697200 \\
\hline $\mathrm{O}$ & 3.35535800 & -4.88601300 & 0.01412800 & $\mathrm{C}$ & 4.17985100 & 0.67431400 & -1.63675800 \\
\hline $\mathrm{H}$ & -0.05732600 & -2.97208900 & -2.09332500 & $\mathrm{C}$ & 5.66107800 & 1.31973500 & 0.65181700 \\
\hline $\mathrm{C}$ & 1.04004900 & -4.62659700 & 2.18862500 & $\mathrm{C}$ & 5.28031600 & -0.09867900 & -1.24007400 \\
\hline $\mathrm{H}$ & 1.98947100 & -4.90052300 & 2.66418200 & $\mathrm{C}$ & 6.03965200 & 0.20528400 & -0.10296200 \\
\hline $\mathrm{H}$ & 0.46743900 & -5.54796600 & 2.00718400 & $\mathrm{H}$ & 6.24383000 & 1.56536500 & 1.54403100 \\
\hline $\mathrm{C}$ & 2.06253200 & -2.48664700 & 1.16167100 & $\mathrm{H}$ & 5.55312900 & -0.96752000 & -1.84311900 \\
\hline $\mathrm{H}$ & 1.32474400 & -1.68277200 & 1.09964100 & $\mathrm{C}$ & -3.77187900 & 1.68716400 & 0.83320000 \\
\hline $\mathrm{H}$ & 2.79761200 & -2.32207800 & 0.36089100 & $\mathrm{C}$ & -4.59167400 & 1.35490800 & -0.27117100 \\
\hline $\mathrm{H}$ & 0.48029700 & -3.99806900 & 2.89439500 & $\mathrm{C}$ & -3.90459500 & 0.97102900 & 2.05379300 \\
\hline $\mathrm{C}$ & 2.73504400 & -2.35930700 & 2.51540300 & $\mathrm{C}$ & -5.52182300 & 0.31054700 & -0.14243500 \\
\hline $\mathrm{C}$ & 3.95149700 & -3.00040900 & 2.80485600 & $\mathrm{C}$ & -4.84181600 & -0.06904700 & 2.11986800 \\
\hline $\mathrm{C}$ & 2.13377100 & -1.57846600 & 3.51668000 & $\mathrm{C}$ & -5.66823600 & -0.41791800 & 1.04089800 \\
\hline $\mathrm{C}$ & 4.54023400 & -2.87713300 & 4.06633900 & $\mathrm{H}$ & -6.15064900 & 0.06667300 & -0.99972500 \\
\hline $\mathrm{H}$ & 4.43273300 & -3.60203100 & 2.03113700 & $\mathrm{H}$ & -4.95424700 & -0.62127700 & 3.05733700 \\
\hline $\mathrm{C}$ & 2.72125200 & -1.45678400 & 4.78065500 & $\mathrm{C}$ & -4.52790700 & 2.12452400 & -1.58616900 \\
\hline $\mathrm{H}$ & 1.20574700 & -1.04581900 & 3.28964400 & $\mathrm{C}$ & -5.85433400 & 2.85446200 & -1.90017100 \\
\hline $\mathrm{C}$ & 3.92467300 & -2.10944400 & 5.06126800 & $\mathrm{C}$ & -4.09819000 & 1.24721400 & -2.78294700 \\
\hline $\mathrm{H}$ & 5.48757200 & -3.38241000 & 4.27363300 & $\mathrm{H}$ & -3.76178000 & 2.90823200 & -1.48423800 \\
\hline $\mathrm{H}$ & 2.23841200 & -0.84266600 & 5.54545800 & $\mathrm{C}$ & -5.76844700 & 3.66203800 & -3.20197900 \\
\hline \multirow[t]{2}{*}{$\mathrm{H}$} & 4.38583600 & -2.01504800 & 6.04798400 & $\mathrm{H}$ & -6.67071400 & 2.11465900 & -1.98479500 \\
\hline & & & & $\mathrm{H}$ & -6.12218600 & 3.51349900 & -1.05721400 \\
\hline ts-3-R-S & & & & $\mathrm{C}$ & -4.01902900 & 2.05818800 & -4.08359900 \\
\hline $\mathrm{C}$ & 1.44332800 & 2.75143600 & -0.69849800 & $\mathrm{H}$ & -4.82081600 & 0.42117800 & -2.91304000 \\
\hline $\mathrm{C}$ & 0.49242000 & 3.72821500 & -0.97959900 & $\mathrm{H}$ & -3.12303900 & 0.78605300 & -2.56241400 \\
\hline $\mathrm{C}$ & 0.72825800 & 4.62363100 & -2.08226000 & $\mathrm{C}$ & -5.33505200 & 2.78614100 & -4.38391600 \\
\hline $\mathrm{C}$ & 1.99880400 & 4.58761000 & -2.74464000 & $\mathrm{H}$ & -6.73913000 & 4.14228600 & -3.41347900 \\
\hline $\mathrm{C}$ & 2.98341300 & 3.66590600 & -2.30136000 & $\mathrm{H}$ & -5.03870300 & 4.48276900 & -3.07241300 \\
\hline $\mathrm{C}$ & 2.73342700 & 2.73307300 & -1.31284900 & $\mathrm{H}$ & -3.74540200 & 1.39726100 & -4.92384200 \\
\hline
\end{tabular}




\begin{tabular}{|c|c|c|c|}
\hline $\mathrm{H}$ & -3.20376600 & 2.80010300 & -3.99849800 \\
\hline $\mathrm{H}$ & -5.23809300 & 3.39538900 & -5.29867800 \\
\hline $\mathrm{H}$ & -6.12507400 & 2.03958000 & -4.58987500 \\
\hline $\mathrm{C}$ & -6.69462400 & -1.52987800 & 1.21344300 \\
\hline$C$ & -7.20582300 & -2.14993100 & -0.09823500 \\
\hline $\mathrm{C}$ & -7.89346800 & -1.07295300 & 2.07957100 \\
\hline $\mathrm{H}$ & -6.18553100 & -2.34019300 & 1.76759400 \\
\hline $\mathrm{C}$ & -8.17453200 & -3.30921100 & 0.16784800 \\
\hline $\mathrm{H}$ & -7.73273400 & -1.37993700 & -0.69088400 \\
\hline $\mathrm{H}$ & -6.35345200 & -2.49966000 & -0.69942200 \\
\hline $\mathrm{C}$ & -8.88121700 & -2.21763500 & 2.34130100 \\
\hline $\mathrm{H}$ & -8.40940900 & -0.24499200 & 1.55897800 \\
\hline $\mathrm{H}$ & -7.53274200 & -0.65704100 & 3.03491900 \\
\hline $\mathrm{C}$ & -9.35867200 & -2.87099000 & 1.03839500 \\
\hline $\mathrm{H}$ & -8.53539600 & -3.72555100 & -0.78802200 \\
\hline $\mathrm{H}$ & -7.62635000 & -4.12527900 & 0.67327700 \\
\hline $\mathrm{H}$ & -9.74172700 & -1.84802800 & 2.92487800 \\
\hline $\mathrm{H}$ & -8.38682900 & -2.98248000 & 2.96887500 \\
\hline $\mathrm{H}$ & -10.01639900 & -3.72924600 & 1.25859400 \\
\hline $\mathrm{H}$ & -9.97373500 & -2.14529800 & 0.47405200 \\
\hline $\mathrm{C}$ & -3.11560700 & 1.35167700 & 3.30519600 \\
\hline $\mathrm{C}$ & -2.48225700 & 0.15954300 & 4.05333100 \\
\hline $\mathrm{C}$ & -3.98610100 & 2.17300000 & 4.28777400 \\
\hline $\mathrm{H}$ & -2.27992300 & 1.99523600 & 2.98981700 \\
\hline $\mathrm{C}$ & -1.68191700 & 0.61744000 & 5.28040300 \\
\hline $\mathrm{H}$ & -3.26958600 & -0.54103200 & 4.38557600 \\
\hline $\mathrm{H}$ & -1.81655100 & -0.38747400 & 3.37192700 \\
\hline $\mathrm{C}$ & -3.19608200 & 2.62854300 & 5.52164300 \\
\hline $\mathrm{H}$ & -4.84431200 & 1.55266000 & 4.60595700 \\
\hline $\mathrm{H}$ & -4.41679900 & 3.04502900 & 3.77080400 \\
\hline $\mathrm{C}$ & -2.53991700 & 1.44570900 & 6.24329700 \\
\hline $\mathrm{H}$ & -1.25684100 & -0.25867700 & 5.79909100 \\
\hline $\mathrm{H}$ & -0.82188000 & 1.22226000 & 4.94063200 \\
\hline $\mathrm{H}$ & -3.85827500 & 3.18069800 & 6.21042600 \\
\hline $\mathrm{H}$ & -2.41268000 & 3.34162600 & 5.20557500 \\
\hline $\mathrm{H}$ & -1.93236700 & 1.80212900 & 7.09256000 \\
\hline $\mathrm{H}$ & -3.32867100 & 0.80072800 & 6.67409600 \\
\hline $\mathrm{C}$ & 3.40881400 & 0.29679700 & -2.89770800 \\
\hline $\mathrm{C}$ & 3.06342400 & -1.20361400 & -2.99029500 \\
\hline $\mathrm{C}$ & 4.14195300 & 0.73863100 & -4.18645400 \\
\hline $\mathrm{H}$ & 2.44561200 & 0.82936300 & -2.86680200 \\
\hline $\mathrm{C}$ & 2.22315700 & -1.50864800 & -4.23625300 \\
\hline $\mathrm{H}$ & 3.98695900 & -1.80766400 & -3.02859700 \\
\hline $\mathrm{H}$ & 2.51986300 & -1.50733600 & -2.08513500 \\
\hline $\mathrm{C}$ & 3.32231200 & 0.42648000 & -5.44590500 \\
\hline $\mathrm{H}$ & 5.11697900 & 0.21945700 & -4.23808900 \\
\hline $\mathrm{H}$ & 4.37022100 & 1.81555300 & -4.14330100 \\
\hline $\mathrm{C}$ & 2.92865100 & -1.05389400 & -5.51982800 \\
\hline $\mathrm{H}$ & 2.00326400 & -2.58884400 & -4.28315900 \\
\hline $\mathrm{H}$ & 1.25057300 & -0.99171400 & -4.14479300 \\
\hline $\mathrm{H}$ & 3.88737800 & 0.71991200 & -6.34744800 \\
\hline $\mathrm{H}$ & 2.40611900 & 1.04522300 & -5.43879800 \\
\hline $\mathrm{H}$ & 2.28753700 & -1.23580600 & -6.39955200 \\
\hline $\mathrm{H}$ & 3.83916800 & -1.66452800 & -5.66801000 \\
\hline $\mathrm{C}$ & 7.24150800 & -0.63226800 & 0.30300500 \\
\hline $\mathrm{C}$ & 8.36380500 & -0.61690300 & -0.75890300 \\
\hline $\mathrm{C}$ & 6.86449500 & -2.08612800 & 0.66200200 \\
\hline $\mathrm{H}$ & 7.65998700 & -0.17366900 & 1.21816300 \\
\hline
\end{tabular}

\begin{tabular}{|c|c|c|c|}
\hline $\mathrm{C}$ & 9.58298600 & -1.43986600 & -0.32199700 \\
\hline $\mathrm{H}$ & 7.97042600 & -1.02538600 & -1.70734200 \\
\hline $\mathrm{H}$ & 8.65772200 & 0.42466300 & -0.97084800 \\
\hline $\mathrm{C}$ & 8.08528100 & -2.90771800 & 1.09700600 \\
\hline $\mathrm{H}$ & 6.39609200 & -2.56370400 & -0.21715600 \\
\hline $\mathrm{H}$ & 6.09932300 & -2.08295800 & 1.45589000 \\
\hline $\mathrm{C}$ & 9.19603600 & -2.87917800 & 0.03965900 \\
\hline $\mathrm{H}$ & 10.34671600 & -1.43531900 & -1.11819700 \\
\hline $\mathrm{H}$ & 10.05088300 & -0.95868900 & 0.55715600 \\
\hline $\mathrm{H}$ & 7.78474800 & -3.94760900 & 1.31154400 \\
\hline $\mathrm{H}$ & 8.47844200 & -2.49952900 & 2.04682400 \\
\hline $\mathrm{H}$ & 10.07914800 & -3.43776600 & 0.39362900 \\
\hline $\mathrm{H}$ & 8.84363600 & -3.39897700 & -0.87102400 \\
\hline $\mathrm{C}$ & 4.23893400 & 3.34176100 & 1.16774700 \\
\hline $\mathrm{C}$ & 5.43535400 & 4.29245700 & 1.39506400 \\
\hline $\mathrm{C}$ & 3.61773400 & 2.92595500 & 2.52132800 \\
\hline $\mathrm{H}$ & 3.47423600 & 3.93403800 & 0.64181400 \\
\hline $\mathrm{C}$ & 5.03775000 & 5.51071100 & 2.24007600 \\
\hline $\mathrm{H}$ & 6.25081000 & 3.75208400 & 1.90795700 \\
\hline $\mathrm{H}$ & 5.84298600 & 4.61538600 & 0.42228200 \\
\hline $\mathrm{C}$ & 3.23193300 & 4.14245000 & 3.37299500 \\
\hline $\mathrm{H}$ & 4.34620800 & 2.30591000 & 3.07615800 \\
\hline $\mathrm{H}$ & 2.73299800 & 2.29728500 & 2.33608600 \\
\hline $\mathrm{C}$ & 4.41681700 & 5.09393200 & 3.57933500 \\
\hline $\mathrm{H}$ & 5.91625300 & 6.15699900 & 2.40754500 \\
\hline $\mathrm{H}$ & 4.30762800 & 6.12080500 & 1.67657800 \\
\hline $\mathrm{H}$ & 2.83130000 & 3.81035400 & 4.34594700 \\
\hline $\mathrm{H}$ & 2.41065000 & 4.68723900 & 2.87226200 \\
\hline $\mathrm{H}$ & 4.10067200 & 5.98431600 & 4.14912900 \\
\hline $\mathrm{H}$ & 5.18642500 & 4.58973800 & 4.19374500 \\
\hline $\mathrm{O}$ & -0.30661900 & 0.07746300 & 1.37113500 \\
\hline $\mathrm{H}$ & -0.67304500 & -1.19777100 & 1.52014500 \\
\hline $\mathrm{C}$ & 2.50772100 & -6.03869300 & 3.75426500 \\
\hline $\mathrm{C}$ & 2.23116300 & -5.27393200 & 2.63893400 \\
\hline $\mathrm{C}$ & 2.43221400 & -3.86411800 & 2.64567300 \\
\hline $\mathrm{C}$ & 2.94793200 & -3.25470700 & 3.84067700 \\
\hline $\mathrm{C}$ & 3.21630600 & -4.07391000 & 4.97366200 \\
\hline $\mathrm{C}$ & 3.00144800 & -5.43491400 & 4.93672900 \\
\hline $\mathrm{H}$ & 2.34969400 & -7.11985500 & 3.72397100 \\
\hline $\mathrm{H}$ & 1.86827000 & -5.75283900 & 1.72962900 \\
\hline $\mathrm{C}$ & 2.16682700 & -3.02260300 & 1.51141900 \\
\hline $\mathrm{C}$ & 3.17949800 & -1.85088200 & 3.86675400 \\
\hline $\mathrm{H}$ & 3.60354900 & -3.59932900 & 5.87945900 \\
\hline $\mathrm{H}$ & 3.21569800 & -6.05063400 & 5.81382800 \\
\hline $\mathrm{C}$ & 2.93799900 & -1.07793400 & 2.75349500 \\
\hline $\mathrm{C}$ & 2.44340900 & -1.67054700 & 1.56753500 \\
\hline $\mathrm{H}$ & 3.56111300 & -1.39744800 & 4.78537700 \\
\hline $\mathrm{H}$ & 3.12771400 & -0.00414300 & 2.76748600 \\
\hline $\mathrm{H}$ & 2.28231800 & -1.05194400 & 0.68525700 \\
\hline $\mathrm{N}$ & 1.60470200 & -3.60114500 & 0.31902000 \\
\hline $\mathrm{C}$ & 2.41854600 & -4.30939400 & -0.59954900 \\
\hline $\mathrm{C}$ & 1.87413000 & -4.88402000 & -1.76442300 \\
\hline $\mathrm{C}$ & 3.79384700 & -4.48357400 & -0.34844800 \\
\hline $\mathrm{C}$ & 2.68059600 & -5.61223300 & -2.64144900 \\
\hline $\mathrm{H}$ & 0.81853900 & -4.75337100 & -1.98941600 \\
\hline $\mathrm{C}$ & 4.58576900 & -5.22196800 & -1.22940800 \\
\hline $\mathrm{H}$ & 4.24718100 & -4.03393300 & 0.53388200 \\
\hline $\mathrm{C}$ & 4.04151700 & -5.79319800 & -2.38297900 \\
\hline
\end{tabular}




$\begin{array}{llll}\mathrm{H} & 2.23024100 & -6.04355300 & -3.53947000 \\ \mathrm{H} & 5.64971200 & -5.34145900 & -1.00817100 \\ \mathrm{H} & 4.66807600 & -6.36541000 & -3.07062400 \\ \mathrm{~N} & 0.42725000 & -3.03955800 & -0.19388100 \\ \mathrm{H} & 0.50607600 & -2.12611700 & -0.68800800 \\ \mathrm{C} & -2.39567700 & -4.89876200 & 1.30442800 \\ \mathrm{C} & -0.89225100 & -4.68428400 & 1.09511000 \\ \mathrm{C} & -2.05740600 & -3.20638200 & -0.52838600 \\ \mathrm{H} & -2.72343800 & -4.66988000 & 2.33126800 \\ \mathrm{H} & -2.73099100 & -5.92737300 & 1.09899800 \\ \mathrm{H} & -0.32570200 & -4.62607400 & 2.02941900 \\ \mathrm{H} & -0.44140100 & -5.48558600 & 0.49008400 \\ \mathrm{C} & -0.77281600 & -3.38251900 & 0.31464900 \\ \mathrm{O} & -1.06662500 & -2.22247700 & 1.69997300 \\ \mathrm{C} & -3.09781300 & -3.93211800 & 0.35098200 \\ \mathrm{O} & -4.28888400 & -3.75792900 & 0.29638300 \\ \mathrm{H} & -2.00214100 & -2.08178500 & 1.91790700 \\ \mathrm{C} & -2.49604100 & -1.79119800 & -0.89363000 \\ \mathrm{H} & -1.75918700 & -1.30284600 & -1.54457100 \\ \mathrm{H} & -2.63997300 & -1.15740500 & -0.01019200 \\ \mathrm{C} & -1.85829600 & -4.06931000 & -1.84192200 \\ \mathrm{H} & -1.52104700 & -5.08095700 & -1.56772200 \\ \mathrm{H} & -1.02913100 & -3.59622100 & -2.38720700 \\ \mathrm{H} & -3.46096600 & -1.83824300 & -1.41252500 \\ \mathrm{C} & -3.05677000 & -4.18575800 & -2.75858900 \\ \mathrm{C} & -3.24743800 & -3.26897400 & -3.80598400 \\ \mathrm{C} & -3.98579500 & -5.22832700 & -2.61102000 \\ \mathrm{C} & -4.33965700 & -3.38202800 & -4.66988000 \\ \mathrm{H} & -2.52709000 & -2.45975700 & -3.95166400 \\ \mathrm{C} & -5.07926300 & -5.34521100 & -3.47262000 \\ \mathrm{H} & -3.85245300 & -5.96567100 & -1.81451000 \\ \mathrm{C} & -5.26173000 & -4.41942200 & -4.50374200 \\ \mathrm{H} & -4.46660400 & -2.65921400 & -5.47984200 \\ \mathrm{H} & -5.78934200 & -6.16533900 & -3.33935000 \\ \mathrm{H} & -6.11548800 & -4.51064400 & -5.17996600 \\ & & & \end{array}$

$\begin{array}{cccc}\text { ts-3-S-R } & & & \\ \text { C } & 1.15793000 & -3.32120200 & 0.12984500 \\ \text { C } & 0.15733200 & -4.25366300 & 0.38924400 \\ \text { C } & 0.44591400 & -5.33569800 & 1.29512100 \\ \text { C } & 1.79012000 & -5.50779000 & 1.76359500 \\ \text { C } & 2.79002200 & -4.58498300 & 1.35911800 \\ \text { C } & 2.50314700 & -3.47604700 & 0.58553700 \\ \text { H } & -1.58547500 & -6.09671100 & 1.46685700 \\ \text { C } & -0.54998000 & -6.23257900 & 1.77915200 \\ \text { C } & 2.09333300 & -6.58805100 & 2.64037000 \\ \text { H } & 3.81618900 & -4.74380000 & 1.69937700 \\ \text { C } & 1.10829400 & -7.44956300 & 3.07228400 \\ \text { C } & -0.22868000 & -7.25740300 & 2.64588000 \\ \text { H } & 3.12626300 & -6.71261200 & 2.97690100 \\ \text { H } & 1.35187800 & -8.27040100 & 3.75146100 \\ \text { H } & -1.01441600 & -7.92481100 & 3.00901500 \\ \text { C } & -3.24932500 & -2.82045300 & -0.63776300 \\ \text { C } & -3.80319000 & -3.91169300 & -1.28027000 \\ \text { C } & -3.06740000 & -5.09749300 & -1.53788600 \\ \text { C } & -1.72428100 & -5.20712800 & -1.04990500 \\ \text { C } & -1.17580200 & -4.12663900 & -0.27006400 \\ \text { C } & -1.91042100 & -2.94883400 & -0.15317000\end{array}$

\begin{tabular}{|c|c|c|c|}
\hline $\mathrm{H}$ & -4.66387200 & -6.07227900 & -2.63765800 \\
\hline $\mathrm{H}$ & -4.83878400 & -3.85626300 & -1.62312100 \\
\hline $\mathrm{C}$ & -3.63281300 & -6.16853500 & -2.28645200 \\
\hline $\mathrm{C}$ & -0.98518200 & -6.37701400 & -1.39156200 \\
\hline $\mathrm{C}$ & -1.55358200 & -7.38944700 & -2.13758900 \\
\hline $\mathrm{C}$ & -2.89589100 & -7.29531000 & -2.57966100 \\
\hline $\mathrm{H}$ & 0.05145600 & -6.46680200 & -1.06734500 \\
\hline $\mathrm{H}$ & -0.95995600 & -8.27107600 & -2.39260000 \\
\hline $\mathrm{H}$ & -3.33728700 & -8.10804000 & -3.16187600 \\
\hline $\mathrm{O}$ & -1.35742600 & -1.88051800 & 0.52124600 \\
\hline $\mathrm{O}$ & 0.86785500 & -2.23502300 & -0.66812200 \\
\hline $\mathrm{P}$ & -0.10863900 & -1.01698400 & -0.13964900 \\
\hline $\mathrm{O}$ & 0.51386900 & -0.22293200 & 0.98262800 \\
\hline $\mathrm{C}$ & 3.58291200 & -2.49557600 & 0.23308600 \\
\hline $\mathrm{C}$ & 4.07488100 & -2.42454500 & -1.09484000 \\
\hline $\mathrm{C}$ & 4.16138800 & -1.68264300 & 1.24370500 \\
\hline $\mathrm{C}$ & 5.13627400 & -1.55275700 & -1.37771400 \\
\hline $\mathrm{C}$ & 5.22370900 & -0.83296500 & 0.90229600 \\
\hline $\mathrm{C}$ & 5.73330600 & -0.75184500 & -0.39860400 \\
\hline $\mathrm{H}$ & 5.52670400 & -1.50568700 & -2.39832500 \\
\hline $\mathrm{H}$ & 5.66877600 & -0.21580100 & 1.68679100 \\
\hline $\mathrm{C}$ & -4.08076400 & -1.58448500 & -0.43566900 \\
\hline $\mathrm{C}$ & -4.65316000 & -1.31779800 & 0.83491100 \\
\hline $\mathrm{C}$ & -4.38830200 & -0.74473500 & -1.53750600 \\
\hline $\mathrm{C}$ & -5.54648400 & -0.24457100 & 0.96830500 \\
\hline $\mathrm{C}$ & -5.29703100 & 0.30844800 & -1.34765300 \\
\hline $\mathrm{C}$ & -5.90437900 & 0.56932900 & -0.11441000 \\
\hline $\mathrm{H}$ & -6.00305500 & -0.06277000 & 1.94477800 \\
\hline $\mathrm{H}$ & -5.55994600 & 0.93985100 & -2.20045800 \\
\hline $\mathrm{C}$ & -4.37144900 & -2.19697000 & 2.04846600 \\
\hline $\mathrm{C}$ & -5.63907300 & -2.91857100 & 2.56075300 \\
\hline $\mathrm{C}$ & -3.68204600 & -1.43103600 & 3.19927200 \\
\hline $\mathrm{H}$ & -3.67083000 & -2.98648900 & 1.73836900 \\
\hline $\mathrm{C}$ & -5.33136600 & -3.83270100 & 3.75414900 \\
\hline $\mathrm{H}$ & -6.39392600 & -2.16987300 & 2.86186700 \\
\hline $\mathrm{H}$ & -6.09127000 & -3.49884800 & 1.73900300 \\
\hline $\mathrm{C}$ & -3.38166800 & -2.34713600 & 4.39331500 \\
\hline $\mathrm{H}$ & -4.33246400 & -0.60125400 & 3.53222100 \\
\hline $\mathrm{H}$ & -2.74998200 & -0.97961600 & 2.82470700 \\
\hline $\mathrm{C}$ & -4.64094400 & -3.06783800 & 4.89018900 \\
\hline $\mathrm{H}$ & -6.25973400 & -4.30602500 & 4.11691900 \\
\hline $\mathrm{H}$ & -4.67405800 & -4.65650200 & 3.41960600 \\
\hline $\mathrm{H}$ & -2.92497400 & -1.76362900 & 5.21080400 \\
\hline $\mathrm{H}$ & -2.62752800 & -3.09654300 & 4.09124600 \\
\hline $\mathrm{H}$ & -4.39025600 & -3.75316300 & 5.71759900 \\
\hline $\mathrm{H}$ & -5.34571100 & -2.32351800 & 5.30646300 \\
\hline $\mathrm{C}$ & -6.95882200 & 1.65830200 & 0.01123600 \\
\hline $\mathrm{C}$ & -6.61303600 & 2.74603500 & 1.05069400 \\
\hline $\mathrm{C}$ & -8.36099500 & 1.07276000 & 0.29808700 \\
\hline $\mathrm{H}$ & -7.01803100 & 2.16276900 & -0.97078800 \\
\hline $\mathrm{C}$ & -7.69274500 & 3.83380600 & 1.13157600 \\
\hline $\mathrm{H}$ & -6.49329400 & 2.27837300 & 2.04502600 \\
\hline $\mathrm{H}$ & -5.64009100 & 3.19950800 & 0.79751300 \\
\hline $\mathrm{C}$ & -9.43791200 & 2.16210200 & 0.37675800 \\
\hline $\mathrm{H}$ & -8.33096100 & 0.51803700 & 1.25350700 \\
\hline $\mathrm{H}$ & -8.61599400 & 0.33327800 & -0.47894500 \\
\hline $\mathrm{C}$ & -9.07922800 & 3.23948700 & 1.40762200 \\
\hline $\mathrm{H}$ & -7.42770000 & 4.57141000 & 1.90769200 \\
\hline
\end{tabular}




\begin{tabular}{|c|c|c|c|c|c|c|c|}
\hline $\mathrm{H}$ & -7.72012000 & 4.38791400 & 0.17498700 & $\mathrm{C}$ & 2.89261900 & -2.47776300 & -3.36585900 \\
\hline $\mathrm{H}$ & -10.41524900 & 1.71016400 & 0.61654000 & $\mathrm{H}$ & 2.71906800 & -3.92060800 & -1.80017500 \\
\hline $\mathrm{H}$ & -9.55107800 & 2.63409400 & -0.61700200 & $\mathrm{C}$ & 4.00303500 & -5.18134600 & -3.86806800 \\
\hline $\mathrm{H}$ & -9.84264400 & 4.03562000 & 1.41443100 & $\mathrm{H}$ & 5.43284500 & -3.70338600 & -3.18768400 \\
\hline $\mathrm{H}$ & -9.08836600 & 2.79192400 & 2.41900300 & $\mathrm{H}$ & 4.99344100 & -4.88390700 & -1.95003300 \\
\hline $\mathrm{C}$ & -3.79049100 & -0.96773700 & -2.92380200 & $\mathrm{C}$ & 2.31637100 & -3.38660500 & -4.46030500 \\
\hline $\mathrm{C}$ & -3.23159600 & 0.31836700 & -3.57044400 & $\mathrm{H}$ & 3.66154100 & -1.81893300 & -3.81056100 \\
\hline $\mathrm{C}$ & -4.79047200 & -1.63378700 & -3.89884800 & $\mathrm{H}$ & 2.10293200 & -1.82806400 & -2.95780600 \\
\hline $\mathrm{H}$ & -2.93618500 & -1.65210900 & -2.80837200 & $\mathrm{C}$ & 3.36538000 & -4.36649100 & -5.00044800 \\
\hline $\mathrm{C}$ & -2.56660200 & 0.02193100 & -4.92068100 & $\mathrm{H}$ & 4.78982500 & -5.84466800 & -4.26642800 \\
\hline $\mathrm{H}$ & -4.05013000 & 1.04313300 & -3.73190500 & $\mathrm{H}$ & 3.23687900 & -5.84253600 & -3.42238800 \\
\hline $\mathrm{H}$ & -2.51326300 & 0.79573900 & -2.89059700 & $\mathrm{H}$ & 1.90405000 & -2.77450000 & -5.28057900 \\
\hline $\mathrm{C}$ & -4.14782100 & -1.92778500 & -5.26125500 & $\mathrm{H}$ & 1.46548300 & -3.95579400 & -4.04344400 \\
\hline $\mathrm{H}$ & -5.65809200 & -0.96154000 & -4.03433800 & $\mathrm{H}$ & 2.91431400 & -5.03861500 & -5.75025400 \\
\hline $\mathrm{H}$ & -5.18884400 & -2.56321500 & -3.46265300 & $\mathrm{H}$ & 4.15541400 & -3.79913500 & -5.52768800 \\
\hline $\mathrm{C}$ & -3.53411600 & -0.67016200 & -5.88868000 & $\mathrm{O}$ & -0.56386200 & -0.28739300 & -1.39089800 \\
\hline $\mathrm{H}$ & -2.18437600 & 0.95672900 & -5.36588800 & $\mathrm{H}$ & -0.76139300 & 1.15425600 & -1.43710300 \\
\hline $\mathrm{H}$ & -1.68589900 & -0.62384100 & -4.75140900 & $\mathrm{C}$ & 3.39753300 & 6.66461900 & -1.38107300 \\
\hline $\mathrm{H}$ & -4.89355300 & -2.37222100 & -5.94265800 & $\mathrm{C}$ & 2.79770000 & 5.61952600 & -0.70859400 \\
\hline $\mathrm{H}$ & -3.35777600 & -2.68917900 & -5.12682200 & $\mathrm{C}$ & 2.81030000 & 4.29847600 & -1.24057700 \\
\hline $\mathrm{H}$ & -3.02190900 & -0.92422600 & -6.83246300 & $\mathrm{C}$ & 3.47125600 & 4.07735300 & -2.49892500 \\
\hline $\mathrm{H}$ & -4.34441800 & 0.03429700 & -6.15565000 & $\mathrm{C}$ & 4.07611000 & 5.18082800 & -3.16538400 \\
\hline $\mathrm{C}$ & 3.67499200 & -1.69427200 & 2.69002400 & $\mathrm{C}$ & 4.04318300 & 6.44677100 & -2.62241600 \\
\hline $\mathrm{C}$ & 3.17761400 & -0.31098900 & 3.16474400 & $\mathrm{H}$ & 3.37229100 & 7.66887900 & -0.95130800 \\
\hline $\mathrm{C}$ & 4.73944600 & -2.24705100 & 3.66568300 & $\mathrm{H}$ & 2.31423100 & 5.80608600 & 0.24877800 \\
\hline $\mathrm{H}$ & 2.80705600 & -2.36719500 & 2.75107600 & $\mathrm{C}$ & 2.20590000 & 3.16900700 & -0.58219700 \\
\hline $\mathrm{C}$ & 2.66720400 & -0.35810800 & 4.61092300 & $\mathrm{C}$ & 3.50744300 & 2.76936700 & -3.05891300 \\
\hline $\mathrm{H}$ & 3.99624300 & 0.42753600 & 3.09539100 & $\mathrm{H}$ & 4.57382300 & 5.00144700 & -4.12217900 \\
\hline $\mathrm{H}$ & 2.37658900 & 0.02492900 & 2.49067200 & $\mathrm{H}$ & 4.51435400 & 7.28281900 & -3.14512100 \\
\hline $\mathrm{C}$ & 4.22559500 & -2.28944700 & 5.11096400 & $\mathrm{C}$ & 2.93708700 & 1.70760800 & -2.39353900 \\
\hline $\mathrm{H}$ & 5.64322200 & -1.61238600 & 3.61830600 & $\mathrm{C}$ & 2.29890900 & 1.90621100 & -1.14549500 \\
\hline $\mathrm{H}$ & 5.05803300 & -3.25338400 & 3.34471800 & $\mathrm{H}$ & 4.00456500 & 2.62183200 & -4.02104500 \\
\hline $\mathrm{C}$ & 3.72165900 & -0.91696600 & 5.57387600 & $\mathrm{H}$ & 2.98055700 & 0.69810300 & -2.80646600 \\
\hline $\mathrm{H}$ & 2.35287400 & 0.65026200 & 4.92945200 & $\mathrm{H}$ & 1.88493300 & 1.04783900 & -0.61706200 \\
\hline $\mathrm{H}$ & 1.76257800 & -0.99258500 & 4.65363500 & $\mathrm{~N}$ & 1.48566900 & 3.36973000 & 0.64623200 \\
\hline $\mathrm{H}$ & 5.01975800 & -2.65623400 & 5.78393600 & $\mathrm{C}$ & 2.18687300 & 3.67282800 & 1.85318600 \\
\hline $\mathrm{H}$ & 3.39887100 & -3.02068500 & 5.17945500 & $\mathrm{C}$ & 1.47169000 & 4.05090200 & 3.00420600 \\
\hline $\mathrm{H}$ & 3.31474800 & -0.98349900 & 6.59754800 & $\mathrm{C}$ & 3.58970800 & 3.61958900 & 1.91192000 \\
\hline $\mathrm{H}$ & 4.57577700 & -0.21547800 & 5.62431100 & $\mathrm{C}$ & 2.14854300 & 4.37314900 & 4.18127000 \\
\hline $\mathrm{C}$ & 6.91140700 & 0.14515800 & -0.74319100 & $\mathrm{H}$ & 0.38309300 & 4.09486000 & 2.98204200 \\
\hline $\mathrm{C}$ & 8.22413200 & -0.33270300 & -0.08151900 & $\mathrm{C}$ & 4.25662700 & 3.95848800 & 3.09165500 \\
\hline $\mathrm{C}$ & 6.66213600 & 1.63474000 & -0.42217800 & $\mathrm{H}$ & 4.16116700 & 3.30505100 & 1.03865900 \\
\hline $\mathrm{H}$ & 7.06029300 & 0.07412700 & -1.83669700 & $\mathrm{C}$ & 3.54526600 & 4.33568400 & 4.23339100 \\
\hline $\mathrm{C}$ & 9.41928500 & 0.54733300 & -0.46994100 & $\mathrm{H}$ & 1.57283300 & 4.66424400 & 5.06344800 \\
\hline $\mathrm{H}$ & 8.09971900 & -0.31793000 & 1.01667500 & $\mathrm{H}$ & 5.34840100 & 3.91224900 & 3.11636100 \\
\hline $\mathrm{H}$ & 8.41402600 & -1.38378200 & -0.35558300 & $\mathrm{H}$ & 4.07219500 & 4.59394000 & 5.15467300 \\
\hline $\mathrm{C}$ & 7.85819300 & 2.51288000 & -0.81374700 & $\mathrm{~N}$ & 0.35298900 & 2.56612200 & 0.84517900 \\
\hline $\mathrm{H}$ & 6.47108300 & 1.74587500 & 0.66079200 & $\mathrm{H}$ & 0.53223900 & 1.56033200 & 1.07078700 \\
\hline $\mathrm{H}$ & 5.74909400 & 1.97513300 & -0.93734700 & $\mathrm{C}$ & -2.62277300 & 2.76301100 & 2.02086200 \\
\hline $\mathrm{C}$ & 9.15815900 & 2.02677100 & -0.16062300 & $\mathrm{C}$ & -1.92791600 & 1.87337300 & 0.97069000 \\
\hline $\mathrm{H}$ & 10.32914400 & 0.20062600 & 0.04885800 & $\mathrm{C}$ & -1.53071100 & 4.20919400 & 0.27948300 \\
\hline $\mathrm{H}$ & 9.61791000 & 0.43282700 & -1.55195900 & $\mathrm{H}$ & -3.71912200 & 2.66573000 & 2.00860200 \\
\hline $\mathrm{H}$ & 7.66107700 & 3.56410800 & -0.54312000 & $\mathrm{H}$ & -2.29711300 & 2.54903300 & 3.05048000 \\
\hline $\mathrm{H}$ & 7.97590500 & 2.49498100 & -1.91336800 & $\mathrm{H}$ & -2.62617100 & 1.53519900 & 0.19644900 \\
\hline $\mathrm{H}$ & 10.00972200 & 2.64517700 & -0.49173500 & $\mathrm{H}$ & -1.45788600 & 0.98700800 & 1.40862900 \\
\hline $\mathrm{H}$ & 9.08521400 & 2.16020400 & 0.93504600 & $\mathrm{C}$ & -0.87878500 & 2.81280000 & 0.37612400 \\
\hline $\mathrm{C}$ & 3.52372900 & -3.29676200 & -2.21720100 & $\mathrm{O}$ & -0.88408700 & 2.19754200 & -1.47011100 \\
\hline $\mathrm{C}$ & 4.58315100 & -4.27564200 & -2.77363500 & $\mathrm{C}$ & -2.21956000 & 4.19184300 & 1.67834700 \\
\hline
\end{tabular}




$\begin{array}{llllllrr}\mathrm{O} & -2.37504000 & 5.15063100 & 2.38973900 & \mathrm{C} & -1.14997700 & 7.10549200 & -1.74882400 \\ \mathrm{C} & -0.58966000 & 5.42162200 & 0.05443200 & \mathrm{C} & -1.86008600 & 7.60323900 & 0.50030400 \\ \mathrm{H} & 0.13327300 & 5.13177700 & -0.71684200 & \mathrm{C} & -1.69952400 & 8.31036000 & -2.19675300 \\ \mathrm{H} & -0.01557100 & 5.59891200 & 0.97437800 & \mathrm{H} & -0.64145100 & 6.45070600 & -2.46260100 \\ \mathrm{H} & -0.06342500 & 2.51433200 & -1.88170600 & \mathrm{C} & -2.40782000 & 8.80967200 & 0.05563500 \\ \mathrm{C} & -2.69264300 & 4.22157700 & -0.74996300 & \mathrm{H} & -1.93268800 & 7.32471800 & 1.55231400 \\ \mathrm{H} & -3.31139200 & 5.11253800 & -0.58087900 & \mathrm{C} & -2.33350100 & 9.16776200 & -1.29415700 \\ \mathrm{H} & -2.29049900 & 4.26600200 & -1.76949800 & \mathrm{H} & -1.62540200 & 8.58108500 & -3.25335700 \\ \mathrm{H} & -3.33002300 & 3.33114600 & -0.68674200 & \mathrm{H} & -2.89513800 & 9.47659000 & 0.77199800 \\ \mathrm{C} & -1.22754500 & 6.72647200 & -0.39809500 & \mathrm{H} & -2.76217900 & 10.11236100 & -1.63894100\end{array}$

\section{References}

1. Gaussian 09, Revision D.01, Frisch, M. J.; Trucks, G. W.; Schlegel, H. B.; Scuseria, G. E.; Robb, M. A.; Cheeseman, J. R.; Scalmani, G.; Barone, V.; Mennucci, B.; Petersson, G. A.; Nakatsuji, H.; Caricato, M.; Li, X.; Hratchian, H. P.; Izmaylov, A. F.; Bloino, J.; Zheng, G.; Sonnenberg, J. L.; Hada, M.; Ehara, M.; Toyota, K.; Fukuda, R.; Hasegawa, J.; Ishida, M.; Nakajima, T.; Honda, Y.; Kitao, O.; Nakai, H.; Vreven, T.; Montgomery, Jr., J. A.; Peralta, J. E.; Ogliaro, F.; Bearpark, M.; Heyd, J. J.; Brothers, E.; Kudin, K. N.; Staroverov, V. N.; Kobayashi, R.; Normand, J.; Raghavachari, K.; Rendell, A.; Burant, J. C.; Iyengar, S. S.; Tomasi, J.; Cossi, M.; Rega, N.; Millam, N. J.; Klene, M.; Knox, J. E.; Cross, J. B.; Bakken, V.; Adamo, C.; Jaramillo, J.; Gomperts, R.; Stratmann, R. E.; Yazyev, O.; Austin, A. J.; Cammi, R.; Pomelli, C.; Ochterski, J. W.; Martin, R. L.; Morokuma, K.; Zakrzewski, V. G.; Voth, G. A.; Salvador, P.; Dannenberg, J. J.; Dapprich, S.; Daniels, A. D.; Farkas, Ö.; Foresman, J. B.; Ortiz, J. V.; Cioslowski, J.; Fox, D. J. Gaussian, Inc., Wallingford CT, 2013.

2. Becke, A. D. Density-functional thermochemistry. III.The role of exact exchange. J. Chem. Phys. 1993, 98, 5648-5652.

3. Barone, V.; Cossi, M. Quantum Calculation of Molecular Energies and Energy Gradients in Solution by a Conductor Solvent Model. J. Phys. Chem. A 1998, 102, 1995-2001.

4. Schäfer, A.; Huber, C.; Ahlrichs, R. Fully optimized contracted Gaussian basis sets of triple zeta valence quality for atoms Li to Kr. J. Chem. Phys. 1994, 100, 5829-5835.

5. Klein, J. E. M. N.; Miehlich, B.; Holzwarth, M. S.; Bauer, M.; Milek, M.; Khusniyarov, M. M.; Knizia, G.; Werner, H.; Plietker, B. The Electronic Ground State of $\left[\mathrm{Fe}(\mathrm{CO})_{3}(\mathrm{NO})\right]^{-}$: A Spectroscopic and Theoretical Study. Angew. Chem. Int. Ed. 2014, 53, 1790-1794.

6. Weigenda, F.; Ahlrichs, R. Balanced basis sets of split valence, triple zeta valence and quadruple zeta valence quality for $\mathrm{H}$ to Rn: Design and assessment of accuracy. Phys. Chem. Chem. Phys. 2005, 7, 3297-3305.

7. Eichkorn, K.; Weigend, F.; Treutler, O.; Ahlrichs, R. Auxiliary basis sets for main row atoms and transition metals and their use to approximate Coulomb potentials. Theor. Chem. Acc. 1997, 97, 119-124.

8. Zhao, Y.; Truhlar, D. G. Density Functionals with Broad Applicability in Chemistry. Acc. Chem. Res. 2007, 41, 157-167. 
9. Zhao, Y.; Truhlar, D. G. The M06 suite of density functionals for main group thermochemistry, thermochemical kinetics, noncovalent interactions, excited states, and transition elements: two new functionals and systematic testing of four M06-class functionals and 12 other functionals. Theor. Chem. Acc. 2008, 120, 215-241.

10. Schäfer, A.; Huber, C.; Ahlrichs, R. Fully optimized contracted Gaussian basis sets of triple zeta valence quality for atoms Li to Kr. J. Chem. Phys. 1994, 100, 5829-5835.

11. Klein, J. E. M. N.; Miehlich, B.; Holzwarth, M. S.; Bauer, M.; Milek, M.; Khusniyarov, M. M.; Knizia, G.; Werner, H.; Plietker, B. The Electronic Ground State of $[\mathrm{Fe}(\mathrm{CO}) 3(\mathrm{NO})]-$ : A Spectroscopic and Theoretical Study. Angew. Chem. Int. Ed. 2014, 53, 1790-1794.

12. Weigenda, F.; Ahlrichs, R. Balanced basis sets of split valence, triple zeta valence and quadruple zeta valence quality for $\mathrm{H}$ to $\mathrm{Rn}$ : Design and assessment of accuracy. Phys. Chem. Chem. Phys. 2005, 7, 3297-3305.

13. Eichkorn, K.; Weigend, F.; Treutler, O.; Ahlrichs, R. Auxiliary basis sets for main row atoms and transition metals and their use to approximate Coulomb potentials. Theor. Chem. Acc. 1997, 97, 119-124.

14. Marenich, A. V.; Cramer, C. J.; Truhlar, D. G. Universal Solvation Model Based on Solute Electron Density and on a Continuum the Solvent Defined by the Bulk Dielectric Constant and Atomic Surface Tensions. J. Phys. Chem. B 2009, 113, 6378-6396. 